\title{
Geometric Perturbation Theory and Plasma Physics
}

\author{
by \\ Stephen Malvern Omohundro \\ Ph.D. Thesis
}

Accelertor and Fusion Research Division Lawrence Berkeley Laboralory and

University of Californiz Berkelev, California 94720

April 4, 1985

This work was supported by the U.S. Department of Energy under Contract Number DF-AC03-76SF00098.

The United States Department of Energy has the right to usc this thesis for any purpose whatsoever including the right to reproduce all or any part thereof.

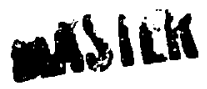


The Goverment reserves for itself and others acting on its behalf a royalty free, nonexclusive, irrevocable, world-wide license for govermental purposes to publish, distribute, translate, cuplicate, exhibj.t, and perform any such data oopyrighted by the contractor.

\section{Geometric Perturbation Theory and Plasma Physics}

Copyright (C)1985

Stephen Malvern Omohundro

\section{DISCLAIMER}

This report was prepared as an accuunt of work sponsoral by an agency of the United Stiates Government. Neither the United States Government nor any ag. ncy thereof, nor any of their employecs, makes any warranty, express or implied, or assumes any legal liability or responsibility for the accuracy, cumpleteness, or usefulness of any information, apparatus, product, or process disclosed, or represents that its use would not infringe privately owned rights. Reference herein to any specific commercial product, process, or service by trade name. tradenlark. manufacturer, or otherwise does not necessarily constitute of imply its endorsement. recommendation, or faroring by the United States Government or any agency thereof. The vieus and opinions of authors expressed herein do nut necessarily slate or renect those of the United States Government or any agency therecf. 
Geometric Perturbation Theory and Piasma Pbyack

Stepher Matrern Omobundro

ABSTRACT

Modem diferential geometrie technirues are used to unify the pbysical asymptotics underlying mechanice, wave theory anj statistical mechanics. The approach gives new insights into the structure of physical : $:-3$ and is suited to the need of modero large-acale computer simulation ald $x$, inbol manipulation sygtems. A coordinate-free formulation of non-gingular perturbation tbeory is given, from which a new Harnitonian perturbation atructure is derived and related to the nuperturbed structure in five different ways. The theory of perturbations in the oresence of symmetry is developed, and the method of averaging is related to reduction by a circle group action. The poeudo-forces and magnetic Poisson bracket terms due to redur. tion are given a ualural asymptotic interpretation. Similar terms due to changing reference $f$ andes are related to the metbod of variation of parameters, which is alon given a Hamiltonian formulation. These methods are ubed to argwer a long at anding question posed by Kraskal about nearly periodic systems The ansa er leads to a new secular perturbation theory tha. contains no ad hos elements. which wa then applied to grromotion. Eikonal wave theory is given a Hamiltonian formulation tha geveratizes Whitham's Lagrangian approach. The evolution of pave action dengity on ray phase space is given a Hamiltomian structure using a Lie-Poisson brarket The relationgbip between dissipative and Hamiltonian gystems is diacussed. A the ory motivated by free electron lasers gives new restrictions on the clange al arpa of projected parallelepipeds under ranunical trangformations A neu IYpe of atrarto: 
Li defined which attracts both forward and backward in rime and is ahown to occur in unfintte-dinuenpional flamiltonian sytems pitb dissipative behavior. The theory of Stnale horyeshoes in apphed to gyronotion in the neighborhood of a cagaetic field reversal and the phenomezon of reinertion in area-preserving barsesboes is introduced. The ceatra' limit theorem is proved by renornalization group techniques. A natural symplectic structure for thermodyamics is ghown to arise asymptotically from the maximum entropy formaliom in the bame way the atructure for clasgical mechasics arises from quantum mechanits vis path integrals. The new etructure for thernodynamics is used to gentralite Maxwell's equal area rule. 


\section{Acknowledgements}

It is a pleasure to here thank those people who made this thesis passible and this period of my life positive, productive, and exrixing.

My mother, Hope G. Wallace, who through ber example bas taught me to be persistent. organized, and to love le rning, and who bas given me ber love and support in every possible way. My father, Stuart $W$. Omobundro, who txught me to pursue philosophical questions and to love oration and vigorous discussion, and who with his wife Astrid Omohundro, bas helped support me financially over the years. My stepfather Dr. Leon Wallace, who by example showed me how to pursue scientific thought and research, and who ! as always given generousl; $t_{1}$ me, both fnancially and emotionally.

To my advisers I owe a great debt of thanks. They have influenced every aspect of this thesis, both in spirit and in content and many of their ideas appear within it.

Allan N. Kaufmen has created a near perfect research environment at the Lawrence Berkeley Laboristory, with just the right mix of individual freedom and group endeavor. He has been an ideal mentor, constantly including me in bis research, beginging with his stimulating year and a balf plasma course, on through the great ferment of ideas he is continually generating ("Let me show $y$ r u this great idea I thought of at 3:00 this morning!") in the development of his unique unified approach to theoretical plasma physics. He has given me immense freedom and bas been extremely supportive of the paths I bave taken, even when they didn' 
scem to be along the main thruat of our group's research (eg. cellular automata knntted dynamical orbits). He has been very innovative in bringing into physics ideas from other disciplines through bis wonderful free-for-all dynamics seminar, in which we tackled head on mome rather abstract mathematics reading, and through his close contart with nathematicians, bridging some traditionally wide gaps. With his dcep insights into physics, his interminable curiosity, and continual probing into the structure of basic theory, Allas has taught ase to be a physicist.

Alan Weinstein, through his course on geometrical mechanics in my first year of graduate school and through his insightful comments and lectured since, has taught me differential geometry and its abolutely central place in physical thinking. Through his example, I have learned that a good picture can be worth a thousand equations and that parsimony and elegance often arise from abatraction. At weekly meetings with his studente, Alan continually makes thoee short insightful remarks that change the whole direction of research. Through his patient explanations and striking insights, Alan has taught me mathematics.

Maureen Saunders for her love and eupport during often stressful times, and for enlivening my life even during her own struggles at medical school.

Peter Blicher, my roommate during all five years of graduate school for buodreds of great discussions about everything (inciuding many of the ideas in this thesis) and for firsthand advice on using TEX for writing a thesis. He and Lynn Hall are responsible for my going out to dinner and seeing roovies many more times than I would have otherwise.

Jerry Marsden for many helpful comments and discussions on the material of 
this thesis, especially for suggesting avcraging as an interesting procedure to look at georetrically, aud for including me in many seminars, gatherings and conferences phich have really shaped my undergtanding of mathematical pbysics His excellent profuse writings and boundless intellectual energy will always serve as at example for me.

Robert Littlejohn, for insightful discussions on many subjects, particularly for his explorations into eikon.I wave theory and for the observation of the many similarities between wave theory and atatistical mechanics. His writings on guiding center theory have served to atimulate me (and many otber people) to think about perturbation theories in new ways.

Richard Montgomery for being a sounding board for virtually everything in this thesis and for his contributions which appear throughout (especially in the sections on peometric perturbation theory and guiding center theory). At our weekly meeting he suggested many useful directions and explained many mysteries to me. Debbie Lewig for her friendship and encouragement, Bruce Boghosian for many discussions and ideas (especially on dissipative Hamiltonian systems, reversibility, infinite dimensional Lie transforms, and relativiatic guiding center theory). Jonathan Yurtele for many discussions and for explaining FEL's to zee. Andy Sessler for sug gesting the questions about the projection of area under Hamiltonian Bow. Pbilippe Similon for many discussions, especially $s:$, ponderomotive stabilization, adiabatic invariants and guiding centers, Ted Courant for discussions of Hamiltoniad tuechanics and guiding ceaters, John David Crawford for many discussions and insights into dyramical systems and plasma physics and for collaborating ou ¡Crawford and 
Omohundro. 1984!. 5teve McDonald for biy insights iato eikonal wave theory, Celso Grebogi for diacussions on dynamical systems and ponderomotive theory, Darryl Holm for bering responsible for a wonderful summer that 1 spent at Los Alamos and for his conutant interest in and encouragement of my work, Doyne Farmer who has given me many insights into life, dynamics and statistics and who has invited me back to Los Alamos for several great stays, Norm Packard ar. 1 Jim Crutchfield for many discussions of dynamical systems theory, Alice Roos for insights into integrability and for sbaring her perspectives on perturbation theory, Bill Baird whose drive for knowiledge and quest for insigbts is truly inspiring, and Yukkei Hui and Ina Chang who are continuing the application of modern mathematical tools to plasma physics.

This thesis was written on the cce machine at Livermore using Donald Knuth's excellent mathematical ty portting language TEX and on an Apple Macintosh computer. These tools have allowed me to proceed much more quickly and with much greater ease than I might have otberwise.

The work reported in this thesis was supported by tbe Office of Basic Energy Scieaces of the U. S. Department of Ecergy under Contract No. DE-AC0376SF00098. This support is due to the far-reaching insight of Oscar Manley who has the vision to support research into basic issues.

The writiog of Vladimir Arnold and, in modera guise, the fundamental ideas of Leonb:.d Euler and Henri Poincaré have constantly served me as inspiration. 


\section{Short Table of Contents}

Chapter 1. Introduction . . . . . . . . . . . . . . . . 1

PART I: MECHANICS . . . . . . . . . . . . . . . . . . T5

Chapter 2. Survey of Geometric Perturbation Theory . . . . . . 78

Chapter 3. Preudo-forces and Reduction . . . . . . . . . . 187

Chapter 4. Bamlltonlan Structures in Perturbation Theory . . 183

Chapter 5. Kruskal'в Theory of Nearly Periodic Systems . . . . 231

Chnpter B. Ponderomotive Force and Gyromotion . . . . . . , 251

PART II: WaVES . . . . . . . . . . . . . . . . . 299

Chapter 7. Asymoptotic Wave Theory . . . . . . . . . . . 300

Chapter 8. A Hamiltonian Appraach to Wave Modulation . . 338

Chepter 9. A Lie-Poisson Bracket for Weve Action Density . . $\mathbf{3 5 6}$

PART III: DISSIPATION AND STATISTICG . . . . . . . . 364

Chapter 10. Imbedailng aad Projection Theorems . . . . . . . 365

Chapter 11. Projected Area and Canonical Transformations . . 378

Chapter 12. Reversibility vg. Irreversibillty . . . . . . . . . 404

Chapter 19. Hamiltonian Disgipation in Inflnite Dimeneions . . \$07

Chapter 14. Reinsertion In Area-Preserving Horseshoes . . . . 424

Chapter 15. Renormalization Group . . . . . . . . . . . . . 439

Chapter 18. Symplectic Thermodyanmica from Maximaum Entropy 458

Chapter 17. Glossary of Terms . . . . . . . . . . . . . . . 528

Chapter 18. Bibliography . . . . . . . . . . . . . . 536 


\section{Table of Contents}

Chapter 1. Introduction

1.1: Summary and Motivation .................... 1

1.2: Philosophical Approach . . . . . . . . . . . . . . . . . . . . 15

1.3: Guide for the Reader . . . . . . . . . . . . . . . . . . . 19

1.4: Intritive Discussion of the Conceptual Framework . . . . . . . . 23

1.4.1: The Analogy Between Entropy and Action . . . . . . . . . . . 25

1.4.2: Adiabatic Invariante and Pseudo-forces . . . . . . . . . 26

1.4.3: Symmetries and Exact Lvariants . . . . . . . . . . . . 29

1.4.4: Thermodynamic Forces . . . . . . . . . . 30

1.4.5: Wave Action . . . . . . . . . . . . . . . . 31

1.4.6: Action, Entropy and Asymptotics . . . . . . . . . . . . 34

1.4.7: Steepest Descents, Stationary Phase, and Averaging . . . . . . . 35

1.4.7.t: Resonance .................... 36

1.4.8: The Key Examples in Mechanics, Waves, and Statistics . . . . . 37

1.4.9: Mechanical Systems . . . . . . . . . . . . . . . . . . . . 38

1.4.10: Mechanical Systems: Separation of Time Scales . . . . . . . . 38

1.4.10.1: Gyromntion and Asymptotics . . . . . . . . . . . . 39

1.4.10.2: Oscillation Centers and Ponderomotive Forces . . . . . . . 41

I 4.10.3: Geometric Perturbation Theory . . . . . . . . . . . . 42

1.4.11: Averaging in Statistical Systems . . . . . . . . . . . . . . . 44

1.4.11.1: Matched Asymptotics . . . . . . . . . . . . . . . 46 
1.4.12: Averaging and Local Fourier Transforms . . . . . . . . . . . 47

1.4.12.1: Scales in Differentiation . . . . . . . . . . . . . 49

1.4.13: Symplectic Asymptotics in Thermodynamics and Mechanjics . . 50

1.5: A Hundred Further Questions, Conjectures, and Suggestions . . . . . 56

PART I: MECHANICS . . . . . . . . . . . . . . . . . . . 75

Chapter 2. Survey of Geometric Perturbation Theory . . . . . . . 76

2.1: Historical Background . . . . . . . . . . . . . . . . . . . . . . 76

2.2: Geometric Perturbation Theory . . . . . . . . . . . . . . . . 81

2.2.1: Manifolds . . . . . . . . . . . . . . . . . . 81

2.2.2: Dynamical Systems . . . . . . . . . . . . . 82

2.2.3: Perturbation Theory . . . . . . . . . . . . . . . 83

2.2.4: First Order Perturbation Equations . . . . . . . . . . . . . . 88

2.2.5: Functions, Covectors, and Cotangent Bundles . . . . . . . . . . 89

2.2.6: Vectors and Tangent Bundles . . . . . . . . . . . . . . . . . 89

2.2.7: The State Space for Firot Order Perturbation Theory . . . . . . 91

2.2.8: Flows and Derivatives . . . . . . . . . . . . . . . . . 92

2.2.9: Dynamics for First Order Perturbation Theory . . . . . . . . . 92

2.3: The Geometry of Jth Order Perturbation Theory . . . . . . . . . 95

2.3.1: The Path Space . . . . . . . . . . . . . . . . 95

2.3.1.1: Spaces of Shorter Paths . . . . . . . . . . . . . . 97

2.3.2: The Space of Germs of Paths . . . . . . . . . . . . . . . . . 98

2.3.3: The Space of Jets of Paths . . . . . . . . . . . . . . . . . 100

2.3.3.1: Coordinates on the Jet Space . . . . . . . . . . . . . 100 
2.3.4: Tangent Vectory to Path Space

2.3.5: Tangent Vectors to the Quotient Spaces ... . . . . . 103

2.3.5.1: Coordinates on the Tangent Bundle to the Jet Space . . 103

2.3.5.2: Coordinate Relation Between Path and Jet Vectors . . . . 104

2.3.6: Dynamics on Path Space . . . . . . . . . . . . . . . 105

2.3.7: Dynamics on Jet Space . . . . . . . . . . . . . . . . 106

2.4: Geometric Bamiltonian Mechanics . . . . . . . . . . . . 108

2.4.1: Poisson Manifolds . . . . . . . . . . . . . . . . 109

2.4.2: Hamiltonians and Hamiltonian Vector Fields . . . . . . 110

2.4.3: Symplectic Manifolds . . . . . . . . . . . . . . . 110

2.4.4: Symplectic Leaves and Bones and Casimir Functions . . . . . 11 .

2.4.5: The Natural Symplectic Structure on Cotengent Spaces . . . . 111

2.5: Hamiltodian Systems with Symmetry . . . . . . . . . . . . . 114

2.5.1: Generalized Noether's Theorem . . . . . . . . . . 115

2.5.2: Circle Actions . . . . . . . . . . . . . . . . 116

2.5.3: Reduction by a Circle Action . . . . . . . . . 11:

2.5.3.1: The Reduced Phase Space . . . . . . . . . . . . . 117

2.5.3.2: The Reduced Hamiltonian . . . . . . . . . . . . . . . 119

2.5.3.3: The Reduced Poiseon Bracket . . . . . . . . . . . . . . 1.9

2.5.3.4: Coordinate Calculation of the Reduced Space . . . . . . . 120

2.छ̀: Example: Centrifugal Force . . . . . . . . . . . . . . . 121

2.6.1: Angular Momentum Generates Rotations . . . . . . . . . . . 121

2.6.2: The Reduced Space and Bracket . . . . . . . . . . . 123 
2.7: Higher Dimensional Synmetries _ . . . . . . . . . $\quad 127$

2.7.1: Hamiltonian Symmetry . . . . . . . . . . . . . 127

2.7.2: The Momentum Map . . . . . . . . . . . 128

2.7.2.1: Lineas and Angular Momentum as Momentum Naps . $\quad 129$

2.7.3: Non-commutativity as the Obstruction to Reduction . . . . . . 129

2.7.4: The Adjoint and Coadjoint Actions . . . . . . . . . . . 130

2.7.4.1: Equivariance of the Momentum Map . . . . . . . . . . 133

2.7.5: Multidimengional Reduction uging a Coadjoint Isotropy Subgroup 134

2.7.6: Multidimensional Reduction using Coad; jint Orbits . . . . . . 135

2.7.7: The Lie-Poikson Bracket and Group Configuration Spaces . . . 135

2.7.8: Euler's Equations for the Free Rigid Body . . . . . . . . . . 137

2.7.9: Euler's Equations for a Perfect Fluid . . . . . . . . . . . . . 139

2.7.10: Gases and Plasmas . . . . . . . . . . . . . . . . . . 141

2.8: Geometric Hamiltonian Perturbation Tbeury . . . . . . . . . . . 145

2.8.1: Linearized Dynamics at a Fixed Point from Jet Bracket . . . . 147

2.8.2: Symmetry and Perturbation Theory . . . . . . . . . . . . . 150

2.9: The Method of Averaging for Hamiltonian Systems . . . . . . . . 152

2.9.1: Appraximate Noether's Theorem . . . . . . . . . . . . . 153

2.9.2: Hamiltonian Averaging as Reduction by a Circle Action . . . . 153

2.9.2.1: Averaging and the Jet Picture . . . . . . . . . . . . 155

2.9.2.2: Extensions from Lrops to Tori and Energy Surfaces . . . . 156

2.9.3: Pseudo-Potentials and Adiabatic Invariants . . . . . . . . . 157 
2 9.3.1: Ping-pong balls and One-dimensional Gases . . . . . . . 159

2.93.2: Oscillatory Stabilization . . . . . . . . . . 160

2.9.3.3: Multiple Space and Time Scales . . . . . . . . . . . . 161

2.10: Exarple: $E \times D$ Drift . . . . . . . . . . 104

Chapter 3. Preudo-forces and Reduction . . . . . . . . . . . . 167

3.1: Pseudo-forces and Reduction . . . . . . . . . . . . . . 167

3.1.1: Fictitious Forces . . . . . . . . . . . . . . . . . . . 168

3.1.2: Rotating Coordinates . . . . . . . . . . . . . 169

3.1.3: Reduction of Sirnple Mechanical Systems . . . . . . . . . . . 171

3.1.4: Circle Actions on Simple Mechanical Systems . . . . . . . . . 173

3.1.5: Fictitious Forces as Reduction . . . . . . . . . . . . . 174

3.1.6: Fictitious Forces with Momentum Shift as Reduction . . . . . 176

3.2: Geometry of the Method of Variation of Parameters . . . . . . . 179

3.2.1: Haniltonian Variation of Parameters . . . . . . . . . . . 181

Cbapter 4. Hamlltonian Structurea in Perturbation Theory . . 183

4.1: Introduction . . . . . . . . . . . . . . . . 183

4.2: First Order Hamiltonian Perturbation Theory . . . . . . . . . 186

4.3: Path and Jet Spaces . . . . . . . . . . . . . . . . . . . 193

4.3.1: Path Space Symplectic Structure and Hamiltonian . . . . . . . 194

4.3.2: The Path Space Dynamics is Hamiltonian . . . . . . . . . . 194

4.4: Coordinate Descr ption of the J-jet Structure . . . . . . . . . . 196

4.4.1: The Jet Hamiltonian . . . . . . . . . . . . . . . . 197

4.4.2: The Jet Poisson Bracket . . . . . . . . . . . . . . . . 197 
4.5: Relation to the Iterated Tangent Bundle

4.5.1: Injectiog Jets iuto the Iterated Tangent Bundle 198

4.5.1.1: Coordinate Description of the Injection 199

4.5.2: Symplectic Structure on the Iterated Tangent Bundle _ . . 200

4.5.2.1: Coordinate Description of Symplactic Structure . . . . . . 200

4.5.3: Pulled Back Symplectic Structure on the Jet Space . . . . . . 201

4.8: Relation to the Path Space Bracket . . . . . . . . . . . . . 202

4.6.1: Weighted Patn Bracket and Bamiltonian . . . . . . . . . . . 202

4.6.2: Jet Bracket Arises from Derivative of Delta Function Weighting . 204

4.6.3: Jet Hamiltonian from Derivative of Delta Function Weighting - . 205

6.7: Jet Space as Derivative . . . . . . . . . . . . . . . . 206

4.7.1: The Sheet Quotient Spaces . . . . . . . . . . . . . . . 206

4.7.2: Sheet Symplectic Structure and Hamiltonian . . . . . . . . . 206

4.7.3: Map Between Sheet Space and Jet Space . . . . . . . . . . . 207

4.7.4: The Pulled Ba-k Sheet Symplectic Structure and Hamilonian ～ 207

4.7.5: Sheet Structures Asymptote to Jet Structures for Small Spacing . 208

4.8: Jets and Symmetry . . . . . . . . . . . . . . . 211

4.8.1: e-dependent Group Actions on $M$. . . . . . . . . . . . . . 211

4.8.1.1: Lift to $G$ Action on the Patb Space . . . . . . . . . . 212

4.8.1.2: Lift to $G$ Action on the Jet Space . . . . . . . . . . . 212

4.8.1.3: Maps from the Lie Algebra to Vector Fields on M. P.M, and J.M 213

4.8.1.4: The Momentum Map . . . . . . . . . . . . . . . . 213

4.8.1.5: Momentun Map on PM . . . . . . . . . . . . . 214 
4.8.1.7: Equjvariance of $J M$ 's Monentum Map 214

4.8.2: The Patb Group: $P G$. . . . . . . . . . . . . . 215

4.8.2.1: The Path Lie Algebra: $P g$. . . . . . . . . . . 216

4.8.2.2: The Dual of the Lin Algebra of the Patb Group . . . . . 216

4.8.2.3: The Action of the Fath Group on the Patb Space . . . . . 216

4.8.3: The jet Group: $J G \ldots \ldots$. . . . . . . . . . . . 217

4.8.3.1: The Lie Algebra of the Jet Group: $J_{g} \ldots . . . . . .218$

4.8.3.2: Homomorphism from $J_{g}$ to Vector Fields on $J M$. . . . 219

4.8.3.3: The Dual of the Jet Lie Algebra: $\mathrm{Jg}_{g^{*}}$. . . . . . . . . . 220

4.8.3.4: The Jet Momentum Mape . . . . . . . . . . . . 220

4.8.4: When $M$ is a Coadjoint Orbit with the KKS Symplectic Structure 221

4.8.4.1: Coadjoint Action of $P G$. . . . . . . . . . . . . . 222

4.8.4.2: The KKS Symplectic Structure on Coadjoint Orbits in $\mathrm{Pg}_{g^{*}}$. 222

4.8.5: Natural Projections and Injections of $G, P G$, and $J G$. . . . . 223

4.8.5.1: Projections and Injections of the Lie Algebras and Duals , . 224

4.8.6: The Lie Poisson Bracket on $g^{\circ}$. . . . . . . . . . . . . . 225

4.8.6.1: The Lie Poisson Bracket on $J g^{*} . . . . . . . . . .225$

4.8.6.2: $j g^{*}$ as $J \cdot$ jets of Paths in $\xi^{*} \ldots . . . . . . . .225$

4.8.6.3: Coadjoint Orbits in $J_{g^{*}}$. . . . . . . . . . . . . . 226

4.8.6.4: $J M$ is a Coadjoint Orbit in $J_{g^{*}}$ as a Manitold . . . . . . 227

4.8.6.5: The KKS Symplectic Structure is the Jet Symplectic Structure 227

4.8.7: $J G$ as a Semi-Direct Product . . . . . . . . . . . . . . . 229 
4.8.8: Jet and Pait ?educed Spacrw are Reduced Jet and Path Space

5.1: In aduction: Kruskal's Approach

5.2: The Neri Approach Expressed in Coordinate:

5.3: The Geometric Version of the Nin Approach

5.4: Extensions and Limitaticns of Kruskal's Theory

5.4.1: Secular Perturbation Theory 243

5.4.1.1: Lie Transforms 245

5.4.1.2: Two-Timing 245

5.4.2: 1/c Time of Validity for Kruskal's Technique 246

5.4.3: Averaging with Mudiple Frequedcies . 248

5.4.4: Averaging Over Ergodic Orbits . . . . . . . . . . . . . . . 249

5.4.5: Non-uniquenesg of Symmetry for Finite Perturbation . . . . . 249

\section{Chnpter 6. Ponderomotive Force and Gyromotion} 251

6.1: Ponderomotive-like Forces . . . . . . . . . . . . . . 251

6.1.1: Intuitive Trea.ment 253

6.1.2: Introducing the Asymptotic Scaling 256

6.1.3: Using the Kruskal-like Perturbation Methor 260

6.1.3.1: The Coordinatea $x, v_{x}, A, \theta$. 260

6.1.3.2: Result of the Method of Averaging 262

6.1.3.3: Calculation of the Roto-rate Vector Field 263

6.1.3.4: The Hamiltonian Structure . . . . . . . . . . . . . . 266

6.1.4: Obtaining the Action to First Order . . . . . . . . . . . 268 
6.2: Some Commeuts on Perturbation Caleulations

6.3: 2D Gyromotion via the Yen Kruskal-like Method . . . . . . . 274

6.3.1: The Four Componeats of $R_{1}$. . . . . . . . . . . . . 278

6 3.2. The Four Components of $R_{2}$. . . . . . . . . . . . 281

6.3.3: Summary of the Calculation . . . . . . . . . . . . 287

6.4: The Hamiltonian Structure of Gyromotion . . . . . . . . . . . 289

6.4.1: The Poison Bracket . . . . . . . . . . . . . . . . 289

6.4.2: The Symplectic Structure . . . . . . . . . . . . . 290

6.4.3: Uniqueness of the Generator for a Vector Field . . . . . . . . 292

6.4.4: Comparison With Robert Littlejohn's Results . . . . . . . . . 293

6.4.5: Prospects for a Fully Hamiltonian Theory . . . . . . . . . . 297

PART П: WAVES . . . . . . . . . . . . . . . . . . . . . . . . 299

Chapter 7. Anymptetic Wave Theory . . . . . . . . . . . . . . 300

7.1: Wave Asy mptotics and Approsimate Symmetry . . . . . . . . 300

7.1.1: Eikonal Waves , . . . . . . . . . . . . . . . . . . 302

7.1.1.1: Sources with Time Scales Generate Eikonal Waves . . . . 305

7.1.1.2: Dispersive Media Create Eikonal Waves . . . . . . . . . 307

7.1.1.3: Whitham's Generalization to Nonlinearity . . . . . . . . 307

7.1.1.4: Sinugoidal Waves . . . . . . . . . . . . . . . . . . . 308

7.1.2: The Local Fouricr Transform . . . . . . . . . . . . . . . . 309

7.1.3: Stati _ary Phase, Laplace's Method, and Steepest Descents . . . 311

7.1.3.1: Heisenberg's Uncertainty Principle . . . . . . . . . . . 314

7.1.3.2: Asymptotic Wares with a Definite $k$ and $y$. . . . . . . . 317 
7.1.4: Eikonal Waves and Lagrangian Submanifolds

7.2: WKB Theory and Asymptotic Equations

7.2.1: The Structure of First Order P.D.E.'s

7.2.2: Hamilton-Jacob: Throry and Symplectic Majifolds

7.2.3: Cotangent Bundles, Contact Spaces, and Jet Spares

7.2.4: The Contact Bundle and the Conormal Bundle .

7.8: Limitations of WKB Theory . . . . . . . . . . . . . . . . . . 335

Chapter 8. A Hamiltonian Approach to Wave Modulation . . , 338

8.1: Introduction . . . . . . . . . . . . . . . . . 338

8.2: Periodic Solutions . . . . . . . . . . . . . . . . . . . . . . 340

8.2.1: The Hamiltonian Regtricted to Periodic Solutions . . . . . . . 340

8.2.2: The Symplectic Structure Restricted to Periodic Solutious . . . 341

8.2.2.1: A Degenerate Poisson Structure on the Prriodic Solutions . 342

8.2.3: The Action of Priodic Orbita . . . . . . . . . . . . 343

8.3: Modulations . . . . . . . . . . . . . . . . . 344

8.3.1: Stationary Prast Integrals . . . . . . . . , 344

8.3.2: The Modulational Poisson Brackets . . . . $\quad 345$

8.3.3: The Moduational Hamiltonian . . . . . . . 346

8.4: Global Symmetry Implies Local Conscriation Law

8.5: The Nonlinear Klein-Gordon Equatiou 350

Chapter 9. A Lie Poisf dn Bracket for Wave Action Denaity 356

9.1: Explicit Calculation of the Ly Poimyon Bracket 350

9.2: The Gcometrical Picture 36 ! 
PART III: DISSIPATION AND STATISTICS

Chapter IU. Imbedding and Projection Theorems 365

10.1: Irnbedding in a Haniltonian System 365

10.2: Projection from a Hamiltonian System 368

10.3: Dangerous Operations with Unbourded Variables 370

10.3.1: Eg : Surreptitiougly Changing Damping to Driving . . . . . 370

10.3.2: Eg.: Pitfalls un the Use of Lie Trangforms . . . . . . . . . 371

10.4: Imbedding in Poisson Systems . . . . . . . . . . 375

Chapter 11. Projected Area and Canonical Trantormaticns . . . 378

11.1: Applizicion to Particle Accelerators . . . . . . . . . . . 379

11.1.1: Courant's Theorem . . . . . . . . . . . . . . 380

11.2: Relation to the Uncertainty Principle . . . . . . . . . . . 382

11.3: Weinstein's Approach . . . . . . . . . . . . 381

11.4: Theorem for Linear Canonical Transformations . . . . . . . 389

11.4.1: The Geounetry of Projected Parillelepipeds . . . . . . 391

11.4.2: The Case of Linear Canonical Transfornations . . . . . . . . 398

Chapter 12. Revergibillty v8. Irreveraibility . . . . . . . . 04

Chapter 13. Hamlltonian Dissipation in Infinite Dimengions . . . 407

19.1: Poincaré Recurrence and Altractors . . . . . . . . . 408

13.I 1. The Lark of Recurrence in Intinite Dimede: thy 409

19.2: Asymptetic ant! Luapunoy St oility . . . . . . . . . 410

13.21 Almnst Attractors . . . . . . . . 410

13.3: Heverwbic Attrartors and Infinite Dinenstodal Hamiltouians . . 411 
13.3.1: Reversible Almost Attractor in the Wave Equation . . . . . 411

13.3.2: The Liouville Equation and Koopmanism . . . . . . . . . 412

13.3.3: Landau Damping . . . . . . . . . . . . . . . . . . 413

1?.3.4: The Boltzmann Equatiou and the BBGKY Hierarchy . . . . . 413

3.3.5: Dissipation from Resonance . . . . . . . . . . . . . . . 414

13.3.6: Resonant Coupling of Eikonal Waves . . . . . . . . . . . 421

13.4: The String with a Spring . . . . . . . . . . . . . . 422

Chapter 14. Relisertion in Area-Preserving Horseshoes . . . . . 424

14.1: The $2 x \bmod 1$ Map . . . . . . . . . . . . . . . . . . . 425

14.2: The Baker's Transformation . . . . . . . . . . . . . 427

14.8: The Borseshoe . . . . . . . . . . . . . . . . . . . . . . . . . . . . 428

14.4: Example of Horseshoer in Gyromotion . . . . . . . . . . 430

14.6: Area Preserving Horseshoes and Reinsertiou . . . . . . . . 435

Chapter 15. Renormalination Group . . . . . . . . . . . . . . 439

16.1: Scaling and Universality . . . . . . . . . . . . 439

15.2: Magnetic Spin Lattices . . . . . . . . . . . . . . . . 442

15.3: The Central Limit Theorem . . . . . . . . . . . . . . 446

15.4: A Poor Man's Feigenbaum Number . . . . . . . . . . . . . 450

Chapier 16. Symplectic Thermodynamice from Maximum Entropy 456

16 1: Previous Approaches to Geometric Thermodynamics . . . . . . 457

8.2: Seven Approaches to the Maximum Ent soy Formalism . . . . . 462

16.2.1: Axiomatic Subjective Approach . . . . . . . . . . . . 463

16.2.2: Counting Sequences of Trials . . . . . . . . . . . 464 
16.2.3: Via Steepest Descents in Two Ways 466

16.2 4: Via Probability in Three Ways. $4: 0$

16.3: The Thermodynamic Limit $\$ 72$

16.3.1. The Density of Stateg . . . . . . . . . . . . 473

16.3.2: Tle Partition Function . . . . . . . . . . . . . . 474

16.4: Maximum Entropy Applied to Statistical Mechanics . . . . . . . 475

16.5: Some Symplectic and Contact Geometry . . . . . . . . . . . . 479

16.5.1: Hypersurfaces Determined by a Function . . . . . . . . . 480

16.5.1.1: The Underlying Manifold $M$. . . . . . . . . . . 482

16.5.1.2: The Graph of a Function . . . . . . . . . . . . . 482

16.5.1.3: The Cotangent Bundle . . . . . . . . . . . . . 482

16.5.1.4: The First Jet Bundle . . . . . . . . . . . . . . 483

16.5.1.5: The Space of Contact Elements . . . . . . . . . . . 483

16.5.2: The Conormal Bundle . . . . . . . . . . . . . . . . 484

16.5.3: The Wavefront Set . . . . . . . . . . . . . . 485

16.5.4: The Space of Tangent Contact Elements . . . . . . . . . . 486

16.5.5: Legendre Transforms and Linear State Spaces . . . . . . . 487

16.5.5.1: The Legendre Map . . . . . . . . . . . . . . . . . . 487

16.5.5.2: The Legendre Transform . . . . . . . . . . . . . . 488

16.5.5.3: The Legendre Transform and a Function's Graph . . . . 489

16.5.5.4: Legendre Transforms and Projective Duality . . . . . . . 490

16.5.5.5: Legendre Transforms and Uncertainty Relations . . . . . 494

16.5.5.6: Legendre Transforms and Jets of Functions , . . . . . . 495 
16.6: The Origin of the Lagrangian Submanifolds in Physics . . . . . . 496

16.6.1: Congtrained Integration and Extremization . . . . . . . . 49j

16.6.2: Paths Constrained on Suriaces . . . . . . . . . . . . . . . . 497

16.6.3: The Wavevector as a Kind of Forec . . . . . . . . . . . . . 498

16.6.4: Distributions Constrained on Subsystems . . . . . . . . 499

16.6.5: Thernodynamic Forces . . . . . . . . . . . . . 500

16.6.6: Lagrange Multipliers and Legendre Maps . . . . . . . . . . 501

16.6.6.1: Constant Force Asymptotic Systems . . . . . . . . . . 502

16.6.7: Lagrangian Submanifolds and Constrained Extremation . . . 503

16.6.7.1: Parametrizing Lagrangian Submanifolds . . . . . . . . . 503

16.6.7.2: Theorem on Pushing Forward Lagrangian Submanifolds . . 504

16.6.7.3: Application of the Theorem to Waves . . . . . . . . . . 505

16.6.7.4: Application of the Theorem to Thermodynamics . . . . 505

16.7: Theorem on the Pushforward of Legendre Subnanifolds . . . . . 507

16.7.1: The Contact Structure for Thermodynamics . . . . . . . . . 508

16.7.1.1: The Contact Form for Jets of Entropy . . . . . . . . . 509

16.7.1.2: The $U, V,(1 / T),(p / T)$ Symplectic Manifold . . . . . . . 509

16.7.1.3: The $(S, V, p, T)$ Symplectic Manifold _. . . . . . . . . 510

16.7.2: Legendre Transforms and Thermodynamic Potentials . . . . . 510

16.8: Phase Transitions and the Geometry of the Equation of State . . 512

16.8.1: Caustics and Phase Transitions . . . . . . . . . . . . 513

16.8.2: Convexity and First Order Phase Transitions . . . . . . 513

16.8.3: A Generalization of Maxwell's Equal Area Rule . . . . . . 517 
16.9: Rolations Between Symplectic Thermodynamics and Mechanics . . 521 16.9.1: A) Eikonal Waves and Stationary Phase . . . . . . . . . . . 521 16.9.1: B) Thermodynamic Limit and Steepest Descents . . . . . . 521 16.9.2: A) Waves and the Feynman Path Integral . . . . . . . . . 522 16.9.2: B) Probability and the Maximum Entropy Formalism . . . . . $\mathbf{5 2 2}$ 16.9.3: A) Wave Path Integrals over a Subspace . . . . . . . . . . . 523

16.9.3: B) Probability Distribution Averages over a Subspace . . . . . 523 16.9.4: A) Lagrange Multipliers and Canonical Conjugacy . . . . . . 524

16.9.4: B) Lagrange Multiplicrs and Thermodynamic Conjugacy . . 525 16.9.5: A) Fourier Transforms and Legendre Transforme . . . . . . . 525 16.9.5: B) Laplace Trangforms and Legendre Transforms . . . . . . . 526 Chapter 17. Gloesary of Terms . . . . . . . . . . . . . . . . . . 528 Chapter 18. Bibllograplyy . . . . . . . . . . . . . . . 536 
This report was done with support from the Department of Energy. Any conclusions or opinions expressed in this report represent solely those of the author(s) and not necessarily those of The Regents of the University of Catifornia, the Lawrence Berkeley Laboratory or the Deparment of Energy.

Reference to a company or product name does not imply eppreval or recommendation of the produce by the University of Califomia or the U.S. Department of Energy to the exclusion of others that may be suitable. 


\section{Chapter 1: \\ Introduction}

"One of the principal objects of theoretic al research in any department of know/edge is to fnd the point of view from which the subject appears in its greatest simplicity "-J.W. Gibbs

\subsection{Summary and Motivation}

This thesis presents the underlying theoretical basis for an ambitious program to develop a unified, coordinate-fren theory of asymptotic perturbation methods in the three major areas of physies: mechan al systems, wave systems. and statistical systems. This program has far-reaching consequences, both practical and theoretical, which we will outline here. It is quite clear that we are entering a new cra in physics and engincering in which powerful computers will play a major role. The tho major applications of the computer will be simulating physical systems and performing symbolic computations. Botb of these areas are fundameutally impacted by out rescarch.

It bas long been known that many of the most important problem: in physe:and enginecring design are analytically intractable lierent theoretical developmenthave shown that this intractability can be inherent its the problem. and not due simply to insufficient mathematical technique. Rigorous remult- from dyanal al

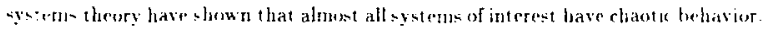


which aleolutely precludes the development of analutic solution [eer for example \{rickeuteimer and Holmes. 1983j). Recent work in cellular autorlata theory has Glown that there are problems whose behavior rannot be predicted by any algnithm which is shorter than direct simulation Wolfram, 1984:.

Computer simulation is therefore destined to play a fundamental role in the study of physical problems. Uufortunately, the very same exponential divergences of neigbboring orbits which cause difficulties in analy tic treatments also plasue computer simulations. For a given accuracy of prediction, the computing power required typically grows exponentially with the tire-scale to be studied measured in units of the smallest important physical time-scale. Advances in parallel computation will increase the power of computers by factors of about a million in the next twenty yaars. While large, this number pajes against the spectre of needed exponential growth and the new scales posed by fully three-dimensional simulations. It is therefore nec isary to develop reliable analytic theorjes for preconditioning problems prior to simulation. An important exaraple of this concept is utilized in studying the gyromotion of a charged particle in slowly-trying magnetic and electric fields. The particle motion consists of fast gyrations neas the gyrofrequency, on top of alow drifts of the center of gyration. If one simulates the particle motion directly, the simulatiun crrors acrumulate on the time-scale of the gyroperiod quickly leading to incurret results on the drift time-scale. By first introducing anymptotics and analytically removing the fast gyration, we obtain the so-called guiding center deacription. Tlee equation bave significant evolution on only the drift time-scal:. and the swoulation retain validity for mush longer times with the same romputing 
resources. A similar motivation is behind the description of a partule til a waw using ovrillation centers and the description of the evoluturn of ats enkoud wave un ternis of modulational equations.

The need for accurate ass mptotics performed to very high order will rventually be met by using symbolic manipulatioi programs such as MACSYMA and S:IP. For this to be possible, it is absolutely essential that we bave methods that are precisely defued with a precisely defined domain of applicability, are systematic. and require no ad hoc choices in their implementation. It is also very important to design simulations to take advantage of any precise knowledge of the dynanics. such as energy or momentum conservation. Insight into the theorctical structure of a physical theory leads to much more efficient and testable computer code and may mean the difference between a successful theory and a useless one.

For these practical rearons, as well as for fundameatal theoretical ones, we have taken a new approach to physical asymptotics. The approach we take is based on some revolutionary new ideas that are changing the underlying mathematical structure of physics. The introduction of the vector calculus by Heaviside in 1882 led not only to the streamlining and simplification of calculaus.'s. but to new conceptual insights into the structure of physical law. The application of coordinate-free differential geometry, developed primarily by Elie Cartan in the $1930^{\circ} \mathrm{s}$, is having an even more profound effect on both the expression and the cofceptual st ructure of pbysics. Coordinates are not physical, and the new formulations encourage the use of only physically relevant concepts, never ad hoc artifacts of sone courdinate description. Haniltonian mechanics has been particularly revolutuntiaed by thi- 


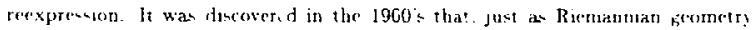
demeribem the structure of spice-time. symplecte geometry describes the st ructure of Hambonian phase space An introduction to the theory in the context of our Hork is presented in chapter 2. The full impact of the reformulation of Hamiler nian mechanics has been folt muly recently. In the past fow years virtually every area of physics has been Hamiltonianized. The new perspective has shed light on the underiying symmeiry structure of these theories (including the elucidation of automatically conserved quantitics, called Casinir functions), has yielded improved novlinear stability results hased on Aroold's stability method, and has given insight into the reasone for the integrability of certain systems.

Hamiltonian structures were originally irt roduced by Lagrange to simplis; and to check perturbation calculations. They will play a similar role in modern computer simulations. Most of the recent developments in Hamiltonian mechanics have dealt. with fundamental models of physical systems and have not studied the structure of perturbation equations. We have initiated a study of such equations and discovered several important result:.

Our first goal was to study ordinary non-singular perturbation theory. Chapter 2 gives a coordinate-free description of this technique in terms of the mathematical theory of jets. This is important for systematizing perturbatio. methods and bringing them under the purview of moders mathematical methods. Many physical systems have state spaces that are naturally manifolds, and many are naturally infinite-dimensionsl. Eefore our work, it was not clear that one obtained the same perturbation expressions when ene worked in different coordinate systemis and there 


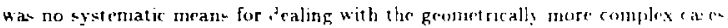

Cbapter 4 studies this perturbation theory an a Hamilonian context. Agan w* find that the theory is expressible in coordinate-free language He have discovered that a remarkable and irmportant Hamiltonian structure goverus the perturbation equations themselves. The fact that the perturbation equations are Hamiltonian means that. for the first time, all of the powerful techniques of Hamiltonian mechanics (including Noether's theorem, energy conservation. Liouville's theorem, and stability techniques) may be applied directly to perturbed systems. We have discovered that the new perturhation structure is related to the key elements of modern Hamiltonian mechanics in five different ways. (We have worked out the direct coordinate description, the relationship with natural structures on the so-called iterated tangent bundle, the relationship with a natural structure on a path space, a new sense in which the perturbation state space can be vieved as a "derivative" which unibes our method witb previous work, and finally an extensive theory of perturbations in the presence of symmetry.) The theory describing the relationstip of perturbations and symmetries extends to perturbation problems what is perflapthe nost important idea of modern Hamiltonian mechanics: reduction Tha is a procedure. formulated by Marsden and Keinstein and discussed a chaperer 2. which is a far-reaching generalizatms of hocther's tbeory of sumplificatust in the" prom we of symetry. Every mode ru Hamiltonian structure. inchuding thone ariing in plasna physics, magneto-bydrodynamics. Huid dynamies getseral relativit.

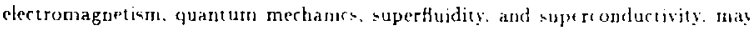

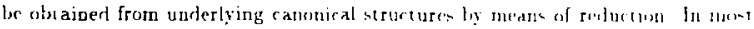




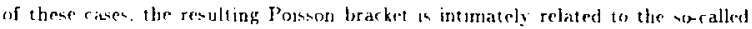

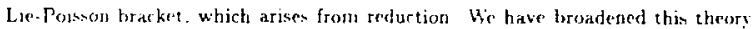
to enconfiase perturbed systenus and nave shewe that the perturbation structures are int imately related to rertain "jet-group" syumeiries. Our Haniltonian perturbation structure arises from the Lie.Poismon bracket of this larger group.

Perhape the most important application of non-singular perturbation theory is as a componert of a singular or secular perturbation calculation. Using geometric methods. we have been able to make fundamental advarces in secular perturbation theory as well. We first demonstrate in section 2.9 that the method of averaging can be viewed as an application of reduction by an approximate circle action. This allows the method of averaging to be incorporated into any situation amenable to reduction. We explicitiy calculate the Poissot structure for $E \times B$ drift using thesz new rnethods in section 2,10 . Finbere in the calculation does or need to introduce the unphysical or ad boc elements usually required. The resultant bracket is derived wich much less calculation than by any previous method.

To rearh higher order than the method of averaging. we reexamine the seminal theory of Kruskal in the light of the acw methods in chapter 5 . He introduced the first systematic, order by order calculaiton of adiabatic invariants to all orders. His technique requires one to make changes of coordinates order by order. Each change, howev $\therefore$ recuuires one to make ceriaiu artitrary choices leading to an ad hoc clement in the perturbatinn algorithm Kruskal was able to show that nonetheless the vector field geuerated by the adiabatir invariant (which we would now call an approximate circle symmetry) wat ungudy defined to all orders Thus vector ficld 
is the real goal of the perturbation icchngue and Kruskal wan mrsod to commert in his paper: "It does not appear obvews whet ber an explicit recursion formula to" determine $R$ the symmetry vectur field in terms of $f$; the dynamical vector field can be found. If so, the whole theory of this paper might be simplitied and rendered less deep." Indeed, our approacb explicitly gives just such a formula ind relates it to the developments in Haraltonian mechanics listed above. Our algorithm is completely weil defined with no id boc elements and so is ideally suited in symbolic implementation on a computer. As an example, we compute the symmetry vector field for two dimensional gyromotion to second order in chapter 6 .

We bave found that Kruskal's results (and others of the same type) are often misunderstood and misused. While Kruskal showed that the accuracy of the adiabatic invariant conservation may be made exponentially small in the small parameter, the time-scaje over which this is valid is only the reciprocal of the small parameter. Kruskal's result is often quoted as: "adiabatic invariants o- preserved to all orders in the small parameter" and wroggly interpreted to mean exponentially small error for exponentially long times. This misinterpretation can be very dangerous in the situations where the theorem is applied. and so we have constructed a number of counterexamples to illustrate the limits of the theory in section 5.4 .

We have also successfully considered the method of variation of parameters in this light and have cxplicitly demonstrated the condection with the notions of "psfudoforces" and "magnetic curvature terms" in the Poisson brackets of reduced systems in reapter 3 . We have stown a new way of interpreting the Coriolik furce (an a term in the Paisson brarket due to reduction) which is exicndible tu any of her 


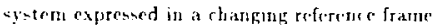

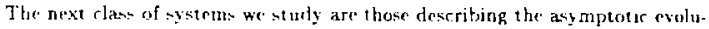
tion of $e^{\prime}$. al waves. We bave dewloped a number of new theoretical constructs ift thi- fielu including a precise astaptotic definitiot of loral Fourier transform. These ideas are presented along with a summary of the geometric approach to eikond wave theory in chapter 7 . We have also succerted in our main goal, which was to develop a systematic method for finding a Hamiltonian description of modulational equations given the underlying Hamiltonian wave system. Our technique is algorithmic and does not depend on linearity, nor on any other sfecial features of the system. We present the theory for the Klein-Gordon equation in detail in chapter 8 . Our approach encompasses the syctems amenable to Whitham's averaged Lagrangian technique. but applies in addition to Hamiltonian systems that do not arise from a Lagrangian (for exanple, aly of the s: stems with Casimirs or any system based on a Lie-Poisson bracket). It also unifies the study of eikonal waves with the other Hamiltonian systems we have discussed.

We also study the common case of linear waves described by a canonical Hamiltonian structure with wave amplitude and phase as conjugate variables. We have shown that these systems are naturally described by a wave action density on the entire $x, k$ phase space. The evolution of this action deasity is itself Hamiltonian, but with respect to a natural Lie-Poisson bracket, as is demonstrated in chapter 9. The symmetry grnup underlyiug this theory is the same as that for the Vlasov equation.

l'sing new mathenatica! techniques, we bave dikcovised streral novel results 


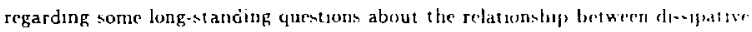

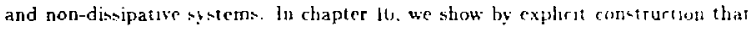
any dynamical system (with any amour: of dissipation) can be imbedded in a Hamilconian system of twice the dimension ar a Poisson system of only one higher dimension. We also show by explicit construetion that there are a Hamiltonian system and a Poisson system of only one dimension greater that project (by ignoring the value of a coordinate) to become any arbitrary dynanical system. These results show at once that Hamiltonian systems are very general and that it can be very dangerous to indiscriminately add new rariables to a system. In particular, we show that by introducing time-dependent changes of coordinates, one can make any system look integrable, or coerce Liapunov axponents tu take any desired values. We give several explicit examples of seemingly harmless yet truly dangerous operations. We show in an explicit example in olving a resonance that, by indiscriminate use of the metbod of Lie transforms, one can inflict mortal injury to the underlying physics.

In chapter 11 we use modern sympleatic geometry to study some questions that arise in the design of free electron lasers and other accrierators. One often wants to force the particles in a beam into a more confined region in phase space. Most devices act on the particles in a (ume-depeudert) Hamiltonan way and so we consider the effect of canoniral transfurmatior on regions of phase space. Liouville: theorem says that it is impossible to chaoge the wolume of the region in phane space Often, though. He are interested in the prosertion of our region nilto sume

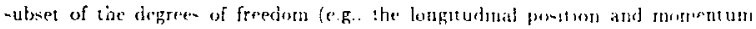




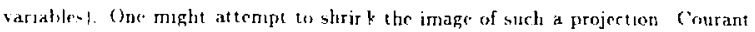
adrienerg thit question for linedr systems. but only under ver: special conditious particle.. in cllipsoirs or parallelepipeds with axcs aligned with the coordinatr axes both before and aftrr the transformation. Weinstein has given a general theorem that afsplu" only for sufficiently small transformations. We generalize Courant s theriy in a fundamental way and obtain results for arbitrary linear casonical trans. formations. This is accomplished by positing and proving a new theorem about the structure of parallelepipeds in high dimensional spaces. We give several examples and counterexamples and discuss the pussibility of a geveralization to statements about projected measures as opposed to voiumes.

In chapter 12 we introduce a new class of attractors into dyamical systems theory that sheds light on classical irreversibility paradoxes. By construction, we exhibit systems possessing a fixed point which is attracting both forward and backward in time. In chapter 13 we show that exactly this behavior occurs in many intinite dimensional $\mathrm{H}$ :miltonian systems and is responsible for the appearance of dissipation in many cases. Examples include a variety of wave systems. Landau damping, resonant coupling. and the decay of correlations in chaotic maps.

The fundamental mathematical structure behind ehaos in dynamical systems is kinow as Smale's horseshoe. This is a very commonly found piece of nonlinear mappings which guarantees the existence of orbits that hos between two regions acrordiug to any sequence of random 1 's and 0 s. Any map with a borseshoe has dyrames whici is as unpredictable as a sequence of coin tosses. In chapter 14 we thes that periodic ally perturbed gy romotion in the neighbortiood of a magnetic 


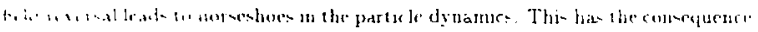

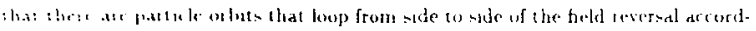

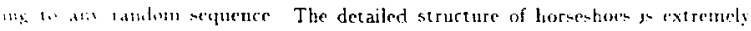

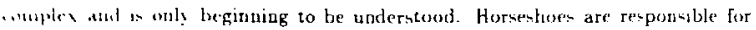

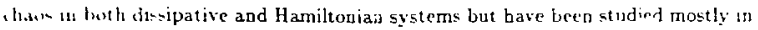
the dissipative case. We baze discovered a fascinating apparent paradox regarding Hamilonian horseshoes whose resolution indicates a much more intricate structure for the Hamiltonian than for the dissipative case. We call the new phenomenon "reinsertion" because it forces the tongues of the unstable manifold to reinsert into other tongues an infoite number of times. It is possible that this new structure is responsible for some extremely complex phenomena that have been recently observed by Holmes and Whitley in the trausition from dissipative to Hamiltonian horseshoes.

In chapter 15 we give a dyamical systems description of the idea of renormaliation and prove the central limit theorem using renormalization group techniques. Using renormalization group methods, Feigenbaum discovered a universal scaling constant for period-doubling cascades. He used a Cray supercomputer to calculate this constant to high accuracy. In section 15.4 we present a poor man's approach that gives the constant to within 25 percent on the back of an cnvelope.

The last type of physical asymptotics that we have incorporated is.to our theoretical structure is the transition from statistical mecbauics to themodynamic. Thi is perhaps the most profound abpect of our work and introduces siructures that are of fundamental physical significance. At discused in chapter 16. we have 


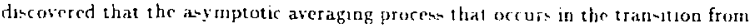
Gtate-thal nechance to thermodynames is exactly analogous to the aurmptote ascraging procese that takes us from pitonal wave theory to tays (e.g., from guantum mechanu to classical mechanics). We show that a thatural symplectic structure For thermudyamics arises from this asymptotics in exactly the same way that the symplertic structure of Hamiltonian mechanics (that bas beet so fruitful in recent applications) arises from wave asymptotics. Our theory now allows the same powerful results which have caused Hamiltonian dynamics to flourish in recent years to be applied to statistical systems.

The underlying statistical foundation for the new theory is the principle of maximum eatropy. We develop several new interpretations for this principle in section 16.2, in which it plays exactly the same role as the principle of least action plays in mechanics. Just as the principle of least action arises asymptotically from the method of stationary phase applied to the Feynman integral over all paths, tne principle of maximum entropy arises from the method of stcepest desents applied to an iniagral over all probability distriuutions. This formulation is new and is expected to lead to the sane benefits in statistical mechanics that are derived from the path integral formulation in quanturn mechanics.

The integral over paths can be done by integrating over paths with a given constraint and then integrating over the constrai'st. This leads to the action de fired on phase space and the description of dynamics in terms of it. The integral over distributions may be done by integrating over distributions obeying a given ronstraint and then integrating over the constraint. This leads to the entropy de- 


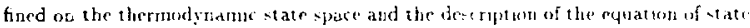

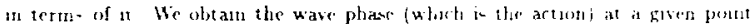
In introducug Lagrange multipliers that are caronically conjugate to the gjettal variable and choosing then so that the extemal action urcurs at the froint of in terest. The value of the multipliers is the wave-vector or monentum conjugate th the position and has a value equal to the derivative of the action. This is the origu of the symplectic structure of mechanics. We obtain the entropy for given valul's of the mechanical quantities by introducing Lagrange multipliess that aro thernodynamically conjugate to the mechanical variables and choosing them to make the maximum entropy occur at the state of in:erest. The value of the multipliers is the derivative of the entropy with respect to the mechanical variables. This is the origin of the smplectic structure of thermodynamics. The Lagrange multipliers cause our path integral to be a Fourier transform and our probability integral to be a Laplace transform. The asymptotic evaluation of these transforms by stationary phase and steepest descents. respectively. leads to the Legendre tranforms that are so central in mecbanics and thermodyamics.

A. a first application of the neu structure of thermodynamich we tecxamme the classical Gibbsian theory of phase transitione in section 10.12. We endous the Maxwell equal area rule with a natural geometric inompretatum withu the ura theory. It is then generalized to describe the phase transuint =urface in all arbitrary direction insicad of only along the jeothermal surfares considered by MiLxwell If the Hood of nou prartical results that arone from the symplectu ituterpretasion of

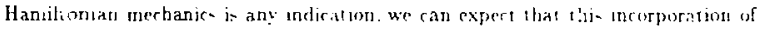




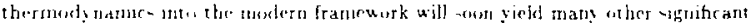
Tr-1: I = 


\subsection{Pbilosophical Approach}

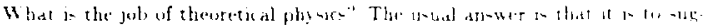

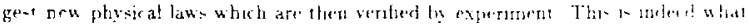
w cured in the great revolutions of Vewtoman mechange. -grerial ard geveral ri: ativity and quantum mechanics. This is not what mont the retical plyyeri-tade however. They start with the fundamental laws (be they quantum electrodyramic or some other modelj and determinc what behaviors these laws imply in special situations. Ideally, this enterprise is one of mattematical deduction. but practically oue must often make intuitive leaps eitber because current mathematical techniques aren't powerful enough or simply because the underlying mathematical model of the pbysical situation is inadequate. The ultimate goals of such studies are the predic. tion and understapding of physical bebaviors. Sometines these rome in the form of numbers to compire - ich experinent, but more oftel one is interested in qualitative features like the stahusty of an equilibrium state or the type of evolution expected of a given system. At the highest level, one bnd gen ral principle which apply to many situations and give reasons for the qualitature hehavion observed.

From this viexpoint the enterprise of theoretical physics may be thought of at the creation of a surcession of models. The highest modeds are extremely general in their dornains of application but are very intractable and because of their gr nerality. give little insight into actual behavior. The lower models spe-ialize the gencral ones to smaller classi of situations and make more and more precise predictions until finally the lowest models descrobe a single cxperinent al motup aud predis t the numerical valuex of individual meavurements. 


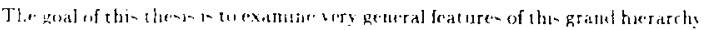

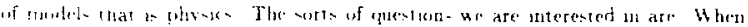

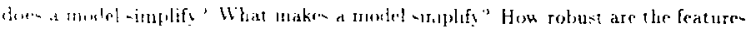

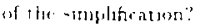

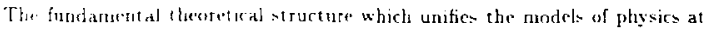
Il levels is the Hamiltouian structure, as may be secn in the beautiful compendium ¿L andin and Lifitulz. 1960 1981'. The fuddamental equations of physics all appear to be Hamitonian and many of the simplifiration procedures respect this Hamiltonian structure. The most basic circumstance whicb allows simplincation is the prewence of symmetry. Emmy Noether diccovered that for systems with a Hamiltonian structure. the preseace of a fimession of symmetry allows one to eliminate two dimensions of state space from cov ideration. Fecently, the reduction of fundamental models to more specibic ones bas been accomplished within a Hamiltonian framework for many exanples using the symmetries preseut in the underlying situation

Basy times, however, we are not exacty in the symmetric situation, but we are close to it in some sense. The physics is described asymptotically using the diverse method of perturbation theory which bave come to be the mainstay of much of themetical physies. One can make a case that all of the actual calculations carried out in quant um electrodyamies. plasma physics. solid state physics and many other held - are expressions of divers perturbation approaches to the underlying equatiors.

The other great simplifying tool is statistics. As for perturbation theory, the fundamertal theoredical justification for using stat istical approximation - i- almos 


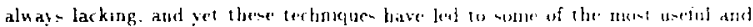
acrutate theorice in any domain of 4 tud.

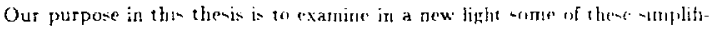
cation procedures at the heart of phyice. Traditionaly, physical a alculatwo hane been carried out in speeial arbitrary coordinate sbotems which may smplofy the calculations but obscure the distinction betueen what is intrinsic to the physical situation and what is arbitrary. The mathematical physics community has recontly been moving to reexpress the fundamental ideas of physics in the coordinate free language of differential gcometry. developed by Elie Cartan. This bas led to some resounding successes and has identitied many new structures that bave direct relevance to physics. Much of the buge body of traditional work in applied mathematics has not yet benefitted from this new viewpoint, however. Books and journal articles on perturbation metbods typically describe these mechods in terms of a specifir example and little or no attmpt is made to delineate what physi al features of the model bave made the metbod worl. The result is a morats of disconnected spectat cases. Horkers in the field bave develope " ntuituons at w what will nork where. but this has not been codified into a theory.

The underlying philosophy here is that there is ne magic If a stubtest -1tn-

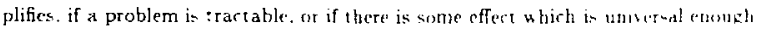
to be given a name, then there nust be a definite physal reatom for at Wa mah to understand these reasons. This thest of couref. to onls a heguring in the

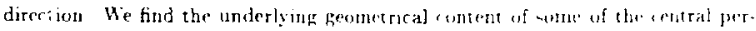

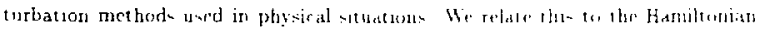




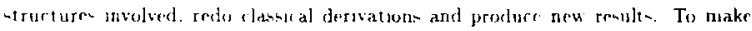

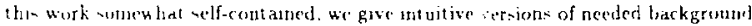

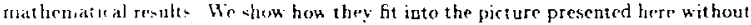
t.we mus he duplieation of matron' that can be found in standard reference works. 


\subsection{Guide for the Reader}

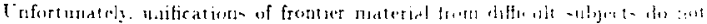

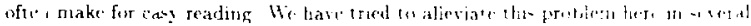

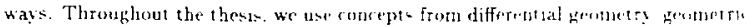

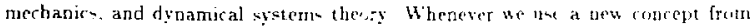
these fields, we give an intuitive discussion of the basic ideas itswolved and retere an to more detailed discussions. Fortunately, this background materal is becorning uidely 'now and used in the physics community, primarly because of the eximence of several excellent texts.

Geometric mechanics is beautifully presented in abraham and Marsen. 1978. iArmold. 1978', and Thirring. 1978'. 'Arnold, 1978' gives the most intuitive dicussions, I-Abraham and Marsden, 1978 is the most complete and martematically. precise, and Thirring, 1978 envers several additional topirs like the kit? theuretn

Both Abrahan and Marsden. 1978, and Thirring, $1978^{\circ}$ begin with interductions to differential gesmetry. An expanded vernion of this intenduction and infinite-dimensional versions of the idea are fiven in Abrabam. Marden. ind Ratiu, 1983'. We give sperific citation to this reference is the fundamental geor metric concepts appear. An intuitively appealing tratmant of differential geometry mar also be found un 'Spivak. 1979'.

The wlede of dypanical systems theory are diccumed in Abrahan and Marden. 1978;, Arnold. 1983', and [Guckenheimer and Holnes, 1953. 'Gukembenurs and Holnes. 1983, gives a very uice treatment of many examules in addition to prementing the pure theory. 


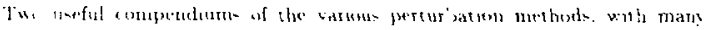

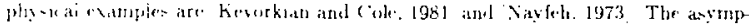

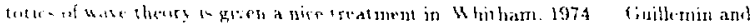

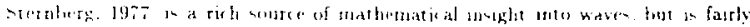

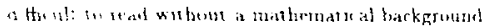

A unified treatment of the classical statistical physics wo ued is presented in

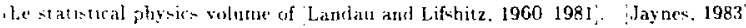
presente the rackomuni entrows viespont that is contral to our approach.

Becauce thre is no iudex, we have made the table of contents rery detailed. We have also providec an alphabetical list of key conrepts and the sections in which they appear in chapter 17.

The remander of this introduction is intended tu be reiblable without extensive mathematica' backgrount. It present" the hasic coureptual structure of the thesis and motiates nome of the mathematical concepts.

The body of the thesis is broker up into three parts: I) mechanics. II) waves. and IIli tissipation dud statistics. While one of our goals is to conneet these disciplines. for the nust part the three parts may be rcad independently of one another.

Chapter 2 is intended to introduce the geometric approach to mechanies while introducing our appruach to non-singular perturbation theory. It gives intuitive dereriptir at of the basic concepts of diferential geometry with pointers to more detailed reference works. We have tried to point out the key ideas of the gecmetric dpproach ind to make our disce sions casier to follow at a heurstic level than the reference work 


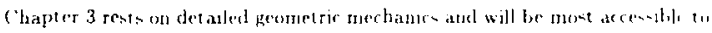
reader with this backgronted So othe'r sections depend on the mater al

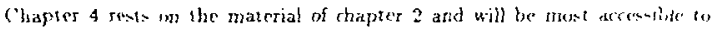
reader: with some mathematical barkground The philusophy and h.oic result: were presented in chapter 2 and chapter 4 may be viewed as a reference for the details and methrids of the approach. The renults are uned in sections 5.3 and 9.3

Chapter 5 excends some standard secular perturbation methods he give an introduction and critique of this theory lut the reader may wish to look at standard references and the paper [Kruskal. 1962] while reading thi: -hapter. Section 5.3 rests on geometric notions from chapter 4 and may be omitted by the uninterested.

Chapter 6 is an application of the methods of etapter 5 to gyromurion. We Lave given fairly complete detail of our calculations so that they may be used on other problems. Later chapters do not depend on this one.

Chapter 7 is an int roduction to ihe geometry of eikotal wave theory. Extensive usc of the symplectic geometry intrefuced in chapter 2 is made in this chapter.

Chapter 8 develops a Hamiftonian perturhaton technique for cikonal wave which is founded in ele geometric ideas of chaper $i$ but takes the iorm of an explicit algorithmic calculation. The example he present mas serve as a model for similar problens.

Chapter 9 agan prenents an explicit calculation wheme theory reat on the

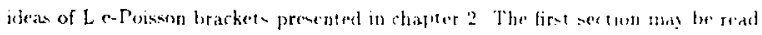

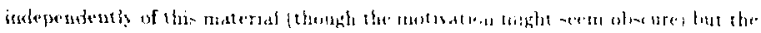
secomi erthon rest: berivily on 1 


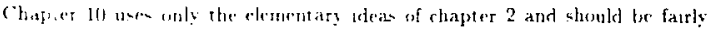
nam 1 ind Int

Chaper 11 sests on some symplectic grometry. but the results are easily underunod and may be userul in general stuations. The proof of the main result uses nnl? 'uncar algelbra and indurtion

"hrupler 12 may be read on its own and serves as the background for chapter 13.

Chepter 14 introduces the dynamical systems concepts necessary aud may be read independertly of the rest of the thesis. It may be uscful for the reader unfamiliar with the ideas of chantic dyamics to consult some of the more detailed work:

Chapter 15 rests os some ideas of probability theory. dy namical systems theory. and renismalization group theory. It may be read independently of the rest of the thes is but consultation of the reference works listed in that section may provide useful background material for the reader.

Shapter 16 makes many references to chapter 7. It also rests on the ideas of imbimum entropy which are quickly sketched here but may be studied in detail from Jayne 1983. It is also necessary to introduce many ideas from contact geometry. whet charify many asperts of thermodynamics. It might be useful to look at the Inote derabled refereaces on this material. 


\subsection{Intuitive Diacuarion of the Conceptual Framework}

Plasma physics is a fascinating discipline it part hec ause it is at the eroweroad. of what $I$ consider to be the three fundamental types of models in physics: mechanics. wave theory, and statistical mechanics. Each of these areas gets its ric leet and most powerful models and clementary concepts from asymptotic approximal insu to the real physical system. It is this asymptotics which allows us to introduce the concepts central to simplified descriptions of physical phenomena. This thesis explores some amazing relatious among these three secmingly disparate disciplines and develops a unified way of understanding the structures that make them work.

We are trying to understand bow systems simplify. The key feature of such systems is that they project onto a sub-piece which moves according to its own dynamics which is approximately independent of the exact state of the forgotten pieces. The interesting physics arises from the fact that the two halves are in no sense decoupled, and the effect of the forgotten piece is felt in the kept piece as a new physical effect. Each of our three main types of system can undergo such a simptification, and together these systems span the breadth of physics. There are simple dynamical systems with fast oscillatory degrees of frecdom. wave systems in the eikonal limit of short wavelength, and chaotic systems of a large number of degrees of freedor in the limit in which statistics is valid. In each, we make a separation of scales by first introducing a formal paraneter (often only itmplicitly) which increases the separation as it vanis'ses, and then by doing asymptutice in that varameire. 


\section{CLASSICAL}

DYNAMICS

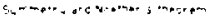

andetion

Acilion son pains

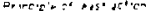

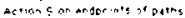

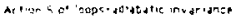

Latarange mulepiers

ihe menod of averaging

cruswals mpthad

Aponaximale symamelry

psoude+fores

Caronically zorivode

prosenspere

\section{$\&$}

THERMO-

DYNAMICS

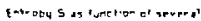
ther modyname quanciles

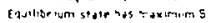

Eneropy sas funeliom of equilibrem

Emlrobu as adabate invariart

bafrege multipliers

rutos s ineory

Thermadynamic forces

inderodyndmically conguadte

age ansuan Subroun ifoles

:eqendre Submaniolds

Tharmadynamic 5!ate Space

5ymolectic monifolds

Contac: Manstolds

Leqendrt irarsiorm

Feuhman Path formulism

surn aver oultes

5idionacy Phase

Four ine Transform

relating wave as for of a dnath

Sotidicathos

Frases otatwe

mareverer is ds:a,

cigr. anp

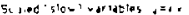

Chand wat

bag angian and tequere marirs's:

\section{wave Action}

roitham s Arertokn

C.ustics
Maximum Eafropy formalism

sum Jue? alste burnons

strepest insepis

L tphar i insform

relating ar uablity as for of and $x$

Colengive atriates $x$

nformation entredy $5: 4$ alstebsetan

rtensive th ters $x=d 5$. .

Thermodynamic , irme

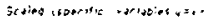

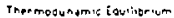

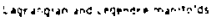

Entropy of a distributionge,

calropi as o tuhe lot. . "

Phase Irans+bions

ST AT ISTICAL

MECHANICS

Figure 1.1: Some links between the three disciplines of mechanics, waves, and statistical mechanics. 


\subsubsection{The Analogy Between Entropy aud Action}

We will forge links between these three disciplines. Let us first st ate the variats relatiousbips and then give int uitive examples of them. Oddly enough, in each casc the classical notation associates the letcer $\$ S^{r}$ with the fundamental unifying quantity. The relation between quantum and classical mechanics is based on the classical action $S$ along a patb is phase space, whicb also represents the quantum phase corresponding to that path. The information entropy $S$ of a probability distribution similarly gives the connection between statistical mechanics aud thermodynamics in the maximum entropy approach. In the asymptotic cikonal limit, we may define the action $S$ as a function on the space of only real dynamiral patbs. In the case of a system all of whose orbits are periodic, we may define the action $S$ as a function on phase space. This function is an adiabatic invariant for slow variations of paramcters, and this constancy forces the exchange of energs between the system moving the parameters and the fast periodic oscillations. This excbange causes the slow syıtem to bebave as if it had new pseudofurces arting on it. which are expressed in terms of $S$ and are Hamiltonian. When we think of wave systems as themselves lueing dynamical systems, we can define a local wave action density. Again. thit ts adiabatically a locally conserved quantity. which catses enetgy to move aroutud so as to stay constant. In the thernodynamic limit, we cab vicu the entropy as a function $S$ on the space of only the real equilitiom distributions. Asymptotically. in the case of ergodicity, we can assiga it to be the function 5 on the eystem phaxe space given by the logaritbm of the volume of particlo orbits. When we think of

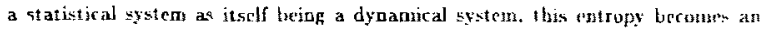


adtakatic invariant and leads to psewdoforces. Thesw are the thermudynamir forces. and they lead wa thernwdymanic symplectis state space on which we can define 5 . nnd in wan of which we get the thermodyoamic equations of state.

Berause this sequence of connections is the central unification around which this thesis revolsm, we will now give some easily understood examples of the concepts involved.

\subsubsection{Adiabatic Invariants and Paeudo-forcea}

Loreatz was first to ask the question that led to the notion of adiabatic invariance. He wondered how" the energy of a simple harmonic oscillator varied as its spring constnnt slowly changed. It was discovered that, although the energy and frequency both change, their ratio remains asymptotically constant for slow variations. This ratio, $H / \omega$, is an adiabatic isvariant fol the oscillator. A precise definition of the concept of adiabatic invariance and some of its limitations are given in sections 2.9 and $5.4 . H / \omega$ is equal to the action of the oscillator over one cycle. The artion of a closed orhit is the area encircled by that orbit in the $(q, p)$ phase plane. It is a quite general result that this area is an adiabatic invariant for slow variations of the parameters of both linear and nonlipear oscillators. We show how to understand this result geometrically in section 2.9 and how to use this understanding to get more refined results in section 5.4. That we consider area as measured in the coordinates $q$ and $p$ is absolutely essential here. If we had instead roordinatized plinse space using velocity ratber than momentum, the arca would hate hat no sperial significance. 
In higher dimensions, the actoon of a closed lowp can be defined at low integral of $p^{\prime} d q_{1}$ around the loop where we adoph the unal Einstein comention that repeated indice are sumined over. This combination $p^{*} d q$, therefore has a derep phy tieal sift. niticance. Geometrically, the choice of individual coordinates $q_{2}$, and their conjughte $p^{1}$ is irrelevant and only the combination $p^{2} d q$, is significant A geometrir entuty that one cam integrate along one-dimensional paths is called a one-form. $p^{\prime} d_{g_{1}}$ is intrinsically built into the structure of physical phase space and is therefore called the canonical one-form and is usually denoted by $\theta$. Another way to obtain the action of a closed loop in phase space is to find the area of a dise whose edge is the loop. -Area" must be defined in a ,pecial way to get an answer that is independent of the means used to obtain it. Applying the (generalized) Stokes theorem to the line integral of $p^{2} d q_{2}$, we see that the action can be defined as the surface integral of $d q_{1} d p^{2}$ over the disc. It is important here to keep track of orientation. A geometric structure that one can integrate oyer two-dimensional surfaces is called a tho-form. The standard notation uses a wedge to kcep track of orientation. The two-form $d q_{1} \wedge d_{p^{2}}$ is usually denoted by $w$ and is called the symplectic form on phase space. It is uniquely defined from $\theta$ and so also is intriusic to the geometry of phase space. Since the evolution of a Hamiltonian system preserves the artion of closed loop: (this is Princarés first integral invariant), the notion of area with respect to the symplectic structure $\omega$ is also preserved. From the modern perspertive of geometry introduced in Eelix Klein's Erlo ager program, a "geometry" is defined by a mathematical structure and the group of symmetry transformations that prencree that structure. For example. Riemannian geometry studies the concepte that are invari- 
ant under the isometries of a metric teneor. The geometry of the phase space of Hamiluruan mechanical systems is stmplectir geometry since it is the symplectic structure which is invariaut under the canopical transformations describing time crolutusi

The real conceptual and computational advantage in the phenomenon of adiabatic invariance is that we can forget about the phase of the fast degree of freedom. Imagine a complex, slowly moving piece apparatus (for example, one of the designs of Rube (ioldberg). There is a small weight attached to a string that hangs out of a bole in the side of the machine. As the apparatus moves, the string is slowly pulled in and out of the bole. The part of the string that is hanging out forms a pendulum witb the weight. The weight rapidly swings back and forth (say thousands of times before the string length varies appreciably). In trying to understand the operation of this device, one first thinks that the exact state of the machine, including the phase of the pendulum, will be essential in determining the time evolution. The idea of adiabatic invariance tells $u 5$ that we only need to know the action of the pendulum (say by observing the initial amplitude of swing) and not the phase in order to determinc the average effect on the apparatus. As the machinc pulls the rope in. the energy of the pendulum will change so as to kcep its action constant. It will thercforc give and take esergy from the rest of the machine. The pendulum energy looks like a function purely of string iength. From inside the machine, we may regard the string as attached wo nonlinear spring whose potential energy represents the entire pendulum euergy. The oscillations have been replaced by a "preurlopotential". 


\subsubsection{Symmetries and Exact Invariants}

One secs similar "pscudopotentials" when one forget about curbedustem in systeme with symnetry. In studying a particle moving in a contral putential. we may ignore the angular position of the particle, sine all angle lead to the satme particie behavior (i.e. there is a rotational symmery). Noether tells us that the symmery leads to a conserved quantity, namely the angular nomentum of the particle. The radial motion must behave in such a way as to keep the angular momentum constant. The energy iu the angular direction is not contistant and so the radial dyamics must supply and receive the extra angular energy. This cones out looking like a new radial pseudopotential that gives rise to the cent rifugal force. The adiabatic invariant case is exactly analogous: we forget about the fast part" phase: the fast part's energy nust change so ae to kecp the adiabatic invariarl constant; this energy must come from the slow part of the system: and the ne: result is a new "pseudopotential" and cotresponding pscudsforce acting on the slow system. We make this conaection precise in section 2.9 .2 and hlow that these "forgetcing operations" are part of a procedure known ith reductum

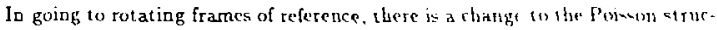
ture. corresponding to the Coriolis force. in atdition to the change in the Hatniltonian In chapter 3 we give the underlying strueture behind this and show hou

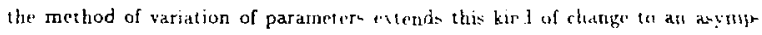
totic setting The simplification procen uhich is ajplied to aymptotic syotetus

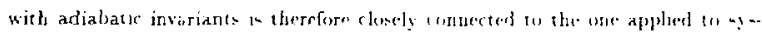

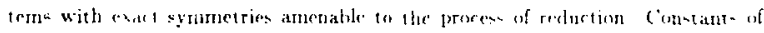


the tostion which resuit from symmetry are rodified geometricaliy in the notion of the momentum may of a group action. The puwerful setting in which this encept makes grtieral suse is discussed in sections 2.5 through 2.7. Adiabatic imvariants are the geferaturs of asymptotic symmetries. This viewpoint is developed into a powerful perturbation method in chapter 5 .

\subsubsection{Thermodynamic Forces}

The next connection is with t'zermodynamic systems. If one slowly varies the parameters of a thermodynamir system in equilibrium, there is again an adiabatically invariant quantity known as the entropy. The entropy rhange along a path in thermodynamic phase space can be defined as the integral of the one-form $(1 / T) d \Gamma^{\prime}+(p / T) \Omega^{*}$ (where $T$ is tempcraturc. $p$ is pressure, $U$ is energy, and $V$ is volume). The choice of coordinates $(1 / T)$ and $(p / T)$. ihermodynamically conjugate to $\mathrm{U}$ and $\mathrm{V}$, is absolutely essential to obtaining an adiabatically invariant integral. $A \leftarrow$ in mechanics, this canonical one-form $(1 / T) d U+(p / T) d T^{*}$ plays a fundamental role in the structure of thermodyzamics. The net change in entropy in a cyelic procesu can be obtained by integrating the corresponding symplectic tro-form

$$
u^{\prime}=d U \wedge d\left(\frac{1}{T}\right)+d l \wedge d\left(\frac{P}{T}\right)
$$

wer aly wo wimensional disc bounded by the loop representing thir cycle in phase space This thermodynamic symplectic structure is discussed in section 16.7.1.2.

Picture a slowly moving apparatus attarhed to a piston that compresses a gas confined in a rylunder. When we first study the system. we might think that we 


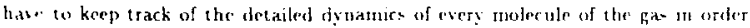
to understand the operation of pus device. The adiabatic invartance of the cut rops telle un. heweser, that we really need only the entropy of the gar fobtained ly intially measuring the internal energy of the gas. say) As the piston moves. the energy of the gas will change so as to keep the cotropy constant This energ. comes from our apparatus, and the effect of the gas is just like a nonlinear spring We can forget the gas by introducing a new "pseudopotential" into the dynamics of the machine. This pseudopotential gives rise to themodyanmic forces. This then connects thermodynamic forces with adiabatic pseudoforces. A more detailed discussion 1, this connection is given in sections 2.9 and 5.4 .4 .

\subsubsection{Wave Action}

The next connection is with wave systems. First consider the example of linear sound waves in a closed room. The wave evolutiun may be expressed as a superpo sition of room eigenmodes, each of which are purely oscillatory at a corresponding eigenfrequency. What kappens if we excite an eigenmode and then slowly vary the shape of the room? In an underlying infinite-dimerisional Haniltonian phase space for the waves, the eigenmode's evolution describes a closed loop (since it is periodic). Just as in the purely mechanical case, we may define the action of the oscillatory wave to be tine integral of the symplectic form over a dise bounder; by this loop. For linear waves. the action is again the energy of the wave divided by the frequency. As we slowly vary the room, the action of the eigenmode remains constant. Because the eigenfrequeucy iypically varies as we change the rom this 
forces the wave energy to change This extra wave energy coine from the work done against the wave in altering the room.

One might think that eur eigennode could excite other eigenmodes as we vary the roont. As long an the eigenfrequencies are distinet, this coupling is exponen. tially small in the .lowness of room variation. For typical onc-parameter room variations it turns out that the eigenfrequencies remain distinct. This phenomenon is sumetimes called "level repulsion" and is duc to the fact that the space of two by two Hermitian matrices (representing the possible couplings of two modes for all room parameters) is 4-dimensionai, while the subspace of matrices with equal eigenvalues is only 2-dimensional (parameterized by the eigenvalue and the imaginary off-diagonal antisymmetric element). It therfore takes two parancters to force a degeneracy.

Imagine a slowly varying apparatus which, as it moves, changes the shape of the room containing the eigenmode. At first we might think that we need to know the phase of the mode to determine the evolution. Adiabatic invariance allows us to get by with just the wave action. The apparatus moves as if it had a neu pseudopotential. When the wave is a light wave, the corresponding force is the radiation pressure.

The same idea may be applied to quantum wave-functions in a slowly varying potential. The classical limit of the wave result applied te a particle in an oscillator potential gives the adiabatic invariance results for mechanical systems that wC discussed first. A square-well potential with slowly varying width corresponds to a particle bouncing back and forth between a stationary and a moving wall. The adj- 


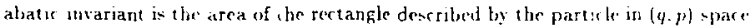
(see section 2.9.3.1). Physically. the particle chergy changes bere and the past as le is bouncing off a moving wall and on cach bounce romes away with a different mar nitude of velority than it had going in. The statisti. al analog is a one-dinensional gas and the adjabatic invariauce is represented by the conservation of $\mathrm{pl}^{-}$, : in wave system may be utuderstood as a gas of quanta (for electromagnetic uavem, a gas of photons), and a quantum changes energy is bouncing off a moving wall duc to the Doppler shift. The de Broglic relation $E=h w$ for a free massless quantum shows thai the action $E / \omega$ is the number of quanta times $\hbar$.

The "normal modes" for a free wave are infinite plane waves. These have infiuite action, but there is a sense in which they have a well-defined action density (i.e. action per unit of volumc). Such i notion becomes asymptotically precise when we study cikonal waves, which are plane waves with slowly varying anplitude and wave-vector. Asymptotically, it begins to make senise to think of the inergy and action of an eikonal wave as being made up of additive contributions from the different regions of space. In chapter 8 we will show how the asymptotic wave energy density and wave action density arise asymptotically. Wr obtain the evoution of the wave action density in time. In the presene of slouly varying potentials. the wave energy density varies but the wave action density evolves as a locally conserved quantity. The pseudeforre on the mediurn due to the giving and taking of wave energ: is the ponderomotive forre. These variou. relations give another connection between our subjects.

We haw seen that the artion in Hamblonian dyasuse and the entrops in 


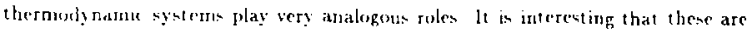
Ehe tho concepts that often give students the trost trouble when learning mechanics ated threnodymamice. Both are adiabatir invarianth under slow variations of the parameter: of a systen. Because of this. they both give rise to the pseudoforces that are a chatracteristic consequence of eliminating degrees of freedom. They are both intinately related to a geometrical symplectic structure on the corresponding phase spaces The action motivates us to introduce canonically conjugate momentum variables to the configuration space variables of a mechanical system. The entropy motivate: us to introduce thermodynamically corjugate variables to the mechanical obervables (like energy, volume, particle number, etc.) of a thermodynamic system.

\subsubsection{Action; Entropy and Asymptotica}

We know, however, that both classical mechanics and thermodynamics are asymptotic theories that are limiting approximations to quanturn mechanics and statistical mechanics respectively. Can we understand the geometrical structure of these liniting theories as arisug from the asymptotic process? Do the key quantities: action and entropy, have any natural meaning in the detailed underlying theorics? The answers to both questions are resoundingly affirmative and much of this the is is devoted to ferreting them out. The essential idea is that quantum mechanics associates to every path a wave amplitude whose phase is the action of that path. This very general action agrees with the classical mechanical action on the pathe that represent real classical motions. These paths are defined asymptotirally wia the methorl of stationary phase and satisfy the principle of least action. 
Statistical mechanes (a) we formulate it here) associates a weight to each probabslity distribution This is an expouential whose exponent is the information entrop. of the prohability distribution and is defined as $-\int p \operatorname{logp} d x$. This very general entropy agrees with the thermodynamic estropy on the distributions that represent real equilibrium thermodynamir states. These distrihutions are defined asymptotically via the method of steepest - escents and satisfy the principle of maximum entropy.

\subsubsection{Steepest Descents, Stalicnary Phase, and Averaging}

The two main matbematical theorems whikh allow the asymptotic simplification are the method of stationary phase for the oscillatory and wave systems, and the method of steepest descents for the statistical systems. These methods connect line integrals in the complex plane of functions with an asymptntic parameter, to expressions that asymptotically depend only on the function in the neighborhood of certain special points. Dependence on the full details is reduced to dependence on only certain special features. Where does the extra eliminated information go? Its contribution is to things that bave no long-term effect on the degrees of frecdom we are isterested in. In each of the domains studied here, we perform some kind of averagiog that eliminates the features which have no !ong-term contribution. Physically, we are ofen interested in the interactions of our system with other systems (like ourselves) that respond only to these averages and so they are really the quantities of interest. The only way a fast degren of freedon can contribute is for it to have a long-term effect. This ean come about only if its mary contributions 
add coherently Thu in turu, can happen if the t tegres of frordom repratedly come bark slene to the same state and bo give a net com ributon to the hlow seale betavio In that caes, our system has an approximate symmetry that maps one fast excursion to the next one.

\subsubsection{Reaonance}

The regions of phase space where fast contributions add coherently are called resonanres in oscillatory systems and correlations in statistical ones. Long-term effects are represented by phenomena whose frequency lies in a region near zero (and with appropriate scaling, the width of this region approaches zere in the asymototic limit, leading to the omnipresent delta-functions in frequency). Nonlinearities 1 make use of rationally related frequencies to create near-zero frequency effects (the dynamics is then defined on tori whose orbits do not cover their surfaces densely); these effects are known as resonant interactions. One can even get them linearly if it is possible to simply add the frequencies to get zero (since then there is an oscillatory coordinate with zero frequency). These rescnant effects are responsible for most of our knowledge of phenomena on time scales smaller than the one on which we normally operate (cg. spectroscopy in atoms, nuclei, particles, etc.). 
1.4.8. The Key Examples in Merhanics, Waves, and Statistics

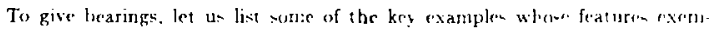
plify the ideas we wish to explore. There is averlap in the technique of the threr domaias (and it is this orerlap that we are particularly interested in). but roughly most models can be categorized as being one of the threc type. In mechanic: We have systems with exact symmetries, such as a planar particle in a rotationally symmetric potential, to which we can apply Noetber's theorem. We also have systems with approximate symmetries to which the method of averaging. Lie transforms, Kruskal's method, and other perturbation techniques wo shall discuss can be applied. Exar,ples include: gyromotion, oscillation center motion, oscillatorily stabilized systems, and the interesting variety of systems with adiabatic invariants.

In wave theory, we have all the situations with short wavelength waves. including plasma waves, propagation of light rays, elastic waves, quant um mechanics, and the various noplinest plasma and fluid waves. The methods of analysis include the classical WB theory, its geometric extension by Maclor to handle caustion, and it: exteusion to nonlinear waves due to Whitham

There are two types of statistical nodels we type is concersed will equilibriuru systems. This includes models of thermodynanues weing either the maxu-

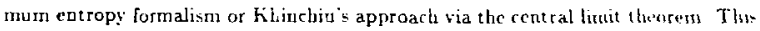
type alse ancludes Brownan motion and Euctuation theory which we relate to the renormalizatiun group The other $y$ pe of model studie non-equbbrium = If 11 at lim-

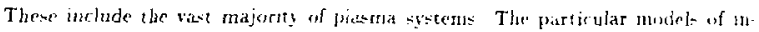

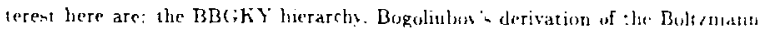


equation the Pustuaturidisipateon theorem the Onsager relatwons, and the tech-

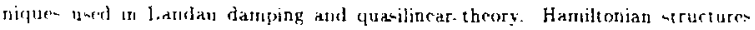
are lurkifg, brhind virtually erery appert of the physies of these systeme and will therefore lie a prime consideration in our exploration.

\subsubsection{Niechanical Systems}

The uriginal example of a Hamiltonian system is given by the dyamics of point particles in a potential. In plasma pbysics, one is interested in charged particles and their Hamiltonian dynamics in electric and magnetic belds. The $t^{2}$ ree nontrivial but trarcable situations one often neeos to study are: the gyration of a particle in a magnetic field, the motion of a particle in an electromagnetic wave; and the scattering of particles off one another (usually all three are present a: the same time but certain aspects dominate the particle's behavior). When there are exact symmetries. we may simplify the system by using reduction as we have discussed. Usually however, systems have only approximate symmetries.

\subsubsection{Mechanical Syetems: Separation of Time Scales}

The aş̣protics in particle mechanics usually arises from a separatiou of time scales One set of degrees of freedom may have dynamics that is considerably faster than the others. In i gic rane, the effect of the iast degrees of freedom on the slow ones tend to be close to the average effect and the effect of the slow degrees of fredom on the fast ones tends to be almost at if we were holding them fixed. If We introduce A-ymptoturs. He may obtain in asymptotic expansion a model of our 


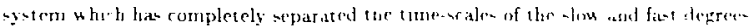

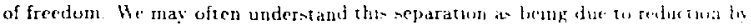

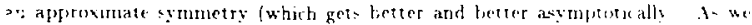

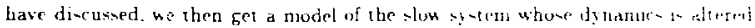

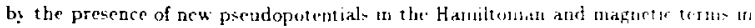
the Poisson brackets. These remain of the forgotlen fesi degress of freadim give rise to the aew physic introduced b, asymptotich

\subsubsection{Gyromotion and Agymptotics}

In the case of a particle in a magnetic feld, we let the asymptotics move the particle faster and faster arouod its gration loops in comparison to the rate of motion of the center of these loops. The asymptotic theory of guiding centers. which forgets the fast gyromotion and describes only the slow drift of the loops. has the neu concepts of: $E \times B$ drif, curvature drift. polarization drift. magnetic momeat as an adiabatic invariant, boupring from a matr-tic mirror. etr. None of these concepts makec precise sease for the physical system as it appears in nature. Tonetheless, they have been extremely important in the design and unorrstanding of plasma devires and represent truly uew physical notions in the given setting To mak! the model of these useful concepts prerise. we must introdure asymptotic: Note that this is far more significant than the usual idea of perturbation theory ar. givin better and better approximations to some underlying cxart model. While it does that. I believe its real importane is in the conceptual advances it allow one to make in understanding and utilizing physics 
Oro often sees (partirularly in older literature) the stated geral of perturbation therest 2- convergent seres and the concumitant lament that mont physically usc ful ertu are anly anyptotic From the conceptual viewpoint we emphasize here. consergerite is ircelevant. Wo never use more than a finite number of terms of our arie- (is-ually the firt urder terms suffice to give the new physies). the underlying model equations we are approximating are based on concepts that themselves are only asymptotic. and convergence is a complex analytic concept requiring apparently physically irrelevant complex structures in our models. Usually we only require our transformations to be smooth and a smooth, non-analytic transformation can destroy the covergence of most scries (a classical construction due to Borel uses $C^{\infty}$-bump functions to construct a smooth function whose derivatives at a given point are equal to the elements of any arbitrary sequence of real numbers: using such a function to change coordinates will convert an analytic function whose first derivative is non-zero into one whose derivatives are any desired sequence; we need only choose one which grows last enough to prevent convergence of the Taylor expansion at any radius). What I feel is important for theoretical understanding is not the actual numbers but rather the physical tendencies. Of course in applications one wants numbers, but asymptotic series often yield a given accuracy of - pproximation to the exact solution with many fewer terms than any corresponding convergent series. 


\subsubsection{Oscillation Centers and Ponderomotive Forces}

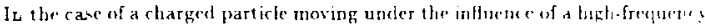

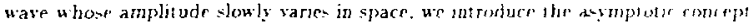
of the oscillatica center. As the wave tscillates. the particle ferl: a fored tirt in one direction and then in the other. causing it to ascillate as well If the rav. were spatially uniform, then the excursions to cach side would exactly cat-cl cach other yielding no net average effect. In a non-uniform wave, the particle fect- a bigger force in the region of larger amplitude and so is pushed more awat from such regions than into them. The average push behaves like a force on the particle pushing it away fron higher wave amplitude regions. The reduced description gives the dyamics of the center of ascillation and includes a remnant of the fast motion through this so-called ponderomotive force. Both ithe guidinf, center equations and the ponderomotive equations are Hamiltonian.

The second chapter shows how to use reduction by a circle symmetry in conjunction with the method of averaging, to obtain the $E \times B$ drift dynames as a Hamiltonian system. The higher order theory, based on the ideas of hruskal. is dicussed in chapter 5, and the relation to the pionering work on the Haniltonidn structure of gyromotion due to Robert Litute ohn in deressed an rhapier 6. Many other sysienis fit into the setting of these asyptotic methus and the physical concepts that conu out of the asyptotic can be cutestriking sy-temwith constraint can exhibit phenomena atalogous to fonderonetive efiects due te

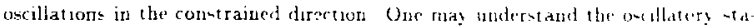

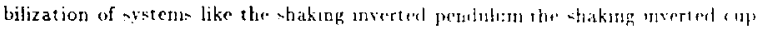


of Hivd. or the of stabilized untable MHD modes as the effert of the asyototir pacudiofurese.

\subsubsection{Geometric Petturbation Theory}

To make thene rotuns procise. we bave developed a syst smatic geomet ric frameHork fur aymptotology based on notions from the mathematical theory oi jets. Because we are interested in systems whose phase spaces are manifolds and we paricularly want to study the asymptotic behavior of geometrically intricate Lis group symmetries, wr develop the various perturbation theories in the coordinate-free languagr of modern differential geometry. While ofen useful (particularly in explieit calculations), coordinates are unphsical and often obscure the underlying content of physical theories. The movement in the mathematical physics community to reexpress the fundamental physical gotions without arbitrary coordinate systems has been very successful in mechunics and has succeeded in dramatically simplifying some of the chassical results (eg. Liouville's theorem, Noether's theorem. Darboux's tbeorem, artion-angle variables, etc.), elarifying the essential structure of the theory (especially the extreme importance of symplectic geometry). and obtaining many new idez and results (eg. the extension to infite dimensions, Noether's theorem for arbitrary lic groups, the KAM theorem, chaotic dynamics. etc.).

Our formulation of perturbation theory is in the spirit of this movement. and wh He sumnarize some of its key ideas in chapter 2 . In thict chapter we also give the nes. Hasniltonian anpert- of this gemetric approach to non-secular perturbation theors and sketch the Hamiltouian results. The Hamiltonian results are given it 


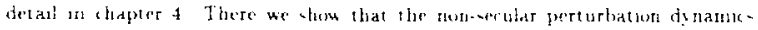

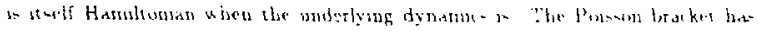

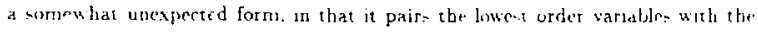
highes order. the next to lowest with the next to wighent and so on Wi shen that it is the natural stucture in five different rays. each of which sheds a different light on thr relationship between the perturbed and unperturbed systems The relationship between symmetry and perturbations is also given in that chapter, and the operation of performing reduction is shown to commute with the operation of performing a perturbative anaysis. Chapter 5 uses the previous non-secular results to do secular perturbation theory. We develop a new technique based on these ideas which is simpler to apply in practice. Its application to guiding center mo:iun is given in shapter 6.

The limitations of this and all gencral secular perturbation theories it not appear to be well known in the physics community. We therefore give examples and explanations of why tive time of validity of this and other theories is only of order $1 / \epsilon$. even thougb the accuracy over this time can be to all orders in , We aist discuss the case of many fast frequeacies and the concomitant resonances Finally. we end chapter 5 with the case in which the fast piorion is ergotic on the energy: surface and begin to make the conuections with statistical mechanics. 


\subsubsection{Averaging in Statiatical Sybtema}

Tisere to an watrotumg way in which the need to average over an intermediate a sile herume apparem in both the statistical and wave systems. Consider the

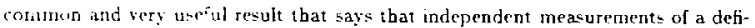
nate qua tu' representative of a complex system will be distrituted as a Gaussian. The very general argument for this assumes only that sinall errors from the many parts of the system will contribute additively to the error in the measured quantity. Regardless of the how the individual errors aic distributed (under some very weak constraints). the centras limit theorem tell us that their sum will be distributed as a Gaussian. Let us try to understand how one applies this atement operationally and so see that a specific type of averaging is required to make sense of the notion of Gaussianity. Let us consider an artual experiment where we bave made, say, 1000 measurements of some quantity (so as not to obscure the argument, let us assume that the results of our measurement are precise real numbers). Taken directly, our measured probability distribution is a sun: of $\delta$-functions, one for each measured value (no Gaussianity bere!). In practice, we "bin" the measurements. i.e. We make a bistogram of the number of measurcments that fall into each of a set of intervals that partition the space of measurement ralues. If we make the bins too small. we get the problem of the $\delta$-dis.ributious, i.e. widely spaced bins with one measurement eact. If we make the bins too large, then all neasurements fall into a single bin. We see something that approxmates a Gaussian only if we hin on an intermediate scale. This is defined using an asymptotic parameter given by the number of meacurements. 


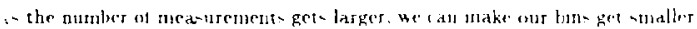

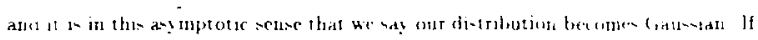

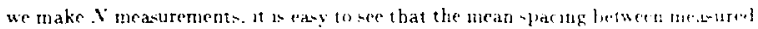

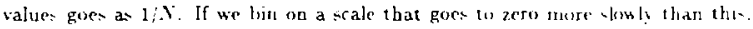
like 1 t $\bar{T}$, then asymptotically there will be all whitute number of neansementin eacb bin. The law of large numbers tells us that asymptotically the number of measurements falling iuto earb bin will agree (with probability one) with the number expected from the Gaussian distribution. Furthermore. since the width of the bins is going to zero, we get arbitrarily tine accuracy. Also note that, while with any given number of ineasurements the observed distribution may be changed by altering the binning, as long as the binuing is on an intermediate scale, the arymptotics is bin independent. Furthermore, the range of bin cholces that yicld values close to the asymptotic result gets wider as $N$ gets larger. Anotber way to think of the asymptotics is in terms of the convergence of the delta-function distribution Iepresenting the measurenents, to the smooth asymptotic distribution from the perspective, the binaing procedure defines a topology on the space of distribut ton

It is only in this atymptotic seuse that the m-lule notion of a probability density makes physical sease at all. There are many other stuatious wh why at sumlar

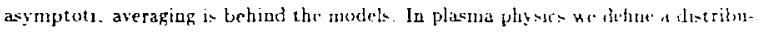

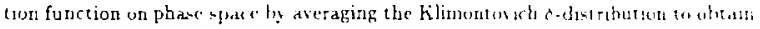

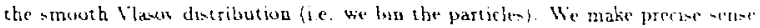

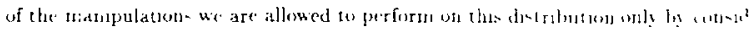

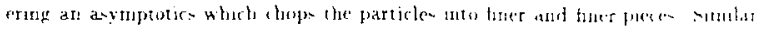




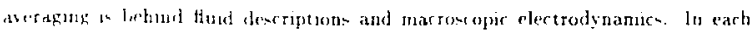

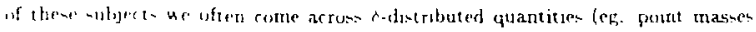
and chargic. Thene are to be nerrpreted in terme of the anymptotics The real stuatsun is furl at the itymptotic limit. but the object of interest is small on the large scale fand we scale it so dt gets ever smaller acyuptotically) and has finite mass for charge. etr.) on the sinall. We then develop consistent rules that are as mptotucally valid and these lead to the Canniliar calculus of $\delta$-functions and other singular distributions. Similarly. real flud velocity tunctions (which are the result of an average over a macroscopically smail region with a large number of particles) cannot validly have ravelengihs shorter than or on the molecular scale. We model the velocity evolution by nonlinear parial differential equations. however. that can (and do) excite arbitrarily shore wavelength Fourier components. If these ever become important. the separation of seales fundanental to our model has broken down and the model becomes invalid.

\section{4-11.1. Matched Asymptotics}

A fundancutal techuique of singular perturbation theory is to insert movels which inclute the physics of the small scale in regions of breakdown. For example, un wry high shear regions of a fluid, on need to include more kinetic effects than ase -epresuml in the muple Naver-Stokes model. The two important cases arise When these = Ingular riginns (with asymptotically small scale physics) are, 1) loc atlerei and get snallet with the ingmptotics and 2) spread ober open region but have

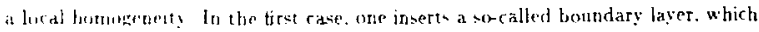




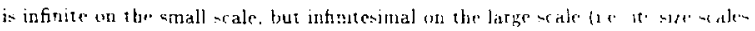

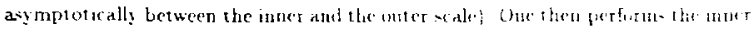

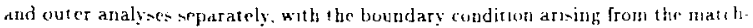

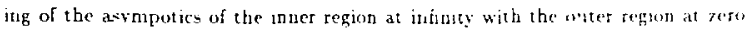
The case where tbe small scale effects are not localized. but are hume renentia teario to the eikoual iectoniques we shall discuse uext, in which we have slowly modulateis Fast bebavior which is regular on the small scale at each large scale point icg loral plane waves.

\subsubsection{Averaging end Local Fourier Trangforms}

The idea of averaging over an intermediate scale that arose in trying to understand the notion of probability dencity also arises in trying to define the frequency or waveiength of a slowly varying wave. When we talk about a wave having a rertain waveleagtb or a sound having a certain frequency, we are always talking about a local Fourier tran: form No souui lasts forever and we usually are not interested in the properties of a wave in the rext galaxy. Hbat do we mean by a lucal Fouric $r$ transfurm? Operationally we usually work with the Finrite trats form of it tignal multipliad by a sindow function.

$$
F(t . \omega) \equiv \int_{-\infty}^{\infty} f(t-r) H(-), \quad+\infty d t
$$

We might bave a spectrum analyser that works on a finte segment of the signa!

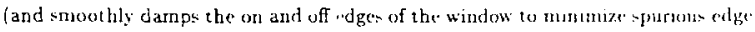

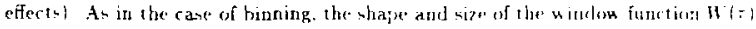




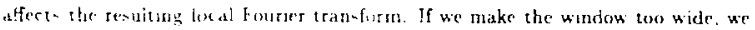

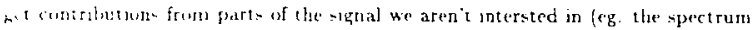
of the cuen- Foadian after the concert) If we make the window too small, we Son t sample enough wavelengta (perhaps not even one) to get a good fix on the frepuensy furmug the radio on and of quickly results in a click with all frequencies). If we are trying to say that a frequency (or frecuencies) is present at a given time (as in describing a piece of music in music notation), th^n that frequency should he distinctive To make precise the manipulations allowed, we embed the given wave in an a-ymptotic family. which makes the frequency of interest mn.e and more distinct as a parameter approaches its limit. We make the number of wavelengths oceuring in a region of significant wavelength change becone infinitc by making the scale length longer for fixed wavelength, the wavelength shorter for fixed scale length, or both. We introrluce a short scale given by the wavelength and a long scale given by the scale length deh. $d$ so that asymptotically their ratio vanishes. The operations and concepts we are allowed to use in describing our wave are those which make Isymptotic sense. As the parameter approaches its limiting value, the domain of validity of such asymptotic concepts gets larger and larger. For any real situation, one must make sure that one doesn $t$ bave plysics which violates the separation of scales and thus the validity of this kind of model.

The iocal Eourier transform above can be made precise asynptotically. We let the window function 6 scale with the asympotics betwern the fast and slow scales. On the slou scale it looks mere and nure like a $\delta$-function aro in the asymptotic calculu- we may treat it as such. OL the fast scale it looks more and nore like 
a ernstant and so the local Fourue, transform asyototirally lonk- focally like the

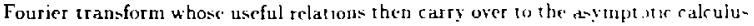

\subsubsection{Scales in Differentiation}

Many other areas of study have this same large us. smaii scale dichotom: Robert Littlejobn suggested the example of optinal algorithms for nunierical differentiation (sce [Stoer and Burlirsh. 1980]). Assume we bave some function represented $n$ a conputer as an algorithm that can calculate values to some aci uracy. What is the optimal way to calculate its deriwative at a point numerically? He can evaluaie the function at two nearby points. take the difference and divide by the distance between the points. How do we choose this distance? If it is too wide then we won't get the derivative at the point of interest, but rather something averaged over a region in which the function may have signifcant change. If it is too small, theu the division by a small number blow up the errors until they are arbitrarily large. Given some criterion of goodness in these twu respects, there is an optimal distance. We identify the asynptotic linit with increasing the numeriod accuracy of the calculation. If we take the distance between the points of evaluatson to shriak on an intermediate scale fl.e. they becume infutcly clone ar far as the variation of the furrtion is conceraed, but are far enough epart that the divison a)goritian bu, umes infinitely arcurate asymptotically) then in the limit we get the

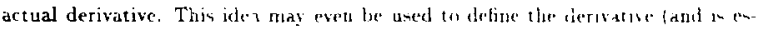
sentially the sabe an the mathematkat definition, guren that real afe befined an

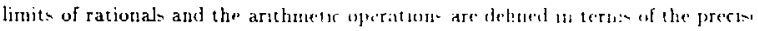


operations on rationals). The ver: same issues are relevant in any physical situation in which he take a derivative, and this kind of approxinatir $\mathrm{n}$ is almost always what we really noan (eg. the strain in the dervative of the displacement in elasticity. but dispiacement as a smonth fuaction is an asymptotic idea as the molecules of material become nore and more numerous).

\subsubsection{Symplectic Asymptotice in Thermodynamic and Mechanic}

The last part of the thesis diccusses the relationship between eikonal wave acymptotics aud the particle number asymptotics of statistical mec' anics. Both theories reduce to asymptotic theories with natural symplectic structures: classical mechanics in the case of waves, and thermodyuamics in the case of statistical mechaules. The description of a state is given by a submanifold of these asymptotic state spaces in both cases. It turns out that this submanifold of states always has a very special relationship with the symplectic structure (the symplectic form actually vanishes when restricted to the submanifold). Such submanifolds are called lagrangian submanifolds and give rise to 1 rirh theory (see section 7.1.4). The role of the Fourier transform in wave tbeuries is played by the Laplace transform in statis:ical mecbanics. The simplification provided by th a method of stationary phase in wave mechanics is provided by the method of steepest descents in statistical mechanich. The rris of the action is played by the entropt. Cauonically conjugats variables correspoud to thermodynamically coujugate variables. The analog of the Heisenberg uncertainty princigle in the asymptotic limit is the fact that the dispersion tensors of thermodyanically conjugate variables are inverses of each other. 
Xon-degenerate critical points give the rays in ine mechanes and the Gatusuans in stat istical mechanics.

Heisenberg. and Fourier before him prevent us from localizing states in $r, k$ space. But. by letting $k$ go to infaity, we can make the relative dispersions in both $I$ and $k$ go to zero (by relative dispersion in $k$ we mean $\Delta k / k$ where $\Delta k$ is the absolute dispersion). So by rescaling (i.e. going to slow variables) we get states whose local Fourier transform si asymptotically a $\delta$-fuuction in phase space

Given a definite mpan energy $U$ (or any other extensive quantity from the underlying mechanical system) the maximum entropy formalism gives an exponential distribution parameterized by the conjugate variable (inverse temperature $\beta$ in the case of energy). A definite temperature corresponds to a (canonical) distribution of energies. A definite energy corresponds to a distribution of temperatures. We may use Bayesian statistics to see that the temperature and energy distributions are related by esse.ucially a Laplace transform. The analog of Heisenberg's principle says that $\mathrm{w}^{+}$cannot localize a distribution in $U . \beta$ space. If we let $U$ go to infinity. however, we can make botb relative dispeisions go to zero. By rescaling, we get an asymptotic delta-fuaction on thermodynamic phase space.

Eikonal waves have $k$ 's at each $I$ that fit Logether into a Lagrangian submanifold. This manifold is locally the graph of the differential of wave phase, which for mechanical systems is the action. If we view $k$ as the base coordinate, this manifold is the graph of the differential of a function of $k$ that is the Legendre transform of the phase. This Legendre transform results from applying stationary phase to the Fourier transform of our eiknal wave. Asymptotically we obtain the Legen- 
dre relation between functions of $s$ and of $k$ berausc only the stationary points rontribute.

The state of an equilibrium statistical mechanical system is restricted to lie in a Lagrangian submanifold in thermodynamic state space. For simplicity, let us just consider (E.S) space (where we have introduced the inverse temperature $B=1 / T$ ). This manifold is locally the graph of the differeatial of the entropy 5 as a function of $U$. In othet words, only those points in $(U, \beta)$ space which satisfy

$$
\beta=\frac{\partial S}{\partial \bar{U}}
$$

correspond to thermodynamic states. To write this as the graph of the differential of a function of $\beta$, we again introduce the Legendre iransform. This Legendre transform results from applying steepest descents to the Laplace transform of our distribution function. Again only stationary points contribute asymptotically. The usual Legendre transforms of thermodynamics are of the energy instead of the entropy. These give the Helmbolz and Gibbs free energies and the enthalpy. They arise from taking the entropy instead of the encrgy as the observable extensive variable in phase space. The two pictures are related to a large one containing the extensive and conjugate intensive variables and the entropy. This has a natural contact structure given by the first law of thernodynamics (conservation of energy). The equation of state is a Legendre submanifold with respect to thiv contact structure and turns into a Lagrangian submanifold when we project onto either of the natural symplectic thermodynamic state spaces.

We may understand the relation between the underlying infinite dimensional wave and probability density spaces and the resulting symplectic phase and ther- 
mon s names spaces better using a theorem about lagrangian manfold. The tetaik

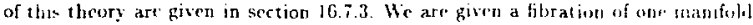
over another (i.e. a projection os that the iverse images of points all lowk the same) and a function $S$ on the first manifold. The graph of the differential dS is at Lagrangian submauifold of the cotangent bundle of the first manifold The pustsforward of this submanifold to the cotangent bundle of the second manifold consists of solutions to a constrained variational principle. that is. it is the push-for $\mathrm{x}$ ard of extrema of $S$ restricted to each of the fibers. From a more general result, one may show that this resulting pushforward is Lagrangian iff all of $S$ 's critical points are non-degenerate (i.e. $\delta^{2} S$ is invertible). The more general formulation is if we are given a map from one spare to another one, then a Lagrangian submanifold of the cotangent bundle of the first space pushes forward to a Lagrangian submanifold in the cotangent b'undle of the second space if and only if it intersects the pull back of the second cotangent bugdle transversally. In the case of a fibration and a Lagrangian submanifold given by the graph of the differential of a function. the extrema of the function restricted to each fiber represent the intersection with the pull-back and so pusb forward to a Lagrangian submanifold (non-degenerary corresponds here to transversal intersection). For the case of waves, we consider the space of patbs originating on some source region. The image spare is $R$ and the projection sends a patb to its final endpoint. The fber orer a point in $\mathbb{R}^{3}$ consksts of all paths that end at that point. For the function $S$ on path spare. we take the action along each path. In the Feyman patb integral formalism. this function is the phase associated with each patb. The differential ds definen a Lagrangian 
-11bmamfold in the cotangent bunde of path space. Doing the Fryman antegral frue 11 a wave on $: R$ whose phase is determined at each point by stationary phase to be that of the pathe of extremal artion. Where we consider variations restricted to the fiber ( $1 e^{\circ}$ to paths that end at top point of interest). This is exactly the pulthforward of the Lagrangian sulsmanifold $d S$ and gives the Lagrangian subrianifuld in the space of $(x, k)$ 's (i.e., the cotangeat bundle of $\mathbb{R}^{3}$ ) which represents an eikonal wrove. For statistical mechanics, our frst space is the space of probability distributions on phase space. The entropy of a distribution (defined by the integral of $-p \log p)$ is a function on this space. The nap to a space of observables given by the mean value is a fibration. The maximum entropy formalism says we extremize the eatropy on the fiber (i.e., all distributions with the given mean values) and the theorem say's that this determines a Lagrangian submanifold in the cotangent bundle of the observables (i.e. thermodynamic state space).

In wave dynamics, we start with a theory of arbitrary waves, and specialize it to the important special case of eikonal waves. To make the ideas of this specialization precise. we imroduce an eikoul parameter that specifies the separation of scales between the local wavelength and its slow variation. We may either think uf the wavelength going to zero for given scale length, or (as I prefer, since the artual wavelength often has physics in it) the seali length going to infinity for given wavelength The waves we want to study the dynamics of anc vi:wed as asymptotic families and we utilize the asymptotology to introduce new physical concepts which apply only approximately to the real waves, but show the tendency of the behavior an the separation of scales becomes greater. The key simplifying idea in the calculus 
of astinptotic waves is the method of stationary phase. Integrals fsuch as thone in the desription of wave prosagation; which really depeted on the entire state of the wave, asymptotically depend on the state only in the lowal neightorthood of a point The key asymptotir information about the wave at a point is the wave-vector $k$ and we obtain ays" in (k, s) space, to which the dependency of a portion of a wave is restricted asymptotically. This is a notion which is precisely defined by the spatial Fourier transform in the case of plane waves, but that bis only asymptotic meaning for our eikonal waves. The asymptotic local Fourier transform makes precise the notion of a wave whose wave-vector spectrum depends on $I$. 


\subsection{A Hundred Further Questions, Conjectures, and Suggestions}

And time for all the works and days of hands

That lift and drop a question on your plate:

Time for you and time for me.

And time yet for a bundred indecisions,

And for a hundred visions and revisions,

Before the taking of a toast and tea.

- Irum The Love Song of J. Alired Prufrock by T. S. Eliot

This section presents a number of questions and suggestions for further work related to the topics covered in this thesis. Some of thern appear to be fairly straightforward and some appear to be quite difficult. They are presented in the order the subjects appear in the thesis and we make reference to the relevant sections for each question. 1. Answer the question posed in section 2.1: Does the KdV Poisson bracket naturally arise from the one for the Boussinesq equations?

2. Is there a natural way of workiag directly with the germs of paths, and can one use this to get information on exponentially small effects in the perturbation parameter c (sucb as tunneling)? (section 2.3.2)

3. Does Newton's approach to centrifugal force, outlined in section 2.6.3, extend to any other situations?

4. Find physical systems that utilize the KKS symplectic structures on the spaces of measured loops and measured Lagrangian submanifolds introduced in section 2.7.10 (sec for example the eikonal wave systems discussed in chapter 9).

5. Vise the symplertir structure on some of the coadjoint orbits of the group of 


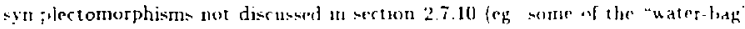
models in plasma physes naturally live in tiese orbit-l.

6. Apply the $J$-jet strue ures of rhapter 2 to a rariety of prothem:

7. Can one extend the Arnold stability method from fixed pount te preriodic orbits (or even orbits which limit on compact ohigets such as tori) by adding Casimirs to make the Hamiltonian quadratically maximal or miuimal on the ortut in question?

8. Richard Montgomery has shown that there is a natural Poisson structure on the space of 2-jets of a Poisson manifold (see section 4.8.6.5). The construction given in section 2.8.1 "explains" the fact that the linearization of a symplectic Hamilenian system about a fixed point is Hamiltonian. Can we apply the same construction to linearize a Poisson Hamiltonian system about a lixed point? I Io linearize at a point in a symplectic leaf, one need only add Casimirs to eliminate any linear piece in the Hamiltonian, take the Poisson structure at the fixed point and the quadratir piece of tbe Hamiltonian. To sec that there are prothloms near bones. just consider the Lie Poisson bracket on the dual of the Lie algebra of the rotation group. This Poisson structure vanisbes at the origin. yet the lincarized dynamics can be non-trivial. The approach suggested here via the jet brarketh may give a solution even at the bones.)

9. Apply the lincarized stractures of section 2.8 .1 and the previous que-tum to any of the numerour physical systems in whirb oue humarize aloout a fixed poim

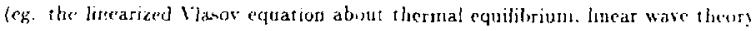
in flud mechanic or elazticity loneat surface waves ete ). 
10. Apply the linearioed Hamiltonian stucture about a given orbit (as opposed

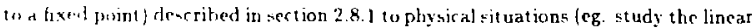
evolutiofi of snall perturbatons akout a nonlinear wave solution. Jerry Marsden is applying this to general relatwity)

11. Ve the same techniques as in the previous question and section 2.8 .1 to understand the IIamiltonian nature of the lift of Hamiltonian dynamics to the symplectic ame bundle which bas arisen in Robert Littlejobn's and Yukkei Hui's work on extending cobereat states by including metaplectic corrections (see also section 7.1 .3 .2$)$.

12. As in section 2.8.1, extend the previous four questions to $J$ th order structures (as opposed to just linearizing) and apply to examples where bigher order effects are important.

13. Use the approach to oscillatory stabilization discussed in section 2.9.3.2 to study various r.1. plasma stabilization schemes in a Hamiltonian manner.

14. lise the connection between averaging and reduction to tieat more problems like that discussed in section 2.10 in a geometric way.

15. Use the method of averaging given in section 2.10 to find the "pseudoputential" [elt by a deforming elastic body due to the presence of elastic waves (i.e. the elastic analog of ponderomotiye forces-as we deform the body the wave spectrum changes, caus ug energy to be transered between the body and the waves).

16. L'se the method of averaging given in section 2.10 to study the Stokes' drift of a fluid partile under the influsnce of surface waves (fluid particies move in approminate circles in the prescner of small amplitude surface gravity waves in 


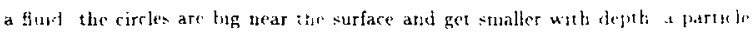

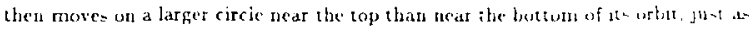
in gy rumotion the resultant pastacle path hat a drift pasallet we rhe -urface of the Hujd).

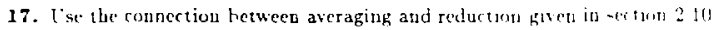
to prove the $K$ - theorem relating the linear susceptibility of a plasma to uave and the ponderomotive pseudo-po ntial in the plasma dynamics due to the raves after reduction.

18. The explicit calculation terhnique of section 2.10 utilized a section of the circle bundle defined by the approximate symmetry. Develop explicit terhniques for treating, nontrivial bundles (these are covered by the abstract theory). An cxample might involve a perturbed rigid body where the circle action is rotation about a given body-fixed axis the nontrivial projection to the orbit space is the Hopf map

19. Can one trmat the conversion from a Lagrangian to an Eulerian description of a lluid with the concomitant introduction of convective terms as an example of the change of reference frame operations introduced in chapter 3 ?

20. (A generalization of the previous question): Can one extend the re:-ult: of cbapter 3 into a general theory connecting the process of reduction and the process of changing reference frames (this is carried out explicitly in section 31.6 for the Coriolis forcel?

21. Apply che methods of se ion 3.1 .0 to develop natural Poison structures for a variety of rotating systeme Jebbje Lewis is currenty studying the infit ite dimencional fluid dynamics of rotating liquid drops (wuch as star or nucleit fom 
this prepective. Another example of interent might be a cold non-neutral elec. itut phantia in a cylundrically symmetric "tin can" with axial magnetic feld the alectrow- $E \cdot B$ drift around the axis making a naturally rotating reicrence frame.

22. The the Hamiltonian structure introduced into the methed of variation of paramerer-in section 3.2 to redo in a geometric way physical derivations based on it (eg. the usual derivation of Fermi's golden rule in quantum mechanics).

23. Does the natural symplectic structure introduced in section 3.2 .1 on the group of (compactly supported) canonical transformations of a symplectic phase space have any otber physical applications?

24. The perspective on $J$ t'd order non-singular perturbation theory that is taken in chapter 4 is to view the perturbation dyaamics as an ordinary vector field on a perturbation extended phase space (i.e. the jet space). Another perfectly valid perspective is to introduce $J$-jets of vector fields on ordinary phase space, whose How is a "J.jet of a diffeomorpbism" and so on for the rest of the objects in a theory. Pursue this alternate route and redo the various calculations of chapter 4 this way. Are there advantages or disadvantages to one or the other of these two perspectives?

25. Docs the binary notation for iterated tangent bundles which was so convenient in section 4.5 haye any other natural applicatioss?

28. The technique of going from the path space bracket io the jet bracket introduced in section 4.6 .1 by quotienting out the degenerate piece is very powerful. A re there other applications of this? In this example when one tried to push forward the original bracket along the projection, one obtained products of delta functions 


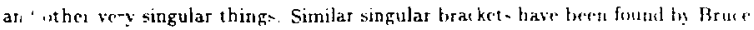

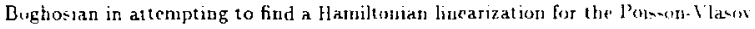
equation. (an one use a similar solution in that situation?

27. The sheet quotient spaces introduced in section 4.7 extend to $J$ th order a const ruction given to first order in /Kijowski and Tule 7 Yjew, 1979!. It that referenee they extend this construction in another direction: by making the shect parameter be larger than one-dimeusional. (In their work they were interested in time as the parameter and the extension is a space-time and feld theories\}. Can one apply the extension to $J$-th order derivatives given here in the field context?

28. is in the previous question, can one introduce more than one parameter but now treating them all as perturbation paraneters. Thus one should redo all calculations in chapter 4 replacing paths by bigher dimensional submanifolds, and path jets by jets of maps of these higher dimensional submanifolds into phase space. Use this extension to treat perturbation theories with more than one small parameter. In particular this should give a context in which to explore various relative scalings of multiple parameters. (All the constructions including jet groups and Lic-algcbras appear to extend in this way.)

29. Can one extead the perturbation structures deveioped in chap.ar 4 to Poisson manifolds, as opposed to symplectic manifolds"? (This is iniportant for appliration to many of the physical systems of interest.)

30. In particular do the jet spares of Poisson manifolds inhrit a Poison structure from the Poiston structure on the iterated cangent hundlet dincil-sed in section 4.8.6.5'? 
31. At-asked in section 4 8.6.5. deres the Lie P'oisnon bracket on the dual of the Lir algebra of ? - jet of paths in a Lie algebra gagree with some prescretton for rxtending $g^{*}:$ Lic Pois bracket to the space of its $J$ - jels? (Thin is important since many physical systems have Hamiltonian stuctures derived from Lie-Poisson bracketw)

32. Can one explicitly write down the "magnetic terms" in the symplectic structures on the jet coadjoint orbits defined in section 4.8 .7 ?

3.?. What is the relationship between the Lie algebra of jets of paths in a Lie algehra ; which arises in perturbation theory) and the Lie algebra of jets of functions on the dual of the Lie algebra which is described in section 4.8 .7 (and is useful in many coutexts such as geometric quantization)?

34. Use the extension of Kruskal's perturbation technique given in section 5.2 to analyse a variety of physical systems.

35. Inplement the explicit algorithm given in section 5.2 on a symbolic manipulation program such as MACSYMA or SMP and carry cut any of the calculations of the last question to arbitrarily high order.

36. Combine the new Kruskal method with its well defined operations with a changr of coordinates (as used in Lie transforms) to obtain a precise method that is easy to carry ut by hand. (section 5.2 )

37. Can on find a coordinate-free interpretation of two-timing or the method of multiple scales". Are there situatious to which this method applies which do not fall under Kruskal's method? (section 5.4.1.2)

38. Rerxamme the attention given to the 1 /e validity of Kruskal s method in 
variou= situations where it is used (for example in the variation of the aliatedie invariagt in magnetic nirrors). If the adiabatic invariat al pears to be marant

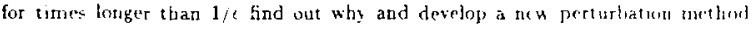
based on tlie reason. (section 5.4.2)

39. Find physical situations with more than one fast frequency in which Arnold's notion of "almost adiabatic invariant" (discussed in section 5.4.3) is really physically appropriate and apply the method of averaging keping track of the ineasure of the "trapped particles".

40. Study [Ott, 1979] and [Kubo et al. 1965] and develop a precise form of the argumeat sketched in section 5.4.4. Ott claims to be able to get an expression for the variation of the adiabatic invariant. Can his argument be made invariantly and hat are the limitations on its validity?

41. Section 5.4 .5 shows that the reduction for finite perturbations may not be unique. What are the physical consequences of this fact and what does it say about the result of chcosing different asymptotic scalings away from the unperturbed system?

42. Chapter 6 discusses the importance of making the unperturbed stytem consist of only periodic orbits when one wants to do singular perturbation theory. Many derivatious in the literatise do not have such an unperturbed systen ('come do not even have unpertu: jed dynamics). Can these systems be converted (say by a change of coordinates) to systems where secular perturbation theory is valid? If not. is the perturbation valid for time $1 / \%$. If it is valid. is there a fundanentally new perturbation meshed hidden in the derimation? 
43. Excend the analysis of gyrumotion in chapter 6 to threedirnenuional. tinfe-varying magnetic gennctries.

44. ("au one undersland the Hamiltonian structure of gyrokinetic equations as redurtion by a "gauged" circle action? (The way the formal structure of gyrokinetics arime from the single particle picture in rhapter 6 is very reminiscent of the way the eikowal theory of chapter 8 is related to strictly periodic waves.)

45. Suction 7.1 sketches heuristically the connection between Kruskal's secular perturbation theory and WKB theory. Can this connection be made precise using the coordinate-frec formulation of chapter 5 ?

46. Develop an analog of the local Fourier transferm of section 7.1.1.3 that is appropriate for nonlinear wave systems (i.e. given the wave family, reproduce the expression in terms of periodic solutions with slowly varying parameters).

47. Apply the variational approach to the Heiseruerg uncertainty principle given in section 7.1.3.1 to other inequalities (and so get new insights into them and perbaps new results).

48. Does the approach to coherent states in terms of momentum maps given in section 7.1.3.2 extend to larger groups (eg. the Heisenberg group semidirect produc the metaplectic group) to give approximations to the waye dyoanics which ara better than elassical mechanics (eg. iaclude the dispersion of the Gaussians in addition to motion of their enters in :hase space)?

49. 'The approach to roherent states given in section 7.1 .3 .2 does not need the base state to be a Gaussian. Can anything be gained by using "coherent stateb" Which art the orbits of states other than Gaussians under the Heisenberg group? 


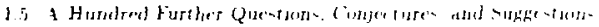

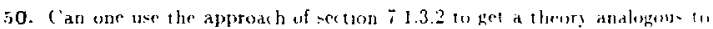
coberent hatce far monlinear equatum:

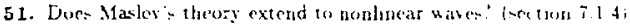

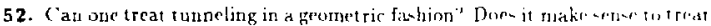
wave phaic space as a complex manifold and so treat evinerent wave" fohaferet 7)

53. Can dissipation be incorporated inte the geonetric $\mathrm{B} K \mathrm{~KB}$ picture?' fohap ier 7)

54. What is the time of validity of the WKB approximatiun? In it lie as conjectured in section 7.3 ?

55. Are there asymptatic theories with asyptotic validity for longer hmen than WKB, perhaps in special situations? (section 7.3 )

58. How does WKB theory' finite time of validity relate to intinite tume concepts such as the eigenvalue spectrum and quantum chans: (section 7.3 )

57. Are there physical situations in which the exatnple of dispersion given in section 7.3 that is no, accounted for by WhB plays an important plysual rols"

58. Is there an analog of gemetric difiaction theory for nonlinear cstoma! waves? (section 7.3 )

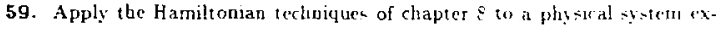

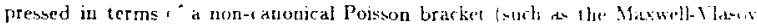
syctem) to obtain result = not available with Whothan - averaged Lagrangian tech-

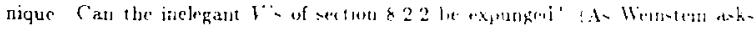
"Aint there nueleganter way wo do i+"*" 


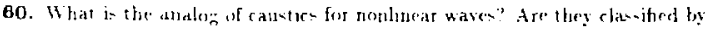

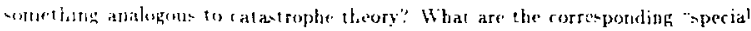

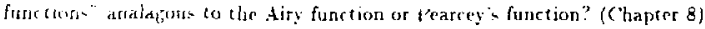

61. Maxe the argument guven in arection 8.2.2.1 relating degeberate symplectic -tructures to Proissun manifolds with Casimirs into a general theor. (a very similar siruation uscurs in the example of guiding center motion given in chapter 5 : In a Poisinon formulation we have a degenerate bracket perturbed by a canonical bracket. In a symplectic formulation we have a degeverate symplectic structure perturbed by a canouical one. In the Poisson case we get zero order dynamics but no unique choice of Hamiltonian or symmetry geverator. In the symplectic case we get a inique Hamjltonian but no zero orde. :-amics. The first order piece somehow defines a natural association between the degenerate zero order structures since the brackez and symplectic structure are uondegeuerate and are inverses of one another when (doesn't vanish.)

62. T'se the Lie Poisson structure obtained in chajter 9 to study a system where wave action density of phase apace is important (for examples see Dewar. $1972 b$,

63. Find examples of dissipa = systems that sit inside Hamiltonian systems as invariant submanifolds as suggested in section 10.1 (John David Crawferd and Bruce Bughosian have suggestel that the dissipation in Landau damping is of this $1: p^{*}+$

64. In if ever uneful to convert dussipative syeteme to Hamiloman ystems

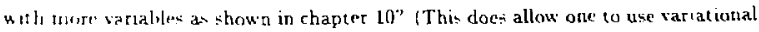


formidatsur) for inetatte :

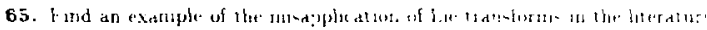

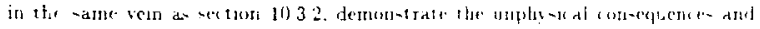
cortert the analy-s

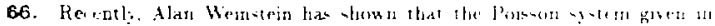
section 10.4 is a sperial cate of a very geared confouction (iven a lie groul, acting on a Poisen manifold, consider the action on the product of that manfold

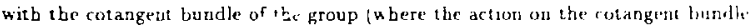
is the lift of left trauslation). Gun obtans a new bracket on the orbis spare of this action by reduction. When the group is the real line acting by the for of a vector field. this reduces to the example in 104 . Lse the more general brackets to underst and ot ber physical situations

67. Can one use the (very elever) technique used in Gromov's proof to get any insights into the physics of the projected area situations of chapter 11 "

68. Can the intuitive argumeut given is section 11.2 for (iromov's theorm based on the uncertainty principle be made precise?

69. Can one make the connection between Bogoliubor's derivation of the Boltzmann equation via BBGKY and projected measures that i-suggested in section 11.4 precise?

70. Can one uesign a better particl acceleratur bised on the abstraxt cont struction for slurinking projected meacure given tn sectinn 114.2 ?

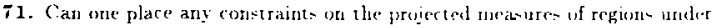

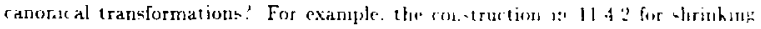




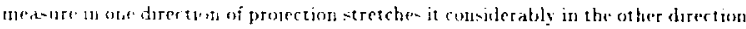

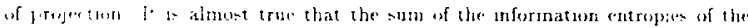

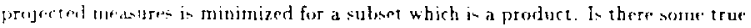

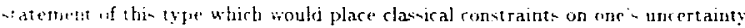
III Imak-urang paurs of projected quantitier"

72. (an you bind finite dimensional t it uation- where the time-reversible almost attractor int roduced in cbapter 12 is directly responsible for irreverible asymptotir behavior in a time-reversal symmetric situation?

73. Implement the approach to the threewave interaction of eikonal waves discussed tn section 13.3.6. Does this invalidate the usual approacb in terms of intinite plane waves?

74. Can one explicitly analyze the Landau damping equations using the notion of an almost attractor in an infinite dimensional Hamiltonian system? (section 13.3.3)

75. Section 13.3.5 introduced a very general mechanism for obtaining dissipative behavior from resonant Hamiltonian systems. Can one find an ahstract settiug in which the precise characteristics of systems exhibiting this phenomenon 1 1 e stated? Can one analyse Landau damping with these tecbniques?

76. Many infinite dimensional linear physical systems bave a continuous spectrum making afalysis difficult (especially bifurcation t,beory). The "eigenfunctions" are often singular for cxample the van Kampen modes in the Poisson-Vlasov systens. Whether the spectrum is continuous or not is physically detectable only aftrr an infinite time. Physically one often is not interested in "normal" modes hut 


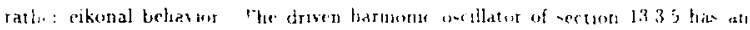

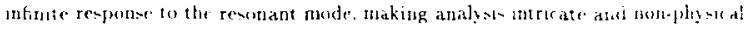
The cikonal analyse of sertion 13.3 .5 dom not $=$ fifer the defent can ane apply a similas analysis to other probleme of thw type'.

77. Can onc analyze the disspation due to bulk viscosty in t manner analu gous to that used in section 13.4? (Bulk viscosity results from the time ligg for the rotational degrees of freedom a gas to reach equilibrum with the fiuear degrees of freedom uder compression. Imagine a gas in a ryliuder with a pi-ion which you quickly move in and out. As you push down the gas bas a kigher pressure than it would in equilibrium since less energy is in the rotational degrees of freedom than should be. When you pull the piston out, the pressure is less han in equilibrum because more of the energy is in rotational degrees of frectom than should be. The net result is tbat the work done on the downstroke is greater than the work returned on the upstroke: the gas absorbs energy and therefore appears distipative. A very similar kind of thing is behind the dissipation due to the string. I

78. Can me use the method of Melnikor to find horithoes in situation- of plasma physics other than the situatiun studied in eection 14.4 feg. other mag-

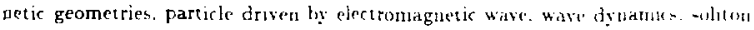
dynamics)?

79. Can one use the kno" n tatetical properties of hor whos studied in

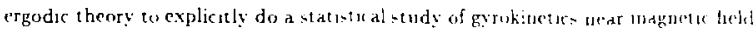
reversals? (mertuon 144 )

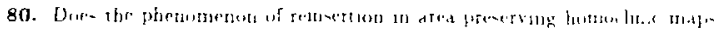




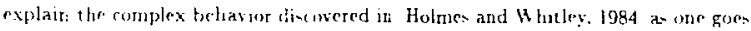

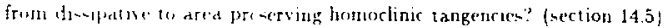

81. (a. you furl uriversal structure in tomoclinic tangles and honoclinic bufurcatob:- using renormalwat un group techaiques? (chapters 14 and 15 ) There "tre many renormalization type mappings at work in homoclinie dynamics. The

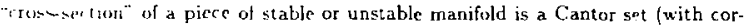
re-pondung scaling proserties) and homoclinic tangencies occur when the stable and uastable tnanifolds Cantor sets intersect. Eacb homoclinic tangency causes an intinite number of period duublings for which we know there is universal behavior.)

82. Study tbe fractal: that arisc :n gature using renormalization. For example. a classic (Canor set (remove the middle third of an interval recursively) corresponds to a periodic orbit as we continuously blow up the scale. A fat Cantor set (eg, remove mic.ile third. then middle vinth, then middle 27 th, etc.: this type of fractal ocrum as the set of parameter values at which hump map orbits are chaotic after criticality asymptotically approaches the stable fixed point represented by a solid line. An unilernourished Cantor set (eg. remove mildle third. middle square root of 3:d. middle cube root of 3rd,etc.) approaches the stable bxed point representing a single point in empty space. The set obtained by removing the rationals from the unit interyal and separating the two sides around each rational $p / q$ by a distance $1 / y^{3}$ (the hAMl tori lie in phase space like the points in this set in an arjitrary perturbation of a Hamiltonan system away from criticality) scales at each point arcurding testerms in the contunued fractuni expansion of the point (Liouville num.

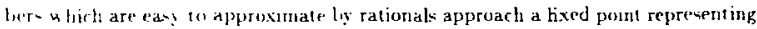




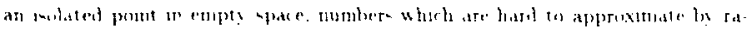

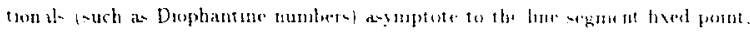

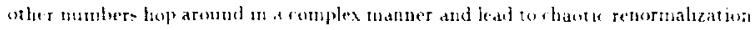
orbit-l (chapter 15$)$

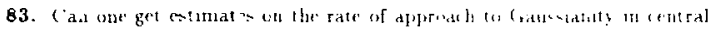

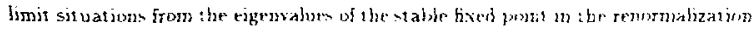
approacb of section $15 \mathrm{~s}$

84. Can one use the renormalization group approdich given in section 153 to derive the Boltzman factor $e^{-3 E}$ using rcuormalization?

85. Sirrilarly, can one derive the standard probability distributions ot her than Gaussians (such as Lorentzians, Zipfians, Bradford's distribution. Lotka's distribution. Pareto distributions, $1 / \int$ noise, $\log$ normal distributions and the other "fong :ail" distributions so important in modern statistical physics) usiug renormalıation but witb a renormalization operator constrained by other criteria than tiaving a prescribed norralization, mean, and dispersion? Do the reoormalization eigenvalues gife scaling rates for these examples: (section 15.3)

86. Some of the distributions of the previous question will correspond to unst able fixed points. Can we understand physical systems with statistics which behave according to one of these distributions for a long range uf scales but ayymptotically behaves diferently. in terms of the renormalization orbit starting pear the stable. manifold of the curresponding fixed point, approaching the hixed point for a lone, time. and finally feeling the effert of the umstable manifold and getting attracted

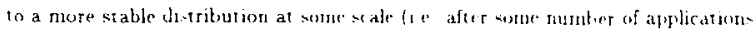


of the reroumdization operatur)". ("an we use the relative values of the renormalizalof agenwalum to pretict the range of wales which are described by the fixed pome? I An example of thit type of behavior occurs in any of the extremely lange

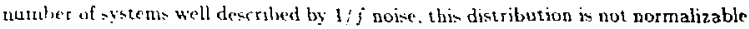

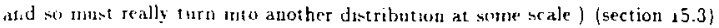

87. Find "posr man" approaches to other renormalization carslations, such LF area-jresetving periou doubling, breakdown of circle maps, breakdown of KA.M tori. \{sertiog 15.4 \}

88. [ee the new formulation of maximum cntropy given in section 16.2 .3 in terms of an integral over all probability distributions to get new insights into statistical nechanics, just as the path integral formulation gave new insights into quantum mechanics ISchulman. 1981!.

89. Fothing in the inaximum entropy formulation (scetion 16.2) either requires c- disallow time-dependence of the studied quantitics. [Jaynes, 1983] derives some aspects of non-equilibrium thermodynamics from the maximum entropy criterion. Can one use this derivation and the symplectic structures introduced in chapter 16 to obtain a symplectic analysis of non-equilibrium thermodynamies?

90. The Onsager relations bave been discussed in terms of Lagrangian subman ifolds in [Abraham and Marsden, 1978]. Can this discussion be given a fundamental basis using the ideas of the last question?

91. Allan Kaufman has discovered many non-equilibrium systems whose evolution is guverned by a Lie Poisson bracket plus a so-called dissipative bracket which i- -ymmetric The generator of the dynamics becomes the Hamiltonian plus the en- 


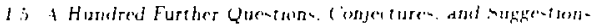

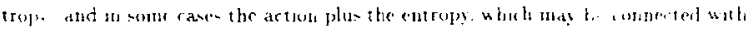

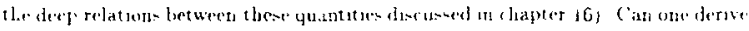

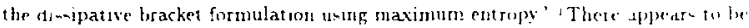

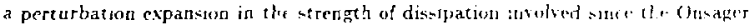
zelations apprar naturally.)

92. Can one introduce "super-reduction" which starts with + large-dimesintorial underlying system, perform reduction by an exact symuet ry w soute variablem, redurtion by an approximate ymmetry it some others, and retuction by a "statistical symmetry" (via maximum ent ropy) in some others, aud end up with ahe standard physical models a plasma physics, gas dynamics and Huid mecbanics?

93. Based on the results of the last few question. can une obtain a geornetrir forrulation of the fuctuation-dissipation thcorem? Is this a statistical analog of the $K-$ theorem".

94. Can one connect the approach of the lant for questions with the test particle theorem and Ead a treatment of this technique an a systematic pertarbation technique?

95. There is a very "symplectio" looking reciprority that artses from thr test-particle theory tbere is $z$ detailed balases betwere cirenkes radiation and Landau damping. between syebrotron emission and colotron damping and betwecn Bremsetrahlung and collisional damping Can one understand the repere" $"$ within a ge'meral theory"?

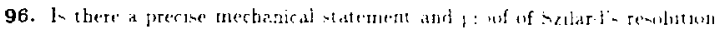

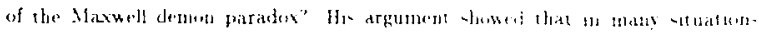


une - mét

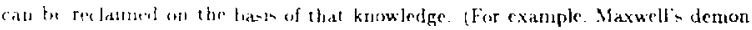
mabht wh-ers the oraterisg of a non-equilibrium photon to detert fast particles

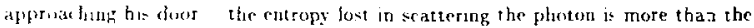
entrops araned in forcmg the fast par.ele to be on une side of the partition.) This argunem is sery similar th the maximum entropy version of the second las of thermoly anme given in section 16 A Can the connection be made precise? What is t.le rciation to projected arca concepts discussed in chapter 11 ? What is the cennection Hith quantum measurernent limitations?

47. Is Young : infuluality for the Legendre transform (sertion 16.5) related to the Heisenterg uncertainty principle (section 7.1 .3 .1 ) in the eikonal limit?

98. Hux much of the geometric theory of first order phase transitions given in section 168.2 extends to the rnore difficult situations where renormalization theory is required (ers. critical points)?

99. Ipply the extension of Maxwell's equal area rule for first order phase irdnations given in section 16.8 .3 to nontrivial problems.

100. Is there a deep reaton for the remarkable parity between the asymptic structure of eikonal wave theory and statustical mechanics as presented in section $109^{\prime \prime}$ 


\section{PART I:}

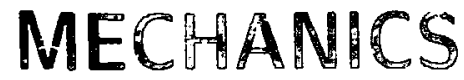

$\because$. he couched his discussion in the mout sophistrated language bnoun to

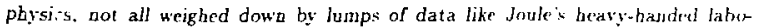
ratory reports, oor confined to the primitive numencal equivalent of Haytr. bist in the graceful, taut, acd lissome differential equations of classical dymanims.." Gillespie referring to Helmholz /Hirsch, 1984" 


\section{Chapter2:}

Survey of Geometric

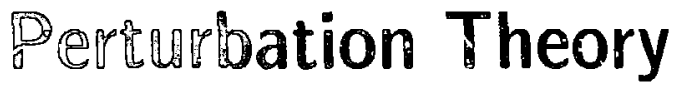

-I completed my conro in enginering and I would like to try to explain the effert of this euginecring training on me. Previously, I was interested only in exact equatings. It seened to we that if one worked with approximations there was an intoler able ugliness in ones's work and I very mucb wanted to preserve mat bematical beausy: Well. the engineering trining wbicb $I$ received did teach me to tolerate approxinations and $I$ Has able to set that even theories based upon approximations could have a considerable amonnt of beauty in them"-P.A.M. Dirac (p. 112 of (Dirac, $\left.1, \because 7_{j}^{j}\right)$

\subsection{Hibturical Background}

In this chapter we survey ideas finm the rest of the thesis, particularly chapter 4. intuitively and beuristicaily. In chapter 4 we assume a background in geometric nech-aits and give detailed proofs. Here we will give the Havor of the siructures and develop the needed background material. We will state results and indicate why they $i$. true without detaled pronf. He begin with some introductory remarks, discus: a geometre picture for non-singular perturbation theory. introduce the needed 


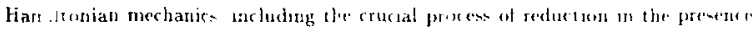

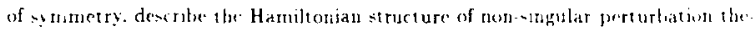

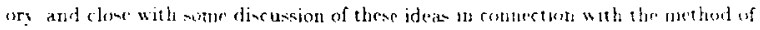

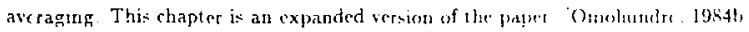

it is of interest to le:1 the seminal idea- that form the backeround of the present work. In 1808. Lagrange introduced the description of the rymamics of cele.tial bodies in terme of what we today call Hamilton is equatome ( Lagrange, 1808 atnd (Weinstein, 198:'). His motivation was the reduction of the enormous fabme involved in a straightforward pert urbation analysis, which required tedious computations to he performed on each component of the dyuanical vector beld. to manipulations of a single function: the Hamiltonian. The description in terms of Lagrange bracket: led to several other bencfits. Las" wnge showed that the value of the Harniltonias and the structure of the brackets were both invariant under the dysamic - leading to a useful cherk of the complex calculations (which at that tume were of courede dene by hand). In addition. he was able to show that the imvariame is the Hamilems. tould be used to prove the stability of cert in equilibria A-the century pregrement

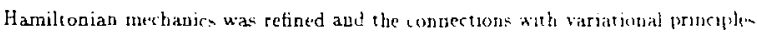

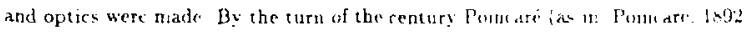
had developed very powerful Aamilonian perturbation methods molizing generat-

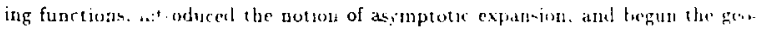

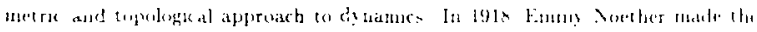

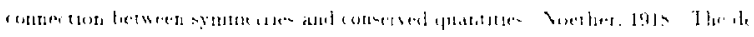

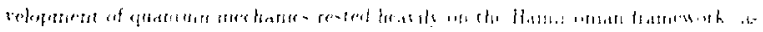




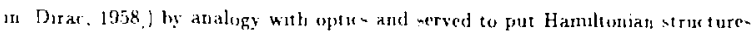

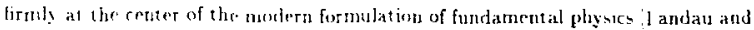

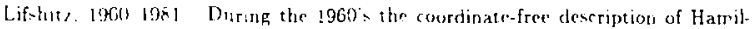

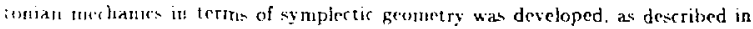
Hermanu. 1966. Sourian. 1970a. Abrabarl and Yarsden. 1978 and Arnold. 1978. About the tume the method of Lic transforms greatly simplitied Hamilinnall perturbatm theory Cary. 1981. The 1970's waw enormous developments u ithe genmetric approach to mechanir ind lasgely as a result of thesc. an ever wider rasıge of physical systems bave been described in Hamiltonian terms. Some

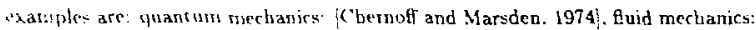

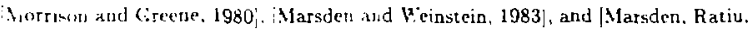
and Weinstein. 1984. Marucll s equations: Pauli, 1933! and Massden and Weinstein. 1992. the Maxwell-Vlasor and Poisson- Vlasor equalions of plasma pbrsics: Morrison. 1980. Marsden aud Wriastein, 1982;, and [Kaufman. 1982], relativis. tic plastla wates Kaufman and Holm. 1984, gyrokinetic models: Kaufman and Uoghokian. 19d4, elastrity theory. Marsden and Hughem, 1983; and (Hoim and

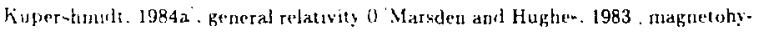

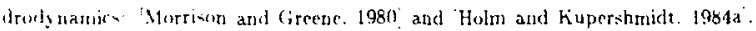
multifluic plismas Spenerer and kaufman, 1982' and Holm and Kupershmidt. 1984a . chromutygdrodỵnatnics: !Gibhous. Holm and Kupershmidt, 1982., superfu-

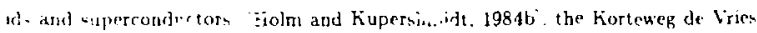
englation Faddeev and Zakharov, 1971', etc These developnents have shed light

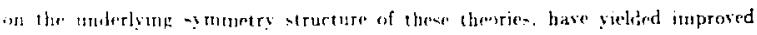




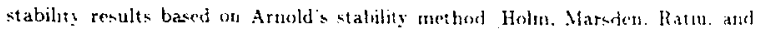
Heinstem. 1984, and have given instgt into the reano for the mestatility af certair, - - atoms, Guillemin and Sternloerg. 1984'.

For the most part. however. these structures describe fundamertal underlyng model $=$ in the various fields. In actual applications wr almost alsays make numerous approximations which may or may not respect the underlyial Hamiltonian structure. It is folklore within the particle physies community and elsewhere that perturbation methods which respect the underlying symmetrics and conservation laws yield much better approximations to the actual system than ihose which do not. It is of interest. then. to try to do perturbation theory within the Hamiltonian framiswork and to obtain structures relevant to the approximate system. Cne may thus hope to understand the relation between the structures of systems which are limiting cases of know' systems (cg. docs the KdV Poisson bracket arisr nat urally from that of the Boussinesq equation:?) [Olver, 1984! The history of Hamitonian mechanics is inextricabl t tied to perturbation methods. For the most part, though. the Hamiltonian strueture was used to simplify thr. perturbation method and the geometric structure of the perturbation method itself was not explored. Win bave found in several examples that taking this structure iuto account leads to simplitications (as in the problem of guiding center motion discussed later in thin-chapter) and to deeper insight into the approximate system (as for the modulational equations for waves in th, eikonal limit studied i:a [Omohundro, 1984c] and chapter 8 )

We have therefore been engaged in a progran of iusestigating the Hanilonian structure of the various perturbation theories used in practice. In the rhaper 
we describe the geometry of a Hanilionan structure for non-singular perturbation theory applied to Haniltu lian stotems on symplectir manifolds and the connection with sing 1 lar perturbation techniques based on the method of averagine Chapter 5 discunew a singular pesturbation technique based on a method introduced by Kru-kal 


\subsection{Feometric Perturbation Theory}

In the spetion we will place perturbation theos unte the context of the gino metre dynamies that has proven so fruitíul in recent years We will give intuiture discussions of the geornetric concepts of dyuamics and explicitly put non-singular general fist-order perturbation theory int this framework. The next section will do the same for higher order perturb:tion the sry and chapters 4, 5, and 6 will for us on Hamiltonian and singular perturbations.

\subsubsection{Manifolde}

The modern setting for describine an evolving system is that of a a dynamical system. The state of the system is represented by a point in a manifold $M A$ manifold is a space which locally looks like Eurlikean space and in which there is a notion of derivative (for more details sec Abrahan. Marse'i. and Ratiu. 1983 p. 122). Globally a manifold may be counected tugethe: in a unotrival way. as ocrurs In the examples $0^{-}$the sphere and the lurus

Many of the standerd systems studied in physe- have state ppaces that are naturally manifolds and have apparent singularitie when on irin. to model them at Euclidean spaces. A simple example of this in given he the rugd hods The standard deseription of the configuration of a rigul body ntitien the Euler angles

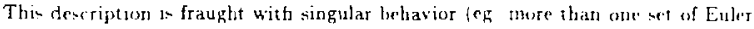

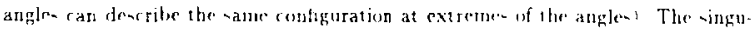

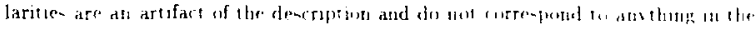




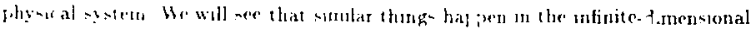

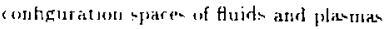

\subsubsection{Dynqmical Systems}

"Among all mathematical disciplines the theory of differential equations is the most important.. It furnishes the explanation of all those elementary manifestations of nafure which invoive time."--Sopbus Lie (1895) [Hirsch, 1984]

If you know where you are in a state space, a dynamical law tells you where you 'r. going.

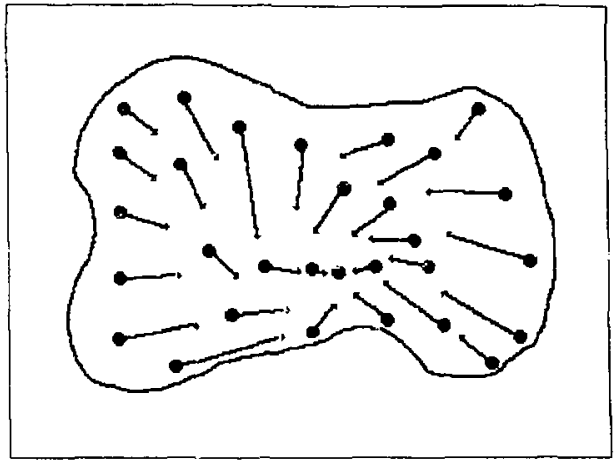

Figure 2.1: A dynamiral sytem 


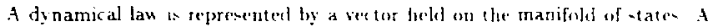

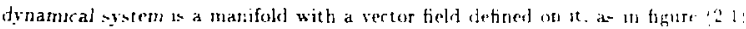

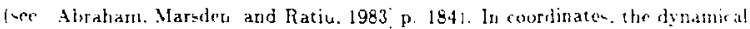

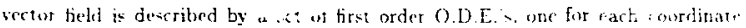

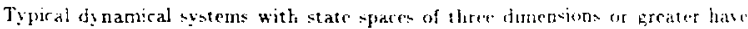
chantic dynamical belavior with extremely cemplicated trajororios In nany awe ode can actually prove that there is an exact description of the solution curve in closed form (sec [Gurkeracimer and Holner. 1983;). If the erolution simplifies then there is some physically relevant special feature, wuch at a symmetry, which rauses the simplification.

\subsubsection{Perturbation Theory}

In important physical applications. we often hud ourselves close to a system which simplifies, and we are interested in the effect of our deviation from it. He express this deviation in terms of the small pararueter 6.

In many physical situations we are faced with an apparently different piohlom in which we have but a sizgle dynamical system and wo are interested in solutions whose initial conditions are close to a known equilibrium point. The parameter - expresses the distance of the initial condition from the equilibrium point One common situation of this type, which appears repeatedly in plasma phyich, his thermal equilitrium as the equilibrium point and studies the time evolution uf deviations of initial sizc e from it. 
We may easily convert this type of problem with asymptotic initid conditions in an unsolvable dynamical aystem, to an asy mptotic system whose liniting cage is solvable. This transformation is commonly made by expanding the dynanical vertur field r.bout the equilibrium and re-expressing the dy namics in terms of scaled quantitie: This leads to a lincar system at tbe lowest order, which is often solvable.

Because thernal eqrilibrium is stable, this lowest order linear evolution is given by (pussibly damped) oscillating nornal modes (i.e. there can be no unstable modes in tbertnal equilibrium). These often take the form of travelling waves. The second order terms in e represent two-wave coupling, the third order terms represent threewave crupling and so on.

To keep a simple model in mind as we proceed, let us focus on the excitation of s. single mode which nonlinearly couples only to itself. This reduces to a system of the form

$$
\ddot{u}=f(u) \text {. }
$$

If $u=0$ is to be the cquilibrium solution of interest, then we bave $f(0)=0$. Let $u$ assurne that $f$ is an odd function in $u$, since this is a common occurance. To write our equation as a dynamical system, we introduce $v \equiv \dot{u}$ and so obtain the system

$$
\dot{v}=v \quad \dot{v}=f(u) .
$$

We want to study the evol itior. of small values of $u$ and $t$, so we choose an asymptotic initial condition:

$$
u(t=0)=\epsilon u^{0} \quad v(t=0)=c v^{0} .
$$


We now have a hard problem with an initial condition that aymptotically approarbes an intitial condition whose evolution we can solve for We want to coavert this to a family of problems that asymptotically approach one withe we can solve. Let us introduce scaled variables.

$$
L^{t} \equiv \frac{u}{c} \quad V \equiv \frac{v}{c}
$$

The in tial condition in terms of $U$ and $V$ is constant:

$$
U(t=0)=u^{0} \quad V(t=0)=v^{0} .
$$

The dynamical system in the new variables bas become an asymptotic family:

$$
\dot{U}=V \quad \dot{V}=\frac{f(c U)}{c}
$$

The limiting system as $c \rightarrow 0$ is

$$
\dot{U}=V \quad \dot{V}=f^{\prime}(0) \cdot U
$$

which is linear. If we express $f$ as an asymptotic series (and remember that $f$ is odd), then we see that our system is asymptotically equivaleat to

$$
\dot{U}=V^{\prime} \quad \dot{V}=f^{\prime}(0) \cdot C+\frac{1}{6} c^{2} f^{\prime \prime \prime}(0) \quad U^{3}+\ldots
$$

If we redefine $c, u$, and $t$ and assume that higher order ternus ; anish. then this system reduces to the Duffing equations for a nonlinear spring:

$$
\dot{i}=v \quad \dot{v}=-u-c u^{3},
$$

with initial conditions that are independent of c, say for example

$$
u(t=0)=G \quad v(t=0)=0 .
$$


We shall usc this system as an ilustrative example as we proceed. Let us return Dow to the abstract settirg which we have just motivated. The class of problems which require study of a small seighborhood of an equilibrium leads to linear zeroth order equations. Examples like gyromotion bave nonlinear zerotb order equations which are nonetbeless solvable due to symu.tries.

In general, we are given a dynamical system of the form

$$
\dot{x}=X_{0}+c X_{1}+\frac{c^{2}}{2 !} X_{2}+\cdots
$$

in terms of the vector fields $X_{2}$ with initial conditions described by

$$
r(c, t=0)=y(c)
$$

We attempt to express the solution as an asymptotic geries in 6 :

$$
x(t)=z_{0}(t)+c x_{1}(t)+\frac{c^{2}}{2 !} x_{2}(t)+\cdots
$$

Choosing coo:dinate $x^{a}(1 \leq a \leq N)$ in a local patch of the staie space manilold and plugging this agsumed asymptotic form into the eyuation of motion gives

$$
\begin{aligned}
\dot{x}_{0}^{a}+c \dot{x}_{1}^{a}+\frac{c^{2}}{2 !} \dot{x}_{2}^{a}+\cdots= & X_{0}^{a}\left(x_{0}+c x_{1}+\frac{c^{2}}{2 !} x_{2}+\cdots\right)+ \\
& +c X_{1}^{\mathbf{a}}\left(x_{0}+c x_{1}+\frac{c^{2}}{\tau_{1}} x_{2}+\cdots\right)+ \\
& +\frac{\epsilon^{2}}{2 !} X_{2}^{\mathbf{a}}\left(x_{0}+\epsilon x_{1}+\frac{\epsilon^{2}}{2 !} x_{2}+\cdots\right)+\cdots
\end{aligned}
$$

Asymptotic expansions are unique (see for example (de Bruijn, 1981i), so we can 
equatc coefficients of equal powers of $t$ to get equations for $x_{n}, x_{1}, x_{2}$.

$$
\begin{aligned}
\dot{x}_{0}^{a} & =X_{0}^{a}\left(x_{0}\right) \\
\dot{x}_{1}^{a} & =\sum_{b=1}^{N} \frac{\partial X_{0}^{a}}{\partial x^{b}}\left(x_{0}\right) \cdot x_{1}^{b}+X_{1}^{a}\left(x_{0}\right) \\
\dot{r}_{2}^{a} & =\sum_{b, c=1}^{N} \frac{\partial^{a} X_{0}^{a}}{\partial I^{b} \partial} \frac{\partial}{x^{c}}\left(x_{0}\right) x_{1}^{b} x_{1}^{c}-\sum_{b=1}^{N} \frac{\partial X_{0}^{a}}{\partial z^{b}}\left(x_{0}\right) I_{2}^{b} \\
& +2 \sum_{b=1}^{N} \frac{\partial X_{1}^{a}}{\partial I^{b}}\left(x_{0}\right) \cdot x_{1}^{b}+X_{2}^{a}\left(x_{0}\right)
\end{aligned}
$$

If $y(t)=y_{0}+c y_{1}+\frac{e^{J}}{2} y_{2}+\cdots$ is an asymptotic expansion for the initial condition $y(c)$, then the initial conditions for these equations are

$$
x_{0}(t=0)=y_{0}, \quad x_{1}(t=0)=y_{1}, \ldots
$$

The Duffing equations yield the following equations by this prescription:

$$
\begin{array}{ll}
\dot{u}_{0}=v_{0} & \dot{v}_{0}=-u_{0} \\
\dot{u}_{2}=v_{1} & \dot{v}_{1}=-u_{2}-u_{0}^{3} \\
\dot{u}_{2}=v_{2} & \dot{v}_{2}=-u_{2}-6 u_{0}^{2} u_{1}
\end{array}
$$

with initial conditions given by

$$
\begin{array}{ll}
u_{0}(t=0)=0 & v_{0}(t=0)=0 \\
u_{1}(t=0)=0 & v_{1}(t=0)=0 \\
u_{2}(t=0)=0 & v_{2}(t=0)=0
\end{array}
$$


If the Duffing model is itself a trupcation of a system with a more general nonlinearity, theu it may not be meaningful to carry out the perturbation analysis to too many orders.

These equations immediately raise a number of questions. They are defined in

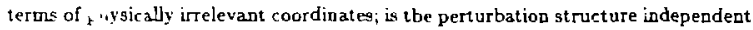
of these coordinates? If the original equations are Hamil thonian, ar? these equations? In $J$ th order perturbation theory, bow are we to interpret this evolution of many variables $I_{0}, I_{1}, \ldots, x_{J}$ ? One goal of this work if to answer these questions.

\subsubsection{Flrst Order Perturbation Equetions}

Let us turn to the geometric interpretation of these equations. It is easiest to underst and the first order perturbation equations.

For the Duffing example, the first order system is

$$
\begin{aligned}
\dot{u}_{0}=v_{0} & \dot{v}_{0}=-u_{0} \\
\dot{v}_{1}=v_{1} & \dot{v}_{1}=-u_{1}-u_{0}^{3} \\
u_{0}(t=0)=a & v_{0}(t=0)=0 \\
u_{1}(t=0)=0 & v_{1}(t=0)=0 .
\end{aligned}
$$

In general, the first order equations have the form

$$
\begin{aligned}
& \dot{x}_{0}^{a}=X_{0}^{a}\left(x_{0}\right) \\
& \dot{x}_{1}^{a}=\sum_{b=1}^{N} \frac{\partial X_{0}^{a}}{\partial x^{b}}\left(x_{0}\right) x_{1}^{b}+X_{1}^{a}\left(x_{0}\right) \\
& x_{0}(t=0)=y_{0} \quad x_{1}(t=0)=y_{1} .
\end{aligned}
$$

We would like to determine the geometric nature of the ruant ties $x_{0}$ and $x_{1}$. To understand what we mean by this, lut us recall the relationship betweed geometric quantities and coordinates. 


\subsection{Functions, Covectora, and Cotangent Bundlee}

A function on a manifold is an intrinsically defined thing; it assigus a real number to each point of the manifold. A coordin ate system on a region of an $N$ dimensional manifold is a collection of $N$ real valued-functions $x^{1}, \ldots, x^{N}$ defined on that region, whose differentids are linearly independent ' . each point. In these coordinates, the gradient of a function is a collection of $N$ numbers: the derivatives with respect to each of the $x^{\circ}$. Geometrically, however, it is wrong to think of tinese as just real nurbers, because they change if we change our coordinate sysiem. For example, if we choose coordinates whoe values at each point of the region are twice those of $x^{1}, \ldots, x^{N}$ then the components of the gradient of a function are halved We introduce a geometric object whose relationahip to the manifold at a given point is lise that of the differential of a function and we call it a covector or one-form (see ¡Abraham, Marsden, and Ratiu, 1983| p.286). In this context, the gradient is usually refered to as the differential of the function. The collection of all covectors at a point is defined to be the cotangent space at that point and the collection of all cotangent spaces taken together form the cotangent bundle (see [Abraham. Marsden, and Ratiu, 1983 p. 285)

\subsubsection{Vectors and Tungent Bundles}

Similarly, the values of the components of a vector at a point are doubled when we double the values oi the coordinates. All vectors at a point taken together form the tangent space at that point and all tangetat spaces taken together form the tangent bundle $T M$ of $M$ (see [Abraham, Marsden. and Ratiu, 1983) p, 150). 
Pictorially, we think of a vextor as a little arrow whose end is at the point of interest as in figure (2.2a). A covector may be thou zht of as a pair of parallel planes representing local level sets of a function whose gradient is that covector ( [Misner, Thorne, and Wheeler, 1973 and [Burke, 1980|) as in figure (2.2b). The distance between planes gets smaller as the gradient gets larger so that the amount of a vector starting at the fust plane that is cut of by the second plane is independent of the scale (and so defines an invarias te pairing between vectors and covectors). Covectors have been referred to as lasagna vectors because of this picture (Jim Napolitano, private commugication).

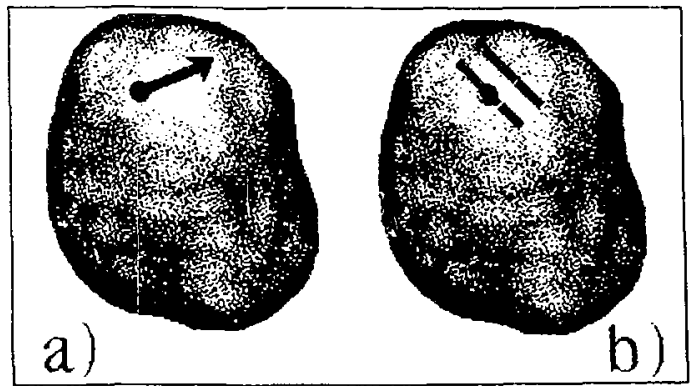

Figure 2.2: a) A picture of a vector, b) A picture of a covector.

Vectors and covectors are different objects when we consider more than one coordinate system, even though they both have $N$ components in any given system. If we have two curves in a manifold through a given point, in ary coordinate system 
Fe may determine if the curves go through the point at the same rate and in the same direcriou (i.e. if they are targent to frst order). It turo out that this determination is independent of which coordinatey are used. In differential geonuctry, one usually defines a tangent vector at a point to be an equivalence clase of curves which are tengent to first order. The invariant pairing betpeen vectors and covectors is then used to define cove tors as elements of the dual space to the tangent space at a point. The dual space $V^{*}$ of a vector space $V$ is the vector space of linear functions on $\boldsymbol{V}$. Our interest here will be to find out whether the quantities $x_{0}^{a}, \ldots, x_{j}^{\circ}$ for $1 \leq a \leq N$ bave any geometne structure that is independent of a given coordinate system

\subsubsection{The State Space for Flrat Order Perturbation Theory}

Int uitively, the first order quantity $x_{1}$ represents a small deviation from the unperturbed quantity $x_{0}$. Because $x_{0}$ can vary over the whole manifold $M$, we expect it to tepresent a point in the manifold. As $\epsilon$ gets smaller, $x_{0}+\epsilon x_{1}$ approaches the point $x_{0}$. The variable $x_{1}$ measures the first order rate of approach to $x_{0}$. Two different paths in the manifold approaching the point $x_{0}$ as $c$ approaches zero bave the same $x_{1}$ if and only if they are tangent at $x_{0}$. This, bowever, is the defining criterion for a vector at the point $x_{0}$. We thus expect $x_{1}$ to lie in the tangent space to $M$ over the point $I_{0}$. The $\left(I_{0}, I_{1}\right)$ dynamirs then lakes place in the tangent bundle $T M$. We will describe this dynamics on $T M$ intrinsically in ter:ns of vertor fields derived from $X(\epsilon)$ on $M$. 


\subsubsection{Flows and Derivatives}

The solution of a system of O.D.E.'s tells us the state at each time $t$ of a system which began with each initial condition. Geometrically, this is a mapping of $M$ to itself for each $t$. If the solution doesn't run off the manifold, then the uniqueness and smootboess of solutions with given initial conditions telis 19 that this map is a diffeomorphism (i.e. a smootb, 1-1, osto map with smooth inverse, A Abraham, Marsden, and Ratiu, 1983] p. 102). This one-paraneter family of diffeomorphisms labelled by $t$ is called the flow of the dynamical vector field (see [Abraham, Marsden, and Ratiu, 1983] p. 185). As $\epsilon$ varies, the corresponding flows of $X(\epsilon)$ will vary. Perturbation theory describes that variation. Any time we have a mapping / from one manifold to another, we may define the derivative map $T f$, called the tangent of $f$ (see [Abraham, Marsdez, and Ratiu, 1983| p. 153). This is a map that takes the tangent bundle of the first manifold to the tangent bundle of the second. It descr.bes how infinitesimal perturbations at a point are sent to infinitesimal perturbations at 'he image point. In coordinates, it acts on the tangent space at a point via the Jacobian matrix of $f$ at that point.

\subsubsection{Dynamice for Flrat Order Perturbation Theory}

Let us denote the flow of the unperturbed vector feld $X_{0}$ by $\tau_{0}(t), \tau_{0}\left(t, y_{0}\right)$ is the point to which $y_{0}$ has flowed in time $t$ under $X_{0}$. A small perturbation in $M$ trom a given orbit will evolve ander $X_{0}$ according to the derivative of this flow: $T x_{0}(t)$. This derivative is itself a flow on the manifold $T M$. The vector field of 
Fibl. th is the fiow may be fritten

$$
\left.\dot{X}_{0} \equiv \frac{d}{d t}\right|_{t=0} T x_{0}(t)
$$

$\tilde{X}_{0}$ is a vector field on $T M$. defined sithout recourse to cuordinates, that repi segts the effect of the unferturbed flow on perturbed orbits. In coordinates, $\bar{x}_{0}$ bas compotents given by

$$
\begin{aligned}
& \dot{x}_{0}^{a}=X_{0}^{a}\left(x_{0}\right) \\
& \dot{x}_{1}^{a}=\sum_{b=1}^{N} \frac{\partial X_{0}^{a}}{\partial x^{b}}\left(x_{0}\right) \cdot x_{1}^{b} .
\end{aligned}
$$

These dynamical equations represent exactly that part of the perturbation equations (2.20) which depends on $X_{\mathrm{C}}$ -

For the Duffing example, these equations give

$$
\begin{array}{ll}
\dot{u}_{0}=v_{0} & \dot{v}_{0}=-v_{0} \\
\dot{u}_{1}=v_{1} & \dot{v}_{1}=-u_{1} .
\end{array}
$$

The last two of these describe the evolution under the zero order equations of a litcle perturbation along $\left(u_{1}, v_{1}\right)$. We now sez that $\left(u_{1}, v_{1}\right)$ gives the coordinateg of a tangeat vector based at $\left(u_{0}, v_{0}\right)$. The $\left(u_{1}, v_{1}\right)$ equations are of the same fortin as the $\left(u_{0}, v_{0}\right)$ equations. This is because the zero order system for the Dufing oscillator is linear and the derivative of a linear $r$ ap is the identity when se identify the linear space with its tangent space.

The part of the dynamirs which depends on $X_{1}$ may also be defued intrinsically For any $x \in M$ and $v \in T_{x} M$, we define

$$
\left.\tilde{x}_{1}(v, r) \equiv \frac{d}{d t}\right|_{l_{1-0}}\left(v-t x_{1}(x)\right)
$$


The $\vec{x}_{1}$ dyuamics for the Duffing system is

$$
\begin{array}{ll}
\dot{u}_{3}=0 & \dot{v}_{0}=0 \\
\dot{u}_{1}=0 & \dot{v}_{1}=-u_{0}^{3} .
\end{array}
$$

This represents the additional effect of the perturbed equation witbout including the effect of the unperturbed system.

The entire first order perturbation dynamica on $T M$ is given by

$$
\tilde{\boldsymbol{X}}_{\mathrm{u}}+\hat{\boldsymbol{X}}_{\mathbf{l}}
$$

We bave therefore succeeded in finding a geovetric, coordinate-free inter retation for first order per arbation theory. 


\subsection{The Geometry of Jth Order Perturbation Throry}

Wis now would like to extend this picture to bigher orders The gmonetric object that arises is called a jet. To underatand the setting. we dsscuss a number of selevant spaces.

\subsubsection{The Patb Space}

How are we to think of the exact equation for the evolution of an e-dependent point $x(\epsilon)$ under $\epsilon$-dependent evolution equation: $I(\epsilon)=X(\epsilon, x)$ witb $\epsilon$-dependent initial conditions $y(\epsilon)$ ? It is useful to think of the $\epsilon$-dependent point $x(t)$ as a curve in the space $I \times M$, where $I$ is the interval (say $[0,1])$ in which 6 takes its values (as in figure (2.3)). We ehall call such curves paths (as this is the standard mathematical terminology). Moving aloug a path corresponds to varying the asymptotic parameter 6 .

Ir we think of $x(\epsilon)$ as a map from $I$ to $M$, then the curre is the graph of this map. The dynamical vector field $X(c)$ naturally lives on $I \times M$ and its $I$ component is zero everywhere. The flow of $\boldsymbol{X}(\boldsymbol{c})$ on $I \times M$ takes paths to paths by letting each point of a path move with the flow as in figure (2.4). Our initial conditions are represented by paths (if they are independent of $\mathrm{f}$ then they are straight lines). The true dynamics takes patbs to paths. Even if the intial conditions are t-independent, the $c$ depcodent dynamics bends the path over as in figure (2.5).

Thus we really should think of our dynamics as living on the infinite dimensional patb space

$$
P_{1} M \equiv\{\text { space of all paths } p: I \rightarrow I \times M \text { of the form } p: t,(c, x(c)\}\} \text {. }
$$




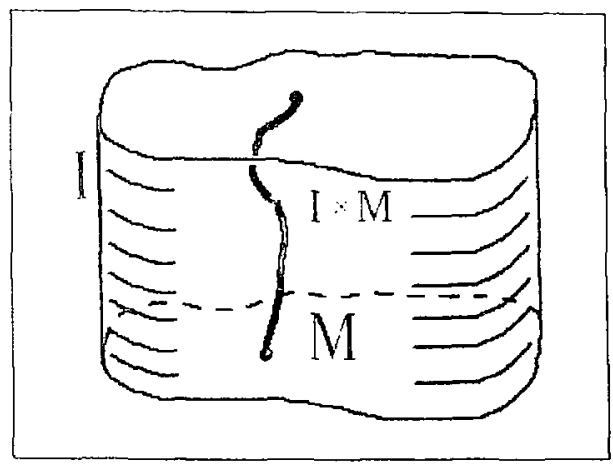

Figure 2.3: Curve in $I \times M$.

where, as before, $I=[0,1]$. For the Duffing example, this is the space of curves in $(u, v, c)$ space that project diffeomorphically onto $c$. Each curve represente $u(c), v(c)$ for $\epsilon$ in $I$.

This space projects taturaliy onto

$$
P_{0} M \equiv\left\{\text { equivalence classes in } P_{1} M \text { where } p_{1} \sim p_{2} \text { iff } p_{1}(0)=p_{2}(0)\right\}
$$

The projection sends a curve to its $\epsilon=0$ endpoint which represents the point about which the perturbati n is taken. $P_{0} M$ is naturally isomorphic to $M$ and represents the domain of the unperturbed dynamics. The equivalence classes forget al] perturbation information and only remember behavior at $c=0$ as shown in figure (2.6). 


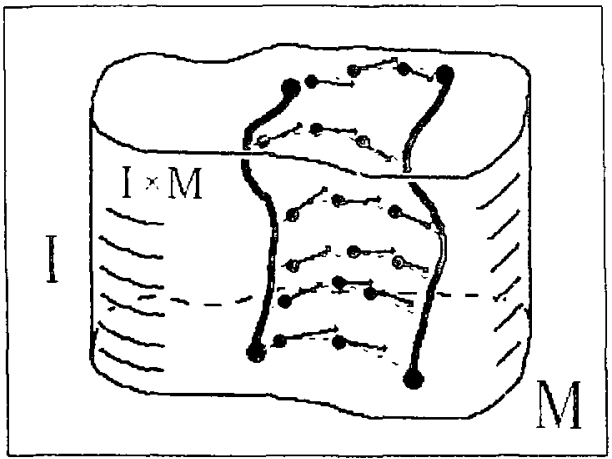

Figure 2.4: A patb moving under the influence of a vector field $X(\epsilon)$.

\subsubsection{Spacea of Shorter Pathe}

We are interested in spaces through which this projection of actual to unperturbed dynamics factors fi.e. spaces which are the image of a projection from the first space and the domain of a projection to the second space, such that the composition of these two projections gives the original projection from the first in the second space). Perturbation theory tries to study behavior infinitesimally close to $t=0$ without actually getting there. For each $0 \leq \alpha \leq 1$ we may defne

$P_{\mathrm{a}} M \equiv\left\{\right.$ equivalence classes in $P_{1} M$

$$
\text { where } \left.p_{1} \sim p_{2} \text { iff } p_{1}(\epsilon)=p_{2}(t) \forall 0 \leq \varepsilon \leq n\right\} \text {. }
$$

Thege allow us to consider more and more rest ricted domains of $t$, but there is always 


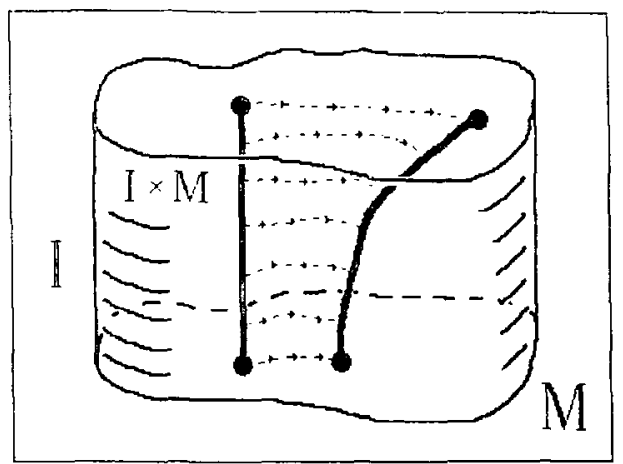

Figure 2.5: An 6 -independent initial condition becoming f-dependent.

a continuum of $e^{\prime} s$ to traverge before reaching $\epsilon=0$. For each $1 \geq \alpha_{1} \geq \alpha_{2} \geq 0$ we bave the natural maps

$$
P_{1} M \rightarrow P_{a_{1}} M \rightarrow P_{a,} M \rightarrow P_{0} M
$$

\subsubsection{The Space of Germs of Paths}

We are interested in structure between "even the smallest $P_{\alpha} M$ witb $\alpha \neq 0^{n}$ and $P_{0} M$. We may introduce germs of paths:

$$
\begin{aligned}
G M \equiv & \left\{\text { equivajence classes in } P_{1} M \text { where } p_{1} \sim p_{2}\right. \text { iff } \\
& \left.\vdots \alpha_{12}>0 \text { sucb that } p_{1}(c)=p_{2}(c) \forall 0 \leq \epsilon \leq \alpha_{12}\right\} .
\end{aligned}
$$




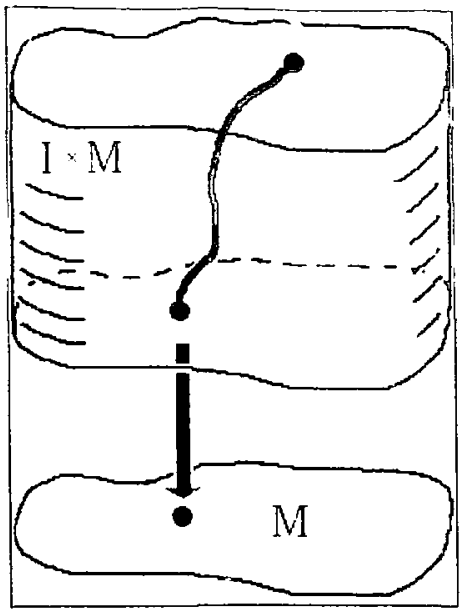

Figure 2.6: The projection of a path to its $\epsilon=0$ endpoint.

For any $\alpha>0$ gre have $P_{a} M \rightarrow G M \rightarrow P_{0} M$. The germs capture bebavior closer to $c=0$ than any given $\epsilon$, but still contain much more information than perturbation theory gives us (gerrs depend on features of functions in a little neighborhood that may not be r.aptured in a Taylor series.) 


\subsubsection{The Space of Jets of Pathe}

Finally He may introduce spaces of jets of paths at $e=0$ with integer $1 \leq J \leq$ $x$ :

$$
\begin{aligned}
& J M=\left\{\text { equivalence clagses in } P_{1} M \text { where } p_{1} \sim p_{2}\right. \text { iff } \\
& \forall C^{\infty} \text { functions } f \text { cn } I \times M \text { we bave } \\
& \left.\qquad\left.\frac{\partial^{\prime}}{\partial c^{4}}\right|_{t=0} f\left(p_{1}(\epsilon)\right)=\left.\frac{\partial^{\prime}}{\partial \epsilon^{1}}\right|_{t=0} f\left(p_{2}(t)\right) \quad \text { for } 0 \leq i \leq J\right\} .
\end{aligned}
$$

Notice that $\infty M$ is the space of infinitc formal power series by this definition. The space of $J$-jets gives thr first $J$ terms in a Taylor expansion of the curve around $c=0$ in any coordinate system. Clearly,

$$
G M \rightarrow \infty M \rightarrow I M \rightarrow J M \rightarrow P_{0} M \quad \text { for } I>J
$$

Thus the jets focus on information cioser to $c=0$ than even the germs.

In the Duffing example, the $J$-jet of $(u(\varepsilon), v(\varepsilon))$ consists of the values of the first $J$ derivatives with respect to $c$ of $u$ and $v$. We called these $u_{0}, v_{0}, u_{1}, v_{1}, \ldots u_{J}, v_{J}$.

\subsubsection{Coordinates on the Jet Space}

If $x^{a}$ for $1 \leq a \leq N$ are coordinates on $M \approx P_{0} M \approx 0 M$, then we may introduce coordinates $\left\{x_{0}^{a}, x_{1}^{a}, \ldots, x_{j}^{a}\right\}$ for $0 \leq J \leq \infty$ on $J M$ to represent the equivalence class of the curve:

$$
I_{0}^{a}+\epsilon I_{1}^{\mathrm{a}}+\frac{\epsilon^{2}}{2 !} x_{2}^{a}+\cdots+\frac{\epsilon^{J}}{J !} x_{J}^{a}
$$

in $I \times M$ (near $\epsilon=0$ this won't leave the chart on which the $I^{a}$ are defined). 


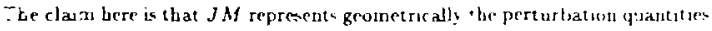
$z$ : If it may seem strange to go tbrugg the infiuite dimeusional space P.M $\therefore$ es: so it, but we shall see (suecially wben looking at the Hamiltonian strurture) ita: it organizes and simplifies the gtructures of interest. It is a completely intrinsic ard natural (or in modern parlance: functorial) operation to go from the original cynamical manifold $M$ to she path space $P_{1} M$ in the jet spare $J M$. We sball now show that the dynamics on $M$ also induces natural dynamics on $P_{1} M$ and then projects fom there down to $J M$ where it is the perturbation dynamics we are interested in. Later we will see that a Hamiltonian structure on $M$ leads to Hafnilisonian structures on $P_{1} M$ and $J M$.

The dynamics $\dot{I}=X(c, x)$ takes elements of $P_{1} M$ to otber elements of $P_{1} M$ and in fact tatos equivalence classes to equivalence classes for each of $P_{a} M, G M$, $x M$. $J M$, and $M$. This is what allows us to obtain an induced dynamics on eacb of these sfraces. To determine this dynamics explicitly, we must understand what a langent vector on each space is.

\subsubsection{Tangent Vectors to Path Space}

Intuitively, a vector represents a little perturbation to a point. We defige it precisely as an equivalence class of tangent curves, where the curve tepresents the direr 10 of perturbation and the equivalence class ensures that only the first order mokion is reflected in the tangent vector. A point in the patb space $P_{1} M$ represeuts a patb in $I \times M$. A small perturbation of this point represents a vearby patb. Each poist of the path is pertusbed a lit tle bit and we are interested in the first order 
perturbation We therefore expect a tangent vector to a point in path space to he a vector fold along the corresponding path as in figure (2.7). By a vector feld along a path. Wir mein a choire of tangent vector to $M$ at each point of the patb. In general. a vertor field along a wap $f$ from a manifold $M$ to a manifold $N$, is a smucels choice of image-spare tangent vector in $T_{f(x)} N$ for each point $I$ in the source spare $M$.

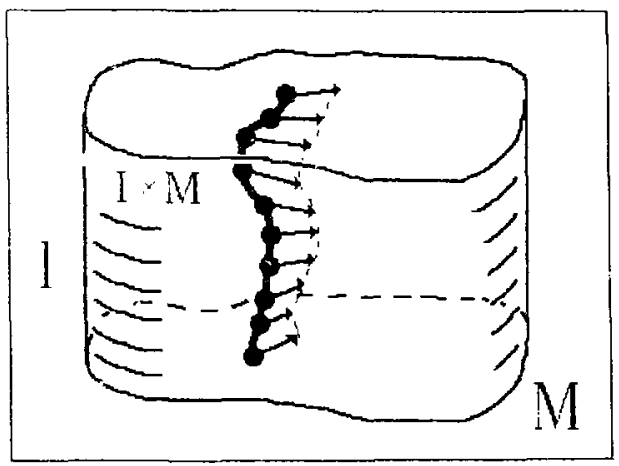

Figure 2.7: A small perturbation of a path in $I \times M$ is given by a vector field along that patb.

A curve $p(\gamma)$ in $P_{1} M$ parameterized by $\gamma$ defines a curve $p(t, \gamma)$ for each $\epsilon$ .hrough $p\left(\varepsilon_{1} \gamma=0\right)$ in $I \times M$. The equivalence class of curves un $P_{1} M$ defining a vector thus reduces to an equivalence class of curves in $M$ fir each $t$. Wit may identify a tangent vector to $p$ in $P_{1} . M$ with a ficid of vectors over $p$ in $I \times M$ sucb 
that each vector has no $\frac{\partial}{d t}$ component. For $p \in P_{1}, 11$ a vector $l T_{p}\left(P_{1}, k\right)$ is a map

$$
\bar{l} I \rightarrow I \times T M
$$

taking $\epsilon \curvearrowleft(\epsilon, \nvdash(\epsilon))$ phere $V(c) \in T_{p(x)} M$ (i. e a path in $\left.J M\right)$

For the Duffing example, a tangent vector based at the path $(u(c), v(t))$ has the form $(\delta u(c), \delta v(c))$ and represents the vector field

$$
\left.\delta u(c) \frac{\partial}{\partial u}\right|_{(u(*), v(\cdot) \cdot}+\left.\delta v(\varepsilon) \frac{\partial}{\partial v}\right|_{(u(c), v(c))}
$$

along the path $(u(c), v(c))$.

\subsubsection{Tangent Vectors to the Quotient Spaces}

The tangent spaces to the quotient spaces are defined by taking the derivatives of the natural projections. Because $P_{0} M \approx M$, we see that $T I_{U} M \approx T M$. Because the first jet space is isomorphic to the tangent bundle: $1 M \approx T M$, we see that $T 1 M \approx T T M$. Thus the first order perturbation space $1 M$ is naturally $T M$ and the dynamical evolution is given by a vector field on $T M$ ag we saw in section 2.2.9.

\subsubsection{Coordinates on the Tangent Bundle to the Jet Space}

As with . I tangent bundles, $T J M$ Lag a natural coordinate chart, derived from the coordinates $\left\{x_{\ell}^{a}, \ldots, x_{j}^{a}\right\}, 1 \leq a \leq N$ on $J M \mathrm{~d} c$ ined carlier. Wic obtain coordinates $\left\{x_{0}^{a}, \ldots, x_{j}^{a}, v_{0}^{a}, \ldots, v_{j}^{a}\right\}$ by writing the corresponding vector as

$$
\sum_{a=1}^{N} v_{0}^{a} \frac{\partial}{\partial x_{0}^{a}}+\cdots+\nu_{j}^{a} \frac{\partial}{\partial x_{J}^{a}} .
$$


We would like to know to which set of components $\left\{0_{0}^{\circ}, \ldots, g\right\}$, the equivalence class of a vector $F(f)$ on $P_{1} M$ corresponds. For the Duffing example, a tangent vectur to the space of $J$-jeth looks like

$$
\delta u_{0} \frac{\partial}{\partial u_{0}}+\delta v_{0} \frac{\partial}{\partial v_{0}}+\ldots+\delta u_{J} \frac{\partial}{\partial \cdot v_{J}}+\delta v_{J} \frac{\partial}{\partial v_{J}} .
$$

\subsubsection{Coordinate Relation Between Path and Jet Vectors}

To the path $x^{a}(t)$ representing a point in $P_{1} M$ corresponds the point coordsnatized by

$$
x_{k}^{a}=\left.\frac{\partial^{k}}{\partial \epsilon^{k}}\right|_{l=0} x^{a}(c), \quad 1 \leq a \leq N, \quad 0 \leq k \leq J
$$

in $J M$. To the curve of paths $x^{a}(c, \gamma)$ in $P_{1} M$ corresponds the curve

$$
x_{k}^{a}(\gamma)=\left.\frac{\partial^{k}}{\partial \epsilon^{k}}\right|_{s=0} x^{a}(\epsilon, \gamma), \quad 1 \leq a \leq N, \quad 0 \leq k \leq j
$$

in $J M$. The rector tangent to this curve in $T P_{1} M$ has coordinates

$$
V^{a}(\epsilon)=\left.\frac{\partial}{\partial \gamma}\right|_{\gamma=0} x^{a}(\epsilon, \gamma)
$$

In $T J M$ this corresponds to

$$
\begin{aligned}
\boldsymbol{v}_{k}^{\mathbf{a}} & =\left.\frac{\partial}{\partial \gamma}\right|_{\tau=0}\left(\left.\frac{\partial^{k}}{\partial \epsilon^{k}}\right|_{\epsilon=0} x^{a}(\epsilon, \gamma)\right) \\
& =\left.\frac{\partial^{k}}{\partial \epsilon^{k}}\right|_{c=0}\left(\left.\frac{\partial}{\partial \gamma}\right|_{\gamma=0} x^{\circ}(\epsilon, \gamma)\right) \\
& =\left.\frac{\partial^{k}}{\partial c^{k}}\right|_{l=0} V^{a}(\epsilon) .
\end{aligned}
$$


For the Duffing equation. the tangent vertor represented by $(6+1+1,4+; i)$ project to the jet space vector

$$
\delta u_{v} \frac{\partial}{\partial u_{0}}+\delta_{v} \cdot \frac{\partial}{\partial v_{0}}++\delta_{J} \frac{\partial}{\partial u_{j}}+\delta_{v_{j}} \frac{\partial}{\partial v_{j}}
$$

where

$$
\begin{aligned}
& \delta_{u_{0}}=\delta u(0) \quad \therefore t_{0}=\partial_{u}(0) \\
& \delta u_{1}=\left.\frac{\partial}{\partial t} \delta u(t)\right|_{\ell=0} \quad \partial v_{1}=\frac{\partial}{\partial t} \delta \tau(t)-0 \\
& \delta u J=\left.\frac{\partial^{J}}{\partial \epsilon^{J}} \delta u(c)\right|_{\epsilon=0} \quad \delta v j=\frac{\partial^{J}}{\partial \epsilon} \delta v(c)_{i e=0}^{k}
\end{aligned}
$$

\subsubsection{Dynamice on Path Space}

Let us now consider the efect of the dyammics $\dot{I}=X(t, x)$ on paths. This lifts to a vector field on $P_{1} M$ given by

$$
\hat{X} \text { where } \vec{X}(p): c-X(\epsilon, p(c)) \text {. }
$$

This is the path space dynamical vector field. Note that this vertor feld is of a very special type and not every vector ficld or $P_{1}, M$ can arise in idis Hay For the Duffing example, the path space vector feld $(\delta u(t), \delta:(t))^{\prime} u, v$ at the point $(u, t)$ is cqual to

$$
b u(c)=n(t) \quad b t(\epsilon)=-u(c) \cdots\left(u^{3}(t) .\right.
$$




\section{3. . Lynamica on Jet Space}

Ii. courdiuates, the corresponding vector fietd on $J P$ is

$$
\begin{aligned}
& v_{k}^{a}\left(x_{0}, \ldots, x_{1}\right)=\left.\frac{\partial^{k}}{\partial t^{k}}\right|_{t=0} x^{\alpha}\left(\epsilon, I_{0}+c x_{1}+\cdots+\frac{t^{j}}{J !} I^{\prime} J\right) \\
& \frac{\partial^{k}}{\partial c^{k}} x_{\epsilon-c}^{-a}\left(\epsilon, x_{0}\right)+\left.k \frac{\partial^{k-1}}{\partial \epsilon^{k-1}}\right|_{\ell=0} \sum_{b=1}^{N} \frac{\partial}{\partial x_{0}^{b}} X^{a}\left(c, x_{0}\right) x_{1}^{b}+\cdots,
\end{aligned}
$$

which is cxactly the perturbation dynamics up to order $J$ obtained in equations $(2.15)^{\prime}$

For exan:ple. we calculate that

$$
\begin{aligned}
V_{0}^{a}\left(x_{2}, \ldots, I_{J}\right)= & X^{a}\left(0, x_{0}\right) \\
V_{1}^{a}\left(x_{0}, \ldots, x_{J}\right)= & \left.\frac{\partial}{\partial c}\right|_{c=0} X^{a}\left(c, x_{0}\right)+\sum_{b=1}^{N} \frac{\partial}{\partial x_{0}^{b}} X^{a}\left(0, x_{0}\right) x_{1}^{b} \\
V_{2}^{a}\left(x_{0}, \ldots, x_{J}\right)= & \left.\frac{\partial^{2}}{\partial c^{2}}\right|_{c=0} X^{a}\left(c, x_{0}\right) \\
& +\left.2 \frac{\partial}{\partial c}\right|_{c=0} \sum_{b=1}^{N} \frac{\partial}{\partial x_{0}^{b}} X^{a}\left(0, x_{0}\right) x_{1}^{b} \\
& +\sum_{b, c=1}^{N} \frac{\partial^{2}}{\partial x_{0}^{b}} X^{a}\left(0, x_{0} ; x_{2}^{b} x_{i}^{c}+\sum_{b=1}^{N} \frac{\partial}{\partial x_{0}^{b}} X^{a}\left(0, x_{0}\right) x_{2}^{b}\right. \\
& \vdots
\end{aligned}
$$

In the Duffing examples, we sec that the jet vector field

$$
\delta u_{v} \frac{\partial}{\partial u_{0}}+\delta_{u_{v}} \frac{\partial}{\partial v_{l}}+\ldots+\delta u_{J} \frac{\partial}{\partial u_{J}}+\delta v_{J} \frac{\partial}{\partial u_{J}}
$$


bas the components:

$$
\begin{aligned}
& \delta u_{0}=\delta u(0):=v(0) \\
& \delta v_{0}=\delta_{v}(0)=-u(0) \\
& \delta u_{1}=\frac{\partial}{\partial \epsilon}\left|\imath=0 \delta u(\epsilon)+\frac{\partial}{\partial u}\right|_{r=0}(\delta u(0)) u_{1}+\left.\frac{\partial}{\partial v}\right|_{\tau=0}\left(\delta u(0) \tau_{1}\right. \\
& =0+0+1 \cdot v_{1}=v_{1}(0) \\
& \delta \mathrm{t}_{1}=\left.\frac{\partial}{\partial \epsilon}\right|_{t=0} \delta \mathrm{v}(\boldsymbol{c})+\left.\frac{\partial}{\partial t}\right|_{t=0}(\delta v(0)) u_{1}+\left.\frac{\partial}{\partial v}\right|_{t=0}(\delta v(v)) v_{1} \\
& =-u^{3}(0)+\left.\left(-1-3 \epsilon u^{2}\right)\right|_{\alpha=0} u_{1}=-u^{3}(0)-u_{1}
\end{aligned}
$$

We have thus found the natural geometric setting for $J$ th order perturbation theory in a certain jet bundle. The picture of the dynamics of paths in $I \times M$ is an extremely fruitful one One can prove that the solution of the perturbation equations (2.15) really is the asymptotic expansion of the true solution just by noting that they are the equations of evolution of the jets of the paths evolving under the true dynamics. The coordinates in which the dynamicg are expressed are irrelevant as regards the perturbation dynamics and therefore we can do perturbation tbcory on manifolds and in infinite dimensions as is required for many physical systems. Next pe will review modern Hamultonian mechanics and indicate why the perturbation dynamics is Hamitonian in a natural way if che unperturbed dynamics is. 


\subsection{Geometric Hamiltonian Mechanica}

"The next worning, I burried along to onc of the libraries as soon as it was open and then I looked up Poissn brackets in Whittaker's 'Analytic Dynamies' and I found that they were just what $I$ needed"--P. A. M. Dirac in [Dirac, 1977] p. 122.

The evolution of mechanical systems is traditionally described in terras of generalizent coordinates $q_{t}$ acd tbeir conjugate momenta $p_{1}$. One introdures the Hamiltonian function

$$
H\left(q_{1}, \ldots, q_{n}, p_{1}, \ldots, p_{n}\right)
$$

and the Poisson bracket

$$
\{f, g\}=\sum_{i=1}^{n}\left(\frac{\partial f}{\partial q_{i}} \frac{\partial g}{\partial p_{i}}-\frac{\partial f}{\partial p_{1}} \frac{\partial g}{\partial q_{i}}\right)
$$

of two functions of $q_{1}$ and $p_{1}$. Any observable $f$ evolves according to the evolution equation

$$
\dot{j}=\{f, H\}
$$

For the Duffing example, we may use the Poisson bracket

$$
\{f, g\}=\frac{\partial f}{\partial u} \frac{\partial g}{\partial v}-\frac{\partial f}{\partial v} \frac{\partial g}{\partial u},
$$

the Hamiltonian

$$
H=\frac{v^{2}}{2}+\frac{u^{2}}{2}+c \frac{u^{4}}{4} .
$$

Togetber these give rise to the correct equations:

$$
\dot{u}=\{u, H\}=\frac{\partial H}{\partial v}=v
$$


an:

$$
\dot{t}=\{v, H\}=-\frac{\partial H}{\partial u}=-u-u^{3} .
$$

\subsubsection{Poisgon Manifolds}

For a detailed description of the modern approach to mechanics see references [Abraham and Marsden, 1978], [Arnold, 1978], and [Marsden, 1981]. The modern perspective regards the particular coordinates $p_{\mathbf{2}}$ and $q_{2}$ as physically irrelevant. Just as general relativity isolates the physically relevant easence of local coordinates in a metric tensor, modern classical mechanics vier's the Poisson bracket structure (not necessarily expressed in any coordinate system) as the physical entity. Just as physics in spacetime is invariant under transformations that preserve the metric, pbysics in phase space is invariant under the canonical transformations which preserve the Poisson bracket. In the modern viewpoint one proceeds axiomatically and does not require canonical coordinates. Dyuamics occurs on a Poisson manj fold. This is a manifold of states with a Poisson bracket defined on it. From this viewpoint a Poisson bracket is a bilinear map from pairs of functions to functions which males the space of function: into a lie algebra and acts on products an a derivative does:

I. Bilinearity:

$$
\begin{aligned}
& \left\{a f_{1}+b f_{2}, g\right\}=o\left\{f_{1}, g\right\}+b\left\{f_{2}, g\right\} \\
& \{f, g\}=-\{g, f\}
\end{aligned}
$$

I1. Anti-symmetry:

III. Jacobi's identity:

$$
\{f,\{g, h\}\}+\{g,\{h, f\}\}+\{h,\{f, g\}\}=0
$$

IV. Derivation property:

$$
\{f, g h\}=\{f, g\} h+\{f, h\} g
$$




\subsubsection{Hamiltonians and Hamiltonian Vector Fields}

The Hamiltonian is a function on the Poisson manifold. The evolution of local coordinates $z^{\prime}$ is obtained from a Hamiltonian $H$ and the Poisson bracket $\{$,$\} via$

$$
\dot{s}^{\prime}=X_{H} \cdot z^{\prime}=\left\{z^{*}, H\right\} \text {. }
$$

$X_{H}$ is the Hamiltonian vector Geld ssociated with $H$ and defines a dynamical system. The fourth property of a Poisson bracket implies the useful expression

$$
\{f, g\}=\sum_{i, j} \frac{\partial f}{\partial z^{*}}\left\{z^{\prime}, z^{\top}\right\} \frac{\partial g}{\partial z^{3}} .
$$

Thus tbe Poisson bracket is equivalent to an antisy mmetric contravariant two-tensor

$$
J^{\prime y} \equiv\left\{z^{i}, x^{\prime}\right\}
$$

\subsubsection{Symplectic Manifolds}

If this tensor is nondegenerate, its inverse $\omega \equiv J^{-1}$ is a closed, nondegenerate tho-form called a symplectic structure. In this case our Poisson manifold is known as a symplectic manifold (see [Abraham, Maroden, and Ratiu, 1983] p. 463). The terminology is due to Herman Weyl. The symplectic group is related to "line complexes ${ }^{n}$ in projective geometry and so w was originally refered to as a complex structure. There is another object that naturally has this name in the study of the complex analysis of many variables, however. To eliminate this confusion, Weyl look the Latin roots com and plex and converted them to their Greek equivalents sym and plectic. 


\subsubsection{Symplectic Leaves and Bones and Casimi Functions}

Berause we do not require nondegencracy, a Poisson manifold is a more general notion than a symplectir manifold. If $l$ is degenerate, thes there are directions it phase space in which no Hamiltonian vector field can point. The available direction lie tangent to submanifolds which fill ou. :he Poisson manifold and on wibich $J$ is nondegenerate. The bighest dimensional of these form a foliation of their unicn and so are knon' as symplectic leaves. The only prior usage of the term symplectic in Engiish is to describe a small bo' ${ }^{-}$in the head of a fisb. Because Poisson is French for fish, the lower dimensional symplectic submanifolds ase sometimes known as symplectic bones (these notions Fere introduced in [Weinstein, 1983a]). Togetber, the symplectic leaves and the symplectic bones fill out the Poisson manifold, and any Hamiltonian dynamics is restricted to lie on a single bone or leaf. Any function which is constant on each bone and leaf Poisson commutes witb every other function. Any function which Poison commutes witb every function is automatically a constant of the motion, regardless of the Hamirtonian and is called a Casimir furction.

\subsubsection{The Natural Symplectic Structure on Co'angent Spaces}

A natural symplectic manifold arises from each Lagrangian mechanical system on a confif,uration space $C$. The Lagrangian $L$ lives on the tangent bundle $T C$ (velocities being tangent to the curves of motion in c: 'jguration space are naturally tangent vectors). Hamiltonian mechanje s takeg place on the cotangent bundle $T^{*} \mathrm{C}$ (momenta, being derivatives of $L$ with respect to velocity, are naturally dual to 
velocities and thus are covectors). At each point $x$ of $C$, the set of velocity vectors $v$ in $T_{x} C$ are identified witb corresponding momenta $p$ in $T_{x}^{*} C$ by a Legendre map defined by $L$ (tbe classical concept of a Legendre map is described in modern terms in section 16.5.11):

$$
p=\frac{\partial L}{\partial v}
$$

The Ha:niltonian is a function on the cotangent bundle which on each cotangent fiber is equal to the Legendre trangform (described in section 16.5.5) of the the Lagrangian restricted to the corresponding tiber of the tangent bundle.

$T^{*} C$ bas the natural symplectic structure

$$
\omega=-d \theta
$$

where $\theta$ is an intrinsically defined one-form on $T^{*} C$ (see /Abrabam, Marsden, and Ratiu, 1983| p. 465). $\theta$ must pair with a tangent vector $v$ in $T T^{*} C$ based at $(x, \alpha)$ in $T^{*} C$ to give a real number. To define this we ure the natural projection

$$
\pi: T^{*} \mathrm{C} \rightarrow \mathrm{C}
$$

which takes a covector to its basepoint in $C$. The differential of $\pi \operatorname{sends} T T^{\circ} C$ to $T C$ and may be applied to $v$ to get a vector tangent to $C . \alpha$ is a one-form on $C$ and may be applied to this vector. Let us defiue the pairing of $\theta$ with $v$ to be the pairing of $\alpha$ with the image of $v$ under $T \pi$ :

$$
\theta(v)=\alpha(T \pi v)
$$

In coordinates $q^{a}$ on $C$, this takes the form

$$
\theta=p_{\alpha} d q^{a}
$$


and, ads to the symplectir form

$$
\text { ir }-d q^{a} \wedge d p_{a} \text {. }
$$

This construction generaizes the usua structure in tern' of canonical $p$ s and $q$ 's $i$ configuration space which are manifolds. Symmetry is responsible for the simpli jed systems about which we perturb and plays an intimate fole in our geometric theory We therefore next introduce some key modern ideas and basic exanples relating to Hamiltonian symmetry. 


\subsection{Hamiltonian Systems with Symmetry}

Perbaps the central advanlageous feature of systems with a Hamiltonian structure Li a grneralizatiou of Noether's theorem selating symmetries to conserved quanuties. Noetber considered symmetries of the Lagranfian under transformations of ronfiguration space. One may introduce generalized coordinates $\left(q_{1}, \ldots, q_{n}\right)$ where $\left(q_{2} \ldots, q_{n}\right)$ are constant under the symmetry transformation and $q_{1}$ varies with the transformation. For example we might take the configuration space to be ordinary Euclidean 3-8pare where the action of the symmetry is translation in the $I$ direction, and wilize the coordinates

$$
q_{1}=x, \quad q_{3}=y, \quad q_{3}=z .
$$

That $L$ is avariant means that it does not depend on $q_{1}$, i.e. $q_{1}$ is an ignorable coordinate. The Euler-Lagrange equations

$$
\frac{d}{d t}\left(\frac{\partial L}{\partial \dot{q}}\right)-\frac{\partial L}{\partial q}=0
$$

show that in this case the momentum

$$
p_{1}=\frac{\partial L}{\partial \dot{q}_{1}}
$$

conjugate to $q_{1}$ is actualiy a constant of the motion 


\subsubsection{Generalized Noether's Theorem}

By going to a Hamiltonian description in terms of Polson brackets we mar extend Noether's theorem in a fundemental pay He may consider a one-parameter symmetry transformation of the phole phase space as opposed to just configuration space. If this transformation preserves the Haniltonian and the Poisson bracket (i.e. is a canonical transformation) then it is associated rich a conserved quantity. We will see that thig extension of Foether's theorem is essential in the case of gyromotion and in other examples.

One-parameter families of canonical transformations of this type may be represented as the "time" evolution generated by some function $J$, treated momentarily as a Hamiltonian. Parametrizing our transformation by and labelling points in phase space by $\underline{z}$, the solution $\underline{z}(0)$ of

$$
\frac{d \underline{z}}{d d}=\{\underline{z}, J\} \quad \underline{z}(0=0)=\underline{z}_{0}
$$

is the family of cajonical transformations generated by $J$.

If the transformations generated by $J$ are symmetries of $H$ then

$$
\begin{aligned}
\frac{d H}{d s}=0 & =\sum_{\cdot} \frac{\partial H}{\partial z^{i}} \frac{d z^{i}}{d s} \\
& =\sum_{i} \frac{\partial H}{\partial z^{i}}\left\{z^{\prime}, J\right\} \\
& =\{H, J\} \\
& =-\{J, H\} \\
& =-j .
\end{aligned}
$$

So $J$ is a conserved quantity. 


\subsubsection{Circle Actions}

We now consider the cage in which the solutions of

$$
\frac{d \underline{z}}{d a}=\{\underline{z}, J\}
$$

are all closed cures with the same period We will call these closed orbits loops. The symmetry irangformation is then sajd to be a circle action on phase space.

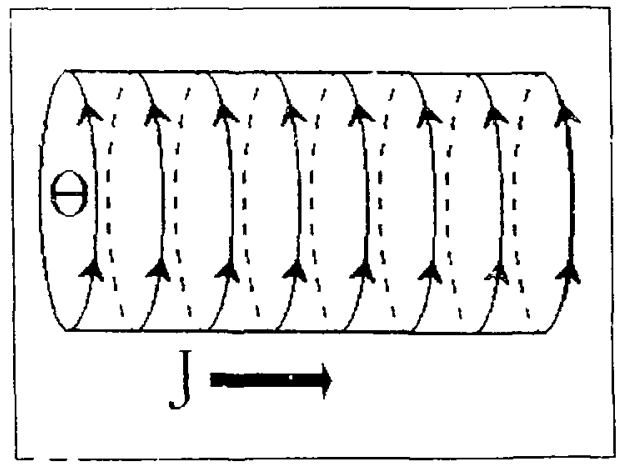

Figure 2.8: The circle action on $J, \theta$ phase space.

For exanple we might congider rotation by $\theta$ in $J, \theta$ space. In this case phase space looks like a cylinder (as shown in figure (2.8). The Poisson bracket is

$$
\{f, g\}=\frac{\partial f}{\partial \theta} \frac{\partial g}{\partial J}-\frac{\partial j}{\partial J} \frac{\partial g}{\partial \theta} .
$$


$J$ gererates the dyamics

$$
\begin{aligned}
& \frac{d \theta}{d A}=\{\theta, J\}=1 \\
& \frac{d J}{d A}=0,
\end{aligned}
$$

which just rotates the cylinder.

\subsubsection{Reduction by a Circle Action}

In studying the dyamics of a Hamitonian $H$ symmetric under a circle action generated by $J$, we may make two simplifications which toge: ber comprise the process of reduction. This procedure was defined in [Marsden and Weinstein, 1974] in a more general setting tbat we will describe sbortly. The process unifies mary previously known techniques for simplifying specific examples of Hamiltonian systems.

\subsection{S.1. The Reduced Phage Space}

1. Because $J$ is a constant of the motion, the surface $J=$ constant in phase space is invariant under the dynamics and 50 re may restrict attention to it.

2. The symmetry property of $B$ implies that if we take a solution curve $z(t)$ of the equation $\underline{\dot{z}}=\{\underline{z}, H\}$ and let it evolve for a "timen $s$ under the dynamiss $\dot{z}=\{\underline{z}, J\}$ then we obtain anotber solution curve of $\underset{z}{\underline{z}}=\{\underline{z}, H\}$. In fact $t\}$.. dyamics of $A$ takes an entire loop into other entire loops.

The dynamies around loops is easy to solve for because

$$
\theta=\frac{\partial H}{\partial J}
$$


Sotice t 1 at $\theta$ is not uniquely defined but $\dot{\theta}$ is. We are interested in the problem of foding ite dynamies from loop to loop. We want to projert the original dy pamics on ph. $=$ pare $P$ dow to a space $P / S^{2}$ whose points represeut whole loops in $P$. Let us all $P / S^{1}$, the space of loops and $\pi: P \rightarrow P / S^{2}$, the projection mapping loops in $P$ to points in $P / S^{1}$. For example, when $P=J, \theta$ space the projectic rapping takes $J, \theta$ to $J$. This projection is shown in Gguie (2.9). Thus the second simplific ation La consider dynanics on the space of loops $P / S^{1}$.

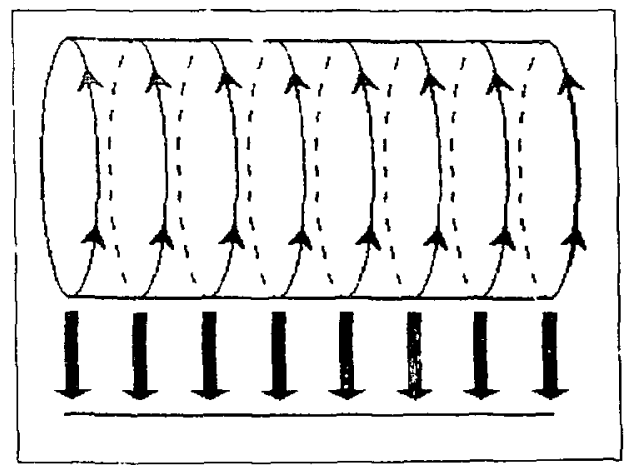

Figure 2.9: The projection of $J, \theta$ spare to the space of loops

Performing both of these operations- restricting to $J=$ constant and considering the space of loops - leaves us with a space,

$$
R \equiv P /\left.S^{1}\right|_{j=\text { eooutas }}
$$


of tro dimenaions leas thar $P$. called the reduced space

Wie bave seen that the dysanics on $P$ uaturally determiges dynamirs on $R$ The bey importance of $R$ is that $R$ 's dyamics is itsell Hamiltowian. For this statement to make sense we need to find a Hamiltonian and a Poisson bracket on $\mathrm{R}$ Tbese are the so-called reciuced Handtonian and reduced Poisso:: bracket

\subsubsection{The Reduced Hamiltonian}

The original Hamiltonian $\boldsymbol{H}$ on $P$ is constant on loops by the symuetry condition. We mat take the value of the reduced Hamilionian at a point of $h$ to be the value of $H$ on the corresponding loop in $P$.

\subsubsection{The Reduced Poisson Bracket}

To obtain the reduced Poisson bracket of two functions $f$ and $g$ on $R$, we consider any two functions $\bar{f}$ and $\hat{g}$ on $P$ which are constant on loops and agree with $f$ and $g$ when rest ricted to $J=$ const ant and projected by $\pi$ to $R$. The Poisson bracket on $P$ of $j$ and $g$ will be constant on loops and its value on $J=$ coustant will be irdependent of how $\hat{f}$ and $\hat{g}$ were extended as functions on $J$ (because they are constant on loops: $\{\dot{f}, J\}=0$ and $\{\hat{g}, J\}=0$ so $\{\dot{f}, \hat{g}\}$ is independent of $\partial \dot{f} / \partial J$ and $\partial \hat{g} / \partial J$ ). Thus the value of the reduced Poisson bracker on $R$ of $f$ and $g$ is the value on the corresponding loop in $P$ of the Poisson bracket of any two exte sions $f, g$ that are constant on loops. 


\subsubsection{Coordinate Calculation of the Reduced Space}

In cxamples we often introduce a courdinate $\theta$ describing the position on a loop. We may then treat $P / S^{\prime}$ as the set $\theta=0$ (at least locally). In this case $R$ is the subset $\theta=$ constant. $J=$ constant of $P$. The reduced Hamiltonian on $R$ is just the value of $H$ on this subset of $F$ To calculate the value of the Poisson bracket of two functions on $P$ on this surface, we need only their first derivatives there.

If the functions are constant on loops (i.e. independent of $\theta$ ), then the derivative $\partial / \partial \theta$ ia zero. The dependence on $J$ is irrelevant, so we may take the derivative $\partial / \partial J$ to be zero. Plugging these two expressions into the Poisson bracket on $P$ gives us the expression for the reduced Poisson bracket on $\boldsymbol{R}$. 


\subsection{I xample: Centrifugal Force}

We consider a particle on a two-dimensional plane moviog in a rotationally symmetric potential The phase space is then $T^{-} \mathbb{R}^{2}$ with coordinates $\left(x, y, p_{x}, p_{v}\right)$ The Poisson bracket is the canonical one:

$$
\{f, g\}=\frac{\partial f}{\partial x} \frac{\partial g}{\partial p_{x}}-\frac{\partial f}{\partial p_{x}} \frac{\partial g}{\partial x}+\frac{\partial f}{\partial y} \frac{\partial g}{\partial p_{y}}-\frac{\partial f}{\partial p_{y}} \frac{\partial g}{\partial y}
$$

The Hamiltonian is taken to be

$$
H=\frac{1}{2 m}\left(p_{x}^{2}+p_{y}^{2}\right)+v\left(\sqrt{x^{2}+y^{2}}\right) .
$$

The symmetry on phase space is given by the evolution of the equations

$$
\begin{array}{ll}
\frac{d x}{d a}=-y & \frac{d y}{d \theta}=x \\
\frac{d p_{x}}{d \theta}=-p_{v} & \frac{d p_{y}}{d \theta}=p_{x}
\end{array}
$$

We may think of a point in phase space as a point in the plane $(x, y)$ with a vector attached $\left(\rho_{x} \cdot \rho_{y}\right)$. The action of the symetry is to rotate the plane about the origin, vector and all as shor'n in figure $(2.10$;

\subsubsection{Angular Momentun Generates Rotations}

The Haniltonian depends only on the radial distance and the magnitude of the womentum vector and so clearly remain invariant under this rotation. The rotation is a cajonical transformation with generator $J$ satiblying

$$
\frac{d f}{d s}=\{f . J\}=x \frac{\partial f}{\partial y}-y \frac{\partial f}{\partial x}+p_{x} \frac{\partial f}{\partial p_{u}} \quad p_{u} \frac{\partial f}{\partial p_{z}}
$$




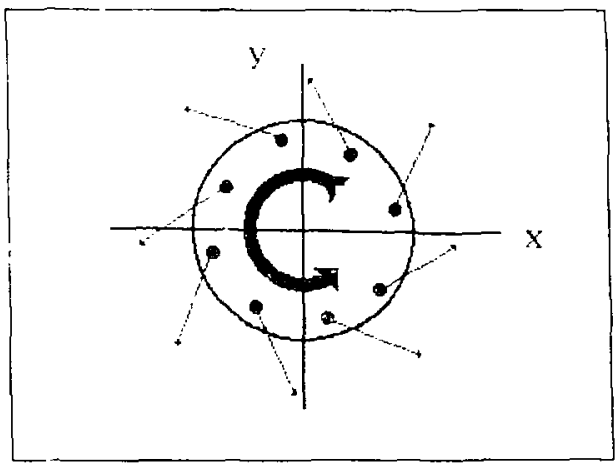

Figure 2.10: The axtion oi the circle symmetry on momentum rectors.

for any $f$. Taking $f=x, y, p_{x}, p_{y}$ gives

$$
\frac{\partial J}{\partial p_{x}}=-y \quad \frac{\partial J}{\partial p_{y}}=x \quad \frac{\partial J}{\partial x}=p_{y} \quad \frac{\partial J}{\partial y}=-p_{x} .
$$

Thus we see that the generator is $J=x p_{y}-y p_{x}$, i.e. the angular momentum. We may label a loop by the value of $x, p_{x}$, and $p_{y}$ when $y=0$ and $x \geq 0 . J$ on this subset is just $x p_{v}$. These then form coordinates on the space of loops $P / S^{1}$. Notice that the value of $x$ on a loop when $y=0$ defines the radial coordinate. For clarity, we will introduce the coordinate functions $r$ and $p r$ on the retuced space for $r a c h$ $J$. A loop is labeled by - if $z=r$ when $y=0$ on that loop. A loop is labeled by $P_{r}$ if $p_{x}=p_{r}$ wen $y=0$ on that loop. 


\subsubsection{The Reduced Space and Bracket}

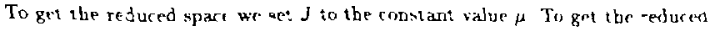
bracket, it is easient to envision the tro-dmessoud redue a spare with correth. natrs (r.p.) ag a submanifold of the four-dumensional total spare with comrinate $\left(I, p_{z}, y, p_{y}\right)$ defined $b y$ :

$$
\begin{array}{ll}
x=r & p_{x}=p_{r} \\
y=0 & p_{v}=\frac{\mu}{r} .
\end{array}
$$

This submanifold intersects each loop transversely in exactly one point since a loop bas $y=0$ with $x \geq 0$ at only one point. It also lies entirely in the level surface $J=\mu$ since

$$
J=I p_{\nu}-y p_{x}=r\left(\frac{\mu}{r}\right)-0 \cdot p_{r}=\mu .
$$

We want to know the rejuced Poisson bracket of functions $f\left(r, p_{r}\right)$ and $g\left(r, p_{r}\right)$ of rand pr. Ag in section 2.5.3.3, we define the bracket of two such functions by introducing $\hat{f}\left(x, p_{x}, y, p_{y}\right)$ and $\hat{g}\left(x, p_{x}, y, p_{y}\right)$ on the four-dimengiona space which are equal to $f$ and $g$ when restricted to the two-dimensional submanifold and which are constant on loops. We take the four-dimengional Poissn bracket of $\hat{f}$ and $\hat{g}$. This bracket is also guaranteed to be constant on loops. The restriction of the bracket of $\hat{f}$ and $g$ to the $\left(r, p_{r}\right)$ submanifold is defined to be the bracket of $f$ and g. Since we only need the full bracket on the submanifold, we don't really need to know $f$ and $g$ everywhere; we ned only their derivatives in each direction at points of the submanifold. We calculate these derivatives as follows. Denivatives along the submanifold are the same as for $\hat{j}$ and $\bar{g}$ :

$$
\frac{\partial \hat{f}}{\partial \boldsymbol{x}}=\frac{\partial f}{\partial \boldsymbol{r}}
$$


and

$$
\frac{\partial j}{\partial p_{r}}=\frac{\partial f}{\partial p_{r}}
$$

with . minilar expressions for $q$. Derivatives of $j$ and $\dot{g}$ along the loops (and so trasiseres to the gubmanifold) auset be zero since $\dot{f}$ and $\dot{g}$ are constant on loops. Tbis implies that on the manifold

$$
\begin{aligned}
\left.\frac{\partial \hat{j}}{\partial \theta}\right|_{\text {.ubmanifold }} & =0 \\
& =\tau \frac{\partial \hat{f}}{\partial y}-y \frac{\partial \hat{f}}{\partial x}+p_{x} \frac{\partial \hat{f}}{\partial p_{y}}-p_{y} \frac{\partial \hat{f}}{\partial p_{x}} \\
& =r \frac{\partial \hat{f}}{\partial y}-0 \cdot \frac{\partial f}{\partial r}+p_{r} \frac{\partial \hat{f}}{\partial p_{y}}-\frac{\mu}{\partial f} \frac{\partial f}{\partial p_{r}} .
\end{aligned}
$$

Finally, we may extend $\vec{f}$ in the fourth direction in any way we like because the result is independent of this extension. For simplicity, let us choose

$$
\frac{\partial \dot{j}}{\partial p_{y}}=0
$$

This leads to

$$
\frac{\partial \dot{f}}{\partial y}=\frac{\mu}{\vec{r}^{2}} \frac{\partial f}{\partial p_{r}} .
$$

Now we can calculate the reduced bracket:

$$
\begin{aligned}
\{f, g\}_{R}\left(r, p_{r}\right) & =\{\hat{f}, \hat{g}\}\left(x=r, y=0, p_{x}=p_{r}, p_{\nu}=\frac{\mu}{r}\right) \\
& =\frac{\partial \hat{f}}{\partial r} \frac{\partial \hat{g}}{\partial p_{x}}-\frac{\partial \hat{f}}{\partial p_{x}} \frac{\partial \hat{g}}{\partial x}+\frac{\partial \hat{f}}{\partial y} \frac{\partial \hat{g}}{\partial p_{\nu}}-\frac{\partial \hat{f}}{\partial p_{\nu}} \frac{\partial \hat{g}}{\partial y} \\
& =\frac{\partial f}{\partial r} \frac{\partial g}{\partial p_{r}}-\frac{\partial f}{\partial p_{r}} \frac{\partial g}{\partial r}+\frac{\mu}{r^{2}} \frac{\partial f}{\partial p_{r}} \cdot 0-0 \cdot \frac{\mu}{r^{2}} \frac{\partial g}{\partial p_{r}} \\
& =\frac{\partial f}{\partial r} \frac{\partial g}{\partial p_{r}}-\frac{\partial f}{\partial p_{r}} \frac{\partial g}{\partial r} .
\end{aligned}
$$

The reduced bracket in this case is just the canonical bracket on $r, p_{r}$ space. 


\subsection{The Reduced Hamiltoniat Gives Certi ifugal Force}

The reduced Hamiltontan is ohtaned by restriesing the origual Hamiltinian subrnanifold and is grven by

$$
H=\frac{p_{r}^{2}}{2 m}+\left(\frac{\mu^{2}}{2 m} \frac{1}{r^{2}}+\downarrow(r)\right) .
$$

Note the effective potential $\mu^{2} / 2 m r^{2}$ d le to reduction, that represents the centrifigal force.

As an aside, it is interesting to note tbat Newton derived the centrifugal force in a more "kinetic" way which is related to averagiug in statistical gas models (Brush, 1983]. He envisioned the motion of a particle along a circular path as the limit of motions on paths on iuscribed polygons as the number of sides becomes infinite. Imagne a circular frictionless billiard table and a billiard ball which refiects from the wall in each traversal about the ed ce of the table. Equivalently a mass attached to a string whose other end is fixed (e.g., a tether ball) can undergo polygonal Inotion where the string is fully extended only at the vertices. The centrifugal force is just the average radial mowentum transfer per unit of time. It turns out that this is independent of the numbs of bounce Nerton considered the average force for an inserived square. Ea-b ir.pact transfers $2 m e / \sqrt{2}$ units of momeatum. The total radial momegt un transfer is then $4 \sqrt{2} \mathrm{mV}$. The distance travelled by the ball in one trat ersal is $4 \sqrt{2} r$. The time this talsen is $v /(4 \sqrt{2} r)$. The average radial momentum transfer per unit of time ic tbeu

$$
4 \sqrt{2} m+\left(\begin{array}{c}
1 \\
4 \sqrt{2} r
\end{array}\right)=\frac{m+}{r}
$$


uhich is the centrifugal force. (The sand result is even easier to obtain with just tro bounce. The momentum transfer is $4 m v$ and the time is $v / 4 r$.) The first correc: arcount of centrifugal force was actually given by Hugens, who is better knowa for bis ideac on waye motion. 


\subsection{Higher Dimenuional symmetrieg}

- The hest and latest mathematkal methods wo appedr on the market Lave beed uatel u bebeser possible In doing this many an old and traterd farorite of throldet generation bas been forsaken. as I deemed it best ant to hand dull and rorn-out rool dond to the vext generation." - Thirrirg. 1978, p iv

Quite ofter phyeical systems are blessed rith more than one dimevsion of symmetry. In keeping with the philosophy of not making unpiysical cbnices, it th natural to consider the process of reduction in the presence of an arbitrary Lie group of sytrmetry. This program was carried out in [Margden and Weinstein, 19/4).

A Lie group is a group which is also a manifold, such that the group operations respect the smoothness structiue. A good example to keep in mind is the group of rotations of three-dimensional Euclidean space. This group is denoted by $S O(3)$. It may be thought of as the space of 3 by 3 matices (this is the 3 in $S O(3)$ ) which are orthogonal (this is the $O$ in $S O(3)$ ) and have unit determinant (this is the $S$, which stands for epecial). The group multiplieation is matrix multiplication, and tbe manifold structure arises from thinking of $S O(3)$ as a submanifold of the nine-dimensional Euclidean space of all $3 \times 3$ matrices.

\subsubsection{Hamiltonian Symmetry}

A Hamiltonian syste-s with symmetry consists of a Poisson manifold $M$, a Hamiltonian $H$, and a group $G$ that acts on $M$ so as to preserve both $H$ and the Poisson bracket $\{$,$\} . The tangent space of G$ at its identity may be identified with the Lie algebra $g$ of the group and represents grour elements infinitesimally close 
to the ideatity. The artion of an infuitesimal element of $G$ on $M$ perturbs each point of 11 by an infinitesinal amount. Thus earh element $v$ of the Lie algebra of $G$ naturally determmes a vertor field on $M$. The action on $M$ of the one-dimensional sutgriouf 14 . Hhich" $"$ is tangent, is given by the flow of this vector field. That the group artion preserves the Poisson brarket, inplies that this vector feld is actually Hamiltonian.

For example, $M$ might be the canonical phase space of a system of particles in a central potential (such as planets around a sun), $B$ is then symmetric under the action of $S O(3)$ which rotates the positions of the particles and the directions of their momenta. Thr Lie algebra $o \circ(3)$ represents infinitesimal rotations and gives rise to a vector feld on $M$. Rotation by a faite angle is the result of flowing along this vector field for a finite time.

\subsubsection{The Momentum Map}

We may therefore associate to each Lie algebra element $v$, a Hamiltonian function wich generates its corresponding vector field (at least locally). If $G$ is $n$ dimensional, and we pick a basis for $g$, then the group action gives $u$ a corresponding Bamiltonian functions on $M$. So as not to prefer one basis over another, we collect these $n$ numbers at each point of $M$ into a vector. This vector naturally pairs with an element of $g$ (to give the value of the function which generates the action of that clement) and so the collection of $n$ Hamitonians is a vector in the dual of the Lie algebra $g^{*}$ at each point of $M$. Thus with every Hamiltonian group artion of $G$ on $M$, there is a natural map called the momentum map from $M$ to 


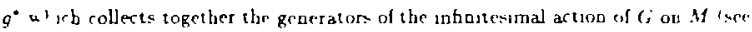
¿Atrah - 7 and Marsden, 1978' p 276)

\subsubsection{Linear and Angular Momentum as Momentum Maps}

For a mechanical system in $R^{3}$ whych is translation invariant, the momentum. map associates with each poirt in phase space the total linear momentum of the system in that state. If the Hamiltonian is rotationally gymmetric, the momentum map gives the total angular momentum in each state (so the angular momentum isn't naturally a vector in $R^{3}$; rather it takes its values in the dual of the Lie algebre of the rotation group $\left.80(3)^{*}\right)$. When we talked about reduction in the one dimensional case above, the generator $J$ of the action was the momentum map.

\section{7.s. Non-commutativity as the Obstruction to Reduction}

Does reduction work for higher dimensionai symmotries? If tbe group is commutative, we may apply the one-dimensional procedure repestedly to eliminate two dimensions of phase space for each dimengion of symmetry. If we are able to eliminate all dimensions of phase space in this pray, the system is called integrable (see [Abrabam and Marsded, 1978] p. 393). If the group orbits are bounded, then one can prove that the group is a torus in this case, dasuming that the "periods" are constant (see $[$ Arnold, 1978] p. 271). Locally we ma: define angle variables on the toroidal group ortits and the correspouding action variables form the momeutum map. Recall that there were two steps in the refuction of systems nijth one di. mension of symmetry, eash of whic belininated ute dimension of the phane space 
and that either could be performed firyt. One was to restrict to a level set of the generating function and the other was to drop down to the orbit space (space of loops) For num-commutative groups, we may again perform either of theve two operations, but each gets in the way of subsequently performing the other. The main issue here is that while the Hamiltonian is invariant under the group action, the momentum map is not. Consider the exarpple of a mechauical system in a spherically aymotric potential so that the rotation group acts on phase space as a symmetry and the momentum map is the total angular momentum While the eperov is left unchanged as we rotate the state, the angular momenturu is rotated just like a vector in $R^{3}$. This action of $S O(3)$ on the dual of its Lie algebra is known as the coadjoint action.

\subsubsection{The Adjoint and Coadjolat Action}

Let us digress a bit on the structure of Lie groups to make this point clearer. We will use the rotation group $S O(3)$ as an example. As shown in the diagram in figure (2.11), every Lie group has three natural actions on itself. If $h$ is an element of $G$, then pie may multiply on the left hy $h$ to get the action $L_{h}$, we may multiply on the right by $h^{-1}$ (the inverse is chosen so that $R_{f h}=R_{f} R_{h}$ ) to get $R_{h}$, and coojugate by $h$ (i.e. $c \multimap h c h^{-1}$ ) to get the action $A D_{h}$.

Let us consider these three actions for $S O(3)$ when $h$ is a rotation by $\pi / 2$ about the $\dot{z}$ axis (say, clockwise looking down $\dot{z}$ ). Let the actions be applied to $c$, a. rotation by $\pi / 2$ about the $\dot{x}$ axis. $L_{h}-c=h c$ means first rotate by $c$ and then by $h$ r r seitud $\bar{x}$ to $\dot{x}$ which $h$ sends to $-\dot{y}$. $c$ sends $\dot{y}$ to $-\dot{z}$ which $h$ sends to $-\dot{z}$, 


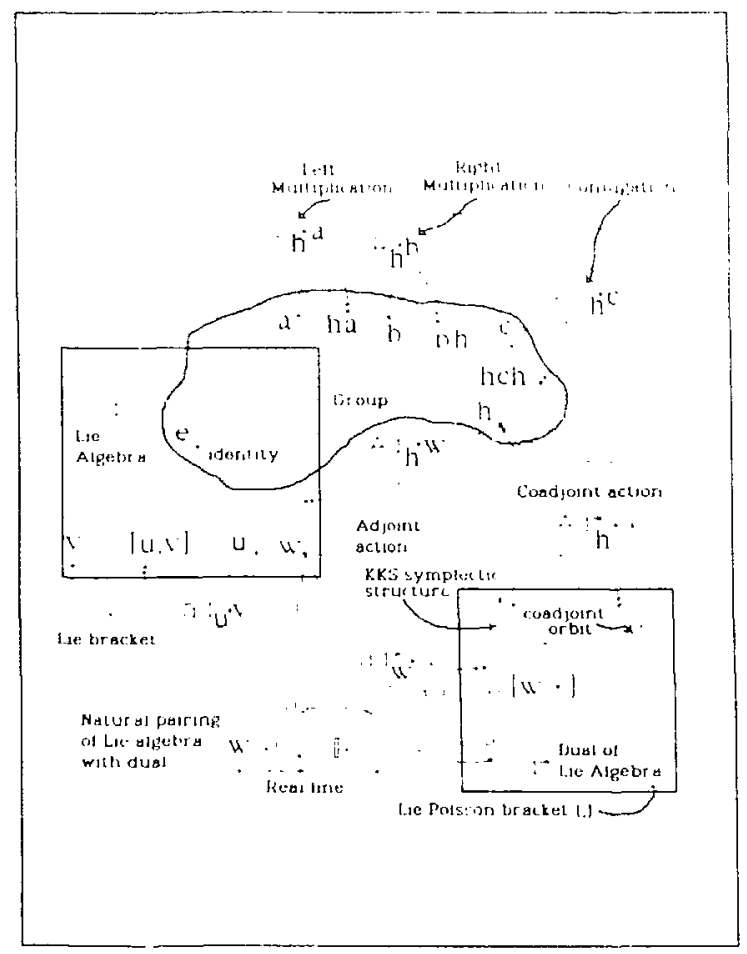

Figure 2.11: Sume Natural Group Actions 
and $c$ sends $\dot{z}$ to $\dot{y}$ phirh $h$ sends to $\dot{r}$ The net result is a rotation ahout the axis contuning $\dot{z} \quad \dot{y}+z$ by arr angle of $2 \pi / 3$.

$R_{h} \cdot r-h^{-1}$ nicaris rotate by $h^{-1}$ and then by $c \cdot h^{-1}$ sends $\dot{x}$ to $\dot{y}$ which $c$ serute to $\hat{z} \cdot h^{-1}$ seuds $\bar{y}$ to $-\bar{I}$ wbicb c gends to $-\dot{j}$ and $h^{-1}$ gends $\dot{z}$ to $\hat{z}$ which $c$ setide $z$, y. The uet result is a rotation about the axis defined by $\dot{x}-i-\dot{z}$ by $2 \pi / 3$. $A L_{h} \cdot c=h r \cdot h^{-1}$ means first to $h^{-1}$, then $c$, and tben $h . h^{-1}$ sends $i$ to $\hat{y}$ which $r$ se nilu to $-\dot{z}$ which $h$ sendy to $-\dot{z}, h$ I sends $\dot{y}$ to $-\dot{I}$ which $c$ sends to $-\dot{x}$ which $h$ sends to $\hat{y}$, and $h^{-1}$ gends $\dot{z}$ to $\dot{z}$ which $c$ sends to $\dot{y}$ which $h$ geads to $\dot{x}$. The net result is a rotation by $\pi / 2$ about the $-\bar{y}$ axis. It is no accident that this is Where $h$ ser ds th aich is c's aris.

Cuniugation saptures the noncommutativity of the group that is at issue here. $A D_{h}$ ' leaves the identity invarianc (gince $h \cdot e \cdot h^{-1}=e$ ). We may therefore take the denrative of $A D_{h}$. at the identity to get a linear map from the Lie algebra to itgelf denoted $A d_{h^{*}}$. Ad is actually a representation of $G$ on its Lie algebra and i. sometimes called the fundamental repregentation. For rotations, $A d_{h}$ takes an inf uitesinal rotation about an axis $v$ to an infinitesimal rotation about the axis $h \cdot v$

If we take the derivative of $A d_{h}$, in the $h$ variable, we get an action ad of the Lie algebra on itself. The action of an element $u \in g$ is none other than Lie bracket with u, i.e.:

$$
\operatorname{ad}_{\mathrm{u}} \cdot v=[u, v]
$$

For rocations, whis action is given by the cross product. An infinitesimal rotation about axis $u$ followed by an infinitesimal rotation about axis $v$ differs from first 


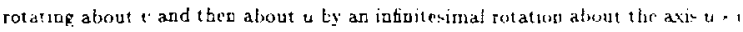

We have seen that the dual of the Lie algebra $g^{*}$ nlays an mportani role m

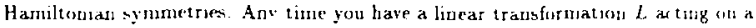
vector space $l^{*}$, you can define its adjoint $L^{*}$ atting on $l^{*}$ by requiring that

$$
\left\langle L^{*} \perp, t\right\rangle=\left\langle a, L_{t}\right\rangle
$$

The adjoint of $A A_{h}$. is called the coadjoint action of $G$ on the duaj of its Lis algebra $g^{*}$ and is pritten $A d_{h}^{*}$. The action of the rotation group on angular momenta tnat we discovered above is an example of this. The rotation it xcts on the angular momentum a to give an angular mumentum $A d_{h}^{*}$ a which is rotated by $h$.

\subsubsection{Equivariance of the Monnentum Map}

One usually requires that a roc nentum mapping be equivariant as in this ex. ample (see [Abrahaw and Marsden, 1978] p. 269). This means that the value of the momentum map varies as the group acts on the phise space according to the coadoint action:

$$
J(g \cdot x)=A d_{g}^{*} \cdot J(x)
$$

The interpretarion of $t^{3}+$ for the rotation group is -5 follows I is a foint in phase space. $g$ is a rotation (1.e. an element of $S O(3)$ ) $g \cdot x$ is the point in phase spare we get to by applying the rotation $g$ (for a mechanical system, $g \cdot x$ just has each of its position and monyentum yariobles rotated by $g) . J(q, x)$ ir the angular mometurn of the rotated state It is na urally an element of the 3-dunensional dual of the Lie algetra of $S O(3) J(5)$ is the angular nometurn if the ongiual state $A, t_{y}$ 
is the cen ijorint action of the rotation $g$ on the dual of the Lie algebra of $S O(3)$. Here thic artion juat rotates the angulas nomentum vector by $g$. That the action in equirariant says that the angular momentum of the system rutated by $g$ is the rudatus by gof the original angular mornentum.

\subsubsection{Multidimengional Reduction using a Coadjoint Lsotropy Subgroup}

thet us now try to minuic the reduction procrdure in this noncommutative case. First we restrict at tention to the sibset of phase space

$$
J=\mu \text {, }
$$

where $\mu$ L a constant element of $g^{*}$. The dynamics restricts to this subset because $J$ is a congtapt of $t h$-motion. The whole group $G$ does not act on this subset lowever, because a general element of $G$ will cbange the value of $J$. The subgroup of $G$ which leaves $\mu$ invanant under the coadjoint action (known as $\mu$ 's isotropy subgroup $G_{\mu}$ ) will act on this subset, and we way drop the dynamics down to its orbit space The resulting space,

$$
J^{-1}(\mu) / G_{\mu}
$$

has a natural symplectic structure, and the Hamiltonian restrict: ${ }^{\prime}$ to it generates the projected dyamies. For the rotation group example, we restrict, to states with a given total angular momenturn (eliminating 3 dimensions) and then forget about the ar gle of rotation about the axis defined by tha: angular momentum (eliminating vale more) The result is a phase apace of four dimensions lower than we started with 
2.7.0. Multidimengional Reduction using Coadjoint Orbits

We may obtain the same result in arother way. Cunsider the orbit of a particular element $\mu$ of the dual of the Lic algebra under the coarjoint artion This coadjoint orbit $O$, has a natural symplecic strurture with we will discusa momentarily (see [Abraham and Marsden, 1978] p. 302). For the rotation group the coadjoint orbite are spheres of constant total angular momentum (and the origin). This is !-ecause the rotation group acts on elements of the dual of the Lie algebra by rotating them, and the eet of all vectors reachable by rotation frora a given vector is a sphcie. The orbit space of $M$ modulo $G$ has a natural Poisson structure (the bracket of $G$ invariant functions is $G$ invariant) which is not typically symplectic. The symplectic leaves of this structure project onto the coadjoint orbits under the moment um map. The inverse image of a whole coadjoint orbit under the momentum map is invariant under the group action on $M$. The orbit space

$$
J^{-1} O_{\mu} / G
$$

is the same reduced space we constructed above. For the rotation group this consists of restricting to states with a given total magnitude of angu'ar momentum and then modding out by the whole rotation group.

\subsubsection{The Lie-Poisan Bracket and Group Configuration Spaceg}

An important example of redurtion applies to wechanical systemu whose configuration space is the symmetry group itselr. We will see that the free rigid body and the perfect fluid are examples of this type in the uext two sections, a fact first 
diacused in [Arnold, 1966]. The phase space $M$ is then $T^{*} G$ and the $G$ action is the canonical lift to $T^{\circ} G$ of left or right multiplication. The $G$ orbits bave one point in each cotangent Eber (i.c. the group action associates with a given momentum in a given configuration exactly one momentum in each other configuration) and so we may iduntify the orbit space with the cotangent space at the identity, i.e. the dual of the Lie algebra. If the group identity represents a reference configration, then we may use the group action to identify momenea in an arbitrary configuration with momenta in the reference. If we apply the process of reduction to this setting, this shows that the orbit space of $G$ acting on $T^{*} G$ may be identified with the dual of the Lie algehra $g^{*}$. If we forget about the configuration and identify momenta with momenta at the reference, then our phare space becomes the space of momenta at the reference.

The mornentum map for the group action is then the identity. The coudjoint orbits receive a natural symplectic structure, being the reduced spaces. These oymplectic structures are known as Kirillov-Kostant-Souriou (KKS) symplectic atructures. If we just consider the orbit space $T^{*} G / G$, theg we obtain a nutrial Poisgon bracket on $g^{*}$ already known to Sophus Lie ([Weinstein, 1983b]) and so -alled the Lie Prisson bracket. Explicitly $t$ is

$$
\{f, g\}(\alpha)=\left\langle a,\left|\frac{\delta f}{\delta \alpha}, \frac{\delta g}{\delta \alpha}\right|\right\rangle
$$

where $\alpha \in g^{*}, f$ and $g$ are functions on $g^{*},[$,$] is the Lie algebra bracket, and \langle$, is the natural pairing of $g$ and $g^{\circ}$. This bracket is behind many of the nontrivial Poisson structures recently discovered in various areas of physics. 


\subsubsection{Eulet'n Equations for the Free Rigid Body}

As an example. Jet us consider Euler's equations for the free rigd horty lare (Abraham and Maroden, 1978i p. 311). To sperify the confeguration of a fere regid body, re give a reference configuration and every other ronfiguration is uniquely sperified oy the eleruent of the rotation group that acts nu the reference to give that configuration. The configuration space is therefore idedtifiable with the group $S O(3)$ itself. As the body rotates in some manner, the representative point in $S O(3)$ moves along a rurve in $S O(3)$, The angular velocity of the body (i.e the velority in this confguration epace) in a given confguration reprcients the first order chango in configiration as we let it evolve for a short time. Two evolytion curves througb a given configua ation point have the same angular yelocity if they are tangent to first order. In section 2.2 .6 we defined a tangent vector to be just such an equivalence class of eurcs. Gcometrically, then, we may identify the angular velocity in a given configuration with a tangent vector to $S O(3)$ based at the point representing that configuration. Therefore the state including the angular velocity is naturally a point in $T S O(3)$.

The angular momentum is obtained by acting on the angular velocity ty the moment of inertia tensor. Since the wonned of inertia tensor pairs with two copies of the angular velocity to give the kinetic energy which as a scalar, we sec that botb of its indices are covariant (i.e. it has two slots for vectors). The angular momentum arisn from flling only one of the slots and is therefore a covector (i.c a one-forw) For general Lagrangiat gystems the momentum is actined in terms of the Lagrangian as $p-\frac{\partial I}{\partial y}$. Since $L$ is a geadar, this agaln shows that $p$ is a ome 
form (wbich pairs with vector, wo give the first-order cbange in $L$ when the velocity is varied along the rector.' In section 16.5 .11 , this kind of map is defined as the Legradre mar gen rated by $L$ from the tangant space to the cotangent space. The moment of inertia teusor (and in general the bilinear kinetic energy form) plays the role of a metric on configuration space which converts velocity vectors to momentum nneformis For example, in the simple free particle relation $p=m v$, we sbould view the mass as a tensor (we can sec that it is pot a scalar by considering multiple particles with different masser). Therefore the state including angular momenturn is a point in $T^{*} S O(3)$. This is then the catural phase space for the rigid body.

A priori, there is no way of comparing the angular velocity or momentum in one configuration pitb that in anotber. Using the growp action, however, we may push all velocitieg to velocities at the identity (i.e. velocities on the reference configuration) which may be identified as elements of the Lie algebra. Both left and righe nultiplication can bring ws to the identity eince they each act on the group trassitively. Consider a path at the identity (for example a rotation ahout the $z$ axis) to which a given element of $g$ is tangent. Left multiplication by $h \in G$ means move along the path and then rotate by $h$. Thus the patb is agsociated with the box, and we get the angular velocity is the body-fixed frame. Multiplying on the right means rotate first by $h$, then follow the path. The path applied is ill lependent of the configuration of the body (degrribed by $h$ ) and so its tangent represents angular velocity in the space-fixed frame. Similarly, left multiplication gives angular momentum in the body-fxed frame and right multiplication gives it in the space-fixed frame. At a configuration represented by $h \in G$, the map from 
$g$ to $g$ that takes spatial angular velocity to body angular velocity is the adjoint action of $h$. Similarly, the map from $g^{*}$ to $g^{*}$ that takes spatial angular momentum to body angular momentum is the coadjoint action.

The energy depends only on the angular momer :um in the body (the orientation in space is irrelevant for a free rigir body) and so the Hamiltonian an $T^{*} S O(3)$ ic invariant under the colangent lift of left muliplication and we are indeed in the situation deacribed above. If we drop down to the orbit space of this left multiplication. we get a Poisson bracket and Hamiltonian on the three dimcosional spare of angular momenta in the body. The dynamics on this space is exactly Euler's equations. The Poiss a bracket is explicitly given by

$$
\left\{J_{x}, J_{v}\right\}=J_{2}
$$

plus cylic permutations. The total angular momentum $J_{x}^{2}+J_{y}^{2}+J_{x}^{2}$ is a Casimir function aud so is automatically conserved. The coadjoint orbits (aud so the symplectic leaves and bones) are the spheres of constant tot - angular momentum and the origin as shown in figure (2.12). The area element on each sptere is the two-form. representing the KKS rymplectic atrucuture.

\subsubsection{Euler'g Equationg for a Perfect Fluid}

In ar exactly analogous way, we may consider the Hamiltonian structure of a perfect Huid. If we chonse a reference cont guration, then to got any other enafiguration we apply a unique diffeomorphism is in Evure (2 13) (volume preserving if the fluid is incompressible). Thus the configurating spare may be ideatifed with 


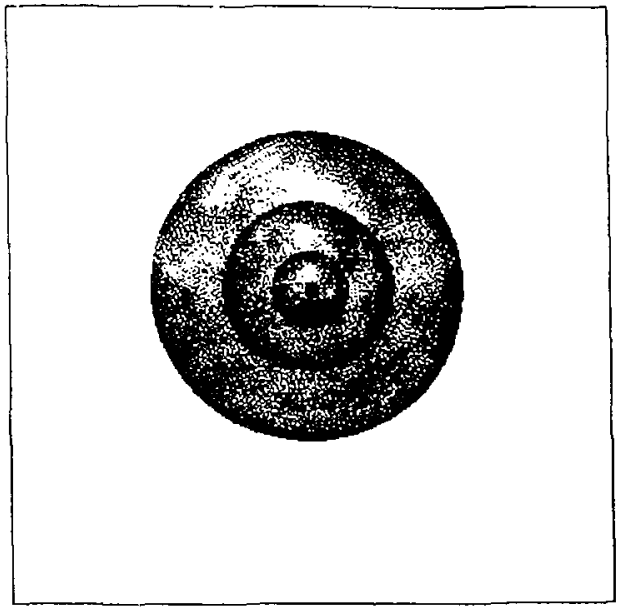

Figure 2.12: The coadjoint orbits of the rotation group.

the group of diffeormophisms of the region in which the fluid resides. The state of the fluid plus its velocity feld is represented by a point in the tangent bundle of this group. Points of the phase space represent the state of the fluid and the momentum density and so lie in the cotangent bundle of the group. Again we may identify velocities and momenta with elements of the Lie algebra and its dual by left or tught multiplication. Right multiplication gives the Eulerian velocity or momentum field in space. Left multiplication gives them for material points in the reference 
confguration. Here, in contrast to the righ body caue. the energy depends noly on the spatial momentum (abich fuid particle where is energetic.liy irrelevan') and so the Hamiltonian is right invariant Dropping to the whlt spare gives us dynames for the spatial momentum density, i.e. Euler's fluid equations, in Hamiltoni. n firm

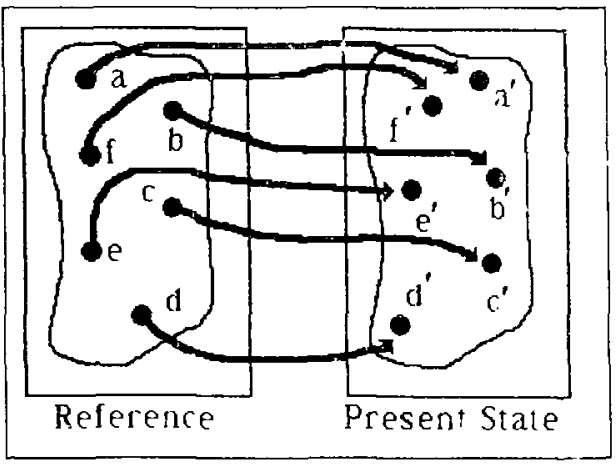

Figure 2.13: A configuraton of the fluid specifier: by a diffeomorphism.

\subsubsection{Gaysg and Plagmag}

For gases and plasmas, the state of the sv tem is represented by the particle distribution function on sinsle-particle phase space. This distritution function evolves $b y$ the action of simplectomorphisms if a anonical transformatioys of this phase spare (see Weinstein, 1984t, for more discussion an 1 referenceil. The 
group of symplectomorphisms has the Haniltonian vector fields as its Lic algebra. We tras irfontify this with the spare of fugr ions on the phase space where the lie brarket is the Poisson bracket of functions. The dual of the Lie algebra is then dencities in phase space. which we may use to describe the kinetic state of plasmas and gates The coadjoint action just pushes the density around by the symplectomorphism One coadjoint orbit comes from considering a delta distribution on phase space. The symplertomorphisms push it all over phase space to give a coadjoin ${ }^{+}$ orbit that is identifable with the original phase space. In fact the KKS symplectic structure is exactly the original symplectic structure. This shows that every symplectic manifold is a coadjoint orbit (albeit in the dual of the Lie algebra of the infnite-dimension $d$ group of canonical transformations of that manifold).

It is interesting to consider some of the other coadjoint orbits for this system. In chapter 9, eikonal waves are associated with certain distributions on $(z, k)$ space Whose support (i.e. the closure of the complement of the region rhere the region vaniches) is a Lagrangian submanifola (an N-dimensional wanifold on which the symplectic structure vanishes, see section 7.1.4). The space of such distributions is a union of coadjoint orbits of the group of symplectomorphisms.

Any local piece of a Lagrangian submanifold can be made to coincide with a local piece of any other Lagrangian submanifold by a canonical transformation [W'einstein, 1977]. In fact, the symplectic structure of a small neighborhood of a small piece of Lagrangian suthanifold is identifiable with the canonical symplectic structure of a small neightworhood of the zero-section of the cotangent bundle of the piece of Lagrangian submanifold In other words, small regions of any Lagrangian 


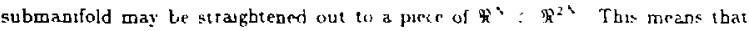

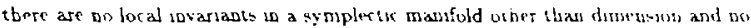
local structure assoctated with a Lagr angan submanifold otbes than the property of beug Lagrangian. (As a sunple example of something with go local cons* rants yet still obeying a global constraint, consider a Fater ballonn The surface of the balloun is arbitrary locally but globally must enclose a grven volume. T This bebavor is in great distinrtion to the case of Riemannian geometry witere local pieces of space are invariantly characterized by various curvatures and even tat submanifold anay sit in space in locally differeat ways The less rigid natwe of symplectic geometry helps to give it its characteristic feel and makes the study of pbase or" * quite different from the study of spacetime.

In section 10.1 we show that an arbitrary diffenorpbigm of a manifold $M$ may be extended to a symple omorphism of $T^{*} M$ which acts on the zero section according to the original diffeurnorphism. We may use this type of map to vary the value of a distribution with Lagrangian support while leaving the support manifold invariant. There are global constraunts on the image of a Lagrangian submanifold under a cinonical transformation as fiell. In $\$^{2 N}$ one can associate with each Lagr angian torus, the actions of each of the non-contrartible joops lying in it. These actions cannot change under a symplectomorphism. In non-trivial topologies. one must talk about the change in action of a lonp under a feformation becauge there may be no disk vitin the loop as its boundary (the change in action is simply the symectir area of the region soupt out by the loop under deformaturil). If the deformations are exact symplectomorphiams (e. g. symplertomorphisma of a 
simply rounerted region), then the artion ranuot change. One may show that, other than this constrant on actjons. earh Lagrangian torus can be taked to every other nearby Lagrangian torus

There are a, she subtleties in generalizing from a delta function at a point to dels a functic ns defiong a submanifold. In general, a distribution is a linear functimal satıfy ng cetain continuity criteria that associatng a real number with each swoolh function (satisfying certain vanishing criteria, see for instance [Hörmander, 1983\}). Ke think of a distribution as something we may integrate smooth fuactions against. A delta function at a point $p \in M$ in a manifold just assigns to eacb smooth function $f$ its value $f(p)$ at the point $p$. The analog of a delta function whose support is a Lagrangian submanifold would associate with each function on $M$ itg integral over the Lagrangian rubmanifold. This requires a measure on the Lagrangian sub. "ifold. The space of Lagrangian supported delta-like distributions may be identified with the gmooth Lagrangian embeddings of it dimensional manifolds with measures on them. Le' va call thesr measured Lagrangian submanifolds. The space of measured Lagrangian tori with given actions is thus a coadjoint orbit. Similarly, the space of measured loops with given artion is a coadjoint orbit and therefore a symplectic manifold. 


\section{R. ' jegmetric Hamiltoniad Perturbation Theory}

Let us now relate this geometric Hamiltonian mechanies to the geometric perturbation theory ne discused earber. We nill see that the Jib order ferturberd dynawes bas a natural Hamiltonian structure if the exart dyamies does More details on the ideas of this section are given in sertion 46

The firgt thing to note is that the patb space dynamiss is Hamilonian This is not surprising if we think of the patb space as a kind of direct isiegral of the phase spaces at each t. The dynamics at different e's are completely independent (except for the fact that the paths are smootb). If we bad the product of only two Hamilonian systems (ingtead of a continuum of them) then we would get the correct dynamics from a syuplectic structure which ts the sum of the pullback to the product of the individual symplectic structures and a Hamiltonian which is the sum of the pulled tack Hamilionians. Fizending this construction ti a continuum of inultiplicands ds to the symplectic structure

$$
\dot{u}_{p}\left(\tilde{V}_{1}, \dot{V}_{2}\right) \equiv \int_{0}^{1}-\dot{p}_{(\ell)}\left(v_{1}(\ell, p(t)), V_{2}\left(\ell, p_{1}(t)\right)\right) d
$$

The analog of the sum of Hamiltonianc is

$$
\dot{H} \cdot p) \equiv \int_{0}^{1} H(\epsilon, p(\ell)) d \epsilon
$$

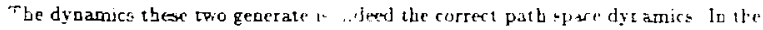
rahe' of a product of a finte number of Hanileonian systens. We are artua'، allowed t. take any linear combiuation of the symplectu strurture (unstrad of a slragkt

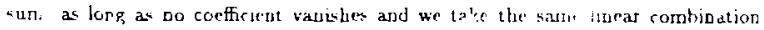


of Hamitonians. If a coefficient vanishes, that factor has no dyaamics. For our [rerturhatmo dyumics then. we want to ignore the region in the interval that is awav from $t=0$

In fact if oc ingart the $J$ th derivative of a delta function into the integrals in (2 101) and (2.102) we get the corrett perturbatic n dyamics on JM. If the Poisson t.rarket on $M$ is $\left\{x^{a}, x^{b}\right\}=J^{a b}$ then the bracket on $J M$ is

$$
\left\{x_{k}^{a}, x_{m}^{b}\right\}=J^{a b} \frac{k ! m !}{J !} \delta_{k, j-m}
$$

and the Hamiltonian 19

$$
\left.\bar{H}\left(x_{0}, \ldots, x_{J}\right) \equiv \frac{d^{J}}{d r^{J}}\right|_{l=0} H\left(\epsilon, x_{0}-\epsilon x_{1}+\cdots+\frac{\epsilon^{j}}{J !} x_{J}\right) .
$$

Togetber these give the correct perturbation dynamics. Notice that the 0th order variables are pared with $J$ th order variables, 1 st order with $J$ - lat order, etc.

From the above coordinate description it is not clear that thiz bracket is in fact intriasic. We may shor this by conaidering the iterated tangent bundle to $M$. The tangent bundle to a symplectic marifold has a natural aymplectic structure. ${ }^{\prime}-$ is the structure on $M$, then we may use it to identify $T M$ and $T^{\bullet} M . T^{\bullet} M$ bas a natural symplectic structure, which we sfined in section 4 . The structure on $T M$ is obtained by pulling $T^{*} M$ ' back using the identification stipplied by $\omega$. This o; cration may be iterated to give symplectic structures on the "erated tangent buajles $T T M, T T T M, T T T T M$, etr The $J$ th order jets naturally embed into the $J$ th iterated tangent hundle. If t , symplectic structure on $T^{J} M$ is pulled back to J.11 we obtain the jet Poisson bracket in equation (2.103). 


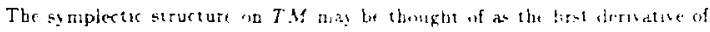

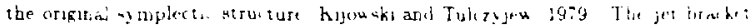

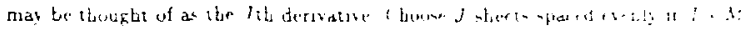

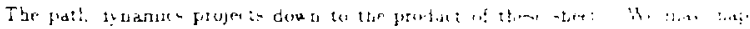

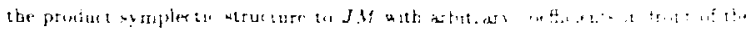

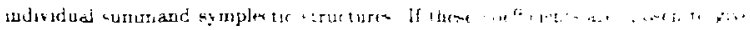

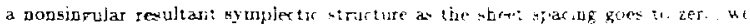

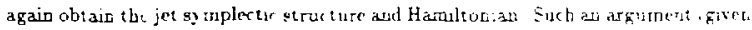

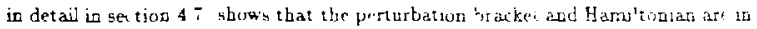

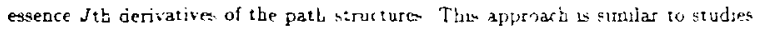
of Gaite differences as approximants of sodmary derivatives

\subsubsection{Lineariyed Dyt malcs at a Pixed Polni from Jet Bracket}

We haw seen that when the Poisson brarket is degenerate. non-degenerate symplectic leaves and bones are injected into the Poisson manifnld as suimanifolds If a closed two-form is degenerate. unen we project ont the degenerate dirortions to obtain a symplectic manifold. The fart that the two-form is closed impires that the nibilated direction satisfy the conditions of Frobetius's theorem and so lie tangent to smootb submanifolds which we may then project along (at least localy). We hav ured an example oi this concruction above. If we insen the $J+t$ derivative of a delta function into the path symplectic integral (2.101). we obtain a degenerate, closed two-form on the patb space $P_{l} M$. The projection elinumating the degenerate directions is exactly abe projection from patb space down to the jet 
apace $J M$ The reaultagas simplerter structure is the get perturbation strurture If

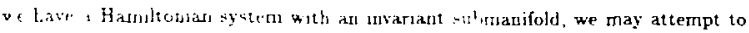

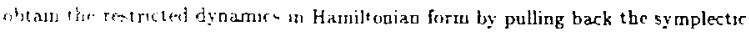
-1fut:a The remulting twi-form will be closer but may not be qou-degenerate If thu r- asc ne givbally we may apply the above projection A special case of

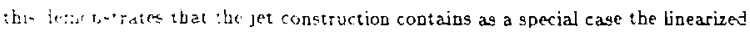
dynamus of a Haniltonas sycuen around a fixed point. We consider the 2-jet space 2.4 The rutmanifoid of jets with base poi . equal to ize fixed point is an invariant Mbmintoli Becaus the zero order base directions are paired with the second order durertions in (2 103), restricting to a given bagepoint makes the second order directions degencrate. Projecting these out leaves us with only the first order jets at the Exed pount (i.e. the tangent space there). These are paired with themselves by the second order bracket according to the original symplectic structure at the fxed point. The second order Hamiltonian (2.104) gives the quadratic piece of the Tay'or expansion in the $x_{1}$ vanables. Together these give the linearzed flow in the tangent spars of the fixed point as a Hamiltonian system. The situation in Poisson manifolds is more complex [Weinstein, 1983a]. If the fixed point is in a symplectic leaf (as opposed to a bone), we take the Poisson bracket at the point, the quadratic part of the Hamiltonian in the leaf direction, and the linear part of the Hamiltonian across leaves. The bones are mnre difficuit.

We can also consider the same approach to the evolution of $J$-jets based at a zero order fixed point. The zero order variablins are now paired with $J$-th order variabies. When we restrict to a given zero order point, the $J$-th order directions 


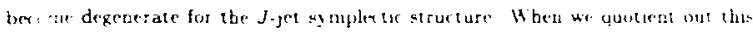

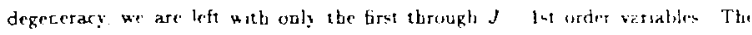

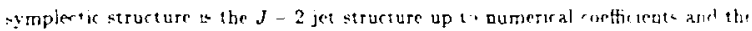
Hamiloned is the $J$-th derivatue

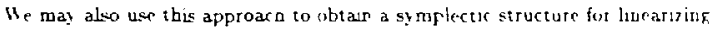
abuut a single non-fixed unperturbed orbit We resenct the spare of 2-jets to jets that originat o the orhit of interest. There is now a $2 Y-1$ dimensional degenerate foliation which we mav quotier: by. The result is a symplectic space whose points indicats position along the unperturbed orbit, an element of the tasgent space at that position, and a single quotiented 2 -jet variable that pars non-trivially with the ortic position variable. The Hamilionian is again the second derivative of the original Bamiltonian:

$$
\begin{aligned}
\bar{H}\left(x_{0}, x_{1}, x_{2}\right)=\left.\frac{d^{2}}{d t^{2}}\right|_{\ell=0} H\left(\epsilon . x_{0}+\epsilon x_{1}+\frac{\epsilon^{2}}{2} x_{2}\right) \\
=\left.\frac{d^{2}}{d \epsilon^{2}}\right|_{\epsilon=0}\left(U\left(\epsilon, x_{0}\right)+\epsilon \frac{\partial H}{\partial x_{0}}\left(\epsilon, I_{0}\right) x_{1}+\frac{\epsilon^{2}}{2} \frac{\partial^{c} H}{\partial x_{0}^{2}}\left(\epsilon, x_{0}\right) x_{1}^{2}+\frac{t^{2}}{2} \frac{\partial y}{\partial x_{0}} I_{2}\right) \\
\left.=\frac{\partial^{2} H}{\partial c^{2}} H\left(\epsilon, x_{0}\right)+\frac{\partial}{\partial x_{0}} \frac{\partial H}{\partial t}, t, I_{0}\right) x_{1}+\frac{\partial^{2} H}{\partial x_{0}^{2}} x_{1}^{2}+\frac{\partial H}{\partial x_{0}} I_{2} .
\end{aligned}
$$

The dependence of this on $x_{2}$ is only through the term

$$
\frac{\partial H}{\partial x_{0}}\left(0, x_{0}\right)-I_{2} \text {. }
$$

$\dot{H}$ is thus constani under variations of $x_{2}$ in the annibilator subspare of $d H$. But these are exartly the dertions symple-ieally orthogonal to the zero order urbit and so span the degenerate $f$ ation. The lnearizen syumics about an unperturbed orbit therefore bas an invariajt formulatus. 


\section{8.z. Symmetry and Perturbetion Theory}

We bave seen hou important symmetry and its related concepts are in Hamiltowian mechanirs. How do the symmetry operations intermix with the perturbation operations" A Hamiltoniw $G$ action on $M$ lifts to both the path spare $P M$ (just pusb the whole path around by the group action) and the jet $=$ pace $J M$ (just push the jet around). The corresponding monentur maps are jugt the integral along a patin of the $A$ mumentun: map and the same integral with the $J$ th derivative of a delta funcion thrown in. Both are equivariant.

When considering reduction we quickly see that these groups are not of bigh cnough dimension. A 4-dimensional phace spare with a 1-dimensional symmetry drops down ", 2 dimensions. The frst order perturbation space has 8 dimensions. In the presence of aymmetry we expect to be able to drop this down to the first order perturbation space of the 2-dimensional reduced space. The above group action can only eliminate 2 dimensions instead of the needed 4 and so we expect a lasger group to act. This is indeed the case. It makes sense to multiply two paths in a group by inultiplying pointwige. Thus $P G$ is an infinite dimensional "Lie" group and its "Lie" algebra is tbe path space of $G$ 's Lie algebra $g$. $P G$ has a Hamiltonian action on the path space $P M$ by multiplying the point $p(c)$ by tbe group element $g(c)$. The momeutuw map sends a path in $M$ to a path in $g^{*}$ gotten by applying $M$ 's momentum map to each $\epsilon$. In an exactly arialogous way, we may define the grcup $J G$ of $J$-jets of paths in $G$ with Lie algebra being $J$-jets of paths in $g$. This acts is a Hamiltonian and equivariant way on the perturbation space $J M$. The morneatum map in obtained by extendiur a jet to auy consistent patb, taking the 
patb momeutum map to $F^{\prime} g^{*}$ and dropping dow"I to $J_{g^{*}}$

The process of reduction commutes with taking the path spare or fet spact The jet or path spare of the reduced spare is the redured space of the jet or prath space by the jet or patis group.

Wic have secr the central importance of the dual of the Lie algebra and the coadjoint orbits with their KKS symplectic atructure for fbysucs the have seen that any symplectic manifold may be thought of as a coadjunt orbit in the dual of the Lie Igebra of some group. It lurns out that if $M$ is a cuadjoint orht is the dual of $G$ 's Lie algebra then the perturbation space $J M$ with the jet symplectic struiture are naturally a coadjoint orbit in the dual of the Lie algebra of the jet group $J G$ and the jet wracket $\{2.103\}$ is the natural KKS symplectic structure. This is show in Bection 4.8.6.

These relations are at the heart of a new framework for singular hie transform perturbation theory about which we oill report in chapter 5 . Here we discuss only the first order method of averaging. 


\subsection{The Mathod of Averaging for Hamiltonian Syotema}

Aary of the interesting plysical regularitin: wo fod in diverge systems are 1 auser by the preseyce of processes that oprate un widely aeparated cime scales. The base minplification that this spasation entails is that the fast degrees of freedom act abnot at if the slow variables are constant and the slow degrees of freedom are afferted only by the average behavior of the fast variables. Bogoliubov in particular has used this separation of scales with great success in many examples. For exarople be obtains the Boltzmann equation from the BBGKY hierarchy of evolution equations for correlation functions by lolding the 1-particle distribution functiong fixed while determining the fast evalution of the higher correlations, and then subatituting the result in as the collision term driving the 1-particle evolution. One makes a similar separation in calculating fluid quantities like viscosity, thermal conductivity, diffusion or electrical conductivity from an underlying kinetic description. In studying complex situations with slow, heavy nuclei and fast, light electrons in molecular and solid state physics, one often holds the nuclei fixed, calculate the electron ground state and energy as a function of the nuclei positions and then uses them to define an effective potential in which the nuclei move (this is the Born-Oppenteimer approach).

We have seen that in the presence of an exact gymmetry, the symmetry directions may be completely eliminated by the process of reduction. We will row discuss how the averaging underlying the systems with separated scales can be viewed as reduction by an approximate symmetry. One often finds that the effect of "forgetting- tbese dcgrees of freedow is to introduce an amended potential into the 


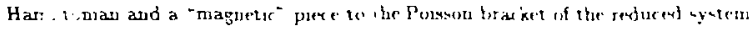

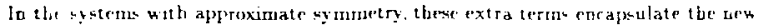

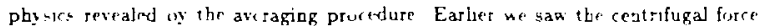

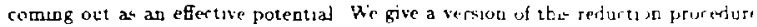
which begiers by including the "angle of the earth" as a dyuanical variable and reduces by the earb's rotation and the rotation of the systen tengrther in the nrext chapter The resulting reduced space gives the centrifuga' force as an amended potestial w the reduced Hamilos an and the Coriolis force as a new term to the Poisson bracket.

\subsubsection{Appraximnte Noet'jer'B Theorem}

When we introduce a perturbation which hreaks a ymmetry we no longer have exactly conserved quantities. It is easy to prove an "approximate Noether's theorem ${ }^{+}$, bozever. which says that the momentum map for a slightly broken symmetr. evolves slowly:

$$
X_{\mathcal{H}} \cdot H=\{H, J\}=\cdot \quad \text { implies } \quad j=\{J, H\}=-\epsilon \text {. }
$$

\subsubsection{Hamiltonian Averaging as R.duction by a Circle Action}

In the special case where the unperturbed dynanics it e.tirely comprosed of periodic orbits, the action of the orbit through each point is the menentura map of a circle symmespy of the unperturbed Hamiltonian. As we turn on a perturbation wharh breake tbu symmetry the motion will stll be promarly around the loops. 
but it pill ylowly drift from lonp to loop Because the symmetry is broked, different point - un a loop fill move toward different loops As the perturbation is made smallf. thesgh. phase points orbit many times near a given loop before drifting atra? Tha suggewts (correct!y) that the $f$ rturbation a point feets will asymptotirally be the same as the average around an unperturbed loop. Bec ause this average in the sane for all points on a loop, for small perturbations entire loops drift onto other entire loops. We may therefore drop the dynamies down to the loop space. In fart one can prove that for a general (even diasipative) aystem where the unperturbed dynamics $X_{0}$ is entirely composed of periodic orbits, the motion of a point under the flow of $X_{0}+c X_{1}$ projected down to the loop space remaing within $\epsilon$ for a time $1 / c$ of the arbit of a corresponding point on the loop space under the flow of the average of $X_{1}$ around each loop projected down [Arnold, 1983]. In the Hamiltonian case we break the circle symmetry of $H_{0}$ to get the perturbed system $\mathrm{H}_{0}+\mathrm{CH}$. We average $H_{1}$ around the loops to get $\bar{H}_{1} \cdot H_{0}+\epsilon \bar{H}_{1}$ is again invariant under the circle action and so we may perform reduction. The reduced dyamics is the slox ¿ynamics on the reruced space and the fact that we may restrict to a constant value of the momertum map shows that it is actually conserved to within order $t$ for time $1 / \epsilon$. The momentum map for the circle group sends each point of phase space to the action of the loop it belongs to. The action of a loop is the integral of the symplectic forw $w$ over a disc whose boundary is the loop. Since $\omega$ is invariant under a canonical transformation, sp is the value of the action of a loop (this is Poincare's first integrai invariant). If the dynamics was represented by a canon transformation that took loops exactiy to uther loops. then the action would be an 
exact contant of the motion The irue dy name = takes loups to only withe of another loop after tmue 1 . Tble says that the as tuon of the loop a phan spat

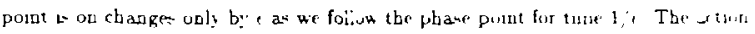

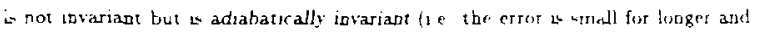

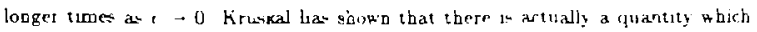
is consered to all orders in e for tume 1/t 'Kruskal. 196'z. (we give a geometrie formulation of thic result in chapter 5) Getting results valid fo: times locger than 1/e ic extremely important physically, but so fas no general tweory exists. Chapter 5 includes sorme discussion of the relevant issues here.

\subsubsection{Averaging and the Jet Plcture}

Let - relate . his procedure $t$ be perturbation struc'ures we drveloped in previous sections. We bave an action of the circle grwip $S^{2}$ on $M$. This lifts to an artion of $P S^{1}$ on $P M$ and $J S^{1}$ on $J M$. The unpercurbed Pamiltonian is invariant under the $S^{1}$ action on $M$, but the path and perturbation Hamiltonians are not invariant under $P S^{1}$ and $J S^{1}$. We would like to change the action of $P S^{1}$ on $P M$ so as to leave the path Hamiltonian invariant and so allow reduction. Fince the resulting action should still be Hamiltonian, we look for an t-dependent canonical transformation of $I \times M$ which is the identity at $\epsilon=0$ and which pushes the $P J^{\prime}$ action into a symmetry. The metbod of Lie transforms (see 0 [Cary, 1981\} ardo [Nayfeh, 1973! p. 200] attempts to do this at the perturbation level, letting the canonical transiorwation be the How of an a-dependeat Hamiltonian, which is tbed obtained orde by order. Here we nem only consider the first urder group artion 
of $1 S^{1}-T S^{1}$ on $1 M-T M$ We know that the axtion will be perturbed so that tise value of the redued Harniltonian is the average of the perturbed Hamiltonian arougd the untridaformed circtes $T M$ bat twice the dimension of $M$. Reducing by TS elminates 4 dimensions. The regulting dynamical vector field has no unpersulted component. One may think of this as the reason for getting results good for time $1 /$ (1t ts the effect of the unperiurbed flow on the perturbation which causes thic level of serularity). In this situation it makes intrinsic sense to project the 1 st order vector field down to $M$, where it represents the slow dynamics.

\subsubsection{Extensions from Loops to Tori and Energy Surfaces}

A lrop in a 2-dimensional phase space (like an orbit of a simple harmonic oscillator) mav be thought of in 3 ways. It is 1-dimensional, 1 dimension less than 2 , and half of 2. Each has an important generalization to higher dimensional Hamiltonian systems. In the presence of a elowly varyigg Hamiltonian, we have already seen that the action of a 1-dimensional loop is conserved. There is an analogous result for half dimensional Lagrangian ..s. Kubo has shown that for a aystem ergodic on an energy surface (which has one dimensiol less tha't phase space), the volume encloged is adiabatically invariant under slow variation sf parameters [Kubo et al., 1965!. Roughy,; since the motion is ergodic, every or bit changes according to the average of the perturbation over the energy surface; thus the entire energy surface charges by the same energy ngt so is taken to another energy surface; but the volume enclosed by a surface is preserved under a canopical transformation by 


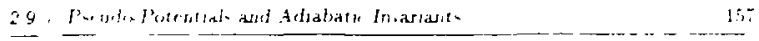

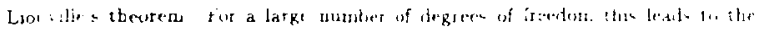

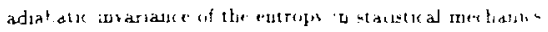

\subsubsection{Peeudo-Potentials and Adiabsicic Invarianta}

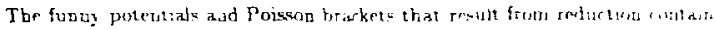

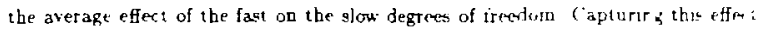
is the cotten: of many physically useful theories. It is interesting to note that in the late nimeteent h century, the idea that all potential energies were really kinetic exergies of bidden or forgotten degrees ui reedom was oup the the main motivation fo: the development of kinetic theory. We may use averaging to see bow this comes about.

Because of itu direct relevance to the ideas preseted here, let us give a long. quotation from Felix Klein's historical account of ninectenth century mathematical ibysies. He first introduces Routh suuction $R$ which was in between the Lagrangan and Ifamitionian in that ouly the fist $m$ of the $n$-opgguration ppare variables bad thet velocities Legendre transforiaed into moncenta. He continnes

"Thus the equations split into two groups, one of tbe Lagr.10gian kind and ist of the Hamiltonias. For $m=0$ the Rowth fuotson, ant therewith the systen of equations, is the sime as th twe Lagrangian case: while for $m=n$ it is the sarte as to the Hanultonsan

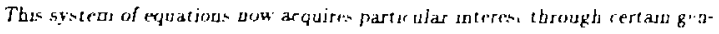
eral fundarsental concepts of merbastes that are comoertal with it liamely. If $R$ 


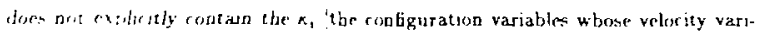

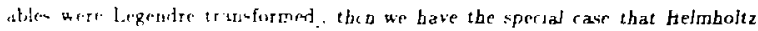

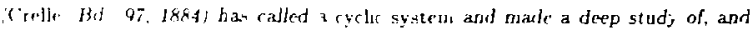

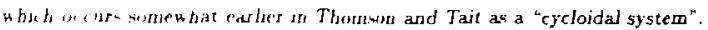

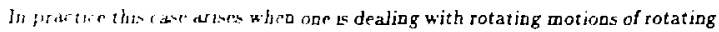

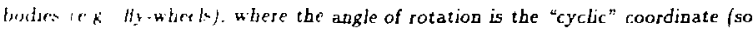

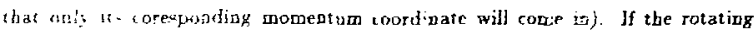

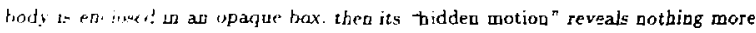
than the usual bebavior that the body as a whole shows as it moves in space (a top or grame opel. In cases like this where outside infuence on the motion of the By-wher is excluded, the momeata corresponding to the cyclic coordinates are constant.

From these facts follow sone remarhable ideas on the nature of potential asergy If we assume that the kinetic energy $T$ decomposes into a part $T(\dot{q})$ that depends ondy on the velocities $\dot{q}$ and into a part $T(\pi)$ that depends only on the cyclic momenta $\pi$ /thus assuming that there are no terms in which velocities $\dot{q}$ are multiplied by momenta $\pi$ ), then the Routb function is

$$
\begin{aligned}
R & =T(\dot{q})-T(q,-\eta \\
& =T(q, \dot{q})-T(q, c)-U(q),
\end{aligned}
$$

if we bear in mund the depencience of all the quantities on the coordinates $q$ and replare the constant momenta $\pi$, by the quantities $c$. The $q_{m+1}, \ldots, q_{n}$ are determined from the differeatial equations

$$
p_{a}=\frac{\partial T(\dot{g})}{\partial \dot{q}_{1}}, \quad \frac{d p_{a}}{d l}=\frac{? \mid T(q)-(U+T(c)) !}{\partial q_{a}}
$$




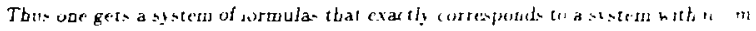

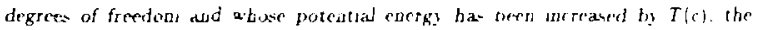

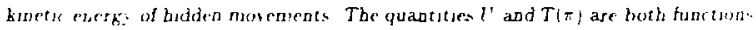
of $q$ with constant coeflicients; they wher wo the sun only together. not apdiately Heace the question arses abere we in any case bave no dea of the ehuence of the potential energy - of whether every quantity that appeds to merbanics as 'potlostia' energy" is actualy a kinetic energy caused by a hidden. cyclic, so-called "ignored" mution. Like a fata morgana, the possibility of a purely kinetic theory of wat'er appears in the distance.

This general idea was first prasented in 1888 by J.J. Thomson in bis book Applications of Dynamics to Physics and Chemistry (Jecture at Cambridge in 1886, then in the Philosophical Thansactions 1886-87). But in spec:al cases it had . eady beed pursued by W' Thomson (=Lord Kelvin), for example in his address to the British Association in Montreal in 1884, which be prudently titled "Strps to a kinetic theory of matter" (Math, and Phys. Papers, Volume 3, p. 366). This idea was fwally worked out for closed systems in Heinrich Hertz's posthwoous work of 1904, Die Prinzipien der Mechanik [The Prisciplei of Mechanics]"

\subsubsection{Ping-pong balls and One-dimensional Gases}

If we slowly move a ping pong paddle up and down from a table with a ping pong ball bouncing very rapidjy between the paddle and the table, then we will feel a varying force due to the average momenta imparted due to the imparts of the ball. Iu phase space the ball describes a rectangle and so the action is given by 
$J$ - $4 L m$ H were $L$ the distance from the paddle to the table and $V$ is the speed of the tall Becaure the is invariant under slow padd e move ments, the ball velocity Rors ac 1 / $L$. The momentum transferred on earb impart is $2 \mathrm{mV}$ and there are $V / 2 L$ tmiparte per unis of tims, so the average force felt goes like $V^{2} / L-1 / L^{3}$. Thus Tatug fith no poteutial energy at all, we end up with a $1 / L^{3}$ effective potential for the pardily:

It is rell known that slow compression of an ideal gas beeps the quantity pir constant, where the ratio of specific heats $\gamma$ depends on the properties of the gas (this folionis from the adiabatic invariance of the entropy). Our single particle result is exactly this requirement for a one-dimensional gas with $\uparrow=2$. Since particles do not interact :- an ideal gas, it makes sense that each particle should reflect the behavior of the entire gas. (A similar result holds for radiation, where the adiabatic compression of a container containing black body radiation actu on each normal mode separately and yet the overall effect keeps the spectrum black body but at a different temperature.)

\subsubsection{Oscillatory Stabilisation}

For a harmonic oscillator, the energy is the product of the action and the frequency: $H=\ldots J$. If we have a weight, hanging on a string and undergoing small amplitude oscillations as we slowly pull the string, the change in pend:alum e. ${ }^{\circ} \Gamma g y$ is the change in $J \omega . J$ remans constant and $\omega \sim \sqrt{g / L}$ so we feel a $1 / \sqrt{L}$ potential. We get other potenticls if we ask for the force we feel if we tune a guitar string as someone plays it or the acoustic pressure ou the water if we fill up a 


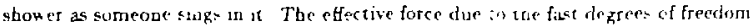

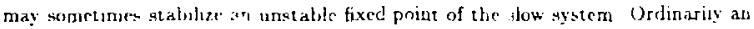
inverted peudulum is unstable aid falls to the position with the veright hangug townard. If we shake the support of the pendulum perodically basd enough and fast enough, the nverted position is stabilized! A c even more spectarilar version of this effect occurs if you shake an inverted cup of fisid and stabilize the Rayleigh-Tay lor inst ability which ordinarily causes the tuid to spill out (it is casiest to actualiy do the experment with a bigh viscosity fluid like notor oil The adea $^{2}$ of RF $\mathrm{s}^{+}$abilization is to stabilize unstable modes of a plasma (say in a tokanak) by bathing it in a bigh frequency radio wave. Some of the modern airplanes with wings in a forward axing delta are actually operated is an aerodynamically uns sble regime shat is stabilizad by the fast dyaamics of a computer controlled feedbrak loop. This aliows for great maneuyerability isince the plane would like to turn 7nyway!).

\subsubsection{Multiple Space end Time Scales}

Quite often it is very useful to split out the main dyuanics of a system and lone arize the rest, treating them as fast oscillatious. Thus one takes a fluid, elastic, or plasma medium and treats its erolution as slop oytrall development of the background mediun with fast skcillatous occuring on top of it. The effect of the oscillations th to change or renormalize the dynamics of the ba kground $\because i$ iats

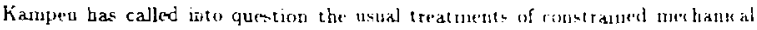

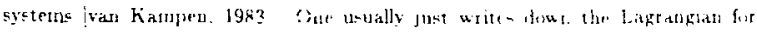


surts a syeters in generalized cuordinates which respece the congtraints. Physucally. though. one supposes that there is some large potential aormal to the constrant surfare The system will execute rapid osrillation in the normal dir zetion and slow rolutun along it If the width of the constraining potential we'l varies with the mechanical coordinates, ibee as we have seen the adiabatic invariance will give rise to a neri paeudopotential which affects the mechanical motion. In a plasma we treat the slowi, varying background as a dielectric mediur $\mathrm{l}$ in which waves propagate according to WKB theory. The waves affect the background (introducing a radiation pressure in the dynamicg) via ponderomotive forces. If we have a charged particle in the presence of a wave with a slowly varying amplitude, the particle will oscillate back and forth with the wave. It feew more of a push in going down an anplitude gradient than in going up one, leading to wrerall average force described by the ponderomotire potentia'. This kind of separation is the basis of plasma quasilinear theory. We bave extended the geometric perturbation theory to some of these singular perturbation problems. Chapter 8 gives a Hamiltonian treatment of an eikonal theory for linear or nonlinear waves (which is related to the averaged Lagrangian treatment of Whitham [Whitham, 1974|). Here let us demonstrate the efticacy of a global gecmetric apprcach only with the simple exanple of $E \times B$ drift. A charged particle restricted to a plaze with a constant perpendicular magnetic field executes periect circles If there is, in addition, an electric field then the radius of the circles is greater in low potential regions and smaller in higb potential regions. Thus the corcular ortits do not close and the particle drifts perpendicularty to the electric held A Hamiltonian treatmeut of more cotrolicated versions of this so-call d guid- 
ing center motion has been previously given [Littlejohn, 1983]. This work required great cleverres in the choice of physically relevant coordinates. We would like to demoustraie, in this simple version, how a coordinate free approach would lead us to the correct answer, with no previous knowledge. 
2.10. Example: $E \times B$ Drift

In the simplest situation we have a charged particle in the $x, y$ plane moving in the presence of a constant magnetic field $B$ which points in the $\dot{z}$ direction and a small constant electric field $\epsilon E$ which points in the $\hat{x}$ direction. We introduce the phase space $P \sim T^{*} R^{2}$ with coordinates $\left(x, y, p_{x}, p_{y}\right)$ (we use mechanical momenta $p=m v$ here). The correct dynamics in the presence of a magnetic field may be described in a Hamiltonian formulation in two ways. The standard approach is to introduce the unphysical vector potential $A$ and to work with canonical mornenta $p=m v-(e / c) A$. Here we use the physical momenta and magnetic field, but a noncanonical Poisson bracket:

$$
\{f, g\}=f_{x} g_{p_{z}}-f_{p_{z}} g_{x}+f_{y} g_{p_{y}}-f_{p_{y}} g_{y}+\frac{e B}{c}\left(f_{p_{x}} g_{p_{v}}-f_{p_{y}} g_{p_{u}}\right)
$$

We obtain the correct dynamics in this case with the Bamiltonian

$$
\boldsymbol{B}=\boldsymbol{B}_{0}+\epsilon B_{1}=\frac{1}{2 \pi n}\left(p_{x}^{2}+\boldsymbol{r}_{y}^{2}\right)-\epsilon E x
$$

The dynamies is then

$$
\begin{aligned}
& \dot{x} .: \frac{p_{x}}{m} \quad \dot{y}=\frac{p_{y}}{m} \\
& \dot{p}_{x}=\frac{e E}{m c} p_{y}+\epsilon e E \quad \dot{p}_{y}=-\frac{e B}{m c} p_{x} .
\end{aligned}
$$

The unperturbec situation here is just a charged particle on a plane in a constant magnetic field. Every orbit in this situation is a closed loop. Thus the unperturbed system bas a circle oymmetry:

$$
\begin{aligned}
\dot{x} & =\frac{p_{x}}{m} \quad \dot{y}=\frac{p_{y}}{m} \\
\dot{p}_{x} & =\frac{e B}{m c} p_{v} \quad \dot{p}_{y}=-\frac{e B}{m c} p_{x}
\end{aligned}
$$


The generator of this symmetry (i.e. the momentum map) is none other than the unperturbed Hamiltonian itself:

$$
H_{0}=\frac{1}{2 m}\left(p_{x}^{2}+p_{y}^{2}\right)
$$

This is because non-relativistic motion in a constant magnetic field has the remarkable property that the period of all orbits is the same (we could introduce a normalization constant to make it 1 or $2 \pi$ if desired). Let us obtain tbe reduced phase space and Poiss a bracket for this symmetry action. Firsi we look at the space of loops $P / S^{1}$. Each circular particle orbit has exactly one point where $p_{y}=0$ and $p_{x} \geq 0$. We may label a loop by the values of $x, y, p_{x}$ at this point. Next we restrict to the set where the momentum map is a constart: $H_{0}=\alpha$. The reduced space is

$$
R=P /\left.S^{1}\right|_{H_{0}=0}
$$

and may be coordinatized by the values of $x$ and $y$ when $p_{x}=\sqrt{2 m \alpha}$ and $p_{y}=0$. The reduced Poiseon bracket $\{,\}_{a}$ of two functions $f(x, y)$ and $g(x, y)$ is obtained by extending them to $P$ in such a way that

$$
\left.\frac{\partial \dot{f}}{\partial p_{x}}\right|_{p_{y}=\sqrt{2 m a}, p_{r}=0}=0
$$

and

$$
\left\{\hat{f}, H_{0}\right\}=0=\frac{\sqrt{2 m \alpha}}{m} \frac{\partial \hat{f}}{\partial x}-\frac{e B}{m c} \sqrt{2 m \alpha} \frac{\partial \hat{f}}{\partial p_{v}}
$$

Thus we replain $\partial / \partial p_{x}$ by 0 and $\partial / \partial p_{y}$ by $(c / \epsilon B) \partial / \partial x$ to get

$$
\{f, g\}_{\alpha}=\frac{c}{e B}\left(f_{v} g_{x}-f_{x} g_{y}\right)
$$


Thus we ser that the original spatial coordinates $x$ and $y$ now play the role of canonically conjugate variables in the reduced space. The factor of $1 / B$ in the bracket appeared in Littlejohn's work [Littlejobn, 1983]. The full system is not invariant under our circle action. If we average the perturbetion Hamiltonian $H_{1}$ around the circles, we do obtain a cire: symmetric aystem. The average of the potential eeEx around a loop is just the value when $p_{y}=0$. Thus the reduced averaged Hamiltonian is

$$
\bar{H}_{a}(x, y)=a-\epsilon e E x
$$

The reduced ayeraged dynamico is then

$$
\begin{aligned}
\dot{x} & =\left\{x, \ddot{H}_{a}\right\}_{\alpha}=0 \\
\therefore \quad y & =\left\{y, \ddot{B}_{a}\right\}_{\alpha}=\frac{c}{c B}(-c e E)=-e \frac{c E}{B} .
\end{aligned}
$$

This is indeed the $E \times B$ drift dynamics. 


\section{Chapter 3: \\ Pseudo-forces and Reduction}

"Philosopby is written in this grand book, the universe, which stands ccretinually open to our gaze. But the book cannot be understood unless one first learns to comprehend the language and read the letters in which it is compased. It is written in the language of mathematics, and its characters are triangles, circlin, and other geometric figures without which it is bumanly impassible to understand a single word of it; without these, one wanders about in a dark Jabyrinth."-Gublo in The Assayer

\subsection{Pceudp-forces and Reduction}

If you have a system that is coupled to some subdynamics and you forget about the subdynamics, the original syatem may evolve with new "pseudo-forces" acting. For simple meche ucal gygtems with symmetry, these take the foro of "maguetic" terms in the Poisson bracket (by analogy with a Hamiltonian description of particles is magotic fields; and nsw "effective potentias" in the Hamiltonian. These forces may stabilize previously unstable dynamics, just as a frec charged particle at 
rest in the plane is stabilized by a perpendicular magnetic field (magnetic stabilization). One finds these pseudo-forces also in situations where the observer's motion is included in the dynamics. In more complex examples these forces seem to lead to "convective" terms in Eulerian continua descriptions, drifts and pondermotive forces, "forces" that bend light rays in inhomogeneous mediz, prezsure and other thermodynamic forces in statistical mechanics, "renormalized" masses for quasiparticles, etc. Near the end of the last century, there was a sehool of thought that held all potential energies to be merely the kinetic energy of "hidden" degrees of freedom. This gave great impetus to the kinetic theory of matter as we discussed in section 2.9.3.1.

\subsubsection{Bletitfou Borces}

Our goal in this chapter is to give a coordinate-free interpretation to the process of chauging reference frames and to the concommitant new physical effects. We first consider the effect of a time dependent change of phase apace. A single state in the new frame corresponds to a time-parametrized curve of states in the old frame (e.g., a given configuration of particles and their velocities fixed in a rotating frame corresponds to $\mathbf{3}$ whole circle of states in a fixed frame as time evolves). We therefore bave a time-dependent identification between the original phase space and the new one. The time dependence of the identification diffeomorphism may be expressed as the low of a vector field $Y$ (for example, a rotating reference frame is described by the vector field we discussed in section $\mathbf{2 . 6}$ ). 
Let $F X_{t}$ be the flow of $X$ for time $t$, and $F Y_{t}$ the flow of $Y$ for time 1 , on $M$. $F X_{1}$ is viewed as the dynamies and $F Y_{1}$ as our "changing point of view", so the dynarical evolution we observe is $F Y_{t} \circ F X_{t}$. This is the flow of the time-dependent vector field

$$
\vec{X}=F Y_{t \cdot} \cdot X+Y
$$

by the chain rule (the lower star means pusb-forward by the map $F Y_{8}$, and represents the image of $X$ at each point under the diferential of $\left.F Y_{t}\right)$. If $X$ is $Y$-invariant, then

$$
\tilde{\boldsymbol{X}}=\boldsymbol{X}+\boldsymbol{Y} .
$$

If $\boldsymbol{X}$ is Hamiltonian $X_{H}$ aud $\boldsymbol{Y}$ is a symmetry generated by $J$, then the combined fow bas Hamiltonian

$$
\check{\boldsymbol{H}}=\boldsymbol{H}+\boldsymbol{J}
$$

$J$ represents fictitious forces in the Hamiltonian due to our changing perspective.

\subsubsection{Rotating Coordinates}

In this section we will demonstrate these ideas on the example of a rotating coordinate system for a particle in the plane. A very important gubtlety arises from the question of what the velocity and momenta are in a rotating frame. One perspective takes some given inertial fra"ne and always talks about velocity $v$ in that frame with corresponding womentum $m v$. The other perspective measures velocities with respeet to the observer's coordinate system. If we do this and still define momentum as mass times velocity, then the Poisson structure will change in 
general as we change coordinates. We take this approach here and will identify the new piece in the Poisson bracket as the Coriolis force. In older times the notion of a non-canouical Poisson bracket was not in widespread use. To keep the bracket defnition invariant, one had to say that momentum did not change under change of reference frame (see for example p. 129 of the mechanics volume of (Landau and Lifshitz, 1960-1981]).

Let

$$
H=\frac{1}{2 m}\left(p_{x}^{2}+p_{y}^{2}\right)+V\left(\sqrt{x^{2}+y^{2}}\right)
$$

be a rotationally eymmetric Familtonias on $T^{*} \Re^{2}$ and

$$
J=\omega\left(x p_{y}-y p_{x}\right)
$$

generate rotazions. Since $B$ is invariant under the symmetry here,

$$
\overline{\boldsymbol{B}}=\boldsymbol{H}+\boldsymbol{J} \text {. }
$$

This captures the observer's rotating reference, but does not include the fact that due to bis motion the oberver will measure a different set of values for the momenta. (In some sense, the trangformation thus far has given us valid orbits of the observed gystem but bas changed which orbit we are looking at). We must change coordinates hy

$$
\begin{aligned}
& \bar{x}=x, \\
& \dot{y}=y \\
& \tilde{p}_{x}=p_{x}-w m y \\
& \bar{p}_{y}=p_{v}+w m x
\end{aligned}
$$


to get the momenta as seeu in the rotating frame. Here we define the monentum $\tilde{p}$ to be $m$ times the observed velocity. The observed $r$-camponent of the velocity will he the velocity $v_{z}$ measured in the fixed frame minus the $x$-component of the velocity of the observation point in the rotating frame, which is w'y. Similarly. the abserved $y$-component of the velocity is $v_{v}$ minus $-w x$. The dynamics is now described by

$$
\overline{\mathbf{B}}=\frac{1}{2 m}\left\{\tilde{p}_{x}^{2}+\tilde{p}_{y}^{2}\right)+V\left(\sqrt{x^{2}+y^{2}}\right)-\frac{\omega^{2} m}{2}\left(\tilde{x}^{2}+\tilde{y}^{2}\right)
$$

with the Poigson bracket

$$
\{f, g\}=\{f, g\}_{\text {cenonical }}+2 \omega m\left(\frac{\partial f}{\partial \tilde{p}_{x}} \frac{\partial g}{\partial \tilde{p}_{y}}-\frac{\partial f}{\partial \bar{p}_{y}} \frac{\partial g}{\partial \tilde{p}_{x}}\right) .
$$

The centrifugal tem

$$
-\frac{\omega^{2} m}{2}\left(\tilde{x}^{2}+\dot{y}^{2}\right)
$$

makes an effective potential or peeudo-force and the Coriolis term in the Poisson bracket is of the "magnetic" type which causes drifts.

\subsubsection{Reduction of Simple Mechanical Systems}

We bave seen how changing coordinates can lead to pseudo-potentials in the Hamiltonian and magnetic terms in the Poisson bracket. Asymprotic systems get pseudo-forces by reduction by an approximate вymmetry. Here sc give the context in which pseudo-forces and magnetic terms in the Poisson bracket may be seen to arise from the process of reduction. We specialize this to circle actions in the next section. In the following 3 sections we show how changing coordinates may be 
vitwed as reduction of a larger space which includes the observer's state. This unifies these two sources of pseudo-forces. The argument is of necessity a bit abstract and so some rcaders may wish to skip the details. Let us start with the general setting.

If the phase space is $T^{*} Q$, where $Q$ is Riemannian with inetric $K$ and the Hamiltonian is of the form

$$
H=K^{\bullet}+\pi^{*} V,
$$

where we moved $K$ to $T^{*} Q$ and

$$
V: Q \rightarrow \infty
$$

is a real valued function on $Q$ which we lift to $T^{*} Q$ along the natural projection

$$
\pi: T^{*} Q \rightarrow Q,
$$

then we have a simple mechanical system (see [Abraham and Marslen, 1978] p. 341). A group action of $G$ on $Q$ by isometries that preserve $V$ lifts to $T^{\bullet} Q$ to preserve $H$. If we reduce at $\mu \in g^{*}$ with the momentum map

$$
J: T^{*} Q \rightarrow g^{*}
$$

the reduced space is

$$
\left(T^{*} Q\right)_{\mu}=J^{-1}(\mu) / G_{\mu}
$$

where $G_{\mu}$ is the isotropy subgroup of $\mu$ under the coadjoint action. We may identify this reduced space wit b the cotangent bundle $T^{*}\left(Q / G_{\mu}\right)$ with a ne $\approx$ Poisson bracket (the old one plus "magnetic terms") and a new Hamiltonjan (the old one with a new "efiective potential"). Using the metric, we choose a one-form $\alpha_{\mu}$ on $Q$ to behave 
on vectors tangent to the orbit of $G_{\mu}$ in $Q$ the way $\mu$ behaves oi the corresponding elemenzs of the Lie algebra, and to angihilate vectors perpendicular to the orbit. $a_{\mu}$ induce $\hat{a}_{\mu}$ on $Q / G_{\mu}$ and by lifting, on $T^{*}\left(Q / G_{\mu}\right)$. The new symplectic structure on $T^{*}\left(Q / G_{\mu}\right)$ is the old one plus the magotic piece $d \bar{a}_{k}$. The gew Hamiltonian is the old one $\left(H\right.$ is $G$ invariant and so is defined on $\left.T^{*}\left(Q / G_{\mu}\right)\right)$ plus a pseudo-potential $K^{\bullet}\left(\alpha_{\mu}(q)\right)$ (for mote details see [Marsden, 1981] p. 33).

\subsubsection{Clrcle Aetions on Simple Mechanieal Systems}

With the notation above, if $G$ is a circle and the vector feld generating its action on $Q$ is $\xi$, then there are some simplifications. The momentum map

$$
J: T^{*} Q \rightarrow \Re
$$

may be taked to be

$$
\alpha_{q} \mapsto \alpha_{q}(\xi)
$$

The G-action on $T^{*} Q$ is then generated by $X_{J} . \alpha_{\mu}$ is then simply

$$
\frac{\mu}{K(\xi, \xi)} K(\cdot, \xi)
$$

so the effective potential is

$$
-\frac{\mu^{3}}{\boldsymbol{K}(\xi, \xi)}
$$

In this case the reduced space is identifiable with $T^{*}\left(Q / S^{1}\right)$ with its modifed gtructures. 


\subsubsection{Fictitious Forces as Reduction}

We now describe a setup which unific some of these constructions. Assume we ar. given, as above, Riemannian $Q$ with metric $K$ and symnetry generated by $\varepsilon$ wich also leaves $V: Q \rightarrow \Re$ invariant. $\xi$ lifts to $X_{J}$, which is generated by

$$
J(q, p)=p(\xi(q))
$$

and leaves $H=K^{*}+V$ on $T^{*} Q$ invariant. Above re reduced by $X_{J}$ to get a lower dimengional aystem. The approach to fictitious forces given in section 3.1 .1 treated tbe rotating phase space as being of the same dimension as the fixed phase space. Here we increase tbe dimension by 2 . Now we introduce a "rotating observer", described by a point $\theta$ of a eirele $S^{2}$ and a rotation action $p_{\theta}$ in $T^{\bullet} S^{1}$. A circle acts on this $S^{1}$ generated by $\partial / \partial \theta$ and lifts to $T^{\star} S^{1}$ generated ty $p_{\theta}$. Call

$$
\hat{\xi}=\frac{\partial}{\partial \theta}+\xi
$$

on $S^{1} \times Q$ and lift it to $X_{j}$ on $T^{*}\left(S^{1} \times Q\right)$ generated by

$$
\hat{J}=p_{\theta}+\boldsymbol{J} .
$$

6 rotates the configuration space without changing $\theta$ (the state of the oberver), $\partial / \partial \theta$ rotate the observer without changing configuration space, $\dot{\xi}$ rotates the two together (twisting togetber the two circle actions), $X$, rotates particle phase space alone, $X_{p t}$ rotates the observer alone, and $X_{f}$ rotates the particle phase space and the observer.

We have the Hamiltonian

$$
\hat{H}=H+\frac{1}{2} p_{\hat{\theta}}^{2}
$$


on $T^{*}\left(S^{1} \times Q\right)$ which generates the real dynamics on $T^{*} Q$ and totaces the obsprver with speed $p_{\theta}$. It Poisgen commutes with $p_{\theta}, J$ (tbought of on $T^{*}\left(\xi^{1} \times Q\right)$ ) and $\hat{J}$. We want to consider the orbit space of the $X_{j}$ action, but to look at the level surfaces of $p_{\theta}$ (as opposed to $\vec{J}$ ) since we want to study a given speed of rotation and ore don't pant to mix up diferent speeds in the same phase space. We may ideatify

$$
\left.T^{*}\left(S^{l} \times Q\right)\right]_{p=\text { =conetant }} / X_{j}
$$

with $T^{*} Q$ by identifying $(\theta, q, p q, p)$ to the $\{\tilde{q}, \tilde{p})$ on the $X_{\mathrm{f}}$ orhit through it at the point $\theta=0$. Since

$$
X_{\hat{B}}=X_{H}+p_{\theta} \frac{\partial}{\partial \theta}
$$

and

$$
X_{J}=X_{J}+\frac{\partial}{\partial \theta}
$$

the dynamics on $T^{*}\left(S^{1} \times Q\right) / X_{S}$ identified in the above way is given by

$$
X_{H}-p_{\theta} X_{J}
$$

The Poisson bracket of two functions is seen to be

$$
\{f, s\}_{T \cdot(s+\times q) / X_{J}}=\{f, g\}_{T \cdot Q}-X_{J} \cdot f \frac{\partial g}{\partial p_{\theta}}+\frac{\partial f}{\partial p_{\theta}} X_{J} \cdot \theta .
$$

If we now reatrict to $p_{\theta}=$ constant. and identify with $T^{*} Q$, get a Hamiltonian system with the original Poisson bracket and Hamiltonian

$$
\boldsymbol{H}-\mathrm{p}_{\theta} J
$$

showing $-p_{t} j$ to be a fictitious force. 


\subsubsection{Fictitious Forees with Momentum Shift as Reduction}

Now we apply the ideas from section 3.1 .4 to the setup in section 3.1.5. The orbit space of $\dot{\xi}$ on $S^{1} \times Q$ is identifiable with $Q$ by sending an orbit to its $q$ value at $\theta=0$, call it $\left(S^{1} \times Q\right) / \dot{\xi}$. We would like to compare the $T^{*} Q$ obtained in section 3.1 .5 by quotienting $T^{\bullet}\left(S^{1} \times Q\right)$ by $X_{\rho}$ and holding $p_{\theta}$ fxed, with the $T^{\bullet} Q$ obtained by laking the cotangent bundle

$$
T^{*}\left[\left(S^{1} \times Q\right) / \dot{\xi}\right]
$$

and so obsain its relation to the original $T^{*} Q$ dynamics. We use the fact that on the cotangent bundle

$$
\left.T^{*}\left[S^{1} \times Q\right) / \bar{\xi}\right] \approx T^{*} Q
$$

with metric $K$ on $Q$, the momentum $\tilde{p}$ should be related to the velocity via $K$ :

$$
\tilde{p}=2 K(\cdot, \dot{q})
$$

Here $\dot{q}$ is the projection of the dynamics on $T^{\bullet} Q$ down to $Q$. This gives us a map

$$
T^{*} Q \rightarrow T^{*} Q, \text { by }(q, p) \rightarrow(q, \bar{p})
$$

where $(q, p)$ is a representative of a point in

$$
T^{*}\left(S^{1} \times Q\right) /\left.X_{\rho}\right|_{p_{\theta}=\text { constant }}
$$

and $(\varphi, \bar{p})$ in

$$
T^{*}\left[\left(S^{1} \times Q\right) / \hat{\xi}\right]
$$


Since the Hamiltonian is now $H-p_{\theta} J$, we see that

$$
\begin{aligned}
\dot{q} & =\frac{\partial}{\partial \boldsymbol{p}}\left(\boldsymbol{H}-\boldsymbol{p}_{\theta} H\right) \\
& =\frac{\partial}{\partial \boldsymbol{p}}\left(\boldsymbol{K}^{*}(p, p)+V(q)-p_{\theta} p(\xi(q))\right) \\
& =\frac{\partial}{\partial \boldsymbol{p}} K^{*}(p, p)-p_{\theta} \xi(q) .
\end{aligned}
$$

$K$ is quadratic, 80

$$
2 K\left(\cdot \frac{\partial}{\partial p} K^{*}(p, p)\right)=p
$$

and so

$$
\begin{aligned}
\bar{\partial} & =2 K\left(\cdot, \frac{\partial H}{\partial p}\right) \\
& =p-p_{\theta} 2 K(\cdot, \xi) .
\end{aligned}
$$

In the case $Q=\mathbb{R}^{2}$,

$$
\varepsilon=x \frac{\partial}{\partial y}-y \frac{\partial}{\partial x}
$$

is a cotation, and

$$
K=\frac{m}{2}\left(d x^{2}+d y^{2}\right)
$$

on $T Q$ and

$$
K^{*}=\frac{1}{2 m}\left(p_{x}^{2}+p_{y}^{2}\right)
$$

on $T^{*} Q$, we see that

$$
\tilde{p}_{x}=p_{x}+p_{\theta} m y
$$

and

$$
\tilde{p}_{u}=p_{y}-p_{0} m x .
$$

If we identify

$$
\omega=-p_{\theta}
$$


then this is exactly the setup in section 3.1.2. Thus the Coriolis force and centrifugal force obtaiged in 3.1 .2 are identified with magaetic terms in the Poisson bracket and a mod:fied potential in the Hamiltonian arising from reduction. 


\subsection{Geometry of the Method of Variation of Parameters}

Let un now considet the powerful and commonly uned perturbution technique known as the method of variation of parameters. A goud description of the technigue may be found in [Nayfeh. 1973] on p. 59. This method defines the crolution of the perturbation in terms of quantities at the unperturbed poiut. We expect to be $O$ (1) away from this point in time $1 / k$ (since the size of the pertorbation is $O(t)$ ) and so cannot get a correct description on this time scale. To extend the time of validity. the orhit of the unperturbed system to which we compare the true fil $w^{*}$ must be allowed to vary. If we label unperturbed orbits by their initial conditions, then we may tewrite the perturbed orbit's drift from unperturbed orbit to orbit in terms of a dris of initial conditions. Often this slow drift may be further simplified (eg. via averaging) and results in a usable perturbation theory for time $1 / c$. Let us formulate this dy namics geometrically.

Any dyamical system

$$
\Sigma=X(x)
$$

on $M$ gives rise to a natural dysamics on the group Diff(M) of diffeomorphisms of $M$. We view $X$ as an element of the Lie Algebra dif $(M)$ and we get the rightinvariant dynamical vector feld by right translation to each point of Diff(M). In general, we get a right-invariant vector field on a group containing the vector $\iota$ at the identir, by considering the first order infinitesinal left trarsiation by the 1-parameter subgroup to which $v$ is tangent. The resulting flow on Diff(M) has the following interpretation: The time- $t$ evolution of $f \in D i f f(M)$ is the diffemorphism obtained by frst applying $f$ and then letting $X$ flow for time-t. 
Now ransider a perturbed vector field

$$
\boldsymbol{r}_{0}+\boldsymbol{X}_{1}
$$

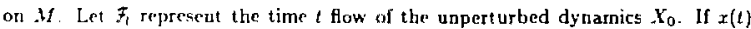
is to be she "fwolution of initial conditions" that variation of parameters produces, then $F_{t}(r(t))$ is a solution to the full equation

$$
\frac{d}{d t} F_{t}(r(t))=X_{0}\left(F_{i}(x(t))\right)+c X_{1}\left(f_{i}(x(t))\right)
$$

By definition, the left hand side is

$$
=X_{0}\left(\mathcal{J}_{k}(x(t))\right)+D \mathcal{F}_{t} \cdot(\dot{x}(t))
$$

Thus

$$
\dot{x}(t)=c D F_{-t}(x(t)) \cdot X_{1}\left(F_{t} x(t)\right)=c F_{-t} \cdot X_{1} .
$$

We may view this as a time dependent evolution equation or consider dynamics on Diff(M) $\times M$ given by

$$
\tilde{X}:(f, x) \mapsto\left(X_{0} \circ f, f^{-1} X_{1}\right)
$$

with initial condition (identity, $x_{0}+\epsilon x_{1}$ ). If $\mathcal{F}_{t}$ is periodic then $\mathcal{F}_{-t+\ldots} X_{1}$ is periodic and the method of averaging is to average it over a period. He discuss the method of averaging in greater de ail in section 2.9 . 


\subsubsection{Hamiltonian Variation of Parametera}

If $M, \omega$ is symplectic and

$$
X_{H_{0}}+C X_{H_{1}}
$$

is Hamiltonian, then we may work on the group $S y m p(M)$ of symplectomorphisms. If $f \in S y m p(M)$ and $X_{H_{0}}$ is Hamiltonian, then so is $X_{H_{0}} \circ f$. We may define a right invariant symplectic structure on $S y m p(M)$ by

$$
\tilde{s}_{f}\left(X_{1} \circ f, X_{2} \circ f\right)=\int_{M} \omega\left\{X_{1}, X_{2}\right) d x \text {. }
$$

We would like to find a Hamiltonian on Symp $(M)$ whose corresponding vector field at $f \in S y m p(M)$ is $X_{H_{0}} \circ f$. We first determine the one-form obtained by inserting. this into w:

$$
\begin{aligned}
\bar{\omega}_{f}\left(X_{H_{0}} \circ f, X \circ f\right) & =\int_{M} \omega\left(X_{H_{0}}, X\right) d x \\
& =\int_{M} d H_{0}(X) d x \\
& =\int_{M} X\left(H_{0}\right) d x .
\end{aligned}
$$

Thus we may take the Hamiltonian on $S y m p(M)$ to be the right invariant function

$$
\bar{H}_{0}(f)=\int H \circ \int d x
$$

To check that this gives the correct dynamics, notics that the rector $\mathrm{X}=f$ acting on this is

$$
\begin{aligned}
\frac{d}{d c} \tilde{H}(f+c X) & =\int \frac{d}{d c} H(f+c X) d x \\
& =\int X\left(H_{0}\right) d x
\end{aligned}
$$

as desired. Now notice tbat

$$
f^{-1} x_{H_{1}}=\left(X_{J} \cdot H_{1} .\right.
$$


So with a symplectic strurture on $S y m p(M) \times M$ given by

$$
\text { is } x \text { w. }
$$

and a Hamiltonian $\dot{H} . S_{y} m p(M) \times M \rightarrow \Re$ given by

$$
\tilde{H}=\left(f, x ;-\int_{M} H_{0} \circ \int d x+c H_{1}(f(x))\right.
$$

We get the variation of parameter dynamics.

We may see from this that if $X_{H_{0}}$ has all periodic orbits then the averaged dyuamics is Hamiltonian with Hamiltonian $H_{1}(f(x))$ averaged over the evolution $f$. 


\section{Chapter 4:}

\section{Hamiltonian}

\section{Structures in \\ Perturbation Theory}

"Schrödinger and I both had a very strong appreciation of matbematical beauty and this dominated all our work. It was a sort of act of faith with us that any equations which describe fundamental laus of Nature must bave great mathematical beauty in them. It was a very proftable religion to hold and can be considered the basis of much of our success."-P. A. M. Dirac on P. 136 of [Dirac, 1977]

\subsection{Intr. duction}

In this chapter we describe the geometry of a Hamiltonian structure for noilsingular perturbation theory applied to Hamiltonian systems on symplectic manifolds. This is limited in two respects: 1) Many systems of physical interest require more sophisticated singular perturbation methods as in [Nayfeh. 1973) and [Kevorkian and Cole, 1981] and 2) the Hamiltonian structures of many systems are given in terms of more general Poisson manifolds (Weinstcin. 1983a: Some extension of the preseut chapter to these cases 15 given in later chapters. The result- 
in this chapter are relevant to these investigations though. Most singular perturbation methods have a nonsingular expansion underlying them. Porsscn manifolds are stratified by symplectic manifolds and many of the symplectic constructions considered here are susceptible to geteralization.

If we are given dynamics in the form

$$
\dot{x}=X_{0}+\epsilon X_{1}+\frac{\epsilon^{2}}{2} X_{2}+\cdots
$$

wher cach of the vector fields $X_{1}$ is Hamiltonian with respect to a common Poisson structure, we may attempt to express the solution as an asymptotic series in e:

$$
x(t)=x_{0}(t)+x_{1}(t)+\frac{t^{2}}{2} x_{2}(t)+\cdots
$$

Plugging this form into the equation of motion and equating coefficients of powers of e gives us equations for $x_{0}, x_{1}, \ldots$. The main result of this chapter is that the equations for $x_{0}, \ldots, x$, form a Hamiltonian system for any $J$.

These resuits were discussed in chapter 2 but are proved here in full detail. The background material and non-Hamiltonian perturbation structures introduced in chapter 2 will be needed in this chapter. The reader not familiar with geometric mechanies at the level of [Arnold, 1978] may find sections of this chapter rough going. Except for parts of chapter 5, the rest of the thesis is independent of the detailed derivations given here.

We study the perturbation Haniltonian structure in Gve different ways, each of which sheds a different light on it. In the second section we do the case of first order perturbation theory explicitly, where it is easied to understand the structure. The extension to arbitrary order necessitates the introduction in the third section 


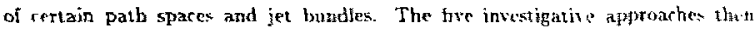
follow. 1) In the fourth section we give the desired Hamiltoniari strurture in local canonical coordinates. 2) The fifth section sbows that this strurture in courdinate independent by imhedding the jet bundle in an itcrated tangent bundle. 3) The sixtb section obtains the structure from a natural one on the infinite dimensional path space. 4) The seventh section shows in what sense the structure is the $J$ th derivative of a product structure, 5) The eighth section shows that if the original symplectic manifold is a coadjoint orbit in the dual of a I.ic algebra with the KirillovKostant-Souriou (KKS) Lie symplectic structure, theo the jet bracket is a KKS Lie symplectic structure for a coadjoint orbit of a certain jet gioup. We close with a discussion of the process of reduction appiled to perturbed systems with symmetry. 


\subsection{First Order Harniltonian Perturbation Theory}

Let us recall the geometric structure of Hamiltonian mechanics. The phase space will be a $2 N$-dimensional symplectic manifold $M$ (many of the constructions work in infinite dimensions and many of the interesting physical examples are infinite dimensional [Marsden, 1981], but this entails technicalities which we will not consider here). This is a manifold with a distinguished closed non-degenerate 2form $w$ which geometrizcs the classical Lagrange bracket. The Hamiltonian is a distinguished function $H$ on $M$ which we take to be a function of a ay well. Usually we assume that \& $E I \equiv\{0,1\}$ and so we can view $H$ as a function on $I \times M$. For each value of $c$ we obtain a vector field $\boldsymbol{X}(\epsilon)$ on $A$ by the Hamiltonian prescription. This says that at a point $x_{0} \in M, X\left(\epsilon, x_{0}\right)$ is the unique (since $\omega$ is nou-degene-ate) vertor at $x_{0}$ which gives the one form $\left.d H\right|_{x_{0}, 2}$ when inserted in $\left.\omega\right|_{=a}$, i.e.

$$
i x(e) \omega=d H \text {. }
$$

We will discuss the flow of $X(c)$ as though it were complete, tnough this need nat be the case (a vector field is complete if solution curies don't run off the manifold in fic.te time). Let us assume that $H$ may $l \sim$ represented in an asymptotic series as $\ell \rightarrow 0$ which is uniform in $x$, i.e.

$$
H(\epsilon, x) \sim H_{0}(x)+\epsilon H_{1}(x)+\frac{\epsilon^{2}}{2 !} H_{2}(x)+\cdots
$$

where the first derivatives of each $H_{1}$ are uniformly bounded in $x$. Because of the linearity in going from functions to their Hamiltonian vector felds, we also bave the asymptotic expansion

$$
X(c . x)-X_{0}(x)+\left\{X_{1}(x)+\frac{c^{2}}{2 !} X_{2}(x)+\cdots\right.
$$


as $\epsilon-0$. In this expression, $X_{i}$ represents the Hamiltonian vector field corresponding to $H_{2}$. Hamilton's equations are

$$
\dot{x}(c, t)=X(c, x(c, t)) \text {. }
$$

it the initial conditions be given as $I(t, t=0)=y(t)$ and assume that $y$ has an expansion $y(\epsilon) \sim y_{0}+\epsilon y_{1}+\left(\epsilon^{2} / 2 !\right) y_{2}+\cdots$.

As we have seen in chapter 2, non-singular perturbation theory asks for the flow representing the solution as an asyptotic series in $c$ :

$$
x(c, t) \sim x_{0}(t)+c x_{1}(t)+\frac{c^{2}}{2 !} x_{2}(t)+\ldots \quad \text { as } c \rightarrow 0 .
$$

In chapter 2 we substituted this representation into the equations of motion, equated coefficients of equal powers of $c$, and so obtained differential equations for $I_{0}, I_{1}, \ldots$ with initial conditions given by $x_{i}(t=0)=y_{i}$. The solution of these equations gave us an asymptotic representation of the true solution, but in general it was nonuniform in $\ell$. To deal with times (like $\frac{1}{\ell}$ ) longer than some bounded value as $\varepsilon \rightarrow 0$, We must use more sophisticated perturbation techniques such as Lie transforms or multiple time scales and so lose the generality of the problems we may treat. We discuss these singular or secular perturhation theories in chapter 5 .

The goal in this chapter is to determine the gemetric nature of the quantities $x_{0}, x_{1}, \ldots$ and to determine a Hamiltonian structure for their evolution equations. Ii is casy to relate these asymptotic expansions for the abstract vector ficlds and their Hamiltonians to a coordinate representation of them. If we introduce a local coordinate system $z^{1}, \ldots, x^{2 N}$ on a chart of $M$, we may express

$$
X(c, x)=\sum_{a=1}^{2 N} X^{a}(\epsilon, x) \frac{\partial}{\partial x^{a}}
$$


and

$$
X_{x}(x)=\sum_{a=1}^{2 x} X_{1}^{a}(x) \frac{\partial}{\partial x^{a}} \quad \text { for } 0 \leq i<\infty .
$$

We will use a convention where the upper index from the beginning of the Latin alphabet (e.g. a,b,c,...) gives the coordinate on $M$ and the lower index from the middle of the Latin alphabet (e.g. $i, j, k, \ldots$ ) gives the order of perturbation. The components of $X$ in this coordinate system bave the asymptotic expansion

$$
X^{a}(\varepsilon, x) \sim X_{0}^{a}(x)+c X_{1}^{a}(x)+\cdots \quad \text { as } \epsilon \rightarrow 0 \text { for } 1 \leq a \leq 2 N
$$

as may easily be seen. The equations of motion are

$$
\dot{x}^{a}=X^{a}(\epsilon, x) \text { with } x^{\circ}(t=0)=y^{a} \text { for } 1 \leq a \leq 2 N .
$$

We saw in chapter 2 that the first order perturbation approximation to these equations is

$$
\begin{aligned}
& \dot{x}_{0}^{a}=X_{0}^{a}\left(x_{0}\right) \\
& \dot{x}_{1}^{a}=\sum_{b=1}^{2 N} \frac{\partial X_{0}^{a}}{\partial x^{b}}\left(x_{0}\right) \cdot x_{1}^{b}+X_{1}^{a}\left(x_{0}\right)
\end{aligned}
$$

with initial conditions $x_{0}^{a}(t=0)=y_{0}^{a}$ and $x_{1}^{a}(t=0)=y_{1}^{a}$. A snlution $x_{0}(t), x_{1}(t)$ to these equations with the correct initial conditions will form a first order asymptotic solution $I_{0}(t)+\epsilon x_{1}(t)$ to the original cquation at each fixed $t$ (and so uniformly over bounded time intervals).

We have seen how to formulate this procedure in terms of coordinate free objects. The true dynamics takes place on $M$ and for each $t$ the flow $x(t)$ is a diffeomorphism of $M$ to itself taking initial conditions to their time $\ell$ evolution. $X_{0}$ and $X_{1}$ are vector fields on $M . x_{0}(t)$ gives the flow of the unperturbed vector 
field $\boldsymbol{X}_{0}$. Becausc $r_{1}$ represents a small perturbation to $I_{0}$ as t vanishes, it livew in the tangent space to $M$ at $x_{0} . x_{0}, x_{1}$ represent an equivalence class of How: parameterized by $c$, where we ideatify flows which asymptote to the unperturhed flow with linear rate $x_{1}$ as $\mathrm{r}$ goes to zero. If we pick a time l and an initial condition then each flow defines a -urve parameterized by $c$, which passes through $x_{0}(t)$ when $c=0$. The equivalence relation on flows leads to an equivalence relation on curves through $x_{0}(t)$ that is exactly the defining relation for a tangent vector based at $x_{0}(t)$. In local coordinates on $M$, we see that $x_{0}^{\circ}, x_{1}^{p}$ for $1 \leq 0, b \leq 2 N$ are coordinates on the tangent hundle $T M$ where $x_{0}$ coordinatizes the base and $x_{1}$ the fiber over $x_{0}$.

Equations (4.12) are to be thought of as locally defining a vector field on $T M$. We showed in chapter 2 that they are actually coordinate independent by defining vector fields $\bar{X}_{0}$ and $\bar{X}_{1}$ on $T M$ from $X_{0}$ and $X_{1}$ on $M$. If $x_{0}(t)$ is the flow of $X_{0}$ on $M$, then its derivative $T x_{0}(t)$ defines a tow on $T M$. We defined the corresponding vector field on $T M$ :

$$
\left.\dot{X}_{0} \equiv \frac{d}{d t}\right|_{t=0} T x_{0}(t) .
$$

$\tilde{X}_{0}$ defines the linearized flow of $\boldsymbol{X}_{0}$ (see [Abraham and Marsden, 1978], page 252;. In coordinates, $\tilde{X}_{0}$ is given by

$$
\begin{aligned}
& \dot{x}_{0}^{a}=X_{0}^{a}\left(x_{0}\right) \\
& \dot{x}_{1}^{a}=\sum_{b=1}^{2 N} \frac{\partial X_{0}^{a}}{\partial x^{b}}\left(x_{0}\right) \cdot x_{1}^{b} \quad \text { for } \quad 1 \leq a \leq 2 N .
\end{aligned}
$$

Because the tangent space at a point is linear, it makes sense to add a vector $v$ in $T M$ to a tangent vector in $T T M$ over $v$. Thus for $(x, v) \in T M$ we may define

$$
\bar{x}_{1}(x, t)=\left.\frac{d}{d t}\right|_{t=0}\left(t+t X_{1}(\tau)\right)
$$


This gives a vertical vector field on the bundle $T M$ which is constant on each fiber. In coordinates $\hat{X}_{\mathbf{I}}$ is given by

$$
\begin{aligned}
& \dot{\mathbf{x}}_{0}^{a}=0 \\
& \dot{\boldsymbol{x}}_{1}^{a}=X_{1}^{a}\left(x_{0}\right)
\end{aligned}
$$

Thus the invariantly defined vector field $\tilde{X}_{0}+\bar{X}_{1}$ on $T M$ gives the correct equations (4.12) in each local coordinate patch.

Let us now assume that $X_{0}$ and $X_{1}$ are Hamiltonian and investigate the Hamitonian nature of the perturbed system $\tilde{X}_{0}+\tilde{X}_{1}$ on $T M . M$ carries the symplectic two-form $w$ and we are given Hamiltonjads $H_{0}$ and $H_{1}$ such that

$$
\begin{aligned}
& i_{X_{0} \omega}=d H_{0} \\
& i_{x_{1} \omega}=d H_{1} .
\end{aligned}
$$

$T M$ carries a natural symplectic structure $\tilde{\omega}$ gotten by using $\omega$ to iejentify $T M$ with $T^{*} M$ and by pulling back $T^{*} M$ 's natural structure tc $T M$ (|Ahraham and Marsden, 1978], page 200, problem 3.31). Berause the unperturbed Hamiltonian is a map from $M$ to the rcals,

$$
H_{0}: M \rightarrow \mathfrak{R},
$$

we see that its differential,

$$
d H_{0}: T M \rightarrow \Re,
$$

may be thought of as a function on $T M$ which is linear on the fibers. If we denote the natural projection of $T M$ to $M$ by $\pi$ then since $H_{1}$ is a function an $M$, we sec $f_{\text {zat }} \pi^{-} H_{1}$ is a function on $T M$. We shall sec that $\dot{X}_{0}$ and $\dot{X}_{I}$ are Harniltonian with respect to is with Hamiltonians given by $d H_{0}$ and $\pi^{*} H_{1}$. Thus

$$
\hat{H} \equiv d H_{0}+\pi^{\bullet} H_{1}
$$


is the desired Hamiltonian on $T M$ for the periurbed rquations (4.12).

We demonstrate these statements in local Larboux (canonical) coordinates $q^{\circ} \cdot p^{\circ}$ for $1 \leq \alpha \leq f$ on $M$. The symplectic structure is

$$
\omega=\sum_{a=1}^{N} d q^{a} \wedge d p^{a}
$$

with correspondiug Poisson bracket:

$$
\left\{q^{\alpha}, p^{B}\right\}=6^{\circ B}
$$

and all other combinations of $q$ 's and p's vanishing. We represent $x_{0}$ by $q_{0}^{\alpha} \cdot p_{0}^{\alpha}$ and $x_{1}$ by $q_{1}^{\alpha}, p_{1}^{\alpha}$. One can see that the natural Poisson bracket on $T M$ corresponding to to is

$$
\left\{q_{0}^{\alpha}, p_{1}^{\beta}\right\}=\delta^{\alpha \beta} \quad\left\{q_{\mathrm{1}}^{\alpha}, p_{0}^{\beta}\right\}=\delta^{\alpha \beta} \quad \text { for } \quad 1 \leq \alpha, \beta \leq N
$$

where all other combinations vanish. In these coordinates

$$
\tilde{H}\left(q_{0}, p_{0}, q_{1}, p_{1}\right)=\sum_{\alpha=1}^{N}\left(\frac{\partial H_{0}}{\partial q_{0}^{\alpha}} q_{1}^{\alpha}+\frac{\partial H_{0}}{\partial p_{0}^{\alpha}} p_{1}^{\alpha}\right)+H_{1}\left(q_{0}, p_{0}\right) .
$$

The corresponding equations of evoiution are

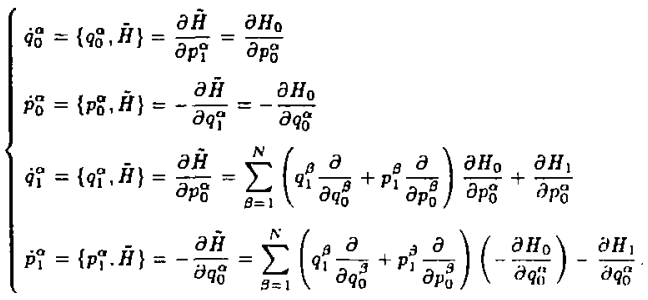


But $\boldsymbol{X}_{0}$ gives the equations

$$
\dot{q}_{0}^{\circ}=\frac{\partial H_{0}}{\partial p_{0}^{\alpha}} \quad \dot{p}_{0}^{\alpha}=-\frac{\partial H_{0}}{\partial q_{0}^{\alpha}}
$$

so we see that

$$
\frac{\partial X_{0}}{\partial x_{0}} \cdot x_{1}=\left(\begin{array}{cc}
\frac{\partial}{\partial p_{0}} \frac{\partial H_{0}}{\partial p_{0}} & \frac{\partial}{\partial p_{0}} \frac{\partial H_{0}}{\partial p_{0}} \\
\frac{\partial}{\partial q_{0}}\left(-\frac{\partial H_{0}}{\partial q_{0}}\right) & \frac{\partial}{\partial p_{0}}\left(-\frac{\partial H_{0}}{\partial p_{0}}\right)
\end{array}\right)\left(\begin{array}{l}
q_{1} \\
p_{1}
\end{array}\right)
$$

$x_{1}$ gives the equations

$$
\dot{q}_{0}^{\alpha}=\frac{\partial H_{1}}{\partial p_{0}^{\alpha}} \quad \dot{p}_{0}^{a}=-\frac{\partial H_{1}}{\partial p_{0}^{\alpha}}
$$

and $\bar{H}$ has indeed given us the desired perturbation equations (4.12). 


\subsection{Path and Jet Spaceg}

We would now like to extrnd the first order results of the last section to arbitrary order $J$. We will extend the Hamiltonian structure from the tangent bundle to the jet bundles introduced in Chapter 2 .

As in that chapter, we introduce the path space:

$$
P M \equiv\{\text { space of all paths } p: I \rightarrow I \times M \text { of the form } p: \epsilon \mapsto(c, x(c))\}
$$

and from this we define the jet spaces with integer $1 \leq J \leq \infty$ :

$$
\begin{aligned}
& J M \equiv\left\{\text { equivalence classes in } P_{1} M \text { where } p_{1} \sim p_{2}\right. \text { iff } \\
& \qquad C^{\infty} \text { functions } f \text { on } J \times M \text { we have : } \\
& \left.\left.\frac{\partial^{i}}{\partial \epsilon^{\prime}}\right|_{\ell=0} f\left(p_{1}(\epsilon)\right)=\left.\frac{\partial^{\prime}}{\partial \epsilon^{\prime}}\right|_{\epsilon=0} f\left(p_{2}(\epsilon)\right) \quad \text { for } 0 \leq \imath \leq J\right\} .
\end{aligned}
$$

If $x^{\alpha}$ for $1 \leq a \leq 2 N$ are ccordinates on $M \approx P_{0} M \approx 0 M$, then we introduce coordinates $\left\{x_{0}^{a}, x_{1}^{a}, \ldots, x_{j}^{a}\right\}$ for $0 \leq J \leq \infty$ on $J M$ to represent the equivalence class of the curve

$$
x_{0}^{a}+\epsilon x_{1}^{a}+\frac{c^{2}}{2 !} x_{2}^{a}+\ldots+\frac{\epsilon^{J}}{J !} x_{J}^{a}
$$

in $I \times M$ (near $\epsilon=0$ this won't leave the chart on which the $I^{\circ}$ are defined).

In chapter 2 we identified the tangent spaces to these and showed how cdependent dynamies on $M$ induces dynamics on these. The induced dynamics on the jet space $J M$ was exactly the perturbation dynamics up to $J$ th order. We will now assume that the dy namics on $M$ is Hamiltonian and try to find Hamiltonian structures for the dynamics on $P . M$ and $J . M$. 


\subsubsection{Path Space Symplectic Structure and Hamiltonian}

The Hamiltonian structurs on $M$ lifts to one on $P_{1} M$. As before, $\omega$ is a symplectic form on $M$ and $H(c, x)$ is a Hamiltonian. There is a natural symplectic form zi on $P_{1} M$. Intuitively, if we think of $P_{1} M$ as a continuous product of $M$ 's corresponding to each value of $\epsilon$, then $\dot{w}$ will just be the continuous sum of the corresponding symplectic structures. At a point $p \in P_{1} M$ and with vectors $\dot{V}_{1}, \dot{V}_{2} \in$ $T_{p} P_{1} M$, we define

$$
\bar{\omega}_{p}\left(\bar{V}_{1}, \bar{V}_{2}\right) \equiv \int_{0}^{1} \omega_{p(\ell)}\left(V_{1}(\epsilon, p(\epsilon)), V_{2}(\epsilon, p(\epsilon))\right) d \epsilon
$$

Similarly, we expect the Hamiltonian to be a continuous sum of the Hamiltonians for each $€$. We define $\bar{H}$ on $P_{1} M$ as

$$
\tilde{H}(p) \equiv \int_{0}^{1} H(\epsilon, p(\epsilon)) d \epsilon .
$$

\subsubsection{The Path Space Dynamica is Hamiltoninn}

We will now show that the Hamiltonian vector field on $P_{1} M$ defined by $\tilde{H}$ and $i$ is exacely the lift $\bar{X}_{H}$ of the Hamiltonian vector field $X_{H}$ on $M$. In finite dimensions, the differential of a function pairs with a vector by taking the sum over components of the product of each composent of the vector with the derivative of the function in the corresponding direction. When we consider functions on a path space. this sum turns into an integral. The differential of $\dot{H}$ thus satisfies

$$
d \tilde{H}\left(\bar{V}_{p}\right)=\int_{0}^{1} V(c, p(c)) \cdot H(c) d \epsilon .
$$


$Y \cdot$ is the directional derivative along $Y$, with $H(t)$ viewed at a function on $M$ and $f(t, p(c))$ viewed as a vector in $T M$. Let us see what ${ }_{x_{H}} \dot{x}^{\prime}$ is

$$
\begin{aligned}
& \bar{\omega}_{p}\left(\dot{X}_{H}, \hat{V}\right)=\int_{0}^{1} \omega\left(y_{H(t)}(p(\epsilon)), V(c, p(c))\right) d t \\
& =\int_{0}^{1} V(c, p(t)) \cdot H(t) d t \\
& =d \tilde{H}\left(\bar{v}_{i}\right) \text {. }
\end{aligned}
$$

Thus $\bar{X}_{H}$ is indeed Hamiltonian on $P_{1} M$.

In chepter 2 we saw that the dynamics on $P_{1} M$ naturally projects down to the desired perturbation dynamics on $J M$. We would like to project the Hamiltonian and symplectic structure as well to make $J M$ 's dynamics Hamiltonian. Unfortunately, functious and forms may only be pulled back functorially and cannot be naturally pushed forward. We may write down the Poisson bracket on $P_{1} M$ corresponding to $\tilde{\omega}$. Poisson brackets can sometimes be pushed forward along a projection by pulling back the bracketed functions. In this case, however, things become too singular and we would be left with "products" of delta functions. In the next section we will find a Hamiltonian structure on $J M$ and in later sections we will relate it to the structure on $P_{1} M$. 


\subsection{Coordinate Deacription of the J-jet Structure}

Let us reiterate the fundamental problem. We have defined a space of $\mathrm{J}$-jets $J M$ with coordinates $\left\{1_{0}^{a}, \ldots, I_{J}^{a}\right\}, 1 \leq a \leq 2 N$. The correct perturbation dynamics is given by the vector feld with components

$$
\begin{gathered}
V_{k}^{a}\left(x_{0}, \ldots, x_{J}\right)=\left.\frac{\partial^{k}}{\partial c^{k}}\right|_{e=0} X^{a}\left(c, x_{0}+c x_{1}+\cdots+\frac{c^{J}}{J !} x_{J}\right), \\
\text { for } 1 \leq a \leq 2 N \text { and } 0 \leq k \leq J .
\end{gathered}
$$

We want to know whether this dynamies on a $2 N(J+1)$ dimensional space is Hamilionian if $X(\epsilon)$ is Hamiltonian on each $2 N$ dimensional space $\epsilon=$ constant.

Darboux's theorem [Arnold, 1978] tells us that we may choose the coordinates $\left\{x^{1} \ldots, x^{2 N}\right\}$ on $M$ to be canonical. Thus the Poisson bracket of any two coordinate functions,

$$
\left\{x^{a}, x^{b}\right\}=J^{a b} \quad 1 \leq a, b \leq 2 N,
$$

is a constant independent of $x$. The dynamics then takes the form

$$
X^{\mathfrak{a}}=\left\{x^{\mathrm{a}}, H\right\}=J^{\mathrm{ab}} \frac{\partial}{\partial I^{b}} H .
$$

The correct perturbation dynamics is then

$$
\begin{gathered}
\left.\dot{r}_{k}^{a}=J^{a b} \frac{\partial}{\partial x^{b}}\left(\left.\frac{d^{k}}{d r^{k}}\right|_{t=0} H\left(c, x_{0}+c x_{1}+\cdots+\frac{\epsilon^{J}}{J !} x^{\prime}\right)\right)\right) \\
\text { for } 1 \leq a \leq 2 N \text { and } 0 \leq k \leq J
\end{gathered}
$$

from th: expression in $\mathbf{4 . 3 6}$ for the perturbation vector field. 


\subsubsection{The Jet Hamiltonian}

This dynamics is Hamilionian with the Hamiltonian function on JM given by

$$
\left.\breve{H}\left(x_{0}, \ldots, x_{J}\right) \equiv \frac{d^{J}}{d e^{J}}\right|_{t=0} H\left(c_{1} x_{0}+\left(x_{1}+\cdots+\frac{J^{J} J J}{J !}\right)\right.
$$

with respect to the Poisson bracket introduced in the next section. All of the various derivatives in 4.39 are contained in this expression and the Poisson bracket picks out the right one for each perturbation variable.

\subsubsection{The Jet Poisson Bracket}

To discover the Poisson structure, we calculate

$$
\begin{aligned}
\frac{\partial \bar{H}}{\partial x_{k}^{a}} & =\left.\frac{d^{J}}{d \epsilon^{J}}\right|_{\ell=0} \frac{\partial}{\partial x_{k}^{a}} H\left(c, x_{0}+c x_{1}+\cdots+\frac{\epsilon^{J}}{J !} x_{J}\right) \\
& =\left.\frac{d^{J}}{d \epsilon^{J}}\right|_{l=0}\left(\frac{\epsilon^{k}}{k !} \frac{\partial H}{\partial x^{a}}\left(c, x_{0}+c x_{1}+\cdots+\frac{\epsilon^{J}}{J !} x_{J}\right)\right) \\
& =\left.\frac{J !}{k !(J-k) !} \frac{d^{J-k}}{d \epsilon^{J-k}}\right|_{\ell=0} \frac{\partial H}{\partial x^{a}}\left(c, x_{0}+\epsilon x_{1}+\cdots+\frac{\epsilon^{J}}{J !} x_{J}\right) .
\end{aligned}
$$

Thus

$$
\dot{x}_{k}^{a}=\left\{x_{k}^{a} \cdot \bar{H}\right\} J M
$$

gives the correct dynamics if the jet Poisson tracket is

$$
\begin{gathered}
\left\{x_{k}^{a}, x_{m}^{b}\right\}=J^{a b} \frac{k ! m !}{J !} \delta_{k, J-m} \\
\text { for } \quad 0 \leq k, m \leq J \text { and } 1 \leq a, b \leq 2 N .
\end{gathered}
$$

Sotice that for $J=1$ this gives $\left\{x_{0}^{a}, x_{1}^{b}\right\}=J^{a b}$. which was the bracket that we found in section 4.2 for first order perturbation theory. 


\subsection{Relation to the Iterated Tangent Bundle}

We now need to show that this construction, defined in terms of coordinates. is really intrinsic. $\bar{H}$ is clcarly intrinsic, being the $J$ th derivative of $H$ along any representative curve in $P_{1} M$ of the point in $J M$ (al) such curves give the same answer by definition). That the structure of the Poisson bracket is intrinsic is not so obvious, but may be seen as follows.

\subsubsection{Injecting Jets into the Iterated Tangent Bundle}

Recall that we are letting $I$ stand for the interval $\{0,1\}$. If we take the $J$ th derivative of the map $I \rightarrow M$, we get a map of the iterated tangent bundles:

$$
T^{J} I \neg T^{J} M
$$

$\left(T^{J} M\right.$ simply means $T(T(\ldots(T M) \ldots))$ where there are $J T^{\prime}$ s. Each time we take the derivative of a map we get a map between the tange.s bundles of the two manifolds.) We may think of this as a curve in $T^{J} M$, since a curve in $M$ lifts to its tangent vector at each point in $T M$, this curve lifts to one in $T T M$, etc. The point $\epsilon=0$ of this curve in $T^{J} M$ is then the image of $\partial^{J} / \partial \epsilon^{J}$. As we look at all curves in $M$, we don't get all points in the $2^{J} \cdot 2 . N$ dimensional $T^{J} M$, but rather only a $2 N(J+1)$ dimensional submanifold identifiable with the jet space. This submanifold is made up of certain diagonals in the iterated tangent bundle which arisc because the derivative of the derivative along a path is the same as the second derivative along a path. We give the details in the next section. 


\subsubsection{Coordinat? Description of the Injection}

If we look in coordinates. wo see that this submanifold is given by cestain diagonals in the iterated tangent bundle:

$$
\begin{gathered}
\left(x^{a}\right)=\left(x_{0}^{a}\right) \in M \\
\left(x^{a},\left.\frac{d x^{a}}{d \epsilon}\right|_{\epsilon=0}\right)=\left(x_{0}^{a}, x_{1}^{a}\right) \in T M \\
\left(x^{a},\left.\frac{d x^{a}}{d \epsilon}\right|_{\epsilon=0},\left.\frac{d x^{a}}{d t}\right|_{\epsilon=0},\left.\frac{d x^{a}}{d \epsilon}\right|_{\epsilon=0},\left.\frac{d^{2} x^{a}}{d \epsilon^{2}}\right|_{\epsilon=0}\right)=\left(x_{0}^{a}, x_{1}^{a}, x_{1}^{a}, x_{2}^{a}\right) \in T T M \\
=\left(x_{0}^{a}, x_{1}^{a}, x_{1}^{a}, x_{2}^{a}, x_{1}^{a}, x_{2}^{a}, x_{2}^{a}, x_{3}^{a}\right) \in T T T M
\end{gathered}
$$

Each time we copy the last list and then copy it again adding one to each subscript. To count how mary of each type of derivative we get, we may write this symbolically as

$$
\left(1+\frac{d}{d \epsilon}\right)^{J}
$$

From the binomial expansion, wc get

$$
\frac{J !}{(J-k) ! k !}
$$

copies of $x_{k}^{a}$ in our list. 


\subsubsection{Symplectic Structure on the Iterated Tangent Bundle}

But now recall that $T M$ has a natural symplectic structure pulled back via from $T^{*} M$. We may use this to obtain a natural symplectic structure on $T T M$ and then $T T^{\top} M$, ete. We have just constructed a natural injection of $J M \rightarrow T^{J} M$, taking: to an appropriate diagonal. We may pullback the symplectic structure on $T^{J} M$ to get a natural one on $J M$.

\subsubsection{Coordinate Description of Symplectic Structure}

Let us introduce coordinates $\left\{y_{0}^{a}\right\}$ on $M,\left\{y_{0}^{a}, y_{1}^{a}\right\}$ on $T M ;\left\{y_{0}^{\circ}, y_{1}^{\circ}, y_{10}^{a}, y_{11}^{a}\right\}$ on $T T M$. etc. Here we are using

$$
y_{d_{1}, \ldots, d}^{a}
$$

on $T^{J} M$ where $d_{1}=0,1$ and leading zeroes are supressed. $y_{1, d, \ldots, d}$ are the coordinates in the fiber over the arara jeseribed by $y_{d, \ldots, d}$. Let the symplectic structure on $M$ be $\omega=\omega_{a b} d y_{0}^{a} \wedge d y_{0}^{b} . T^{*} M$ pairs $y_{0}$ with $y_{1}$ and on $T M$ the $y_{1}$ factor is twisted by $w$. Thus the symplectic structure on $T M$ is

$$
\omega_{a b} d y_{0}^{a} \wedge d y_{1}^{b} \text {. }
$$

$T \cdot T M$ would pair $y_{0}$ w'ith $y_{10}$ and $y_{1}$ with $y_{11}$. On TTM $y_{10}$ and $y_{11}$ are twisted by $T M$ 's symplectic structure. Thus the structure for $T T M$ is

$$
\omega_{a b} d y_{0}^{a} \wedge d y_{11}^{b}-\omega_{a b} d y_{1}^{a} \wedge d y_{10}^{b}=\omega_{a b}\left(d y_{0}^{a} \wedge d l_{11}^{b}+d y_{10}^{a} \wedge d y_{1}^{b}\right)
$$

If we think of the subscript as a binary number, then the prescription is to pair each $y$ with the $y$ whose difits have 1's and 0's switched. Thus it pairs $y_{m}$ with 
$y_{2} s:-m$ such that the even binary number is the first in the wedge product. This: the symplectir structure on $T^{J} M$ is given hy

$$
\sum_{a, b}^{2 N} \sum_{m=0}^{2^{m}-1}(-1)^{m} \frac{1}{2} \omega_{a b} d y_{m}^{a} \wedge d y_{2}^{b},-1-m
$$

How does the jet bundle map into $T^{J} M$ in these coordinates? Each time there is a 1 in the binary expansion of $m$, it indicates that $y_{m}^{a}$ coordinatizes another tangent to a tangent, i.e. another derivative in $\epsilon$. Thus the injection of $J M$ into $T^{J} M$ is given by

$$
y_{m}^{a}=x_{\text {sum }}^{a} \text { of binary digite of } m \text {. }
$$

\subsubsection{Pulled Back Symplectic Structure on the Jet Space}

We may get a symplectic form on $J M$ by pulling back the one on $T^{J} M$. This amounts to substituting the appropriate $x$ for each $y$. Since $2^{J}-1-m$ is $m$ with all $I^{\prime}$ 's and $O$ 's switched, the sum of the l's $12^{J}-1-m$ is $J$ minus the number of $l$ 's in $m$. Thus $x_{k}^{a}$ is paired with $w_{a b} x_{J-k}^{a}$. We see that there ase $J ! /[k !(J-k) !$ : ways of choosing $m$ with $k l^{\prime} s$, and so $d x_{k}^{a} \wedge d x_{j-k}^{b}$ will get this coefficient. The pulled back symplectic form on $J M$ is thus

$$
\left.\sum_{a, b=1}^{2 n} \sum_{k=0}^{J} \frac{J !}{k !(J-k) !} \frac{1}{2}\right\lrcorner_{a b} d x_{k}^{a} \wedge d x_{J-k}^{b}
$$

The corresponding Poisson bracket is exactly the one we abtained in the previous section. We bave therefore shown that this brarket really is coordinate independent 


\subsection{Felation to the Path Space Bracket}

In this section we rill show how $J . M$ s symplertic strurture is related to $P_{1}, I^{\prime}$ s. The result is smmething like the $J$-th derivative of the path structure. This interpretation will be made explicit in the next section. We saw in section 4.3 that $P_{1} M$ was essentially a direct integral of the spaces $M_{\mathrm{c}}$ as $\mathrm{g}$ goes from 0 to 1 . The path space symplectic structure and Hamiltonian are integrals of the corresponding structures on $M$.

\section{B.1. Weighted Path Bracket and Hamiltonian}

Because the spaces do not interact for diJerent values of $c$, we may obtain equally viable structures by putting a weighting factor $\beta(\epsilon)$ into the integrals. Assuming that $\beta(\epsilon)$ doesn't vanish anywhere. we get the correct dynamics on $P_{1} M$ with

$$
\begin{gathered}
\dot{\omega}_{B p}\left(\dot{V}_{1}, \bar{V}_{2}\right) \equiv \int_{0}^{1} \beta(\epsilon) \omega_{p(\epsilon)}\left(V_{1}(\epsilon, p(c)), V_{2}(\epsilon, p(c))\right) d \epsilon \\
\dot{H}_{\beta}(p) \equiv \int_{0}^{1} \beta(c) H(\epsilon, p(\epsilon)) d \epsilon .
\end{gathered}
$$

When we project to one of the smaller spaces, we essentially take $\beta$ to vanish on some domain. $P_{a} M$ comes from taking $\beta(\epsilon)=1$ for $0 \leq \epsilon \leq a$ and $\beta(\epsilon)=0$ for $a<c \leq$ I. Taking $\beta$ to be a delta function $\beta(c)=\delta(c)$ gives the original structure on $P_{0} . \mathbf{M} \sim M$.

In genera!, if we have a closed two-form on a manifold, it may not be symplectic due to degenerate directions (i.e. there exist tangent yectors such that the one-form that result, from inserting them into the two-form vanishes). The set of degenerate 
directions forms a subspare of the tangent space at earh point of the manifold Tear pwints where the degenerate subspaces dov't change in dimension. we may attempt to find a foliation by degenerate submanifolds (i.e. a smooth collection of disjoint submanifolds called leaves of the same dimension as and tangent to the degenerate subspaces, whose union is the whole manifold). Usually this is not possible even locally. Conditions under which it is possible locally are given by Frobenius' theorem (see [Spivak, 1979] p. 257). In the situation we are considering, the condition that the two-form be closed is suficient to guarantee that the requirements of the Fsubenius theorem are satisfied by the degenerate subspaces. We would like to consider the quotient of our manifold hy the degenerate foliation. The quotient is a manifold whose points are the leaves of the foliation. It is always possible to form such a quotient locally and sometimes it is possible globally. The original degenerate two-form gives rise to a non-degenerate symplectic two-form on the quotient. The value of this two-form on two tangent vectors on the quotient is defined to be equal to the value of the original two-form applied to any two vertors on the original manifold that project to the two quotient vectors. The result of this is independent of the point we lift the vectors to Decause the original two-form is closed. It is independent of the vectors we choose at that point because the different choices differ by degenerate vectors on which the two-form vanishes.

These very general considerations apply to the path symplectic structure $v_{j}$ defined in terms of $\beta$. A degenerate vector on the path space at $p$ is a vector field along $p$ on which $\tilde{\partial}_{\theta}$ vanishes. Examining $\tilde{\omega}_{\beta}$ 's defining integral and using the fact that $w$ is non-degenerate, we see that if $\beta$ is a function (as opposed to a 
distribution) then a degenerate $r$ is non-zero only at those $($ where 3 vanishes. If $s=1$ for $0 \leq c \leq a$ and $=0$ for $a<c \leq 1$, a degenerate vector is described by a $I(\epsilon)$ which is non-zera only $\mathrm{f}_{\mathrm{r}} \mathrm{f}>\alpha$. The degenerate foliation has leaves that are some given $p(\boldsymbol{f})$ for $0 \leq \ell \leq \alpha$ and all possible extensions for $\alpha<, \leq 1$. The quotient by the degenerate foliation is then exactly $P_{\alpha} M$ and the quotient symplectic structure is $\tilde{\omega}_{\beta}$ viewed as acting on vector fields along paths defined for $0 \leq c \leq \alpha$. The case of distributional $\beta$ 's ray be studied in a similar way.

\subsubsection{Jet Bracket Arises from Derivative of Delta Function Weighting}

We claim that taking $\beta$ to be the $J$ th derivative of a delta function gives us the $J$-jet structure. Consider

$$
\begin{gathered}
\bar{\omega}_{J p}\left(\bar{V}_{1}, \bar{V}_{2}\right) \equiv \int_{0}^{1}\left((-1)^{J} \frac{d^{J}}{d \epsilon^{J}} \delta(\epsilon)\right) \omega\left(V_{1}(\epsilon, p(\epsilon)), V_{2}(\epsilon, p(\epsilon))\right) d \epsilon \\
\bar{H}_{J}(p) \equiv \int_{0}^{1}\left((-1)^{J} \frac{d^{J}}{d \epsilon^{J}} \delta(c)\right) H(c, p(\epsilon)) d \epsilon .
\end{gathered}
$$

This structure does respect the jet equivalence classes. We take symplectic coordinates on $M$ so that $\omega^{+}=\frac{1}{2} \omega_{a b} d x^{a} \wedge d x^{b}$ and use the coordinates $\left\{x_{0}^{a}, \ldots, x_{j}^{a}\right\}$ as defined before on tbe $J$-jet space. Recall that a vector

$$
\sum_{a=1}^{2 N} \sum_{k=0}^{J} v_{k}^{a}-\frac{g}{c} x_{k}^{a}
$$

corresponds to a vector $X$ along a curve with the same jet according to

$$
V_{k}^{a}\left(x_{0}, \ldots I_{J}\right)=\left.\frac{d^{k}}{d \epsilon^{k}}\right|_{t=0} X^{0}\left(c, x_{0}+c_{1}+\cdots+\frac{\epsilon^{J}}{J !} x_{J}\right) .
$$


We wre that $i J$ really depends only on the $J$-th jot of the path and the $J$-ih jet of the vector field:

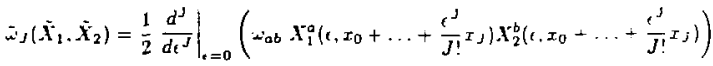

$$
\begin{aligned}
& =\frac{1}{2} \sum_{k=0}^{J} \frac{J !}{k !(J-k) !} \omega_{a b}\left(\left.\frac{d^{k}}{d c^{k}}\right|_{c=0} X_{1}^{a}\left(c, x_{0}+\ldots+\frac{c^{J}}{J !} x_{J}\right)\right) \\
& \left(\left.\frac{d^{J-k}}{d e^{J-k}}\right|_{\epsilon=0} X_{2}^{b}\left(\epsilon, x_{0}+\ldots+\frac{\epsilon^{J}}{J !} !^{x}\right)\right)
\end{aligned}
$$

by the Leibniz rule for derivatives.

But these derivatives give the components of the jets of $X$ :

$$
\tilde{\omega}_{J}\left(\tilde{X}_{1}, \bar{X}_{2}\right)=\frac{1}{2} \sum_{k=0}^{J} \frac{J !}{k !(J-k) !} \omega_{a b} V_{1, k}^{a} \dot{r}_{2, J-k}^{\prime b}
$$

So is is really the pultback along $P_{1} M \rightarrow J M$ of the form which we discorered before:

$$
\sum_{a, b=1}^{2 N} \sum_{k=0}^{J} \frac{J !}{k !(J-k) !} \frac{1}{2} \omega_{a b} d x_{k}^{0} \wedge d x_{J-k}^{b}
$$

\subsubsection{Jet Hamiltonian from Derivative of Delta Function Weighting}

Similarly,

$$
\bar{H}_{J}(p)=\left.\frac{d^{J}}{d \epsilon^{J}}\right|_{\imath=0} H\left(\epsilon, I_{0}+c_{1}+\cdots+\frac{c^{J}}{J !} I J\right)=\bar{H}\left(x_{0} \ldots \ldots x_{J}\right)
$$

is the pultback of $\bar{H}$ along $P_{1} M \rightarrow J M$. 


\subsection{Jet Space as Derivative}

In this section wr will make more explieit the sense in which the symplectic structure on $J M$ is a derivative of 2 . Tulczyjew and Kijowski have shown how the natural structure on $T M$ is a first derivative in [Kijowski and Tulczyjew, 1979].

\subsubsection{The Sheet Quotient Spaces}

We will need the spaces defined by

$$
\begin{array}{r}
P_{0, \delta} M \equiv\left\{\text { equivaleuce classes in } P_{1} M \text { where } p_{1} \sim p_{2}\right. \\
\text { iff } \left.p_{1}(0)=p_{2}(0) \text { and } p_{1}(\delta)=p_{2}(\delta)\right\}
\end{array}
$$

and in geveral by

$$
\begin{array}{r}
P_{0, \delta, \ldots, J \delta} M \equiv\left\{\text { equivalence classes in } P_{1} M \text { where } p_{1} \sim p_{2}\right. \\
\text { iff } \left.p_{1}(\epsilon)=p_{2}(\epsilon) \text { for } \epsilon=0, \delta_{1} \ldots, J \delta\right\} .
\end{array}
$$

We require the curves to agree on sheets $\epsilon=0, \delta, \ldots, J \delta$ spaced by $\delta$. Let us call the coordinates on these sheets $\left\{z_{0}^{a}, z_{1}^{a}, \ldots, z_{J}^{a}\right\}$.

\subsubsection{Sheet Symplectic Structure and Hamiltonian}

We get the correct dyamics on these sheets if we take the symplectic structure and Hamiltonian to be

$$
\begin{gathered}
\dot{\omega}_{\delta} \equiv \sum_{k=0}^{J} \frac{1}{2} \beta_{k} \omega_{a b} d z_{k}^{a} \wedge d z_{k}^{b} \quad \beta_{k} \neq 0 \\
\ddot{H}_{\delta}\left(z_{0} \ldots z_{j}\right) \equiv \sum_{k=0}^{J} \beta_{k} H\left(k \delta . z_{k}\right)
\end{gathered}
$$


These are discrete versions of the infinite dimensional structures mentioned in the last section. We want to map $J M$ into this space and pull l,ack $\dot{z}_{\text {, }}$ and $\dot{H}_{\hbar}$. We choose the $3_{k}$ as functions of $\delta$ so that the limit $\delta-0$ is both non-singular and non-trivial.

\subsubsection{Map Betwesen Sheet Space and Jet Space}

With coordinates $\left\{x_{0}^{a}, \ldots, x_{J}^{a}\right\}$ on $J M$ we can define the map

$$
z_{k}^{a}=\sum_{m=0}^{J} k^{m} \frac{\delta^{m}}{m !} x_{m}^{a}
$$

This identifies $\left\{x_{0}, \ldots, x_{J}\right\}$ with the points where the curve $x_{0}+c x_{1}+\ldots+\frac{c^{J}}{j !} x_{J}$ intersects the sheets introduced above.

\subsubsection{The Yui: ad Back Sheet Symplectic Structure and Hamiltonian}

The pulled back $\breve{\omega}_{0}$ on $J M$ is then

$$
\omega_{\delta}=\frac{1}{2} \sum_{k, m, n=0}^{J} k^{m+n} \frac{\delta^{m+n}}{m ! n !} \beta_{k} \omega_{a \Delta} d x_{m}^{a} \wedge d x_{n}^{b} .
$$

The pulled back Hamiltonian is

$$
H_{\delta}\left(x_{0}, \ldots, x_{J}\right)=\sum_{k=0}^{J} \beta_{k} H\left(k \delta, x_{0}+k \delta x_{1}+\cdots+\frac{(k \delta)^{J}}{J !} x_{J}\right)
$$


4.7.5. Shret Structures Aaymptote to Jet Structurea For Small Spacing

Loskinf; at the expression for $H_{6}$. we sce that there is no hope for $I_{j}$ dependence as $c \rightarrow 0$ if $j_{k}-\infty$ slower than $\delta^{-t}$ as $\delta \rightarrow 0$. We therefore assume that $3_{k}=b_{k} c^{-J}$ were $b_{k}$ is independent of $\delta$. 6 then only appears in the expression for wit as $\delta^{m+n-1}$. Terms with $m+n>J$ will vanish when $\delta \rightarrow 0$. For $\omega_{\delta}$ to be defiaed as $\delta \rightarrow 0$. we must choose $b_{0}$ so that the sum of terms with $i=m+n<J$ must bave a vanishing coefficient. Thus the $b_{k}$ must satisfy

$$
\sum_{k=0}^{J} \sum_{m+n=1} k^{m+n} \frac{b_{k}}{m ! n !}=0 \quad 0 \leq i \leq J-1 .
$$

This may be rewritten

$$
=\sum_{k=0}^{J} \sum_{m=0}^{1} k^{k} \frac{b_{k}}{m !(i-m) !}
$$

and the binomial theorem gives $\sum_{m=0}^{*}[m !(i-m) !]^{-1}=2^{i} / i$. Thus

$$
\sum_{k=0}^{J} \frac{2^{i} k^{k}}{i !} b_{k}=0 \quad \text { for } 0 \leq i \leq J-1
$$

or

$$
\sum_{k=0}^{J} k^{2} b_{k}=0 \quad \text { for } 0 \leq i \leq J-1 .
$$

This is $J$ equations for $J+1$ unknowns. We may take $b_{0}=1$ and remember that an arbitrary multiplicative factor is allowed. We solve the equations by introducing a generating function

$$
f(x) \equiv \sum_{k=0}^{1} b_{k} x^{k}
$$

The condition $b_{0}=1$ becomes $f(0)=1$. Natice tbat

$$
\left(x \frac{d}{d x}\right)^{\prime} f=\sum_{k=0}^{J} k^{2} b_{k} x^{k} .
$$


So

$$
\left.\left(x \frac{d}{d x}\right)^{*} f\right|_{x=1}=0 \quad \text { for } 0 \leq i \leq J-1 .
$$

This easily implies that

$$
\left.\frac{d^{i}}{d x^{i}} f\right|_{x=1}=0 \quad \text { for } 0 \leq i \leq J-1
$$

Using these equations we may Taylor expand $f$ about $x=1$

$$
f(x)=0+0+\cdots+\frac{C_{J}}{J !}(x-1)^{\jmath}+\cdots
$$

Because $f$ is a $J$-th order polynomial and $f(0)=1$, we see that $C,=J !(-1)^{d}$ and 50

$$
f(x)=(1-x)^{J}
$$

From the binomial expansion

$$
b_{k}=(-1)^{k} \frac{J !}{k !(J-k) !} \text { and } \sum_{k=0}^{J} k^{J}=\left.\frac{d^{J}}{d x^{J}}(1-x)^{J}\right|_{x=1}=J ! .
$$

The only terms left in $\omega$ bave $m+n=J$ and give us

$$
\begin{aligned}
\omega_{b} & =\sum_{k, m=0}^{J} \frac{1}{2} \vdash^{J} \frac{1}{m !(J-m) !} b_{k} \omega_{a b} d x_{m}^{a} \wedge d x_{J \rightarrow m}^{b} \\
& =\frac{1}{2} \sum_{m=0}^{J} \frac{J !}{m !(J-m) !} \omega_{a b}^{b} d x_{m}^{a} \wedge a x_{J-m}^{b}
\end{aligned}
$$

just as before.

Now

$$
\begin{aligned}
& H_{i}\left(x_{0} \ldots \ldots x_{J}\right) \\
& \quad=\sum_{k=0}^{J} \frac{1}{\delta !}(-1)^{k} \frac{J !}{k !(J-k) !} H\left(k b_{0} x_{0}-k s_{s_{1}}+\ldots-\frac{(k-\delta)^{j}}{j !} x_{J}\right) .
\end{aligned}
$$


If we call $S_{\delta}$ the operator which shift: a function of $\epsilon$ by an amount $n$. so $S_{\delta} f(t)=$ $\int(t+8)$, then we soe that

$$
\begin{aligned}
H_{A}\left(x_{0} \ldots x_{J}\right)= & \left.\sum_{k=0}^{J} \frac{1}{\delta^{J}}(-1)^{k} \frac{J !}{k !(J-k) !} S_{\delta}^{k}\right|_{\ell=0} H\left(c . x_{0}+\epsilon x_{1}+\cdots+\frac{c^{J}}{J !} x^{J}\right) \\
& =\frac{\left(1-S_{\delta}\right)^{J}}{\delta^{J}} H\left(c, x_{0}+c x_{1}+\cdots+\frac{c^{J}}{J !} x_{J}\right) .
\end{aligned}
$$

As $\delta$ goes to zero, the operator $\left(1-S_{\delta}\right) / \delta$ becomes $d / d t$. In the limit we bave

$$
\bar{H}\left(x_{0}, \ldots, x_{J}\right)=\left.\frac{d^{J}}{d \varepsilon^{J}}\right|_{\varepsilon=0} H\left(\epsilon, x_{0}+\epsilon x_{1}+\cdots+\frac{\epsilon^{J}}{J !} x_{J}\right)
$$

just as beforc. Our jet strurture thus comes out of a limiting process almost uniquely. Similar kinds of arguments arise in the theory of finite differences used to approximate derivatives numerically. A good reference written from a theoretical perspective is [Stoer and Burlirsb, 1980]. 


\subsection{Jete and Symmetry}

In this section we study Hamiltonian group actions in the pert urbatut context we have been considering. We seed to understand the perturbation analog of group actions and the process of reduction. We first show that a Hamiltonian group action of $G$ on $M$ lifts to the path space $P_{1} M$ and jet space $J M$. We calculate the momentum maps and show that they are equivariant. We then introduce the group $P G$ of paths in $G$ and the group $J G$ of their $J$-jets. These too act on $r$ it and $J M$ and we find their momentum maps. Next we consider the case where $M$ is a cuadjoint orbit in $g^{*}$ and show that $P M$ is a coadjoint orbit in $P g^{*}$ and $J M$ in $J g^{*}$. We caleuiate the corresponding Lie symplectic structures and show that they give the path bracket and jet bracket that we discovered earlier. We then study the process of reduction and show that the reduced path space is the path space of the reduced space and the reduced jet space is the jet space of the reduced space.

\subsection{1. t-dependent Group Actions on $M$}

Our starting point is an $t$-dependent group action on the manifold:

$$
\rho: I \times G \times M-M
$$

Here $I$ is the interval $[0,1], G$ is the group, and $M$ is the manifold. If $e$ is the identity of $G$ then

$$
\rho(c, L, m)=m
$$

and

$$
\rho\left(c, g_{1}, \rho\left(c, g_{2}, m\right)\right)=\rho\left(c, g_{1} \cdot g_{2}, m\right)
$$


For a compact group $f$ acting on a compact manifold.$W$, we can elıminate the r-dependence of the group action by an e-depcudent coordinate change in $M$ (this result is dive to Palais). This is not true for non-compact $G$ as the example of the real line demonstrates. In this case group actions are the flows of vector felds. Even if we restrict attention to arbitrarily small neighborhoods of $\epsilon=0$, only the socalled "structurally stable" vector fields can be made c-independent by an e-dependent coordinate change.

\subsubsection{Lift to $G$ Action on the Path Space}

This actiou lifts to the space of paths $P M$ by defining

$$
\tilde{\rho}: G \times P M \rightarrow P M \quad \text { by } \quad \tilde{\rho}(g, p)(\epsilon) \equiv \rho(\varepsilon, g, p(\epsilon))
$$

\subsubsection{Lift io $\subseteq$ Action on the Jet Space}

This respects the equivalence classes that define the $J$-jet space $J M$. We introducc coordinates $x^{a}$ on $M$ and $x_{i}^{a}, i=0,1, \ldots, J$ on $J M$ as before. The components of $\rho$ will be written $\rho^{a}$. The action of $G$ on $J M$ is then given by

$$
\left.\vec{\rho}_{\mathrm{a}}^{a}\left(g,\left(x^{0}, x^{1}, \ldots, x^{J}\right)\right) \equiv \frac{d^{\prime}}{d \epsilon^{x}}\right|_{\epsilon=0} \rho^{a}\left(c, g,\left(c, x_{0}+\epsilon x_{1}+\cdots+\frac{c^{j}}{J !} x^{J}\right)\right) .
$$


4.8.1.3. Maps from the Lie Algebra to Vector Fields on $M, P M$, and $M$

The artion of 6 on $I \times M$ gives us a map from the Lie algebra $g$ of $/$ te weretor fe.ld $=$ on $I \times M$ that leave $e$ invariant. If $v \in g$ is tangent to a curve $l \ldots(;, s)$

$$
v=\left.\frac{d}{d t}\right|_{t=0} g(t) .
$$

then $x_{1}$ on $I \times M$ is defined by

$$
X_{v}(t, m)=\left.\frac{d}{d t}\right|_{t=0} \rho(t, g(t), m)
$$

This induces a map from $g$ to $T P M$ defived by

$$
\bar{X}_{v}(p)(c) \equiv X_{v}(\epsilon, p(c))
$$

and to $T J M$ defined in coordinates by

$$
\begin{aligned}
& \left.\left(\dot{X}_{v}\right)_{1}^{d} \equiv \frac{d^{\prime}}{d c^{\prime}}\right|_{k=0} X_{v}^{a}\left(c,\left(c, x_{0}+t x_{1}+\cdots+\frac{e^{j}}{J !} I^{\prime}\right)\right) \\
& =\left.\left.\frac{d^{\prime}}{d \epsilon^{j}}\right|_{t=0} \frac{d}{d t}\right|_{t=0} p^{a}\left(c, g(t) \cdot\left(c_{0}+I_{0}+\cdots+\frac{t^{j}}{J !} x_{l}\right)\right) \text {. }
\end{aligned}
$$

\subsubsection{The Momentum Map}

In the case where $M . w$ is symplectic and our $G$ action has an 6 -dependent equivariaat momentum map

$$
y: 1 \times .11+g^{*}
$$

then

$$
i x_{w}=\{d\} \cdot v
$$

on each $\imath=$ constant. 
4.8.1.5. Momentum Map on F.M

This gives us a mornentum map for $("$, artion on $P . M$ with the symplectic structure ì given by

$$
j: P M \rightarrow g^{*} \text { by } \rho \mapsto \int_{0}^{l} J(t, p(t)) d t .
$$

It's easy to see that this gives the correct action and is equivariant because $J$ is.

\subsubsection{Momentum Map on $J M$}

I'sing coordinates $\left(x_{0}^{a}, \ldots, x_{j}^{a}\right)$ on $J M$ as before where the $x^{a}$ 's are canonical, we see that we nay defne a momentum map from $J M \rightarrow g^{*}$ by

$$
j\left(x_{0} \ldots, x_{j}\right)=\left.\frac{d^{J}}{d t^{J}}\right|_{t=0} J\left(c,\left(\varepsilon, x_{0}+\epsilon_{1}+\cdots+\frac{\epsilon^{J}}{J !} x_{J}\right)\right) .
$$

\subsubsection{Equivariance of $J M^{\prime}$ 's Momentum Map}

The equivariance follows from that of $J$, as follows. Equivariance of $J$ says

$$
J(c, p(c, g, m))=A d_{g}^{*} \cdot J(c, m)
$$

On $J M$ we see

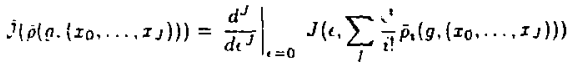

$$
\begin{aligned}
& =\frac{d^{J} !}{d t^{j} !} g\left(\left.t \cdot \sum_{t=0}^{J} \frac{t^{2}}{i !} \frac{d^{*}}{d t^{2}}\right|_{t=0} \rho\left(c \cdot g \cdot\left(t \cdot x_{0}+\tau_{1}+\cdots+\frac{c^{J}}{J !} x_{J}\right)\right)\right) .
\end{aligned}
$$


The $d^{\prime} /\left.d \epsilon^{J}\right|_{\ell=0}$ allows us to neglect terms in $e$ of higher that we $J$ th order. But to this order any function $f(t)$ is equal to its $J$ th order Taylor serese

$$
\left.\sum_{i=0}^{J} \frac{t^{t}}{i !} \frac{d^{2}}{d t^{2}}\right|_{t=0} f(t) \sim f(t) .
$$

Thus

$$
\begin{aligned}
& =\left.\frac{d^{J}}{d \epsilon^{J}}\right|_{\epsilon=0} J\left(\epsilon, p\left(\epsilon, g,\left(\epsilon, x_{0}+\epsilon x_{1}+\cdots+\frac{\epsilon^{J}}{J !} x_{J}\right)\right)\right) \\
& =\left.\frac{d^{J}}{d c^{J}}\right|_{\epsilon=0} A d_{g}^{*} \cdot J\left(\epsilon,\left(\epsilon, x_{0}+\epsilon x_{1}+\cdots+\frac{\epsilon^{J}}{J !} x_{J}\right)\right) \\
& =A d_{g}^{*} \cdot \bar{J}\left(x_{0}, \ldots, x_{J}\right)
\end{aligned}
$$

as desired.

\subsubsection{The Path Group: $P G$}

When we do reduction, we'll want a much larger group to work with. In essence, we want a symmetry that can act on different level sets of $\epsilon$ independently. Thus we define

$$
P G \equiv\{\text { all paths } \bar{g}: I \rightarrow G, \bar{g}: \epsilon \mapsto \bar{g}(c)\}
$$

The product in this space is defined as

$$
\tilde{g}_{1} \cdot \tilde{g}_{2}(c)=\tilde{g}_{1}(c) \cdot \tilde{g}_{2}(c)
$$

The identity in $P G$ is the constant path at the identity è(c) $=e$ in $G$. 
4.8.2.1. The Path Lic Algebra: $P g$

Taking infinitesimal elements, we see :hat the Lic algebra is

$$
P g \equiv\{\text { all paths } \tilde{v}: I \rightarrow g, \quad \tilde{v}: \iota \mapsto \tilde{v}(\epsilon)\}
$$

The notation $\mathrm{Pg}$ would be ambiguous in that it could mean the path space of the Lie algebra or the Lie algebra of the path group, except that these two spaces are naturally isomorphic. The Lie bracket of two elements is defined pointwise

$$
\left\{\tilde{v}_{1}, \tilde{v}_{2}\right\}(\epsilon)=\left\{\tilde{v}_{1}(\varepsilon), \tilde{v}_{2}(\varepsilon)\right\}
$$

\subsubsection{The Dual of the Lie Algebra of the Path Group}

The dual of the Lie algebra is all (distributional) paths in $g^{*}$ :

$$
P g^{*}=\left\{\tilde{a}: I \rightarrow g^{*}, \dot{\alpha}: \epsilon \mapsto \tilde{\alpha}(\epsilon)\right\} .
$$

The pairing is giv:n hy

$$
\langle\overline{\mathrm{u}}, \dot{\alpha}\rangle \equiv \int_{0}^{1}\langle\overline{\mathrm{v}}(\epsilon), \bar{\alpha}(\epsilon)\rangle d \epsilon .
$$

\subsubsection{The Action of the Path Group on the Path Space}

$P G$ acts on $P M$ by

$$
\tilde{R}: P G \times P M \rightarrow P M \quad \bar{R}(\bar{g}, p)(\epsilon) \equiv \rho(\epsilon, \bar{g}(\varepsilon), p(\epsilon)) .
$$

$G$ is the subgroup of $P G$ with $\bar{g}(\epsilon)=g$ and this action of $P G$ on $P M$ extends the action of $G$. The momentum map for this action is $P M \rightarrow P g^{*}$ by $p \curvearrowleft J(\cdot, p(\cdot))$ where is the parameter $c$ to be inserted in an clement of $\tilde{g}^{*}$ to get $I \rightarrow g^{*}$. 


\subsubsection{The Jet Group: $J G$}

Consjer paths in $G$ which begin at the identity and remain there to order ${ }^{t}$ i.e. $\bar{g}(0)=e, \partial^{k} /\left.\partial c^{k}\right|_{t=0} \bar{g}(c)=0$ for $1 \leq k \leq J$. The product of two such paths is such a path as are inverses and the ideutity. Let us call this subgroup of $P G$. $P G_{J-f l a t}$ (since a function whose derivative at $\epsilon=0$ vanishes is flat there and one whose higher derivatives vanish is very fat there, i.e. $J$-fat). With the action $\dot{h}$ on PM. we see that this subgroup leaves invariant the $J$ - jet equivalence classes, so we are naturally interested in

$$
P G_{J} \equiv P G / P G_{J-\text { flat }}
$$

assuming that this is a group. It is easier to show $P G J-f_{\text {lat }}$ is a normal subgroup of $P G$ by considering Lie algebras (recall that a normal subgroup $H \subset G$ satisfics $g \mathrm{Hg}^{-1}=\mathrm{H}$ for every $\mathrm{g} \in \mathrm{G}$ and that this is a neiessary and sufficient condition for the quotient $G / H$ to be a group)

The Lie algebra of $P G J-f i a t$ is clearly

$$
P g J \text {-flat } \equiv\left\{\tilde{v} \in P g\left|\frac{d^{t}}{d \epsilon^{2}}\right|_{\ell=0} \bar{v}(\varepsilon)=0 \text { for } \quad 0 \leq i \leq J\right\} \text {. }
$$

To check for normality, we want to show that

$$
\{P g, P g J-\text { flat }\} \subset P g J-\text { flat }
$$

(this is the Lie algebra analog of normality). But if

$$
\hat{u}(c)=\tilde{u}_{0}+r \tilde{u}_{1}+\cdots \in P y
$$


and

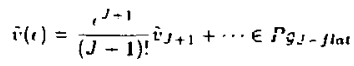

the'

$$
\left.[\tilde{u}, \tilde{i}](\iota)=\frac{\iota^{J+1}}{(J+1) !} \mid \tilde{u}_{0}, \tilde{v}_{J+1}\right]+\cdots \in P g J-\text { flat }
$$

as desired. Ordinarily. there are problems in relating results about infinitedimensional Lic algebras to results about the corresponding infinite-dimensional groups (for example, the image of the exponential map of the diffeomorphism group does not contain any neighborbood of the identity). Path groups are especially wellbehaved, however, and the argument here is valid in this context (for more discussion see Appendix $A$ of [Freed and Uhleabeck, 1984j]). Thus we may introduce the group of $J$-jers of paths in $G$ :

$$
J G \equiv P G / P G_{J-\mu l} \text { lot }
$$

\subsubsection{The Lie Algebra of the Jet Group: $J_{g}$}

Its Lie algebra is

$$
J_{g} \equiv P_{g} / P_{g J-\text { flot }}
$$

As for $P g$, the potentially ambiguous notation is not. by a simple theorem. We may put coordinates $u_{0}^{c}, u_{1}^{c}, \ldots, u_{j}^{c}$ on $J_{g}$ by associating $\left(u_{0}, u_{1}, \ldots, u_{j}\right), u_{1} \in g$ with the equivalence class of $u_{0}+\left(u_{1}+\cdots+\left(c^{J} / J !\right) u_{J}\right.$. The bracket is then

$$
\begin{aligned}
& {\left[\left(u_{0}-u_{1}, \ldots, u J\right)_{+}\left(v_{0}, v_{1}, \ldots, v_{J}\right)\right]} \\
& \quad=\left[u_{0}+c u_{1}+\cdots+\frac{c^{J}}{J !} u_{J}, v_{0}+\epsilon v_{1}+\cdots+\frac{c^{J}}{J !} v^{\prime} J\right] \\
& \quad-\sum_{k=0}^{J} \frac{c^{k}}{k !}\left(\sum_{i=0}^{k} \frac{k !}{i !(k-i) !}\left[u_{1}, v_{k-2}\right]\right) .
\end{aligned}
$$


So

$$
\left.\left[\left(u_{0}, u_{1}, \ldots, u_{J}\right),\left(v_{0}, v_{1} \ldots, v_{J}\right)\right\}_{k}=\sum_{i=0}^{k} \frac{k !}{t !(k-i) !} i u_{1}, v_{k-1}\right]
$$

This is an explicit formula for the Lie bracket of the jet Lis algebra. We shall need it in determining the Lie-Poisson bracket.

\subsection{3.:. Homomorphiem from $J_{g}$ to Vector Fields on $J M$}

The group $J G$ acts on $J M$ and so we get a Lie algebra homomorphism from $J_{g}$ to vector fields on $J M$ (these will generate symmetries that hold up to order $c^{J}$ ior the full dynamics but are exact symmetries on $J M$ ). Recall that $X_{v}$ is the image of a map from $v \in g$ to vector fields on $I \times M$. When $v$ depends on $\epsilon$ as above, we see that

$$
\begin{aligned}
& \left(\tilde{X}_{\left(v_{0}, v_{1}, \ldots, v_{s}\right)}\right)_{i}= \\
& =\left.\frac{d^{i}}{d \epsilon^{i}}\right|_{\epsilon=0} X_{\left(u_{0}+z u_{1}+\cdots+\left(\epsilon^{s} / J !\right) v_{j}\right)}\left(\epsilon, x_{0}+\epsilon x_{1}+\cdots+\frac{\epsilon^{J}}{J !} x_{J}\right) \\
& =\left.\frac{d^{i}}{d \epsilon^{i}}\right|_{\epsilon=0} X_{v_{0}}\left(\epsilon, x_{0}+c x_{1}+\cdots+\frac{\epsilon^{J}}{J !} x_{J}\right)+ \\
& +\left.\frac{d^{\prime}}{d \epsilon^{2}}\right|_{\iota=0} \epsilon X_{v^{\prime},}\left(\epsilon, x_{0}+c x_{1}+\cdots+\frac{\epsilon^{J}}{J !} x_{J}\right)+ \\
& +\cdots+\left.\frac{d^{\prime}}{d \epsilon^{\prime}}\right|_{\ell=0} \frac{\epsilon^{J}}{J !} X_{w_{J}}\left(\epsilon, x_{0}+\epsilon x_{1}+\cdots+\frac{c^{J}}{J !} x_{J}\right) \\
& =\left.\sum_{k=0}^{i} \frac{d^{j}}{d \epsilon^{i}}\right|_{\epsilon=0} \frac{\epsilon^{k}}{k !} X_{v_{k}}\left(\epsilon, x_{0}+\epsilon x_{1}+\cdots+\frac{\epsilon^{j}}{J !} x_{J}\right) \\
& =\left.\sum_{k=0}^{i} \frac{i !}{k !(i-k) !} \frac{1}{k !} \frac{d^{n-k}}{d c^{1-k}}\right|_{\epsilon=0} X_{v_{k}}\left(\epsilon, x_{0}+\epsilon x_{1}+\cdots+\frac{\epsilon^{J}}{J !} I^{J}\right) \text {. }
\end{aligned}
$$

So:

$$
\left(\hat{\boldsymbol{X}}_{\left(\nu_{0}, v_{1}, \ldots, v\right)}\right)_{t}=\sum_{k=0}^{\dot{0}} \frac{i !}{k !(i-k) ! k !}\left(\tilde{X}_{v_{k}}\right)_{1-k} .
$$


This gives the action of a jet Lic algebra element on the jel space of $M$ in terms of - he e-dependent action of the group on $M$. If we have an approximate symmetry in the sense that our e-uependent action preserves some structure to order $J$, then this $J$-jet action will be an exact symmetry.

\subsubsection{The Dual of the Jet Lie Algebra: $J g^{\circ}$}

The dual of the Lie algebra, $J g^{*}$, inay be coordinatized by $J$ elements of $g^{*}$ with the pairing

$$
\left\langle\left(\alpha_{0}, \alpha_{1}, \ldots, \alpha_{J}\right),\left(v_{0}, \ldots, v_{J}\right)\right\rangle=\sum_{i=0}^{J}\left\langle\alpha_{i}, u_{i}\right\rangle \text { for } \alpha_{i} \in g^{*}, v_{i} \in g .
$$

\subsubsection{The Jet Momentum Maps}

By the definition given in section 2.7.2 of momentum maps on $M$ we have in geueral that

$$
X_{v}^{a}(\epsilon, m)=\omega^{a b} \frac{\partial}{\partial x^{b}}\langle J(\epsilon, m), v)
$$

In this case

$$
v=v_{0}+c v_{1}+\cdots+\frac{c^{J}}{J !} v_{J}
$$

is t-dependent, but nonetheless,

$$
\left\langle J(\epsilon, m), t_{0}+\cdots+\frac{\epsilon^{J}}{J !} v_{J}\right\rangle
$$

is an $\varepsilon$-dependent Hamiltonian on $M$ for the correct action. We know from section 4.4.2 that with

$$
\left\{x_{1}^{a}, x_{k}^{b}\right\}=\omega^{a b} \frac{i ! k !}{J !} \delta_{1, J-k}
$$


we will get the correct dynamics from the $J$ th derivative of the Hamiltoman. So

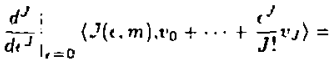

$$
\begin{aligned}
& =\left.\sum_{i=0}^{J} \frac{d^{j}}{d c^{J}}\right|_{c=0} \frac{c^{2}}{i !}\left(J(e, m), v_{2}\right) \\
& =\left.\sum_{i=0}^{J} \frac{J !}{i !(J-i) ! i !} \frac{d^{J-2}}{d t^{J-i}}\right|_{\epsilon=0}\left\langle J(\epsilon, m) \cdot v_{i}\right\rangle
\end{aligned}
$$

generates the $\left(v_{0}, \ldots, v_{J}\right)$ dynamics on $J M$. Thus the momentum map is

$$
\tilde{J}_{2}\left(x_{0}, \ldots, x_{J}\right)=\left.\frac{J !}{i !(J-i) ! i !} \frac{d^{J-1}}{d \mathrm{c}^{J-1}}\right|_{\ell=0} J\left(c, x_{0}+\epsilon x_{1}+\cdots+\frac{\epsilon^{J}}{J !} I_{J}\right) .
$$

This momentum map is again equivariant. This is important hecause this is the approximate constant of motion corresponding to an approximale symmetry. For example, we will see in chapter 5 that adiabatic invariants may be viewed $i_{*}$ this way. If we can find an exact symmetry of the dynamics on $J M$, then this gives a constant of the motion up to order $J$ in $C$ Ior the full system.

\section{4,8.4. When $M$ is a Coadjoint Orbit with the KKS Symplectic Structure}

Thus we have discovered two groups, $P G$ and $J G$, that contain $G$. Let us consider the special case in which $M$ is a coadjoint orbil of $G$ in $g^{*}$ with the canonical Kostant-Kirillov-Souriou symplectic structure. (This is really no limitation since we may take $G$ to be the group of symplectomorphisms of $M, g$ is then Hamiltonian functions. $g^{*} i$ distributions and we may identify $M$ and its symplectic structure with the orbit of a $\delta$-function and its KKS structure). 
4.8.4.1. Condjolnt Action of $P G$

Because multiplication in $P G$ is e-wise, the adjoint action of $P G$ on $P g$ is just

$$
\left(\bar{A}_{\bar{q}} \cdot \bar{v}\right)(\epsilon)=A d_{\overline{\mathrm{a}}(\epsilon)} \cdot \bar{v}(\epsilon)
$$

and the coadjoint action is similar.

4.8.4.2. The KKS Symplectic Stracture on Coadjolnt Orbita in $P q^{*}$

Consider any path

$$
\tilde{\alpha}: J \rightarrow M \subset g^{*}
$$

Since $M$ is a coadjoint orbit of $G$, under the coadjoint action of $P G$ on $\overline{\mathbf{a}}$, at each $\epsilon$ we will sweep out a copy of $M$. Since the different e's are nearly independent, it is easy to see that the coadjoint orbit of $P G$ tbrough $\bar{a}$ is the path upace $P M$ of $M$.

What is the Lie symplectic structure? Given an element $\bar{v}$ of $g$, we determine a curve of tangent vectors to $M$, i.e. an element $\bar{v}$ of $T_{p} P M$, by identifying a patb p with an element $\bar{\alpha}$ of $\boldsymbol{P g}^{*}$. We let

$$
\bar{a}(\epsilon)=p(\epsilon)
$$

and take

$$
\bar{V}(p)=\bar{a} d_{j} \bar{\alpha}
$$

or

$$
V(p(\epsilon)):=a d_{(t)}^{*} \alpha(\epsilon) .
$$


Sinilarly ansociate $\hat{a} t \bar{g}$ with $\dot{l} \in T_{p} \Gamma M$. The Jie symplectic structure is the

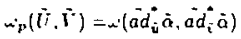

$$
\begin{aligned}
& =\langle\bar{a} \cdot\{\bar{u}, \bar{v}|\rangle \\
& =\int_{0}^{1}\langle\alpha(r),\{\bar{u}(\tau), \hat{v}(r)|\rangle d \tau \\
& =\int_{0}^{1}\left(\omega_{p(t)}(\tilde{U}(\epsilon), \bar{V}(\epsilon)) d \epsilon\right.
\end{aligned}
$$

since $\omega_{p(\varepsilon)}$ on $M$ was the Lie symplectic structure for $G$. Thus we obtain our previous path space symplectic structure via a coadjoint orbit of the group of paths in $G$.

4.8.5. Natural Projections and Injections of $G, P G$, and $J G$

We are interested in the groups $G, P G$, and $J G . G$ is naturally a subgroup of $P G$ given by constant patbs:

$$
\tilde{g}(c)=g .
$$

We also bave a projection $P G \rightarrow G$ which sends a path to its endpoint:

$$
\tilde{g} \mapsto \tilde{g}(0),
$$

which is a dso a homomorphism. $P G$ also projects to $J G$ by $J G$ 's definition as $P G / P G_{J-f l a x}$. $G$ 's image in $P G$ gets sent to a subgroup of $J G$ containing those points with zero jets. $J G$ also projects onto $G$ but is not naturally a subgroup of $P G$.

The picture summarizing these ratural maps is

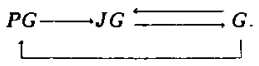




\subsubsection{Projections and Injections of the Lie Algebras and Duals}

Taking derivatives of these maps also gives us maps between the corresponding Lie algebras

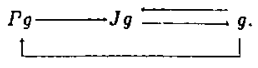

where - means a projection and $\leftarrow$ an injection. Elen ents of $\mathrm{Pg}$ are paths in $\boldsymbol{g}$. They project to their $J$-jets in $J g$ and their value at $c=0$ in $g$. Elements of $g$ are sent to $J$-jets whose 0 -jet is the element and whose higher order jets vanish. They are sedt to constant paths of the given value in $P g$.

Taking duals gives

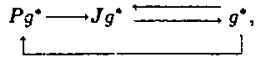

where $\rightarrow$ means a projection and $\leftarrow$ means an injection. Elements of $P g^{*}$ are distributional patbs in $g^{*}$. They are sent to $g^{\bullet}$ by letting them act on an element of $g$ by integrating their value on that element over $c$ :

$$
\int_{0}^{1}\langle\alpha(t), v\rangle d t .
$$

Elements of $J g^{*}$ are in the dual to the $J$-jets of paths in $g$. We may coordinatize it by $J-1$ copies of $g^{*}$ and give the pairing

$$
\left\langle\left(\alpha_{0}, \ldots, \alpha_{J},\left(v_{0}, \ldots, v_{J}\right)\right\rangle=\left\langle\alpha_{0}, v_{0}\right\rangle+\cdots+\left\langle\alpha_{J}, v_{J}\right\rangle .\right.
$$

$\left(\alpha_{0}, \ldots, \alpha_{J}\right)$ is sent to the element

$$
\alpha_{0} \delta(\epsilon)-\left.\alpha_{1} \frac{d}{d c}\right|_{\ell=0} \delta(\epsilon)+\cdots+\left.\alpha_{J}(-1)^{J} \frac{d^{J}}{d \epsilon^{J}}\right|_{\ell=0} \delta(\epsilon)
$$

of $P g^{*}$ and to the element $\alpha_{0}$ of $g^{*} . g^{*}$ injects into $J g^{*}$ to send $a_{0}$ to $\left(\alpha_{0}, 0, \ldots, 0\right)$. 


\subsubsection{The Lie Poisgon Bracket on $g^{*}$}

The Lie-Poisson bracket on $g^{*}$ is

$$
\{f, g\}(a)=\left\langle a \cdot\left[\frac{\delta f}{\delta a} \cdot \frac{\delta g}{\delta a}\right]\right\rangle
$$

and $M$ is a symplectic leaf for this bracket.

4.8.6.1. The Lie Poisson Bracket on $\mathrm{Jg}^{*}$

The Lie-Poisson bracket on $J_{g^{*}}$ may be defined with the help of our coordinates

as

$$
\begin{aligned}
\{f, g\}\left(\alpha_{0}, \ldots, \alpha_{J}\right) & =\left\langle\left(\alpha_{0}, \ldots, \alpha_{J}\right),\left[\left(\frac{\delta f}{\delta \alpha_{0}}, \ldots, \frac{\delta f}{\delta \alpha_{J}}\right),\left(\frac{\delta g}{\delta \alpha_{0}}, \ldots, \frac{\delta g}{\delta \alpha_{J}}\right)\right]\right\rangle \\
& =\sum_{i=0}^{J}\left\langle\alpha_{1}, \sum_{k=0}^{2} \frac{i !}{k !(i-k) !}\left[\frac{\delta f}{\delta \alpha_{k}}, \frac{\delta g}{\delta \alpha_{k}-k}\right]\right\rangle \\
& =\sum_{i=0}^{J} \sum_{k=0}^{i} \frac{i !}{k !(i-k) !}\left\langle\alpha_{1},\left[\frac{\delta f}{\delta \alpha_{k}}, \frac{\delta g}{\delta \alpha_{t-k}}\right]\right\rangle .
\end{aligned}
$$

\subsubsection{2. $J g^{*}$ ag $J$-jets of Pathe in $g^{*}$}

We would like to identify some coadjoint orbit in $J g^{*}$ as $M$ s $J$-jet space $J M$. For this we would like to identify $J$-jets of paths in $g^{*}$ with elements of $\mathrm{Jg}^{*}$. Whai is a natural pairing of $J$-jets of patbs in $g$ with $J$-jets of pat!ts in $g^{*}$ ? If we change the measure on our pairing of paths to

$$
\langle\alpha, v\rangle_{J} \equiv \int_{0}^{1}\langle\alpha(c) \cdot v(t)\rangle(-I)^{j} \frac{d^{j}}{d c^{j}} \delta(c) d \epsilon
$$


where $\alpha$ is a path in $g^{*}$ and $r$ a path in $g$. we see that the result only depends on the $J$-jet of $\alpha$ and the $J$-jet of t: i.e. it defines a pairing of the appropriate jet space. Yow we would like to find the identification of $J$-jets of paths in $g^{*}$, say $\alpha_{0}+n_{1}+\cdots+\frac{s}{j} \alpha_{f}$, with our previous coordinatization which gives duals of the components $\left(v_{0}, v_{1}, \ldots, v_{J}\right)$ of the je: of the path $v_{0}+e v_{1}+\cdots+\frac{\varepsilon^{J}}{J !} v_{J}$. We see that

$$
\begin{aligned}
& \left\langle\alpha_{0}+\alpha_{1}+\cdots+\frac{\epsilon^{J}}{J !} \alpha_{J}, v_{0}+\epsilon v_{1}+\cdots+\frac{\epsilon^{J}}{J !} v_{J}\right\rangle_{J}= \\
& =\left.\frac{d}{d c}\right|_{c=0}\left\langle\alpha_{0}+\left(\alpha_{1}+\cdots+\frac{c^{J}}{J !} \alpha_{j}, v_{0}+c v_{1}+\cdots+\frac{c^{J}}{J !} v^{\prime} j\right\rangle\right. \\
& =\left.\left.\sum_{x=0}^{J} \frac{d}{d t}\right|_{t, 0} \frac{d}{d t}\right|_{t=0}\left\langle\frac{c^{2}}{i !} \alpha_{i}, \frac{\epsilon^{d-i}}{J-i !} v_{j-i}\right\rangle \\
& =\sum_{i=0}^{J} \frac{J !}{i !(J-i) !}\left\langle\alpha_{x}+t_{J+i}\right) \text {. }
\end{aligned}
$$

Thus the $J$-jet of $\alpha_{0}+c \alpha_{1}+\cdots+\frac{c^{2}}{J !} \alpha_{J}$ is associated with the element

$$
\left(\frac{J !}{J ! 0 !} \alpha_{J} \frac{J !}{(J-1) ! 1 !} \alpha_{J-1}, \cdots+\frac{J !}{0 ! J !} \alpha_{0}\right) .
$$

The $J$-jet space $J M$ of $M$ gets turned upside down when we put it into $J g^{*}$.

\subsubsection{Coadjoint Orbits in $J g^{*}$}

With this identification of $J$-jets of $g^{*}$ with $J_{g^{*}}$, the coadjoint action of $J G$ an $J$-jets of $g^{*}$ is just the obvious one: pick a representative path in $G$ with the right $J$-jet: let it ast at cach $t$ according to the coadjoint action of $G$ on $g^{*}$ and then take the $J$-jet of Le resulting path.

By definition this is the way the adjoint action of $G$ or $g$ works. We would like

$$
\left\langle a \dot{d}_{u} \mathbf{z}^{*}, a \dot{d}_{u} \cdot \alpha\right\rangle_{s}=\langle v, \alpha\rangle
$$


for all $\alpha \in g$. But since

$$
\left\langle a d_{u(c)} v(c) \cdot a d_{u(c)}^{*} \alpha(c)\right\rangle=\langle v(c) a(c)\rangle
$$

for all $c$, it is clear by integrating over c against $(-1)^{J} \frac{d^{\prime}}{d t} \delta(c)$ that it is true for the jets.

\subsubsection{4. $J M$ is a Cogdjoint Orbit in $J g^{*}$ as a Manifold}

Because the coadjoint orbit in $P g^{*}$ of a $p^{x t h}$ which lies solely in $M$ is the space of all paths in $M$, the coadjoint orbit in $J g^{*}$ of a $J$-jet of a path in $M$ is the space of all $J$-jets of paths in $M$, i.e. the $J$-jet space $J M$.

4.8.6.5. The KKS Symplectic Structure is the Jet Symplectic Structure

So $J M$ is a coadjoint orbit. What is the Lie symplectic structure" Again every $J$-jet of a path in $g$ determines a $J$-jet of a patb of tangent vectors to $M$, i.e. an element of $T J M$, by

$$
\begin{aligned}
& V_{1}\left(\alpha_{0}+\left(\alpha_{1}+\cdots+\frac{\epsilon^{j}}{J !} \alpha_{j}\right)\right. \\
& =\left.\frac{d^{n}}{d c^{2}}\right|_{c=0} a d^{*} v_{r}+c v_{1}+\cdots+\frac{j}{J} v_{s}\left(\alpha_{0}+c \alpha_{1}+\cdots+\frac{c^{J}}{J !} \alpha_{J}\right)
\end{aligned}
$$


The lie symplectic structure is then

$$
\begin{aligned}
& \nu^{\prime}\left(\alpha_{11} . \ldots,\right)\left(\left(u_{0} \ldots, u J\right),\left(v_{0} \ldots v_{J}\right)\right)= \\
& =\left(\left(\sigma_{1}, \ldots, \alpha_{J}\right),\left\{\left(u_{0} \ldots, u_{J}\right) \cdot\left(r_{0}, \ldots, r_{j}\right)\right\}_{j}\right. \\
& =\int_{0}^{1}\left\langle a_{0}+c a_{1}+\cdots+\frac{\epsilon^{\prime}}{j !} o_{j},\right. \\
& \left.\left[u_{0}+\epsilon u_{1}+\cdots+\frac{\epsilon^{J}}{J !} u_{J} v_{0}+\epsilon_{1}+\cdots+\frac{\epsilon^{J}}{J !} v_{J}\right]\right\rangle(-1)^{J} \frac{d^{J}}{d \epsilon^{J}} \delta(\epsilon) d t \\
& =\left.\int_{0}^{l} \frac{d^{n}}{d c^{2}}\right|_{\epsilon=0} \omega_{a}\left(U_{0}+c U_{1}+\cdots+\frac{c^{J}}{J !} U_{J}, V_{0}+\epsilon V_{1}+\cdots+\frac{\epsilon^{J}}{J !} V_{J}\right) d \epsilon,
\end{aligned}
$$

but we have seen that this is exactly the $J$-jet bracket that we obtained before.

Jerry Marsden has pointed out that some of these symplectic cons rur'ions immediately generalize to corresponding constructions on Poisson manifolos. For example, to get a symplectic structure on $T M$ from $\omega$ on $M$, we use $\omega$ to identify $T M$ with $T^{*} M$ and pull back the canonical structure. While a Poisson structure does not define an isomorphism ${ }_{y}$ it does define a map from $T^{*} M$ to $T M$. We may push forward the canonical Poisson structure on $T^{*} M$ to obtain a non-canonical one on $T M$. Iterating this gives Poisson structures on each of the iterated tangent buadles. Presumably one can use these to define a Poisson structure on each of the jet spaces as well. It appears likely that the jet structure derived from a Lie-Poisson bracket on $g^{*}$ is the Lie-Poissoz bracket on $J g^{*}$. Richard Montgomery has recently shown that the second order perturbation jet structure may be extended in this way to arbitrary Poisson manifolds and it appears that the same methods will work to all orders. 


\subsubsection{JG as a Seml-Direct Product}

Xotice that the set of elemer of $J g$ of the form

$$
\left(0.0 \ldots, 0, u_{1}, u_{t+1}, \ldots, u_{J}\right)
$$

form a nilpotent ideal. say $J g_{v}$. We sec that $i g \sim J_{g} / J g_{1}$. Thus $J g$ is a semi-direct product of $i g$ and $J g_{1}$. Note in particular that $I G$ is $T G$ and has the group structure of the semi-direct product of $G$ with $g$ with the adjoint action. Alan Weinstcin has pointed out th..t this provides an "explanation" of why most of the coadjoint orbits of the Euclidean group (which is the tangent group of the rotation group) are the tangent bundle of the 2-spherc (most of the coadjoint orbits of the rotation group are 2-spheres). Note, bowever, that the symplectic structure on these orbits agrees with the jet symplectic structure only on the orbits whose tangent vertors are tangent to the spheres. The other orbits have extra "magnetic terms" in their symplectic forms. If the conjecture at the end of the last section is correct. then the general coadjoint orbits of $J G$ are the symplectic leaves of the jet lift of the Lie Poisson structure $(t) g^{*}$ to $J g^{*}$. It is interesting that there is another natural jet type Lie algebra associated with an arbitrary Lie algebra $g$. Consider $J$-jets at the origin of real valued functions (as opposed to paths) defineci on $g^{*}$. The Lie Peisson bracket gives this jet space a natural Lie algcbra structurc. Its dimension is much larger than that of $J g$ and the relacion hetween the two Lie algebras is not clear. 
4.8.8. Jet and Path Reduced Spaces are Reduced Jet and Path Spaces

Teet us now return to the general setting of an d dependent action of $G$ on $M, \lambda$. If we hatr an e-dependent invariant function $H(c)$, it is easy to sce that if the orbit sparem for all e are differmorphic (for example if the $G$ action is e independent or if $G$ is compact and the variation is small enough) then $P G$ leaves $\tilde{H}$ (n $P . M$ invariant. Its orbit space is the space of paths in the orbit space of $G$ on $M$. Similarly, the inverse image of a point in $\mathrm{Pg}^{*}$ under the momentum map $\tilde{J}$ is the space of paths that lie in the corresponding inverse image of $J$ at each $c$. Thus tbe reduced space of $P G$ acting on $P M$ is the path space of the reduced spaces at each $\epsilon$.

Similarly $J G$ acts on $J M$ leaving

$$
\left.\frac{d^{n}}{d c^{\mathbf{2}}}\right|_{l=0} H(\epsilon, p(c))
$$

invariant. Again the reduced space for this action is the jet space of the reduced space for the action of $G$ on $M$. 


\section{Chapter 5:}

Kruskal's Theory of

Nearly Periodic

\section{Systems}

"All nature and the graceful sky are symbolized in the art of Geometria.".Kepler. Tertius Interveniens

\subsection{Introduction: Krugkal's Approach}

In 1962 Martin Kruskal published "Asymptotic Theory of Hamiltonian and other Systems with all Solutions Nearly Periodic" [Kruskal. 1962]. In this paper he generalized and unified previous results due to himself and others showing that many specific Ihysical systems had adiabatic invariants to all orders in a small parameter expressing the separation of slow and fast time seales. This work is the theoretical foundation for many concepts in plasma physics and elsewhere. It is therefore of interest to re-examine the underlying structure of the theory in the light of recent developments in geometric mechanits and dynamical systems. We have presented in earlier chapters a geometric formulation for ordinary perturbation thecry and showed that it leads to deeper insights into the Hamiltonian nature of 
perturbed systems. Hrre we will present a new version of Kruskal's result which le'ad to a completely unambiguous prescription for the perturbation analysis. We then show how the new procedure is expressed in coordinate-froe language using the geonetric structure of ordinary perturbation theory. The procedure is intimately tied with the process of reduction in the Hamilionian case and so is connected with recent developments in finding Hamiltonian structures in plasma and other physical contexts.

Let us begin by setting up the problem in geometric language and then discussing Kruskal's method in this framework. We are interested in studying dynamical systems whose dynamical vector field depends on a small parameter $c$ in such a way that when $c=0$ all orbits are periodic and the period is a smooth non-vanishing function on state space. Kruskal called the closed unperturbed orbits loops. These loops naturally give the state space the structure of a circle bundle. Locally we may express this bundle as a product: $S^{1} \times \Re^{n}$. It is easy to see that we may choose coordinates $\theta$ and $x^{a}, \quad a=1, \ldots, n$ such that the unperturbed dynamical vector field is independent of $\theta$ and has no $x$ component. In these coordinates the dynamics is given by the vector field

$$
X=t(x) \frac{\partial}{\partial \theta}+\epsilon x_{1}+\frac{c^{2}}{2} X_{2}+\ldots,
$$

where $v(x)$ describes how the frequency varies with $x$. As we turn on the perturbation by letting $\mathrm{e}$ be nonzero, the dynamical vector field no lor ger points along loops and the orbits in state space drift from loop to loop along a helical path. If we are uninterested in the dynamics around the loops then the unperturbed system projects to trivial dynamics on the base of the bundle. i.e. the $I$ coordinate 
dy not exolve. If the perturbed vector feld did not depend on $\theta$. then again in: ignoring the fast 0 motion we could project the dynarics to the base space leaving only tbe interesting slow dynamies. Unfortunately, typical perturbation: will not be independent of $\theta$ and different points on a loop will evolve to different lnops. precluding any well defined slow dynaricic on the base. Kruskal attempts to find t-dependent coordinates $\dot{\theta}$ and $\tilde{x}^{a}$ which reduce to $\theta$ and $x^{a}$ when $\varepsilon$ vanishes, such that the dynamical vector field expressed in these coordinates is independent of $\tilde{\theta}$. In general this will not be possible for finite $\epsilon$, but Kruskal was able to sbow that such $\dot{\theta}$ and $\tilde{x}^{a}$ exist as asymptotic series to all ordet $\varsigma$ in $\epsilon$. His technique involves an intricate "bootstrap" argument which links two expansions sogether and obtains terms in one from lower order terms in the other.

The choice of coordinates is not unique beca"se we can always apply an s dependent diffeomorphism to the base and rotate the fibers (i.e. choose coordinates $\tilde{y}(\tilde{x})$ and $\tilde{\theta}+f(\tilde{x}))$ without altering the desired properties. Kruskal called the $\epsilon$ depeedent loop obtained by holding $\bar{z}$ fixed and letting $\dot{\theta}$ run from 0 to $2 \pi$, a ring. The set of rings determines a fibration of phase space for each $c$ and reduces to the original fibration when $c=0$. The vector field

$$
R=\frac{\partial}{\partial \hat{\theta}}
$$

is tangent to the rings and was denoted the roto-rate by Kruskal. He showed that as an astmptotic series, $R$ is uniquely defined to all orders in $c$. In the paper he rakes the interesting comment: -It does not $>$ repar obvious whether an explicit recursion formula to determice $R$ in terms of $f$ fthe dynamical vector firld: can be fount. If so. the whole theory of this paper might be simplified and rendered less deep." In 
this chapter we will sxhibit such a formula and stow its geometric significance. 


\subsection{The New Approach Expressed in Coordinates}

Many perturbation methods. beginning with the Poincaré-Lindsted method and leading up to the metbod of Lic transforms, are like Kruskal's approach in that they rork by looking for an $\epsilon$-dependent diffeomorphism of phase space which takes the perturbed system into a simpler one (equivalently one thinks of finding new coordinates in terms of which the dynamics looks simple). This bas the advantage that closed loops are automatically taken to closed loops and if the system is Hamiltonian and the diffeomorphism is a canonical transformation, it preserves the Hamiltonian structure. In the present setup we wil see that it is easier to explicitly require the rings to be closed as a constraint that belps determine terms in the expansion. In Kruskal's technique a similar constraint is required to eneure that the change of coordinates is periodic in $\theta$. One advantage of the present ap 1 roach over methods which change coordinates is the uniqueness of the desired expansion. This leads one to suspect (correctly as we will show in section 6.3) that the whole procedure has an intrinsic coordinate-free interpretation. Because the coordinate change is not vaiquely specified in the standard approach, there will always $k$, 17 arbitrary and unphysical choice to be made at some point. This becomes especially critical in infinite-dimeusions where one wishes to apply these techniques to fields (we will see in chapter 13 that wfinite-dimensional coordinate changes may involve many subtle phenomena).

Wic will now see that as asymptotic expansion for $R$ can be uniquely determined on che basis of two constraints. The coordinate-free way of expresting the dynamical 
vertor field $X$ independence of $\bar{\theta}$ is to say that the Lie bracket of $X$ and $R$ vanishes:

$$
\{R \cdot \mathbf{r}\}=0
$$

The second requirement on $R$ is that its integral curves all be closed. Thus

$$
\text { if } y(t)=R \text { then } y(0)=y(2 \pi) .
$$

If we assume an expansion for $R$ and $X$ as above then equation (5.3) taken orfer by or te* in $\epsilon$ gives us a hierarchy of equations for the terms in $R$ :

$$
\begin{aligned}
& \left\{R_{0}, X_{0}\right]=0 \\
& \left\{R_{1}, X_{0}\right\}=-\left\{R_{0}, X_{1}\right\} \\
& {\left[R_{2}, X_{0}\right\}=-2\left[R_{1}, X_{1}\right]-\left\{R_{r} X_{2}\right]}
\end{aligned}
$$

Sotice that cach right hand side is known from before as we determine successive terms in $R$. On the left hand side we alwars find the bracket of a term in $R$ with $X_{0}=t(x) \partial / \partial \theta$. The $x$ components and the $\theta$ components of this bracket have a different structure and must be dealt with separately.

We use $y^{2}, 1 \leq i \leq n+1$ to represent $x^{a}, \theta, 1 \leq a \leq n$ together. Recall that the coordinate expression for the Lie tracket of two vector fields $A$ and $B$ takes the form

$$
[A, B]^{2}=\sum_{j=1}^{n+1}\left(A^{x} \frac{\partial}{\partial y^{3}} B^{x}-B^{j} \frac{\partial}{\partial y^{2}} A^{x}\right) .
$$

$X_{0}$ is special in that it has only a $\theta$ component, which depends only on $I$. Denoting the $I$ components of a vector by an $x$ superseript and the $\theta$ component by a $\theta$ superscript, we find

$$
\left\{R_{1}, X_{0}\right\}^{x}=-\because(x) \frac{\partial R_{1}^{x}}{\partial \theta}
$$


Since $u(x)$ doesn t vanish by assumption, and assuming we have alrealy obtaned all lower order terms, we may integrate this equation and find the solution up to an arbitrary n-component function $F(x)$ :

$$
K_{1}^{x}=-\frac{1}{y(x)} \int_{0}^{\theta}(\text { known terms) } d \theta+F(x)
$$

The $\theta$ component of the Lie bracket is slightly more complicated:

$$
\left[\boldsymbol{R}_{\mathrm{z}}, \boldsymbol{X}_{\mathbf{0}}\right]^{\theta}=-\psi(x) \frac{\partial R_{i}^{\theta}}{\partial \theta}+\frac{\partial \psi}{\partial \theta} R_{\mathrm{i}}^{\theta}+\sum_{a=1}^{n} \frac{\partial \psi}{\partial x^{a}} R_{\mathrm{i}}^{a}
$$

The second term vanishes because $\psi$ has no $\theta$ dependence. If we are able to determine $R_{i}^{x}$ first then we may obtain $R_{2}^{\theta}$ by integrating up to an arbitrary function $G(x)$ :

$$
R_{1}^{\theta}=-\frac{1}{\psi(x)} \int_{0}^{\theta}\left(-\sum_{a=1}^{n} \frac{\partial \psi}{\partial x^{\alpha}} R_{s}^{a}+(\text { known terms })\right) d \theta+G(x) .
$$

To determine $F(x)$ and $G(x)$ we employ the periodicity condition order by order. We must find $F(x)$ first without the help of $R_{2}^{\theta}$ so that we may use $R_{1}^{r}$ in its determination as above. The tow of $\dot{y}=R$ satisfes the integral equation

$$
y(t)=\int_{0}^{t} R\left(y\left(t^{\prime}\right)\right) d t^{\prime}
$$

Let us expand $y(t)$ as an asymptotic series in $\epsilon$ and substitute this into this equation:

$$
y_{0}(t)+\epsilon y_{1}(t)+\ldots=\int_{0}^{t}\left\{R_{0}\left(y_{0}\left(t^{\prime}\right)\right)+c\left(\frac{\partial R_{0}}{\partial y_{0}} \cdot y_{1}+R_{1}\left(y_{0}\right)\right)+c^{2} \ldots\right\} d t^{\prime}
$$

He again get a hierarchy of equations by collecting terms urder by urder in a. For reference. let us work out the integrand to order $\epsilon^{3}$. We firt expand the terme in 
$R$ s asymptotic expansion in Taylor serien to the needed order:

$$
\begin{aligned}
R(y)= & R_{v}\left(y_{0}\right)+\frac{\partial R_{0}}{\partial y_{0}} \cdot\left(c y_{1}+\frac{c^{2}}{2} y_{2}+\frac{c^{3}}{6} y_{3}\right) \\
& -\frac{1}{2} \frac{\partial^{2} R_{0}}{\partial y_{0}^{2}}\left(c y_{1}+\frac{t^{2}}{2} y_{2}\right)\left(t y_{1}+\frac{c^{2}}{2} y_{2}\right)+\frac{1}{6} \frac{\partial^{3} R_{0}}{\partial y_{0}^{3}}\left(c y_{1}\right)^{3} \\
& +c\left(R_{1}\left(y_{0}\right)+\frac{\partial R_{1}}{\partial y_{0}} \cdot\left(c y_{1}+\frac{\epsilon^{2}}{2} y_{2}\right)+\frac{1}{2} \frac{\partial^{2} R_{1}}{\partial y_{0}^{2}}\left(c y_{1}\right)\left(c y_{1}\right)\right) \\
+ & \frac{\varepsilon^{2}}{2}\left(R_{2}\left(y_{0}\right)+\frac{\partial R_{2}}{\partial y_{0}} \cdot\left(y_{1}\right)\right. \\
+ & \frac{c^{3}}{6} R_{3}\left(y_{0}\right) .
\end{aligned}
$$

Now let us collect terms to get the vector field to the various orders. To order $\epsilon^{0}$ we bave simply

$$
R_{0} \text {. }
$$

To order $\epsilon^{1}$ we have

$$
\frac{\partial R_{0}}{\partial y_{0}} \cdot y_{1}+R_{1}
$$

To order $e^{2}$ we have

$$
\frac{1}{2} \frac{\partial R_{0}}{\partial y_{0}} \cdot y_{2}+\frac{1}{2} \frac{\partial^{2} R_{0}}{\partial y_{0}^{2}} \cdot y_{1} \cdot y_{1}+\frac{\partial R_{1}}{\partial y_{0}} y_{1}+\frac{1}{2} R_{2}
$$

Finally $t o$ order $\mathrm{c}^{3}$ we have

$$
\begin{aligned}
& \frac{1}{6} \frac{\partial R_{0}}{\partial y_{0}} \cdot y_{1}+\frac{1}{2} \frac{\partial^{2} R_{0}}{\partial y_{0}^{2}} \cdot y_{1} \cdot y_{2}+\frac{1}{6} \frac{\partial^{3} R_{0}}{\partial y_{0}^{3}} \cdot y_{1}^{3} \\
& +\frac{1}{2} \frac{\partial R_{1}}{\partial w_{1}} \cdot y_{2}+\frac{1}{2} \frac{\partial^{2} R_{1}}{\partial y_{0}^{2}} \cdot y_{1}^{2}+\frac{1}{2} \frac{\partial R_{2}}{\partial y_{0}} y_{1}+\frac{1}{6} R_{3},
\end{aligned}
$$

In the expression for $y_{1}(t)$, the term $y_{3}(t)$ appears only in the form $y_{1} *\left(\partial \mu_{0} / \partial y_{0}\right)$ atud all other terms are of lower order and therefore known. But $R_{0}=(\partial / \partial \theta)$ is constant and so its derivative vanishes. Thus each $y_{\text {s }}$ is a well defined integral over 
known quautities:

$$
\begin{aligned}
\theta_{0}(t) & =\theta_{0}(t=0)+t \quad I_{0}(t)=x_{0}(t=0) \\
y_{1} & =\int_{0}^{t} R_{1}\left(x_{0}, \theta\right) d \theta \\
y_{2} & =\int_{0}^{t} 2\left(\frac{\partial R_{1}}{\partial y_{0}} \cdot y_{1}+\frac{1}{2} R_{2}+\frac{1}{2} \frac{\partial^{2} R_{0}}{\partial y_{0}^{2}} \cdot y_{1} \cdot y_{1}\right) d \theta \\
& \vdots
\end{aligned}
$$

We may now impose the constraint that the orbits be periodic:

$$
\begin{aligned}
y_{1}(2 \pi)-y_{1}(0)=0 & =\int_{0}^{2 \pi} R_{1} d \theta \\
\int_{0}^{2 \pi} R_{2} d \theta & =-\int_{0}^{-2 \pi} \frac{\partial R_{1}}{\partial y_{0}} \cdot y_{1} d \theta \\
& =-\int_{0}^{2 \pi} \frac{\partial R_{1}}{\partial y} \cdot\left(\int_{0}^{\theta} R_{1} d \theta^{\prime}\right) d \theta
\end{aligned}
$$

The elementary asymptotic series for $y$ is valid since we need it only for finite time (in fact, only time one). Each of these equations has an $x$ compinent and a $\theta$ component. The asymptotic series for $R$ is uniquely determined as follows: i) $R_{0}^{\theta}=1$ and $R_{0}^{x}=0$, ii) assumigg $R_{j}$ known for $j<i$, we obtain $R_{i}^{x}$ up to the function $F(x)$ by means of equation (6.8), iii) we determine $F(x)$ by means of the I component of equation (6.14), iv) using the $R$, 's and $R_{1}^{x}$ we determine $R_{1}^{0}$ up to the function $G(x)$ by means of equation $(6.10), y)$ and fnally we determine $G(x)$ by using the $\theta$ component of equation (6.14) giving us the entire $R_{t}$ and allowing us to continue the iteration to $R_{\mathrm{a}+1}$. 


\subsection{The Geometric Veraion of the New Approach}

Let us now formulate the new procedure completely invariantly. in terms of the geometric picture introduced in chapter 4 . Given a vector field $X(c)$ on $I \times M$ with no $\partial / \partial c$ component and $X(0)$ having all periodic orbits, we would like to find $R(c)$ on $I \times M$ with no $\partial / \partial c$ romponent such that $X(0)$ and $R(0)$ are tangent. $[X(\epsilon), R(\epsilon)]=0$, and all of $R(\epsilon)$ 's orbits are periodic of period $2 \pi$. We cannot do this for finite c (due to homoclinic bebavior, for example, which is discussed in chapter 14 ), but $R$ is determined uniquely to all orders in $\epsilon$.

Let $\psi: M \rightarrow \Re$ be the period function of $X(0)$ and assume that it does not vanish anvwhere. Let $R(0)=\boldsymbol{X}(0) / \psi$. We have the hierarchy of jets of paths at $c=0$ :

$$
\infty M \rightarrow \ldots \rightarrow J M \rightarrow \ldots \rightarrow 2 M \rightarrow 1 M \rightarrow M .
$$

$X(c)$ determines vector fields on each of these spaces. We are looking for $R$ 's on each space such that each projects to the one below. The bracket of two vector fields lifts to a vector field on $J M$ which is the bracket on $J M$ of the lifts to $J M$ of the vector fields. Thus $[X(c), R(c)]=0$ lifts to a condition on each $J M$. The flow of a vector field for time 1 is a diffeomorphism of $I \times M$ preserving $c$. It therefore lifts to diffeomorphisms of each $J M$ which project into one another. On $J M$ the diffeomorphism is the time-1 flow of the lift of the vector feld to $J M$. The condition $\dot{y}=R(y)$ implies $y^{\prime}(1)=y(0)$ lifts to similar conditions on the lifts to each $j M$.

We know from the coordinate calculations that to determine a given order of $R$, we first have to determine it modulo $R_{0}$ and then use this to get the full $R$. Thus we introduce the drift tangent bundles $D T J M$ which at each point of $J M$ has fiber 
$T_{p} J U / \hat{R}_{0}(p)$, where $\bar{R}_{0}$ is the dift to $J U$ of ${ }^{J} R_{0}$. Wr ger the natural projertions

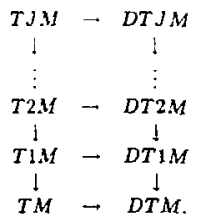

The key relation is

$$
\left[\boldsymbol{R}_{j}, \boldsymbol{I}_{\mathbf{0}}\right]=\left\{\boldsymbol{R}_{j}, \psi \boldsymbol{R}_{0}\right\}=\left(\boldsymbol{R}_{j} \cdot \psi\right) \boldsymbol{R}_{0}+\psi\left[\boldsymbol{R}_{j} . \boldsymbol{R}_{0}\right]
$$

The fi-st term is along $R_{0}$ and so is killed by the $D$ operation, the second may be solvea for $R_{3}$ by integrating along the flow of $R_{0}$ the quantity $1 / \psi$ times the right band side. The integral relation on $D$ space then gives the constant of integration. Next, since $R_{0} \cdot \psi=0$, we see that

$$
\left(R_{3}+f R_{0}\right) \cdot \psi^{\prime}=R_{3} \cdot \psi
$$

So the first term depends only on $D R_{y}$. So the $R_{0}$ component of $R$, may be found by integrating: $\frac{1}{v}\left(-\left(D R_{j} \cdot w\right) R_{0}+r . h . s.\right)$ and then the integral gives the const ant. We will see several examples of this procedure it the next chapter. 


\subsection{Extengions ano Limitatione of Kruskal's Theory}

In this wection let ue describe some of the limitations and extensions of hruskal': and other secular perturbation theories. Most of the isymptotic theories of physics are based upon asymptotic rather than convergent expansions. An asymptotic expansion of a function of $\epsilon$ is a formal power series in $\epsilon$ such that the eruncation after the $N$ th term approximates the function to order $e^{N}$. Typically, as $N$ gets larger. one must go to smalier ( to get a good approximation and there may be no c for which all the terms in an asymptotic series are helpful (and in fact most such series are divergent). Most of the techniques one applies in perturbative analyses yield asymptotic series because the exact solution we ase approxinating does not have a convergent expansion. The notion of convergence rests on a complex analytic structure, while smooth non-analytic coordinate changes, wbich should he physically irrelesant, can destroy the convergence of an asymptotic expression. There arn two limiting processes here, letting $e$ go to zero and letting the number of terms go to infinity. Asymptotic series have nice limits when they are done in that order; convergent series allow us to switch the order of tive limiting processes. From a practical point of view, one never uses more than a finite number of terms of an expansion in any cane and often there are asymptotic series which approximate an expression much more quickly than a corresponding cr jvergent series. 


\subsubsection{Secular Perturbation Theory}

If the coefficients of an asymptotic series are in addition a function of orher parameters $x$, then one may inquire into the uniformity of the asymptotic approximation. We say that a series is asymptotic uniformly in $I$ if the coefficient of $e^{m}$ in the error term of a truncation may be chosen to be independent of $x$ for each $m$. How small we ave to make $\epsilon$ to get a given approximation should be specifiable independently of $x$. Most of the asymptotic expansions in physics are nonuniform, otherwise known as singular or secular. If the nonuniformity occurs near a finite point, we may often insert a boundary layer expansion defined on an 6 dependent region about the singular 2 which is uniform over that region, and patch it to the singular expansion using the method of matched asymptotic expansions (see [Nayfeh, 1973] p. 111). Such a technique is used for example in matching a thin boundary layer where viscosity is important in an almost inviscid fluid to the inviscid solution in the interior.

The most cummon occurrance ff nonuniformity, however, takes place as $\boldsymbol{x}$ goes to infinity (again note that the order of the I limit and the $c$ limit may not be exchanged). A key example of this is where $x$ represents the time in the solution of ordinary differential equations. We have seen that the most pedestrian perturbation technique is to simply expand the supposed solution of an O.D.E. in an asymptotic series, plug this into the equation, and solve for the terms order by order. We saw that this technique gave asymptotic solutions over times independent of to all orders if $c$. For even the simplest problems with recurrent behavior. however. these expansions are non-uniform in time and in fact are no longer asymptotic 
expancions at all when written in terms of a "slow" time: $\tau \equiv t$. We have not made any mistakes in our analysis of the equation as is sometimes suggested; rather this nonuniform behavior is a property of the exact solution.

Consider for example the simple equation

$$
\ddot{x}=-(1+c)^{2} x .
$$

This is just a harmonic oscillator of frequency $(1+c)$ and so bas solutions like

$$
x(t)=\sin (1+\epsilon) t
$$

If we expand this in an asymptotic expansion, we obtain

$$
x(t) \sim \sin (t)+c t \cos (t)+\cdots
$$

This is the asymptotic expansion of the true solution, and yet we see that for times of order $1 / c$, the second term does not go to zero as $c$ does. This is thus non-uniform in $t$ and the non-uniformity is on a scale of $t=1 / \epsilon$. For this example we may make a change of independent variable to $s=(1+\epsilon) t$ and we see that we get a completely uniform (and even convergent) expansion in terms of $s$. The effect of this is to do our asymptotics along the lines $s=$ constant in the $(\epsilon, t)$ plane instead of along $t=$ constant. 
5.4.1.1. Lie Traneforms

The general technique of all secular perturbation theories is to int roduce a new asymptotic procese as in this example. For example, the method of Lic transforms makes an e dependent canonical transformation of an underlying phase space, such that in terms of the new variables the standard perturbation method yields solution:s that are asymptotic for longer than bounded times. This technique may be shown to work for nearly periodic dynamical systems for time $1 / \mathrm{c}$ (see for example [Cary, 1981] and [Nayfeh, 1973] p. 200). Even here it is not completely specified what Lie transform one should make (since there is always extra freedom in making changes of coordinates). There is no general theory describing other circumstances in which the methad works and even when the method does work, there is no algorithmic procedure for cariying it out.

\subsubsection{Two-Timing}

The multiple time scale approach (two-timing) writes the solution in terms of two variables $t$ and $r$ (see $[$ Nayfeh, 1973\} p. 228). The expression in lerms of $t$ is obtained by replacing $\tau$ by $c t$, but the limiting process is performed with both $t$ and $\tau$ beld fixed. We choose the representation so that the dependence or: $t$ is nonsecular on times of order $1 / \mathrm{c}$ by writing the secular parts in terms of $\tau$. The result is asymptotics good for times of order $1 / 6$. This procedure is not given algorith, mically, it is not clear what systems this approarb works for and it is not clear how to procerd order by order even on systems where it works (though in specific examples the technique is often quite useful). One may sometimes get expressions for longer 
times by introducing more slow times: $t, \tau=1 / \mathrm{r}, \tau^{\prime}=1 / \mathrm{f}^{2} \ldots$. but again the theory seetns to be restricted to sperial examples. It appears that when we expres: quantitie as functions of more than one variable that we introduce an essential non-uniqueness which may limit the precision with which one can specify what one is doing.

\subsubsection{1/c Tịme of Validity for Kruskal's Technique}

We have scen that Kruskal's method, while restricted to perturbations of an exactly periodic system, is perfectly general within this context and uniform to all orders in $\epsilon$ for times of order $1 / \epsilon$. That the time of validity is $1 / \epsilon$ is a significant point, clearly stated in Kruskal's original paper, but not often repeated when this paper is quoted. One often hears the phrase: "Kruskal showed that adiabatic invariants exist to all orders in $\epsilon .^{n}$ This then leads one to confuse the accuracy of the approximation with the time of validity.

Why is the theory good for even time 1/C (we have seen that the simplest examples make the basic perturbation technique fail on this scale)? The idea is to convert our system, by hook or hy crook, to one of the form

$$
\dot{x}=\epsilon X,
$$

where $X$ is an asymptotic vector field. In this case we may make the change of variables to $\tau=\epsilon t$ and obtain the equation in the usual form:

$$
\frac{d x}{d \tau}=X
$$


We may apply standard perturbation theory to this to get an expression for the solution that is asymptotic for bounded $r$. But going back to $t$, this is valid for $t$ of time 1/e. In Kruskal's technique, we get rid of the fast oscillations by sucressive coordinate changes and so the resulting dynamics has only the slow drifts which are of order $\epsilon$ and so susceptible to the above technique. There is no way to get longer times out of this technique in general.

One sometimes hears the plausible argument: "Since the adiabatic invariant's time derivctive is zero to all orders in $\epsilon$, it will be constant for expopentially long times." As an example to see that this reasoning is faulty, consider the expression

$$
j=e^{-1 / c t} \text {. }
$$

If we take derivatives of this with respect to $\epsilon$, while holding $t$ fixed, we see that $J$ 's time derivative is indeed zero to all orders in $\epsilon$. Nonetheless, $j$ is of order 1 on times of order $1 / c$. Since when $J$ is of order $1, J$ can change in times of order 1 , we see that for this example $J$ can undergo an order 1 change in times of order $1 / \mathrm{c}$.

To see that there are physical examples that fit into Kruskal's (and so everbody else's) framework and yet do not have preserved adiabatic itsariants on times of order $1 / c^{2}$ we need only consider parametric resonance. As in Arnold. 1983]. the simple harmonic oscillator with slowly varying frequency:

$$
\ddot{x}=-\omega^{2}(1+a \cos (c t)) x,
$$

may be shown to be unstable for arbitrarily small $c$ since therc are resonances of arbitrarily high order. (An example is the child who pumps up a swing by 
resonantly varying its length, and so its natural frequency.) It is easy to see that in this circumstance, the action will change significantly in tirnes of order $1 / \mathrm{c}^{2}$.

\subsubsection{Averaging with Multiple Frequencies}

If. instead of a single fast degree of freedom, we have several fast degrees of freedom. the motion is close to being on tori that fill up the phase space. It appears at first that one could just average over the tori and obtain as adiabatic invariants the artions correspending to the fundamental loops of the tori. This is fine if the unpert urbed dynamics covers each torus ergodically and so the average perturbation over a corus really reflects the time average. If any of the frequencies are rationally related. however, then the orbit covers only a piece of the torus and we have no reason to expect that the average over the whole torus should have anything to do with the average over the orbit. Indeed one finds that one may have "trapping" at these so-called resonances, where the adiabatic invariant changes drastically in time 1/c. Fortunately, for generic Hamiltonians, the measure of the trapped regions is small and goes to zero as $\sqrt{\ell}$. [Arnold, 1983] introduces the notion of an almost adiabatic invariant which is a quantity that is constant to first order in $C$ for times of order $1, \epsilon$ except for a set of phase space whose measure goes to zero with $c$. 


\subsubsection{Averaging Over Ergodic Orbita}

When the unperturbed system is ergodic on its energy surfaces, Kubo ct al.. 1965:- and later [Ott, 1979], showed that the volume contained insid" an cuergy surface is preserved to order $\epsilon$ for time $1 / c$. This is based on an averaging similar to the single frequency averaging case. This is of intercst because as the dimension gets larger, the volume being preserved is equivalent to the entropy being preseryed. This gives a mechanical justification for the adiabatic invariance of the entropy in thermodynamics. When we forget ahout the oscillatory directions, we have seen that the constancy of the adiabatic invariant in an oscillatory system forces it to give and take energy under a variation of its parameters in such a way that it simulates a potential, leading to the pseudoforces we have discussed earlier. The same circumstance in the thermodynamic case lcads to thermodynamic forces. We shall see in the last chapter of this thesis that there is a symplectic structure and Lagrangian submanifold that describes these forces in a Hamiltonian way exactly analogous to the pseudo forces of the mecuanical systems.

\subsubsection{Non-uniqueness of Symmetry for Finite Perturbation}

Even though Kruskal showed that in a nearly periodic system there is a unique cicle action on the perturbed dynamics to all orders in $\epsilon$. for finite perturbation there may be none or the circle action may not be unique. Hete is give a simple example to demonstrate this non-uniqueness. Our system will be a cylinder with $\operatorname{coordinates} \theta$ and $y$. The unperturbed system will just rotate in 0 :

$$
x_{0}=\frac{\partial}{\partial \theta} .
$$


A typical perturbayion shilts the orbits on the cylinder so that almost all of them are beli-es. For example, we get orbits that look like the stripes on a barber pole for finite with

$$
\boldsymbol{x}=\frac{\partial}{\partial \theta}+\epsilon \frac{\partial}{\partial y} .
$$

Since this vector field does not depend explicitiy on $\theta$, the unperturbed vector feld generates a circle action which is a symmetry of this one. There are an infinite number of others. bowever.

Let $R$ be a vector feld on the cylinder, all of whose orbits are circles. By the relationship of the flow of a vector feld to the Lie derivative along it, $R$ will generate a circle symmetry of $X$ if, and only if the Lie derivative of $X$ by $R$ vanishes. But for vector felds this is just the Lie bracket: $[R, X]$, which is antisymmetric- and so the Lie derivative of $\boldsymbol{R}$ along $\boldsymbol{X}$ must vanish as wein. This says that if we know $\boldsymbol{R}$ at any place on an $X$ orbit, for it to be a symmetry its value anywhere else on this orbit must be obtained by pushing it forward along the flow of $X$. The orbits of $X$ are a circle of belices flling up phase space. If we choose any closed loop around the cylinder that intersects each belix exactly once (it is easy to see that there are an infinite number of ways of doing this), then we can make that loop one of the orbits of $R$. For $R$ on the loop, just choose $R$ tangent to the loop, and to get $R$ everywhere else, we push this forward by $X$ 's flow. 


\section{Chapter6:}

\section{Ponderomotive Force \\ and Gyromotion}

"The purpose of computing is insight, not numbers."-Richard W. Hamming

\subsection{Panderomative-like Forces}

In this chapter we will apply the perturbation techniques of the last chapter to some example problems. The physical plasma phevomena that underlie our examples are the ponderomotive force and gyromotion. We wish to keep the physics of these asymptotic physical effects in the foreground and so we do not work with the most general situation in which these effects arise. Instead, we focus on the simplest situations which contain the relevant physical effects, and discuss the underlying asymptotic process in detail. This approach allows us to explicitly compare aspects of these problems which are obscure in more complex settings. The insights and intuitiuns gained are of course applicable to more general settings.

First we discuss a model problem that contains the essence of ponderomotive force. We consider a time-indepes lent problem with two degrees of freedon. One degree of freedom behaves like $\rightarrow$ fast oscillator and the other evolves slow!y in an 
arbitrary potential. The example we will use is

$$
\begin{aligned}
\dot{I} & =v_{x} \\
\dot{y} & =v_{y} \\
\dot{v}_{x} & =-\frac{\partial v(x)}{\partial x}-\frac{1}{2} \frac{\partial W(x)}{\partial x} y^{2} \\
\dot{v}_{y} & =-W(x) y
\end{aligned}
$$

with the initial conditions

$$
\begin{array}{cl}
x(0)=x_{0} & y(0)=y_{0} \\
v_{x}(0)=v_{x 0} & v_{y}(0)=v_{w 0} .
\end{array}
$$

This system is Hamiltonian with the canonical Poisson bracket in $x, y, v_{x}, v_{y}$ and the Hamiltonian

$$
H\left(x, y, v_{x}, v_{y}\right)=\frac{1}{2} \mathrm{e}_{x}^{2}+\frac{1}{2} v_{y}^{2}+V(x)+\frac{1}{2} W(x) y^{2} .
$$

A physical model with this dynamics is a ball rolling in a trough. The trough is a surface whose height over the $(x, y)$ plane is given by $V(x)+W(x) y^{2} / 2 .\left(v_{x}, v_{v}\right)$ represent the $x$ and $y$ velocities respectively. Along each line $x=$ constant, the potential has a minimum at $y=0$ and grows quadratically with strength $W(x)$ as $|y|$ increases. If the rolling particle starts at $y=0$ with $v_{y}=0$, then it remains on the line $y=0$ for all time. In this case the system behaves like a one degree

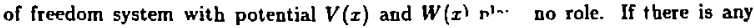
displacement froin $y=0$, then the particle continues to wisillate back and forth about the $x$-axis. The $y^{2}$ in the potential acts like a restoring force toward $y=0$ and the strength $W(x)$ varies with $x$. Exactly this kind of model is hehind mechanical systems with constraints. When we say we have a particle in the plane constrained 
to the - -axis (like a bead on a wire), we jcally mean that there is a still sestoriseg force transverse to the axis. It is sometimes implicitly assumed that if the restoring force is sufficiently strong, the motion of the particle is well approximated by the equations restricted to the constraint manifold (in this case the I-axis). To make this statement precise one must introduce asymptotics in the strength of the restoring force. We will see in fact that if $W(x)$ is not constant, then the transverse motions add a new pseudo-potential to the const rained motion.

We are interested, then, in the case where the transverse $y$ motion is very fast compared to the $x$ motion. We want to explicitly introduce asymptotic scaling with a parameter $c$ into our equatione of motion to make this analysis precise. Usually one is somewhat sloppy and simply proceeds intuitively. Sometimes this gives a usaful answer but the chances of this go down as the complexity of the problem goes up. It is also important to explicitly state the intended scaling as this represents the physical effect one is trying to study. Let us give an intuitive argument and then see what must be done to make it precise.

\subsubsection{Intuitive Treatment}

Because the $y$ motion is supposed to be fast compared to the I motion, the' motion initially behaves a: If $x$ is frozen at $x_{0}$. The equations for the $y$ motion are now those of a simple harmonic oscillator with frequenry $\sqrt{H\left(x_{0}\right)}$;

$$
\dot{y}=v_{y} \quad \dot{t}_{y}=-W\left(x_{0}\right) y .
$$


For simplicity, we take the $y$ initial condition to be zero: $y(0)=0$. In this case the solution is

$$
y(t)=\frac{t y 0}{\sqrt{W\left(x_{0}\right)}} \sin \left(\sqrt{W\left(x_{0}\right)} t\right)
$$

The $v_{x}$ evolution equation

$$
\dot{v}_{x}=-\frac{\partial V(x)}{\partial x}-\frac{1}{2} \frac{\partial W^{\prime}(x)}{\partial x} y^{2}
$$

depends on $y$. Because $y$ traverses its periodic arbit many times be [ore $?$ or $v_{z}$ evolve appreciably, $y$ 's effect on $v_{x}$ 's evolution will be almost the same as its average over $y$ 's orbit. Because the average of $\sin ^{2}(\theta)$ over $0 \leq \theta \leq 2 \pi$ is $1 / 2$, the average of $-\frac{\partial W(x)}{\partial x} y^{2} / 2$ will be

$$
-\frac{1}{2} \frac{\partial W(x)}{\partial x} \frac{v_{v 0}^{2}}{2 W\left(x_{0}\right)}
$$

If we now let $u$ be the value of $v_{v}$ when $y=0$, we see that the same argument applies to any time (not just the initial time) to give the $v_{x}$ equation:

$$
\hat{v}_{x}=-\frac{\partial V(x)}{\partial x}-\frac{1}{2} \frac{\partial W(x)}{\partial x} \frac{u^{2}}{2 W(x)} .
$$

Thus tells us how $v_{x}$ varies if we know u (i.e. $y$ 's maximum velocity).

We may determine $u$ from the constraint that the total energy must remain corstant. Ac an arbitrary time, the average energy in the $y$ motion is $u^{2} / 2$, a result we obtaio by holding $I$ and $v_{x}$ fixed and realizing that all of the oscillatory energy is kinetic when $y=0$. The total energy (averaged over the $y$ motion) is tben

$$
H=\frac{1}{2} v_{z}^{z}+\frac{1}{2} u^{2}+v(x) \text {. }
$$

The time derivative of $H$ must vanish, so

$$
\hat{H}=v_{x} \dot{i}_{x}+v \dot{u}+v_{x} \frac{\partial V}{\partial \tau}=0 .
$$


We substitute in the expression for $i_{x}$ to obtain

$$
\begin{aligned}
\dot{H} & =0 \\
& =-v_{x} \frac{\partial \psi}{\partial x}-\frac{1}{2} v_{x} \frac{\partial \psi}{\partial x} \frac{u^{2}}{2 W}+u \dot{u}+v_{x} \frac{\partial r}{\partial x} \\
& =u \dot{u}-\frac{1}{2} \dot{W} \frac{u^{2}}{2 W} .
\end{aligned}
$$

This has an integrating fartor of $1 /(2 \sqrt{W})$ by which we multiply to obtain:

$$
\begin{aligned}
0 & =\frac{u \dot{u}}{2 \sqrt{W}}-\dot{W} \frac{u^{2}}{8 W^{3 / 2}} \\
& =\frac{d}{d t}\left(\frac{u^{2}}{4 \sqrt{W}}\right) .
\end{aligned}
$$

We have found a constant of the motion, a multiple of 2 of which we shall call $J$ (tince it is equal to the action of the transverse oscillations):

$$
J=\left(\frac{u^{2}}{2 \sqrt{W(x)}}\right)=\left(\frac{v_{\text {yo }}^{2}}{2 \sqrt{W\left(x_{0}\right)}}\right) .
$$

This may be solved for $u$ :

$$
u=\sqrt{2 J \sqrt{W}}
$$

and then substituted into the equation for $v_{x}$ :

$$
\begin{aligned}
\dot{v}_{x} & =-\frac{\partial V(x)}{\partial x}-\frac{1}{2} \frac{\partial W^{\prime}(x)}{\partial x} \frac{u^{2}}{2 W(x)} \\
& =-\frac{\partial V(x)}{\partial x}-\frac{1}{2} \frac{\partial W(x)}{\partial x} \frac{J}{\sqrt{W}} \\
& =-\frac{\partial V(x)}{\partial x}-\frac{\partial}{\partial x}\left(J \sqrt{W^{\prime}(x)}\right) .
\end{aligned}
$$

The $x$ motion behaves just like a particle in the potential

$$
V(x)+J \sqrt{W(x)}=V(x)+\frac{v_{y 0}^{2} \sqrt{W(x)}}{2 \sqrt{W\left(x_{0}\right)}}
$$

The ordinary putential $V(x)$ is augmented by a:. extra pseudo-potential $J \sqrt{W^{\prime}(x)}$ which is non-zero when there is transverse motion and has an effect on the $x$ motion when $W$ varies with $x$. 


\subsubsection{Introducing the Asymptotic Scaling}

This argument is intuitively appealing but has not been systematic. It is difficult to see how to get more accurate evolution equations. We therefore wish to introduce an explicit $\&$ into our equations which captures the physical assumptions used in the intuitive discussion above as $\epsilon \rightarrow 0$ and which gives the correct equations for $\epsilon=1 . y$ and $v_{y}$ are supposed to be fast variables. If we view them as varying by order $l$ on a time scale of $c$ (so the fast motion gets faster and faster as $c-0$ l, then $x$ and $v_{x}$ should vary by order 1 on times scales of order 1 . In modelling constrained motion, one typically makes the constraining force stronger asymptotically. We therefore replace $W(x)$ with $W(x) / \epsilon^{2}$. If we keep the initial conditions $y(0)=y_{0}$ anó $v_{v}(0)=v_{\nu 0}$, then as $c \rightarrow 0$ the energy of the transverse motion becomes infinite. Because the transverse energy is teally of the same order as the energy of the constrained motion, we must scale the transverse displacement with $c$ as $y(0)=c y_{0}$. This leads to a system given by

$$
\begin{aligned}
\dot{x} & =v_{x} \\
\dot{y} & =v_{v} \\
\dot{v}_{x} & =-\frac{\partial V(x)}{\partial x}-\frac{1}{2 c^{2}} \frac{\partial W(x)}{\partial x} y^{2} \\
\dot{v}_{y} & =-\frac{1}{c^{2}} W(x) y
\end{aligned}
$$

with the initial conditions

$$
\begin{array}{cc}
x(0)=x_{0} & y(0)=\epsilon y_{0} \\
v_{x}(0)=v_{x} 0 & v_{y}(0)=v_{y 0} .
\end{array}
$$

From the equations, it might appear that $v_{x}$ can really evolve by order $1 / \mathrm{c}$ on times of order c contrary to assumption. This doesn't happen. however, because 
the maximal excursion in $y$ scales as $c$ and so counteracts the $1 / \mathrm{c}^{2}$ in the equation for $v_{x}$. This is because tive initial $y$ has this scaling and because the total energy in of order 1 . That the traniverse patential energy $W y^{2} / 2 s^{2}$ is of order 1 means that $y$ 's maximal excursion is of order $\epsilon$. This argument shows that the time lerivative of $v_{y}$ is of order $1 / c$. This system is Hamiltonian with the canonical Poisson bracket in $x, y, v_{x}, v_{y}$ and the Harniltonian is

$$
H\left(x, y, v_{x}, v_{y}\right)=\frac{1}{2} v_{x}^{2}+\frac{1}{2} v_{y}^{2}+V(x)+\frac{1}{2 c^{2}} W(x) y^{2} .
$$

This scaling has the fast motion getting very fast as $\mathrm{C} \rightarrow 0$ as is physically reasorable. The interesting pselidr-potential bas its effects in tine 1 (which is presumably the time scale used by the observer). Unfortunately, this scaling is not a perturbation around any well understood system for $c=0$. As $c$ approaches zero, the equations of motion become singular. It is also not apparent how the operations we performed in our intuitive approach may be expressed systematically witl this sraling (e.g.. we held the $x$ variables fixej while studying $y$-why could we do this?)

One way to resolve these difficulties is to redo our study on a stretched time scale (i.e. imagiue the clocks in your sensory apparat us getting faster as $t \rightarrow 0$ ). We introduce $i \equiv t / c$. If we interpret dot to mean derivative with respect to $i$ (while keeping the interpretation of the velocities $v_{x}$ and $t_{y}$ as derivarives with respect to t), then the equations of motion become

$$
\begin{aligned}
\dot{x} & =\iota v_{x} \\
\dot{y} & =\epsilon v_{V} \\
\dot{i}_{r} & =-c \frac{\partial V(x)}{\partial x}-\frac{1}{2 \epsilon} \frac{\partial W(x)}{\partial x} y^{2} \\
\dot{i}_{y} & =-\frac{1}{i} W^{\prime}(x) y
\end{aligned}
$$


with the initial ronditions

$$
\begin{gathered}
x(0)=I_{0} \quad y(0)=y_{0} \\
v_{x}(0)=v_{x} \quad v_{y}(0)=v_{y} 0 .
\end{gathered}
$$

To get rid of the apparently singular terms. we may introduce a rescaled $y$ defined by $Y \equiv y / t$. The initial conditions are now $\epsilon$ independent and the equations are non-singular:

$$
\begin{aligned}
& \dot{x}=c v_{x} \\
& \dot{Y}=v_{y} \\
& \dot{v}_{x}=-c \frac{\partial V(x)}{\partial x}-\frac{\epsilon}{2} \frac{\partial W(x)}{\partial x} Y^{2} \\
& \dot{v}_{y}=-W(x) Y
\end{aligned}
$$

with the initial conditions

$$
\begin{array}{cc}
x(0)=x_{0} & Y(0)=y_{0} \\
v_{x}(0)=v_{x} 0 & v_{\nu}(0)=v_{y 0} .
\end{array}
$$

These equations are perfectly set up for the non-Hamiltonian versions of the methads given in the last chapter. They limit as $c \rightarrow 0$ on a system with only periodic orbits:

$$
\dot{x}=0, \quad \dot{Y}=v_{y}, \quad \dot{v}_{x}=0, \quad \dot{v}_{y}=-W(x) Y .
$$

We will study these equations momentarily. Unfortunately, they are not Hamiltonian with respect to the canonical Poisson bracket. They are Hamiltonian with respec: to the bracket

$$
\{f, g\}=\epsilon \frac{\partial f}{\partial x} \frac{\partial g}{\partial v_{x}}-\epsilon \frac{\partial f}{\partial v_{x}} \frac{\partial g}{\partial x}+\frac{\partial f}{\partial Y} \frac{\partial g}{\partial v_{y}}-\frac{\partial f}{\partial v_{y}} \frac{\partial g}{\partial Y}
$$

with the Hamiltonian

$$
H\left(x, y, v_{x}, v_{y}\right)=\frac{1}{2} v_{x}^{2}+\frac{1}{2} v_{y}^{2}+V(I)+\frac{1}{2} W(I) Y^{2} .
$$


This bracket becomes degenerate (and functions of $r$ and $v_{x}$ becone C'asinirs / when $\imath=0$

One way to preserve the Hamiltonian nature with respeci $i t$ the canonical bracket is to scale the potentials $V(x)$ and $W(x)$ to vary more and more slowly, the strength of the restoring potential to grow and the contribution of $v_{x}$ to the kinetic energy to vanish as $t-0$. This leads to the Hamiltonian

$$
H\left(x, y, v_{x}, v_{\nu}\right)=\frac{1}{2} \epsilon v_{x}^{2}+\frac{1}{2} v_{y}^{2}+V(c x)+\frac{1}{2} W(c x) y^{2} .
$$

The equations of motion are

$$
\begin{aligned}
\dot{x} & =\epsilon v_{x} \\
\dot{y} & =v_{y} \\
\dot{v}_{x} & =-\epsilon \frac{\partial V}{\partial x}(\epsilon x)-\frac{c}{2} \frac{\partial W}{\partial x}(\epsilon x) y^{2} \\
\dot{v}_{v} & =-W(\epsilon x) y
\end{aligned}
$$

with the initial conditions

$$
\begin{gathered}
x(0)=x_{0} \quad y(0)=y_{0} \\
v_{x}(0)=v_{x 0} \quad v_{y}(0)=v_{y 0} .
\end{gathered}
$$

Again this has nice limiting behavior as $\epsilon \rightarrow 0$. Physically, the picture is that we are stretching the $z$ coordinate, so that the trough becomes flatter and latter in the $x$ direction. If we hold $v_{x}$ fixed, then it takes a longer and longer time to make W vary substantially. 


\subsubsection{Using the Kruskal-like Perturbation Method}

Let us now obtain the adiabatirally invariant action to first order in $r$ using the method outined in rhapter 5 . The dynamical vector field is:

$$
\begin{aligned}
x & =x_{0}+c X_{1} \\
& =\left(v_{y} \frac{\partial}{\partial Y}-W(x) Y \frac{\partial}{\partial v_{y}}\right) \\
& +c\left(v_{x} \frac{\partial}{\partial x}-\left(\frac{\partial V(x)}{\partial x}+\frac{1}{2} \frac{\partial W(x)}{\partial x} Y^{2}\right) \frac{\partial}{\partial v_{x}}\right) .
\end{aligned}
$$

The solution curves of the unperturbed piece $\boldsymbol{X}_{0}$ are all closed:

$$
\begin{aligned}
x(t) & =x_{0} \quad v_{x}(t)=0 \\
Y(t) & =A \cos (\sqrt{W(x)} t) \\
v_{y}(t) & =-A \sqrt{W(x)} \sin (\sqrt{W(x)} t) .
\end{aligned}
$$

8.1.3.1. The Coordinateg $x, v_{x}, A, \theta$

Let us define the angle on these orbits as $\theta$ and introduce an anplitude $A$ :

$$
Y=A \cos \theta \quad v_{y}=-A \sqrt{W(x)} \sin \theta
$$

Writing these relations in the other direction jives

$$
\tan \theta=-\frac{v_{y}}{Y \sqrt{W(x)}} \quad A^{2}=Y^{2}+\frac{1}{W(x)} v_{y}^{2}
$$

Comparing these expressions with the earlier discussion we expect the zero order action to be given by

$$
J_{0}=\frac{(A \sqrt{W(x)})^{2}}{2 \sqrt{W(x)}}=\frac{1}{2} A^{2} \sqrt{W(x)}
$$


He may take the time derivatives of these expressions to obrain the dynameal sector ficld in the new coordinates.

$$
\begin{aligned}
\left(1+\tan ^{2} \theta\right) \dot{\theta} & =-\frac{\dot{t}_{y}}{y \sqrt{W(x)}}+\frac{v_{y} \dot{y}}{Y^{2} \sqrt{W(x)}}+\frac{v_{y}}{2 \xi W(x)^{3 / 2}} \frac{\partial W^{\prime}}{\partial x} \dot{x} \\
& =\sqrt{W(x)}+\tan ^{2} \theta \sqrt{W(x)}-\epsilon v_{x} \frac{\tan \theta}{2 W(x)} \frac{\partial W}{\partial x} .
\end{aligned}
$$

And so

$$
\dot{\theta}=\sqrt{W(x)}-c v_{x} \sin \theta \cos \theta \frac{1}{2 W(x)} \frac{\partial W}{\partial x} .
$$

Similarly for $A$ :

$$
\begin{aligned}
2 A \dot{A} & =2 Y \dot{Y}+2 \frac{v_{y}}{W(x)} \dot{v}_{y}-\frac{v_{y}^{2}}{W(x)^{2}} \frac{\partial W}{\partial x} \dot{r} \\
& =2 Y v_{y}-2 v_{y} Y-\epsilon v_{x} \frac{v_{y}^{2}}{W(x)^{2}} \frac{\partial W}{\partial x} \\
& =-\frac{c v_{x} A^{2}}{W(x)} \sin ^{2} \theta \frac{\partial W}{\partial x}
\end{aligned}
$$

leading to

$$
\dot{A}=-\frac{\epsilon v_{x} A}{2 W(x)} \sin ^{2} \theta \frac{\partial W}{\partial x} .
$$

Thus the dynamical vector feld in these coordinates is

$$
\begin{aligned}
X & =\sqrt{W(x)} \frac{\partial}{\partial \theta}+\epsilon v_{x} \frac{\partial}{\partial x}-\epsilon\left(\frac{\partial V(x)}{\partial x}+\frac{1}{2} \frac{\partial W(x)}{\partial x} A^{2} \cos ^{2} \theta\right) \frac{\partial}{\partial v_{x}} \\
& +\epsilon v_{x} \sin \theta \cos \theta \frac{1}{W^{\prime}(x)} \frac{\partial W}{\partial x} \frac{\partial}{\partial \theta}-\epsilon \frac{v_{x} A}{2 W(x)} \sin ^{2} \theta \frac{\partial W}{\partial x} \frac{\partial}{\partial A} .
\end{aligned}
$$


6.1.3.2. Regult of the Metbod of Averaging

The method of averaging says that the projection to $x, v_{x}, A$ space of the integral curves of $X$ agrees with the evolution on that space of the averaged vector ficld:

$$
\tilde{X} \equiv<\left(v_{x} \frac{\partial}{\partial x}-\left(\frac{\partial V}{\partial x}+\frac{A^{2}}{4} \frac{\partial W(x)}{\partial x}\right) \frac{\partial}{\partial v_{x}}-\frac{v_{x} A}{4 W(x)} \frac{\partial W}{\partial x} \frac{\partial}{\partial A}\right)
$$

to first order in $\epsilon$ for a time $1 / \epsilon$. From this we again (though this time it is rigorous) see that for the averaged dynamics

$$
\begin{aligned}
j_{0} & =\frac{d}{d t}\left(\frac{1}{2} A^{2} \sqrt{W(x)}\right) \\
& =A \dot{A} \sqrt{W(x)}+\frac{A^{2}}{4 \sqrt{W(x)}} \frac{\partial W}{\partial x} \dot{x} \\
& =-\frac{v_{x} A^{2}}{4 \sqrt{W(x)}} \frac{\partial W}{\partial x}+\frac{v_{x} A^{2}}{4 \sqrt{W(x)}} \frac{\partial W}{\partial x} \\
& =0 .
\end{aligned}
$$

So $J$ is exactly a constant of the motion for the averaged dynamics. Tbis implies that $J_{0}$ is conserved to within $\mathrm{e}$ for time $1 / \mathrm{C}$, i.e. it is an adjabatic invariant. If we consider a leve! set of $y_{0}$ then the averaged vector field restricted to that level set has the form

$$
\bar{X}_{J}=\ell\left(v_{x} \frac{\partial}{\partial x}-\frac{\partial}{\partial x}(v(x)+J \sqrt{W(x)}) \frac{\partial}{\partial v_{x}}\right)
$$

and so is Hamiltonian with an extra pseudo-potential $J \sqrt{W(x)}$. 


\subsubsection{Calculation of the Roto-rate Vector Field}

Let us now find the roto-rate symmetry vector field to first orde ' in a. Wi know that

$$
\boldsymbol{R}_{0}=\frac{\partial}{\partial \theta}
$$

from the general theory. We wish to impose the requirement that

$$
\left[R_{1}, X_{0} \mid=-\left[R_{0}, X_{1}\right]\right.
$$

Let us solve this for $R_{1}$ componeut by component. Since

$$
X_{0}=\sqrt{W(x)} \frac{\partial}{\partial \theta}
$$

we find that

$$
\begin{aligned}
{\left[R_{1}, X_{0}\right]^{x} } & =-\sqrt{W(x)} \frac{\partial R_{1}^{x}}{\partial \theta} \\
& =-\left[R_{0}, X_{1}\right]^{x} \\
& =-\left[\frac{\partial}{\partial \theta}, X_{1}\right]^{x} \\
& =-\frac{\partial}{\partial \theta} X_{1}^{x} \\
& =0 .
\end{aligned}
$$

We conclude that

$$
R_{1}^{x}=\int_{1}^{x}\left(x, v_{x}, A\right)
$$

is a constant. The next step in the method is to impose the constraint that the orbit $\subseteq$ close after time $2 \pi$ :

$$
\int_{0}^{2 \pi} R_{1} d \theta=0 .
$$

We conclude that the constant vanishes and that

$$
R_{1}^{x}=0
$$


Similarly. we obtain the t'x component:

$$
\begin{aligned}
\left|R_{1}, X_{0}\right|^{\omega_{x}} & =-\sqrt{W(x)} \frac{\partial R_{1}^{\prime \prime x}}{\partial \theta} \\
& =-\left|\frac{\partial}{\partial \theta}, X_{1}\right|^{*,} \\
& =-\frac{\partial}{\partial \theta} X_{1}^{\nu_{s}} \\
& =-\frac{\partial W^{\prime}(x)}{\partial x} A^{2} \cos \theta \sin \theta .
\end{aligned}
$$

So

$$
\begin{aligned}
R_{1}^{v_{x}} & =\frac{1}{\sqrt{W(x)}} \int_{0}^{\theta} \frac{\partial W(x)}{\partial x} A^{2} \cos \tilde{\theta} \sin \tilde{\theta} d \tilde{\theta}+f_{1}^{\nu_{x}}\left(x, v_{x}, A\right) \\
& =-\frac{1}{4 \sqrt{W(x)}} \frac{\partial W(x)}{\partial x} A^{2} \cos 20+\bar{f}_{1}^{v_{x}}\left(x, v_{x}, A\right) .
\end{aligned}
$$

Again the integral condition forces the constant of integration to vanish and we obtain

$$
R_{1}^{v_{s}}=-\frac{1}{2} \frac{\partial}{\partial x}(\sqrt{W(x)}) A^{2} \cos 2 \theta
$$

We proceed to find $R_{1}^{A}$ :

$$
\begin{aligned}
{\left[R_{1}, X_{0}\right]^{A} } & =-\sqrt{W(x)} \frac{\partial R_{1}^{A}}{\partial \theta} \\
& =-\left[\frac{\partial}{\partial \theta}, X_{1}\right]^{A} \\
& =-\frac{\partial}{\partial \theta} X_{1}^{A} \\
& =\frac{v_{x} A}{W(x)} \sin \theta \cos \theta \frac{\partial W}{\partial x}
\end{aligned}
$$

Thus sie see that

$$
\begin{aligned}
R_{1}^{A} & =-\frac{1}{2 \sqrt{W(x)}} \int_{0}^{\theta} \frac{v_{x} A}{W(x)} \frac{\partial W}{\partial x} \sin 2 \dot{\theta} d \dot{\theta}+f_{1}^{A}\left(x, V_{x}, A\right) \\
& =\frac{v_{x} A}{4 W(x)^{3 / 2}} \frac{\partial W}{\partial x} \cos 2 \theta+\tilde{f}_{1}^{A}\left(x, v_{x}, A\right) .
\end{aligned}
$$

Imposing the integral condition gives

$$
R_{1}^{A}=\frac{v_{x} A}{4 W(x)^{3 / 2}} \frac{\partial W}{\partial x} \cos 2 \theta .
$$


Finally we obtain $R_{1}^{\theta}$.

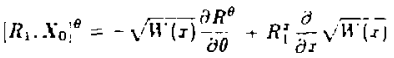

$$
\begin{aligned}
& =-\sqrt{H(x)} \frac{\partial R^{\theta}}{\partial \theta} \\
& =-\left[\frac{\partial}{\partial \theta},\left.X_{\mathrm{L}}\right|^{\theta}\right. \\
& =-\frac{\partial}{\partial \theta} X_{1}^{-\theta} \\
& =\frac{1}{2} v_{x} \cos 2 \theta \frac{1}{W(x)} \frac{\partial W^{\prime}}{\partial x} .
\end{aligned}
$$

Thus we find that

$$
\begin{aligned}
R^{\theta} & =\frac{-v_{x}}{2 W(x)^{3 / 2}} \frac{\partial W}{\partial x}-\int_{0}^{\theta} \cos 2 \bar{\theta} d \bar{\theta}+f_{1}^{\theta}\left(x, v_{x}, A\right) \\
& =\frac{-v_{x}}{4 W(x)^{3 / 2}} \frac{\partial W}{\partial x} \sin 2 \theta
\end{aligned}
$$

where we have already imposed the integral condition.

Thus to first order in $\epsilon$, the roto-rate vector field is

$$
\begin{aligned}
R & =R_{0}+\epsilon R_{1} \\
& =\frac{\partial}{\partial \theta} \\
& -\epsilon \frac{1}{2} \frac{\partial}{\partial x}\left(\sqrt{W^{\prime}(x)}\right) A^{2} \cos 2 \theta \frac{\partial}{\partial v_{z}} \\
& +\epsilon \frac{v_{x} A}{4 U^{*}(x)^{3 / 2}} \frac{\partial u}{\partial x} \cos 2 \theta \frac{r}{\partial A} \\
& -\epsilon \frac{v_{x}}{4 H^{\prime}(x)^{3 / 2}} \frac{\partial U}{\partial x} \sin 2 \theta \frac{\partial}{\partial \theta}
\end{aligned}
$$




\subsubsection{The Hamiltonian Structure}

In the original coordinates. the non-trivial Poisson bracket relations are

$$
\left\{r, r_{z}\right\}=t \quad\left\{Y, v_{y}\right\}=1 .
$$

Let us determine the Poisson bracket in terms of the variables $x, v_{x}, A$ and $\theta$. Since neither $A$ or $\theta$ depend on $v_{x}$, we bave that

$$
\{A, x\}=\{\theta, x\}=0
$$

We may find the other relations most simply by using the derivation property of the bracket:

$$
\begin{aligned}
2 A\left\{A, v_{x}\right\} & =\left\{A^{2}, v_{x}\right\} \\
& =\left\{Y^{2}+\frac{1}{W(x)} v_{y}^{2}, v_{x}\right\} \\
& =-\frac{v_{v}^{2}}{W(x)^{2}} \frac{\partial W(x)}{\partial x}\left\{x, v_{x}\right\} \\
& =-\frac{\epsilon v_{y}^{2}}{W(x)^{2}} \frac{\partial W(x)}{\partial x} \\
& =-\frac{\epsilon A^{2}}{W(x)} \sin ^{2} \theta \frac{\partial W}{\partial x} .
\end{aligned}
$$

Thus we obtain the relation

$$
\left\{A, v_{x}\right\}=-\frac{\epsilon A}{2 W(x)} \sin ^{2} \theta \frac{\partial W}{\partial x}
$$

Similarly

$$
\begin{aligned}
\left(1+\tan ^{2} \theta\right)\left\{\theta, v_{x}\right\} & =\left\{\tan \theta, v_{x}\right\} \\
& =\left\{-\frac{v_{y}}{Y \sqrt{W(x)}}, v_{x}\right\} \\
& =-\frac{c \sqrt{W(x)} \sin \theta}{2 A \cos \theta} \frac{1}{W(x)^{3 / 2}} \frac{\partial W}{\partial x} .
\end{aligned}
$$


so

$$
\left\{\theta, v_{x}\right\}=-\frac{1}{2 U^{+}(r)} \sin \theta \cos \theta \frac{\partial U}{\partial x} .
$$

Lastly

$2.4\{A \cdot \theta\}\left(1+\tan ^{2} \theta\right)=\left\{A^{2}, \tan \theta\right\}$

$$
\begin{aligned}
& =\left\{Y^{2}+\frac{1}{W(x)} v_{v}^{2},-\frac{v_{y}}{Y \sqrt{W(x)}}\right\} \\
& =-2 Y\left\{Y, v_{v}\right\} \frac{1}{Y \sqrt{W(x)}}+\frac{2 v_{y}}{W(x)}\left\{v_{y}, Y\right\} \frac{1}{Y^{2}} \frac{v_{y}}{\sqrt{W(x)}} \\
& =-\frac{2}{\sqrt{W(x)}}-\frac{2 v_{v}^{2}}{Y^{2} W^{\prime}(x)^{3 / 2}} \\
& =-\frac{2}{\sqrt{W(x)}}-\frac{2}{\sqrt{W(x)}} \tan ^{2} \theta
\end{aligned}
$$

so

$$
\{A, \theta\}=-\frac{1}{A \sqrt{W(x)}}
$$

In the new coordinates the Hamiltonian is

$$
\begin{aligned}
H & =\frac{1}{2} \nu_{x}^{2}+\frac{1}{2} v_{v}^{2}+V(x) \cdot \frac{1}{2} W(x) Y^{2} \\
& =\frac{1}{2} v_{x}^{2}+\frac{1}{2} A^{2} W(x) \sin ^{2} \theta+V(x)+\frac{1}{2} W(x) A^{2} \cos ^{2} \theta \\
& =\frac{1}{2} A^{2} W(x)+\frac{1}{2} v_{x}^{2}+V(x) .
\end{aligned}
$$

One easily checks that this Hamiltonian generates the dynamical vector fielr. in these coordinates using the above Poisson bracket relations. 
6.1.4. Obtaining the Action to First Order

Let us now use the Poisfon bracket relations to find the function $J_{0}+U_{1}+$ .. which generates the roto-rate vector ficld $R_{0}+c R_{1}+\ldots$ From the defining expression

$$
R_{0}+\epsilon R_{1}+\ldots=\left\{\cdot J_{0}+\epsilon J_{1}+\ldots\right\}_{0}+\left\{J_{0}+\epsilon J_{1}+\ldots\right\}_{1}
$$

for the action we find a hierarchy of equations

$$
\begin{aligned}
R_{0} & =\left\{\cdot, J_{0}\right\}_{0} \\
R_{1} & =\left\{\cdot, J_{1}\right\}_{0}+\left\{\cdot, J_{0}\right\}_{1} \\
& \vdots
\end{aligned}
$$

A pricri each $J_{2}$ is only determined up to Casimirs for $\{,\}_{0}$ until we get to the next stage. In this example, we may always take these Casimirs to vanish (is this a general phenomenon?).

The zero-order vector field generated by $J$ is

$$
\left\{{ }_{1} J_{0}\right\}_{0}=-\frac{\partial J_{0}}{\partial \theta} \frac{1}{A \sqrt{W(x)}} \frac{\partial}{\partial A}+\frac{\partial J_{0}}{\partial A} \frac{1}{A \sqrt{W(x)}} \frac{\partial}{\partial \theta} .
$$

Comparing this with the desired

$$
R_{0}=\frac{\partial}{\partial \theta}
$$

we obtain the relations

$$
\frac{\partial J_{0}}{\partial \theta}=0 \quad \frac{\partial J_{0}}{\partial A}=A \sqrt{W(x)}
$$

Up to possible Casimirs, we obtain the expected zero order action:

$$
J_{0}=\frac{1}{2} A^{2} \sqrt{W(x)} .
$$


The fistworder vertor ficld generated by $J$ han thw preces. The first is

$$
\begin{aligned}
& \left\{\cdot J_{0}\right\}_{1}=\{\cdot, A\} A \sqrt{1(x)}+\{\cdot, x\} \cdot \frac{A^{2}}{4 \sqrt{W(x)}}-\frac{\partial W^{2}(x)}{\partial x} \\
& =\frac{A}{2 W^{\prime}(x)} \sin ^{2} \theta \frac{\partial H^{*}}{\partial x} A \sqrt{W^{*}(x)} \frac{\partial}{\partial v_{x}}-\frac{A^{2}}{4 \sqrt{W(x)}} \frac{\partial \|^{*}}{\partial x} \frac{\partial}{\partial v_{x}} \\
& =\vec{A} \cdot \frac{A^{2}}{\overline{W(x)}} \frac{\partial \psi}{\partial x}\left(\sin ^{2} \theta-\cos ^{2} \theta\right) \frac{\partial}{\partial v_{x}} \\
& =-\frac{A^{2}}{4 \sqrt{W(x)}} \frac{\partial u^{\prime}}{\partial x} \cos 2 \theta \frac{\partial}{\partial v_{x}} \text {. }
\end{aligned}
$$

The second term is $\left\{, J_{1}\right\}_{0}$ and has the same form as in the zero order calculation.

Together the two terms give

$$
\begin{aligned}
\left\{s_{1} J_{1}\right\}_{0}+\left\{\cdot, J_{0}\right\}_{1} & =-\frac{\partial J_{1}}{\partial \theta} \frac{1}{A \sqrt{W(x)}} \frac{\partial}{\partial A}+\frac{\partial J_{1}}{\partial A} \frac{1}{A \sqrt{W(x)}} \frac{\partial}{\partial \theta} \\
& -\frac{A^{2}}{4 \sqrt{W(x)}} \frac{\partial W}{\partial x} \cos 2 \theta \frac{\partial}{\partial v_{x}} .
\end{aligned}
$$

We must choose $J$, so that this vector feld agrees with $R$. Comparing the $\partial / \partial v_{x}$ terms. we see that they alreaty agree (this just says that the undetermined Casimirs in the zero order step were actirally zero). For the $\theta$ and $A$ terms to agree we must bave:

$$
-\frac{\partial J_{1}}{\partial \theta} \frac{1}{A \sqrt{W(x)}}=\frac{1}{4 W(x)^{3 / 2}} \frac{v_{I} A}{\partial I} \cos 2 \theta
$$

and

$$
\frac{\partial J_{1}}{\partial A} \frac{1}{A \sqrt{11(x)}}=-\frac{\nu_{x}}{A W^{\prime}(x)^{3 / 2}} \frac{\partial W^{\prime}}{\partial x} \sin 2 \theta .
$$

These are satisfied by

$$
J_{1}=-\frac{v_{x} A^{2}}{8 W(x)} \frac{\partial W}{\partial x} \sin 2 \theta .
$$

We have thus shown that the f:nction

$$
J_{0}+r J_{1}=\frac{1}{2} A^{2} \sqrt{\mathscr{H}(r)}-\iota \frac{v_{x} A^{2}}{8 H(x)} \frac{\partial H^{*}}{\partial x} \sin 2 \theta .
$$

varie: only by arder $e^{2}$ over times of arder $1 / t$ 


\subsection{Some Comments on Perturbation Calculations}

Let un use the opportunity of this example to make explicit some very important point: about physical perturbation theory which are implicitly contained in the rest of the thesis. Since the time of Galileo, the scientific method has thrived on theorists making all assumptions explicit and basing the acceptance or rejection of a theory on the testable validity of the assumptions and conclusions of a theory. For science to advance, it is important to be precise about the distinction hetween physical assumptions and rigorous mathematical deduction. In the asymptotic physical throries discussed in this thesis there are two distinct pbases of analysis. The first is the "putting the $i$ 's in". This process requires physical intuition as to which aspects of a problem are physically important and should be emphasized in the scaling with c. One must make the assumed scaling precise at the beginning of the problem if one is to have bope for a self-consistent theory. Too often workers eliminate terms haphazar Jly as a calculation is progressing and the resulting theory does not have its assumptions made explicit. Without this one cannot build anything else on top of the theory and tire result is the fragmentation of a feld.

Once the e's bave been inserted, the rest of a durivation should be rigorous mathernatics. One must state precisely what problem is to be solved and in particular u bat time scalc of validity is desired and obtained. Let us try to clearly state some facts about coordinates. When we are giv:n a problem in terms of physical variables incluting $\ell$, cach physical state is associated with a well-defined point in the state space manifold with these coordinates. The dynamics is a precisely defined vectur field on this manifold. When we start with a definite physical state. its time 
evolution goes through other definice physical states. regardless of any coordinate system. By changing coordinates. one canuot change the evolution of the system. We have seen in chapter 2 that perturbation theory is independent of coordinates as well. This means that if we change our coordinate system, do nonsingular perturbation theory in the new coordinates and change back to the original system. we always get the same result as working with the original conrdinates all along. If the asymptotic expansion of a solution is secular in time (i.c. the coefficients in the expansion hlow up as $t \rightarrow \infty$, usually on a time scale of order $1 / \ell)$, then this is a physical fact which cannot be changed by going to another coordinate system. (One can introduce fake coodinates which shrink with time making the system apparently non-secular, but rewriting this solution in the original coordinetes shows that it really is secular. If the orhits are bounded, then Oseledec's thcorem (see section 10.3) shows that one caraot do even this.)

If one is happ.' with time scales of order 1 then one may use simple non-secular perturbation theory (as discussed in cbapter 2) in any coordinate system one desires (in particular there is no advantage or need to do anything fancy like Lie tronsforms or Kruskal's method). If one wishes to do secular perturbation theory, then again there is a physical operation involved which is independent of coordinates. One must eliminate the variable that gives rise to the secularity, say by using the method of averaging or by reduction by an approximate symmetry. This is a physical operation whicb changes the identification of physical states witb points in state space. One must lose the information that causes the secularity. Merely changing coordinates does not lose any information and cannot eliminate secularity by itself. (Changing 
coordinates can make the physical operation of reduction or averaging much easier to carry out in pratice, but one must not lose sight of the fart that it is mere convenience and has no fundamental signifigance.)

We have secn only two methods for eliminating secularity expressed independently of the coordinate system: the method of averaging and Kruskal's method. Botb of these rest in an absolutely essential way on the unperturbed system being made up of purely closed orbits. If we wish to use these methods, we must scale our system to be perindic at $\epsilon=0$. Sometimes one sees the argument made that while the unperturbed system isn't periodic, we are only interested in the case where orbits almost come back to their starting point, validating averaging. If this is the case, it should be put into the scaling with $\epsilon$ ! The whole point of the scaling is to make explicit what variation is small compared to what other variation; in a self-consistent theory all such assumptions are put in at the beginning.

One otber point in connection with these polemics is that one must he careful not to expend great effort producing a long time theory for a system of equations which are themselves only an approximation for a short time. One place where this issue must be clarified is in plasma physies. One often begins with the Vlasov equation, assumes wave amplitudes are small, and then works with the linearized Vlasov equation. This is just the first order of non-secular perturbation theory, where the disturbance amplitude is scaled with $c_{\text {, }}$ and so is valid only for bounded times as $t \rightarrow 0$ as we have seen. One then often proceeds with an analysis of the linearized system using secular perturbation theory (say in analysing ponderomotive effects) which purportedly will give asymptotic answers uniformly on long time 
sralue If the long time scale in longer than the bounded time seale for which the linear theory is correct, then as far as the actual physical behavior is conserned liwe result i not correct. This is not to say that such studies are srong on!y that there is a further assumption behind them (that the scaling of the process studied using secular theory is such that the secular time scale is bounded as far as the original livearization is ccncerned) which must be made explicit. Higher order non-secular theory gives the two-wave, three-wave, etc. interaction equations. Again these are often studied using fancy secular techniques. Again the scaling which makes this a worthwhile endeavor must be made explicit. This will also give restrictions on the domain of validity of the thecry. 


\subsection{D Gyromotion via the New Kruskal-like Method}

In this section we will apply the perturbation technique developed in ehapter 5 to the problem of two-dineasional gyromotion. We will carry out all calculations in explicit detail. so as to provide a model for other calculations done with this method. This work was done in collaboration with Richard Montgonery. The problem we wish to consider is the motion of a charged particle in the $(x, y)$ plane, in the presence of a purely perpendicular magnetic field:

$$
B(z, y) \dot{z} \text {. }
$$

The Loreatz force law:

$$
m \frac{d v}{d t}=q(\mathbf{v} \times \mathbf{B})
$$

implies that when we set $g=m=c=1$, the exact non-relativistic equations of motion arise from the dynamical vector field:

$$
X=v_{x} \frac{\partial}{\partial x}+v_{y} \frac{\partial}{\partial y}+B v_{y} \frac{\partial}{\partial v_{x}}-B v_{x} \frac{\partial}{\partial v_{y}}
$$

To do a perturbation analysis, we must introduce the scaling factor $\epsilon$. There are a variety of ways of doing this, but we choose $\varepsilon$ so that the unperturbed orbits have their velocity vectors rotatiog at the gyro-frequency and the particle position remains stationary. This scaling is equivalent to making the charge to mass ratio infinite (though we rescale time so that the unperturbed system has well defined dynamics). This scaling is the standard one used in plasma physics. More dis cussion may be found in [Northrop, 1963] and in the papers [Littlejohn, 1979: and [Littlejohn. 1981! whicb are also good references for the rest of this section. 
This dynamical vector teld has only an unperturbed part. $X_{0}$. and a first-order perturbation, $\boldsymbol{x}_{1}$

$$
\boldsymbol{X}=\boldsymbol{X}_{0}+\epsilon \boldsymbol{X}_{1}=\boldsymbol{B}\left(v_{v} \frac{\partial}{\partial v_{x}}-v_{x} \frac{\partial}{\partial v_{y}}\right)+c\left(v_{x} \frac{\partial}{\partial x}+v_{y} \frac{\partial}{\partial y}\right)
$$

If $B$ doesn't vanish, the unperturbed system $X_{0}$ has all of its orbits periodic. In this case, we are in the appropriate situation for the application of the method of chapter 5 . The goal of the perturbation analysis is to find the (roto-rate) symmetry vector field $\boldsymbol{R}$, order by order in $\epsilon$ :

$$
R=R_{0}+\epsilon R_{1}+\frac{\epsilon^{2}}{2} R_{2}+\ldots
$$

The technique used here requires no special knowledge and makes no arbitrary choices. It is therefore suitable for a computer implementation which could work symbolically order by order to as high an accuracy as desired.

Recall that the condition for $R$ to be a symmetry of $\mathrm{X}$ is that their Lic bracket vanish:

$$
[R, X]=0
$$

We write this equation order by order. This example has only wo terms in $X$. and 
(4) earh equation contains two brackets:

$$
\begin{aligned}
& \left\{R_{0}, X_{0}\right\rfloor=0 \\
& \left\{R_{1}, X_{0}\right\}=-\left[R_{0}, x_{1}\right\} \\
& \left\{R_{2}, X_{0}\right\}=-2\left[R_{1}, X_{1}\right] \\
& \left\{R_{3}, X_{0}\right\}=-3\left\{R_{2}, X_{1}\right] \\
& \vdots \\
& \left\{R_{j}, X_{0}\right\}=-j\left[R_{-1}, X_{1}\right] \\
& \vdots
\end{aligned}
$$

We saw in chapter 5 that it is convenient to introduce the coordinate $\theta$ describit. the phase on the unperturbed orbits. This makes it simpler to do the integrals, but is in no way required for any fundamental reason. We introduce polar coordinates in the $\left(v_{x}, v_{y}\right)$ plane at each point:

$$
\theta \equiv \tan ^{-1}\left(\frac{-v_{x}}{v_{y}}\right) \quad v \equiv \sqrt{v_{x}^{2}+v_{y}^{2}} .
$$

The inverse relations are

$$
v_{x}=v \cos \theta \quad v_{y}=-v \sin \theta
$$

Expressed i- these coordinates, the unperturbed dynamical vector feld is

$$
\boldsymbol{X}_{0}=B \frac{\partial}{\partial \theta}
$$

The first order perturbition of the dynamies is

$$
x_{1}=r \cos \theta \frac{\partial}{\partial x}-t^{\prime} \sin \theta \frac{\partial}{\partial y} \text {. }
$$


T:e frequency of the unperturbed ortits is $B$. A: discuned it rhapter 5 . the zero ordet symmetry $R_{0}$ is parallel to these orbits, but normaluzed so that all orluthave period 2-:

$$
R_{n} \equiv \frac{\partial}{\partial \bar{\theta}}=\frac{1}{B} \mathbf{X}_{0}
$$

The four components of the Lie bracket of $R_{1}$ with $X_{\mathrm{C}}$ are given by

$$
\begin{aligned}
& \left.\left[R_{3}, X_{0}\right\}^{I x}=-B \frac{\partial R_{j}^{x}}{\partial \theta}=-j \mid R_{j-2}, X_{1}\right]^{x} \\
& \left.\left[R_{j}, X_{0}\right]^{y}=-B \frac{\partial R_{j}^{y}}{\partial \theta}=-j \mid R_{j-1}, X_{1}\right]^{v} \\
& \left.\left[R_{3}, X_{0}\right]^{v}=-B \frac{\partial R_{j}^{v}}{\partial \theta}=-j \mid R_{,-1}, X_{1}\right]^{v} \\
& {\left[R_{3},\left.X_{0}\right|^{\theta}=-B \frac{\partial R_{j}^{\theta}}{\partial \theta}+\frac{\partial B}{\partial x} R_{j}^{x}+\frac{\partial B}{\partial y} R_{j}^{y}=-j\left|R_{j-1}, X_{1}\right|^{\theta} .\right.}
\end{aligned}
$$

These are the expressions that we use to explicitly perform the integral to determine cach cumponent of $R_{\text {, }}$

$$
\begin{aligned}
& R_{,}^{z}=\frac{j}{B} \int_{0}^{\theta}\left[R_{3-1}, X_{1}\right]^{x} d \tilde{\theta}+f_{3}^{x}\{x, y, x\} \\
& R_{j}^{y}=\frac{j}{B} \int_{0}^{\theta}\left|R_{j-1}, X_{1}\right| y_{d}\left(\tilde{\theta}+f_{j}^{y}(x, y, v)\right. \\
& R_{j}^{*}=\frac{j}{B} \int_{0}^{\theta}\left[R_{j-1}, X_{1}\right]^{v} d \dot{\theta}+J_{j}^{*}(x \cdot y \cdot v) \\
& R_{j}^{\theta}=\frac{1}{B} \int_{0}^{\theta}\left(j\left|R_{3}-1 \ldots y_{1}\right|^{\theta}+\frac{\partial B}{\partial z} R_{j}^{s}\right. \\
& \left.+\frac{\partial B}{\partial y} R_{j}^{\nu}\right) d \tilde{\theta}+f_{j}^{\theta}\left(x, y, t^{\prime}\right) \text {. }
\end{aligned}
$$

The right hand sides of the Lie bracket equations are all of the form

$$
\left[R,-1, x_{1}\right]=\left[R_{j-1},+\cos \theta \frac{\partial}{\partial y}-\gamma \sin \theta \frac{\partial}{\partial y}\right] .
$$

Let us work out the four compoisenis of this in general, wo do make later work a 
matter of simple substitution:

$$
\begin{aligned}
& R_{j}, x_{1}^{r}=R_{j}^{\prime}, \cos \theta-R_{j-1}^{\theta} r^{r} \sin \theta-v \cos \theta \frac{\dot{\partial}}{\partial x}\left(R_{j, 1}^{x}\right)+r \sin \theta \frac{\partial}{\partial y}\left(R_{j-1}^{x}\right) \\
& { }_{i}^{\prime} R_{j-1} X_{i}^{y}=-R_{j-1}^{*} \sin \theta-R_{j-1}^{0} v \cos \theta-1 \cos \beta \frac{\partial}{\partial x}\left(R_{j-1}^{y}\right)+v \sin \theta \frac{\partial}{\partial y}\left(R_{j-1}^{y}\right) \\
& { }_{i} R_{2-1} \cdot x_{1}^{\prime \prime}=-t \cos \theta \frac{\partial}{\partial x}\left(R_{j-1}^{\prime}\right)+r \sin \theta \frac{\partial}{\partial y}\left(R_{j-1}^{*}\right)
\end{aligned}
$$

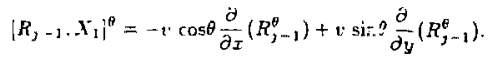

\subsubsection{The Four Componente of $R_{1}$}

From the general theory, we know that $R_{0}$ is given by

$$
\boldsymbol{R}_{0}=\frac{\partial}{\partial \theta}
$$

Let us now $t$ :occed to find the four components of $R_{2}$. The constants of integration: $f_{1}^{z}, \int_{1}^{y}: f_{1}^{*}, f_{1}^{\theta}$. will be determined by the condition that the orbits of $\boldsymbol{R}$ close to first order. We saw in chapter 5 that this implied that

$$
\int_{0}^{2 \pi} R_{1} d \theta=0 .
$$

We will apply thic condicion to each component of $R_{1}$ after evaluating it. The components of the Lie bracket $\left[R_{0}, X_{1}\right]$ are

$$
\begin{aligned}
& {\left[R_{0}, X_{1}\right\}^{x}=-v \sin \theta} \\
& \left\{R_{0_{2}} X_{1}\right]^{y}=-v \cdot \cos \theta \\
& \left\{R_{0}, X_{1}\right]^{v}=0 \\
& \left\{R_{0}, X_{1}\right]^{\theta}=0 .
\end{aligned}
$$


Substituting thee into the general expression give:

$$
\begin{aligned}
R_{1}^{r} & =\frac{-1}{B} \int_{0}^{\theta} v \sin \dot{\theta} d \dot{\theta}-f_{1}^{x}(r, y, v) \\
& =\frac{1}{B} v \cos \theta+j_{1}^{x}(x, y, v) .
\end{aligned}
$$

Since $\cos \theta$ has zero average over the interval $\mid 0,2 \pi !$, the integral condition

$$
\int_{0}^{2 x} R_{1}^{x} d \theta=0
$$

implies that the constant of integration is

$$
\bar{f}_{1}^{x}=0
$$

The $x$ component of $R_{1}$ is therefore

$$
R_{1}^{x}=\frac{1}{B} v \cos \theta
$$

Similariy,

$$
\begin{aligned}
R_{1}^{y} & =-\frac{1}{B} \int_{0}^{\theta} v \cos \bar{\theta} d \bar{\theta}+\int_{1}^{y}(x, y, v) \\
& =-\frac{1}{B} v \sin \theta+f_{1}^{y}(x, y, v) .
\end{aligned}
$$

The integral condition

$$
\int_{0}^{2 \pi} R_{1}^{y} d \theta=0
$$

implies that the constant of integration is

$$
f_{l}^{y}=0
$$

The $y$ component of $R_{1}$ is therefore

$$
R_{1}^{y}=-\frac{1}{B} \cdot \sin \theta
$$


Similarly

$$
\begin{aligned}
R_{\mathrm{i}}^{*} & =\frac{1}{\bar{B}} \int_{0}^{e} 0 d \bar{\theta}+\int_{i}^{v}\left(x, y, u^{\prime}\right) \\
& =\int_{l}^{v}(x, y, v) .
\end{aligned}
$$

The integral condition

$$
\int_{0}^{2 \pi} R_{1}^{2} d \theta=0
$$

implies nat the constant of integration is

$$
\int_{1}^{v}(x, y, v)=0 .
$$

The $v$ component of $R_{!}$is therefore

$$
\boldsymbol{R}_{1}^{v}=\mathbf{0} .
$$

The $R_{1}^{\theta}$ equation uses these results:

$$
\begin{aligned}
R_{1}^{\theta} & =\frac{1}{B} \int_{0}^{\theta}\left(0+B_{x} R_{1}^{x}+B_{y} R_{1}^{y}\right) d \tilde{\theta}+f_{1}^{\theta}(x, y, v) \\
& =\frac{1}{B} \int_{0}^{\theta}\left(\frac{B_{x}}{B} v \cos \tilde{\theta}-\frac{B_{y}}{B} v \sin \tilde{\theta}\right) d \tilde{\theta}+f_{1}^{\theta}(x, y, v) \\
& =\frac{1}{B}\left(\frac{B_{x}}{B} v \sin \theta+\frac{B_{y}}{B} v \cos \theta\right)+\tilde{f}_{1}^{\theta}(x, y, v) .
\end{aligned}
$$

The integral condition

$$
\int_{0}^{2 \pi} R_{1}^{\theta} d \theta=0
$$

implies that the constant of integration is

$$
\tilde{f}_{1}^{\theta}(x, y, v)=0
$$

The $\theta$ component of $R_{1}$ is therefore

$$
R_{1}^{\theta}=\frac{B_{x}}{B^{2}} v \sin \theta+\frac{B_{y}}{B^{2}} v \cos \theta
$$


These expressions may be simplined by introduring a variable reprectint the reciprocal of the magnetic field:

$$
C \equiv \frac{1}{B}
$$

We see that

$$
C_{x}=-\frac{B_{x}}{B^{2}} \quad \text { and } \quad C_{\nu}=-\frac{B_{y}}{B^{2}}
$$

We may then write the entire expression for $R_{1}$ as

$$
R_{1}=C v \cos \theta \frac{\partial}{\partial I}-C v \sin \theta \frac{\partial}{\partial y}+\left(-C_{x} v \sin \theta-C_{y} v \cos \theta\right) \frac{\partial}{\partial \theta} .
$$

\subsubsection{The Four Components of $R_{2}$}

We will now proceed to obtain $R_{2}$. The algebraic manipulations are more tedious than those for $R_{1}$, but conceptually the calculation proreeds identically.

We begin by calculating the four components of $\left\{R_{1}, X_{1}\right\}$. In these expressions it is convenient to use the double angle trigonometric relations (purely for case of notation):

$$
\sin ^{2} \theta-\cos ^{2} \theta=-\cos 2 \theta
$$

and

$$
\sin \theta \cos \theta=\frac{1}{2} \sin 2 \theta
$$

The $x$ component is

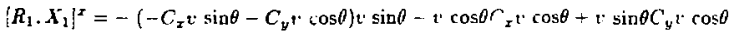

$$
\begin{aligned}
& =v^{2} C_{x}\left(\sin ^{2} \theta-\cos ^{2} \theta\right)+2 v^{2} C_{y} \sin \theta \cos \theta \\
& =-r^{2} C_{x} \cos 2 \theta+r^{2} C_{y} \sin 2 \theta
\end{aligned}
$$


The $y$ component is

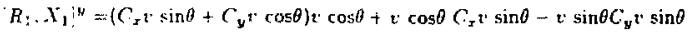

$$
\begin{aligned}
& =2\left(C_{2} v^{2} \sin \theta \cos \theta+C_{y} x^{2}\left(\cos ^{2} \theta-\sin ^{2} \theta\right)\right. \\
& =C_{x} x^{2} \sin 2 \theta+C_{y} v^{2} \cos 2 \theta \text {. }
\end{aligned}
$$

The $v$ component is

$$
\left|R_{1}, X_{1}\right|^{\nu}=0
$$

The $\theta$ component is

$$
\begin{aligned}
{\left[R_{1}, X_{1}\right]^{\theta} } & =-v \cos \theta\left(-C_{x x} \mathrm{v} \sin \theta-C_{y x} v \cos \theta\right)+v \sin \theta\left(-C_{z y} v \sin \theta-C_{y v} v \cos \theta\right) \\
& =\frac{v^{2}}{2} C_{x x} \sin 2 \theta+v^{2} C_{x y} \cos 2 \theta-\frac{v^{2}}{2} C_{u y} \sin 2 \theta
\end{aligned}
$$

As we saw in chapter 5 , the condition that the integral curves of $R$ close to second order is

$$
\int_{0}^{2 \pi} R_{2} d \theta=-\int_{0}^{2 \pi} \frac{\partial R_{1}}{\partial y_{\alpha}} \cdot\left(\int_{0}^{0} R_{1} d \tilde{\theta}\right) d \theta .
$$

This aill eventually determine the constants of integration. To prepare for that we will evaluate the right hand side using the value of $R_{1}$ obtained above. First find the components of the

$$
\int_{0}^{\theta} R_{1} d \hat{\theta}
$$

integral:

$$
\begin{gathered}
\int_{0}^{\theta} R_{1}^{r} d \bar{\theta}=\int_{0}^{\theta} C v \cos \dot{\theta} d \tilde{\theta}=C v \sin \tilde{\theta} \\
\int_{0}^{\theta} R_{1}^{y} d \bar{\theta}=\int_{0}^{\theta}-C v \sin \dot{\theta} d \dot{\theta}=C v \cos \tilde{\theta} \\
\int_{0}^{\theta} R_{1}^{v} d \tilde{\theta}=0
\end{gathered}
$$




$$
\begin{aligned}
\int_{0}^{\varepsilon} R_{1}^{\theta} d \dot{\theta} & =\int_{0}^{\theta}\left(-C_{r} \cdot \sin \dot{\theta}-C_{y} \cdot \cos \dot{\theta}\right) d \bar{\theta} \\
& =C_{x}{ }^{2} \cdot \cos \bar{q}-C_{y^{2}} \sin \dot{\theta}
\end{aligned}
$$

We now substitute in these results to obtain the general expresciun:

$$
\begin{aligned}
\int_{0}^{2 \pi} R_{2}^{5, y \cdot v \cdot \theta} d \theta=-\int_{0}^{2 \pi}\left(\frac{\partial R_{1}^{x \cdot y \cdot u, \theta}}{\partial x} C v \sin \theta+\frac{\partial R_{1}^{x, y, x \cdot \theta}}{\partial y} C v \cos \theta\right. \\
\left.+\frac{\partial R_{1}^{x, y, v, \theta}}{\partial \theta}\left(C_{z} v \cos \theta-C_{y} v \sin \theta\right)\right) d \theta .
\end{aligned}
$$

We now explicitly calculate the four components of this expression.

The $x$ component yields

$$
\begin{aligned}
\int_{0}^{2 \pi} R_{2}^{x} d \theta= & -\int_{0}^{2 \pi}\left(C_{x} v \cos \theta C v \sin \theta+C_{y} v \cos \theta C v \cos \theta\right. \\
& \left.-C v \sin \theta\left(C_{x} v \cos \theta-C_{y} v \sin \theta\right)\right) d \theta \\
= & -\int_{0}^{2 \pi}\left(C C_{\nu} \mathrm{r}^{2} \cos ^{2} \theta+C C_{y} v^{2} \sin ^{2} \theta\right) d \theta \\
= & -2 \pi C C_{y} v^{2}
\end{aligned}
$$

The $y$ component yields

$$
\begin{aligned}
\int_{0}^{2 \pi} R_{2}^{y} d \theta & =-\int_{0}^{2 \pi}\left(-C_{x} v \sin \theta C v \sin \theta-C_{v} v \sin \theta C v \cos \theta\right. \\
& \left.-C v \cos \theta\left(C_{x} v \cos \theta-C_{v} v \sin \theta\right)\right) d \theta \\
& =\int_{0}^{2 \pi} C C_{z} v^{2}\left(\sin ^{2} \theta+\cos ^{2} \theta\right) d \theta \\
& =2 \pi C C_{\tau} v^{2}
\end{aligned}
$$

The $v$ component $y$ ields

$$
\int_{0}^{2 \pi} R_{2}^{\prime \prime} \mathrm{d} \theta=0
$$


The $\theta$ component yields

$$
\begin{aligned}
& \int_{0}^{2 \pi} R_{2}^{\theta} d \theta=-\int_{0}^{2 r}\left(1-C_{x x^{2}} \cdot \sin \theta-C_{y x} x^{*} \cos \theta\right) C \cdot t \sin \theta+ \\
& \left(-C_{x y} x \sin \theta-C_{y y^{2}} v \cos \theta\right) C v^{\prime} \cos \theta \\
& \left.+\left(-C_{x} v^{2} \cos \theta+C_{y} v \sin \theta\right)\left(C_{x} v^{2} \cos \theta-C_{y} v \sin \theta\right)\right] d \theta \\
& =-\int_{0}^{2 \pi} 1-C C_{x x} v^{2} \sin ^{2} \theta-C C_{y y} u^{2} \cos ^{2} \theta \\
& \left.-C_{x} C_{x} v^{2} \cos ^{2} \theta-C_{y} C_{y} v^{2} \sin ^{2} \theta\right] d \theta \\
& =\pi v^{2}\left(C C_{x x}+C C_{v y}+C_{z}^{2}+C_{y}^{2}\right) .
\end{aligned}
$$

We may finally solve for the four components of $R_{2}$. The $x$ component is

$$
\begin{aligned}
R_{2}^{x} & =\frac{2}{B} \int_{0}^{\theta}\left[R_{1}, X_{1}\right]^{2} d \tilde{\theta}+f_{2}^{x}(x, y, v) \\
& =\frac{2}{B} \int_{0}^{\theta}\left(-v^{2} C_{x} \cos 2 \bar{\theta}+v^{2} C_{y} \sin 2 \tilde{\theta}\right) d \tilde{r}+f_{2}^{x}(x, y, v) \\
& =-v^{2} C C_{x} \sin 2 \theta-v^{2} C C_{\nu} \cos 2 \theta+j_{2}^{x} .
\end{aligned}
$$

We vext determine the constant of integ 'ion $f_{2}^{x}$. We have seen that

$$
\begin{aligned}
-2 \pi C C_{v} v^{2} & =\int_{0}^{2 \pi} R_{2}^{x} d \theta \\
& =\int_{0}^{2 \pi} \tilde{f}_{2}^{x} d \theta \\
& =2 \pi \tilde{f}_{2}^{x} .
\end{aligned}
$$

Thus

$$
\bar{f}_{2}^{x}=-C C_{\mathrm{y}} v^{2}
$$

Finally we obtain

$$
R_{2}^{x}=-v^{2} C C_{x} \sin 2 \theta-v^{2} C C_{y} \cos 2 \theta-C C_{y} v^{2}
$$


The y component of $R_{2}$ is

$$
\begin{aligned}
R_{2}^{y} & =\frac{2}{B} \int_{0}^{\theta}\left[R_{1}, x_{1}\right]^{y} d \vec{\theta}+f_{2}^{y}(x, y, v) \\
& =\frac{2}{B} \int_{0}^{\theta}\left(C_{x} v^{z} \sin 2 \theta+C_{y} \mathrm{t}^{2} \cos 2 \theta\right) d \tilde{\theta}+f_{2}^{y}(x, y, v) \\
& =-v^{2} C C_{x} \cos 2 \theta+v^{2} C C_{y} \sin 2 \theta+\int_{2}^{y}(x, y, v) .
\end{aligned}
$$

The constant of integration comes from

$$
\begin{aligned}
2 \pi C C_{x} v^{2} & =\int_{0}^{2 \pi} R_{2}^{y} d \theta \\
& =\int_{0}^{2 \pi} \int_{2}^{y} d \theta \\
& =2 \pi \rho_{2}^{y} .
\end{aligned}
$$

Thus

$$
f_{2}^{y}=C C_{x} r^{2}
$$

Finally we obtain

$$
R_{2}^{\nu}=-v^{2} C C_{x} \cos 20+v^{2} C C_{\nu} \sin 2 \theta+C C_{x} v^{2} .
$$

The $v$ component of $R_{2}$ is

$$
\begin{aligned}
R_{2}^{v} & =\frac{2}{B} \int_{0}^{\theta}\left|R_{1}, X_{1}\right|^{*} d \bar{\theta}+f_{2}^{v} \\
& =\int_{2}^{v} .
\end{aligned}
$$

The constant of integration comes from

$$
\int_{0}^{2 x} R_{2}^{v} d \theta=0
$$

Thus

$$
f_{2}^{*}=0
$$


Finall; tre obtain

$$
R_{2}^{*}=0
$$

The $\theta$ compenent of $A_{2}$ requires the above results and is given by

$$
\begin{aligned}
& R_{2}^{\theta}=\frac{1}{B} \int_{0}^{\theta}\left(2\left[R_{1}, X_{1}\right]^{\theta}+B_{x} R_{2}^{x}+B_{v} R_{2}^{y}\right) d \tilde{\theta}+f_{2}^{\theta}(x, y, v) \\
& =\frac{1}{B} \int_{0}^{0} t v^{2} C_{x x} \sin 2 \theta+2 v^{2} C_{x y} \cos 2 \theta-v^{2} C_{y y} \sin 2 \theta \\
& +B_{x}\left(-v^{2} C C_{x} \sin 2 \theta-v^{2} C C_{v} \cos 2 \theta-C C_{y} v^{2}\right) \\
& \left.+B_{v}\left(-v^{2} C C_{x} \cos 2 \theta+v^{2} C C_{v} \sin 2 \theta+C C_{z} v^{2}\right)\right) d \bar{\theta}+f_{2}^{\theta} \\
& =\frac{1}{B}\left(-\frac{1}{2} v^{2} C_{x x} \cos 2 \theta+v^{2} C_{z y} \sin 2 \theta+\frac{1}{2} v^{2} C_{\mathrm{uy}} \cos 2 \theta\right. \\
& +\frac{1}{2} v^{2} B_{x} C C_{x} \cos 2 \theta-\frac{1}{2} v^{2} B_{x} C C_{y} \sin 2 \theta-B_{x} C C_{y} v^{2} \theta \\
& \left.-\frac{1}{2} v^{2} B_{v} C C_{x} \sin 2 \theta-\frac{1}{2} v^{2} B_{z} C C_{\mathrm{p}} \cos 2 \theta+B_{y} C C_{x} v^{2} \theta\right)+\tilde{f}_{2}^{\theta}(x, y, v) \\
& =-\frac{1}{2} v^{2} C C_{x x} \cos 2 \theta+v^{2} C C_{x y} \sin 2 \theta+\frac{1}{2} v^{2} C C_{y v} \cos 2 \theta \\
& -\frac{1}{2} v^{2} C_{x}^{2} \cos 2 \theta+\frac{1}{2} v^{2} C_{x} C_{v} \sin 2 \theta+C_{x} C_{v} v^{2} \theta \\
& +\frac{1}{2} v^{2} C_{y} C_{x} \sin 2 \theta+\frac{1}{2} v^{2} C_{\nu}^{2} \cos 2 \theta-C_{y} C_{x} v^{2} \theta+f_{2}^{\theta}(x, y, v) \\
& =-\frac{v^{2}}{2}\left[\left(C C_{x x}-C C_{v y}+C_{x}^{2}-C_{y}^{2}\right) \cos 2 \theta-\left(2 C C_{x y}+2 C_{x} C_{y}\right) \sin 2 \theta\right]+\tilde{f}_{2}^{\theta} \text {. }
\end{aligned}
$$

To determine the constant of integration, we use

$$
\begin{aligned}
\pi v^{2}\left(C C_{\mathrm{zx}}+C C_{y v}+C_{x}^{2}+C_{\mathrm{y}}^{2}\right) & =\int_{0}^{2 \pi} H_{2}^{\theta} d \theta \\
& =2 \pi f_{2}^{\theta} .
\end{aligned}
$$

Thus

$$
f_{2}^{\theta}=\frac{u^{2}}{2}\left(C C_{x z}+C C_{y y}+C_{x}^{2}+C_{y}^{2}\right)
$$


Finally we obtain

$$
\begin{aligned}
R_{2}^{6}=-\frac{r^{2}}{2} & \left(C^{\prime} C_{y}-C C_{y y}-C_{x}^{2}-C_{y}^{2}\right) \cos \theta+r^{2}\left(C C_{z y}+C_{z} C_{y}\right) \sin 2 \theta \\
& +\frac{y^{2}}{2}\left(C C_{x x}+C C_{y y}+C_{x}^{2}+C_{y}^{2}\right) .
\end{aligned}
$$

\subsubsection{Summary of the Calculation}

We have thus succeeded in fading the roto-rate vector feld up to second order:

$$
\begin{aligned}
R & \sim R_{0}=C R_{1}+\frac{\epsilon^{2}}{2} R_{2} \\
& =\frac{\partial}{\partial \theta}+ \\
\epsilon & C v \cos \theta \frac{\partial}{\partial x} \\
& -\epsilon C v \sin \theta \frac{\partial}{\partial y} \\
& +\epsilon\left(-C_{x} v \sin \theta-C_{y} v \cos \theta\right) \frac{\partial}{\partial \theta} \\
& \frac{\epsilon^{2}}{2}\left(-v^{2} C C_{x} \sin 20-v^{2} C C_{\nu} \cos 2 \theta-C C_{y} v^{2}\right) \frac{\partial}{\partial x} \\
& \frac{\epsilon^{2}}{2}\left(-v^{2} C C_{x} \cos 2 \theta+v^{2} C C_{v} \sin 2 \theta+C C_{x} v^{2}\right) \frac{\partial}{\partial y} \\
& \frac{\epsilon^{2}}{2}\left(-\frac{v^{2}}{2}\left(C C_{x x}-C C_{y y}+C_{x}^{2}-C_{y}^{2}\right) \cos 2 \theta+v^{2}\left(C C_{x y}+C_{x} C_{y}\right) \sin 2 \theta\right. \\
& \left.+\frac{v^{2}}{2}\left(C C_{x x}+C C_{y y}+C_{x}^{2}+C_{y}^{2}\right)\right) \frac{\partial}{\partial \theta} .
\end{aligned}
$$

Let us summarize what we have done. We began with the exact equations of motion for a two-dimensional particle in a magnetic field. We introduced the scaling parameter $t$ to empbasize the physically important dynamics in sucb a way that the limiting system for $\mathrm{c}=\mathrm{O}$ has only periodic orbits. We then used the procedure presented in chapter 5 to find the roto-rate vector field order by order. This required zo special coordinate system. though we did introduce $\theta$ to make the integrals pasier 
to write. There may exist coordinate systems is which the calculation is eimpler and If one is clever enough to find them certainly one should certainly ure them. The virtue of our method is that it requires no cleverness and by slugging away doing more integrals like the above we could continue order by order. This explieitnoss is very important when we want to delegate this labor to machines. We showed in chapter 5 (and Kriskal showed it before) that this roto-rate vector field is the unique vector Geld whose orbits are all closed loops of period $2 \pi$ to all arders in $\epsilon$ and which commutes to all orders in $\epsilon$ with the dynamical vector field $X$. If we wish to introduce gujding center coordinates $\hat{X}, \bar{Y}, \tilde{J}, \tilde{\theta}$, there is a lot of freedom in the choice. What is not Iree, if the dynamies is to be independent of $\bar{\theta}$, is that $\bar{X}$ $\ddot{\boldsymbol{Y}}$ and $\bar{J}$ must be constant on the orbits of $\boldsymbol{R}$. We bave calculated these orbits in the course of the calculation.

In a Hamiltonian context, the roto-rate vector feld is generated by the adiabatically coneerved action, which we may then determine order by order (in any coordinateg). We turn to this issue in the next section. 


\section{B.4. The Homiltonien Stmetnre of Gyromotion}

In the previous section we etudied gysomotion without using any Hamiltonian gtructures. Here we would like to introduce auch structures and so wake the connection with adiabatic invariance. There are a variety of ways to Hamiltonianize the equations of motion for a particle in a magnetic field. The standard approach introduces canonical momenta which depend on the (unphysical) vector potential $A$. In the paper [Littlejobn, 1979] an approath is developed based on a non-canonical Poisson bracket which it aelf depends on the magnetic field. The points in phase space represent the true particle position and velocity and the Eamiltonian is just the kinetic energy $v^{2} / 2$. This approach allows a particularly nice formulation of the perturbative acaling used in the last section. The subtlety is that the Poisson bracket itself depends on $\epsilon$ and become degenerate when $c=0$. This makea for a much more interesting analyeis and is one motivation for extending the Hamiltonjan perturbation results of chapter 4 to singular Poisson gyatems.

\subsubsection{The Poisson Bracket}

Explicitly the bracket for the two dimensional ayatem is

$$
\begin{aligned}
\{f, g\}= & \{f, g)_{0}+c\left\{f ; g h_{\mathrm{X}}\right. \\
= & B\left(\frac{\partial f}{\partial v_{x}} \frac{\partial g}{\partial v_{v}}-\frac{\partial f}{\partial v_{x}} \frac{\partial g}{\partial v_{x}}\right)+ \\
& \epsilon\left(\frac{\partial f}{\partial x} \frac{\partial g}{\partial v_{x}}-\frac{\partial f}{\partial v_{x}} \frac{\partial g}{\partial x}+\frac{\partial f}{\partial y} \frac{\partial g}{\partial v_{y}}-\frac{\partial f}{\partial v_{y}} \frac{\partial g}{\partial y}\right) .
\end{aligned}
$$

With tbe Bamiltonian

$$
H=\frac{1}{2} v_{x}^{2}+\frac{1}{2} v_{y}^{2}
$$


this gives tbe scaled equations of motion used in the last section:

$$
\{\cdot, H\}=\{\cdot, H\}_{0}+c\{\cdot H\}_{1}=X_{0}+c X_{1} .
$$

When $\rightarrow 0$, the bracket reduces to $\{,\}_{0}$. This bracket is singular and any function of $x$ and $y$ alone is a Casinir.

\subsubsection{The Symplectic Structure}

It is interesting to look at the $\epsilon$-dependent symplectic structure $w$ which corregponds to our bracket. Since the bracket is non-singular when $\epsilon$ ign't zero, we may invert it to give a well defined symplectic form. Since the bracket becomes singular as $\epsilon$ spproaches zero, the symplectic form must become infinite in this limit. It is easiest to introduce the matrix $J^{i j}$ representing the components of the contravariant tensor that defines the bracket:

$$
\{f, g\}=\sum_{i, j} \frac{\partial f}{\partial z^{i}} J^{i j} \frac{\partial g}{\partial z^{j}} .
$$

In the coordinates $x, v_{x}, y, v_{y}$, the watrix $J$ has the form

$$
J=\left(\begin{array}{cccc}
0 & \epsilon & 0 & 0 \\
-\epsilon & 0 & 0 & B \\
0 & 0 & 0 & \epsilon \\
0 & -B & -\epsilon & 0
\end{array}\right)
$$

If we introduce a matrix representing $\omega$ by

$$
\omega=\sum_{i j} d z^{i} w_{i j} d z^{\prime}
$$


then it will be the inverse of the matrix correspondiag to $J$. We easily see (say by Gauss' method) that

$$
\left(\begin{array}{cccc}
0 & \epsilon & 0 & 0 \\
-\epsilon & 0 & 0 & B \\
0 & 0 & 0 & \epsilon \\
0 & -B & -\epsilon & 0
\end{array}\right)\left(\begin{array}{cccc}
0 & -1 / \epsilon & B / \epsilon^{2} & 0 \\
1 / \epsilon & 0 & 0 & 0 \\
-B / \epsilon^{2} & 0 & 0 & -1 / \epsilon \\
0 & 0 & 1 / \epsilon & 0
\end{array}\right)=\left(\begin{array}{llll}
1 & 0 & 0 & 0 \\
0 & 1 & 0 & 0 \\
0 & 0 & 1 & 0 \\
0 & 0 & 0 & 1
\end{array}\right) .
$$

This shows that the symplectic structure is

$$
\omega=\frac{1}{\epsilon^{2}}\left(B d x \wedge d y+\epsilon d x \wedge d v_{x}+\epsilon d y \wedge d v_{y}\right)
$$

This is inded singular as $\epsilon \rightarrow 0$, but if we rescale by multiplying by $\epsilon^{2}$, we get a well defined form for all $\epsilon$. Now, buwever, when $\epsilon \rightarrow 0$ the form becomes $d x \wedge d y$ which is degenerate. This vanishes when we insert any vector which is a linear combination of $v_{x}$ and $v_{y}$. The correct dynamics is obtained by the usual Hamiltcrian prescription for any $c \neq 0$ :

$$
\begin{aligned}
i X^{w}= & -B v_{y} \frac{1}{\epsilon} d x+B v_{x} \frac{1}{\epsilon} d y+v_{x} d v_{x}-\frac{B}{\epsilon} v_{x} d y \\
& +\frac{B}{\epsilon} v_{y} d x+\frac{1}{\epsilon} v_{y} d y \\
= & v_{x} d v_{x}+v_{y} d v_{y} \\
= & d H .
\end{aligned}
$$




\subsubsection{Uniqueness of the Generator for a Vector Field}

The Poisson atructure we bave given becomes degenerate when $\epsilon=0$. We must tberefore study its properties carefully. First, it is not at all obvious that the association of vecto: fields with Hamiltonians is unique with this bracket (since to zero order, say, there are Casimirs which may be added to any Hamiltonian without changing the dynamies). We will show that if we have two Hamiltonian vector fields with respect to this bracket, which are asymptotically equal to all orders in $\epsilon$, then their Hamiltonians are also asymptotically equal to all orders in $\epsilon$. Let us subtract the two vector fieldo in question to obtain a vector field which vanishes to all orders in $c$. We want to show that only a Hamiltonian which vanishes to all orders can produce such a vector field.

The Poisoon bracket has the form

$$
\{,\}=\{,\}_{0}+\epsilon\{,\}_{1}
$$

The zero order piece $\{,\}_{0}$ is drgenerate and the Casimirs are exactly the functions of $x$ and $y$ alone. The first order piece \{\}$_{1}$ is canonical and so non-degererate. Let us assume that $\boldsymbol{H}$ generates the zero vector field to all orders in $\epsilon$. Expanding $\boldsymbol{B}$ in an asymptotic series

$$
\boldsymbol{B} \sim \boldsymbol{H}_{0}+\epsilon \boldsymbol{H}_{1}+\frac{1}{2} \epsilon^{2} \boldsymbol{B}_{2}+\ldots
$$

and riorking out the generated vector field order by order gives us a bierarchy of 
eqtations:

$$
\begin{aligned}
\left\{, H_{0}\right\}_{0} & =\eta \\
\left\{\cdot, H_{1}\right\}_{0} & =-\left\{\cdot, H_{0}\right\}_{1} \\
\left\{\cdot \frac{1}{2} H_{2}\right\}_{0} & =-\left\{\cdot, H_{1}\right\}_{1} \\
\vdots & \\
\left\{\cdot \frac{1}{k} B_{k}\right\}_{0} & =-\left\{\cdot, H_{k-1}\right\}_{1}
\end{aligned}
$$

The only thing the zero order bracket can produce is derivatives with respect to $p_{x}$ and $p_{y}$. These derivatives must be equal to the right hand sides. If any of the $H_{t}$ depended on anything but $x$ and $y$, then the right hand gide of its equation would bave a derivative which could not be matched on the left. So each $H_{k}$ depends only on $x$ and $y$. But these are then Casiming for the left hand sides which therefore vanisb. But the right hand brackets are non-degenerate and so they only vanish when the corresponding $H_{k}$ is constant. Adding a meaningless constant to the energy, we see that $\boldsymbol{H}$ must vanish order by order.

\subsubsection{Comparison W1th Robert Littlejohn's Results}

Let us now show that our results agree witb the results obtained by Robert Littlejohn. The two references of interest here are [Littlejobu, 1979] and [Littlejoho, 1981|. To avoid confusion, we $\nabla$. al use the notation of these papers and refer to formulas within them. To help otber workers make explicit comparisons, we will describe manipulations in detail. Readers without these papers available may want to skip this section. 
Let us begin with [Lit1lejohn, 1979]. In this paper Littlejobn introduces a very clever method based on Darhoux's theorem to manipulate the two-dimeosional guiding ccnter problem into a form suitable for the application of Lie transforms (the Lie trausform is clever too, becauge the bracket is tependent). His Poisson bracket is $1 / \epsilon$ times the one we have used and his variable $\theta$ is rotated by $\pi / 2$ from ours (ours is the clockwise angle of the velocity vector from the $\hat{x}$ axis, his is the clockwise angle of the gyro-radiug vector from the $\hat{x}$ axis in a uniform field). Using the Darboux algorithm introduced in the paper, he defines a set of variables $(X, Y, \theta, J)$, where $\theta$ is the original $\theta, J$ is a function whose Poisson bracket with $\theta$ is $-1 / \epsilon$ to all orders in $c$ and $X$ and $Y$ are coordinates that Poisson commute with both $J$ and $\theta$ to 11 orders in $\epsilon$. These variables are the vatural ones for a uniform magnetic freld, but other than that have no dependence on the Hamiltooian. These functions are obtained as asy mptotic series in 6 . Using his formulas 4.8, 4.21, 4.20, and 4.29 and letting $C$ denote $1 / B$, we find that to first order in $\epsilon$ they are

$$
\begin{aligned}
& X=x-\epsilon v C \cos \theta+\ldots \\
& Y=y+\tau \nu C \sin \theta+\ldots \\
& J=\frac{\pi}{2} C-\frac{\epsilon v^{3}}{6}\left(C \cos C x-C \sin C_{v}\right)+\ldots
\end{aligned}
$$

Be then performs the Lie tranform to find new variables $\bar{X}, \bar{Y}, \bar{J}, \vec{\theta}$ which have the same Poisson bracket relations and auch that the Hamiltonian is independent of $\ddot{\theta}$ to all orderg in 6 . This theo implies that ${ }^{7}$ is the adiabatic ivvariant to all orders in $\epsilon$. We are interested only in this adiabatic invariant (since it is the only quantity that is uniquely determined). By the Poisson bracket relations, the vector field which $\vec{J}$ generates must be simultaneously tangent to the level sets of $\tilde{X}, \vec{Y}$, and $\vec{J}$. 
The closed orbitg of this vector field are parameterized by $\bar{\theta}$ and the Hamiltonias is constant on them (implying that the Hamiltonian vector field commutes with the field generated by $\bar{J}$ ). These conditions are exactly the ones imposed on our roto rate vector feld $R$ and it is the unique vector field which satisfies them. Thus the adiabatic invariant $\bar{J}$ must generate the roto-rate vector field. We will show that the adiabatic invariant introduced by Littlejohn does indeed generate the roto-rate vector field we calculated (at least to first order).

The expression 5.27 in the paper gives $\bar{J}$. To first order in $\epsilon$, in is

$$
\bar{J}=J+\epsilon-\frac{(2 B J)^{3 / 2}}{3 B^{3}}(\hat{\alpha} \cdot \nabla B)
$$

Using the expressions for these quantities in terms of $\theta, x, y$, and $v$, we fixd:

$$
\begin{aligned}
\bar{J} & =\frac{v^{2}}{2 B}-\frac{\epsilon v^{3}}{6}\left(-\cos \theta \frac{B_{x}}{B^{3}}+\sin \theta \frac{B_{u}}{B^{3}}\right) \\
& +\frac{\epsilon v^{3}}{3 B^{3}}\left(-\cos \theta B_{x}-\sin \theta B_{v}\right) \\
& =\frac{v^{2}}{2 B}+\frac{\epsilon v^{3}}{2 B^{3}}\left(\cos \theta B_{x}-\sin \theta B_{v}\right) .
\end{aligned}
$$

Let us check that this agrees with the much more general results presented in [Littlejohn, 1981]. Formula 82e of that paper gives the expresgion for the adiabatic invariant:

$$
\bar{M}=\left[\frac{w^{2}}{2 B}+\frac{\epsilon}{B^{2}} \mid w G_{6}+\frac{w^{2}}{4}\left(G_{8}-2 G_{2}\right)+\frac{w^{3}}{2} F_{6}\right]
$$

To compare whth our much simplified situations we utilize his expressiors $17 \mathrm{~g}, 17 \mathrm{i}$, $17 \mathrm{c}, 16 \mathrm{~g}, 9 \mathrm{~b}, 8$, and 11 . These show that $w=v, G_{\dot{r}}=0, G_{8}=0, G_{2}=0$, and $F_{6}=\hat{a} \cdot \nabla B / B$. Substituting these in we obtain to first order in $\epsilon$ that

$$
\bar{M}=\frac{v^{2}}{2 B}+\frac{\epsilon v^{3}}{2 B^{3}}\left(\cos \theta B_{x}-\sin \theta B_{v}\right) .
$$


This agrees with the first paper.

Let us now determine the vector field which $\bar{J}$ (or $\bar{M}$ ) generates. For this we need the Poisson bracket relations:

$$
\begin{array}{ll}
x, v=-\sin \theta & y, v=-\cos \theta \\
x, \theta=-\frac{1}{v} \cos \theta & y, \theta=\frac{1}{v} \sin \theta \\
\theta, v=\frac{B}{c v} . &
\end{array}
$$

We then the dynamical vector field generated by $\bar{J}$ to lowest two orders is

$$
\begin{aligned}
J= & \frac{l}{\epsilon} \frac{\partial}{\partial \theta}+ \\
& \frac{B}{v}\left(\frac{3 v^{2}}{2 B^{3}}\left(\cos \theta B_{x}-\sin \theta B_{v}\right) \frac{\partial}{\partial \theta}-\frac{v^{3}}{2 B^{3}}\left(-\sin \theta B_{x}-\cos \theta B_{v}\right) \frac{\partial}{\partial v}\right) \\
& -\sin \theta \frac{v}{B} \frac{\partial}{\partial x}-\cos \theta \frac{v}{B} \frac{\partial}{\partial y}+ \\
& \sin \theta \frac{v^{2}}{2} \frac{\partial}{\partial x}\left(\frac{1}{B}\right) \frac{\partial}{\partial v}+\cos \theta \frac{v^{2}}{2} \frac{\partial}{\partial y}\left(\frac{1}{B}\right) \frac{\partial}{\partial v} \\
& +\frac{v^{2}}{2} \frac{1}{v} \cos \theta \frac{\partial}{\partial x}\left(\frac{1}{B}\right) \frac{\partial}{\partial \theta}-\frac{1}{v} \sin \theta \frac{v^{2}}{2} \frac{\partial}{\partial y}\left(\frac{1}{B}\right) \frac{\partial}{\partial \theta} \\
= & \frac{1}{\epsilon} \frac{\partial}{\partial \theta} \\
& -\frac{v}{B} \sin \theta \frac{\partial}{\partial x}-\frac{v}{B} \cos \theta \frac{\partial}{\partial y} \\
& \left(\frac{v^{2}}{2 B^{2}} \sin \theta B_{x}+\frac{v^{2}}{2 B^{2}} \cos \theta B_{y}\right. \\
& \left.-\frac{v^{2}}{2 B^{2}} B_{x} \sin \theta-\frac{v^{2}}{2 B^{2}} B_{v} \cos \theta\right) \frac{\partial}{\partial v} \\
& \left(\frac{3 v}{2 B^{2}} \cos \theta B_{x}-\frac{3 v}{2 B^{2}} \sin \theta B_{y}\right. \\
& \left.-\frac{v}{2 B^{2}} \cos \theta B_{x}+\frac{v}{2 B^{2}} \sin \theta B_{v}\right) \frac{\partial}{\partial \theta} \\
= & \frac{1}{\epsilon} \frac{\partial}{\partial \theta}-\frac{v}{B} \sin \theta \frac{\partial}{\partial x}-\frac{v}{B} \cos \theta \frac{\partial}{\partial y}+ \\
& \left(\frac{v}{B^{2}} \cos \theta B_{x}-\frac{v}{B^{2}} \sin \theta B_{y}\right) \frac{\partial}{\partial \theta} . \\
&
\end{aligned}
$$


If we make the conversion (due to the diferent definitions of $\theta$ ):

$$
\begin{aligned}
& -\sin \theta \rightarrow \cos \theta \\
& -\cos \theta \rightarrow-\sin \theta
\end{aligned}
$$

and multiply by $\epsilon$, we find that this agrees to order $\epsilon$ with the roto-rate vector field $\boldsymbol{R}$ that we obtained in the last section.

\subsubsection{Prospects for a Fully Hamiltonlan Theory}

Wie may essentially apply the argument of the last gection in reverse to obtain the adjabatically invariant action from the roto-rate vector feld. Because the generating function is unique, we can solve for it order by order using the express:on for the Poisson bracket and the roto-rate vector field. It is interesting that because the zero order piece of the Poisson bracket is degenerate, we can determine a given order of $J$ from the corresponding order of $R$ only up to Casimirs. These become determined ty the aext order term in $R$ (only a piece of this higher order term is actually needed, so the fult calculation need not be carried out). When one inserts $R$ into the aymplectic form $\omega$, the $\epsilon^{-2}$ term and the $\epsilon^{-1}$ terms vanish, though this is by ro means obvious a priori. Can this be shown in general? [Kruskal, 1962j proves that if the dynamical vector field is Hamiltouizn with respect to an $\epsilon$ independent symplectic structure, then the corresponding roto-rate vector field $R$ is as well, leading to an adiabatic invariant. We have seen that the same is true $i_{\mathrm{i}}$ this example, even though the Poisson bracket was $\mathrm{c}$-dependent and became degenerate at $\epsilon=0$. Is it always glaaranteed that the symmetry vector Geld is Hamiltonian in such degener ate cases? 
One would like to implement the perturbation analygis we bave given directly in terms of the Hamiltonian structures involved instead of fog through the essentially non-Hamiltonian andygis. Such a theory would look like the one developed in chapter 4, but requires some extensions, in particular, I expect that:

1. We must do everything with Poisson brackets that have a singular structure and Casimir functions.

2. We roust deal with approximate group actions, i.e. we bave an action on the $J$-jet space which does not arise from a true action. Thus, for example, our "circle action" is generated by a vector field given as an asymptotic series in $\epsilon$. The orbits of the $\epsilon^{J}$ truncation of this vectortield are only closed to order $e^{J+1}$. We may still reduce asymptotically in this setting.

3. The reduction map, which for $\epsilon=0$ takes the form $M \rightarrow N$, was previously cifined from $J M \rightarrow J N$. Here we would like to work with the $J$-jet of diffesmorphisms from $M$ to $N$. Thus the reduction map is given an asymptotic sering, not the phwe space and the reduced space. In the example at hand this will give us the guiding center coordinates as agymptotic series in the original coordinates. 


\section{PART II:}

\section{WAVES}

"Before Maxwell, people conceived of pnysical reality-insofar as it is supposed to represent events in nature-as material points, whose changes consist exclusively of motions, which are subject to total differential equations. After Maxwell they conceived of physical reality as represented by continuous fields, not mechanically explicable, which are subject to partial differential equations."-Albert Einstein (1931) [Hirsch, 1984] 


\section{Chapter 7:}

\section{Asymptotic Wave \\ Theory}

Let w now turn to the next large class of systems: waves. These systems are particulasly interesting from a foundations point of view, because the symplectic Btructure of rlassical mechanies arises from a natural yymplectic structure occuring in asymptotic wave theory as applied to quantum mechanics. All of the elegant and physically important Hamiltonian notions of mechanics may be seen as examples of the new conceptual otructures that can arise from asymptotics, an we have discussed tbroughout this thesis. The concepts of momentum, position, energy, action, rays, and Lagrangian submanifold do not make sense for a given wave or wavepacket. As we separate the wavelength from the ocale length, however, they take on asymptotic meaning and give us classical mechanics as we know it.

\subsection{Wuye Arymptotics and Appraximate Symmetry}

A crucial element of this simplifieation proceedure is the idea of approximate symmetry. Part of the asymptotic stretching we will perform on our system will make it approximately symmetric under translations. In any given region of space, an eikonal wave looks like a plane-wave. Consider the translation group acting 
on a plane-wave. Tranglations in a direction contained in the wave-front leave the wave isvariant. Translations transverse to the wave-front bring the wave back to its initial state at integral multiples of some distance. The space of distinct planewave obtainable from the initial one by tracklation is identifiable with tbe quotient of the group of translations by the subgroup which leaves the wave invariant. This quotient is a circle, and we call the angle on the circle the phase of the translated wave relative to the reference wave.

For an asymptotically eikonal wave, we have an approximate symmetry given by translation. On the large scale this is not a real translation because we must translate by different amounts at different points in space. Asymptotically, however, it becomes closer and closer to a true translation. On the small scale a translation that alowly varies cannot wake neighboring wave crests exactly line up. Asymptotically, bowever, they will match up more and more closely, We may therefore think of an asymptotic circle action on our space of eikonal waves (using the philosophy of chapters 4 and 5, way define an asymptotic symmet ry to be an exact symmetry on a jet space). It is in this zrase tbat we may assign a unique asymptotic phase to our eikonal wave. Our eikonal methods to eliminate the wavelength scale details and to obtaty scale-length sized dy namics and concepts may be seen as asymptotic reduction by this circle action.

The bey technical tool which allows us to carry out this asymptatic simplifcation is the method of atationary phage. On a large ecale only average behavior is important. Functions that obey the eikonal geparation bave zero average. The systematic effects that mount up near places where the wavevector vasighes are ac. 
counted for by the method of stationary phase and so allow us to obtain largescale behavior. These cikonal structures are intimately connected witb the esentially mechanical material of the earlier chapters. We sball see in the last chapter that there is also an intimate connection with the statistical averaging that leads to thermodynamics. We will develop the needed wave structures to make this conoection clear. This is the reason we discuss contact structures and Legendre submanifolds in places where aymplectic structures and Lagrangian submanifolds alone could have sufficed for waves.

\subsubsection{Elkongl Waves}

A beautiful description of eikonal wave theory for the example of light waves in the geometric optics limit is given in [Born and Wolf, 1970]. Let wo begin by defining what we mean by an eikonal wave. Intuitively we mean a physical feld in space which is locally like a plane-wave. The local plane-wave is deacribed by a one-form, called the navevector $k$, which is the differential of the wave phase. As discussed above, these notions are not precise for a real wave. To make them precise, we introduce an asymptotic class of waves parameterized by $c$. When $\epsilon$ is 1 this should give the real wave. As c goes to 0 , the class should emphasize the tendency that We feel to be physically important for the behavior of the real wave. For the systems we have in mind, this tendency is the separation of wavelength and scale length. We therefore introduce $\epsilon$ in such a way as to separate them infigitely as it goes to zero. 
There are two natural ways to do this (Lhough any combination of them is also possible). We may let the wavelength go to zero while holding the scale lengtb fixed or we may let the scale length go to infinity as we hold the wavelength fixed as in figure (7.1). I prefer the second tactic because the wavelength often determines the type of wave and its fundamental physical behaviot (eg. in plasmas, how close the wave is to the Debye length plays a fundamental role, sinilarly for electron wavelength compared to the atomic sparing of a crystal in solid state physics). The scale length is often something quite variable (i.e. determined by engineering rather than physics). We may change the size of a tokamak, silicon chip, or auditorium. When we feel eikonal methods provide a valid description, we are saying that we bave made things large enough so that the waves propagate in an eikonal faghion. Of course we may let the wavelength go to zero without changing the wave's evolutiouary behavior by altering the physical constants in the equation. One common example of this if to say that letting $h$ go to zero in the Schrödinger equation is the way to take the classical limit (in reality we make the potential the particle moves in very slowly varying). We ehall see, however, that regardluss of how we do the asymptotics, it is often useful to introduce coordinates on the slowly varying scale, and many of the asymptotic concepts will make sense only in these coordinates.

From the geometric viewpoint we have heen emlohasizing, it is important to

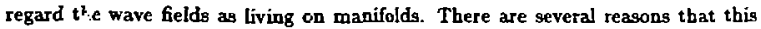
generality is important. General relativity says that spacetime is really a curved manifold, and so studies of quantum mechanics o. vectromagnetic radiation on a cosmic scale must utilize a geometric formulation. We bave seen in our discussion of 
mechanics that even when the mavifold structure of spacetime is not important, the natutal spaces of interest are products (for more than one component), quotients (by symmetries and ignored variables), and submanifolds (for constrained systems and invariant subsystems) of regions of spacetime and these often have a non-trivial manifold structure. (One class of rich examples arises in the study of linkages. e.g., a system of 3 rigid links in three-dimensions joined in a line by universal joints and constrained to have the two ende fixed I 2 space, has a state space that is naturally a 3-sphere and rotations about the line joining the ends define the Hopf fibration.) Corresponding operations on wave systems lead to the study of waves on nontrivial manifolds (for example, tbe $Y_{t m}$ spherical harmonics that arise in systems with rotational syametry are the normal modes of a wave operator on a ophere). As we have been emphavizing, formulations of a theory that make explicit which structures are essential for which phenomena (e.g., what aspects of a coordinate system are used in an essential way in a derivation) give ingight into the underlying physics. Lastly, coordinate-free expressions msy be evaluated in any coordinatea. This allows us to work in the system most convenient for the problem at hand. This is important for systems based on a complicated geometry (e.g., modern plasma fusion devices).

How are we to incorporate the asymptotic scaling into the geometric structure of the manifold in an invariant way? The manifold structure represents slow-scale behavior. We therefore want to "blow up" the manifold asymptotically. For example, most invariant P.D.E.'s ube a Riemannian structure on the underlying state space (e.g., the Laplace-Beltrami operator, which is the invariant Laplacian). The 
metrit should depend on $\epsilon$ in such a way that the distadce between two points grows indefinitely as $c \rightarrow 0$. The curvature of the manifold then goes to zero with c. Asymptotic fast-acalc (c-independent) objects may be defined near a point of the slow manifold as if they were defined on a linear space (which invariantly is the tangent space at the point of interest). One way to see this witbout a metric is to use the Whitney embeding theorem to embed the manifold smootbly in $7^{2 N+1}$ with the point of interest sent to the origin. In $x^{2 N+1}$ we do the scaling by sending $x \in x^{2 N-1}$ to $x / c$. If we look in a fixed neighborhood of the origin as $c \rightarrow 0$, our manifold approaches a linear space identifiable with the tangent space. If we change the embedding by a map $f$ that leaves the origin invariant, the asymptotic lincar space is changed by the Jacobian of $f$ at the point. This show that the asymptotic linear approximation space transforms under coordinate changes just like the tangent space. One advantage of using the other type of scaling (where iast-scale obj cts are scaled smaller and smaller) is that the geometric structure is more immediately recognizahle. Two points that are fixed on the fast-scale (for example two crests of an eikonal wave) asymptotically approach one another in the physical manifold as $\epsilon \rightarrow 0$. The equivalence class of all points that approach a given point with a given fint order rate may be identifect as a tangent vector.

\subsubsection{Sources with Time Scales Generate Eikonal Waveg}

Eikonal waves appear in nearly every discipline of physics and are me of the most useful andytic tools. Why do they arise in practice? One reason is simply that the systems we wish to study have a fast natural time scale and we vary this 


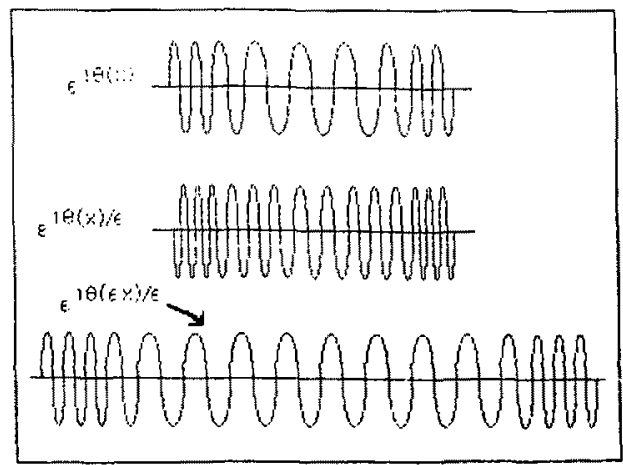

Flgure 7.1: Two waye of making a wave asymptotically eikonal.

slowly. The waves that are generated are thus eikonal. For exarr ile, a tuning fork has a natural period of oscillation and the dissipative processes that make the amplitude decay and the period change are much slower than this. The tuning forie's generated sound wave is then eikonal. Murical instruments are usually considered to be playing with a definite pitch. The amplitude and timbre (tbe barmonic mix which determines the characteristic sound of an instrument) vary slowly during the note and distinguie'a different instrumente. Similar examples abound in plasina wave generation processes, solid st ste waves, atomic light emmibsion, etc. 


\subsubsection{Dlnpersive Media Create Eikonal Waves}

The other common reason for the prevalence of eikonal waves is the fact that in linear dispersive media, any bounded initia' wavepacket will eventually turn into an eikonal wave, and the eikonality will get better and better with time. Imagine throwing a stone into a large lake. This will create a bounded disturbance with some spectrum of wavevectors. For surface gravity waves on water, the long wavelengths have a greater group velocity than the short ones. As our disturbance evolves, the long wavelengths *ill congregate at the outer edge of the spreading wave. After a long enough time, the different wavelengths present in the intial disturbance will have sorted tbemselves out radially. As time goes on, the fastest waves will go further than the slower ones and new wave crests will be formed in between. As we wait asymptotically long times, we get agymptotically long stretches of wavetrain close to each wavelength.

\subsubsection{Whitham's Generalleation to Nonlinearity}

Whitham has shown that many of the notions of linear eikonal theory carry over to nonlinear waves as well [Whitham, 1974]. The nonlinear systems, the basic periodic solu tions are not necessarily sinusoidal. When we make eikonal waves, they will not be slowly varying sine waves; instead, they will be locally like the nonlinear periodic wavetrains.... wit! parameters that slowly vary. The nonlinearity aiso leads to amplitude dependent dispersion relations, which lead to some interesting effects. We will develop parts of this theory to encompass Whitham's ideas in later sections, but let us here focus on linear waves for clarity. 


\subsubsection{Sinusoidal Waves}

In this case re strall be interested in slowly varying sinusoids. In a typical situation, we ate given a wave of the form

$$
A(x) e^{i \theta(x)}
$$

and are told that the amplitude $A(x)$ and the wavevector $k \equiv d \theta(x)$ are slowly varying functions of $x$ compared to the wavelength $\lambda=1 /|k|$. Using the two schemes discussed above we may write down an asymptotic family es either

$$
A(x) e^{i \theta(x) / \epsilon}
$$

or

$$
A(\epsilon x) e^{i \theta(t x) / \epsilon} .
$$

Both of these give the original wave when $c=1$ and the first sbrinks the wavelength, while the second stretches the scale length. We will obtain expressions and concepts relevant to $A(y)$ and $k(y)$ which are slow scale variables (note the absence of $\epsilon$ ), if we take $y=c x$. Eikonal waves of this form are described by $k$ as a function of $y$, an overall phase (since $k$ doesn't set the zero of phase), and the anplitude as a function of $y$. If we don't care about the pbase (and on the large scale we shouldn't, since it changes by order 1 on scales of order c) then we can represent the asymptotic features of our wave as a distribution on $y, k$ space, with support in the surface $k=k(y)$. 


\subsubsection{The Lacal Fourier Trandorm}

This representation will result from talring a local Fourier transform. This is a notion one would often like to use in physical situations, but that is usually either il] defined or has very arbitrary components to it. When we introduce the asymptotics as above, however, it becomes precisely defined. The idea is to take an ordinary Fourier transiorm, but to restrict the domain of integration to the neighborhood of the point $y$ we are interested in in such a way that the domain shrinks to zero on the large scale, but grows to infinity on the small-scale, asymptotically. We can implement this with a window function $W_{c}(y)$ that asymptotes to a delta function of $y$, but a constant function of $x$. A convenient choice is to use a family if Gaussians:

$$
W_{k}(y)=e^{-y^{2} / \epsilon}
$$

Given an arbitrary agymptotic family, we would like to explicitly obtain its local Fourier transform. Let us define the local Fourier trangform of an eikonal family $\psi_{c}(x)$ to te

$$
\hat{\psi}_{c}(y, k) \equiv \frac{1}{2 \pi} \int_{-\infty}^{\infty} e^{-\iota x^{2}-1 k x} \psi_{c}\left(x+\frac{y}{c}\right) d x
$$

This definition is related to ideas presented in [Guillemin and Sternberg, 1977] on page 394 and in [Weinstein, 1978]. If we ignore the asymptotic aspect of this defnition which makes the Gausgian convenient but arbitrary, this definition is related to the so-called lagolnitzer transform (see [lagolnitzer, 1975]). This expression 5 ss many of the nice properties we desire of a local Fourier transform asymptotically. As one can easily see from the manipulations below, the only properties of the window $\exp \left(-\operatorname{cI}^{2}\right)$ that are actually needed are that it is 1 when $x$ is zero, and that it 
grow' frith a scaje greater than 1 but less than 1/c. Here the width of the Gaussian is : order $1 / \sqrt{ }$ as far as $x$ is concerned.

A specific nite property of this definition is given in the following lemma:

Lemme 7.1. If a eikonal wave is of the form:

$$
\psi_{c}(y)=A(\epsilon y) e^{i \theta(c y) / c},
$$

then the modulus of its locaj Fourier transform is asymptotic to:

$$
|\hat{\psi}(y, k)| \sim A(y) \delta\left(k-\theta^{\prime}(y)\right)
$$

Procf. We will show that $\hat{\psi}(y, k)$ itself is asymptotic to $A e^{i \theta / c} \delta\left(k-\theta^{\prime}\right)$ from which the lemma follows. Choose any smooth test function $f(k)$. Then, letting $f(x)$ represent the ordinary Fourier translorm of $f(k)$,

$$
\begin{aligned}
& \int_{-\infty}^{\infty} \hat{\psi}_{k}(y, k) f(k) d k= \\
& =\frac{1}{2 \pi} \int_{-\infty}^{\infty} e^{-\left(x^{2}\right.} \hat{f}(x) A(e x+y) e^{i \theta(e x+y) / e} d x .
\end{aligned}
$$

Now change variables to $X=\boldsymbol{C}$ :

$$
=\frac{1}{2 \pi \varepsilon} \int_{-\infty}^{\infty} e^{-x^{2} / \mu} \hat{f}\left(\frac{X}{\epsilon}\right) A(X+y) e^{i \theta(X+y) / \epsilon} d X .
$$

Since the lagt three factors are bounded in magnitude at each $\boldsymbol{X}$ as $\in$ goes to zero, the first factor allows us to replace the integral by one over an arbitrarily small interval about sero, asymptotically. In fact, we get a contribution to the integral only when $X$ is of order $\sqrt{\epsilon}$. We may thus expand $A$ and $\theta$ in Tzylor series and keep only the highest order asymptotic contribution, when $X$ is of this order. We obtain

$$
=\frac{1}{2 \pi c} A(y) e^{i \theta(y) / c} \int_{-\infty}^{\infty} e^{-X^{2} / \epsilon} \bar{j}\left(\frac{X}{c}\right) e^{2 X \cdot \theta^{\prime}(t) / e} d X .
$$




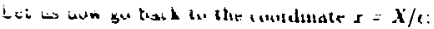

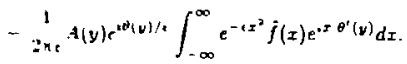

Sun let: +If in the tutegral to obtain the desired result:

$$
\int_{-\infty}^{\infty} \bar{V}_{\varepsilon}(y, k) f(k) d k \sim A(y) e^{i \theta(y) / c} f\left(\theta^{\prime}(y)\right)
$$

Sinc: $f$ was arbitrary, $\hat{\psi}_{k}(y, k)$ is weakly asymptotic to (i.e. agrees when integrated against test functions)

$$
A(y) e^{i \theta(r) / z} \delta\left(k-\theta^{\prime}(y)\right)
$$

Q.E.D.

\subsubsection{Stationary Phase, Laplace'日 Method, and Steepest Descents}

The method of stationary phase is the central tool in doing wave asymptotics. It and steepest descents will be the central theoretical tools of chapter 16. There are many different cases and situations where one might apply this method, but we will restrict ourselves to the simplest cases. The basic idea of the metbod is that the integral of a short wavelengtb wave against a slowly varying function will vanish asymptotically. In fact, such integrals vanish to all orders in the asymptotic parameter $\epsilon$, as is quite easy to show. Consider, for instance, the integral

$$
\int_{-\infty}^{\infty} f(y) \cos (y / \epsilon) d y
$$

or equivalently in terms of $x=y / \epsilon$,

$$
\int_{-\infty}^{\infty} f(c x) \cos (x) \operatorname{cdx}
$$


where $f(x)$ is assumed to be smooth and to die of at infinity. If we integrate by parts, we obtain

$$
\left.c f(\epsilon) \sin (x)\right|_{-\infty} ^{\infty}-\epsilon \int_{-\infty}^{\infty} f^{\prime}(\epsilon x) \sin (x) \epsilon d x
$$

The first term vanishes and the second is $c$ times an integral of the type we are considering. Repeatiog this procedure puts as many $\epsilon$ 's out in front as we desire, showing that the integral vanishes to all orders in $\epsilon$.

If we have a slowly varying frequency in the cosine, ouch as $\cos (g(x) / \epsilon)$, and if $d g$ never vanishes, we may change coordinates using $g$ to get an integral with $\cos (x)$ and a slowly varying Jacobian of the transformation. This is the situation above, and we may again conclude that the integral vanishes to all orders. Thus if an integral of this type is to have a non-zers asymptotic value, it must arise from the regions where $d g=0$. In fact, it is easy to see, by chopping our integral into one on ar. interval around $d g=0$ and one on its complement, that the width of the non-uniform region for the above argument is of order $\sqrt{\epsilon}$. This shows tbat if $g$ 's second derivative is non-zero, the value of the integral will be of order $\sqrt{\epsilon}$ and the only terms in the Taylor series of $g$ that can contribute to leading order are those of the second order. The integral then becomes one over a Gaussian, which may be evaluated by elementary methods. That this gives the highest order abymptotics may also be seen by using the Morse lemma to make a slow change of coordinate to a quadratic (see [Guillemin and Sternberg, 1977] p. 16).

The s.me idea may be used in integrals over an arbitrary number $n$ of dimensions, and assuning a single stationary point at $y=0$ leads to the formula (see 
[Guillemin and Sternberg, 1977] p. 6)

$$
\begin{aligned}
& \int f(y) e^{u v(v) / \epsilon} d y= \\
& \frac{f(0)(2 \pi \epsilon)^{n / 2}}{\sqrt{\left|\operatorname{det} g_{y v}(\overline{0})\right|}} e^{i g(0) / c+(t r / 4) \text { Sigo } o_{v y}(0)}+O\left(\epsilon^{j+n / 2}\right) .
\end{aligned}
$$

Hert we assume that the Hessian $g_{v v}$ is nou-degenerate and "Sign" denotes the signature which is the number of positive eigenvalues minus the number of negative ones. The term in the exponential with this factor gives rige to the "extra phase shifts in going through caustics" and is respousible for the notorious correction factors in the Bohr-Sommerfeld expression for energy eigenvalues in the old quantum theory (eg. the extra $h \omega / 2$ for the barmouic oscillator).

If we have a real exponent instead of an imaginary one, then we may use Laplace's method. Maxima of the exponent tend to completely dominate under exponentiation, asymptotically. In this situation, the Taylor expansion of the exponent near the maxima (and possibly the endpoints) completely determine the agymptotic expansion of the integral (though one typically has to consider terms of order $2 j$ in the Taylor expansions to get terms of order $j$ in the expansion of the integral). To bighest order, if $\phi$ has a maximum at the point $a<c<b$ and $\phi^{\prime \prime}(c)<0$ then asymptotically

$$
\int_{0}^{b} f(t) e^{\phi(t) / \epsilon} d t \sim \sqrt{\frac{2 \pi \epsilon}{-\phi^{\prime \prime}(c)}} f(c) e^{\phi(\epsilon) / \epsilon} .
$$

We will use this result when we look at statistical mechanics in a way that is analogous to the use of stationary phase in wave mechanics.

The method of steepest descents generalizes statiouary phase and Laplace's method to saddle points occuring anywhere in the cornplex plape. The idea is to 
deform the contour of integration in the ueighborhood of the sardle point so that the phase oi the integrand has constant imaginary part and a maximum in the real part. Wi can then use Laplace's method asymptotically.

Lastly, the stationary phase situation with no stationary points or maxima, may often be converted to a situation where we can use the Riemann-Lebesgue lemma. This says that

$$
\int_{a}^{b} f(t) e^{i t / e} d t \rightarrow 0 \text { as } \quad \epsilon \rightarrow 0
$$

if

$$
\int_{a}^{b}|f(t)| d t
$$

exists. We don't have to assume any differentiablity for integration by parts in this situation.

\subsubsection{Heisenberg's Uncertainty Principle}

Heisenberg's uncertainty principle puts limitations on how tightly one can localize a function and its Fourier transform at the same time. In quantum mechanics this puts fundamental limitations on how accurateiy one may neasure the position and the momentum of a particle at the same time. As re discuss in section 11.2, one wan think of quantum state as corresponding to a region in phase space of volume $h^{n}$ (where $h$ is Planck's constant). The usual proofs of the uncertainty pr aciple rely on inequalities that are hard to remember. Let us demonstrate it here using functional derivatives. 
We us: the Ditar notation where $\langle\phi \mid v\rangle$ represents the $L^{2}$ pairing of 0 ard $t$. Vie wow with a normalized fo oction $\psi$ defined on the real line:

$$
\langle\psi \mid \psi\rangle=\mathbf{1}
$$

By a transformation of the form

$$
\psi(x) \rightarrow e^{1 k_{0} x} \psi\left(x-x_{0}\right)
$$

we can put the mean values of $x$ and $k$ to zero without affecting the dispersions.

We therefore assume that

$$
\langle\psi|x| \psi\rangle=0=\langle\psi|k| \psi\rangle
$$

The dispersions on which we want to put bounds then take the form

$$
(\Delta x)^{2} \equiv\left\langle\psi\left|x^{2}\right| \psi\right\rangle \quad(\Delta k)^{2} \equiv\left\langle\psi\left|k^{2}\right| \psi\right\rangle
$$

One easily evaluates the functional derivatives

$$
\begin{aligned}
& \frac{\delta\langle\psi \mid \psi\rangle}{\delta \psi}(x)=\psi^{*}(x) \\
& \frac{\delta(\Delta x)^{2}}{\delta \psi}(x)=x^{2} \psi^{*}(x) \\
& \frac{\left.\delta_{i}^{\prime} \Delta k\right)^{2}}{\delta \psi}(k)=k^{2} \psi^{*}(k) .
\end{aligned}
$$

Fourier transforming this last expression gives

$$
\frac{\delta(\Delta k)^{2}}{\delta \psi}(x)=-\frac{d^{2}}{d x^{2}} \psi^{\cdot}(x)
$$

We want to show that the product of the disper:ions reaches some minimum value on normalized functions. We do this by showing that the Gaussian has the minimum 
uncertainty of any wave and then evaluating its uncertainty. We formulate the minimum uncertainty normalized wavepacket problem as a variational expression with Lagrange multiplier $\alpha$. With the function $F$ defined as

$$
F=(\Delta x)^{2}(\Delta k)^{2}-\alpha\langle\psi \mid \psi\rangle,
$$

a minimal uncertainty wavepacket will be a point where $F$ bas a vanishing functional derivative with respect to $\psi$. So

$$
0=\frac{\delta F}{\delta \psi^{\prime}}(x)=x^{2} \psi^{*}(x)(\Delta k)^{2}+(\Delta x)^{2}\left(-\frac{d^{2}}{d x^{2}} \psi^{*}(x)\right)-\alpha \psi^{*}(x) .
$$

Since $(\Delta k)^{2}$ and $\left(\Delta x^{2}\right)$ are just real numbers, this yields the differential equation

$$
\frac{d^{2}}{d x^{2}} \psi^{\bullet}=\left(\frac{\alpha}{(\Delta x)^{2}}-\frac{(\Delta k)^{2}}{(\Delta x)^{2}} x^{2}\right) \psi^{\bullet} .
$$

We may easily solve this, and imposing the normalization condition determines a; imposing zero means for $x$ and $k$ and choosing a phase factor so that $\psi$ is real determines the constants of integration and gives a relation between $\Delta x$ and $\Delta k$. We are left with the solution (which is easily checked by plugging into the equation):

$$
\psi(x)=\left[\left.2 \pi(\Delta x)^{2}\right|^{-1 / 4} e^{-x^{2} / 4(\Delta x)^{2}} .\right.
$$

To see that th is extremal is really a minimum, we calculate the second functional derivative:

$$
\frac{\delta^{2} F}{\delta \psi^{2}}(x, y)=x^{2} \psi^{\bullet}(x)\left(-\frac{d^{2}}{d y^{2}} \psi^{*}(y)\right)+y^{2} \psi^{*}(y)\left(-\frac{d^{2}}{d x^{2}} \psi^{\bullet}(x)\right) .
$$

When we plug in the Gaussian, both terms are positive, showing that the Gaussian is indeed the minimuin uncertainty packet. We need only find the uncertainties for this packet, which entails doing some Gaussian integrals. We see that

$$
\int_{-\infty}^{\infty} x^{2} \psi^{2}(x) d x=\left(2 \pi(\Delta x)^{2}\right)^{-1 / 2} \int_{-\infty}^{\infty} x^{2} e^{-x^{2} / 2(\Delta x)^{2}} d x=(\Delta x)^{2}
$$


and

$$
\begin{aligned}
(\Delta k)^{2} & =\int_{-\infty}^{\infty} k^{2} \dot{\psi}(k)^{2} d k \\
& =\int_{-\infty}^{\infty} k^{2}\left(\frac{2(\Delta x)^{2}}{\pi}\right)^{1 / 2} \epsilon^{-2(\Delta x)^{2} k^{2}} d k \\
& =\frac{1}{4(\Delta x)^{2}} .
\end{aligned}
$$

Thus we find the relation

$$
\Delta k \Delta x>\frac{1}{2}
$$

which is Heisenberg's uncertainty relation (with $\hbar$ set to 1 ).

\subsubsection{Anympto:ic Waves with a Deflnite $k$ and $y$}

Heisenberg's uncertainty principle tells us that the product of the absolute uncertatinty in $I$ space and $k$ space (i.e. the dispersion in $x$ and $k$ without regard for the size of $x$ or $k$ ) is bounded from below. The minimum uncertainty wave packet centered at a given $I_{0}$ and $k_{0}$ is given by a complex Gaussian:

$$
\left[2 \pi(\Delta x)^{2}\right]^{-1 / 4} \exp \left\{-\frac{\left(x-x_{0}\right)^{2}}{4(\Delta x)^{2}}+i k_{0} x\right\}
$$

The dispersion in $x$ is given by $\Delta x$ and in $k$ it is $1 / 2 \Delta x$. The relative dispersions are $\Delta x / x$ and $\Delta k / k$. If we let either $x$ or $k$ get asymptotically large, we may make both of these relative dispersions $q$, to zero. In the stretched cordinates: $(x, K)$ or $(y, k)$ where $\epsilon x=y$ and $\epsilon k=K$, the local Fourier transforw can be an asymptotic $\delta$-function in both directions. Any eisonal wave may be ay ymptoticaly decomposed into these $\delta$ states. 
For example, in quantum mechanics $h$ is often used as the asymptotic parameter. The momentum operator $\dot{p}$, defined as

$$
\bar{p} \equiv \frac{\hbar}{i} \frac{\partial}{\partial x}
$$

is exactly a stretched version of the wave vector $k$ as $\hbar$ asymptotes to zero. We may therefore create wavepackets that have a definite $x$ and $p$ as $\hbar$ goes to zero. Heisenberg's uncertainty principle for $x$ and $p$ takes the form:

$$
\Delta x \cdot \Delta p \geq \frac{1}{2} \hbar
$$

We see then that as $h \rightarrow 0$ we may make both $\Delta x$ and $\Delta p$ vanish.

These asymptotic states are intimately rehated to the theory of coherent states and have been connected with the Lie Poisson structures associated with the Heisenberg group. Let us quickly sketch some of the results in [Yaffe, 1982], but reformulate them in terms of momentum maps. The idea is to consider an asymptotic family of "quantum mechanics's" labeled by the parameter $h$. For each value of $\hbar$ we have a Hilbert space $B_{n}$ of $L^{2}$ wavefunctions on $x^{n}$ and each operator (like $\hat{p}$ above) is defined for each $\hat{h}$ on $\boldsymbol{H}_{\boldsymbol{n}}$. We choose a special state $|0\rangle_{h}$ in each $\boldsymbol{B}_{\hat{h}}$ which will asymptotically represent the state witb zero poeition and zero momentum. This is chosen to be one of our special states with vanishing position and momentum dispersions as $h \rightarrow 0$, such as

$$
\langle x \mid 0\rangle_{h} \equiv(\pi h)^{-1 / 4} e^{-\left(x^{2} / 2 n\right)} .
$$

Quantum mecbanics has a natural Hamiltonian structure. We may consider $1 / \hbar$ times the imaginary part of the Hermitian inner product $(\|)$ as a symplectic 
structure on the Hilbert space (being a linear space, pe may lift this structure to each tangent space). The Schrōdinger evolution defined by the Hermitian Hamiltonian operator $\dot{H}$ is Hamiltonian with respect to this symplectic structure and a Hamiltonian function given by the expectation value of $\dot{H}$ :

$$
\boldsymbol{H}(\psi) \equiv\langle\psi|\hat{\boldsymbol{H}}| \psi\rangle
$$

There is a natural $2 n+1$ dimensional Lie group that is intimately connected with the asymptotics of quantum mechanics, called the Heisenberg group. We may consider group elements to the in $\mathrm{M}^{n} \times \mathrm{M}^{n *} \times \mathrm{M}$ with coordinates $(q, p, \alpha)$ (sometimes the $\alpha$ factor is taken to lie in a circle). The multiplication law is

$$
(q, p, \alpha) \cdot\left(q^{\prime}, p^{\prime}, \alpha^{\prime}\right)=\left(q+q^{\prime}, p+p^{\prime}, \alpha+\alpha^{\prime}-\left\langle q, p^{\prime}\right\rangle\right) .
$$

Here we use $\langle$,$\rangle to mean the pairing of W^{n}$ with $\mathfrak{R}^{n *}$. This is just the tranglation group on $\Re^{2 n}$ with the extra $\alpha$ factor twisted into the multiplication. The Heisenberg group naturally arises through an irreducible representation on the Hilbert spaces $B_{n}$ defined by the mapping to unitary operators:

$$
(q, p, \alpha) \mapsto e^{i \alpha / h} e^{i(p, x) / h} e^{-i\langle q, p\rangle / h} .
$$

$(q, 0,0)$ translates pavefunctions by $q$ in position space, $(0, p, 0)$ translates the Fourier transform of wavefunctions by $p$ in momentum space, and $(0,0, \alpha)$ changes the phase.

The elements of the orbit of the special state $\mid 0>n$ under this group action are called coherent states and are iabeled by the $(q, p, \alpha)$ which acts to produce 
them. The irreducibility of the group action may be used to immediately prove many interesting relations such as the decomposition of the identity:

$$
\overline{\mathrm{I}}=c_{\mathbf{h}} \int|q, p, \alpha\rangle\langle q, p, \alpha|,
$$

where the integral is over an invariant measure on the Heisenberg group. This group action is Hamiltonian and we may ask for its momentum map. Each element $u$ in the Lie algebra $g$ of the Heisenberg group has an associated Hermitian operator $\hat{t}_{\mathrm{h}}$ defined on $H_{n}$ which generates the action of the one-parameter subgroup tangent to u. As for the Hamiltonian operator, the corresponding Hamiltonian function simply associates to each state $|\psi\rangle$ the expectation value $\left\langle\psi\left|\bar{u}_{n}\right| \psi\right\rangle$. The momentum map $J$ then sends $\psi$ to that element of the dual of the Lie algehra $g^{*}$ which satisfies

$$
\langle J(\psi), u\rangle=\left\langle\psi\left|\hat{u}_{\mathbf{n}}\right| \psi\right\rangle
$$

for each $u$ in $g$. We may easily see that this is equivariant since if $\beta$ is an element the Heisenberg group, then

$$
\begin{aligned}
\langle J(\hat{\beta} \psi), u\rangle & =\left\langle\hat{\beta} \psi\left|\dot{\mathbf{u}}_{\mathbf{h}}\right| \hat{\beta} \psi\right\rangle \\
& =\left\langle\psi\left|\hat{\beta}^{-1} \hat{u}_{\mathrm{h}} \hat{\beta}\right| \psi\right\rangle \\
& =\left\langle\psi\left|A \widetilde{d_{\theta}} \cdot u\right| \psi\right\rangle \\
& =\left\langle J(\psi), A d_{\beta} \cdot u\right\rangle \\
& =\left\langle A d_{\theta}^{*} \cdot J(\psi), u\right\rangle .
\end{aligned}
$$

The dual of the Lie algegra $g^{*}$ is $2 n+1$ dimensional. The coadjoint orbits of the Heisenberg group in $g^{*}$ consist of $2 n$ dimensional planes labeled by a parameter when that parameter is non-zero and an entire plane of individual points when the 
parameter wobes. On each of these spaces the KKS symplectic structure is a multiple of the canonical structure on $\Re^{n} \times \Re^{n *}$. Because the coherent states are an crbit of 'te Heisenberg group, their image under the monnentum map $J$ is exactly one coadjoint orbit. It turns out that coherent states that get sent to the same element in $g^{*}$ are not distinguishable by means of operators that have a nice classical limit as $h \rightarrow 0$ (the eliminated degree of freedom is the phase). Asymptotically, the $2 n$-dimensional coadjoint orbit is the natural areas for dynamics. Associated with each operator with a nice classical limit is a function on this coadjoint orbit whose value on an element is the expectation value of the operator in any of the coherent states corresponding to that element (they all give the same value). This reai-valued function on the coadjoint orbit is called the symbol of the operator. The symbol of the product of two such operators is simply the product of their symbols as $h \rightarrow 0$. The symbol of the limit of $i / h$ times the commutator of two sperators is the Lie-Poisson bracket of the symbols of the operators. It would be interesting to extend these definitions to the entire dual of the Lie algebra (say by giving a family of $\mid 0>$ 's, one for each coadjoint orbit).

\subsubsection{Eikonal Wavea and Lagrangian Submanifius}

"The correspondence is as illustration of what I might call the "symplectic

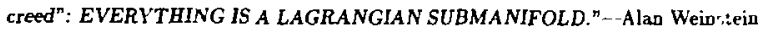
on p. 5 of [Weinstein, 1981]

We have seed that the result of our aysmptotic local Fourier transform on an eikonal wave family is a distribution on $(y, k)$ space whose support is on the set 
$k=d \theta$. We see that $k$ is naturally a one-form based at the point $y$, it being equal to the differential of the phase function there. Geometrically we are to think of $(y, k)$ space as the cotangent bundle of $y$ space. We have seen that this has a natural symplectic structure that in coordinates is minus the differential of the canonical one-form: $k_{0} d y^{a}$. That the aurface defined by our wave's singular support is the graph of the differential of a function is locally equivalent to the symplectic property of being Lagraugian (if the submanifold projects diffeomorphically to $y$ space).

We may see this connection geometrically as follows. If we think of an arbitrary one-form $\alpha$ on $y$ space as a mapping from $y$ space to its cotangent bundle, then the pullback of the canonical one-form hack to $y$ space yields the form $\alpha$. (In coordinates: $\alpha=\alpha_{i} d y^{i}$ and the canonical one form is $k_{i} d y^{i}$. The mapping defiued by $a$ takes the point with coordinates $y^{i}$ to the point with coordinates $\left(y^{i}, a_{i}\right)$. The canonical one-form on the image is $a_{i} d y^{i}$ which pulls back to $a$ as desired.) The canonical one-form restricted to the graph of the differential of a function has zero exterior derivative, eince its pullback to $y$ space does (since $d \circ d=0$ and exterior differentiation and pullback commute). Thus the symplectic form restricts to zero on the graph. This graph is of the same dimension as the base and so is Lagrangian. If a submanifold is Lagrangian and projects diffeomorphically to the base, then by Poincare's lemma the canonical sue-form in locally the differential of a function. But then so is the one-form whose graph the manifold is.

Thus eikonal waves are asymptotically associated with Lagrangian submanifolds that don't "bend over" in the cotangent space and so don't have a singular projection. When we allow an eikonal waye to evolve in time, the dynamics may 
bend the corresponding Lagrangian submanifold over. At such times, the originally eikonal wave bas ceased to be eikonal. The image of the points with a singular projection forms the caustic of the wave (so naned because such places have a high intensity and so tend to get hot in optical fields). The straightforward asymptotics of traditional WKB theory breaks down at these points and, a priori, one migbt not expect the corresponding Lagrangian gubmanifold to have anything more to do with the wave. Maslov introduced the concept and name of Lagrangian subrnanifolds io [Maslov, 1965] while generalizing earlier one-dimensional work of Keller in [Keliet, 1958). Maslov was able to show that there is a more general asymptotic class of asynptotic lamilies than eikonal waves which is associated with arbitrary Lagrangian submanifoids. The asymptotic dynamics of an eikonal wave does not leave thig larget class and in fact the bent over Lag:angian manifold continues to represent the wave. The hasic idea is to treat our wave as being on a higher dimensional space where the correponding Lagrangian submanifold is not singular, but atill projects onto the eingular one over the space we are really interested in. Projection of one space onto another corresponds to integrating the wave over the fibers of the projection. Since tice evolution equations are linear, one may introduce dyoamics on the large space which projects to the correct dydamice on the space of interest. On the large space, everything is eikosal and so we get representatives of the caustic wave fields as integrals of eisonal waves on a higher dimensional space ( [Guillemin and Steraberg, 1977] p. 428). 


\subsection{WKB Theory apd Agymptotic Equationg}

Let uy quickly sketch the theory of eikonal waves for linear P.D.E.'s. This may be gencralized to vector equations, asymptotic series in fractional powers of the amplitude, and bigher order terms (see for example [Guillemin and Sternherg, 1977 p 50), but I want to focus on the bare esentials here.

In the mathematical literature on this subjext (Alich aso [Hōrmander, 1983]), one usually is looking for asymptotic solutions to an asymptotic partial differeptial equation on a manifold. One makes geometric sense of a partial differential operator as a certain class of mappings between apaces of sections of bundles over the manifold. One introduces a small parameter $\epsilon$ and introduces the usual asymptotic equivalence classes of c-dependent operators to define asymptotic operators. An asymptotic P.D.E. is given by requiring an asycoptotic operator to vanish on an agymptotic function. One usually assumes that the higher derivative terms have coefficients with higher powers of $\epsilon, 80$ that to make the terns balance, a solution must oscillat $f$ more and more as $c$ vanishes. The resulting class of solutions are of the form of an asymptotic amplitude times an ever more quickly oecillating exponential. One finds a Harnilton-Jacobi equation for the phase. In addition, one obtains series of transport equations, defined along the characteristics of the Hamilion-Jacobi equation, for the terms in the amplitu $\lrcorner$ e's expansion. The Hamiltonian is the so-called principal symbol of the operator, which invariantly is a function on the cotangent bundle. Because the wavelength is getting ever smaller, the local asymptotic behavior of the paves is unaffected by the global atructure of the manifold.

We are interested in scaling our system the other way. We want to make the 
coefficients of our equation slowly varying as $t \rightarrow 0$. If we are on a manifold, then the manifold should "grow asj mftotically, to become more and more like $x^{n}$ locally. This type of scaling is often the physically relevant one. Most of the manifolds used in physics are really only manifolds in some asymplotic sense. Even if spacetime really is a manifold (which is very unlikely on scale lengtbs of order of the Plauck length), the state spaces for our systems come from large products of space with itself (representing the state of many particles) followed by projections and constraints. There is always some "kidth" in the constraint direcion, and the inanifold picture breaks down on this scale. Similarly, quantum mechanics imposes finest scales on which it is reasonable to look at the eitronal state of a wavepacket as being a point in a manifold. Ingtead of letting this physically determined small scale shrink, we often mean to say that the large acale structure of the state spacz is not strongiy affecting local behovior. We may represent this asymptotically as in section 7.1.1.

We also want to say only that the a:oitrary scale lengths in our problem get large, and not to charge the physical relations of the equa: 100 . For exanple, assume we are studying internal waves in the ccean and want to consider slow salt gradient variations. We introdur asymptotics which makes the gradient variation more and more gradual asymptoucally. If we were to shrink the wavelengh instead, we would be changing the physics of interad waves.

We are therefo-a interested in equations of the form

$$
P\left(x, \frac{1}{i} \frac{\partial}{\partial x}\right) \cdot \psi_{c}(x)=0 \text {, }
$$

whe:e $P(y, k)$ is a smooth function on the cotangent bupdle of $y$ space, and we 
ass, me its growth in $k$ is bounded by some power of $k$ (so we have a finite order equation). Notice that we are treating $y$ as an elemegt of a vector space for the reasons discussed in section 7.1.1.

As an example, we will consider the Klein Gordon equatico in 3-dimensions: This could have slowly varying coefficients, but for simplicity we demonstrate only the effect of slowly varying initial conditions. The base space is 4dimensional and is parametrized by $(x, y, z, t)$. The equation may be written

$$
\left(-\left(\frac{1}{i} \frac{\partial}{\partial t}\right)^{2}+\left(\frac{1}{i} \frac{\partial}{\partial x}\right)^{2}+\left(\frac{1}{i} \frac{\partial}{\partial y}\right)^{2}+\left(\frac{1}{i} \frac{\partial}{\partial z}\right)^{2}+1\right) \psi=n
$$

So the function $P$ on the cotangent bundle is given by

$$
P\left(x, c y, c z, d t, k_{x}, k_{y}, k_{z}, k_{z}\right)=-k_{i}^{2}+k_{x}^{2}+k_{y}^{2}+k_{x}^{2}+1
$$

We look for solutions of the form

$$
\psi_{\mathrm{e}}=A(\epsilon) e^{i \theta(\epsilon x) / \epsilon}
$$

In taking a derivative of this, we get $\mathrm{scne}$ terms that come from differentiating $A(\epsilon x)$, and these will have as many powes of $e$ as there were orders of differentiation. In contrast, derivatives of $\exp (i \theta(c x) / c)$ do not bring down any extra c's (though once a $\theta^{\prime}(c x)$ has come down, any higber derivatives of it fill get extra $c$ 's). If $P \cdot \psi$ is to vanish to all orders in $c$, it must vasush term by term. The lowest order term has all derivatives hitting the exponential and looks like

$$
P(y, \partial g(y)) \psi_{c}=0,
$$

where as before $y=\epsilon x$ and $d \theta$ is the exterior derivative of $\theta$ on $y$ space. 
If our solution doesn't vanish, then $\theta$ must sat:sfy the Harilton-Jacobi equation:

$$
P(y, d \theta(y))=0 \text {. }
$$

The analysis of such equations leads to a rich theoretical struct 1 re. To see wherc this comes from, in the next section we consider arbitrary frst order P.D.E.'s and then specialize to those of Hamilton-Jacobi type.

For the Klein-Gordon example, the Hamilton-Jacobi equation is

$$
-\left(\frac{\partial \theta}{\partial t}\right)^{2}+\left(\frac{\partial \theta}{\partial x}\right)^{2}+\left(\frac{\partial \theta}{\partial y}\right)^{2}+\left(\frac{\partial \theta}{i z}\right)^{2}+1=0 .
$$

\subsubsection{The Structure of Flrat Order P.D.E.'s}

Most of the symplectic structures in phyeics may trace their origin to a natural structure that arises with any (nonlinear) frst order P.D.F. By a frst order P.D.E. we mean that we are given an equation of the form

$$
F\left(x, u, u_{x}\right)=0
$$

where $x$ represents a point in the $n$-dimensional manifold $M$ on which the P.D.E. lives, $u$ is the function on this manifold we are trying to solve for, $u_{x}$ represents all its first derivatives, and $F$ is a smooth function of $2 n+1$ variables. Genmetrically the space un which $F$ lives is the first jet space of $M$ ( [Arnold, 1983] p. 66). This is a manifold whose points are equivalence clases of functions defined on neighborhoods of points in $M$. Two functions have the same 1 -jet at a point in $M$ iff they bave the same value and the same differential there. Let us use the coordinates $p$ to 
represent the differential directions of which there are $n$ and $t$ to represent the value direction.

Given a function, the graph of it and its first differential determine an $n$ dimensional surface in this first jet space. Just as we saw in section 7.1.4 that the property of being the graph of the differential of a function in the cotangent space was equivalent to being a lagrangian submanifold, being the graph of a function and its differential is locally equivalent to being a Legendre manifold in this jet space. Just as there is a natural symplectic structure on the cotangent bundle, there is a natural contact structure on the first jet space ( A Amold, 1978) p. 349). This may be defined as a smooth choice of a ny perplane in each tangent space of a $(2 n+1)$-dimensional manifold, that is locally annihilated hy a one-form $\theta$ (called a contact form), with the property that $\theta \wedge d \theta^{n}$ is a volume form (here $d \theta$ is a two-form and $d \theta^{n}$ means $d \theta \wedge \ldots \wedge d \theta$ with $n$ factors). If we think of the jet space as the cotangent bundle with an extra direction tacked on to represent the value of functions, then its natural contact form is a on-form that is the differential of the value coordinate minus the curnonical one-form on the cotangent bandle. In the coordinates $\left(x^{i}, p_{i}, u\right)$, it is given by

$$
\theta=d u-p_{i} d x^{i}
$$

A Legendre aubmanifold ([Aroold, 1978] p. 365) is an n dimensional aubmanifold on which the contact form vanishes (the hyperplanes defined by the contact form just contact the surface). It is easy to see that this is exactly the condition that the $p$ coordinates really represent the derivatives of a function whose jet graph is supposed to be the surface in question. 
The $2 n$-dimensional distribution (smooth field of subspaces cbost $\mathrm{n}$ from the tangent spaces at each point) defined by the vectors that $\theta$ annibilates is maximally non-integrable. It would be integtable if thr contact space conld be filled with smootb $2 \pi$-dimensional submanifolds (which together define a foliation) that were tangent to the specified planes ai each point. It requires very special circumstances that are spelled out in Frobenius' theorem (see [Spival, 1979] p. 257) for $\theta$ to define such 2 -dimensional surfaces (in fact. $\theta$ must be the differential of a function locally for these surfaces to exist). It turns out, however, that there always exists an adimensional foliation everywhere tangent to $\theta$ 's annihilator. For the contact planes, this is in fact the largest dimensional foliation you can find (this is the meaning of maximally non-integrable). The two form $d \theta$, acting on vectors in a characteristic plane, gives a measure of the nonintegrability there. If $d \theta$ doesn't vanish on two vectors, then it is not possible to deform the parallelogram they form to be tangent to the contact planes. That a contact structure is maximaliy nondegenerate says that $d \theta$ is nondegenerate on each contact plage (i.e. is a eymplectic bilinear form on each piane).

The P.D.E. in this picture simply states that the jet graph of the solution functinn $u$ must lie in the $2 n$-dimensional set given by $F=0$. That an $n$-dimensional surface is a jet graph of a function says that it is tangent to the contact planes at eacts of its points. If both of theoe conditions are satiafied and the surface projects down to $M$ diffeomoptically, then we have a local solution to the P.D.E.

Any such surface must include a certain direction in its tangent plane at each point. called the characteristic direction. This direction is defined as follows. The 
$2 n$-dimensional tangest plane to the surface $F=0$ at each point intersects the $2 n$ dimepsional contact plane generically in a $(2 n-1)$-dimensional subspace. When $d \theta$ is restricted to this subspace it bas a single degenerate direction (just as in Hamiltonian mechanics, where the degenerate direction of the symplectic structure restricted to an energy level surface gives the direction of the dynamics). This direction is the characteristic direction that mist be included in any solution (since $d \theta$ is nondegenerate on the $(n-1)$-dimensional quotient by the characteristic direction and must vanish on our n-dimensional surface, that surface must include the characterigtic direction).

It is now easy to bee bow to solve the Cauchy initial value problem for our P.D.E. The initial surface on which the value of $u$ is given, is an $(n-1)$-dimensional submanifold of $M$. The initial data plus the constraint that it lie in $F=0$, determines an (n-1)-dimensional aubmanifold in the jet space which our solution surface must include. As it must also include the characteristic direction, if these are aot tangent to the intial manifold, we get a local solution by just flowing the intial manifold aloog the integral curves of the characteristic direction field (see [Guillemin and Sternberg, 1977] p. 34).

\subsubsection{Hamilton-Jacobi Theory and Symplectic Manifolda}

In the special case of a first order P.D.F. which does not explicitly depend on the value of the function $u$, we obtain the Bamilton-Jacobi theory (ace [Abrabam and Marsden, 1978| p. 381). We saw above that it is exactly this kind of equation that arises from eikonal solutions of lineas P.D.E.'s. The whole point to the separation 
of scales is that the value of the fast phase itself is irrelevant and only its slow derivatives contribute to the average dynamics in the eikodal limit. It is for this reason that mechanics may be formulated in terms of symplectic manifolds instead of coztact manifolds.

In this case, the function $H(x, p)$ whose zero set gives our P.D.E. may be called the Hamiltonian. Since $H=0$ includes the entirety of the $t$ fibers, and the contact form $\theta$ is invariant under translation in the $u$ direction, we may forget about the $u$ direction completely in our theory. What is left is the cotangent bundle $T^{*} M$ with its caronical one-form and corresponding symplectic form. Thie characteristic directions project to a line feld on the set $H=0$ in $T^{*} M$ (since both $H$ and $\theta$ were symmetric -Jong $u$, so were the characteristic directions). The Hamiltoniar vector feld determined from $H$ and the symplectic structure in the usual way is along this direction. Under this projection, Legendre subrratifolds transverse to the $u$ fbers project down to Lagrangian submanifolds. As we have seen in section 7.1.4, this is the condition in $T^{*} M$ that an $n$-dimensional submanifold be the graph of the differential of a function. The Cauchy problem now becomes like Hamiltcuian mechanics. Given an (n-1)-dimensional intitial surface in $M$ pith the intial data of $u^{\prime} s$ value on it, we get au $(n-1)$-dimensional initial surface in $T^{*} M$ as the only submanifold in $H=0$ consistent with the differential of the intial data. The solution surface is then made up of the integral cur'ts of $H^{\prime}$ 's Hamiltonian vecte. field tha: pass through the initial menifold.

We saw in sectivn 8.2 that the Hamilton-Jacobi mquation for eikonal solutions 
of the Klein-Gordon equation is defined by the function

$$
H=-k_{t}^{2}+k_{x}^{2}+k_{\nu}^{2}+k_{x}^{2}+1
$$

on $\left(x, y, z, t, k_{x}, k_{y}, k_{x}, k_{t}\right)$ space. The characteristics are the orbits of the Hamiltonian vector field defined by $\boldsymbol{H}$. This vector field is given by

$$
\begin{array}{cc}
\dot{x}=\frac{\partial H}{\partial k_{x}}=k_{x} & \dot{y}=\frac{\partial H}{\partial k_{y}}=k_{y} \\
\dot{z}=\frac{\partial H}{\partial k_{x}}=k_{x} & \dot{i}=\frac{\partial H}{\partial k_{t}}=-k_{t} \\
\dot{k}_{x}=-\frac{\partial H}{\partial x}=0 & \dot{k}_{y}=-\frac{\partial H}{\partial y}=0 \\
\dot{k}_{x}=-\frac{\partial H}{\partial z}=0 & \dot{k}_{\mathrm{t}}=-\frac{\partial H}{\partial t}=0 .
\end{array}
$$

We only use these curves in the surface $B=0$.

The physical P.D.E.'s we are often interested in considering (such as the KleinGordon example) have a distinguished time direction. The phase space discussed above is really an "extended" phase space in that it includes the time direction. If the Hamiltonian (and so the original P.D.E.) is time independent, we may apply reduction along the time direction to get a symplectic manifald of dimension $2 n-2$. Now our intial wave is given over the entire base manifold. This is the picture we have been using in the earlier sections of this chapter. The time evolution of the Lagrangian manifold is given by letting it flow along the Yamiltonian trajectories of the reduced Haniltonian. As in ordinary mechanics, these trajectories are now important on the whole phase space, not just on a subset like $H=0$. As we have discussed earlier, the Hamiltonian dynamics can bend the Lagrangian submanifold over and make its projection singular. 
I: the Klein-Gordon example, ve eliminate $t$ and set the gencrator of $t$ transiation: $k_{t}$, to a constant $w$. The characteristics ase now defined on the full $\left(I, y, z, k_{x}, k_{v}, k_{2}\right)$ space and are the orbits of the Hamiltonian vertor feld if

$$
\omega=\sqrt{1+k_{x}^{2}+k_{y}^{2}+k_{z}^{2}} .
$$

\subsubsection{Cotangent Bundles, Contact Spaces, and Jet Spaces}

Contact spaces are associated witb odd dimensions and sywplectic spuces with even dimensions. We bave seen that the sywplectic cotangent space has a natural generalization to the first jet space, and that the canonical one-form generalizes to the contact form. We may also obtain a contact space of one dimension lower than the cotangent bundle by forgetting about the magnitude of a covector. A covector without its length is given by saying only which vectors it annihilates (and not what it does to other vectors). This is a hyperplane called a contact element in each tangent space of our original manifold (see [Aroold, 1978] p. 354). The space of tangent byperplanes of a manifold, is itself a contact manifold. The name "contact" makes the most sense bere, since these planes represent elements of first order contact with surfaces in the manifold. The contact structure on this space is given quite analogously to the definition of the canonical one-form. The $(2 n-1)$ dimensional space of contact elements naturally projects to the base manifoid by sending a contact element to the point it is based at. A tangent vector to tbr space of contact elements is in the contact plane at uat point if its projection lies in the contact element it is based at. We will use this in our study of thermodynamics in chapter 16. 
7.2.4. The Contact Bundle and the ('onormal Bundle

As important class of Lagrangian and Legendre submanifolds arises from submanifolds $N$ of the base manifold $M$. The contact bundle of $N$ is just the set of all contart elements which are tangent to $N$. This is clearly a Legendre submanifold since any vector tangent to it projects to a vector tangent to $N$ and so is in the contact plane at that point. The conormal bundle of $N$ is the set of all euvectors that annihilate the tangent space of $N$. This is a Lagrangian submanifold of the cotangent bundle $T^{*} M$ since a vector tangent to it must project to a vector tasgent to $N$ wich is annihilated by the form it was based at and so by the canonical one form. Thus we that the zero section of a cotangent bundle is Lagrangian in one limit ( $N$ equals $M$ ) and the fiters of the cotangent bundle are as well, in the other limit ( $N$ equals a point). If $N$ is a source of light, then the conormal bundle gives the rays that are emanating from $N$. Thus a point source radiates in all directions while a plane radiates only normal to itself. The Buygens construction gives wavefronts as the envelope of the manifold formed by projecting those points that are a given distance along the tays. 


\subsection{Limltathons of WKB Theory}

It is well known that ordinary WKE theory breaks down whes diffartion occurs (i.e. Where the medium scale length is as small as the wavelength) and near turning points (where the ravelength goes to zero). Keller bas developed a beautiful theory of geometric diffaction theory wibich uses geometric optics (i.e. WKB) away from the bad regions in the medium and glues in the extra rays due to diffraction emanating fron these regions using matched asymptotics. The gr' at simplification is that as the wavelength becomes smaller, any "edge" (or any other type of bad region) affecte a ray over a smaller and smaller portion of it. Asymptotically, the effect of a distontinuity is identical ts one of only a few "canonical problems" (eg. the adge of an infinite half plane, the tip of a cone, etc.). TI are solved once and for all and it is their solution that is glued into the problem. Turning point problems were dealt with classically ir a similar way io one dimeosion. Near the turning point the potential asymptotically becomes more and rnore like a linear potential as far as the asymptotic wave can tell. The exact solution for a linear pr :ential (i.e. an Airg' function) is glued in using matched asymptotics again. As we discussed in section 7.1.4, Maslov generalized WKB theory to situations with caustics in higher dimensions. Associated with each of the elemeutary catastrophes of Thom (which classify the generic caustics) is a special function which is a higher dimensional analog of the Airy function. The wave field around a caustic may be obtained by glueing iv these special functions at the caustics of the WKB solution. One important phenomenon that has not to my knowledge received a geometric treatment is tungeling. This is the propagation of real waves into regions that the 
WKB rays cannot get to. The problem is that eikonal waves decay exponentially in clasically forbidden regions (i.e. regions without rays) as the eikonal parameter vanishes. Straight WKB cannot detect this, but by asymptotically matching solutions on opposite sides of the barrier, one can estimate the transmission coefficient.

As in the case of perturbation theory for mechanical systems, there has been little discussion of the time of validity of the WKB asymptotic expansion (i.e. the time-seale on which it is uniform). We san in the mechanical case that using special zechniques such as Kruskal's method could give us expansions uniform over time $1 / \epsilon$ but that getting longer times was problematic. Based on the picture of WKB as reduction by an approximate symmetry in wave space, we expect exactiy the same phenomena for waves. Long times have become important in recent years as much study has been devoted to quantum chaos (i.e. the behavior of eikona) vaves when the corresponding tays are chaotic). Chaos is an inrinite time con:ept.

In fact it is easy to construct cxamples where all the requirements of WKB are satisfied everywhere and for all time and yet the WKB solution becomes invalid on times of order 1/c. Jeff Lerner has suggested to me the example of a translation invariant dispersive wave equation with initial condition $A(\epsilon x) e^{i k x}$ where $k$ does not depend on $x$ and $A(x)$ is a single hump. Because $k$ is constant, all rays are parallel and WKB predicts no spreading of the wave packet with time. However, we might have alternatively represented our wave packet in terms of its Fourier trangform. Because $A$ varies, the Fourier spectrum will be a smooth bump containing a band of wavevectors centered at $k$. This shows that the dispersive character of the equation will indeed be noticed by the packet which then in fact will spread with time. Let 
us extimate how long it takes for the pasket to spread to twice its ridtb. Because Fourier transforms live os the dual space. the peak in $\mathrm{r}$-space bis widib of order c. The greatest difference in group s 'scity at different poirt of the rave packet can then be at most of order $c$ Thus our packet takes time $1 / c$ to spronad 1 unit in $I$-space. But the packet bas width $1 / t$. To spread to twice its width. we must let the packet evolve for tirne $1 / c^{2}$. Given a real giave, there is alorays ambiguity in the choice of splitting into amplitude and wa"e-vector parts The differences between theie alternative scalings lead to a significant difference in the corresponding WKB predictiong on time scales of order $1 / \epsilon^{2}$. This may be seen by angurdent exartly analogous to the one used in this example. 


\section{Chapter 8:}

\section{A Hamiltonian}

\section{Approach to Wave Modulation}

"In mechanics the setting is the theory of slow modulations for vibrating systems... The classical thecty is usually develnoed by Familonian methods, which are not directly applicable to iraves, but we may instead derive the simplest of the classical results by the methods developed bere." - [Whitham, 1974] p. 506.

\subsection{Introduetion}

If we are given a wave system with a Hamiltonian structure, we would like to find a Hamiltonian structure for the evolution of slow modulations of the amplitude and wave number. We want an algorit: ic procedure completely independent of any previous knowledge or special features of the systım (for example, nothing should depend on linearity). We work out the cage of the inear Kleio-Gordon equation with this constraint in mind The same procedure stould rork for any sys tem: nonlinear, multi-field, integ,al equations, etc. We rish to extend the beautiful work of Whitham and later Lighthill which is based on Lagrangian averaging (see 
the discussion and references in the excellent text [Whitham, 1974]) to Hamiltonian systems. which we have seen are more general. [Dubrovin and Novikov. i983, gives a Hamiltonian treatment for a special class of systems (thougb their perspective is quite different from ours) and may be of interest to readers as well.

Here we will work trith tie one-dimensional Klein-Gordon equation:

$$
p_{t e}-p_{x x}+\varphi=0
$$

To represent this as a Hamiltonian system we introduce the conjugate field $\Pi$ with the equations of motion:

$$
\begin{aligned}
& \varphi_{t}=\Pi \\
& I_{t}=\varphi_{x x}-\varphi .
\end{aligned}
$$

This is Hamittonian with the Poisson bracket

$$
\{F, G\}=\int\left(\frac{\delta F}{\delta \varphi} \frac{\delta G}{\delta \bar{\Pi}}-\frac{\delta F}{\delta \Pi} \frac{\delta G}{\delta \varphi}\right) d x
$$

and the Bamitionian

$$
\boldsymbol{B}=\int h d x
$$

where $h$ is the Hamiltonian density

$$
h=\frac{1}{2}\left(\Pi^{2}+\varphi_{=}^{2}+\varphi^{2}\right) .
$$




\subsection{Periodic Solutions}

There is a three dimensional submanifold of periodic solutions in this $\varphi, \Pi$ space. We may label points in this submanifold by the three coordinates $(A, k, \theta)$ via

$$
\begin{aligned}
& \varphi_{A, k_{\theta} \theta}(x) \equiv A \sin (k x+\theta) \\
& \Pi_{A, k, \theta}(x) \equiv \sqrt{k^{2}+1} A \cos (k x+\theta) .
\end{aligned}
$$

For convenience we define

$$
w \equiv \sqrt{k^{2}+1}
$$

The dynamies restricted to this manifold is given by

$$
\begin{aligned}
\left(\begin{array}{c}
\dot{\varphi}_{A k \theta}(x) \\
\dot{\Pi}_{A k \theta}(x)
\end{array}\right) & =\left(\begin{array}{c}
\omega A \cos (k x+\theta) \\
-\omega^{2} A \sin (k x+\theta)
\end{array}\right) \\
& =\left(\begin{array}{c}
\omega \frac{d}{d \theta}[A \sin (k x+\theta)] \\
\omega \frac{d}{d \theta}[\omega A \cos (k x+\theta)]
\end{array}\right) \\
& =\omega \frac{d}{d \theta}\left(\begin{array}{c}
\varphi_{A k \theta}(x) \\
\Pi_{A k \theta}(x)
\end{array}\right) .
\end{aligned}
$$

Thus the dynamics on $A, k, \theta$ space is

$$
\left(\begin{array}{c}
\dot{A} \\
\dot{k} \\
\dot{\theta}
\end{array}\right)=\left(\begin{array}{l}
0 \\
0 \\
\omega
\end{array}\right) .
$$

\subsubsection{The Hamiltonian Restricted to Periadic Solutions}

Now restrict the Hamiltonian to the submanifold and integrate over a large volume $V$ :

$$
B_{V}\left(\varphi_{A k \theta}, \therefore I_{A k \theta}\right) \sim \frac{1}{4} A^{2} V\left(k^{2}+!\right)+\frac{1}{4} A^{2} V k^{2}+\frac{1}{4} A^{2} V=\frac{1}{2} A^{2} \omega^{2} V
$$

Notice that this is asymptotically true as $V \rightarrow \infty$ and neglects an order 1 contribution at the ends. If we were only interested in the periodic case it would perbaps 
be more elegant to introduce the average bere and not to introducr the $[$ at al] In fact. He are studying the periodic case only as a model for the eikonal case. We want to think of the eiku nal waves as limiting on the submanilold of exactly periodic -aves as the eikonal parameter vanishes. It is for this reason that we do not take the mean over the whole line and are therefore st "ck with the inclegant $V$ 's.

\subsubsection{The Symplectic Structure Restricted to Periodic Solutions}

Poissce brackets can be pushed forrard along projections but cannot in general be pulled back along injections like we have bere. We therefore work with the symplectic structure, wbich can be pulled back:

$$
\left.\Pi\left(\mid \delta \varphi_{1}, \delta \Pi_{1}\right]_{1}\left[\delta \varphi_{2}, \delta \Pi_{2}\right]\right)=\int d x\left(\delta \varphi_{1}(x) \delta \Pi_{2}(x)-\delta \Pi_{1}(x) \delta \varphi_{2}(x)\right)
$$

This two-form is the differential of a one-form $a$, which is easier to work rith:

$$
\begin{gathered}
\Omega=-d a, \\
\alpha([\delta \varphi, \varepsilon \pi]) \equiv \int \pi(x) \delta_{\varphi}(x) d x .
\end{gathered}
$$

To pull this back to $(A, k, \theta)$ space. we push forward z vector $(1, A,(k, \delta \theta)$ to $(\delta \varphi, \delta I)$. We only need the of component:

$$
\delta_{\varphi} \tau_{A k \theta}(x)=\delta A \sin (k x+\theta)+\delta k A x \cos (k x+\theta)+\delta \theta A \cos (k x+\theta) .
$$

Thus

$$
\dot{\alpha}(\delta A, \delta k, \delta \theta]) \equiv \alpha\left(\delta \varphi_{A H}, \delta \Pi_{A k \theta}\right)
$$




$$
\begin{aligned}
& =\int_{V} A w \cos (k x+\theta)[\delta A \sin (k x+\theta)+ \\
& \quad+x \delta k A \cos (k I+\theta)+\delta \theta A \cos (k x+\theta)] d x \\
& \quad-\frac{r}{2} A^{2} w \delta \theta .
\end{aligned}
$$

So the two-form $\omega$ on $k, k, \theta$ space is

$$
\overline{\mathrm{A}}=-d \theta=-V A \omega d A \wedge d \theta-\frac{V}{2} A^{2} \frac{d \omega}{d k} d k \wedge d \theta .
$$

$\hat{\Omega}$ is closed but degenerate (as it must be in three dimensions), and it annihilates the vector

$$
A * \frac{\partial}{\theta \cdot A}-2 \omega^{2} \frac{\partial}{\partial t} \text {. }
$$

\subsubsection{A Degenerate Paleson Structure on the Periodic Solutions}

If we ch sose a function whose level sets are transversal to this vector field, we may make this function a Casimir for a Poisson structure agreeing with $w$ on the level sets. If, furthermore, the function is a constant of the motion, then $B$ restricted to the level sets must give the correct dynamics. Here we take this function to be $k$, which we now hold constant. Then on $A, \theta$ space,

$$
\tilde{\Omega}=V A \omega d \theta \wedge d A
$$

and

$$
H_{V}=\frac{V}{2} A^{2} \omega^{2}
$$

But

$$
d H=A V \omega^{2} d A
$$


so

$$
X_{\omega}=* \frac{\partial}{\partial \theta} \text {. }
$$

which is the correct dynarnics.

\subsubsection{The Action of Periodic Ortits}

We wuuld like to work in canonical coordinates. From the expression for $\tilde{\Gamma}$, we see that $\theta$ is conjugate to

$$
J \equiv \frac{1}{2} V A^{2} w
$$

In terms of $J, \theta$ the structure in quite nice:

$$
\begin{aligned}
& \overline{\mathbf{\Omega}}=d \theta \wedge d J \\
& H=J \omega .
\end{aligned}
$$

This gives the dynamics

$$
\boldsymbol{X}_{H}=\omega \frac{\partial}{\partial \theta}
$$

or equivaltotly

$$
\dot{J}=0 \quad \dot{\theta}=\omega .
$$




\section{B.3. Modulatione}

Modulations essentially "gauge" this theory. We introduce the space of pairs of functions: $(A(I), \theta(x))$. For each value of $e$ we map this into $(\varphi, \Pi)$ space via

$$
\begin{aligned}
& \varphi_{c, A(x), \theta(x)}(x) \equiv A(\epsilon x) \sin \left(\frac{1}{\epsilon} \theta(\epsilon)\right) \\
& \Pi_{\ell, A(x), \theta(x)}(x) \equiv \omega(c) A(\epsilon x) \cos \left(\frac{1}{\epsilon} \theta(\epsilon)\right),
\end{aligned}
$$

where we bave defined for convenience

$$
k(x) \equiv \theta^{\prime}(x) \quad \omega(x) \equiv \sqrt{k^{2}(x)+1}
$$

This time the dynamics leaves the submanifold invariant only asymptotically as $c \rightarrow 0$ (but does so to all orders). We would like to consider this asymptotic dynamics on $(A, \theta)$ space, which represents the mudulational equations, as a Hamiltonian system. We do this by pulling back the Hamiltonian and the symplectic structure for each $c$ and then do asymptotics in $\epsilon \rightarrow 0$.

\subsubsection{Stationary Phase Integrals}

The method of stationary phase tells us that, as long as $\theta^{\prime}$ doesn't vanish, integrals of the form

$$
\int f(\epsilon x) \cos \left(\frac{1}{\epsilon} \theta(\epsilon x)\right) d x \sim 0
$$

vanish to all orders in $\epsilon$ as $\epsilon \rightarrow 0$. This tasily implies that

$$
\int f(c x) \cos \left(\frac{1}{\epsilon} \theta(\epsilon x)\right) \sin \left(\frac{1}{\epsilon} \theta(c x)\right) d x \rightarrow \hat{\jmath}
$$

and

$$
\int f(\epsilon x) \cos ^{2}\left(\frac{1}{\epsilon} \theta(\epsilon x)\right) d x \sim \frac{1}{2} \int f(\epsilon x) d x
$$


to all orders in e provided $f$ is smootb and dies rapidly at infinity.

\subsubsection{The Modulational Poisson Brackete}

The modulational ?oisson brackets are obtained as before by cou verting (A.0) perturbations to $(\varphi, \Pi)$ perturbations. If we call the map from $(A, \theta)$ to $(\varphi, \Pi)$, a $_{\text {, }}$, then

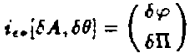

$$
\begin{aligned}
& =\left(\begin{array}{c}
\delta A(c x) \sin \left(\frac{1}{e} G(\epsilon x)\right)+\delta \theta(x) \frac{1}{\varepsilon} A(c x) \cos \left(\frac{1}{\varepsilon} \theta(c x)\right) \\
\text { not needed }
\end{array}\right) \text {. }
\end{aligned}
$$

All gin's and coe's have the argument: $(\theta(c x) / c)$. We will leave this argument out of our expressions for clanty. So

$$
\left.i_{\varepsilon} \alpha([\delta A, \delta \theta])=\alpha\left(i_{x} \mid \delta A, \delta \theta\right]\right)=\int(\omega A \cos )\left(\delta A \sin +\delta \theta \frac{1}{\epsilon} A \cos \right) d x
$$

By stationary phase, assuming $d \theta \neq 0$ anywhere, we have that to all orders in $\epsilon$ :

$$
i, \alpha([\delta A, \delta \theta])=\frac{1}{\epsilon} \int \frac{1}{2} \omega A^{2} \delta \theta d z \text {. }
$$

Motivated by the periodic case, we introduce

$$
J(x) \equiv \frac{1}{2} \omega(x) A(x)^{2} .
$$

This is the wave action density. Th山s the one form is $\left(1 / \epsilon^{2}\right) \int J \delta \theta d x$ (the $\epsilon$ is squared since $J$ and $\delta \theta$ are evaluated at $(x)$ and the corresponding Poisson bracket is canonical:

$$
\{F, G\}=\epsilon^{2} \int a x\left(\frac{\delta F}{\delta \theta} \frac{\delta G}{\delta J}-\frac{\delta F}{\delta J} \frac{\delta G}{\delta \theta}\right) .
$$


8.3.3. The Modulational Hamiltonian

346

8.3.3. The Modulational Hemiltodian

The modulational lianiltonian is sicularly ottained by pulling back $H$ :

$$
i^{\prime \prime} H=\int d x\left(\frac{1}{2} \omega^{2} A^{2} c \cos ^{2}+\frac{1}{2} A^{2} k^{2} \cos ^{2}+2 \epsilon A k A^{\prime} \operatorname{cossin}+\epsilon^{2} A^{\prime 2} \sin ^{2}+\frac{1}{2} A^{2} \sin ^{2}\right) .
$$

To all orders in $\epsilon$, this is

$$
=\int d x\left(\frac{1}{4} \omega^{2} A^{2}+\frac{1}{4} A^{2} k^{2}+\frac{1}{2} \epsilon^{2} A^{\prime 2}+\frac{1}{4} A^{2}\right)=\int d x\left(\frac{1}{2} \omega^{2} A^{2}+\frac{1}{2} \epsilon^{2} A^{\prime 2}\right) .
$$

Or in terms of $J$ :

$$
=\int d x\left(J \omega+\frac{1}{2} \epsilon^{2} A^{\prime 2}\right)
$$




\subsection{Global Symmetry Implieg Loca] Conaervation Law}

Let us now show that the preseuce of a global symmetry implies the existence of a local conservation law. Given a pair of canonically conjugate fields $(J(x), \theta(x))$. so

$$
\{F, G\}=\int\left(\frac{\delta F}{\delta \theta} \frac{\delta G}{\delta J}-\frac{\delta F}{\delta J} \frac{\delta G}{\delta \theta}\right) \mathrm{dx}
$$

and a Hamiltonian $\boldsymbol{H}(J, \theta)$ that is invariant under a global change of $\theta$ by a constant $\theta_{0}$ everywhere:

$$
\text { i.e. } H\left(J, \theta+\theta_{0}\right)=H(J, \theta) \text {, }
$$

we may apply a generalization of Noether's theorem kwown as reduction. This entails rewrit $L \mathrm{~g}$ everything in serms of

$$
k(x) \equiv \nabla \theta(x)
$$

which contains all information in $\theta$ except for a constant $\theta_{0}$. We

$$
\begin{aligned}
& \int \frac{\delta G(J, k)}{\delta \theta} f(x) d x=\frac{d}{d \beta} G(J, k+\beta \nabla f) \\
= & \int \frac{\delta G}{\delta k} \cdot \nabla f d x=-\int\left(\frac{d}{d x} \cdot \frac{\delta G}{\delta k}\right) f(x) d x .
\end{aligned}
$$

So

$$
\frac{\delta G}{\delta \theta}=-\frac{d}{d x} \cdot \frac{\delta G}{\delta k}
$$

for $G^{\prime} s$ independent of $\theta_{0}$. Thus the reduced Poiason bracket is

$$
\{F, G\}=\int d x\left(\frac{\delta G}{\delta J} \frac{d}{d z} \cdot \frac{\delta F}{\delta x}-\frac{\delta F}{\delta J} \frac{d}{d x} \cdot \frac{\delta G}{\delta k}\right) .
$$


The evolutinn of $J$ is thus

$$
j(x)=\{J, H\}=-\frac{d}{d x} \cdot\left(\frac{\delta H}{\delta k}\right)
$$

This is the degired conservation equation for $J$, with the flux of $J$ given by $\delta H / \delta k$. Similarly

$$
\dot{k}(x)=\{k, H\}=-\frac{d}{d x}\left(\frac{\delta H}{\delta J}\right)=-\frac{d}{d x} \omega(x) .
$$

In our case, to first order in $\epsilon$ we have

$$
\boldsymbol{H}=\int h d x \quad h(x)=J(x) \omega(x),
$$

which is independent of the value of $\theta$ (it depends only on the gradient). We may thus apply the previous theorem to ohtain

$$
\dot{J}(x)=\{J, H\}=-\frac{d}{d x}\left(J \frac{\partial \omega}{\partial k}\right)=-\frac{d}{d x}(U J)
$$

where we have introduced the group velocity:

$$
U \equiv \frac{\partial \omega}{\partial \boldsymbol{k}}
$$

Thus the wave action $J$ is conserved and the fiux is $U J$.

We have therefore successufully obtained the correct mo:dulational equations using a purely Bamiltonian framework. Traditionally, obtaining modulational equations is a very complex task fraught with traps for the unwary. Whitham and Lightlill brought order to this task during the 1960's using Lagrangian methods. A fine account of this work occupies most of the second half of Whitham's book on waves: [Whitham, 1974]. The quote at the beginning of this chapter indicates 
that Whitban did not thiuk that this cruld be accomplished within a Hamiltonian framework. Wo see that it can indeed. This is of both theretical interest (particularly because of the explicit connection with the process of reduction) and practical interest. As we discussed in chapter 2. many of the recent systems which bave received a Hamiltonian formulation have done so only in the context of Poissod manifolds and therefore have no Lagrangia. analog. Our theory should be applicable to waves in these systems as rell. 


\subsection{The Nonlinesr Kloin-Gordon Equation}

Thin section describes work done in collaboration with Richard Montgomery. Recently, Richard Monigomery bas proven that the method described in previous secrions of this chapter for the hnear Klein-Gordon equation really is quite general and applies to arbitrary systems (preprint, March 1985). Let us bere sketch the extension to the nonlinear Klein-Gordon equation:

$$
\phi_{t}=\phi_{x x}-V^{\prime}(\phi)
$$

The arguments are almost identical to the linear case. We first introduce a momentum II conjugate to $\phi$ :

$$
\begin{aligned}
& \phi_{t}=\Pi \\
& \Pi_{t}=\phi_{x x}-V^{\prime}(\phi) .
\end{aligned}
$$

Ths system is Hamiltonian with the canonical bracket given in section 8.1 and Aamiltonian

$$
B=\int\left(\frac{1}{2} \Pi^{2}+\frac{1}{2} \phi_{x}^{2}+V(\phi)\right) d x
$$

We search for periodic functions of the form

$$
\bar{\phi}_{1}^{\prime}(x, t)=F(k x+\omega t)
$$

where $F$ is a function of one variable. Let us denote $k x+\omega t$ by $\theta$. We want $F$ to be: $2 \pi$-periodic:

$$
\boldsymbol{F}(\theta+2 \pi)=\boldsymbol{F}(\theta)
$$

The corresponding momentum will bave the form

$$
\tilde{\Pi}(x, t)=\omega F^{\prime}(\theta)
$$


(whese we uxe a mune to denote the $\theta$ derivative). Substat utug tha ansatz into the rojustums of motion gives us a siruple O.D.E. for $F$

$$
\left(\omega^{2}-k^{2}\right) \frac{\partial^{2} F}{\partial \theta^{2}}(\theta)+v^{\prime}(F(\theta))=0
$$

Anytime one searches for travelling pave solutions (or similarity solutions) one obtains a simple O.D.E. even when the origiual system is a complex nonlinear P.D.E.. In this case the resulting O.D.E. is the equation of motion for a "particle" of "mass" $\left(\omega^{2}-k^{2}\right)$ moving in a potential well defined by $V$ if we treat $\theta$ as "time". The corresponding "energy" of this particle is

$$
\frac{1}{2}\left(\omega^{2}-k^{2}\right)\left(\frac{\partial F}{\partial \theta}\right)^{2}+V(F)=A
$$

which we set to the constant value $A$ (because it varies with wave amplitude).

It is interesting to note that the actual energy of the wave is

$$
\begin{aligned}
H & =\int\left(\frac{1}{2} \tilde{\Pi}^{2}+\frac{1}{2} \tilde{\phi}_{x}^{2}+v(\bar{\phi})\right) d x \\
& =\int\left(\frac{1}{2} \omega^{2}\left(\frac{\partial F}{\partial \theta}\right)^{2}+\frac{1}{2} k^{2}\left(\frac{\partial F}{\partial \theta}\right)^{2}+v(F(\theta))\right) d x
\end{aligned}
$$

which is the integral over $I$ of the "particle" energy. (TLL, is connected with the use of the Klein-Gordon equation in paticle physics).

The energy equation gives

$$
\left(\frac{\partial F}{\partial \theta}\right)^{2}=\frac{2(A-V(F))}{\omega^{2}-k^{2}}
$$

wbict may be integrated to give the solution

$$
\theta=\frac{1}{2}\left(\omega^{2}-k^{2}\right) \int \frac{d F}{\sqrt{A-V}(\bar{F})} .
$$


The congtaut of integration is the initial phase of the wave. When $V$ is a simple harmonic oscillator, corresponding to the linear system, this gives sin's and cos's as solutions. When $V$ is a pendulum potential (as in the sine-Gordon equation) this is an elliptic integral and when $V$ is quartic we get the solution to the Duffing oscillator. For the linear case we see that the constant $A$ is half the amplitude squared (we continue to use $A$ to farilitate the comparison with Whitham).

The nonlincar dispersion relation is obtained by requiring that $F$ be $2 \pi$ periodic in $\theta$ :

$$
\begin{aligned}
2 \pi & =\int_{0}^{2 \pi} d \theta \\
& =\int_{\text {pariod }} \frac{d \theta}{d F} d F \\
& =\frac{1}{2} \sqrt{\omega^{2}-k^{2}} \int_{\text {period }} \frac{d F}{\sqrt{A-V(F)}} .
\end{aligned}
$$

This gives one relation among $A, k$, and $\omega$. This is analogous to the fact that we could write $w$ as a function of $k$ in the linear case. Here, though, $w$ depends on $A$ as well, which leads to characteristically nonlisear effects fust as in a nonlinear oscillator where the frequency depends on the amplitude). The submanifold of periodic solutions is thus three-dimensional again and we inay coordinatize it by $A$, $k$ and $\theta$ as in section B.2.

As in the linear case, we find the action for a periodic solution by integrating the canonical one-form

$$
\int \Pi(x) \delta \phi(x) d x
$$

around a periodic orbit. $\delta \phi(x)$ acting on a unit tangent vector to the orbit will give

$$
\frac{1}{\omega} \frac{\partial}{\partial t} \tilde{\phi}(x, t)=\frac{\partial F}{\partial \theta}(k x+\omega t)
$$


since $c$ poves around its orbit in tine $1 / \mathrm{u}$. The total action is thereforc

$$
\int \omega^{\prime} \frac{\partial F}{\partial \theta}\left(k I+\omega^{\prime} t\right) \frac{\partial F}{\partial \theta}(k I+\omega t j d x .
$$

As in the linear case, we must really integrate only over a volume $\tilde{v}$, divide by $\tilde{v}$, and let $\dot{v}-\infty$, leaving w with a well defined action density. Since the integrand is periodic in $x$, we need only integrate ove $r$ one period. The action density $J()$ i then

$$
\begin{aligned}
J(x) & =\frac{1}{(2 \pi / k)} \int_{0}^{2 \pi / k} \omega\left(\frac{\partial F}{\partial \theta}(k x+\omega t)\right)^{2} d x \\
& =\frac{1}{(2 \pi / k)} \int_{0}^{2 \pi} \omega\left(\frac{\partial F}{\partial t^{2}}(\theta)\right)^{2} \frac{d x}{d \theta} d \theta \\
& =\frac{1}{2 \pi} \int_{0}^{2 \pi} \omega\left(\frac{\partial F}{\partial \theta}(\theta)\right)^{2} d \theta .
\end{aligned}
$$

Let us denote the average over a period of $\theta$ by $\leftrightharpoons$.

$$
\langle\cdot\rangle \frac{1}{2 \pi} \int_{0}^{2 \pi} \cdot d \theta .
$$

The action density may then be written

$$
J(x)=\omega\left\langle F^{\prime 2}\right\rangle
$$

We may use the equation satisfied by $F$ to write this as

$$
\begin{aligned}
J(x) & =\frac{\omega}{2 \pi} \int_{\text {periad }} \frac{\partial F}{\partial \theta} d F \\
& =\frac{\omega}{2 \pi} \int_{\text {periad }} \frac{\sqrt{2(A-V(F))}}{\sqrt{\omega^{2}-h^{7}}} d F \\
& =\frac{\omega}{2 \pi \sqrt{\omega^{2}-k^{2}}} \int_{\text {period }} \sqrt{2(A-V(F))} d F .
\end{aligned}
$$

When aic look at the special case where $V(F)=-\cos F$, this agrees with the expression given in [Forest anis Minaugbliw, 1982]. 
Let us now jump right into the modulational equations. We , ain consider the space of functions $(A(x), \theta(x))$ and for each $c$, map this into $(\phi, \Pi)$ space ria

$$
\begin{aligned}
& \phi_{t, A(x), \theta(x)}=F_{A(t x)}\left(\frac{1}{\epsilon} \theta(c x)\right) \\
& \Pi_{\{, A(x), \theta(x)}=\omega(e x) F_{A(t x)}^{\prime}\left(\frac{1}{\epsilon} \theta(c x)\right)
\end{aligned}
$$

where $F_{A}$ is the periodic $F$ with constant set to $A$ and $\omega$ is the known function of $k=\theta_{x}$ and $A$. We use $F_{A}^{\prime}$ to denote the $\theta$ derivative of $F_{A}$ and $\partial F_{A} / \partial A$ the $A$ derivative. Exactly as in section 8.3.2, the canonicel one-form $\alpha$ is pulled back to $(A, \theta)$ space by

$$
\begin{aligned}
i_{\varepsilon}^{*} \alpha\left(\left[\delta A_{3} \delta \theta\right]\right) & =\alpha\left(i_{\delta},\left[\delta A_{i} \delta \theta\right]\right) \\
& =\int \omega F_{A}^{\prime}\left(\delta A \frac{\partial F_{A}}{\partial A}+\delta \theta \frac{1}{e} F_{A}^{\prime}\right) d x .
\end{aligned}
$$

Now we use stationary phase to replace inurgs ou uver periodic quantities like $\boldsymbol{F}$ and its derivatives by integrala over the corresponding quaurtities averaged over $[0,2 \pi]$. We change to the slow scale $X \equiv c x$ :

$$
\begin{aligned}
i_{e} \alpha([\delta A, \delta \theta]) & =\frac{1}{\epsilon^{2}} \int \omega(X)\left(F_{A}^{\sqrt{2}}\right)(X) \delta \theta(X) d X+\frac{1}{\epsilon} \int \omega(X)\left(\frac{\partial F_{A}}{\partial A} F_{A}^{2}\right)(X) \dot{\nu} A(X) d X \\
& =\frac{1}{\epsilon^{2}} \int J(X) \delta \theta(X) d X+\frac{1}{\epsilon} \int \omega(X)\left(\frac{\partial F_{A}}{\partial A} F_{A}^{\prime}\right)(X) \delta A(X) d X .
\end{aligned}
$$

Similarly, we see that the Harniltonian is

$$
\begin{aligned}
H & =\int\left(\frac{1}{2} \pi^{2}+V(\phi)+\frac{1}{2} \phi_{x}^{2}\right) d x \\
& =\int\left(\frac{1}{2} \omega^{2} F_{A}^{\prime 2}+V(F)+\frac{1}{2} \theta_{x}^{2} F_{A}^{2} \frac{\partial F_{A}}{\partial A} A_{x}+\epsilon^{2}\left(\frac{\partial F_{A}}{\partial A} A_{x}\right)^{2}\right) d x .
\end{aligned}
$$

Yiow we use stationary phase again and change to $X=t x$ to see that to leading 
order

$$
\begin{aligned}
H & \sim \frac{1}{\epsilon} \int\left(\frac{1}{2}\left(\omega^{2}+k^{2}\right) F_{A}^{\prime 2}(X)+V\left(F_{A}(X)\right)\right) d X \\
& =\frac{1}{\epsilon} \int\left(\frac{1}{2}\left(\omega^{2}+k^{2}\right) \frac{2\left(A-V\left(F_{A}\right)\right)}{\omega^{2}-k^{2}}-\frac{\omega^{2}-k^{2}}{\omega^{2}-k^{2}}\left(A-V\left(F_{A}\right)-A\right) d X\right. \\
& =\frac{1}{\epsilon} \int\left(k^{2} \frac{2\left(A-V\left(F_{A}\right)\right)}{\omega^{2}-k^{2}}+A\right) d X \\
& =\frac{1}{c} \int\left(k^{2} F_{A}^{\prime 2}+A\right) d X \\
& \sim \frac{1}{\epsilon} \int\left(k^{2}\left(F_{A}^{2}\right)(X)+A(X)\right) d X \\
& =\frac{1}{\epsilon} \int\left(\frac{k^{2}}{\omega(X)} J(X)+A(X)\right) d X .
\end{aligned}
$$

Again we see that $\boldsymbol{H}$ is asymptotically independent of $\theta$. Since $J$ generates $\theta$ to leading order (as may be seen by looking at the asynptotic expression for the canonical one-form $\alpha), J(X)$ is asymptotically a locally conzerved quantity as for the linear system. 


\section{Chapter 9:}

\section{A Lie Poisson Bracket}

\section{for Wave Action}

\section{Density}

\subsection{Explicit Calculation of the Lle Polagon Bracket}

This section represents joint work of Allan Kaufman, Steve McDonald, and myself. $J$ and $\psi$ are real valued functions of $x \in \mathrm{x}^{3}$. We consider the canonical Poisson bracket on functionals of $J$ and $\psi$ :

$$
\{A(J, \psi), B(J, \psi)\}=\int\left[\frac{\delta A}{\delta J}\left(x^{\prime \prime}\right) \frac{\delta B}{\delta \psi}\left(x^{\prime \prime}\right)-\frac{\delta A}{\delta \psi}\left(x^{\prime \prime}\right) \frac{\delta B}{\delta J}\left(x^{\prime \prime}\right)\right] d^{3} x^{\prime \prime} .
$$

We shift attention to functic rals of the distribution $I$ on $(x, k)$ space. We obtain the Poisson bracket of such functionals $T_{1}$ and $T_{2}$ by requiring that it reduce to the above canonical bracket on distributions of the form

$$
I(\mathbf{x}, \mathbf{k})=J(\mathbf{x}) \delta^{3}(\mathbf{k}-\nabla \psi(\mathbf{x}))
$$

Thus can really only give the value of the wave Poisson bracket evaluated at distributions of this form. We may implicitly assume linearity in $I$, however, (and the 
Lie P'oisson bracket in linear) and thereby extend tbe definition to multiple cikonal waves and then by continuity to all waves. Calling $(x, k) \equiv y$ and $\left(x^{\prime}, k^{\prime}\right) \equiv y^{\prime}$, the chain rule gives

$$
\begin{aligned}
\left\{\mathrm{T}_{1}(J), \mathrm{T}_{2}(I)\right\} & =\int d^{6} y d^{6} y^{\prime} \frac{\delta \Upsilon_{1}}{\delta I}(y) \frac{\delta \Upsilon_{2}}{\delta I}\left(y^{\prime}\right)\left\{I(y), I\left(y^{\prime}\right)\right\} \\
= & \int d^{6} y d^{6} y^{\prime} d^{3} x^{\prime \prime} \frac{\delta \Upsilon_{1}}{\delta I}(y) \frac{\delta \Upsilon_{2}}{\delta I}\left(y^{\prime}\right) \\
& {\left[\frac{\delta I(y)}{\delta J}\left(x^{\prime \prime}\right) \frac{\delta I\left(y^{\prime}\right)}{\delta \psi}\left(x^{\prime \prime}\right)-\frac{\delta J(y)}{\delta \psi}\left(x^{\prime \prime}\right) \frac{\delta I\left(y^{\prime}\right)}{\delta I}\left(x^{\prime \prime}\right)\right] . }
\end{aligned}
$$

We calculate the needed functional derivatives:

$$
\begin{aligned}
\int \frac{\delta I(y)}{\delta J}\left(x^{\prime \prime}\right) \cdot f\left(x^{\prime \prime}\right) d^{3} x^{n} & =\lim _{t \rightarrow 0} \frac{I(y)[J+\epsilon J]-I(y) \mid J]}{c} \\
& =f(x) \delta^{3}(k-\nabla \psi(x))
\end{aligned}
$$

So

$$
\frac{\delta I(y)}{\delta J}\left(\mathbf{x}^{\prime \prime}\right)=\delta\left(x-\mathbf{x}^{\prime \prime}\right) \delta(\mathbf{k}-\nabla \psi(\mathbf{x}))
$$

Sinilarly,

$$
\begin{aligned}
& \int \frac{\delta I(y)}{\delta \psi}\left(x^{\prime \prime}\right) \cdot \phi\left(x^{\prime \prime}\right) d^{3} \mathbf{x}^{\prime \prime}=\lim _{\ell=0} \frac{J(y)[\psi+\epsilon \phi]-J(y)[\psi]}{c} \\
& \quad=\lim _{\ell \rightarrow 0} \frac{1}{\epsilon} J(\mathbf{x})\left(\delta^{3}(\mathbf{k}-\nabla \psi(\mathbf{x})-\nabla \phi(\mathbf{x}))-\delta^{3}(\mathbf{k}-\nabla \psi(\mathbf{x}))\right) \\
& \quad=-J(\mathbf{x}) \nabla \phi(\mathbf{x}) \cdot \frac{\partial}{\partial \mathbf{k}} \delta^{3}(\mathbf{k}-\nabla \psi(\mathbf{x})) .
\end{aligned}
$$

So

$$
\frac{\delta I(y)}{\delta \psi}\left(\mathbf{x}^{\prime \prime}\right)=\frac{\partial}{\partial \mathbf{x}^{\prime \prime}} \cdot \delta\left(\mathbf{x}-\mathbf{x}^{\prime \prime}\right) J\left(\mathbf{x}^{\prime \prime}\right) \frac{\partial}{\partial \mathbf{k}} \delta\left(\mathbf{k}-\nabla \psi\left(\mathbf{x}^{\prime \prime}\right)\right)
$$

Fint substitute in $\frac{\delta l}{\delta f}$ and do the $x^{\prime \prime}$ integral:

$$
\begin{aligned}
&\left\{\mathbf{T}_{1}, \mathbf{T}_{2}\right\}=\int d^{3} \mathbf{x} d^{3} \mathbf{k} d^{3} \mathbf{x}^{\prime} d^{3} \mathbf{k}^{\prime} d^{3} \mathbf{x}^{\prime \prime} \frac{\delta \Upsilon_{1}}{\delta I}(\mathbf{x}, \mathbf{k}) \frac{\delta \Upsilon_{2}}{\delta I}\left(\mathbf{x}^{\prime}, \mathbf{k}^{\prime}\right) \\
& {\left[\delta^{3}\left(\mathbf{x}-\mathbf{x}^{\prime \prime}\right) \delta^{3}(\mathbf{k}-\nabla \psi(\mathbf{x})) \frac{\delta I\left(\mathbf{x}^{\prime}, \mathbf{k}^{\prime}\right)}{\delta \psi}\left(\mathbf{x}^{\prime \prime}\right)\right.} \\
&\left.-\delta\left(\mathbf{x}^{\prime}-\mathbf{x}^{\prime \prime}\right) \delta\left(\mathbf{k}^{\prime}-\nabla \psi\left(\mathbf{x}^{\prime}\right)\right) \frac{\delta I(\mathbf{x}, \mathbf{k})}{\delta \psi^{\prime \prime}}\left(\mathbf{x}^{\prime \prime}\right)\right] .
\end{aligned}
$$


Thus

$$
\begin{aligned}
&\left\{\Upsilon_{1}, \Upsilon_{2}\right\}=\int d^{3} \mathbf{x} d^{3} \mathbf{k} d^{3} \mathbf{x}_{1}^{\prime} d^{3} \mathbf{k}^{\prime} \frac{\delta \mathbf{I}_{1}}{\delta I}(\mathbf{x} \cdot \mathbf{k}) \frac{\delta \Upsilon_{2}}{\delta I}\left(\mathbf{x}^{\prime}, \mathbf{k}^{\prime}\right) \\
& {\left[\delta^{3}(\mathbf{k}-\nabla \psi(\mathbf{x})) \frac{\delta I\left(\mathbf{x}^{\prime}, \mathbf{k}^{\prime}\right)}{\delta \psi}(x)\right.} \\
&-\delta^{3}\left(\mathbf{k}^{\prime}-\nabla^{\prime} \psi\left(\mathbf{x}^{\prime}\right)\right) \frac{\delta I(\mathbf{x}, \mathbf{k})}{\delta \psi}(\mathbf{x}) .
\end{aligned}
$$

Now substirute in $\frac{\delta I}{\delta \psi}$ :

$$
\begin{aligned}
& =\int d^{3} x d^{3} k d^{3} x^{f} d^{3} k^{\prime} \frac{\delta \Upsilon_{1}}{\delta I}(x, k) \frac{\delta \Upsilon_{2}}{\delta I}\left(x^{\prime}, k^{\prime}\right) \\
& {\left[\delta^{3}(\mathbf{k}-\nabla \psi(\mathbf{x})) \frac{\partial}{\partial \mathbf{x}} \cdot \delta^{3}\left(\mathbf{x}^{\prime}-\mathbf{x}\right) J(\mathbf{x}) \frac{\partial}{\partial \mathbf{k}^{\prime}} \delta^{3}\left(\mathbf{k}^{\prime}-\nabla \psi(\mathbf{x})\right)\right.} \\
& \left.-\delta^{3}\left(\mathbf{k}^{t}-\nabla \psi\left(\mathbf{x}^{t}\right)\right) \frac{\partial}{\mathbf{x}^{t}} \cdot \delta^{3}\left(\mathbf{x}-\mathbf{x}^{t}\right) J\left(\mathbf{x}^{t}\right) \frac{\partial}{\partial \mathbf{k}} \delta^{3}\left(\mathbf{k}-\nabla^{t} \psi\left(\mathbf{x}^{t}\right)\right)\right] .
\end{aligned}
$$

Now integrate by parts with respect to $\mathbf{k}^{\prime}$ in the first term and $\mathbf{k}$ in the second:

$$
\begin{aligned}
=\int d^{3} \mathbf{x} d^{3} \mathbf{k} d^{3} \mathbf{x}^{7} d^{3} \mathbf{k}^{\prime}\left[-\frac{\delta \mathbf{T}_{2}}{\delta I}(\mathbf{x}, \mathbf{k})\left(\frac{\partial}{\partial \mathbf{k}^{\prime}} \frac{\delta \mathbf{T}_{\mathbf{2}}}{\delta I}\left(\mathbf{x}^{\prime}, \mathbf{k}^{\prime}\right)\right)\right. \\
\delta^{3}(\mathbf{k}-\nabla \psi(\mathbf{x})) \cdot \frac{\partial}{\partial \mathbf{x}} \delta^{3}\left(\mathbf{x}^{\prime}-\mathbf{x}\right) J(\mathbf{x}) \delta^{3}\left(\mathbf{k}^{\prime}-\nabla \psi(\mathbf{x})\right) \\
+\left(\frac{\partial}{\partial \mathbf{k}} \frac{\delta T_{1}}{\delta I}(\mathbf{x}, \mathbf{k})\right) \frac{\delta \mathbf{T}_{2}}{\delta I}\left(\mathbf{x}^{\prime}, \mathbf{k}^{\prime}\right) \\
\left.\delta^{3}\left(\mathbf{k}^{\prime}-\nabla \psi\left(\mathbf{x}^{\prime}\right)\right) \cdot \frac{\partial}{\partial \mathbf{x}^{\prime}} \delta^{3}\left(\mathbf{x}-\mathbf{x}^{\prime}\right) J\left(\mathbf{x}^{\prime}\right) \delta^{3}\left(\mathbf{k}-\nabla^{\prime} \psi\left(\mathbf{x}^{\prime}\right)\right)\right] .
\end{aligned}
$$

Now change the variableg of integration: exchange $\mathbf{k}$ and $\mathbf{k}^{t}$ in the first term and $x$ and $x^{\prime}$ in the second:

$$
\begin{aligned}
&=\int d^{3} \mathbf{x} \delta^{3} \mathbf{k} d^{3} \mathbf{x}^{\prime} d^{3} \mathbf{k}^{\prime} {\left[-\frac{\delta \mathbf{Y}_{1}}{\delta I}\left(\mathbf{x}, \mathbf{k}^{\prime}\right)\left(\frac{\partial}{\partial \mathbf{k}} \frac{\delta \mathbf{Y}_{3}}{\delta I}\left(\mathbf{x}^{\prime}, \mathbf{k}\right)\right)\right.} \\
& \delta^{3}\left(\mathbf{k}^{\prime}-\nabla \psi(\mathbf{x})\right) \cdot \frac{\partial}{\partial \mathbf{x}} \delta^{3}\left(\mathbf{x}^{\prime}-\mathbf{x}\right) \mathbf{J}(\mathbf{x}) \delta^{3}(\mathbf{k}-\nabla \psi(\mathbf{x})) \\
&+\left(\frac{\partial}{\partial \mathbf{k}} \frac{\delta \mathbf{Y}_{1}}{\delta I}\left(\mathbf{x}^{\prime}, \mathbf{k}\right)\right) \frac{\delta \mathbf{T}_{2}}{\delta I}\left(\mathbf{x}, \mathbf{k}^{\prime}\right) \\
&\left.\delta^{3}\left(\mathbf{k}^{7}-\nabla \psi(\mathbf{x})\right) \cdot \frac{\partial}{\partial \mathbf{x}} \delta^{3}\left(\mathbf{x}^{\prime}-\mathbf{x}\right) J(\mathbf{x}) \delta^{3}(\mathbf{k}-\nabla \psi(\mathbf{x}))\right] .
\end{aligned}
$$


Collext terms:

$$
\begin{aligned}
&=\int d^{3} \mathbf{x} d^{3} \mathbf{k} d^{3} \mathbf{x}^{\prime} d^{3} \mathbf{k}^{\prime} {\left[-\frac{\delta \mathbf{T}_{1}}{\delta I}\left(\mathbf{x}, \mathbf{k}^{\prime}\right)\left(\frac{\partial}{\partial \mathbf{k}} \frac{\delta \mathrm{T}_{2}}{\delta I}\left(\mathbf{x}^{\prime}, \mathbf{k}\right)\right)\right.} \\
&\left.+\left(\frac{\partial}{\partial \mathbf{k}} \frac{\delta \mathrm{I}_{1}}{\delta r}\left(\mathbf{x}^{\prime}, \mathbf{k}\right)\right) \frac{\delta \mathbf{T}_{2}}{\delta I}\left(\mathbf{x}, \mathbf{k}^{\prime}\right)\right] \\
& \cdot \delta^{3}\left(\mathbf{k}^{\prime}-\nabla \psi(\mathbf{x})\right) \frac{\partial}{\partial \mathbf{x}} \delta^{3}\left(\mathbf{x}^{\prime}-\mathbf{x}\right) J(\mathbf{x}) \delta^{3}(\mathbf{k}-\nabla \psi(\mathbf{x})) .
\end{aligned}
$$

Now recall $J(\mathbf{x}) \delta^{3}(\mathbf{k}-\nabla \psi(\mathbf{x}))=I(\mathbf{x}, \mathbf{k})$ and integrate by parts in $\mathbf{x}$

$$
\begin{aligned}
=\int d^{3} \mathbf{x} d^{3} \mathbf{k} d^{3} \mathbf{x}^{\prime} d^{3} \mathbf{k}^{\prime}\left\{\left[\left(\frac{\partial}{\partial \mathbf{x}} \frac{\delta \mathbf{\Upsilon}_{1}}{\delta I}\left(\mathbf{x}, \mathbf{k}^{\prime}\right)\right)\left(\frac{\partial}{\partial \mathbf{k}} \frac{\delta \mathbf{\Upsilon}_{2}}{\delta I}\left(\mathbf{x}^{\prime}, \mathbf{k}\right)\right)-\right.\right. \\
\left.-\left(\frac{\partial}{\partial \mathbf{k}} \frac{\delta \mathbf{\Upsilon}_{1}}{\delta I}\left(\mathbf{x}^{\prime}, \mathbf{k}\right)\right)\left(\frac{\partial}{\partial \mathbf{x}} \frac{\delta \mathbf{\Upsilon}_{2}}{\delta I}\left(\mathbf{x}, \mathbf{k}^{\prime}\right)\right)\right] \\
\\
\quad\left[-\frac{\delta \Upsilon_{1}}{\delta I}\left(\mathbf{x}, \mathbf{k}^{\prime}\right)\left(\frac{\partial}{\partial \mathbf{k}} \frac{\delta \mathbf{r}_{2}}{\delta I}\left(\mathbf{x}^{\prime}, \mathbf{k}\right)\right)+\left(\frac{\partial}{\partial \mathbf{k}} \frac{\delta \Upsilon_{1}}{\delta I}\left(\mathbf{x}^{\prime}, \mathbf{k}\right)\right) \frac{\delta \Upsilon_{2}}{\delta I}\left(\mathbf{x}, \mathbf{k}^{\prime}\right)\right] \\
\left.\quad \times \nabla \nabla \psi \cdot \frac{\partial}{\partial \mathbf{k}^{\prime}} \delta^{3}\left(\mathbf{k}^{\prime}-\frac{\partial}{\partial \mathbf{x}} \psi(\mathbf{x})\right) \delta^{3}\left(\mathbf{x}^{\prime}-\mathbf{x}\right) I(\mathbf{x}, \mathbf{k})\right\} .
\end{aligned}
$$

In the first term, the $\delta^{3}(\mathbf{k}-\nabla \psi(\mathbf{x}))$ in $I(\mathbf{x}, \mathbf{k})$ Iets us replace $\delta^{3}\left(\mathbf{k}^{\prime}-\nabla \psi(\mathbf{x})\right)$ by $\delta^{3}\left(\mathbf{k}^{\prime}-\mathbf{k}\right)$. In the second term we integrate by parts in ' $\mathbf{k}^{\prime}$ and then do the same replacement.

$$
\begin{aligned}
& \left\{\Upsilon_{1}, \Upsilon_{2}\right\}=\int d^{3} \mathbf{x} d^{3} \mathbf{k} d^{3} \mathbf{x}^{\prime} d^{3} \mathbf{k}^{\prime}\left\{\left[\left(\frac{\partial}{\partial \mathbf{x}} \frac{\delta \Upsilon_{1}}{\delta I}\left(\mathbf{x}, \mathbf{k}^{\prime}\right)\right)\left(\frac{\partial}{\partial \mathbf{k}} \frac{\delta \Upsilon_{2}}{\delta I}\left(\mathbf{x}^{\prime}, \mathbf{k}\right)\right)\right.\right. \\
& \left.-\left(\frac{\partial}{\partial \mathbf{k}} \frac{\delta \Upsilon_{1}}{\delta I}\left(\mathbf{x}^{\prime}, \mathbf{k}\right)\right)\left(\frac{\partial}{\partial \mathbf{x}} \frac{\delta \Upsilon_{2}}{\delta I}\left(\mathbf{x}, \mathbf{k}^{\prime}\right)\right)\right] \delta^{3}\left(\mathbf{k}^{\prime}-\mathbf{k}\right) \delta^{3}\left(\mathbf{x}-\mathbf{x}^{\prime}\right) I(\mathbf{x}, \mathbf{k}) \\
& +\left[\left(\frac{\partial}{\partial \mathbf{k}^{\prime}} \frac{\delta \Upsilon_{1}}{\delta I}\left(\mathbf{x}, \mathbf{k}^{\prime}\right)\right)\left(\frac{\partial}{\partial \mathbf{l}} \frac{\delta \Upsilon_{2}}{\delta I}\left(\mathbf{x}^{\prime}, \mathbf{k}\right)\right)\right. \\
& \left.\left.-\left(\frac{\partial}{\partial \mathbf{k}} \frac{\delta \Upsilon_{1}}{\delta I}\left(\mathbf{x}^{\prime}, \mathbf{k}\right)\right)\left(\frac{\partial}{\partial \mathbf{k}^{\prime}} \frac{\delta \Upsilon_{2}}{\delta I}\left(\mathbf{x}, \mathbf{k}^{\prime}\right)\right)\right]: \nabla \nabla \psi \delta^{3}\left(\mathbf{k}^{\prime}-\mathbf{k}\right) \delta^{3}\left(\mathbf{x}^{\prime}-\mathbf{x}\right) I(\mathbf{x}, \mathbf{k})\right\} .
\end{aligned}
$$

We do the $x^{\prime}$ and $k^{\prime}$ integrals using the delt 3 functions. The second term vanishes 
and the first becomes the Lic-Poisun bracket:

$$
\begin{aligned}
& \left\{\Upsilon_{1}(I), \mathbf{T}_{2}(I)\right\}=\int d^{3} \mathbf{x} d^{3} \mathbf{k} I(\mathbf{x}, \mathbf{k})\left[\left(\frac{\partial}{\partial \mathbf{x}} \frac{\delta \Upsilon_{1}}{\delta I}(\mathbf{x}, \mathbf{k})\right) \cdot\left(\frac{\partial}{\partial \mathbf{k}} \frac{\delta \Upsilon_{2}}{\delta I}(\mathbf{x}, \mathbf{k})\right)\right.
\end{aligned}
$$

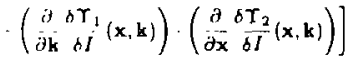

$$
\begin{aligned}
& =\int d^{\sigma} y I(y)\left[\begin{array}{ll}
\delta I_{1}, & \delta I_{2} \\
\delta J & \delta J
\end{array}\right]_{v} \text {. }
\end{aligned}
$$




\subsection{The Geometrical Picture}

Alan Weinstein has given the following genuetric interpretation of this sesult On the one hand, as in section 2.7 .10 we may consider the group of symplertor morphisms of a symplectic manifold $M$. An infinitesimal symplectomorphism in represented by a Hamilionian vector field (at least locally). We may think of the Lic algebra of the group of aymplectomorphisms as being all Hamiltonian vector fields on $M$ with the Lie bracket of vector felds being the Lie algebra bracket (per. haps with a minus sigo, depending on coLverions) $\bar{\tau} \cdot$ ivalently (up to a constant), we may view it as the space of functions (Har., (t)L.ars) on $M$ with the Lie algebra bracket being the Poisson bracket of tro functions (defined using $M$ 's symplectic structure). The coadjoint action of a symplectomorphism on such a function is simply given by pullback. The dual of the Lie algebra is then the space of distributions on $M$. We therefore have the natural Lie-Poison tracket (given above) on functionals of such distributions.

The coadjoint action of a symplectomorphism on a distribution is given by pushing the distribution forward along the symplectomorphism. The coadjoint orbits, whic, have a natural KKS symplectic structure, consist of all distributious obtainable from a given one by canonical transformatuos In particular. the ortut of is $\delta$-function looks just like $M$ and bas the same symplectic structure (points of $M$ correspond to the $\delta$-function at that point). Lagrangiau submanifolds (i $r$ balf-dimensional subranifolds on which the symplectic form vanishes) of $M$ are taken to other Lagrangian submanifolds under canonical transformations (since the symplectic form is preserved) Locally, any small enough picce of a Lagrangian sub- 
manifold ran be taken to a corresponding piece of any other Lagrangian submanifoi.s by a ranonical trangformation. So up to global issucs, the space of measured Lagrangian sutımanifolds on $M$ is itself a symplectir manifold (see section 27.10 for nore details.

A bove, we saw that the phase fuction which determines the manifold and the amplitude which determines the distribution on that manifold, may be treated as ramonirally conjugate variables in a way that is consistent with the Lie-Poisson gtrurture on all distributions. We would like to understand this fact geometrically. We will show that the cotangent bundle of the space of Lagrangian submanifolds of 11 may be naturally identified with the space of distributions with Lagrangian support. The cotangent space at $\mathbf{s}$ given Lagrangian submanifold is identifiable with the the space of distributions defined on that Lagrangian suhmanifold. This is then identifiable with the corresponding space of $\delta$-like distributions or $M$ supported on that manifold (see section 2.7.10). Thus the canouical conjugacy of the distribution and the manifold ariges in a natural way.

What is the tangent space to a Lagrangian submanifold of $M$ in the space of Lagrangian submanifolds? A tangent vector will be a small deformation of the manifold o.bicb is itself Lagrangian. All such deformations come from Hamiltonian vector fields. The tangent space may thus be identified with the space of Hamiltonan vector felds on $M$ modulo those which leave the Lagrangian aubmanifold invariant. A Hamiltonian vector feld leaves a Lagraggian manifold invariant if and only if it is constant on it.

We see thin as follows. If a Hamiltonian vector field $X_{H}$ is tangent to our 
Lagrangian manifold, then $d H=w\left(X_{H}, \cdot\right)$ must aunihilate all tangent vertor: to the manifold, since it is Lagrangian and so its cangent spaces are symplectucally orthogonal to themselves. Thes says that $H$ is constant on it (if it is connerted). If $H$ is constant on the Layrangian submanifold, then it annibilates all tangent vectors. Thus $X_{H}$ is symplectically orthogonal to the whole tangent space. But un a Lagrangian submanifold, the only such vectors are themselves tangent to it. Thus $X_{H}$ preserves the Lagrangian manifold.

We may therefore identify the tangent space to the space of Lagrangian submanifolds at a given such manifold with the space of functions on $M$ modulo those functions which vanish on the manifold (again ignoring constants). But this is exactly the space of functions on the Lagrangian submanifold. Taking the dual we see that the cotangent space to the space of Lagrangiausubmanifolds at a given Lagrangian manifold is indeed isomernhic to the space of distributions on that manifold.

This apace of distributions is identifiable with the delta-like distributions on $M$ supported on the manifold. The cotangent bundle of the space of Lagrangian submanifolds obtainable by deforming a given one is thus isomorphic to the coadjoini orbit of the symplectomorphigm group which contains any delta-like distribution supported by the given Lagrangian Bubmanifold. The KKS coadjoint orbit symplectic structure is exactly the canonical structure obtained from looking at the orbit as is cotangent bundle. 


\section{PART III:}

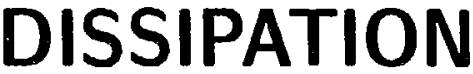

and

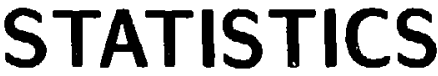

"These two branches, mechanjcs and thermodynamics, can be joined only from a higher standpoint, that of the statistical mechanjes of molecular systems." [Klein, 1928] p. 203 


\section{Chapter 10: \\ Imbedding and \\ Projection Theorems}

In this chapter we will demonstrate a number of relations between dissipative and non-dissipative systems. We will show, in particular, that any system can be embedded in a Hamiltunian system of twice the dimension and in a Poisson system of only one dimension more. These constructions are of interest because they help to delineate what operations lead one to artificial structures. Since they can arise from any system, the Hamiltonian structures of this section cannot be expected to give any new ingights into the original system. They may be useful in understanding bow dissipative dynamics muy arise from an underlying Hamilonian system, however, since this always involves some find of projectioc. We will also give examptes of some seemingly harmless "dangerous operations" which can completely destroy the physical content of a model.

\subsection{Imbedding in a Hamiltonian System}

Consider an arbitrary dynamical system given by a vector field $X$ on a manifold $Q$. We will construct a Hamiltonian system of twice the dimension which bas an invariant submanifold diffeomorphic to $Q$ and on witich the restricted dynamirs 
in exactly that given by $X$. The madifold will be the cotangent bugdle of $Q$, i.e. $T^{*} Q$. The dyoanics will be the cotangent lift of $\dot{A}^{*}$ to $T^{*} Q$. This is defined as follows the tlow of $X$ is a one parameter family of diffeomorphisms of $Q$ to itgell. A one-form may be pushed fortiard along a diffeomorphism (just pull it back along the inveme of the diffeomorphism). Thus at every moment of time each point in $T^{*} Q$ is mapped to another such point. Taking these together gives us a natural one-parameter family of diffomorphisms from $T^{*} Q$ to itself which covers the flow of $X$ (this is a point trangfornation). This family is actually the flow of a vector field on $T^{*} Q$ which is Hamiltonian with respect to the natural symplectic atructure on $T^{*} Q$. The Hamiltonian is the function on $T^{*} Q$ given by

$$
H(q, p)=\langle p, X(q)\rangle
$$

Notice that $H$ is lizear on each fber of $T^{*} Q$ and that the zero section is in the zero set of $H$. It is eagy to check that this gives the correct dynarnics. With the symplectic structure

$$
\omega=\dot{d q} \wedge d p,
$$

the dyoamics is

$$
\begin{aligned}
& \dot{q}^{\prime}=X^{\prime}(q) \\
& \dot{p}^{\prime}=-p^{\prime} \frac{\partial X^{\prime}(q)}{\partial q^{i}} .
\end{aligned}
$$

The zero section is indeed invariant (siber. if $p$ starts out zero, it semains so) and the dynamics restricted to it in the original dynamics. Thus by restricting the class of initial conditions, we may have dyaamies inside a Hamiltonian system looking like a dirsipative system of any type. 
Notice further that under the natural projection

$$
\pi: T^{*} Q \rightarrow Q \text { by }(q, p)-q
$$

the Hamiltonian dyamics projects down to the given dynamics. Thus if we observe only certain variables in a Hamiltonian system, it may look just like a dissipative system of any type.

Linear Hamiltonian vector fields must have their eigenvalues ditributed in the complex plane so as to be symmetric under reflection about both the real and inaginary axes. Thus if we start with a linear atracting fixed point, like

$$
X^{i}(q)=-q^{i}
$$

which has all of its eigenvalues in the left half-plane, we must double the dimension to imbed it in a Hamiltonian system. Thus the above construction is the smallest Hamiltonian space we might congtruct in general. 


\subsection{Projection from a Hamiltonian System}

Tbis argument for a lower bound on dimension does not apply to the case of projection, bowever, A fixed point of projected dynamics need not be a fixed point of the unprojected dy namics, so the eigenvalue argument doesn't hold. In fact, we may construct a Hamiltonian system that projects to our system by adding only a single dimension if the original space is odd dimensional, or two more dimensions if it is even dimensional. The construction sheds light on a dangerous operation one sometunes sees being performed in the physics literature. If one has unbounded motion, one may smootbly untwist the orbitg and hide any features of the dynamics that one desires.

Let us extend our given space $Q$ by a single dimension to get $Q \times \mathbb{R}$. Let us denote the time $t$ flow of the given dynamics $X$ on $Q$ by $\mathcal{F}_{t}$. It is quite natural to consider the extra dimension in $Q \times 9 R$ as time and to cousider the original dynamics on $Q$ augmented by a time which flows uniformly:

$$
\bar{X}(q, t) \equiv(X(q), 1)
$$

Because this has no fixed points and no recurrent orbits, we may now perform the following seemingly harmless operation. We define a diffeomorphism from $Q \times \Re$ to itself by

$$
(q, t) \mapsto\left(\mathcal{F}_{-\mathrm{e}}(q), t\right)
$$

By the definition of flow,

$$
\frac{\partial}{\partial t} F_{t}(q)=X(q)
$$


so the image of our dyaamical vector field $\tilde{X}$ under this diffeomorphism is simply:

Thus we have completely trivialized the dynamies by a coordinate change that unraveled the original orbits. In particular all dynamics can be made to look the same on extended state spaces. For our Hamiltonian construction, we may choose a symplectic structure and Hamiltonian that give the trivial dynamics of (10.9) and pull them back to the original cxtended state space by our diffeomorphism. There they give the extended dynamics as a Hamiltonian system and so project onto the original dynamics. The upshot is that one must beware of time-dependent coordinate changes because they can easily de many non-physical things. such as making any system look Hamiltonian. 


\subsection{Dengeroug Operations with Unbounded Variables}

This same construction is sometimes used in disguige to sbow that all dyarmical systems (or any particular system an autbor is interested in) are integrable. Tturougb a series of suitably complex coordinate chinges, the autbor succeeds in adding an extra variable with unbounded dy namics (the next two sections present explicic examples). This is then used as above to irivialize the system, where constant: of the rootion are pi. ziful. The congtants are pulled back and appear magically to have simplified the original gygtem.

Other dangeroug thing one can do are to make attractive ayotems look repulsive (as in figure (10.1)) and in fact get Liapunov exponents to be anything one wishes. (Oneledec's theorem shows that they are well defined for bounded systems, in the sense that all amooth metrics give them the same values as discusaed on $p$. 284 of [Guckenheimer and Holmes, 1989], but they have no intrinsic meaning for unbounded systems).

\subsubsection{Eg: Surfeptltlougly Changüg Damping to Driving}

Let us canvert a damped hamonic oscillator to one with negative friction. We start aith the Bystem

$$
\begin{aligned}
& \dot{x}=v \\
& \dot{v}=-x-k v .
\end{aligned}
$$

We make the thange of coordinate

$$
\begin{aligned}
& \vec{x}=e^{k t} x \\
& \bar{v}=e^{t t} v+k e^{t t} x .
\end{aligned}
$$


We see that the neB coordinates satisfy the tquations

$$
\begin{aligned}
\frac{d}{d t} \tilde{I} & =k e^{k t} x+e^{k t} \dot{x} \\
& =k e^{k t} x+e^{k t} x \\
& =\hat{v} \\
\frac{d}{d t} \bar{v} & =k e^{k t} v+e^{k t} \dot{v}+k^{2} e^{k t} x+k e^{k t} \dot{x} \\
& =k e^{k t} v-e^{k t} x-k e^{k t} v \\
& =-\tilde{x}+k \tilde{v}
\end{aligned}
$$

as desired.

In perturbation theory, if one makes time dependent coordinate changes, one must make certain that they remain close to the identicy for large times (for example by requiring that they be periodic or quasiperiudic in time) or else one may sweep any undesirable dynamics under the rug.

\subsubsection{Eg.: Pitfalle in the Use of Lie Trangforms}

Int us illustrate some of these dangers with a simple example using the method of Lie transforms. It is becoming fashionable to use Lie transforms in very complex situations, where it is hard to keep one's physical intuition about the problem (eg. in systems with many-particle dynamics or even infinite-dimensional wave systems). It is therefore worthwhile to point out some pitfalls for the unwary in a simple example. Such examples will hopefully help us to avoid misapplying Lie transforms in more complex circumstances. 


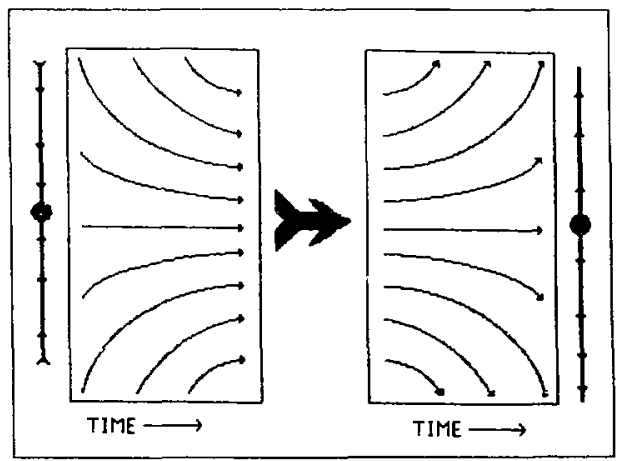

Figure 10.1: Attraction changed to repulsion by a time dependent change of coordinates.

In this method we assume given a Hamiltonian as an agymptotic series in $c$ whose zero order term has been brought to action-angle form and depends only on the actions. Let us examine the example:

$$
H=H_{0}+C H_{1}=\omega_{1} J_{1}+\omega_{2} J_{2}+\cos \left(\theta_{1}-\theta_{2}\right),
$$

on the phase space $\theta_{1}, J_{1}, \theta_{2}, J_{2}$ with the canonical bracket: [,]. The method of Lie transforms seeks to find an E-dependent canonical transformation which becomes the identity as $\epsilon \rightarrow 0$ and which converts $H$ to a new Hamiltonian $K$, which depends only on the actions. We represent the canonica' transformation as the time-one flow generated by the $\epsilon$-dependent Hamiltonian:

$$
-W=\epsilon W_{1}+c^{2} W_{2}+\ldots
$$


$-W$ 's hamilionien vector field, yiewed as a differential operator on functions on phase space, is denoted by

$$
L=\mid W, \cdot \cdot
$$

The action of the flow on functions is then the operator:

$$
e^{L} \text {. }
$$

The transformed Hamiltonian is then given by

$$
K=e^{L} \cdot H=H_{0}+e\left(\left[W_{1}, H_{0}\right]+H_{1}\right)+\ldots
$$

We want to choose $W$ order by order to eliminate the $A$ dependence in $K$. At first order we would like to soive for $W_{1}$ :

$$
\left\{w_{1}, H_{0}\right\}=-H_{1}
$$

This just says that the derivative of $W_{1}$ along the unperturbed dyamics (gencrated by $H_{0}$ ) should be equal to $-\theta_{1}$. One sometimes ses the solutions written formally:

$$
W_{1}=-\int_{\text {uaperturbed orbita }} H_{1}
$$

This leads one to think that the transformation is always possible aud that the qualitative behavios of $K\left(\bar{J}_{1}, j_{2}\right)$ 's dynamics, which has all orbits periodic or quasiperiodic, is representative of the behavior of $H$ 's. ln the example wr are considering. this is indeed true if $\omega_{1}$ and $\omega_{2}$ are irrationally related. In this case, the Lie transform remains close to the identity for all time (and gets cluser as $c \rightarrow 0$ ) and so the change of variables doesn't do much damage. 
Consider the case $\omega_{1}=\omega_{2}$, however. For this system

$$
\dot{\theta}_{1}=\omega_{1}=\dot{\theta}_{2}
$$

and so $\theta_{1}-\theta_{2}$ is constant in time. This means that

$$
j_{1}=\operatorname{csin}\left(\theta_{1}-\theta_{2}\right)
$$

is congtant as well, and so $J_{1}$,rows steadily in time. This is in great variance with the prediction of $K$ which says that $J_{\mathfrak{l}}$ remains constant in time. How is it that by a change of variables we converted a system with monotonically changing action to one with constant action? The seemingly harmless integral over unperturbed orbits created a canonical tranpformation which wag unbounded in time. As we have discusged, such transformations allow one to convert any system to any other, regardless of the actual physics involved. As this example demonstrates, one must be eqpecially careful when dealing with systerns tbat have resonances (different unperturbed degrees of freedom with the same frequency or with rationally related frequeacies). Notice that in this example, the error over bounded time is only of size $c$ and so we bave done as well as näive perturbation theory. The whole poia of Lie transforms, though, is to get behavior on a timescale of order $1 / \mathrm{e}$. On this scale the method has failed in the example above. 


\subsection{Imbedding in Poigson Systemg}

Because Harniltouian dynamics on Poisson manifolds is more geveral than on symplecti: nauifolds, we may imbed arbitiary dynamics into Poisson dynamics of only one dimension higher. As above, assume given an arbitrary manifold $Q$ and dynamical vector field $X$. We construct the manifol: $\mathbb{R} \times Q$ (this is realiy $(T \cdot \Re \times Q) / \Re$ as diacussed in section 1.5 , question 66$)$. Let $h$ be a function on $\Re$ defined by

$$
h(x)=x
$$

Detine the vertical vector feld $Y$ on $\$ \times Q$ to be

$$
y=\frac{\partial}{\partial h}
$$

We will use $h$ pulled up to $\mathbb{R} \times Q$. We may define a Poisson bracket on $\mathfrak{R} \times Q$ as follows (this Poisson structure may be viewed as the bivector $X \wedge Y$ ):

$$
\{f, g\} \equiv(X \cdot f)(Y \cdot g)-(X \cdot g)(Y \cdot f) .
$$


This satisfies ihr Jarobl identity, as may be routinely checked:

$$
\begin{aligned}
& \{f,\{g, h\}\}+\{g,\{h f\}+\{h,\{f, g\}\}= \\
& X f y\left(X g Y h-X h Y_{g}\right)-X(X g Y h-X h Y g) Y f \\
& +X g Y(X h Y f-X f Y h)-X(X h Y f-X f Y h) Y g \\
& +X h Y(X f Y g-X g Y f)-X(Y f Y g-X g Y f) Y h \\
& =X f Y X g Y h+X f X g Y Y h-X f Y X h Y g-X f X h Y Y g \\
& -X X g Y h Y f-X g X Y h Y f+X X h Y g Y f+X h X Y g Y f \\
& +X g Y X h Y f+X g X h Y Y f-X g Y X f Y h-X g X f Y Y h \\
& -X X h Y f Y g-X h X Y f Y g+X X f Y h Y g+X f X Y h Y g \\
& +X h Y X f Y g+X h X f Y Y g-X h Y X g Y f-X h X g Y Y f \\
& -X X f Y g Y h-X f X Y g Y h+X X g Y f Y h+X g X Y f Y h \\
& =0
\end{aligned}
$$

since the Lie bracket of $X$ and $Y$ :

$$
[X, Y]=X Y-Y X=0,
$$

since $Y$ is constant. Because

$$
Y \cdot h=\frac{\partial}{\partial h} h=1
$$

we see that for any $f$

$$
\{f, h\}=X \cdot f \text {. }
$$

Thus with this Poisson bracket and $h$ as Hamiltonian we obtain the original dynamics given by $X$. Here, each of thr level sets of $h$ has the original dynamics and so 
both each injected level set and the projection along give the original $X$ dyuamics on $Q$. The symplectic leaves are the products of $\Re$ and the non-fixe-point orbits of $X$. Each fixed point of $X$ yields an interval of point symplectic bones along $\Re$. We have shown that by adding one extra dynamical variable, we can make any system into a Hamiltonian system on a Poisson manifold.

Let us give an explicit example. The simplest disipative dynamical system is given by

$$
\dot{x}=-x,
$$

where $x$ is a point on the real tine. This type of system is used as a model for linear relaxation in nog-equilibrium thermodynamies, where $x=0$ is $x$ 's equilibrium value. If we introduce another variable $y$, then the $(x, y)$ plane is a Poisson manifold with a Poiegon bracket given by

$$
f, g=x\left(\frac{\partial f}{\partial y} \frac{\partial g}{\partial x}-\frac{\partial f}{\partial x} \frac{\partial g}{\partial y}\right) .
$$

If we consider the Hamiltonian

$$
\boldsymbol{H}=\boldsymbol{y} \text {, }
$$

then the Hamiltonian dynamics is

$$
\dot{f}=f, H=-2 \frac{\partial I}{\partial r}
$$

This gives the dyamical equations

$$
\dot{x}=-x \quad \dot{y}=0 .
$$

The original dissipative system: $\dot{x}=-x$, is both imbedded as the dynamics on any of the submanifolds: $y$ =constant, and the result of piojecting along $y$ (i.e forgetsing the value of $y$ ) 


\section{Chapter 11:}

\section{Projected Area and}

\section{Canonical}

\section{Transformations}

Tourist: Can you give me the directions to Omaha?

Farmer, scratching his bead: You can't get there from here.-Anon.

Much of this thesis has been about simplifying the description of physical systems by projecting their dynamics down to various subspaces. A classical theorers of Licuville utates that the dyuamics of a Hamiltonian system preserves a certain canonical volume element in phase space. In modern parlence this theorem is very easy to prove. The Hamiltonian fow preserves a symplectic structure $\omega$ on phase space (i.e. it is equal to its pullback along th " How for any time). Since the operation of pullback of differential forms commutes with the operation of wedging tbem togetber, the Hamiltonian flow also preserves $\omega \wedge \omega, \omega \wedge \omega \wedge \omega, \ldots$, and finally in $N$ dimensions: $\omega^{N}$. It is easy to see that this last is a volume element, and in fact is the generalization to manifolds of the one dt ribed by Liouville. This volume prescrvation property of Hamiltonian Hows has many significant consequences for pbysical systems. It is the key ingredient for the Poincaré recurrence theo- 
rem [Arnold, 1978)and is responsible for the absence of attrartors in Hamiltonian systems. It is also the basis for much of atiistical mechanics.

\subsection{Application to Particle Acceleratorg}

When we project our dynamies to a smaller space, the volume preservation is in : Meral lost, but some remnant remains to impose constraints on the projected dynamics. There are many physical situations where these constraints lead to very interesting consequences. Andy Sessler has described to me the relevance to the design of free-electron lasers of any theorems constraining the ability of a Harniltonian system to change the volume of projection of a region. The time evolution of the particles moving through an FEL is often described by linearizing ahout some known orbit, leading to a time dependent linear canonical transformation on single particle phase space. If the particles in the beam are sufficiently noninteracting, the device applies this Hamiltonian transformation to all particles simultaneously. The phase space for a particle is a product of a 2-dimensional longitudinal phase space and a 2 or 4 dimensional transverse phase space. The source of the beam going into the FEL typically produces a particle distribution with longitudinal and transverse distributiong uncoupled, and a uniform spread over some range of momenta and some range of positions. Thus we can think of the incoming beam as a uniformly filled in parallelepiped lined up along the transverse and longitudinal phase spaces. We send these particles through the FEL and get a distorted and bent over parallelepiped in phase space out from the other end. Quite often we are more interested in some of the phase space coordinates than the other ones. For instance, we may 
want a be $m$ with very small transverse spread in space and mosentum, while not caring about the longitudinal spatial spread, to get a well defined beam axis. (Or sometimes the other way around if we want well defined buckets to increase the efficiency of encrgy transfer between a wave and the beam). While we know that the total volume in the parallelepiped mugt remain constant, pre are interested in the possibulity of trading some transv'rse phase space volume for longitudinal phase space volume.

\subsubsection{Courant's Theorem}

[Courant, 1966] has looked at this question and arrived at the following theorem: "An ellipsoid in phase space whose principal axes are the canonical coordinate and momentum axes can be transformed by a linear canonical transformation into anotber such normally oriented ellipeoid only if the areas of the projections of the Frst elipsoid on each of the $\left(q_{i}, p_{i}\right)$ planes are separately equal, one by one, to the corresponding projections of the second ellipsoid. The transformation is then the direct sum of $N$ separate area-preserving, two-dimensional transformations." This statement of his theorem assumes that all the projected areas are differcnt. When two are the same, the symplectic transformation may couple them. It is also important to note that the "corresponding" projections that have the same area need not be along the same sets of axes. In particular, we may exchange two sets of axes. This ruay be all that is needed for certain circumstances in the accelerator setting. If the phase space is $\left(q_{1}, p_{1}, q_{2}, p_{2}\right)$, then the Hamiltonian

$$
H=q_{1} p_{2}-q_{2} p_{1} \text {, }
$$


has a flow which just rotates the $\left(q_{1}, q_{2}\right)$ and $\left(p_{1}, p_{2}\right)$ planes (this is the angular mer mentum for a point particle in the plane and we know its action is jut rutat:ous) Thus after an appropriate amount of time we will have exchanged $q_{1}$ with $q_{2}$ and $p_{1}$ with $p_{2}$. Courant then states that the same throrem is true for the rectangular pa. allelepipeds tangent to and surrounding the ellipsoids Linfortunately. this therorem is restricted to the case wuere the final sct is lined up the same way as the initial set is, which is likely to be a rather rare orcurener. We would like to gearralize it to the projections under arbitrary transformations.

(Note added in proof: In a very recent preprint, M. Grnmov has show that the unit ball in $\Re^{2 N}$ cannot be mapped into a cylinder over a disc in $\left(x, p_{y}\right)$ space with radius less than one by any canonical transformation. This landmark work resolven many unsolved classical problems in symplectic geometry using very sophisticated arguments combining minimal surface throry from Riemannian geometry. elliptic P.D.E. theory. complex analysis in many variables. and the Atiyah-Singer index theorem. It is available as a preprint dated January 1985: "Pseudo-hulomorphic Curves in Symplectic Manifolds" from Institut des Hautes Etudes Srientifiques. 35. route de Chartres. 91440-Pures-sur-Yvette, France.) 


\subsection{Relation to the Uncertainty Principle}

Semi-classical mechaniss allows us to construct certain asymptotic relations between cikonal saves, say evolving under the Schrödinger equation, and Hamiltonian dyamis on a rorresponding elissical phase space. Every wave function has a corresponding Wigner distribution on the classical phase space. We may use this to aw-mptotically assign to wave functions, those regions in phase space which contain most of the density of their Wigner function. It is a folk theorem in physics that in $\mathcal{A}^{*}$ dimensional systems, these regions are of volume $h^{N}$ (where $h$ is Plank's constant). A elassical theorem of Weyl show5 that as the energy $E$ approaches infinity, the number of eigenstates of a Hamiltoman corresponding to a bounded classical system, with energy less than $E$, asymptotically approaches the volume of the region in classical phase space with energy less than $E$, measured in units of $h^{N}$. The exact relation between the classical regions and the quantum idtes is being clarified with the techniques of micro-loral analysis [Fefferman, 1983]. Even though the volume of a wave-packet is always the same in phase space, we are usually interesied in its extent in position space or in its Fouricr transform's extent in momentum space. As the wave packet crolves, it typically stretches out in a "Ciagonal" direction in phase space, making both of these projections grow. This is the well known quantum spreading of wave packets (or any other waves with disfersion). There is a rigorous lower bound on 'sow tightly we can compact a wave packet in both a iratial direction and the corresponding momentum direction, given by the uncertainty principle. This says that the prirduct of the $q$ dispersion and the $p$ dispersion mut be greater than $h$ for cach coordinate. In particular. we may not arbitrarily 
give uj phase volume in one ser if coortinate at the behest of atwother set Thus to the extent that the semiclassical connection is valid. wo would expect there to be limitations on shrinking projected vohmes under Haniltonian flow: If we could find such a shrinking flow. we could apply the corresponding quantum Hismiltonian to a wave packet and make measurements that give mone information than allowed by the uncertainty principle about position and momentum at an carlier time. 


\subsection{Weingtein's Approach}

The baric ided of Weinstein's approarh !unpublished is to reduce the questim of reducing projected area to a known theorem abcut Lagrangian tori. He hinds a Lagrangian torus in the region of interest which projects to a circle. If the area enclosed by this circke shrinks under a ranonical transformation. w may cause the image of the circle to be disjoint from the circle itself. This implees that the Lagrangian torus is also disjoint from its image. This is disallowed by a kuown theorem for sufficiontly small canonical transformations. Therefore the projected area cannot shrink for small ejough canonical transformations. Let us now go through this argument more precisely.

Consider the symplectic manifoid $S$, formed as the product of two symplectic manifolds $S_{1}$. wl and $S_{2}, w_{2}$. Take $S_{1}$ to be two-dimensional and $S_{2}$ to be arbitrary. For the product symplectic struct:re we use $\omega=\omega_{1}+\omega_{2}$ (here we are identifying $\omega_{1}$ and $\omega_{2}$ with their pullbacks along the natural projections). Consider connected. simply-connected regions $R_{1} \subset S_{1}$ and $R_{2} \subset S_{2}$. Their produc?

$$
R \equiv R_{1} \times R_{2}
$$

will he our initial region. Its projection down to $S_{1}$ has area equal to the area of $R_{1}$. Weinstein has shown that under arbitrary (i.e. nonlinear) canonical transformations, that are sufficiently close to the identity, the projection of the transformed $R$ to $S_{1}$ has an area that is greater than or equal to the initial area. Furtbermore. there appears to be a "rigidity" theorem which says that if the area is the same. then. in fart. our transformation is a product of a symplectic transformation on $S_{1}$ 
and or" on $S_{2}$. Any coupling between the two sets of degrees of freedom must cause the projerted area to increibe. This is reminisecnt of the increase of conaregrained or prifieted cftropy in statistical mechanics.

The proof of the first part rests on some Lagrangian intersection theory. Let us sketcls the basic idea bere. The boundary of $R_{1}$ is topologically a circle. Sinse $S_{1}$ is 2 dimeusioual, we may apply a canonical transformation that takes any such region to any other one with the same area as was shown iv [Banyaga. 1977\}. The circle is a Lagrangian submanifold of $S_{1}$ (i.e. $\omega_{1}$ vanishes on it) as are all 1 dimensional submanifolds. Choose a Lagrangian torus in $R_{2}$ (say by taking a product of sufficiently small circles in each of the canonical planes formed by a canonical basis in some little region). The product of these two tori will be a Lagrangian torus in $R$ with respect to w. This corus projects down to the circular boundary of $R_{1}$ in $S_{1}$. Assume we could apply a near ide..tity canonical transformation to $R$ such that its projection to $S_{1}$ had a smaller area tha $R_{1}$. By a canonical transformation of $S_{1}$ we can force this inage to be stric:ly inside the boundary of $R_{1}$ (say by making it look like a smaller circle concentric to the boundary of $R_{1}$ in some coordinates). But this mans we bave a near-identity canonical distortiun of a Lagrangian torum whome projection doesn't intersect the ivitial torus. I[ the projections don't intersect, then neither do the cori. But this is known to be impossible.

Io fact, recent technical advances have shown that even (i) inall canonical deformations of Lagrangian cori must intersect the initial torue (conley and Zehndet. 1983 and Chaperon. 1983: This result is one of a number of related result atout

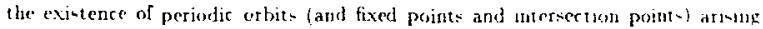


from a rembination of topological and symplectic properties. These ideat had their beginning in Poincare conjecture that all area prescrving map of an annulus to itself that thisted the bounding cirsles in opposite directious must have at least two fixed puint- 'Poincare. 1912'. Poincare used hiv theorem to show the existence of infinitely many periodic orbits in the neighborhood of an elliptic periodic orhit in relestial mechanirs. Puincares theorem was proved in [Birkhoff, 1913] and partially generalized to certain compact symplectic manifolds in [Arnold, 1965]. Since then many fascinating developments have occurred as surveyed in [Weinstein. 1984a].

Let us sketch the proof for $C^{\prime}$ small deformations (as in [Arnold, 19:8] p.420). A small acighborbood of any Lagrangian submanifold is symplectomorphic to a neighborhood of the cotangent buadle of that Lagrangian submanifold with the canonical cotangent symplectic structure [Wejnstein, 1971]. In this representation, the initial Lagrangian torus is the zero section. A sufficicntly close Lagrangian torus will project diffeomorphically onto this under the canonical cotangent projection. Io fact it is actually the graph of the differential of a smooth function on the initial torus. Pecause it is Lagrangian it must be the graph of a closed form. (It is easy to check that the pullback of the canonical one-form on a cotangent bundle from the graph of a one-form o back to the zero secticn is exactly that one-form $\alpha$. Being Lagrangian meaus that the differential of the canonical one-form vanishes on the manifold. and so its pullback's differential must also vanish. which just says that $a$ is clo: ed.) Because the canonical transformation extends to an open ball containing the torus. this form must actually be exact.

Poincarc's lemma tells us that locally every closed one-form is the differential 
of a function. The only problem might be that shen we go armund the fumdamentat uncontractable loops of the torus, the function might not returi th the initial valuet If te think of the initial torus and its deformation in the original phase apare, the canonical transformation is defined over a topological ball containing them. We may thus find 2-dimensional discs whose boundaries are the fundament al loow on the torus. The integral of the symplectic form over the initial disc is thercfore equal to its integral over the deformed disc. If we make a closed cylinder (no boundary) from the two discs and the sheet swept out by a loop under the deformation, Stokes theorem says that the integral of $د$ over the cylinder is zero. Since the two dise contributions cancel, the integrai of $\nu$ over the cylinder swept out by the loop is itself zero. Stokes' theorem then tells us that the integral of a one form whose differential is $\omega$ in the region the sheets sweep out, must have the same integral over the loop and its deformation. In general, canonical Jeformations with this property are known as exact deformations. The canonical one-form in our cotangent representation bas $\omega$ as its differential in this region. But since the intial torus is the zero section. the integral of $p d q$ around any loop must be zero. We may then conclude that its integral around any loop of the deformed torus is also zero. There is therefore no obstruction to finding a function on the deformed torus whose differential is the canonical one-form.

The points of intersection of the deformed torus with the initial torus are exactly the places where this differential vanishes (and so its graph hits the zero section). But these are the critical points of the function on the torus. But being confact, the torus forcen an function to have a maximum and a mimimum (and 
at least one poweibly degenerate saddle by Lusterntk Shuireman categery theoryl which force interecetion. ('haperon given the criterum that any exact deformation of the torus on which the $q_{1}^{2}+p_{\mathrm{a}}^{2}$ all remain powitive (and so there is some loop that ian t puifed through $z$ rol must aluse the image to intersect the initial torus. 


\subsection{Theorem for Linear Canonical Transformations}

We would like to generalize this rosult from near-identity transformations to arbitrary transformations. Unfortunately, it cannot be true in general as the folluw. ing example shows. Let us consider $R^{2} \times \Re^{2}$ and take our region to be a large area in $S_{1}$ tirnes a small area in $S_{2}$. There is a canonical transformation which exchanges $S_{1}$ and $S_{2}$ and so makes the projection ontu $S_{1}$ smaller. This exchange is a "large transformation" and by the above, the projection must first increase and then decrease. We can get around this cxample in a number of ways. We may consider regions whose projected areas are the same or wo may study the sum of the arcas or the minimal area under the different projections. Below we prove a theorem for arbitrary linear transformations on the increase of the sum of the projected areas. This generalizes Courant's theorem to arbitrary linear canonical transformations (but so far only in $\Re^{9}$ ) and is not restricted to be near-identity. One would like to extend it to higher dimensions, non-parallelepiped initial conditions, and nonlinear transformations. We develop the theory of projected parallelepipeds for arbitrary dimensions. The nonlinear situations are locally linear and we may chop our region into parallelepipeds to which the theorem applies. Unfortunately, the little parallelepipeds obscure one another under projection and this must be understow for a nontinear theory.

Another lack to take is to not look at the projected area. but retter the projected measure (i.c. we want the yolume in the region that sits over each little arca in the two-dimensional space). Pbysically it is often important to know not jusi that some particle's state project to a given region. but also hou manv particles. 
Perhape the entropy (i.e. integral of $(-p \log p)$ over the projected region) of this distribution furtetion must itscrease. In this situaticn as well the geometry of parallelepuped-appears relevant. One place to see the possible condection is through the central limit theorem. If we project a cube to a real line parallel to its longest diagonal. then the projected measure along the line is the multiple convolution of a rectangular pulse, with one pulse for each dimension of the cube. In the limit of large dimension, this approaches a Gaussian. The measure of lines on which the projection is close to a Gaussian asymptotes to one as the dimension grows. Futhermore. the eptropy of a Gaussian is a maximum for all distributions with the same dispersion. A cube projected to its diagonal is in some sense maximally sensing each of the degrees of freedom (perturbing a point along any of the orthogonal axes of the cube is reflected by a perturbation in the image of the projection; if the projection isn't diagonal, then some axes are short-changed because their projections are less: important than others). Any rotation which lines the projection up more along an edge sill decrease the entropy of the projected measure.

If such a theorcm guaranteeing the increase of entropy under coupling is true in infinite dimensions, then it might shed light on the increase of entropy under the evolution of Boltzmann's equation. Bogoliubov derived Boltzmann's equation as the first order term in an asymptotic expansion of the BBGKY hierarchy. The essential part of the argument obtains the evolution of the two particle distribution function as the Liouville equation fo: two interacting particies (ihe contribution of the three-particle and bigher distributions is higher order in the ordering seheme). The evolution of the two partirle distribution function is then Hamiltonian and 
linear (albeit infinite demensional). In Bogolinbuv s argutuent. we are grven as spatially uniform one partule distribution whose evolution we wish to find (at) covlution cyuation depends on the two particle distribution). We take the two particle distribution which is simply an uncorrelated product of this one-particle one with itself as initial conditions for the 2-particle Liouville equation. Uader evolution the two particles will collide reating a correlation and the infinite time asymptotics of this (i.e. when the two particles become widely separated) is used as the 2-particle driving term in the 1-particle rolution, which becomes the Boltamaan collision term. Boltzmann shows that under this evolution, the entropy of the ope-particle di:tribution must increase.

\subsubsection{The Geometry of Projected Parallelepipede}

When we project a parallelepiped down to a lower dimensional space. the result is no longer a parallelepiped as in figure (11.1). We show here, though, that it may be decomposed into parallelepipeds in a unifor m way, allowing us to get a formula for the volume of the projection. We demonstrate this decomposition using induction and a couple of simple initial lemmas. Let us be given $n$ vectors $l_{1}, 1 \leq i \leq n$ in the $k$-dimensional linear space $\mathfrak{R}^{k}$. We are interested in the region $R$ consisting of points of the form

$$
\sum_{i=1}^{n} a_{i} v_{i} \text { for } 0 \leq 0_{1} \leq 1
$$

Let us call this region the [0.1/ syan of the $F$, 's.

Lemma 11.2. For any $n$ and $k . R$ is convex. 


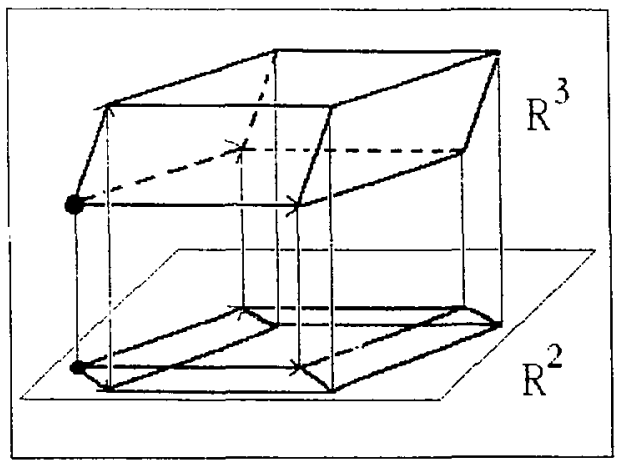

Figure 11.1: A three-dimensional parallelepiped projects to a hexagon in two dimensions.

Proof. Consider any two points $\sum_{t=1}^{n} a_{1} V_{1}$ and $\sum_{t=1}^{n} b_{2} V_{t}$ in the region $R$. The line betwen them consists of all points of the form

$$
\begin{array}{r}
\sum_{i=1}^{n} a_{1} V_{1}+t\left(\sum_{i=1}^{i=} b_{1} V_{1}-\sum_{i=1}^{n} a_{i} V_{i}\right) \\
=\sum_{i=1}^{n}\left[(1-t) a_{i}+t b_{i}\right) V_{i}
\end{array}
$$

where $0 \leq t \leq 1$. But since $t$ and $1-t$ are non-negative and $a_{1}$ and $b_{1}$ are less than one, we see that

$$
(1-t) a_{1}+t b_{1} \leq(1-t) \cdot 1+t \cdot 1=1 .
$$

But thit shows that all poiuts on tbe line between the two given points satisfy the defining criterion for $R$ and therefore belong to $R$. Heace $R$ is convex. Q.E.D. 
Definition 11.1. Let us define a vertex of a subset of $R^{k}$ to be any poist of the set such that there does not exist any open interval of a straight line in $\Re^{k}$ which contains the point and lies completely in the set.

Lemma 11.3. All vertices of the set $R$ have a unique representation as a sum $\sum_{i=1}^{n} c_{2} V_{2}$ and all of the $a_{z}$ 's are either $I$ or 0 .

Proof. Assume we can represent a vertex as $\sum_{1=1}^{n} c_{8} V_{v}$ where some $a_{j}$ is not 1 or 0. There is then an open interval around $a$, which is contained in $[0,1]$, and therefore the correspouding vectors form an interval of a straight line lying in $R$, violating our assumption of verticity. Now assume that there are two representations: $\sum_{t=1}^{n} a_{2} V_{\mathrm{r}}$ and $\sum_{t=1}^{n} b_{2} V_{1}$ for thr vertex, where all the $a_{1}$ 's and $b_{1}$ 's are neccessarily 0 or 1 . The point may then also be represented as

$$
(1-t) \sum_{i=1}^{n} a_{1} V_{1}+t \sum_{i=1}^{n} b_{1} V_{1},
$$

where $0 \leq t \leq 1$ or equivalently as

$$
\sum_{i=1}^{n}\left((1-t) a_{1}+t b_{1}\right] V_{1}
$$

But unless all the $b_{\mathrm{z}}$ are equal to the corresponding $a_{1}$, we can thereby get a coeffi. cient which is not ! or 0 , violating the above. Thus vertices bave unique representations. Q.E.D.

Lemma 11.4. a) Let us assume that the number of vectors $n$ is greater than or equal to the dimension $k$ of the ambient space. The region $R$ may thes be decomposed as a unjon of parallelepipeds formed by rigid translations of the 0.1 . spans of $k$-element subsets of the set of $n$ vectors, such that they intersect onfy in 
$(k-1)$ and lower diniensional sets (which are of Lasbegue measure zero). There are $n^{\prime}(k)(n-k) !$ such parallelepipeds.

b) Let us assurne that $R$ is k-dimensional. The boundary of $R$ may then the decomponed as a union of paralielepipeds formed by rigid tras lations of the $[0,1]$ spans of $(k-1)$-clement subsets of the set of $n$ vectors, where each such span is included twice and the intersections of the translates are of dimension $k-2$ and lower.

Proof. We prove these two parts together using a double induction in $k$ and $n$. The essence of the proof is showa in figure (11,2). We bave shown the effect of adding an extra vector to the projection of a 3-dimensional parallelepiped's projection onto 2 dimensious (to give the projection of a hyper-cube). It adds an extra parallelogran to half of the boundary edges formed from the extra vector ard 1 each of the original 3 vectors. We may generalize this same construction to arbitrary dimensions as follows.

Both a) and b) have assumptions that prevent $n$ from being less than $k$. To start the induction, assume $n=k$, whete $k$ is arbitrary. a) is now trivial since there are exactly $k$ vectors and $R$ is indeed equal to their $[0,1]$ span. In case $b), R$ is actually a paralleiepiped and its faces are obtained by taking the $[0,1]$ span of eack set of $k-1$ vectors and basing it at zero and at the end of the remaining vector, uthich agrees with the statement in the lemma. We also ueed the case $k=1$ for arbitrary $n$ (see figure $(11.3)\}$. This case is also easy, since we may just line up the $n$ yectors starting at zero, each representing its own $[0,1]$ span and the boundary is just two endpoints which we may take as the union of the ends of the vectors. 


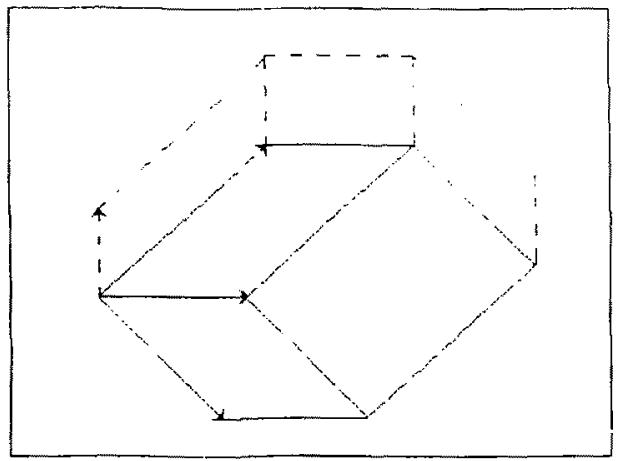

Figure 11.2: The esseace of the lemma. Adding a fourth vector adds three parallelograms foumed from that vector and each of the original three vectors.

Let us now assume the theorem true for all $n$ 's when $k$ is less than $k_{0}$ and for $n$ 's less than or equal to $n_{0}$ when $k$ equals $k_{0}$ and prove it for $k_{0}, n_{0}+1$. By induction this will prove the lemma as stated for .ll allowed $k$ 's and $n$ 's.

Let us begin with part a). We may assume that $n_{0}+1$ is strictly grrater than $k$ (since we already did the equal case). Thus $R$ is the 0,1 , span of $n_{0}-1$ vectore. Consider the $[0, I]$ span of the bist $n$ of these and call it $S . R$ is obtained from $S$ by adding $|0,1|$ multiples of the $\left(n_{0}+1\right)$ st vector to each point in $S$. In fact, we get all the extra points in $R$ that aren't in $S$ by adding such multiples: to anly boundary points of $S$ isince $t 0$ get a new point the ver tor must intersect 1 liz* boundary and we can get the same point by adding a smaller multiple to that point 


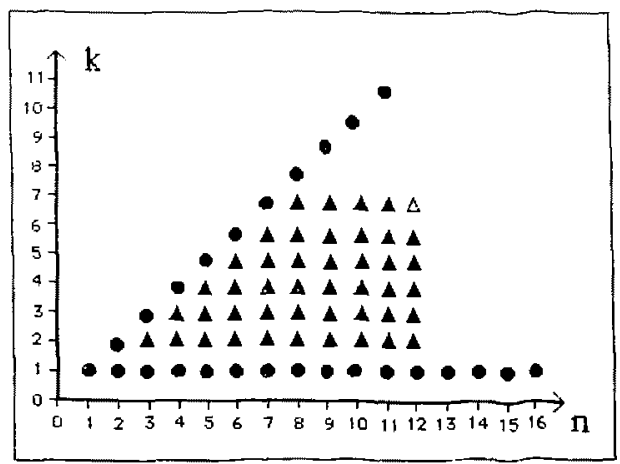

Figure 11.3: The $n, k$ plane.The circles are the base induction cases. The open triangle is an example case. The closed triangles are the cases we must use in its proof by induction in addition to the circles.

of intersection). By induction, $S$ decomposes into translates of the $|0,1|$ spans of the $k$-element subsets of the first $n_{0}$ vectors. We geed only sbow that the region in $R: x$ contained in $S$ is the union of tranglates of $[0,1]$ spans of the $\left\{n_{0}+1\right)$ st vector fith all $k-1$ element subsets of the first $n_{0}$ vectors (up to sets of smaller dimension) to prove part a). But we may now apply part b) to $S$ by the inductive hypothesis. Consider each set of $k-1$ vectors from the first no in turn. By part b) the buundary of $S$ has cwo regions that are translates of their span, which are parallel (since they are rigio translates of each other). By the convexity of $S$ (from the lenma above), $S$ lics extircly in and on one side of the byperplane in $\mathbb{R}^{k}$ which 
contains such a picce of the boundary. If the two regions are distinet. S muti lie on opposite sides of their repective hyperplanes. The ractor $v_{\text {matl }}$ either lie: in such a hyperplane or points to one side of it when its basepoint is contained in it If it lies in the hyperplane or if the $k-1$ vectors span a space of lower than $k-1$ dimensions, then the parallelipiped it forms with the $k-1$ vectors is degenerate and so of lower dimension than $k$ and irrelevant for our union. Otherwise, take the piece of S's boundary such that $S$ is on the opposite side of the hyperplane as the vector. This parallelepiped and the vector $\{0,1\}$ span a $k$-parallelepiped which is in $R$ but whose intersection with $S$ is of lewer dimension (since they can only intersect in the hyperplane because they are on opposite sides of it). Because $S$ 's boundary is filled out by such $k$ - 1-parallolepipeds, and $R$ is obtained by adding $[0,1]$ multiples of the $\left(n_{0}+1\right)$ st vector to the boundary, $R$ is the union of these $k$-parallelepipeds as desired. It remains only to show that they intersect each other in scts of dimension lower than $k$. Since these parallelepipeds are obtajncd by sweeping the $(k-1)$. parallelepipeds in half the boundary of $S$ along the last vector, if two of them intersect in a regiton of dimension $k$, the corresponding $(k-1)$-parallelepipeds must intersect in a region of dimension $k-1$, but this ts not allowed by the statement of b).

Let us now prove b) under the same inductive assumption as above and using the result of a) (this is uk since the proof of a) only used b) on lower inductive cascs). Since we have added $k$-parallelepipeds to $S$ to get $R$, half of the original $k-1$ parallejepipeds in $S$ 's boundary have remajoed ard the other balf have just been rigidly translated by the last vector. Thus we have two parallel copies of each 
of alue $(k-1)$-parallelepipids generated by the first $n_{0}$ vectors, in the boundary of $R$ as desired. Extra boundary sufaces arcse when we translated one parallelepiped by the last vector but not its neighbor. Consifer the projection from $5 \Re^{k}$ to $h^{k-1}$ along the last vector. Both $S$ and $R$ bave the same imnge and the extra part of $R$ 's houndary is exactly formed by translating by the last vector that part of $S$ which projects to the boundary of the image. We may apply part b) to the projection of $S$ to see that this boundary may be decomposed into pairs of translates of all $[0,1]$ spans of the projection of $k-2$ vectors (since the induction hypothesis applies to this lower dimensional space). The subset of $S$ that projects onto this boundary is then decomposed into unions of $[0,1]$ spans of $k-2$ vectors up to things which point along the last vector and are therefore degenerate with it. So indeed the extra part of the houndary of $R$ is decomposable into translates of parallelepipeds [ormed from the last vector and all $k-2$ element sets of vectors from the first no. Together with the original decomposition of $S$ 's boundary these give us the desired decomposition of part b). Q.E.D.

\subsubsection{The Care of Linear Canonical Transformations}

The way we wisl to use this lemma is as follows. We get subsets that are $[0,1]$ spans of vectors as the projected image of a parallelepiped in a lower dimensional space. The volume of this projection: is the sum of the volumes of all parallelepipeds formable by these vectors, by the lemma. For $2 N$-dimensional symplectic vector spare, the volume of a $2 N$-dimensional parallelepiped is given by ioserting the generating vectors into the $N_{i b}$ wedge product of the symplectic form with itself. 
giving a natural colume element. Let us now une the to prewe the theores of interest.

Let $S_{1} \approx \mathbb{R}^{2}$ bave the coordinates $\left(q_{1}, p_{1}\right)$ and the symplectic form: $-1=$ $d q \wedge d p$. Let $S_{2}, \nu_{2}$ be defined sirilarly. Consider the symplectic manifeld

$$
S \equiv S_{1} \times S_{2}
$$

with the symplectic structure

$$
\omega^{\prime} \equiv \omega_{1}+\omega_{2} .
$$

Here the $\omega_{2}$ are thought of as pulled back to $S$. We are interested in how the area of the projection of a set down to $S_{1}$ changes under canonical transformations. We may prove the following theorem for product parallelepipeds under linear symplectic transformations. This is a physically relevant setting for many physical situations and is the local picture for the general case.

Theorem 11.5. Let us be given two vectors $u_{1}$ and $v_{1}$ in $S_{1}$ whose $\{0,1\}$ span is a parallelogram of a ${ }^{3} 3 A_{1}$ and similarly for $u_{2}$ and $v_{2}$ in $S_{2}$ with area $A_{2}$. Consider the parallelepiped $I$ in $S$ which is the product of these two parallelograms. Inder an arbitrary linear canc nical transformation $L$ of $S$, the sum of the projected areas of $L \cdot P$ in $S_{1}$ and $S_{2}$ is always greater than or equal to $A_{1}+A_{2}$. Furthermore equality only bolds if a certain restrictive condition listed below hold.

Proof. We have sten above that the projer'ed area of $L \cdot P$ in $S_{1}$ is equal to the sum of the areas of the parallelograms spanned by the projections of $\left(L \cdot u_{1}, L \cdot u_{2}\right)$. $\left(L \cdot u_{1}, L \cdot v_{1}\right),\left(L \cdot u_{1}, L \cdot v_{2}\right),\left(L \cdot u_{2}, L \cdot v_{1}\right),\left(L \cdot u_{2}, L \cdot v_{2}\right)$, and $\left(L \cdot v_{1}, L \cdot v_{2}\right)$. The area is just the absolute value of the result of inserting the projected vectors inte 
-1 But this is the same as inserting the vectors intu the pullback of w $w^{+}$along the projecture which wr are denoting again by in $S_{1}$ and $S_{2}$ is given by

$$
\begin{aligned}
& \left.\operatorname{lin}_{1}\left(L \cdot u_{i} L \cdot u_{2}\right),-\mid \omega_{1}\left(L \cdot u_{1}, L \cdot v_{1}\right)\right\}+\left|-w_{1}\left(L \cdot u_{1}, L \cdot v_{2}\right)\right|+ \\
& \left|-\dot{\prime}_{1}\left(L \cdot u_{2}, L \cdot u_{1}\right)\right|+\left|-1\left(L \cdot u_{2}, L \cdot \tau_{2}\right)\right|+\left|w_{1}\left(L \cdot v_{1}, L \cdot v_{2}\right)\right|+ \\
& \left|u_{2}\left(L \cdot u_{1} \cdot L \cdot u_{2}\right)\right|+\left|u_{2}^{\prime}\left(L \cdot u_{1}, L \cdot u_{1}\right)\right|+\left|-u_{2}\left(L \cdot u_{1}, L \cdot v_{2}\right)\right|+ \\
& \left|\omega_{2}\left(I \cdot u_{2}, L \cdot r_{1}\right)\right|+\left|\omega_{2}\left(L-u_{2}, L \cdot v_{2}\right)\right|+\left|\omega_{2}\left(L \cdot v_{1}, L \cdot v_{2}\right)\right| \text {. }
\end{aligned}
$$

Applying this formula in the original situation, where $L$ is the identity, and using the fact that $w_{1}$ annibilates $u_{2}$ and $v_{2}$, we see that

$$
A_{1}+A_{2}=\left|\omega_{1}^{\prime}\left(u_{1}, v_{1}\right)\right|+\left|w_{2}\left(u_{2}, v_{2}\right)\right| .
$$

Because $L$ is symplectic on $S, \omega$ is preserved by it. This means that for any two vectors $u_{1}^{\prime}$ and $w_{2}$ in $S$, we bave

$$
\begin{aligned}
& \omega\left(L \cdot w_{1}, L \cdot w_{2}\right)=\omega\left(w_{1}, w_{2}\right) \\
& =\omega_{1}\left(L \cdot w_{1}, L \cdot w_{2}\right)+\omega_{2}\left(L \cdot w_{1}, L \cdot w_{2}\right) \\
& =\omega_{1}\left(w_{1}, w_{2}\right)+w_{2}\left(w_{1}, w_{2}\right) .
\end{aligned}
$$

Let's assume that $u_{1}, v_{1}$ and $u_{2}, v_{2}$ are in the right order so that $\omega_{1}$ and $w_{2}$ give positive answers when acting on them. Then we see that

$$
\begin{aligned}
& A_{1}+A_{2}=\omega_{1}\left(u_{1}, v_{1}\right)+\omega_{2}\left(u_{1}, v_{1}\right)+\omega_{1}\left(u_{2}, v_{2}\right)+\omega_{2}\left(u_{2}, v_{2}\right) \\
= & \omega_{1}\left(L \cdot u_{1}, L \cdot v_{1}\right)+\omega_{2}\left(L \cdot u_{1}, L \cdot v_{1}\right)+\omega_{1}\left(L \cdot u_{2}, L \cdot v_{2}\right\}+\omega_{2}\left(L \cdot u_{2}, L \cdot v_{2}\right) \\
\leq & \left.\left|\omega_{1}\left(L \cdot u_{1}, L \cdot v_{1}\right)\right|+\mid\right\lrcorner_{2}\left(L \cdot u_{1}, L \cdot v_{1}\right)|+| \omega_{1}\left(L \cdot u_{2}, L \cdot v_{2}\right)|+| \omega_{2}\left(L \cdot u_{2}, L \cdot v_{2}\right) \mid .
\end{aligned}
$$

But in the expression above for the new sum of projected areas. this last expression appear with some positive or zero terms added to it. Thus the sum of the new 


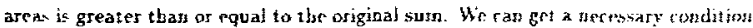
for the sum to be equal. since all the extra terms in absolute value must scparately vanish. So $\left(L-u_{1}, L-u_{2}\right), \omega_{1}\left(L-u_{1}, L \cdot v_{2}\right), \omega_{1}\left(L \cdot u_{2}, L \cdot v_{1}\right), u_{1}\left(L-v_{1}, L \cdot v_{2}\right)$. $\omega_{2}\left(L \cdot u_{1}, L \cdot u_{2}\right)_{1} \omega_{2}\left(L \cdot u_{1}, L \cdot *_{2}\right), \omega_{2}\left(L \quad u_{2}, L \cdot v_{1}\right)$, and $\left.\nu_{2} L \cdot v_{1}, L-v_{2}\right)$ must all varish. Q.E.D.

Courant's paper contains an apparent counterexample to this kind of theorem for aonlinear dy edged septum into a recirculating beam to strip of a than outer band and cause the inner region to continue to recirculate. In this way one can make the spatial width of the beam smaller, without increasing its momentum spread. The knife edge is really a very steep potential. Even though the number of particles that hit it is small, they are given a very large momentum kick (this sounds like some of the classical arguments for the uncertainty principle). Thus the actual region in phase space is probably very spread out. The physically important quantity in this example, however, is the particle distribution function. If tesigaed properly. one could presumably make the measure of the wild particles snall. This kind of example indicates that one must be very careful in any distribution function version of these theorems.

Another construction that such a theorem will bave io beat is as follows it is weil known that there exists a canonical transformation that moves aily $N$ distinet points of phase space to any other $N$ distinct points. If we force most of the measure to be near these points, then we can line them up to project to any kind of distribution we desire. How do we get the measure to be near points in a product 
distributixan? Consider the phase spare to be $\mathbb{R}^{6}$. All canonical transformation: will take plare en only the $\Re^{a}$ iurmed by the first 4 coordinates. The lest two coordicates are needed only to make the measure large near the specified points in the firat four dincessions. We choose points in the first and second $\Re^{2}$ so that the points in the product $\mathbb{R}^{4}$ don't all lie over single point in the first $\Re^{2}$ (for cxample se can choose 9 points arranged in three rows of three). We choose a volume in $R^{6}$ which is very extended in the last two dimensions near these points. This may be constructed as a product of a region in the first 2 dirrensions times a region in the last 4 . We now use a canunical transformation in the first 4 coordinates which makes the points line up along a single fiber of the projection to the first two coordinates, letting the last two dimensions just go along for the ride. The projected distribution can then be made to vary in any way we wish.

An even simpler example can be constructed in $\Re^{4}$ which we assume to be coordinatized by $\left(x, p_{x}, y, p_{y}\right)$. Let our initial set be the unit ball in $\Re^{4}$. We chop the ball into 3 pieces: the part $B_{1}$ with $-1 \leq x \leq-\ell$, the part $B_{2}$ with $-c \leq$ $x \leq t$ and the part $B_{3}$ with $\& \leq x \leq 1$. By making $\&$ small enough, we can make the measure of $B_{2}$ arbitrarily small. By a simple canonical transiation and rotation defined on a region including $B_{3}$ bur in the complement of $B_{1}$, we can make $B_{3}$ "sit above" $B_{1}$ so that they project to the same region in the $\left(x, p_{x}\right)$ plane. This is easily extended to a canonical transformation on all of $\mathbb{M}^{4}$ which leaves $E_{\mathfrak{l}}$ fixed (say by extending the tinge dependent Hamiltonian vector Geld defining the transformation using a partition of unity). By Gromov's theorem, we know that $B_{2}$ must be stretched in some wild way so that its projected area is large for at 


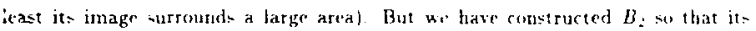
measure in $\mathrm{R}^{\mathrm{s}}$ is small By repeating this construction. we can make an arbitrariy large percentage of the measure of the ball projest to arbitrarily small regions by a canonical transformation. 


\section{Chapter 12:}

\section{Reversibility vs. Irreversibility}

The seeming paradox of reversible underlying dynamics leading to ir reversible macroscopic behavior has been wrestled with since the time of Boltzmann. For the case of gas dyamics, Boltzmann derived his famous equation on the basis of the questionable statistical assumption of "Stosszahlansatz". From the Boltzmann equation one may derive the " $\mathrm{H}$ theorem" which says that the time derivative of the entropy is greater than or equal to zero. The "Stosszahlansatz" or "moiecular chaos assumption" says that the probability distributions for colliding molecules should be uncorrelated. As has been pointed out many times (see for example: pp. 46-88 of [Chapman and Cowling, 1958] and pp. 28-32 of the Statistical Physics volume in iLandau and Lifshitz, 1960-1981]), because the underlying dynamics is reversible, for every state wit' its entropy increasing. there is a corresponding state with its entropy decreasing. In fact, one may show from this argument that the stosszahlansatz can hold only when the time derivative of entropy is zero. There is no int rinsically special direction in time: If one starts with a random state with low ent ropy, the ntropy increases if one follows the cvolution of the state either backward-or forwards in time. There are many more states with bigh entropy than 
lox. and so a system is likely to be in a high entropy state regardless of the time.

There is a simple dyuamical system which exbibits some of the secmingly paradoxical feat ures of these complex statistical systems. Consider the differential equa* tivas

$$
\begin{aligned}
& \dot{I}=-x y \\
& \dot{y}=x^{2}-y^{2} .
\end{aligned}
$$

A sketch of the dyaanics in the $x-y$ plane is shown in figure (12.1).

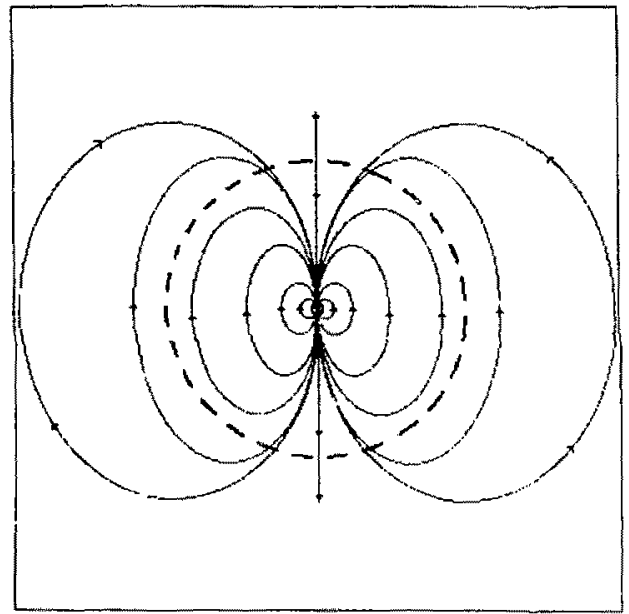

Figure 12.1: The phase portrait of the equations in the $x$ "y plane. The origin acts like an ariractor both forward and barkward in tims. 
Cotsider the erolution of the radius: $\sqrt{x^{2}}+\overline{y^{2}}$. For every point $(x, y)$ where the radius is getting smaller, there is a correspondigg point $(x,-y)$ where it is getting larger. That is, if you look on a circle of radius $r$, the length of are where the vectorfield points outward is the same as where it points inward. Nonetheless almast every initial condition (excluding the $y$ axis, which you have zero probability of hitting) leads to the eventual decrease of the radius asymptotically to zero. Notice that this decrease takes place not only forward in time but backwards. The origin is almost an attractor for both the true and the time-reversed dynamics. 


\section{Chapter 13:}

\section{Hamiltonian}

\section{Dissipation in Infinite}

\section{Dimensions}

"We now have an example of a so-called irreversible thermodynamic process, such as does not occur in mechanics: in all natural processes the entropy increases. ... Irreversible processes can in no way be simulated by purely mechanical processes."-[Klein, 1928] p.203.

In this section we will examine some interesting facets of the dynamics of infruite dimensional Hamiltonian systerns. One of the key simplifying aspects of dissipative dynamics is the presence of attractors whose behavior dominates the time asymptotics of all nearby initial conditions. In fiute dimensions. Liouville's theorem regarding the volume preservation of Hamiltonian dynamics leads to the Poincaré reccurence theorem. This says that under the time-one map of a bounded Hamiltonian system every seighborbood of every initial condition has points that return to that neighborhood and furthermore almost every point comes back infinitely often arbitrarily close to its startiug point. The proof rests on the pigeon hole principle which savs that with $x$ pigeon holes and $x+1$ pigeons, there mist 
be at least one pigron hole with more than one pigeon. In a finite rolume region. there is only room enough for a finite nutrber of images of a non-zero volume region milet a volume preserving mapping. Eventually two images must intersect and by mapping the intersection region back to the starting region, we find points that recur. This precludes the presence of attractors in finite dimensional Hamiltonian systems (no small region can be special since almost all points go back to where they came from). We will see that infuite dimensional Hamiltonian systems can have attractors of a certain kind.

\section{1. Poincaré Recurrence and Attractora}

The fact of recurrence is bebind Ze.'melo's objection to Boltzmano's $\boldsymbol{H}$ theorem proving the increase of entropy for the Boltzmann equation. The fact of the matter is that the recurence time is quite loog. For merely 10 barmonic oscillators with frequencies about 1 cycle per second and irrationally related. the room in the corresponding 1 in-torus is so vast that the typical time to returo to within one percent of their initial conditions is $10^{20}$ seconds which is longer than the age of the universe. Boltzmann's retort to Zermelo was purportedly: "You should live so long", [Kac, 1959]p, 62 (in response to Losthmidt who objected to getting an irreversible equation from reversible underlying dynamics he replied: "Go ahead, reverse them! !'). These long times also indicate that true ergodicity is not responsible for the experimental validity of statistival mechanics (if we consider a state of a cubic meter of air to be tie number of molecules in each cubic millimeter and if 
the dyoamics is ergodic, the gas visits every state only once in about $10^{100}$ times. the age of the universe).

\subsubsection{The Lack of Recurrence in Infinite Dimentions}

Reccurrence is a chararteristically finthe dimensional phenonmenon. As the dimension gets higher the recurrence time-scale grows exponentially. If we are interested in Guite (but perhaps long) times, it is often physically valid to introduce asymptotics even if it leads to infinite dimensional models. Sometimes the analysis of infinite dimensional systems is easier because the structure "at infinity" organizes the dynamics. The wave equation is easier to deal with than the uvderlying molecular dynamics. When we make such models we must he careful not to befieve them when they depend crucially on asymptotic aspects beyond the value of the limiting parameter at which the modelled system really is. (For an analysis of some very interesting phenomena that occur near molecular wavelengths in a systera asymptotically approximated by the wave equation, see [Masiov, 1976] p. 58). The time to recur grows with the dimension and infinite dimensional systems nced sot rexur. There is no general Liouville theorem in infuite dimensions and orbits need not recur (they can "head of to infuity" along higher and higher dimensions still staying close to the origin). While we have argued that when the system is in too high a dimension the model beromes unphysical, the behavior on the way there ridl reflect the real behavior and properties of the infinite dimensional system may be properties of the real system for long times. 


\subsection{Agymptotic and Llapunov Stability}

For finte dimensional dyuamical systems there are two standard notions of sta* bility of an invariant subset. The weaker of the two is Liapunov stabifity which says that given any neighborhood of the set we can find a (possibly) smaller neighborhood all of whose points have future time orbits lying inside the given neighborhood. This sayg tbat if we perturb a point slightly from our set, it hangs around forever. An example is the equilibrium of a simple harmonic oscillator. The stronger notion is that of asymptotic stability. This says that there is some peighborhood $\boldsymbol{V}$ of our set whose forward time images all lie in $V$ and such that each orbit asymptotes into our st. Asymptotic ally stable sets are called attractors and the poibts which limit on them form the basin of attraction. If an attracting set is a fractal (with respect to your favorite deo nition of dimension), then it is a strange attractor.

\subsubsection{Almogt Attractora}

Recent work of Grebogi, Ott and Yorke has shown that strange attractors can exist without chaotic dynamics (Grebogi, Ott, and Yorke, 1984). For this they needed a definition of attractor which we shall also use. We call a set an "almost attractor" if tor small enough neighborhoods, almost every point eventually asymptote to our set. We have seen in the example of a vector field on the plane alang dipole field lines that it is possible for a point to be an almost attractor both forward and backward in time. By Poincaré reccurrence, this is not passible for finite dimensional Hamiltonian systems. 


\subsection{Reveraible Attractors and Infinite Dimensionai Hamiltoniang}

Infnite dimensional Hamiltonian systens. on the other hand. can haye almost attractors and if the system is time reversible (as most physical syctems are) then it is of the time reversible type. This tytre of structure is responsible for most of the dissipative models in physics that I know of. The resolution of Loschmidt's paradox here lies in the fact that the infinite dimensional model is valid only asymptotically and becomes a bad representative of the underlying finite dimensional system after a long but finste time. Until that time it represents the system well and its dissipation represents real tendencies of the underlying system (when they ultimately break down due to recurrence, we call it a fuctuation).

\subsubsection{Reversible Almost Attractor in the Wave Equation}

A simple example to think about merely consists of the wave equation on a one-dimensional string. It is well known that this system is Hamiltonian. Let us define the state space of the system, to be those displacement and velocity fields which die off exponentially at infinity. This class is preserved by the time evolution for Gaite time (which j:5i translates a wave). Let us assume we are more interested in what the wave is doing under our noses near the origin than what it is doing far away. We will thus put a norm on our space which says that the size of a wave is the integral of the sum $r$, the absolute values of the displacement and relocity ficlds over the line weighted by a Gaussian centered at the origin:

$$
\int_{-\infty}^{\infty}(\mathrm{i} f(x)|+| v(x) \mid) e^{-x^{2}} d x
$$


Since the dynamies is y st translation. cventually every wave in our elass gets "pushed into the wings" far enough so that its norm decays nianotonically to zero. Sote that this happer both forward and backward in time. Thus the quiescent string is a reversible almost attractor for this system. In higher dimensions, compactly supported waves decay even in the uniform norm (i.e. the size of a function is the maximum of its absolute value over all space).

This example has many of the characteristics of the dipole vector field in an infuite dimensional Hamiltonian context. While everything eventually comes into the origin, for any given time there are always states whicb baven't come close yet.

\subsubsection{The Liouville Equation and Koopmeniom}

A natural situation in which this sort of system arises occurs when we consider the Liouville equation of a dy namical system, which is known to be Hamiltonian (albeit with respect to a Lie Poisson bracket). This looks at the evolution on the space of probability distributions on a manifold given by pusbing a distribution forward along a measure preserving map. Koopman suggested studying dynamical properties of the underlying system by the spectral properties of the Liouville operator(see [Cornfeld, Fomin, and Sinai, 1982] p. 323). John Cary and Jobu David Crawford have looked at the Liouville evolution of probability distributions on Arnold's cat map. What one finds is that the riap's effect on distributions is merely to shift Fourier components along hyperbolas in Fourier space except for the zero wavevector component which is invariant. If the original distribution is smooth. then its Fourier series dies off exponeutially. If we put a norm on the distributions which 
weighs the low Fourier components more heavily (say our data is run through a low pass filter. i.e. we perform some kind of averaging or binging), then as our distribution's spectrum goes trotting off to infinity, its porm goes to zero leaving only the coustant part and we again have a reversible attractor up to a constant. They show that this leads to the exponential decay of the autocorrelation of smooth functions,

\subsubsection{Landau Damping}

Similar phenomena are responsible for Landau damping (which damps both forward and backward in time) of waves in plastnas. We start with a distribution of particles (as in figure (13.1)) in the bottom half of the "eye" of the pendulum dynamical vector field (as in starting with particles in a strip in velocities slower than the wave, and eveniy spread out in phase relative to the wave). As time goes on the dynamics inside the eye is like a sbearing harmonic oscillator, which soon smears the inital distribution uniformly through the eye. The effect of this is that the average energy of the particles has gone up (and so the energy of the wave must go down causing damping). This garne shearing will occur backwards in time.

\subsubsection{The Boltzmann Equation and the BBGKY Hierarchy}

The same kind of phase mixing is responsible for the dissipative aspects of the Boltzmann equation as derived from the Hamiltonian and reversible BBGKY hitrarchy (see volume 10, Physical Kinctics of [Landau and Lifshitz, 1960-1981]).

We rewrite the Liouville equation for the distribution function of all the particles 


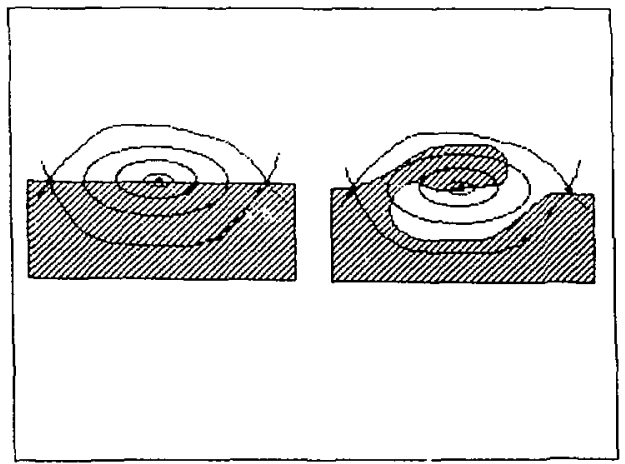

Figure 13.1: The evolution of the distribution function near the wave velocity.

as a hierarchy of equations for 1-particle, 2-particle, ... etc. distribution functions. If we truncate at any stage, we obtain the Liouville equation on the phase space of that many particles. The contribution of the higher order correlations phase mixes away as above leaving only the Boltzmann collision term to first order.

\subsubsection{Disgipation from Resonance}

The same scenerio applies to the random phase approximation for interacting waves. The essential idea here may be seen by considering resonances. One of the most interesting examples is the simple resonantly driven barmonic oscillator. The solution to this problem is given in most elementary mechanics texts but 1 have never seen any discussion of its rather paradoxical properties. Let us consider a 
particle in one-dimension moving in a unit-frequency harmonic oscllator potential which is driven sinusoidally at the same frequency:

$$
\ddot{x}=-x-\cos t \text {. }
$$

For this kind of linear differential equation with an inhomogereous term, the usual metbod of solution is to find a particular solution to the inbomogeneous problem and then to add to this an arbitrary solution of the bomogeneous one. In this case this procedure leads to the general solutiou:

$$
x(t)=A \sin t+B \cos t+\frac{1}{2} t \sin t
$$

This solution is reaily quite remarkable. Regardless of the initial conditions (which are specified $t_{y} A$ and $B$ ), the $t$ sint term eventually dominates and the amplitude of oscillations grows indefinitely with time. But the equation is invariant under $t \rightarrow-t$ ! This means that the same behavior occurs as $t$ approaches $-\infty$.

It is interesting to try to visualize the orbits in the three dimensional $(r, v, t)$ space. On the one hand we lnow that each orbit winds to larger and larger $x$ and $v$ values as $I$ goes toward botb positive and negative infinity. This means that an individual orbit winds on what looks like a one-sbeeteu hyperboloid of revolution along ceatered on the $t$ axis (or a cone if ever both the position and velocity vanish on its trajectory). On the other hand, the entire system is invariant under the translation $t \rightarrow t+2 \pi$. To get some feel for the way this orbits can fi: together in this way, consider all orbits that intersect the $t$ axis. These form an invariant 2 dimensional submanifold "hich includes the $t$ axis. Imagine taking the $t, v$ plane and twisting it about the $t$ axis so that it makes one twist every $2 \pi$ in $t$. The dỵamies 
on the unwound plase is made of parallel straight lines that intersect the $t$ axis at mone angle. They leave the $t$ axis linearly for both positive and negative time and yet the whole field is trasslation invariant in time. When we wind this plane up. the orbits lie on cones. Th- other orbits do a similar thin" on hyperboloids.

Let us think of the harmonic osrillator as being a piece of a larger system and the driving as arising from the rest of the system. Time asymptotically the oseillator only sucks energy from the rest of the system and this ts tiue both forward and barkward in cime. Let us calculate the time variation of the average energy in the oscillator for long times:

$$
\begin{aligned}
E= & \frac{1}{2} x^{2}+\frac{1}{2} x^{2} \\
= & \frac{1}{2}\left(\frac{1}{2} \sin t+\frac{1}{2} t \cos t+A \cos t-B \cos t\right)^{2} \\
& +\frac{1}{2}\left(A \sin t+B \cos t+\frac{1}{2} t \sin t\right)^{2} .
\end{aligned}
$$

For times large compared to $A$ and $B$ (we can make this comparison because we have made everything dimensionless), only terms with an unadorned $t$ in them are important:

$$
\begin{aligned}
E & =\frac{1}{8}\left(t^{2} \cos ^{2} t+t^{2} \sin ^{2} t\right) \\
& =\frac{t^{2}}{8} .
\end{aligned}
$$

Thus the oscillator energy grows without bound as $t$ goes to either positive or negative infinity.

In real closed Hamiltonian systems, the energy that the oscillator is suckir 6 out of the rest of the system will eventually cause the damping to change. In nonlinear systems the frequency of driving will i,e pushed away from resonance. In linear systems the amplitude of driving will go dow until eventually the ascillator 
drives rie rest of the system leading to oxtillation on long time seales. The slowly changing amplitude of driving can also be viewed as having energy in frequencies slightly away from resonance. As long as we are in a situation where the rffect of the oscillator on the rest of the system is small, however, the solutions will be close to the resonant one. In such cases we get time-reversible dissipative behavior fur long times.

This is exactly the sense in which non-equilibrium thermodynamics is dissipative. If we are far from equilibrium then there are many more ways to be perturbed closer to equilibrium than away from it. Statistically the motion appears to go in one direction (eg. entropy increases). The same increase occurs backward in time, however, since the same statistics applies. If we are in a state with low entropy, then it is most fikely that a few moments ago we had bigher entropy and that a few moments hence we will also have bigher entropy. As we get closer to equilibrium. the imbalance in the number of perturbations toward and away from it diminshes. Exactly at the bighest entropy state the ody way you can go is down. In equilibtium the dissipative properties dissappear and instead we have fluctuations. Since exactly the same mechanisms cause dissipation far from equilibrium and fluctuations in equilibrium, the corresponding rates are related. This is the content of the Huctuation-dissipation theorem. (As an example, a Brownian particie satisfying the Langevin equation:

$$
\frac{d v(t)}{d t}=-\nu v(t)+\delta F(t)
$$

in a teat bath at temperature $T$ which causes the partirle's notion to be damped at rate $\nu$ and driven with random force $F(t)$, the fluctuation-dissipation throron 
says that

$$
\nu=\frac{M}{2 T} \int_{-\operatorname{in}\left\langle t_{\psi}\right.}^{\infty} d t\langle\delta F(0) \delta F(t)\rangle .
$$

)

The mechanism of time-reversible dissipation due to resonance underlies many important plysical processes. In many of these situations, the driver changes frequency so as to slowly pass through resonance. An example might be an eikonal light wave passing through a medium whose electrons are hound like harmonic oscillators. The light wave loses energy to the resonantly excited oscillators (note that bere it might be that the wave is of constant frequency but the ascillator frequency changes slowly as we progress through the medium; the physics of this situation is identical). For frequeacies of driving which are sot exactly resonant, the oscillator begins by removing energy, but eventually gives it back (on a time scale of the reciprocal of the frequency deviation from resonance) leading to an oscillatory overall behayinr which does not change the average energy of the driver. If we watch the systum for tine $T$, then all frequencies within order $1 / T$ of the resonant. frequency will behave as if they are resonant. As we slow the frequency variation of our eikonal wave asymptotically and watch the effect for longer time we have two effects: 1) we are in resonance for a longer time causing a larger dissipation and 2) since we are watching longer, less of the nearby frequencies contribete any dissipa tion. As time goes to infinity there is net dissipation which arises from arbitrarily small peighborhoods of the tesonant frequency.

Let us see this explicitly in an example. We consider the driven oscillator:

$$
\ddot{I}=-I+F(t) \cos (w(k t) t) .
$$


Where we assume that the resonance occurs at ! - (and nowhere che)

$$
-\{(1)-1
$$

and that we pass through resonance with non-zero speed:

$$
\left.\frac{d+{ }^{\prime}}{d t}\right|_{t=0} \equiv \omega^{\prime}>0 .
$$

Let us denote $\dot{x}$ by $v$. We wish to solve this asymptotically as $t-0$. We begin (as in the methods discussed in chapter 3) by going to a "rotating" system of courdinates in phase space:

$$
X=x \cos t-v \sin t \quad V=v \cos t+x \sin t .
$$

The dynamics of these coordinates is

$$
\begin{aligned}
\dot{X} & =\dot{x} \cos t-x \sin t-\dot{v} \sin t-v \cos t \\
& =-F \cos (\omega t / \sin t \\
& =\frac{-F}{2}(\sin ((\omega+1) t)+\sin ((\omega+1) t))
\end{aligned}
$$

and

$$
\begin{aligned}
\dot{V} & =\dot{v} \cos t-v \sin t+\dot{i} \sin t+x \cos t \\
& =F \cos (\omega t) \cos t \\
& \left.=\frac{F}{2}(\cos (\omega+1) t)+\cos ((-1) t)\right) .
\end{aligned}
$$

W. may integrate these equations using the method of stationary phase. which was: discussed in section 7.1.3. Introduring the rescaled time $\bar{c} \equiv$ a and using stationary phase. we see that to leading order the change in $X$ in pasing through the resonance 
is:

$$
\begin{aligned}
& \Delta x=c+\int_{T / /}^{T / t}-\frac{F}{2}(\sin ((-+1) t)+\sin ((\omega-1) t)) d t \\
& C+\int_{-T /,}^{T / r} \frac{-F}{2} \sin ((\omega-1) t) d t \\
& \sim C+\int_{-T / e}^{T / r} \frac{-F}{2} \sin \left(\omega^{\prime}\left(t^{2}\right) d t\right. \\
& \sim C-\frac{1}{\epsilon} \frac{F(0)}{2} \int_{-\tau}^{\tau} \sin \left(\omega^{\prime} \frac{\tau^{2}}{\epsilon}\right) d \tau \\
& \sim C-\frac{1}{\epsilon} \frac{F(0)}{2} \int_{-\infty}^{\infty} \sin \left(\omega^{\prime} \frac{\tau^{2}}{\epsilon}\right) d \tau \\
& \sim C-\frac{1}{c} \frac{F(0)}{2} \Im \int_{-\infty}^{\infty} \exp \left(\frac{i \omega^{\prime} \tau^{2}}{\tau}\right) d \tau \\
& \sim C-\frac{1}{\epsilon} \frac{F(0)}{2} \Im \int_{-\infty}^{\infty} \exp \left(\frac{i \omega^{\prime} \tau^{2}}{\epsilon}\right) d \tau \\
& \sim C-\frac{1}{\epsilon} \frac{F(0)}{2} \Im\left(\frac{\sqrt{2 \pi \epsilon}}{\sqrt{2 \omega^{\prime}}} e^{2 \pi / 4}\right) \\
& =C-F(0) \sqrt{\frac{\pi}{8 \cdot \omega^{\prime} c}} \text {. }
\end{aligned}
$$

Similarly,

$$
\Delta Y=C_{2}+F(0) \sqrt{\frac{\pi}{8 \omega^{\prime} c}} .
$$

Thus asymptotically there is a net dissipation which grows as the reciprocal of the square root of the eikonal parameter. Notice that for any real system the initial conditions will determine whether the oscillator takes or gives up energy for a time independent of $\epsilon$. The asymptotics we have introduced always beats this time for some value of $\ell$. Thus the asymptotic system can have dissipation without constraint on the initial conditions whereas any real system may not have this behavior. I think this is a good example for sceing why an asymptotic approximation to a system (say Boltzmann's equation) may have propertics like irreversibility and lack of reccurence 
whirli are not possessed by any real system. These are new anyuptour concepte whirh validly apply to the system whenever the asymptotirs is valid

\subsubsection{Resonant Coupling of Eikonal Waves}

For this kind of experiment ve must really use eikonal driving since we are interested in the response as we turn on the oscillations. Traditionally in plasma physici one uses a linear analysis in a uniform system and so finds the normal modes for the evolution operator. These will be unphysical infinite plane waves and lead to subtleties like a singular spectrum (as in van Kampen modes) which obscures the physics of the analysis. It is interesting to rethink these analyses in terms of eikonal waves. For example, the response function of a harmonic oscillator at its natural frequency is infinite. If we excite it with a slowly varying frequency, we may use stationary phase to study the passage through resonance and we see that there is no infinity. Only in the asymptotic limit does the response lead to a pole on the natural frequency. In tradicional analyses of the three wave interaction (which arises by retaining third order terms in the nonlinear coupling of waves) one studies the response of an infinite plane wave to the piesence of two other infinite plane waves. In reality, given two eikonal waves in three dimensions, the resonance condition can only be satisfied on a 2-dirneusional spatial surface which moves through space. The generated wave will inciude these geometrical aspects. 


\subsection{The String with a Spring}

Let us nou give an interesting example which shows bow the effect of an infinite dimensional system on a finite dimensional piece say sometimes be modeled by dissipation. The idea is that, asymptotically in time. the infinite number of modes will only suck energy out of the system, learang to the appearance of dissipation.

The simple system we have in mind is a half infinite string whose end is attached to a mass on a spring (or any other shaking system). If we consider only string displacements which are damped at infinity, then asymptotically the string behaves as if it were quiescent (any energy going away from the end doesn't affect us, and all the energy going toward it refiects and is eventually leaving it).

Let the string's displacement $w$ and its velocity $v$ be functions of the position $I$ along the string. Let the tension in the string be $T$ (i.e. the force along the string) and the mass density be $\rho$. The transverse force that the string applies at its end is given by the transverse component of the tension, and for small displacements this is just the tension times the slope:

$$
T \frac{\partial w}{\partial x}
$$

The wave equation arses from Newton's equation for a little piece of string. where the transverse force is the difference of the force due to the string on the left a id th - string on the right. Asymptotically this is proportional to the second derivative of the displacement, leading to the usual wave equation:

$$
\rho \frac{\partial^{2} w}{\partial t^{2}}=T \frac{\partial^{2} w}{\partial x^{2}} .
$$


This is known to be Hamilnonian. The solutions aray from the chel are sume of a function translating rigidly to the left and to the right witl veloe ity

$$
c=\sqrt{\frac{T}{\rho}}
$$

For a quiescent string. if we shake the end according to

$$
u(\mathbf{0}, t)=y(t),
$$

then we get only a wave travelling away and the string displacement for all time is

$$
w(x, t)=y(t-I / c)
$$

What then is the force the string applies to our shaking apparatus? From the above it is

$$
\left.T \frac{\partial u}{\partial x}\right|_{x=0}=\left.T \frac{d}{d x} y(t-x / c)\right|_{x=0}=-\frac{T}{c} \dot{y}(t) .
$$

If the shaker was a barmonic oscillator

$$
m \ddot{y}=-k y \text {. }
$$

then we could forget about the string if we changed the equation to

$$
m \ddot{y}=-k y-c \rho \dot{y} .
$$

This is just a damped oscillator. Thus an infinite dimensional piece of $₫$ Hamiltonian system is replaced by an effective dissipation in the cvolution of a finite dimensional piece. Notics that the exact form of the system to which the string is coupled is completely irrelevant. It is this fact which makes such replacement physically relevant (if it behaved dissipatively only in extremely special circumstances we wo sld have no reason to think of it as a replaceable piece during simplification). 


\section{Chapter14: \\ Reinsertion in \\ Area-Preserving Horseshoes}

"It is said that Newton communicated to Leibniz an anagram somewhat like this: azaaabbbecceii, etc. Leibniz naturally understood nothing at all of this; but we Who have the key know that this anagram meant, translated into modern language: I know how to integrate all differential equations; and this suggests to us that ejther Newton was very lucky or else be beld a peculiar illusion."-Poincaré (1908) [Hirsch, 1984!

Over the last ten years, the idea of intrinsic stochasticity in the deterministic dynamies of low dimensional dynamical systems has joined the mainstream of physical thought. Every month physics journals carry many reports of chaos (as the phenomenon has come to be known) in new pbysical situations. Applications to piasma physics are given in (Srrith and Kaufman, 1975]. [Smith and Kaufman, 1978!, and [Kau[man, 1979]. The chaos has always been there, but until recently the mathematical tools for noticing it were not well known in the physics community. Every example of chaos that $I$ know of has within it a construction dubbed 
by Smale the borseshoe in ịtrale. 1967. The essentual phenomena nay be seen in a taffy puller (and indeed Ot to Rössler designed his attractor based on it) The key feature of a taffy puller is its continual stretching of the taffy. To keep it in a finite voiume, the tafy must also be bent over. These two features lead to dynamics where the taffy is tboroughly mixed togetber. (This may be secn soniewhat grotesquely by considering the fate of a fly which lands on the sweet melling taffy. In on the order of ten cycles, there will be a piece of the fly in each piece of the taffy.)

\subsection{The 2x mod 1 Map}

The basic mathematical model of this stretching and mixing is the map from the circle to itself given by

$$
x \rightarrow 2 x \bmod 1 .
$$

This dynanics bas periodic orbits of every period, dense orbits, and orbits which hop from the interval: $|0,1 / 2|$ to the interval: $[1 / 2,1\}$ according to any desired (possibly random) sequence of $L$ 's and $R$ 's (p. 106 of 'Cornfeld. Fomin, and Sinai. 1982]). This is easy to see by the method of symbolic dynamics. Call the teft interval 0 and the right one 1. The interval in which a point lies is given by the first digit in the binary expansion of the uum er corresponding to that point. Since $2 x \bmod 1$ just shifts the binary point and lops of the integer part, we see that the sequence of hops is given by the sequence of digits in the binary expansion, but this is arbitrary. This map cannot appear in ien? dynamics since it is 2 to 1 (but variants 
of it appcar in prujected dynamics all the time, for example, Lorenz's equations as in Civckenturimer and Holmes. 1983) p. 276). 


\subsection{The Baker's Transformation}

The trouble, of course. is that we are continually losing information about our intitial condition, so knowing where we are isn't enough to tell us where we canne from. We can remedy this by adding another variable ubich keeps track of the lost information. The symbolic dyamics model is the space of doubly infirite sequences of 1's and 0's with the map being a shift. Tbis clearly has the same stochastic properties, but is invertible. A dynamical realization may be found in the so called baker's transformation, named because of the similarity of the map to the experiences a baker puts dough through (particularly filo dough). The baker first rolls the dough out so that it is thinner and longer, he then cuts the elongated dough into two pieces, places one atop the other and repeats the process. The corresponding map is from the unit square to itself:

$$
\begin{aligned}
& x \neg 2 x \quad \bmod 1 \\
& y \vdash \quad \frac{y}{2} \text { if } 0 \leq x<\frac{1}{2} \\
& \frac{y+1}{2} \text { if } \frac{1}{2} \leq x<1 .
\end{aligned}
$$

If we take $y$ 's binary expansion backward and adjoin it to $x$ 's binary expansion. then this map is exactly the shift on the doubly infinite sequence of the combines digits ( (Cornfeld, Fomin, and Sinai. 1982] p. 9). Unfortunately, this still cannot appear as a return map because it is not continuous (due to the baker's knifc). 


\subsection{The Horseshoe}

If instead of pushing the two pieces of dough all the way together, we leave a gap. then we may initued this in a smooth map which has all the interesting stochastic properties ( Guckenheiner and Holmes, 1983\} p. 230). The horseshoe is such a map as shown in figure (14.1). Because of the gap (which under the map gets scot to a skinnier gap. etc.) the invariant set associated with the symbol sbift is a Can'or set.

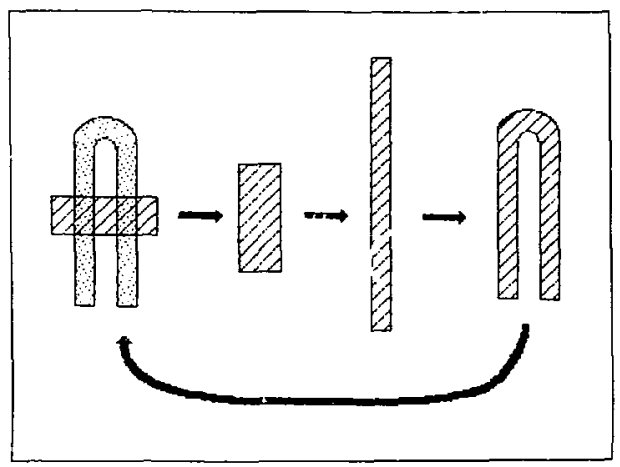

Figure 14.1: Smale's borsesboe map.

That such a map might appear in any natural systems is at first perhaps surprising, but in fact it is quite common as we now sbow. Consider a dynamical system whose state space is threc dimensional (for example, the three dimensional 
energy surface of a two degree of freedom Hamiltonian system). We may study the neighborhood of a periodic orbjt by means of the two dimensional return map induced by the dynamics on a two dimensional surface transverse to the orbit. The orbit is a fixed point of the map and we consider the case where its linearization is byperbolic with one eigenvilue inside the unit circle of the complex plane, and one outside. The stable manifold theorem states that there exists a smootbly inbedded two dimensional manifold in our state space consisting of all points which asymptote to our orbit as time goes to positive infinity. It must approach the orbit tangent to the linear attracting direction and the orbit. The corresponding unstable manifold theorem says a similar thing about the points that asymptote to our orbit under the time reversed dynamics. We will now see that the Poincaré return map must contain a harseshoe if the stable and unstable manifolds intersect transversally. The first image of a point of intersection must also asymptote to the orbit both forward and backward in time, implying that the stable and unstable manifolds must intersect again, as in figure (14.2) (repeating this argument shows that tbey must intersect an infinite number of times). As in the figure, if we choose an appropriate rectangle. some iterate of it gives us a horseshoe imbedded in our dynamics. 


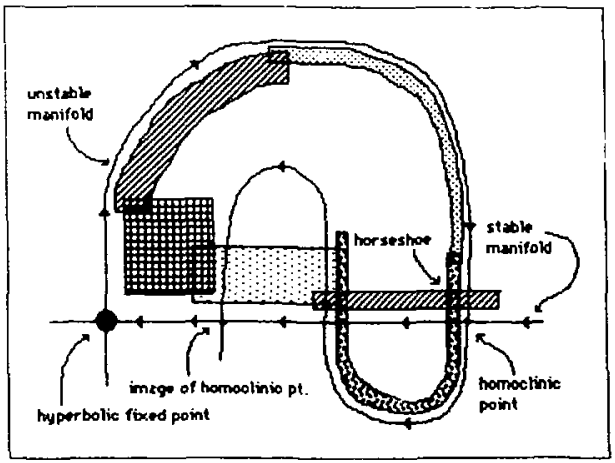

Figure 14.2: A transversal intersection of the stable and unstable manifolds implies the presence oi a horseshoe for some iterate of the map.

\subsection{Example of Horseshoes in Gyromotion}

Let us give a plasma example which has horseshoes in the dynamics. Consider the two-dimensional $x, y$ motion of a charged particle in a linear magnetic field

$$
B_{2}=\alpha y,
$$

near the region where it vanishes. Define the vector potentiar by taking $A_{y}=0$, so that

$$
B_{z}=\frac{\partial A_{y}}{\partial x}-\frac{\partial A_{x}}{\partial y}=-\frac{\partial A_{x}}{\partial y}=\alpha y,
$$

leading us to take

$$
A_{x}=-\frac{Q}{2} y^{2} .
$$


As usuad. let us set $e-m=r-1$. The Hamiltomisun is then

$$
\left.H=\frac{1}{2}\left(t_{z}-\frac{\alpha}{2} y^{2}\right)^{2}+p_{y}^{2}\right) .
$$

and since it is r-translation symmetrir. $p_{x}$ is constived. The equatuons of morion are

$$
\begin{gathered}
\dot{x}=\frac{\partial H}{\partial p_{x}}=p_{x}+\frac{a}{2} y^{2} . \\
\dot{y}=\frac{\partial H}{\partial p_{y}}=p_{y} . \\
\dot{p}_{x}=-\frac{\partial H}{\partial x}=0 .
\end{gathered}
$$

and

$$
\dot{p}_{y}=-\frac{\partial H}{\partial y}=-\left(p_{x}+\frac{\alpha}{2} y^{2}\right) \alpha y .
$$

Let us denote $p_{x}$ 's constant value by

$$
p_{x}=p_{x}^{0}
$$

We know that by reducing by the $x$ translation symmetry we may get equations involving only $y$. The $y$ crolution is given by

$$
\ddot{y}=\dot{p}_{y}=-\alpha p_{x}^{0} y-\frac{\alpha^{2}}{2} y^{3} \text {. }
$$

This is Duffing:s equation, whicb bas been well-studied. It represents the Hamiltonian dyuamics of a particle muving in a poteutial given by

$$
V(y)=\frac{a}{2} p_{x}^{0} y^{2}+\frac{a^{2}}{8} y^{4}
$$

Let us assume that $a$ is positive. If

$$
p_{x}^{0}=\dot{i}-\frac{\alpha}{2} y^{2}
$$




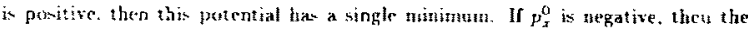
porential woll has two mimma as stou is in figure (14.3a). The phase portrait for this case is shown in figure (14.3b). Far from the origin, all orbits are periodic and encircle the origith. As we move in, we see that there are two stable elliptic fixed point: and an unstable bype-bolic fixed point. The fyperbolic fixed point bas two bomoclinic sepuratrices which are susceptible to horseshoes under periodic driving. In fart using the method of Melnikov, Holmes and Marsden have shown that the driven Duffing equation has transversal homoclinic points and therefore horseshoes ( Guckenheimer and Holmes, 1983] p. 184). If we force our particle in this magnetic held with a low amplit tde wave in the $y$ direction, it too will have horseshoes.

What do the orbits look like? For the unforced system, the particle can move in a straight line along the $I$ axis with any velocity, as in fgure (14.3c). Since the Duffing oscillator is bounded in $y$, every orbit has a maximal excursion in $y$, where $\dot{y}=0$. Let us then study the orbits with initial condition

$$
y=y_{0} \quad \dot{y}=0
$$

for various values of $p_{x}^{0}$ and any $I$. When $p_{x}^{0}$ is positive, the $y$ motion is represented by a Duffing oscillator with a single minimum in its potential. The $x$ velocity

$$
\dot{I}=p_{x}^{0}+\frac{a}{2} y^{2}
$$

never goes negative and so : he particle moves inexorably in the positive $x$ direction as in figure $(14,3 \mathrm{~d})$. When $p_{x}^{0}=0, \dot{i}$ vanishes at $y=0$, but is positive elsewhere. This leads to particle motion which crosses the feld reversal perpendicularly at in tigure (14.30). As $p_{x}^{0}$ becomes negative, the orbits actually meve in the negative $x$ 
ditection whis aver

$$
y_{1}<\sqrt{-\frac{2}{a} p_{x}^{3}}
$$

as in figure (14.3f). The Duffing equation now has two minima but we are in the oscillatory region that cucircles them. As $p_{x}^{0}$ gets more degative, the hops overlap one anotber as in figure $(14.3 \mathrm{~g}$ and b) until successive loops actually coincide to give a "figure eight ${ }^{*}$ orbit as sbown in figure (14.3i). As $p_{x}^{0}$ gets still more negative. the net motion is in the negative $x$ direction, whike still in the positive direction at the tops and the bottoms of the loops. The particle path is made of alternating curliques about the ficld reversal line as shown in bgure $(14,5)$ and $k)$. When

$$
p_{x}^{0}=-\frac{\alpha}{4} y_{0}^{4}
$$

we are on $x$ separatrix of the Duffing equation and the particle makes but a single loop, asynuptoting to $y=0$ both forward and backward in time as shown in tigure (14.31). There is another orbit eorresponding to the other separatrix which has $y$ nesative as in thgure $(14.3 \mathrm{~m})$. For

$$
p_{x}^{0}<-\frac{a}{4} y_{0}^{2}
$$

we are inside the separatrix and the particle executes ordinary gyration as in higure (14.3n). When

$$
p_{x}^{0}=-\frac{\alpha}{2} y_{0}^{2}
$$

we are at a thathe fixed point of life Dulfing equation, $\dot{I}=0$, and particle notion has stopped. For $p_{x}^{u}$ more negative, the particle gyrates in the region $y>$ yn. 
Under oscillatory perturbation, there are orbits ncar the separatrix that go around one and then the other separatrix according to any (possibly random) sequence of $0:$ and 1 s. The resulting particle orbit has corresponding upward and dor nward curliques, as shown in figure (14.3p).

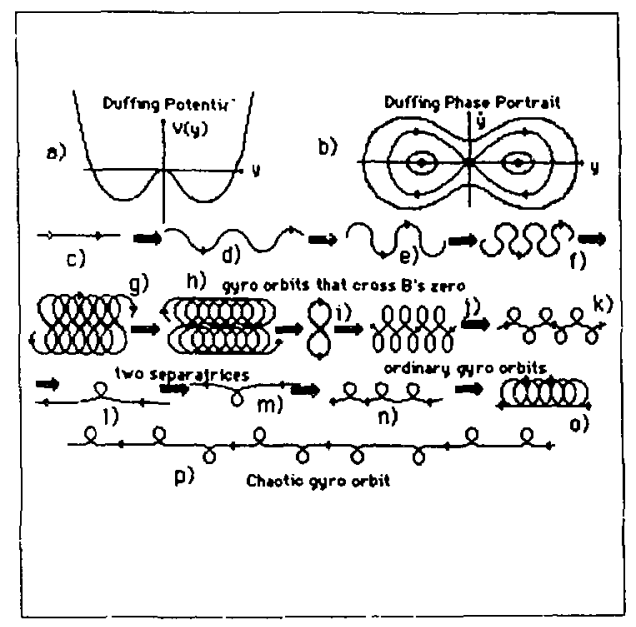

Figure 14.3: The Duffing potential, phase portrait, corresponding particle orbits, and a chaotic orbit. 


\subsection{Area Preserving Horbebhops and Rpingertion}

The issue that we would like to address here concerss the structure of the stable and unstable manifolds in the area preberving (as arising from a Hamiltonian xystem) as opposed to the dissipative cage. As we extend the stable and unstable manifolds in the dissipative case, we obtain the intricate pattern shown in figure (14:4). We will see that the area preserving case must be more complicated.

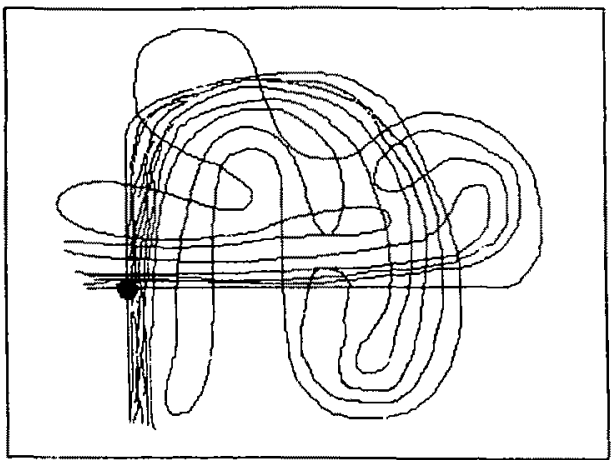

Pignre 14.4: The structure of the st thle and unstable manifolds in the dissipative case.

If we $t \Gamma_{y}$ this same construction in the area preserving case (figure (14.5)), we run into problems. As in that figure, the region $A$ bounded by the stable and unstable manifolds is taken to the region $A^{\prime}$ of the same area. This is tsken to $A^{\prime \prime}$ and so on. In real gystems, this structure is often bounded between two curves 


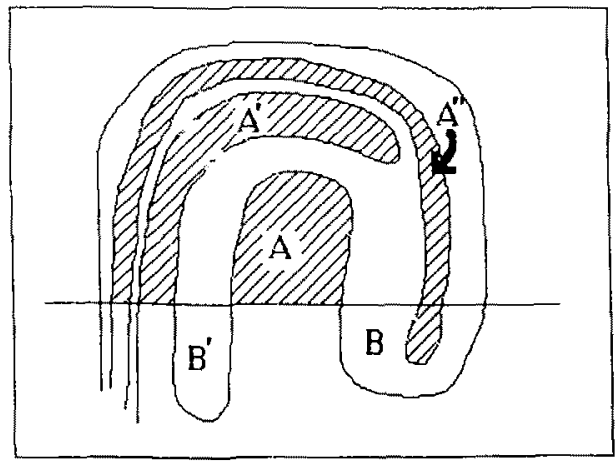

Figure 14.5: The seemingly paradoxical tongues which must intersect.

corresponding to Kolmogorov-Arnold-Moser tori ( [Abraham and Marsden, 1978] p. 582) and so is constrained to a finite area. But because the tounges all have the same area and there are an infinite number of them, they must intersect one another (and in fact an iufinite number of them inust intersect). Because the whole picture is taken to jeself under the map, each tongue must intersect an infinite number of others, including both an infinite number of images and preimages (if tongue $T$ interserts $M^{2} \cdot T$, then it must intersect $M^{-i} \cdot T$ as well as one can sec by applying $M^{-4}$ to both sets). Upon first examining the figure it is a mystery how these tongues can possibly intersect. Neither the stable or the unstable manifolds ran cross themselves (since they are injectively immersed copies of the real hine). One therefore concludes that if tongue $A^{N}$ is going to intersect $A$, it cannot come 
in through the top segment of $\boldsymbol{A}$ (which is a piece of the unstable manifuld) bitt must come in through the bot tom (which is a piece of the stable manifold and so is allowed to intersect the top of $A^{N}$ s tongue). The question is: how does $A^{x}$ get down to the bottom border of $A$ ? The unstable manifold makes escentially a complete loop (it includes the origin as it leaves vertically, and it limits on the origin on the right), leaving no room for $A^{N}$ to sneak through. We quickly come to the conclusion that if $A^{N}$ is going to get inside $A$, then one of the lower tongues $B^{M}$ must as well and $A^{N}$ gets in via $B^{M}$.

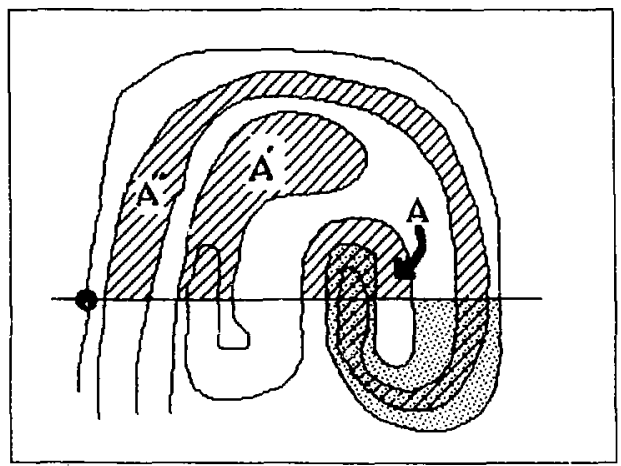

Figure 14.6: The Re-iasertion of tongues forced by area preservation.

This implies the much more complex strurture shown in figure (14.6) than? have seen described in the literature. The number of tongues between a tongue 
and the first lower tongue to insers into it is an invariant of the map. Furthermore as we constrain the area within which this structure lies, relative to the area of a tongue, fhe point at which reinsertion must take place gets nearer and nearer (since a tongue must intersect more of its colleagues), until no structure is possible when the area of a congue is larger than the total allot ted area. Thus we see that Hamiltonian systems are forced to have a very intricate tongue structure. Recent work of Holmes and Whitley, 1984| has studied the change in bifurcation sequences in "shoemaking" in going from the dissipative to the non-dissipative case. They find a continuum of different bifurcation sequences. It would be interesting to see how the intersection structure of the horsesboe tongues evolves in this sequence. 


\section{Chapter 15: \\ Renormalization

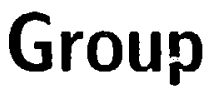

Recentls a number of previously intractable problems in several verv different areas of physics have been successfully tackled using renornalization group techniques. Running through tbese treatments is a beautiful set of ideas which are fairly easy to understand, even though the actual calculations can become quite complex. The goal af this section is to present the essential concept in simple physical situations, where it is easy to see what is going on. We sketch the pbysical idea of renormalization in the original context of the statistical mechanics of spin lattices and then use it to prove the central limit theorem.

\subsection{Scaling and Univeraality}

It is a quite common physical occurrence that certain forms appear over and over in many different contexts. In satistical pbysics we see the Gaussian distribution $e^{-\tau^{2}}$ and the Boltzman distritrution $e^{-E / k T}$ appearing again and again. In electromagnetism we have the monopole and dipole poteutial functions, for example. In radiation theory there are the plane wave. dipole radiatiout. and the firy 
function near saustics In quantum mechanics we continually see the free particle. the hamonic oscillator, and the rigid rotator. In dynamics there exist certain canonical forms for change (eg. the Hopf bifurcation, the saddle-node bifurcation. period-doubling cascades, and the breakdown of $\mathrm{KAll}$ tori) which appear in many rontext:

In many cases the effect of a physical system on the rest of the world is only felt on a large scale in space-time which averages over the detailed structure. It is a common tool in physics to study a particular instance of a system by thinking of it as a member of a family of relaied systems. Sometimes by looking at the family, as opposed to the individual, we see new regularities. This is the basis for perturbation theory which studies the rase of interest by finding an easily soluble case nearby and studying bow tbe differences change tbe bebavior.

Often, one may formalize these beurstic notions by introducing a "spacer, which is often infinite dimensional, whose points represent physical systems. For the statistical systems, we "step back" from the system (i.e. look at it from a greater distanre), and treat all bebavior as if it were occuring on a smaller scale. For example. we might consider photographs of the ocean's surface taken at different beights. The rescaling is called repormalization. In the situations of interest, the renormalized system is again a member of our space, but corresponds to different values of th parameters. The ocean with a tida! wave may, from a distance, bebaye just like a ripple in the tide pools on the shore. The ripple may need to be in water with a different surface tension or viscosity, however. (Such ideas ace actually used for special effects in movies. Since one usually has control of the seale of space and 
time but not of viscosity, the small-scale waves in orean scenes and the small-scale eddies in explosions often do not look right.) Tne renormalization map that rescales a system sometimes bas a Exed point whose properties can he used to study nearby systems. For example, as we get further and further from the surface of the ocean. it looks calmer ald calmer.

We shall not discuss the examples of renormalization in dynamical systems here. They work in much the same way, but the renormalization usually corresponds to looking at the system through a finer and finer microscope. For example, if we zoom in on a byperbolic fixed point of a vector field, the flow becomes more and more like its linearization about the fixed point. A linear system is taken to itself under rescaling and so is a fixed point of the renormalization map. 


\subsection{Magnetic Spin Lattices}

As an example, let us look at the Ising spin model for which Kadanof introduced many of these scaling ideas (see, for example, the review article [Wilson and Kogut. 1974] and the references given there). We imagine an infinite checkerboard with a spin at the center of each square which may have a magnetic moment pointing ejther up or down. We imagine that gearby squares feel each otber's magnetic felds and that it is energetically favorable for them to have their spins pointing in the same direction.

The whole system is in contact with a heat bath with temperature $T$. This tends to cause the spins to jostle between up and down. The bigher the temperature, the more violent the jostling. The spin-spin interaction tends to prohibit the relative jostling of neighbors. If the temperature is very high, then the average kinetic energy of the degrees of freedom of the heat bath will be much greater than the aligument potential energy of a spin due to its interaction with its neighbors. In this case the spins will piggle between up and down relatively independently of the state of their neighbors.

If the temperature is very low, then the average thermal energy will be much less than the interaction potential energy an 1 the spins will nnly rarely be able to overcome it and Bip relative to their neighbors.

When $T=\infty$ the interaction is completely negligible and the stat istics of a spin are independent of its neigbors. The probabilities for up and down are the same and there is no correlation between the probability distributions $f$ or different spius. When $T=0$ the thermal energy is completely negligible compare, to the interaction 
potential energy. The spins all point in the same direction, say up. The probabilit! distribution for an individual spin has spin up with probability one and down with probability zero. Again the distributions for the individual spins are unrorrclated Even if a spin bappened to he pointing down (an infinitely improbable event). the probability distributions for the otber spins would remain uncbanged.

Now we know the statistics of the spins in two states: $T=0$ and $T=\propto$. What do states near these two look like? If we are at a high but finite temperature, then spins are still kicked between up to down. the probability of each being $\frac{1}{2}$. Now: however, the statistics of one spin depend slightly on the state of its neighbors. There is some tendency for neighboring spins to flip together and one will sce little patches of spins pointing the same direction. If we kncw a spin is up, then its nearest neighbors are slightly more likely to point up than down. Next nearest neighbors are affected even less, and one can show that the correlation decays exponentially as we move away from the upward pointing spin. The patches of coherent spirs are finite in extent and get smaller as the temperature gets bigher.

When the temperature is low but not zero, spins are still much more likcly to point up than down. Now, howeyer, if a spin is flipped to down this slightly affects the neighboring spins. Nearest neighbors bave a slightly higher probability for Elppiag than usual. Again the effect dies off exponentially. The state looks like a sea of upward pointing spins with little islands of spins which poine down. As th. temperature is decreased, the islands get smaller.

We now introduce the idea of renortalization. If nur eyes are blurry, when we steg away fron the infinite chec rbourd we will only notice the average spin 
over a segion. If we are near $T=x$. then as we step back. neightworing spins becorme a single spin to us and we are ever more unable to resolve the last vestiges of corrclation among neighbors. The further we go the more the sybin's statisties look like those of $T=x$. We may codify this idea, by introducing a (typically infinite dimensional) space whose points represent the statistical state of our spins (i.e. the space of probability distributions on the space of spins). The operation of "stepping back" or rescaling takes one distribution and gives us another one. We may therefore think of this as a mapping on this hig space and we denote it by $R$ which stands for "renormalization group operator". The infinite temperature state (i.e. the probability distribution is just an uncorrelated product of half up and half down distributions for each spin) is a fixed point of this mapping. We have just indicated that all nearoy states get even closerto this fixed point under the renormalization map, and so it is a stable fixed point.

Similarly, the state representing zero temperature (again an uncorrelated product of distributions for each spin, each of which is probability one for being up (actually the real one is a sum of two uucorrelated states on all spins; one for up and one for down)) is a fixed point. If we are near zero, then as we step back, the "cloud" of spins that an errant spin takes with it on the rare occasion of a lip gets smaller and smaller until in the limit there is no such cloud. So zero temperature is also a stable fixed point.

There is clearly a path from zero to infinite temperature labeled by the temperature itself. At some point on this path we must lcave zero's hasin of attraction and enter infinity's (it is not hard to show that there are 'r stable fixed points). 
He expect there to be a codimension-one surface that forms the boundery het wren these two basins. There is and it is the stable manifold of a third fixed point with a 1-dimensi. 1 unstable direction. While the correlation length of neighboring spina was zero for the other two fixed points, it is infinite for this one (it must be either zero or infinity, since these are the only two numbers that are equal to themselves when multiplied by a rescaling factor). The corresponding temperature is called the critical temperature and the point on a path where it intersects the separatrix is called a critical point. Because there are fluctuations on all scales there, it is often associated with such phenomena as critical opalescence (where a normally clear substance becornes milky white due to fluctuations which can scatter all wavelengths of light). If we consider the place at which our path pierces this codimension 1 stable marifold under applications of $R$, we see that the path asymptotes to the 1-dimensional unstable manifold (see figure (15.1)). Thus this represents a universal path from zero to infinite temperature, and the way it crosses the separatrix will represent that of any path seen from far enough away. In particular, the unstable eigenvalue will tell how fast things scale as we cross the critical surface and may be used to calculate the universal critical exponents corresponding to this fixed point. 


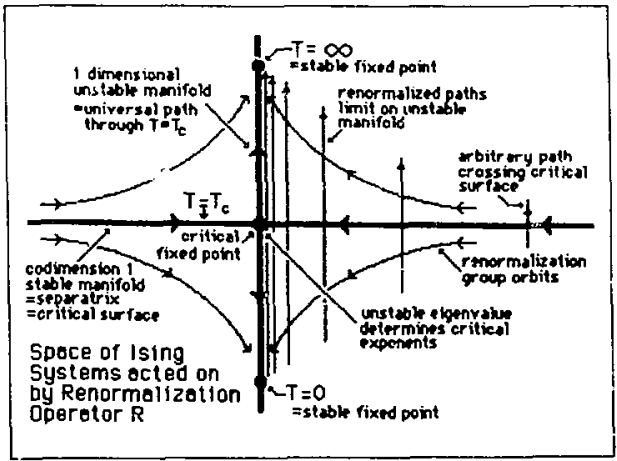

Figure 15.1: The space of spin probability distributions with two stable fixed points corresponding to zero and infinite temperature and a co-dimension one unstable fixed point corresponding to the critical point.

\subsection{The Central Limit Theorem}

One very old example of universality is the prevalence of Gaussian probability distributions in the statistical description of physical phenomena. The mathematical theorem descr:hing the approach of the distribution of a sum of random variables to Gaussianity is the central limit theorem. Khinchin based his development of statistical mechanics on this theorem ( [Kbinchin, 1949|). It is of interest to look at this theorem from a renormalization group perspective.

A typical physical example, like Brownian motion, involves some distribution of random forces on a small time scale whose average effect on a long time scale 


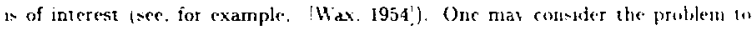

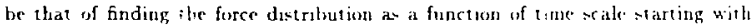
the given one on the smallest scale and hopefully aurnuteting to a univeral one on long time scales. In our renormalization group approach we define a mapping $R$ from the space of distributions $D$ to itself, which ictegrates out the next wialler time scale and renormalizes the variables so as to return the problem to one of the original form. We will show how a simple version of the central limit theurem fits into this context.

$D$ will he the space of distributions $\rho(x)$ which are normalized:

$$
\int_{\infty}^{\infty} \rho(x) d x=1
$$

have zero mean:

$$
\int_{-\infty}^{\infty} x \rho(x) d x=0,
$$

and a constant nonzero finite dispersion:

$$
\int_{-\infty}^{\infty} x^{2} \rho(x) d x=\sigma^{2}
$$

The distribution of $x=x_{1}+x_{2}$ where $x_{1}$ and $x_{2}$ are individually and independently described by $\rho$ is the convolution

$$
\rho^{(1)}(x)=\int_{-\infty}^{\infty} \rho(x-y) \rho(y) d y .
$$

since $x=(x-y)+(y)$ for $-\infty<y<\infty$ describes all ways $r$ decomposing $I$ into a sum. It's easy to see that the dispersion squared of $\rho^{(1)}$ is i wice that of $\rho$. Thus

$$
\sigma^{2}=\frac{1}{2} \int_{-\infty}^{\infty} x^{2} \rho^{(1)}(x) d x=\int_{-\infty}^{\infty} x^{2} \rho^{(1)}(\sqrt{2} x) \sqrt{2} d x .
$$


Tu kecp the normalizatuon and the disperand comet ant we define the senormalization operator as

$$
R \rho(x)=\sqrt{2} \int_{-\infty}^{\infty} \rho(\sqrt{2} x-y) \rho(y) d y .
$$

We would like to study $R$ by looking for fixed points and studying their stability. By two changes of coordinates on $D$ we will actually turn $R$ intu a linear operator. We first label a distribution by its Fourier transform:

$$
\tilde{p}(k)=\int_{-\infty}^{\infty} e^{\star k x} p(x) d x .
$$

The moment conditions on $p$ tura into

$$
\hat{\rho}(0)=1 \quad \frac{d}{d k} \hat{\rho}(0)=0 \quad \frac{d^{2}}{d k^{2}} \hat{\rho}(0)=-\sigma^{2} .
$$

We see that $\dot{\rho}$ has a quadratic maximum of value $l$ at $k=0$. Because convolution turns into multiplication under Fourier transform, in these coordinates the reaormalization operator $R$ becomes

$$
R \hat{\rho}(k)=\left(\hat{\rho}\left(\frac{k}{\sqrt{2}} i\right)^{2}\right.
$$

It is already easy to see why Gaussians will arise. Squaring emphasizes large values compared to small ones. After repeated squaring, only the quadratic maximum of $\dot{\rho}$ will play any role and so $\dot{\rho}(k)$ 's behavior will be the same as that of $1-\frac{\sigma^{2}}{2} k^{2}$. But

$$
\text { as } N \rightarrow \infty, \quad\left(1-\frac{\sigma^{2}}{2}\left(\frac{k}{\sqrt{2}^{N}}\right)^{2}\right)^{2^{N}}-e^{\frac{-\sigma^{2} k^{2}}{7}} \text {. }
$$

This is very similar to the case of period doubling in one dimensional maps where the Feigerbaum sraling is determined by the quadratic maximum. 
To make $R$ linear we now ehows as our coordinate

$$
\eta(k)=\log (\hat{\rho}(k))
$$

7 satisfies the conditions

$$
\gamma(0)=0, \quad \frac{d \gamma}{d k}(0)=0, \quad \frac{d^{2} \gamma}{d k^{2}}(0)=-\sigma^{2}
$$

and $R$ rakes the form

$$
R_{\gamma}(k)=2 \gamma\left(\frac{k}{\sqrt{2}}\right)
$$

which is indeed linear. The "eigenfunctions" of rescaling are powers of $k$ and so we expand $\gamma(\dot{k})$ in a Taylor series:

$$
\gamma(k)=-\frac{a^{2}}{2} k^{2}+a_{3} k^{3}+a_{4} k^{4}+\ldots
$$

$R$ takes $\left(a_{3}, a_{4}, \ldots\right)$ into $\left(a_{3} / \sqrt{2}, a_{4} / \sqrt{2}^{2}, \ldots\right)$. Thus $\left(a_{3}=0, a_{4}=0, \ldots\right)$ is the unique attracting fixed point. This fixed point in the other condinates is

$$
\begin{aligned}
\gamma^{*}(k) & =-\frac{\sigma^{2}}{2} k^{2}, \\
\dot{\rho}^{*}(k) & =e^{\frac{a^{2} k^{2}}{2}}, \\
\text { and } \quad \rho^{*}(x) & =\frac{1}{\sqrt{2 \pi} \sigma} e^{-\frac{x^{2}}{2 \sigma^{2}}} .
\end{aligned}
$$

Thus every distribution asymptotically approacbes a Gaussian with the same dispersion under this mapping. 


\subsection{A Poor Man's Feigenbaum Number}

One of the great discoviries of the last decade was the universality c certain aspecte of period-douhling cascades. Many physical systems undergo a series of bifurcations or sudden changes in their behavic: with the increase of some parameter. Typical parameters, like the Reynold's number, represent the strength of driving or energy input to the system. An example to think about is the beharior of the stream of water from a faucet as the water pressure is increased. Initially the flow is steady but at some point oscillations set in and eventually the stream dynamics becomes chantic. Another example is a flag waving in the wind. As the wind gets stronger, the flag begins fluttering and then waving chaotically. A last example is river meauders. A high viscosity river (made of honey, say) goes straight down a hill. As the viscosity is lowered, the river's path meanders frcm side to side. As discovered hy unfortunate farmers who built their farms on the rich soil hetween meanders of rivers, the meanders slowly move downstream. The state of a meanferiug river is therefore periodic in time (at any point on the bill the rive, nosition moves periodically from side to side). I don't know if any further bifurcations bave been observed in this example.

For small enough values of the parameter, each of these systems is in a stable equilibrium state with no time variation. As the parameter increases, a Hopf bifurcation occurs at a particular valuc und the system undergoes oscillations described by a stable limit cycle. As the paraneter is further increased in many systems, the period of the oscillation doubles at some point. On every other cycle. the system doesn 1 quite come hack to the starting point causing the period to be twice 


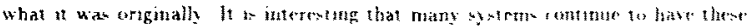

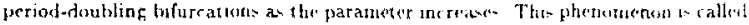

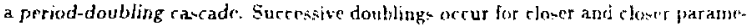

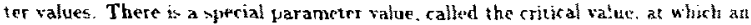
infinite number of doublings have orcured and beyond which the dynatmies is wery complicated and often chaotic. Feigenbaym discovered that the perior doubling parameter values approach this lifniting point geometrically. and that the rave of approach is a universal number, called Frigenbaurn's number: $4.669 \ldots$

[Crawford and Omobundro, fsot: gives a geometric picture of period-doubling in the state space of the system and discusses the phenomenon of knotted period. doubled orbits. Here we sould like tu sketci Feigenbaum's renormalization argu* ment (for more information, see [Guckenheimer and Holmes, 1983, p. 346 and the references given there). Feigenbaum obtained his number to tigh arcurary using a Cray supercomputer. We will sbow bow to obtain it to within 25 percent on the back of an envelope.

Period-doubling is usually studied by looking at the Poincaré cortum map for a periodic orbit. This is the mapping obtained by contmidering the cffert of the dynamics on points near the periodic orbit that lie on a codimengivm-one shect which cuts the orbil transversally. Each point on the sher: fiaw alugg until it hits the sheet again. We wish to study the mapping of the sheet co itself that this defiries. A periodic urbit with a period near that of the one under study is represented by a hied point of the maging. Orbits of higher period are fixed points of some it erate of the mapping. Peried doubling occuss when an eigenvalue of the linkarization 
at the napping at a wable tixed point geen throwgli -1 . This bas the effert of

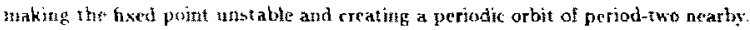
All af the interesting behavion orrurs along the eigendirectios of the rigenvalue whici goe through $w$. It is for this reason that period-doubling of systems with thany-dimensional state spaces nay be effectively studied by considering only onedimensional maps.

The key features of the period doubling castade arise in any onc-dimensional map with a quadratic maximum. We may consider

$$
f_{v}(x)=1-\mu x^{2}
$$

on the interval $I \in \mid-1,1]$. As $\mu$ varies, the width of the hump changes and period doubling ensues. Let us use the term hump map to denote even maps of the interval $[0$, 1] to itself, which have a single quadratic maximum at $x=0$ witb value 1 .

The renormalization picture of period-doubling is based on the observation that for any hump map $f$, its first iterate $f$ of again looks like a bump map when we consider only a smaller range of $x$ 's and invert and nagnify the value. Furthermore. when $f$ is undergoing the nth stage of period-doubling, $f \circ f$ is undergoing onty the $n-1$ st stage. We are therefore motivated to introduce a renormalization operator $R$ on the space of bump maps that takes a map to a rescaled first iterate:

$$
T[f](x)=0 f\left(j\left(\frac{x}{a}\right)\right.
$$

The rescaling parameter $\alpha$ is chosed so tzat given a hump map, $T$ produces another one. Siuce $f(0)=1$, we see that $f(f(0))=f(1)$. For this to be onc. we must define

$$
\alpha=\frac{1}{f(1)} \text {. }
$$


Sincs period-doubling requires only one cigenvalue to be pushed through -1 . the set of hump maps undergong any order of period doubling is codimension-one (i.t. ante dimension less than the full space of hump maps). We noted that the renormalization operator $T$ takes the sbeet of order $n$ period doubling to the sheet of order $n-1$. $T$ has a fixed point $f^{*}$ in the space of maps. Since the renormalization halves the period of periodic orbits, a fixed point either has no periodic orbits or orbits of every power of two period. It turns out that $f^{*}$ has these periodic orbits and is at the accumulation point for period-doubling (where it has just doubled an infinite number of times). $f^{*}$ has a I-dimensional unstable manifold and a codimensionI stable manifold. All maps that approach $f$ under repeated application of the renormalization operator must also bave just period-doubled an infinite number of times. Thus $f^{\text {"'s }}$ stable manifold is the codimension-one sbeet of maps at criticality: $T^{-1}$ takes sheets of period-doubling to sheets of higher and higher order period-doubling, which must eventually limit on $f$ 's stable manifold. The rate at which the approach to this manifold occurs is given by the unstable eigenvalue of the linearization of $T$ at $f^{*}$, which is therefore Feigenbaum's number.

To actually calculate this number, one may employ various nunerical trich. which amount to projecting the entire bunp space onto some finite dimensional approximating space and carrying out the analysis there. We may actually carry this out by hand for an extremely crude one-dimensional approximation. We consider the family

$$
f_{\mu}(x)=1-\mu x^{2}
$$

for different values of $\mu$ as the approxirnating one-dimensional space. We project 
arbitrary hump maps to this space by truncating their Taylor series about 0 at the quadratic turm. The action of the projerted $T$ on this space is then

$$
\begin{aligned}
T\left|f_{\mu}\right|(x) & =\alpha f_{\mu}\left(f_{\mu}\left(\frac{I}{\alpha}\right)\right) \\
& =\alpha\left(1-\mu\left(1-\mu\left(\frac{x}{\alpha}\right)^{2}\right)^{2}\right) \\
& =\alpha-\alpha \mu+2 \alpha \mu^{2} \frac{x^{2}}{\alpha^{2}}-\alpha \mu^{3} \frac{x^{4}}{\alpha^{4}} \\
& =(\alpha-\alpha \mu)+\frac{2 \mu^{2}}{\alpha} x^{2}-\frac{\mu^{3}}{\alpha^{3}} x^{4} .
\end{aligned}
$$

To get this to have 1 as the constant term we must choose the renormalization factor as above:

$$
\alpha=\frac{1}{f(1)}=\frac{-1}{\mu-1} .
$$

ing this and truncating away the cubic terms gives

$$
T^{\prime} \int_{\mu} \mid(x)=1+2 \mu^{2}(1-\mu) x^{2}
$$

Our space can be coordinatized by $\mu$ and $T$ then has the form

$$
T(\mu)=(\mu-1) 2 \mu^{2}=-2 \mu^{2}+2 \mu^{3} .
$$

The fixed point is labelled by $\mu^{*}$ which satisfies

$$
\Re\left(\mu^{*}\right)=\mu^{*}=2 \mu^{* 3}-2 \mu^{* 2} \text {. }
$$

We find the solution to the resulting quadratic equation to be:

$$
\mu^{*}=\frac{1+\sqrt{1+2}}{2}=\frac{1+\sqrt{3}}{2}
$$

The eigenvalue is then obtained by taking the derivative of $T$ at this fixed point:

$$
\left.\frac{d T}{d \mu}\right|_{\mu^{*}}=\left.\left(6 \mu^{2}-4 \mu\right)\right|_{\mu^{*}}=4+\sqrt{3}-5.7 .
$$




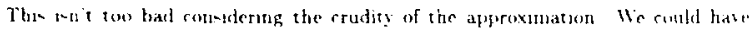
kept quarte or higher terms to evettually get any dested accurary lunfortunately theere requare finding root of quartic and higher order polynomials. Which is hast without a corsputer). 


\section{Chapter 16:}

\section{Symplectic}

\section{Thermodynamics from} Maximum Entropy

"The formulation is mathematically equivalent to the more ucual foundations. There arc, therefore, no fundamentally new results. However, there is a pleasure in recognizing old things from a new point of view. Also, there are problems for which the new point of view offers a distinct advantagc."--R. P. Feynman introducing path integrals in [Feyoman, 1948].

We have seen that the underlying geometry of elassical mechanies is symplectic geometry and that many physically important structures result from this. We have also sect that these geometric structures arise in a natural way as the marhematics of the asymptotic limit of an underlying wave tbeory. When one looks at classical thermodynamics, one sees many tantalizing indications of synplectic geometry. The Legendre transform $\Gamma^{\prime}$ dys an essential role, thermodynamically conjugate variables remind us of canonically conjugate variables, differential one-forms and their integral around loops (suggesting important two-forms) abound in the theory of Cartort cycles, ete Thermodyuamics also arises as the mathematical structure of 
the ansmptotic linit of an underlying statistical mechanical theory. We will show that indeed the structure of thermodynamics is intimately based on sy mplectus geometry and that this structure arises naturally from the underlying statistical mechanics in a way that is suprisingly analogous to thw wave case. Let us begin by describing some earlier inquiries into the geometry of thermodynamics and possible reiations with wave asymptotics.

\subsection{Previous Approaches to Geometric Thermodynamics}

In his book [Tisza, 1966] of collected oapers, Tisza describes his lifelong attempt to develop a unifed theory of thermodynamics. His sixth paper on p. 235 in this volume is entitled: "The Geometrical Interpretation sf the Formalism of MTE" (Macroscopic Thermodynamics of Equilibrium). In this chapter be points out that there is no natural metric on thermodynamic state space but that there is a natural volume element. He clains that there is more structure than just a volume and so introduces an affine structure and attempts to fiud symmetry groups of the theory. He claims to be unable to find out anything about the group he finds. He gives the affine geometric interpretation of Legendre transforms duc to Pluecker. in ternıs of representing a curve in the plane by either its points or by the tangent lines to it (i.e. its image in the the dual projertive space as we have discusseri). He finally attempts to relate a so called "stiffness moduli" to the curvature, but makes the comment that curvature in a theory with no underlyiug metric is puzzling (there is curvature in affine geometry. however). 
Gilmore. 1981 j p. 229 attempts to introduce a metrir structurr into thermodynamics using the Hessian of a certain generating fuuction as the metric. He makes sone interesting connections, but the fundamental basis of his metric appears ubscute to me. He eurls with a section on page 247 entitled: "Additional Questions". There he mentions the classical limit of quantum mechanics and the reconstruction of quanturn mechanics in terms of path integrals. He asks if there is a similar way to reconstruct statistical mechanics from thermodynamics. He then gives Hamilton's equations of motion, writes some thermodynamic equations with a similar form and asks: "Is there an intrinsic geometric structure in $\mathfrak{R}^{n} \times \Re^{n}$ associated with this variational formulation of thermodynamics? Is this geometry associated with the sy mplectic or orthogonal group $S p(2 n)$ or $S O(2 n)$ or some related real form?" but does zot go any further in their elucidation.

In [Poston and Stewart, 1978| p. 237 they discuss thermodynamics and phase transitions in terms of catastrophe theory (apparently with the consultation of Gilmore, as indicated in the preface). They do not discuss the underlying geometry of thermod: namics, but noting the similarity of their analysis of certain phase transitions to caustics in asymptotic optics, comment: "It is interesting to speculate on the possibility of a unified asymptotic analysis, treating phase transitions as caustics in the 'matter wave' everything is made of."

In a series of papers beginning with [Sourina, 1970b], Souriau has addressed certain aspects of statistical mechanics and thermodynamics from a geometric point of view. He focusses on relativistic and cosmological issues and does not appear to concider the questions addressed here. 


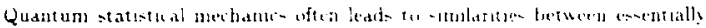

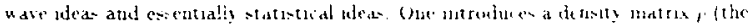

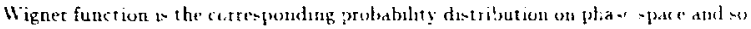
was incroduced with satuc(io in mind!). The time eselution of the demit: matrix is given by

$$
\rho=i, \rho, H i
$$

where $\boldsymbol{H}$ is the Hamiltovian operator |Feyman. 1972!. The sanonical density matrix at given temperature $T=k / 3$ is

$$
\rho(3)=e^{-\beta H} / \operatorname{Tr}\left(e^{-3 H}\right) .
$$

If we introduce an ur.oormalized $\rho$. then it satisfies the equation

$$
\frac{\partial \rho}{\partial 3}={ }_{1}, H_{\mathrm{i}}
$$

This looks like the evolution equation where $t$ times the inverse tem, crature 7 plays the role of the time. This formal si. ilarity is behind all the uses that I have seen of path integrals in statistical mechanics $\omega^{4}$ in leyman and Hibbs. 1965. 20 [Feyman. 1972 , and 0 [Schulman. 198:. This is greal for talrulating partition functions, but it is hard to see any decp physical significance for their relation We will present an alternative approach based on the maxinum cntropy formalism where it is very easy to see the physicai sigaificance.

The last connection between waves and statistics (hat I have seen mentioned by other authors is tbat the cikonal first amplitıde transport equation: may he writern in a form that looks like the mass and momentum denaty fluid aran-port 
"quatwos: of Euter (which arise from monent: of the statistical BBCikil hierarchy. Ond might atgut blat rikonal wave's represent photun gases for which a Huid theory is apperuprate

The book [Kijow'tki and Tulczyew', 1979] describes a symplectic structure

$$
\dot{x}=d N \wedge d p \div d T \wedge d S
$$

on the the four-dinensional state space of thermodynamics, where $(V, p, T, S)$ are volume. pressure, temperature, and entropy. The authors show ibat in the case of . $\mathbf{n}$ ideal git. the equations of state,

$$
\begin{gathered}
p V=R T, \\
p V=k e^{S / c V},
\end{gathered}
$$

define a Lagrangian submanifold with respect to this symplectic structure. If we view this symplectic manifold as a cotangent bundle with basc coordinatized by $(V, S)$ or $(V, T)$ or $(p, T)$ or $(S, p)$, this Lagrangian submanifold is the graph of the differentian of the interual energy, the Helmboltz free energy, the Gibbs free energy, and the enthalpy, respectively. Unfortunately. the authors do not give any reason for this stiuiture.

We will show here that the prineiple of maximunı cntropy as applied to statistical mechanics leads naturally to this symplectic structure. We can sec quite easily why the equation of state manifold should be Lagrangian. If we describe a loop ot states on this manifold (i.e. a Cara'st cycle), then the integrai over the surface bounded by this loop of $d r^{\circ} \wedge d p$ gives the work done and the integral of $d T \wedge d S$ given the heat gained. The first law of thermodynamics (energy conservationj says that theme must be equal and opposite so the symplectic structure which is their 
sum must vapish on our manifold, which in thu: Lagrangian it anterefing thit each term in the symplectic structure has its own physical interpretation 


\section{2. Seven Approachea to the Maximum Entropy Formalism}

The maximum entropy furmalism is a very powerful statistical tocl. introduced in the papers reprinted in $\{$ Jaynes, 1983\}, which gives a prescription for finding the "least biased" probability distribution consistent with any known data. If we have a discrete number of possibilities and no data to distinguish them, then symmetry forces us to choose the distribution which makes them equiprobable. If we have some information about the distribut:on, say its mean value, then we would like a prescription to choose among all possible distributions with that mean value.

Assuming there is such a prescription that always gives the same answer in the same situation and is uniform across number of possibilities, it bas been sbown to be unique (p. 16 of [Jaynes, 1983|). In fact one must choose that distribution coosistent with any krown data which maximizes the entropy defined as the sum over states of

$$
-p \log p \text {, }
$$

where $p$ is the probability of a state. It is easy to see that if nothing is known, this gives the equiprcbable distribution. The basic requirement in the general case is that if we partition the elementary events into subsets and rall membership in these subsets the elementary events of a new distribution, then applying one's prescription should give the same distribution in each situation. This is in some respects a renormalization group idea. 


\subsubsection{Axiomatir Subjective Approach}

The work wheh introduced information theory Shannom. 1948 give an axiomatic rharacterization of the information entrepy which applies equally well w the case at hand. Given $k$ possible outcomes of an experiment with the probutilites $p_{1} \ldots \ldots p_{k}$. one would like a measurn of the utcertatuly th the value mezisured un cach trial. Shannon requires of such a measure $H\left(p_{3}, \ldots p_{h}\right)$, that it satisfy three axioms. 1) $H$ should be continuous as a function of the $p_{2}$ 's. 2)If all the $p_{1}$ 's are equal. then $H$ should be a monotone increasing function of $k$ (more equally likely outcomes means more uneertainty). 3) If a choier is broken into two choices. then $H$ should be a weighted sum of the individual choices. Shannon gives the example

$$
H\left(\frac{1}{2}, \frac{1}{3}, \frac{1}{6}\right)=H\left(\frac{1}{2}, \frac{1}{2}\right)+\frac{1}{2} H\left(\frac{2}{3}, \frac{1}{3}\right)
$$

Here se have three possible outcomes, say A, B, and C. with probabilities of $1 / 2$. $1 / 3$, and $1 / 6$ respectively. We may alternatively view this as two events: $A$ and $D$. with probabilities $1 / 2$ and $1 / 2$. D represents tie occurance of either $B$ or $C$. The total uncertainty is the uncertainty in the A rs. D choice. plus the uncertainty in choosing $\mathrm{B}$ v. C weighted by a factor of $1 / 2$ (since this choice only arises half the time).

By approximating the probabilities by rational numbers and breaking the elementary events up iato a number (the least common multiple of the denominators) of equally likely events, we may reduce the problem to equiprobable distribution:But for them the third property forces $H$ to be a logarithinir function of the mumber. For arbitrary distributions. Whe find that $-p$ log $p$ (or some multiple of $i t$ ) 
in the untulue $H$ satiufying the requirements. 'To to unbiased. we should choose That di-griburiots which has the least information about our experiment that is still consistent with the kutwn data. He should therefore choose that consistent distribution that maximize - this entrops. This approarh chooses the distribution on the bask of not assuming infornation that we do not have and may therefore be considered "subjective". This allows it to be applied in many circumstances where the "objective" view of probability as frequency in large number of trials doesn't have any meaning. For example, we may ask for the best prediction of something on the basis of one ofmervation.

\subsubsection{Counting Sequences of Triale}

To see another place whet the fo:mula for the entropy comes from, we consider as elementary events, sequences of $N$ trials of the basic experiment and let $N$ go to infinity. The law of large numbers says that it is extremely likely for the number of trials with a given value in a sequence divided by $N$ to be the probabilty of that value. Let us therefore consider all sequences with $n_{1}=p_{1} \cdot N$ eniries with the first value, $n_{2}=p_{2} \cdot N$ entries with the the second value, and so on up to the number of possible measured values $k$. The least biased choice of $p_{1}, p_{2}, \ldots, p_{k}$, is that which is consistent with the known data and which maximizes the number of allowed measurement sequences. How many such sequences are there for given $p, s$ ? We may lay our required measurements down in $N^{\prime}$ ! ways, but permutations of the $n$, with the same value don't change the measurement sequence. Thus the number 
of scquences is

$$
=\frac{N !}{n_{1} ! n_{2} ! \ldots n_{k} !}
$$

Maximizing this is equivalent to maximizing its logarithm. Berause the $p_{1}$ is are fixed as $x^{r}$ gets large, all the $\pi_{1}$ 's get large as well (if they are not zero). We may therefore asymptotically use the crudest Stirling approximation:

$$
\log n ! \sim n \log n-n,
$$

for $N$ and each of the $n_{i}$ 's as $N$ goes to infinity. We thus want to maximize

$$
\begin{aligned}
& (N \log N-N)-\left(n_{1} \log n_{1}-n_{1}\right)-\cdots-\left(n_{k} \log n_{k}-n_{k}\right)= \\
& =\left(n_{1}+\cdots+n_{k}\right) \log N-n_{1} \log n_{1} \cdots \cdots-n_{k} \log n_{k} \\
& =-n_{1} \log \left(\frac{n_{1}}{N}\right)-\cdots-n_{k} \log \left(\frac{n_{k}}{N}\right),
\end{aligned}
$$

since

$$
\mathbf{n}_{h}+\cdots+\mathbf{n}_{k}=N .
$$

Equivalently we want to maximizc

$$
\sum_{i=1}^{k}-p_{1} \log p_{i} .
$$

which is the maximum entropy prescription. 


\subsubsection{Via Steepeat Deacenta in Two Ways}

The ustal proof of Stirting's Cormula used above. uses steepest descents on the integral formula for the gamma function.

Because it in perbaps the simplest example of a combinatorial quantity giving rise to nice andytical asymptotics, it is worth examining the classical application of steepest descents to the integral formulation of the gamma function to obtain Stirling's expression for $x$ ! as $x$ gets large. It is interesting that the form of the integral is quite similar to those appearing in statistical mechanics.

The garnma function of $x+1$ is the Laplace transform with respect to $t$ of $t^{x}$ evaluated at 1 (and so the $x$-fo,d convolution of the Laplace transform of $t$ ). For large $x$, the expression

$$
\begin{aligned}
I ! & =\Gamma(I+1) \\
& =\int_{0}^{\infty} t^{x} e^{-t} d t \\
& =\int_{0}^{\infty} e^{x \log t-t} d t .
\end{aligned}
$$

is of a form ripe for Laplace's method. The exponent is

$$
I \log t-t_{1}
$$

with derivative with respect to 1 given by

$$
\pm-1 \text {, }
$$

and so has its maximum at

$$
t=x
$$


We expana to secont order about this maximum and extemd the nutegranon te infinity to get

$$
=e^{x \log z-x} \int_{-\infty}^{\infty} e^{\cdot \frac{1}{2 y}(t-x)^{2}} d t .
$$

This vields an Stitling approximation

$$
x ! \sim \sqrt{2 \pi x} x^{x} e^{-x}
$$

We - all show here two ways in which to view maximum entropy as coming fron a steepest descents argument directly. These will allow us to make connections with eikonal wave theory and path integrals which use stationary phase.

In the first picture, we realize chat our system is coupled to the rest of the world. The probability distribution of our system is determined by the state of the rest of the world and all possible such states must be considered in our choice of probability distri: utio. Thus we think of our desired probability distribution as being an "integtal" over tIl possibie distributions consistent with the known data. The distributions mus; be weighted by the number of external conditions that can produce t'iem. TH $\$$ is the number of ways of rearranging states and is given by the integral over all states of the weight

$$
\exp \left(\sum-p \log p\right)
$$

For the syst' ans we ar interested in, the thermodyamic limit makes the exporient grow asymptotically (since changing the scale from $x$ to $X=$ ex makes $d x=\frac{1}{e} d X$ : equivalently, th. number of states grows exponentially with the number of particles) and so "stecpest desents" tells us that only the maximum europy distribution con- 
tribute: (The integral wor all distributions must of course be interpeted asympfot ally in torm, of apuroximating shms, themgh Simon, 1979, discusscs igorous applicationw of stecpect derents on intinite-dimensional spaces).

Thin is in the spirit of (ibbsian cusembles. but applied to the distributions themselves (i.c, an ensernble of probability distributions). One might imagine many copies of our systern and each ooe has its phase space populated with a swarm of particles whose density is qoverged by some distribution. How many particle swarms correspond to a given distribution? We get multiple systems by exchauging particles but must divide by the number of exch inges between particles of the same probability. Think of chopping phase space into bins. Each probability distribution places a certain number of particles in each bin. The number of distinct ways of obtaining a given distribution is obtained by counting all permutations of the particles and dividing by the number of exchanges which leave the same particies in the bins (and so don't count as a distince way of obtaining a distribution). Again the exponential of the entropy gives the numbet of possibilities in the limit as the number of particles becomes infinite and the binning becomes infinitesimal.

The second approach is an empirical one. We say that probability distributions are experimentally determitued by measurement sequences and if we know only the distribution. its multiplicity should be the number of distinct measurement sequences that give ris, to it. We may make the conneztion with path integrals in the following ray. Consider the space of onr observables $M$ crossed with an interval in $\Re$. We can tbink of paramirtrized famities of measurements ax being path= in this spare. With appropriate binning (as discussed in the introduction). 
each path determines a probability distrihution by integrating along the intersal. Some paths are consistent witb the known data, and we must consider them equally likely, others are not and they bave zcm probability. The expected dintribution in then an integral over those distributions corresponding to the possible paths. Wi may convert this to an integral over possible distributions, if we include a wrighting factor equal to the "measure" of paths corresponding to each distribution. But wo have seen above that this is just $\exp \left(\sum-p \log p\right)$. Again we use "steepest descents" to conclude that the maximum entropy distribution is most likely.

It is perhaps artificial to think of measur ements as parameterized by a real parameter (though time might serve this role). We onight just as well consider sequences of measurements which asymptotically determine distributions. It is really the distributions that play the role of paths in Feymman's theory in any case In fact, when oce does quantum field theory via path integrals, the integral is over fields and so is quite similar to our integral over distributions.

Let us explicitly write down the formula for the "averagc" distribution which is analogous to the Feynman path integral. We want to sum over all allowed $p(Z)^{\text {'s }}$ weigbted by the factor

$$
e^{-S t o s i *}
$$

\{the $c$ arises from the scaling discussed earlicr in this section). We want to normalize the resulting distribuion as well. If we let $D(\rho)$ represent the "mearure" an distribution space, and let $\Gamma$ be the subset of distribution whymg an: imponed 
constratnts, then

$$
\text { Favetage }(Z)-\frac{\int_{C} \rho(Z) e^{-\int \rho(Z) \log \rho(Z) d Z / 4} D(\rho)}{\int_{C} e^{-\int \rho(Z \log \rho(Z) d Z / t} D(\rho)} .
$$

Integrating over $Z$. we sce that this expression is correctly normalized (since each $\rho$ in $C$ is). Applying steepest descents as $c \rightarrow 0$, we pull out bie $\rho$ with the maximum entropy from the integral in the numerator and the remaining integrals cancel leaving

$$
\rho_{\text {aversge }}(Z) \sim p_{\text {max }} \text { tatropy }(Z) \text {. }
$$

We can get the expected value of any functional of $\rho$ by inserting it in place of $\rho$ in the integrand of the numerator. In each case we may use steepest descents to pull it out of the integrand by evaluating it on the maximum entropy distribution. For example, by integrating over the constant energy surfaces each distribution $\rho$ or phase space determines a digtribution of energies (i.e. the density of states). By this argument, the average distribution over energies is exactly the one determined by the maximt in eatropy phase space distribution.

\subsubsection{Vis Probability in Three Waye}

In the very interesting reference: [Tikochinsky, Tishoy, and Levine, 1984], the authors provide three "objective" justifications for the maximum entropy procedure to complement Jaynes' more "subjectiv' ' philosopby. Their first technique is to consider the known data to be a sequence of experimental samples and from consistency conditions and the reproducibility of the experiment, they deduce the maximum entropy criterion. This argument is very much like the sequence space 


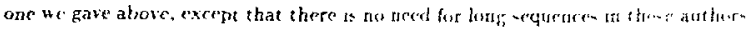
itork.

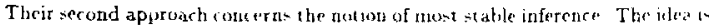
that since the data inferred from real samples in likely to be slightly off. one hrouki choose that consistent distribution that is least spenstive to errors in the data. This too leads to the maximum antropy distribution. Intuitively, this distrilistion : the most spread out that in can be, consist at with the datan and so changes the teart as the data varies.

Their last approdch uses the notion of sufficic. statistics. In later sections we shall need to use Bayes theorem. which allows one to calculate the probability dis. tribution of a parameter shat paraneterizes a family of distribution functions. given the actual distribution. A sufficient t tatistic is a function of some number of sample p jints which contains all the intrormation that the samples do as far as determining tiae value of the parameter. If the sauple averages of the observed parameters serve as sufficient statistics for the mean value of thosc paraneters, then the probability distribution of those parameters mist in faci be the maximum entropy one. Thut if the sample average is all that can uscfully be used in determining expectation values we must have the maxinum eatropy distribution. 


\subsection{The Thermodynamic Limit}

(Bu aeymptrace will consist of taking the thermodynamic limit. We want the obervable to be certain mechanical quantities. like the volume $I$. the total energy $\checkmark$. the numbers of variuus particles $X_{1}$. the magnetic moment $\mu$. etc. These will all be taken at constant $X$. the total number of particles, because this will play the role of an asymptotic parameter. If we have $n$ particle species only $n-1$ of the quantities $Y_{1}$ are really independent variables en our space (so pick the first $n-1$ say). We will thus assume that $N$ is precisely known when applying maximum entrop: Let us symbolize the rest of the observables by the variable $x$, which is a Fector in the observable vector space $O$. We will assume that experimentally only the mean values of the $I$ after many measurement trials are known. We introduce the asymptotic parameter $c$ and let the total particle number scale as: $N=1 / \epsilon$. As $N$ gets large, the boundary effects shrink and so the $r$ really become extensive. and so proportional to $N$. We therefore introduce the "slow" rescaled (intensive) quantitics: $y \equiv t I$. The behavior of the system expressed in terms of $y$ as $\ell \rightarrow 0$ will give us the thermodynanic limit.

In our discussions we will often want to distinguish the mechanical variables $y$ and their thermodynamically conjugatc variables. Since the mechanical variable: are additive when we couple systems, we will sometimes refer to them as "the extensive variabl:s" (even though they are intensive with respect to the se lling of $d$ ) and their thermodymamic conjugates (like temperature and pressice) as "the intensive variatles" since these equalize in coupled sytems. This nomet.clature is introduced merely to keep from repeating the awkward phrase "and ther thermadynamically 
conjugate variables".

\subsubsection{The Density of Stater}

The density of states arailabie to the system with given $r$ 's Hill be denoted by $\Omega(x)$. So $\Omega(x) d x$ is a density on $O$ whose integral over a region represents the number of microstates represented by that region. If the I's are large, the.. the number of states of a system is equal to the product of the number of states in each of two subsystems into wich it decomposes (since interaction becomes irrclevant asymptotically). Thus

$$
\Omega(y)=\Omega(y-\xi) \Omega(\xi),
$$

as $c \rightarrow 0$. We may find the asymptotic dependence of $\Omega$ or $c$ by taking the logarithm:

$$
\log \Omega(y)=\log \Omega(y-\xi)+\log \Omega(\xi)
$$

and taking $\xi=y / 2$ to get

$$
\log \cap(y)=2 \log \Omega(y / 2),
$$

and by extending this to first binary fractious:

$$
\left.\log \Omega(y)=2^{n} \log \Omega\left(2^{-n} y\right)\right)
$$

and then by continutity, to all reals:

$$
\left.\log \Omega(y)=e_{e}^{l} \log \Omega(\iota y)\right)
$$

and we obtain finally

$$
\Omega(y)=e^{(\log x) /(x) y / t} .
$$


l'his shows that as $\rightarrow 0$. the density of states scales as an exponential with a $1 / \mathrm{c}$ in the exponent.

\subsubsection{The Partition Function}

The partition function $Z(X)$ corresponding to the density of stases $\Omega(x)$, where $X \in O^{*}$ is in the dual space (i.e. the space of linear functions) to $x \in O$, is given by the multiple Laplace transform:

$$
\begin{aligned}
Z(X) & =\int_{0}^{\infty} \cdots \int_{0}^{\infty} e^{-(x, X)} \Omega(x) d^{n} x \\
& =\frac{1}{c} \int_{0}^{\infty} \cdots \int_{0}^{\infty} e^{(l o g \Omega(y)-(y, X)) / c} d^{n} y .
\end{aligned}
$$

Let us now use steepest descents to get the $c \rightarrow 0$ asymptotic behavior. The exponent is a maximum at that value of $y$ where

$$
X=\frac{\partial \log \Omega(y)}{\partial y} .
$$

La: us call this point $y_{0}(X)$. Then asymptotically we have

$$
Z(X)=\frac{1}{c} \frac{\sqrt{2 \pi}}{\sqrt{-\partial^{2} \log \Pi /\left.\partial y^{2}\right|_{\nu_{0}}(x)}} e^{\left(\log n\left(\nu_{0}(x)\right)-\left(y_{0}(x), x\right)\right) / \varepsilon}
$$

So we see that the partition function, like the density of states, also scales as an exponential with a $1 / \mathrm{C}$ in the exponent asymptotically, Notice that $y_{0}(X)$ defines a Legendre transformation from $y$ space to $X$ space geuerated by the function $\log \Omega(y)$ and that the exponents of $\Omega$ and $Z$ are the Legendre trasslorms of each other. 


\subsection{Maximum Entropy Applied to Statiatical Mechanics}

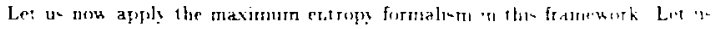

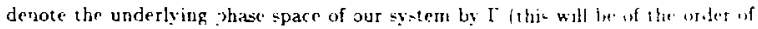
$10^{23}$ dimensional). We have the space $P$ of probablity dist ributume ou $I$ and at map $0: \Gamma-2$ which represents the value of the observables of mert't wi a given mirroscopic state $(O$ is a linear spare of observables discusspd abowe) He may integrate the $O$ valued function $o$ with respect to edch probability distribution to get a map

$$
m P \rightarrow O
$$

giving the mean values of the ubservables for each probability distribution. We also have the information eatropy

$$
S: P \rightarrow \mathfrak{R}
$$

which is a positive real valued function on $P$ obtained by integrating - $p$ log $p$ over $\Gamma$ for each measure $p \in P$. Our goal is to detine a map

$$
E: O-P \text {. }
$$

representing the must likely distribution with the given mean valur of " Thr image of $y \in O$ lies in $m^{-1}(y) \subset P$. We define it tu be the maxmutn of 5 rentricted 1, this set. We may then pull back $S$ along $E$ to get the entropy as a furmion on $O$.

The constrained extremization required is must eisjlu carried out ising lat.

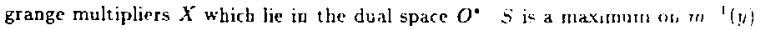
at $p \in P$ if and only if there exists a Lagrange multiplier $I \div O^{*}$ surh that

$$
5-\mathrm{X} \circ \mathrm{m}
$$




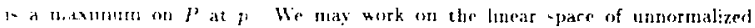

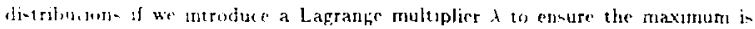
nos nalizers Thus we ohtain the requirement

$$
\therefore\left(-\int_{I} p(z) \log r(z) d z-\langle x \cdot m(p)\}-\lambda \int_{I} p(z) d z\right)=0 \text {. }
$$

Inserting the definition of $m$ and carring out the functional derivative gives

$$
\begin{aligned}
& =\frac{i}{\delta p}\left(-\int_{\Gamma} p(z) \log p(z) d z-\left\langle X \cdot \int_{\Gamma} o(z) p(z) d z\right\rangle-\lambda \int_{\Gamma} p(z) d z\right) \\
& =\frac{\partial}{\delta p}\left(-\int_{\Gamma} p(z) \log p(z) d z-\int_{\Gamma} p(z)\langle X, o(z)) d z-\lambda \int_{\Gamma} p(z) d z\right) \\
& =\frac{\delta}{\delta p} \int_{\Gamma} p(z)(-\log p(z)-(X, o(z)\rangle d z-\lambda) d z \\
& =-\log p(z)-\langle X .,(z)) d z-1-\lambda .
\end{aligned}
$$

Let ut rall

$$
\mathrm{e}^{-1-\lambda} \equiv \frac{1}{Z}
$$

We must choose $\lambda(X)$ and therefore $Z(X)$ to ensure that $p$ is a properly normalized probability distribution. Solving for $p(z)$, we find

$$
p(z)=\frac{1}{Z(X)}:-\{X, o(z)\} .
$$

The normalizetion condition shows us that

$$
z(x)=\int_{T} c^{-(x, o[z))} d z
$$

is the partition function.

Given $y \in O$, we solve for $X \in O^{*}$ by requiring that the corresponding distrihution give $y$ at its mean vajue of 0 . Looking at the expression for $\mathcal{Z}$. we see that thi- in equavalent to requiring that

$$
y=-d(\log Z) i x
$$


Here we are identifying $O=O^{*}$. This map $L: O \rightarrow O^{*}$ is the Legrndre trafsform generated by $\log Z$. We may pull bark the entropy on $P$ by $E$ to give a function on $O$. We see that this is

$$
S \circ E(y)=\log (Z \circ L(y))+\{X, y\rangle
$$

And so the surface

$$
\boldsymbol{X}(y)=L(y)=d S(y)
$$

is a Lagrangian submanifold in $O \times 0^{\circ}$.

Jaynes has given a nice demonstration of the second law of thermodynamins using maximum entropy [Jaynes, 1983]. We need oniy assume that the measured eneropy for a given set of thermodynamic parameters is the entropy of the maximum entropy distribution with mean values given by the measurements (we dave just seen that this is equivalent to the Gibbs distribution giving the correct value--the basic assumption in traditional statistical mecbanics). W'e will show that if we start with a caronical distribution corresponding to one set of thermodynamic parameters and push it forward by any canonical transformation of the underiying phase space, then the values of the thermodyuamic parameters abtained from the pushed forward distribution cortespond to an entropy which is larger than that of the first set. We first recall that the information entropy of the pushed forward distribution is the same as the entropy corrcsponding to the initial parameters. This is because the integral $\int-p$ logp doesn't change under volume preserving difeomorphismand canonical transformations preserve volume. Next, the entrofy corresponding to the new parameters is the information entropy of the maximal entropy distribution with them as mean values (i.e. the Gibba canonical distribution). Since 
this entropy is maximal and the pushed forward distribution is another distribution with the now parameters as mean values, the new et tropy is greater than or equal to the inforration ent ropy of the pushed forward distribution. But ahis shows that the new eatropy is greater than or equal to the old entropy. Since information entropt measures out ignorance, this interpretation of the second law simply says that if we begin with a knowa (canonical) distribution, follow it in detail under a canonical transformation, and then forget everytbing but the mean values of some thermodynamic parameters, we are bound to lose information for at kast not gain it). 
16.5. Sone Symplectir and Contact Geometry

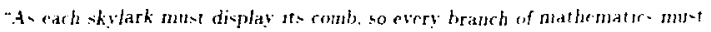
finally displas stmplertsation." p 74 of Aruold. 1984

In this section we will collect together some of the definition and remiste of symplectic and cotact geometry and give some motivation for their use in the contexts we have in mind. We have seen that in wave theory we get asymptotic interak over

$$
p^{15 \%}
$$

where $S$ is the action and that in statistical mechanies we get asymptotic integrals over

$$
e^{5 / 1}
$$

where $S$ is the entropy. By using stationary phase or steepest descents. we asymptotically reduce these expressions to ones involving only regions with specified differential $d S$. When we are studying families of values parameterized by $y$ (eg. the point in space we are observing our wave or the thermodynamic observablen). we often obtain $S$ as a function of $y$ and are interested in points whete $d S$ haw a value equal to a Lagrange multiplier in tbe dual space of $y$. The level sets of si alsu often have physical interest (eg. the wavefront or the inentropic ntater). Thu. we are motivated to study the geometric structures associated with the differentiai and level sets of functions. 


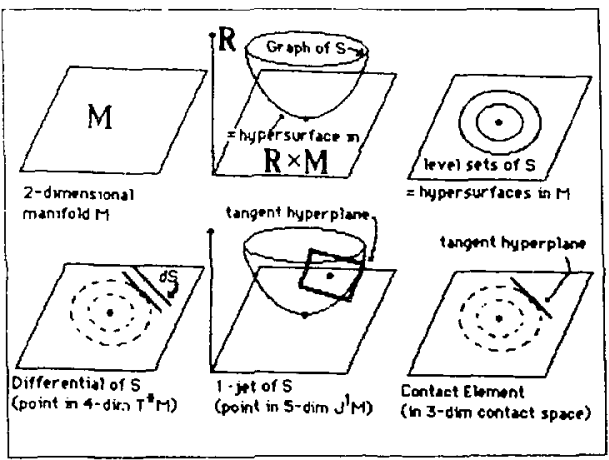

Figure 16.1: Spaces associated with a function on a manifold.

\subsection{Hy persurfaces Determined by a Function}

Every function $S$ on a manifold $M$ of dimension $\boldsymbol{m}$, determines two natural kinds of hypersurface (a hypersurface is a codimeusion-1 submanifold (i.e. of one dimcusion less than the ambient soace it lite in)). For example, consider the function $S(r, y)=J^{2} \div y^{2}$ defined on tie 2 -dimensional plane coordinatized by $I$ and $y$. Its level sets form a family of hypersurfaces of $M$ parametrized by $S$ (with occasional non-submanifolds tbat are of measure zero generically, by Sard's theorem). For $S=x^{2}+y^{2}$, the level sets are the curcles $x^{2}+y^{2}=$ ronstant. On the other band. $S$ graptl is a hypersurface in $M \times \Re$. For $S=x^{2}+y^{2}$, the graph is a paraboloid of revolution in $(x, y, S)$ space. The differcutial $d S$ of $S$ is a cue-form on $M$ (which 
geometrically represents the gradient of $S$ ). For $S=s^{2}+y^{2}$. we ses that $d S$ $2 x d x+2 y d y$. This gives the first order behavior of $S$ near earh point of .11 . The first order behavior of a hypersurface at a point in a manifold in represented by a hyperplane in the tangent space of the manifold at that point (i.e. a codimension one subspace of the tangert space). We may thus form the set of all hyperplanes in $T M$ that are tangent to level sets of $S$ and the set of all hyperplanes in $T(M \times \Re)$ tbat are tangent to the graph of $S$. The tangent hyperplanes to the level sets are exactly those vectors which $d S$ annihilates. Thus this set of byperplanes contains all the information that $d S$ does except its length. For $S=x^{2}+y^{2}$, tbe vectors which are scalar multiples of

$$
y \frac{\partial}{\partial x}-x \frac{\partial}{\partial y}
$$

are annihilated by $d S$. At each point, this vector spans the tangent space to $S^{\text {ts }}$ level set. The tangent hyperplanes to the graph of $S$ give all the information of $d S$, but in addition, the place they are based at 10 lls ws the value of $S$ fwhich isn't known from just $d S$ ). The tangent byperplane to the graph of $S=x^{2}+y^{2}$ at the point $(x, y, S\}$, assumed to be away from $z=0, y=0$. is spanned by the vectors.

$$
y \frac{\partial}{\partial z}-x \frac{\partial}{\partial y}
$$

and

$$
\frac{1}{2 x} \frac{\partial}{\partial x}+\frac{1}{2 y} \frac{\partial}{\partial y}+2 \frac{\partial}{\partial S}
$$




\subsubsection{The Underlyine Manifold $M$}

Wi thut get speral natural additional spaces of interest when we begin to ronsider functions on a manifold. Let the coordinates $q$ represent points in $M$. The space of all $g$ 's is $M$ and is of dimension $m$.

\subsubsection{The Graph of a Function}

The space whose points give both $q$ and the value of a function $S$ at $q$ is $M \times M$. This is $(n+1)$-dimensional and is where the graph of $S$ lives.

\subsubsection{The Cotangent Bundle}

The space whose points represent $q$ and the diflerential $p=d S$ of a function at $q$, is the cotangent bundle $T^{*} M$. This is $2 \pi$ dimensional and as we bave seen earlier has the canonical one-form $p d q$ and a natural symplectic structure $d q \wedge d p$ defined on it. We defined an $m$ dimessional submanifold of $T^{*} M$ to be Lagrangian if the symplectic form vanishes on it. We bave seen in section 7.1.4 that the graph of $d S$ 15 a Lagrangian submanifold. Fot waves this represents the local waverector as a function of position in an eikonal wave (in spacetime the manifold represents the solution to the intial value problem). It is important to represent this in the space of both $y$ 's and $k$ 's, because even though this surface is smooth, its projection may not be, and our wave can develop multiple branches and caustics. For thermodynamics it gives the intensive variables as a function of the extensive ones (recall the nomenclature convention from 16.3.1). We may think of it as the equation of state. 


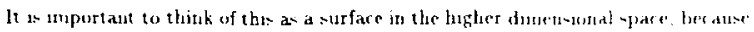
1t - projection onto the mtencive variables beromes sughlar at phase transtione

\subsubsection{The First Jet Bundle}

The space wbose points represent $g$, the differential $p=d S$ of functions, and the value $S$ of functions. is the first jet bundle $J^{1} M$. This is $(2 m+1)$-dimensional and we saw earlier that this bas a natural contact structure on it. defined as the set of tangent hyperplanes annibilated by the one-form: $d S-p d q$. Any function $S$ defines an $m$ dimensional submanifold of $J^{1} M$ by $q \sqcap(q, d S, S)$. An $m$ dimensional submanifold of a contact manifold is called a Legendre submanifold if it is tangent to the contact planes at each point. The submanifold of $J^{\prime} M$ determined by a function $S$ is Legendre. For waves this means including the value of the phase with the position and waverector. When we forget about it (by reduction), we get the Lagrangian submanifold above. For thermodynamics. this gives the relation between the cutropy and the intensive and extensive merhanical varibles.

\subsubsection{The Space of Contact Elements}

The set of hyperplanes iu a linear space of dimension m forms a smooth manjold of dimension $m-1$ (eg. the set of lines through the origit in a plane may be thougit of as a circle). A byperplane of the tangent space of a manifold at some point is called a contact element at that point. The set of all contart elements of.$M$ forms a manifold of dimension $2 m-1$ whose points reprement a pout $q$ of 11 and a tangent hyperplane there. We have sren that this manfold of contact element- is 
Iineli a contiet matifold A tangent verter to the yace of contart elements, which

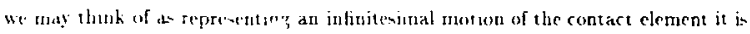
liand at. 19 wh the contact atrurture if the velocity of the basepoint of the contart . Henuent lirs in ihe contart elen ent. The set of contact elements which are tangent to a level set of 5 forme a submanifold of the space of contact elements of $M$ that is of dimensiot $m$ and is in fact a Legendre submanifold. We may thank of this as parameterizing the wavefionts, including the direction. When a wavefront begins to cross itself (as it a caustic), it is important to keep the direction of the wavefront as well as the position. Thi Legendre submanifold is always smooth, even though the wavefront may derelop cusps and self intersections.

Sirnilarly, the set of contact elements of $M \times \Re$ is a contact manifold of di*ension $2 m+1$. The set of contact elements that are tangent to the graph of $S$ forms an $m$ dimensional Legendre submanifold. This set will be important to our understanding of the Legendre transform.

\subsubsection{The Conormal Bundle}

If we are given a codinension $n$ submanifold $N$ of $M$ (that is thus of dime : : ion $m-n$ ). We may think of it as the sinultaneous level set of $n$ linearly independent functions (lorally) This motivates us to consider the set of all covectors in $T^{*} M$ based on $N$, which annihilate the tangent space to $N$. This is called the conormal buudle of $X$ in $M$. (lf $M$ had a metric, then this would be all vectors that are perpendicular to $\mathrm{Y}^{\mathrm{y}}$. This is a Lagrangian submanifold of $T^{*} M$. In the limiting case where $A$ is a point of $M$. the conormal bundle is just the set of covectors based 
at that point If $N$ is the whole of $K$, then it in the zeruscetion of $T \cdot M$ If $M$ were Riemannian, then there is a natural projection of the [co)vormal burdle of $X$ into 11 giren by se.jing ( $q, p$ ) in the normal bundle to the point a distans $p$ along the geodesic in $M$ starting at $q$ in the direction $p$. This gives the set of rays in $M$ that are traversed by light emitted by $X$, where the metric represents the (atisotropic) index of refraction. The singularities of the projection from the normal bundle to $M$ represent the caustics. They are the points which lic at the center of curvarure of some direction on the surface ( $\mid$ Artoold, 1983| p. 83).

\subsubsection{The Wavefrout Set}

This map is also related to the wave front set of a distribution $d$ on 4 introduced by Hörmander ( [Hörmander, 1983) p. 252). We associate with $d$ a Lagrangian su'trnanifold of $T^{*} M$ by saying that a covector $p$ is in $d^{\prime}$ s wavcfront set if the pushforward of $d$ to $\mathbb{R}^{3}$ along any smooth function whose differential is $p$ is still singular (i.e. there exists a smooth function on $\Re^{1}$ whose integral with respect to the pushforward of $d$ doesn't approach zero as the region of integration vanisher . Thus a puint $\delta$-function at $q$ on $M$ has a wavefront set that uncludes all cowctor: at $q$ wile a $\delta$-function supported on a submanifold $X$ has a wavefront set that includes only the conormal bundle of $N$ This is of interest because the singularitir: of the solution of a byperbolic P.D.E. with singular intial conditions must lie on the projection to $\boldsymbol{M}$ given above of the wavefront set. Thus for the wave equation on a Riemnatian manifold. a b-function intial condition will lead to singularites on a growing sphere (with respers to the metric) which we recongize as lices of 
the light rone. A singular hypersurface moves like a waveliont and propagates only in the directon of the rays. We can understand this close relation between high frequancy asymplotics and the evolution of situgular distributions by recognizing that the singular aspects are due to the infinitely high frequencies, and a singular distrihution can be represented as an integral over the asymptotic parameter of a family of eikonal waves. This can be related to Huygens principle. The fact that singularities move on rays is behind a beautiful discussion on p. xi of /Guillemin and Steraberg, 1977; explaining why the frequescy of a bowed violin is the same as that of a strummed one (a priori, the frequencies of driver oscillations should bave nothing to do with free oscillations). The explanation is that when the string snaps away from the bow, a singular kink is generated which goes down the string and back to kick tue string off the bow again. Bererating a frequency equal to that of the normal mode corresponding to that periodic ray. The reference gives figures showing the string motion.

\subsubsection{The Space of Tangent Contact Elements}

He may also consider the set of all contact elements of $M$ which are tangent to Yi.e. which contain $N$ 's tangent space). This set is a Legendre submanfold of the space of contact elements of $M$. This represents the local piewes of the wavefront that will be emitted from $N$. Even if $N$ is lower dimensional, like a point, the emitted wavefront will be $n$ dimensional (like a sphere about the point). 
16.5.5. Legendre Tranyforms and Linear State Spaces

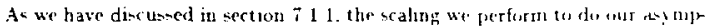
totics stretches the underlying manifold $M$ in both the wave case (where we wert 10 a slow space) and in the statistical mechanical case (where we went to rescajed mechanical variables). Asymptotically, any non-trivial manifold structure disispear: and we are left with $\Re^{m}$. In this case the cotangent bunale becomes $R^{m} \times \mathrm{R}^{\mathrm{m}}$. When the hase space is linear, there are more geometrical uperations which we ma! perform. The new' freedom is to project not only "vertically" to $\Re^{m}$, but also "horizont ally" to $\mathfrak{R}^{\mathrm{m} *}$. Essentially wi have decided how to identify all the co:angent spaces at different points of $\boldsymbol{M}$. We may do this by choosing coordinates on $M$. which gives such an identification but de sends on coordinate choice. As $<\rightarrow 0$. however, all smonth coordinate system lead to the same asymptotic identification. This asymptotic identification of cotangent spaces is non-uniform in $q$. but all our operaticns. like local Fourier transform. always include a window which scales so ar to eliminate the nou-uniform parts.

\subsubsection{The Legendre Map}

Given any function $S$ on $\Re^{m}$, its differential takes its values in $\mathbb{R}^{m *}$. Thus a's is a map from $\Re^{m}$ to its dual space, which we may call the Legendre map. In the case of waves, this maps y space into $k$ space. For thermodyuamics, it taken an net of extensive variables into their thermodynamically conjugate intensive variables. We have seen that these are the stationary points for the Fourier and Laplace transforms respectively. 


\subsubsection{The Legendre Trangfot $\mathrm{m}$}

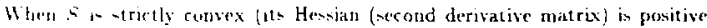
detinite) then the map is one to one. If $S$ is bounded below by some quadratic form, the it is a diffeomorphism. In this asie it nyakes sense to ask for the function $T(p)$ on $\mathrm{K}^{\mathrm{m} *}$ whose corresponding Legendre map is the inverse of the one generated by $S(q)$. One sec, that in this case:

$$
T(p) \equiv\langle p, q(p)\rangle-S(q(p))
$$

where $q(p)$ is the inverse of the first Legendre map, generates the inverse.

Let us show this explicitly in the soordinates $q^{2}$ where $1 \leq i \leq N$. Let us use $L_{S}$ to denote the Legendre map $L_{S}(q)=d S(q)=p$ defined by $S$. In coordinates this reads

$$
p_{x}=\left(L_{S}(q)\right)_{2}=\frac{\partial S}{\partial q^{\prime}}
$$

By the condition imposed on $S$, this map is invertible. We denote the inverse by $L_{S}^{-1}$ and the function $T$ we defined is then given by

$$
T(p)=\left(L_{S}^{-1}(p)\right)^{\mathrm{t}} p_{1}-S\left(L_{S}^{-1}(p)\right)
$$

We want to show that the Legendre map $L_{T}$ defined by the function $T$ is actually the inverse of $L_{S}$. This Legendre map is expressed in coordinates as

$$
\begin{aligned}
\left(L_{T}(p)\right)^{*} & =\frac{\partial T}{\partial p_{k}} \\
& -\left(L_{s}^{-1}(p)\right)^{2}+\mu, \frac{\partial\left(L_{s}^{-1}(p)\right)^{j}}{\partial p_{i}}-\frac{\partial S}{\partial q^{j}}\left(L_{s}^{-1}(p)\right) \cdot\left(\frac{\partial}{\partial p^{\prime}}\left(L_{s}^{-1}(p)\right)^{j}\right) .
\end{aligned}
$$


We have used the Leibniz rute to do the deriwative of the first ten in $T$ and the chain rule to do the second term. Now recognize that

$$
\frac{\partial S}{\partial q^{\prime}}\left(L_{\underline{L}}^{-1}(p)\right)=p
$$

to set chat the last two terms can.el. We are finally left with

$$
L_{T}=L_{S}^{-1}
$$

as desired.

In gineral we may define:

$$
T(p)=\sup _{q}[(p, q)-S(v)\}
$$

to be the Legendre transform of $S$ (|Arcold, 1983; p 19). The previous definition agrees with this one in the situations to which it applics. If $S$ is (strictly) convix then so is $T$. We shall see that this is important for thermodynamics. uince $-\$$ must be a convex function of the extensive variables.

\subsubsection{The Legendre Trangform and a Function's Graph}

If we ara given a number $T$ and a vector $p \in \Re^{m *}$. then the equat lun.

$$
T=\langle p, q\rangle-S
$$

debnes a byperplane $\left\{(q . S)\right.$ space (i.e. $\left.\Re^{m} \times \Omega\right)$. The equation above say that the graph of $S$ in $\mathrm{h}^{\mathrm{m}} \times \mathrm{F}$ hits this hyperplane at the point where th ha the shy $p$ The value of $T$ is misus the $S$ intercept of this hyperplane (1.c. The point where 
it hits the axis $q=0$ ) as is shown in figurc $(16.2 \mathrm{a})$. We paraneterize the space of nun-ver ${ }^{*}$ al (i.e. they don't contain lines parallel to the $q=0$ axis) byperplanes it (q.S) space by (p.T) as above. These are called Pl. ker coordinates (see for example p. 88 of [lennes, 1963]). The map that sends points of the graph of $S$ in ( $q . S$ ) space to the hyperplane tangent to the graph there, goes to $p=\partial S / \partial q$ which is image of $q$ under the Legendre map and $T$ which is the value of the Legendre transform of $S$ at $p$.

\subsubsection{Legendre Traneforms and Projective Duality}

The map which seuds points of a hypersurface to the byperplane tangent to the surface there has been the object of mathematical study for a long time. It is behind the notion of projective duality where, for example, all theorems of geometry in the (projective) plant may bave the worcis "point" and "line" exchanged (eg. two points determine a line, two lines detrmine a point). To make this work out, one must tack on "directions at infinity" to $R^{m}$ so that parallel lines really interse:at infuity. This leads to projective geometry, where the $m$ dimensional projective spare $R P^{m}$ is defied as the space of lines through the origin of $\mathrm{R}^{\mathrm{m}+1}$. A line through the origin of $5 \Re^{m+2 *}$ defines a linear form on $\Re^{m+1}$ up to magaitude, wbic ${ }^{L}$ may be identified with thr hyperplane through the origin of $\mathfrak{R}^{m+1}$ on which it vanishes. This in turn is made up of lines through the origin, and may be thought of an an arbitrary hyperplane in $P^{m}$. Therelore we call the space of hyperplanes in $\rho^{m}$ it:- projectivel, tual spare $P^{m}$. 


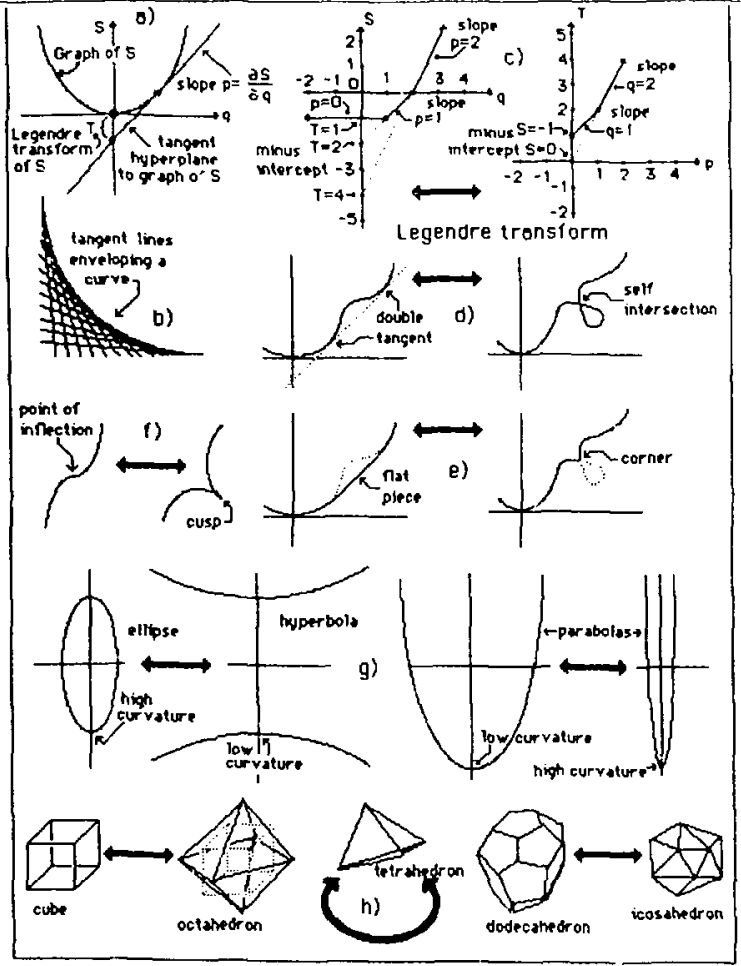

Figure 16.2: Various asfects of Legendre transforms and penjective duality: explanations are given in the text. 


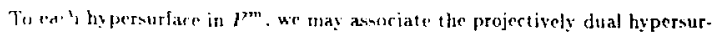
farc "f $f^{m}$ defined as all hyperplanes that are tangent to the first hypersurface. The uriginal hypersurface is the envelope of the planes defined by its dual (e.g. the tangen lines to a curve as shown in figure (16.2b) themselves form a curve in the space of linas). This relat innship is involutive in the sense that the dual of the dual bring you back to the original. The graph of the Legende transform of a function is the dual of the graph of the function in ihis sense (p. 20 of [Arnold. 1983|).

Flat places in the surface, where it includes straight line segments and so there is an interval of different points with the same slope, currespond to corners in the dual surface, which has an interval of different slopes at the same point. For example, in figure (16.2c) the graph of $S$ is made of 3 straight segments joined at two corners. The graph of $T$, its Legendre iransform, is made of 2 straight segments (corresponding to the corners in the graph of $S$ ) and 3 zorners (corresponding to the segments of $S$ ). The entire graph of $S$ for $q \leq 1$ bas slope 0 and $S$ intercept -1 and therefore corresponds to the single point $p=0$ and $T=1$. As we follow $S$ 's graph around the corner at $q=1, S=-1$, the slope goes from 0 to 1 and the intercept from -1 to -2 . This single point therefore corresponds to the whole line segment over $0 \leq p \leq 1$ in the graph of $T$. The line segment corresponding to $1 \leq q \leq 2$ in $S^{\prime}$ graph again bas a single slope and intercept and corsesponds to the point $p=1, T=2$. The corner at $q=2, S=0$ gives rise to the line segment over $1 \leq p \leq 2$. Finally the entire line over $2 \leq q$ corresponds to the single point $p=2 . T=4$. This entire analysis may be applied in reverse to go from $T(p)$ to $S(q)$ showing that Legendre transforms are involutive. For example. the line segment in 
$T$ : graph over $1 \leq p<2$ has slope 2 and intercept 0 and so corre: ponds to the single point $q=2, S=0$ in the graph of $S$. This is important in thermodynamics where flat places in the graph of the entropy as a function of the cxtensive variablen correspond to phase transitions.

Double tangents (i.c. when a hy perplane is tangent at two points of the surface) correspond to points of self intersection of the dual surface (an example is shown in figure (I6.2d)). In thermody namics, we take the convex hull of the region below the graph of entropy, and so places with double tangents get turned into Hat regions as shown in figure (16.2e). The dual surface replaces the intersection of two surfaces by their coming together at a corner and stopping.

Surfaces defined by algebraic equations have duals defined by algebraic equations. In the 2-dimensional plane, a curve with an inflection point (i.e. flat to the second order) has as its dual a cusp (whose edges are tangent to the second order) as siown in figure (16.2f). A conir section in the plane gets taken to a ronic section. As shown in figure $(16.2 \mathrm{~g})$, ellipses go to hyperbolas and parabolas go to parabolas. The duals of polybedra in 3 dimensions have vertices corresponding to the original faces and faces corresponding to the original vertices ( $\mathrm{gg}$. a cube and an octabedron, an icosabedron and a dodecahedron. and a tetrahedron and itself are dual as shown in figure $(I G, 2 \mathrm{~h}))$. The graphs of $q^{a} / a$ and $p^{b} / b$ are dual when $1 / a+1 / b=1$ and so they are Legendre transforms of each other. A norm $J(q)$ on a linear space may be defined by the unit sphere it defines. There is a natural norm on the dual space given by $g(p)=\max _{f(x) \leq 1} !(p, r)$. It unit sphere is the dual of the original one (this exemplifies the relationsbip betwen hypersufaces defined by 
Irvel scts and by graphs of functions).

\subsubsection{Legendre Transforms and Uncertainty Relations}

Since $q$ and $p$ are dual variables, acting on one by an invertible linear transformation $A$ is equivalent to acting on the other by the inverse $A^{-1}$ of that transformation. This means that the Legendre transform of a function $S(A \cdot q)$ is equal to $T\left(A^{-1} \cdot p\right)$ if $T(p)$ is the Legendre tra+.sform of $S(q)$. This is the asymptoric formulation of uncertainty principles for the Fourier and Laplace transforms. If $S(q)$ is a quadratic form, then its Legendre transform is also a quadratic form. In fact these are the unique functions for which the value of the Legendre transform is equal to that of the function at the corresponding point. The widths of the forms (and so the volume of the unit spheres they define) are inverses of each other. Since the exponential of a quadratic form is a Gaussian, this says that in thermodynamics, when the probability distribution of an extensive variable is Gaussian (as is commonly the case in fluctuation theory), then the asymptotic distribution over the conjugate intensive variable is also Gaussian with the inverse dispersion. The more precisely you know the temperature, the less precisely you know the energy and vice versa. 
16.5.5.6. Legendre Transforms and Jets of Funct in:

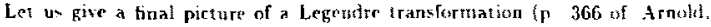
1978 , Let us call a map from one contact manifold to another of the same dinurension that takes cou:act planes to contart planes, a contact transformation. If we consider the first jet space of M. then the map

$$
(q, p * S)-(p, q,(p, q)-S)
$$

is a contact transformation which takes the graph of $d S$ and $S$ into the graph of ith Legendre transform. 
16.6. The Origin of the Lagrangian Submanifolds in Physicg

In the wh toun we will state the theorem from symplertic geometry which may: be viewal a being responsible for the lagrangian subnanifolds is both wave theory and themodynamics In both these cases we have reduced the quantitics of interest to integrals of asymptotic exponentials over large spaces which we then reduce to a variational principle for the exponent by stationary phase or steepest descents. In the wave case. we obtain the wave at a given point as an integral over all paths of an exfonential with the action $S$ of a path in the exponent (see50 [Schulman. 1981 ), leading to the priaciple of least actic a (or actually stationary action). In the statistical casc we obtain the probability of a given set of measurable quantities as an integral over all distributions of an exponential with the entropy $S$ of a distribution in the exponent, leading to the priaciple of most entropy (maximum eatropy).

\subsubsection{Congtrained Integration and Extremization}

In both cases we have an integral over some space, typically defined by some constraints (the end of the path is at the observation point, Jr the distributions have given mean values for the quantities of interest ?. If we project this space to a smaller one, we may first integrate over the fibers of the projection. and then over the smaller space. This leads to a variational principle where we first extremize $S$ over the fibers, giving a function $S$ on the smalicr space whose extrema represent the contributions we are interested in.

We discuss the physical examples in the next few- sections. To sce what is going sn geosnetrically, consider the projection from $\mathbb{R}^{\vec{x}}$ to $\mathbb{R}$ taking $(x, y) \rightarrow x$. If we want 
the extretnal value of

$$
S(x \cdot y)=15+(x-1)^{2}+(y-2)^{2} .
$$

we may first extremize $S(I, y)$ at each $x$, bolding $I$ foxed and lettug y vary. The stationary points undes this constrained variation satisfy

$$
\frac{\partial}{\partial y} S(x, y)=0=2(y-2)
$$

The surface $y=2$ is made up of the constrained critical points. $S$ rest ricted to this surface is

$$
S_{c}(x)=15+(x-1)^{2}
$$

which wie may think of as living on the projerted space. We now extremize over I yielding

$$
\frac{d}{d x} S_{c}(x)=0=2(x-1)
$$

Thus $x_{c}=1$ anc the critical value of $S$ is $S_{k}(1)=15$.

\subsubsection{Pathe Conetrained on Surfaces}

For example as in figure (16.3\}. we may firs sum over path which go threugh given points on the surfacs: $P_{1} \ldots P_{1}$ in space before rcaching the point of otr servation. We extrenize over patlus subject to these constrints and so notain the action as a functiou on

$$
P_{1} \times \cdots \times P_{3}
$$


We may now find the critical points on this space to find the actual paths taken. This finitr dimensional integral and variational principle make good mathematiral serise. We actually define the "integral over all paths" in terms of finer and figer approximations by sucb pierewise patbs as the asymptotic parameter vanishes. Physically, the rays don't mean anything on a scale smailer than a wavelength, and as wir do our scaling, the pieces of path we sum over should get smaller and smaller while including more and more wavelengths.

As we let the number of constraint surfaces on which we specify the point of intersection with a path increase to infinity, we more and more precisely constrain the ray. One can imagine this limiting to the case where giving a point in the surface product space uniquely specifies a path. This is the sense in which the path space can be thought of as an infinite product of interposed surfaces (that foliate space).

\subsubsection{The Wavevector as a Kind of Force}

We see in this example that the true paths wiil be those which come into and leave a surface with the same slope, hinting that the dual space of a r trace is important. For an extremal ray, when we perturb the point on the surface. the change of $S$ on the incoming part exactly cancels the change of $S$ on tile outgoing part to first order. Thus the derivative of the action of a part of the ray with respect to changes in its endpuint acts as a kind of "force". For a valid ray the "forces" on the incom: 1 g ray and outgoing ray must balance. We will see the analogy with thermodynamic forces momentarily. 


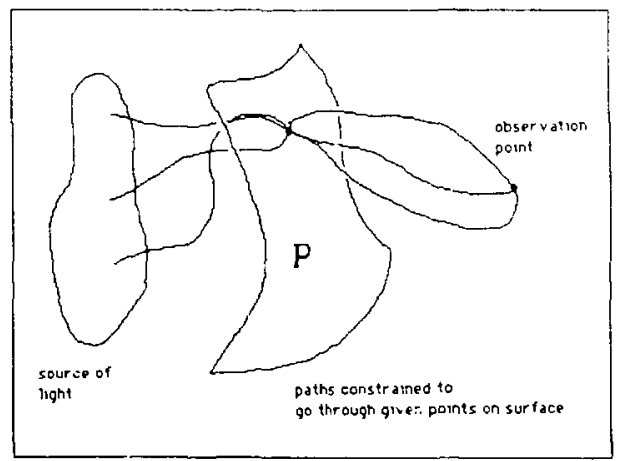

Figure 16.3: Some paths that go through a given point on the surface $P$.

\subsubsection{Distributions Constrained on Subsystems}

In the statistical case, we may imagiae diferent picces of our thermodyuanic system to be forced to have given values for their exteusive quantities. For example as in fgure (16.4). We might bave a box with a movable partition which allows the transfer of volume between its two balves and is thermally conducting and so also allows the transfer of eaergy. We may first do our integral over distributions with a given energy and volume in the left portion. We maximize the entropy subject to this constraint and so obtain an eptropy on the finite dimensional space of values of the left side's energy and volume. We extremize this entropy on a firite dimensional space to find the actual equilibrium values of the constrained quantities. 
Thin finite dincisioral integrat and variatimal principle makes good mathematical sonse. Nis arcual!. Jofine the "incegral ower all distritutions" in terms of finer and finer partitions of our system at the asymptotic parameter vanishes. Physically, the tistributions dont nean anytbing for too few particles, and so as we do our scaling the distributions we sum over should be constrained to give definite values to smaller and smallcr parts of the system while including more and more degrees of frecdom. This kind of averaging was discussed in the introduction.

As we let the regions of phase space over which the probability distribution averages are specified become smaller and smaller, we more and more precisely constrain a distribution. One can imagive this limiting to the case where giving a point in the region average product space uniquely specifies a probability distribution. This is the sense in which the space of distributions can be thought of as an infinite product of spaces of avet agcs at points of phase space.

\subsubsection{Thermodynamic Force日}

We ser in this example that the true energy and youme of the left system will be those such that the variation of the left portion's entropy is equal and opposite to the variation of the right portion s entropy to first order, tinting that the dual space of the coustrained uhservables is iriportant. The derivative of the entropy of the left k. If with respect to the constraint acts like a "force" and the left and right forces muse be balanced in equilibrium. 


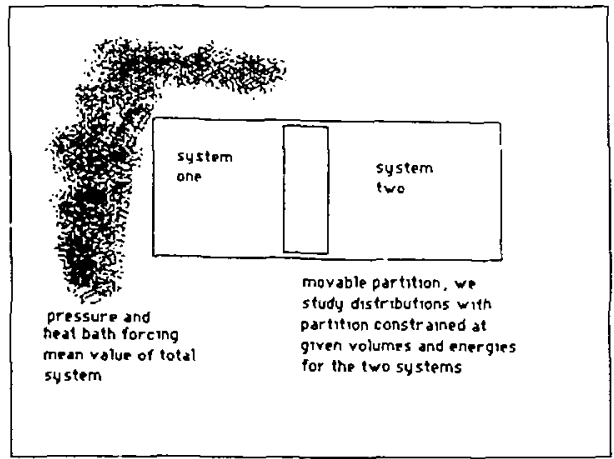

Figure 16.4: A distribution is constrained to give the left region a defir te energy and volume.

\subsubsection{Lagrange Multipliers and Legendre Maps}

This same idea is captured in the notion of Lagrange multiplier, which acts like a system with given "force" instead of given value for any const rained quantity. To maximize a function over a space with an imposed constraint. we may instead maximize over a new system on the whole spare with an additional linear piece with given "force" that allows us to a postiori make the critical point satisfy the constraint.

For example. if we want to maximize

$$
S(x, y)=2-\tau^{2}-y^{2}
$$

with the constrain: that $y=1$, we might consider the rontrained variational prob- 
irm of maximizing

$$
S_{r}(x)=2-r^{2}-1=1-r^{2}
$$

over $t^{\prime}$ constraint surfare. This yiclds $I=0$ and $S=1$. Alternatively, we may maximizer

$$
S_{L}(x, y)=2-x^{2}-y^{2}-\alpha(y-1)
$$

over all $I$ and $y$ yielding $x=0$ and $y=-\alpha / 2$. The proper force $a$ to push the maximum to $y=1$ is $a=-2$. This again yields $x=0$ and $S=1$. The reason for doing this is that it is often easier to do the unconstrained variations (even with the free parameter a) than to impose the constraint explicitly. We bave seen that the relation between the states and the conjugate forces is just the Legendre map geverated by $S$. There is a corresponding function on the dual variables which is the Legendre traosform of $S$. If we think back to our asymptotic integrals. imposing as given $\partial S / \partial y$ in the wave case makes the integral into a Fourier transform with specified wavevector. In the statistical case we get a Laplace transform with specified intensive variables.

\subsubsection{Constant Force Asymptotic Systems}

These systems with given "forces" may often be thougbt of as asy mptotic imits of real systems. Thus for exanple, a very extended weak spring acts like a constant force (a very strong inextended spring acts like a constant position (wall)), a large system in thermal equilibrium acts like a heat bath with constant temperature and infinite beat capacity. (a small system acts like a thermal iasulator, it bas zero heat capacily and anything conted to it has almost constant energy l. a large battery 


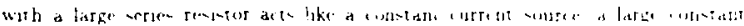

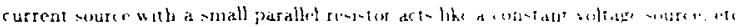

\subsubsection{Lagrangian Submanifolds and Constrained Extremization}

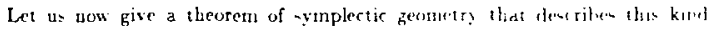

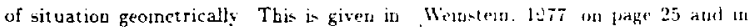
'Guillemin and Siernberg. 1977 on page 149. Abewe he have seth that we often want to projec: a space onto a smaller the while contidering the critical ponte of a function $S$. The following thenern (16.7) cells us that if we have a pojection of If onto $N$. then those poitsts in $T \cdot Y$ which pullback to points of the grapt. of $d S$ in $T^{*} M$ actually form a Lagrangian submanfold of $T^{*}, Y^{-}$These points sil over the critical points of $S$ restricted to each hiber of the projert il (i.c. inverse image of a point in $N$ ).

\subsubsection{Parametrizing Lagrangian Submanifolda}

This theorem is particularly interesting when ther. $\$$ more than one criteral point of $S$ on the fiber over $x \in X$. This means that the correnponding lagrangan submanifold in $T \cdot N$ has more than oue st set stting over $x$. Thus wit may ohain "folded" veer Lagrangian submanifolds from perfectly nice ones (i.e. the graph of $d S$ in $T^{*} M$ ). Weinstein show that this may alway be dene locally and giveconditions for the global version. This is the kry 11 Malns approach to waw 
anyprotu - In dong als eikunal sudy of a linear wave equation, we may represent a wate at a - ar-called oncillatory integrai.

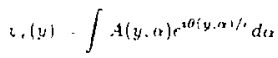

If n. ver our equation as detined on $(y, a)$ space by ignoring $a$, solutions on the (y. (s) cpace projert to solutions on y space (bx- linearity). We bave just seen that even whers the Lagrangiau submanifold in the cotangext bundle of $y$ space becomes folded over and the astmprotics becomes iuralid (i.e. nonuniform at the fold). there is a nice wave on $(y, \alpha)$ space that projects to it. We may do our asymptotics there and project the answer via stationary phase to see that even the folded over Lagrangian submanifold is a representative of the wave.

\subsubsection{Theorem on Pushing Forward Lagrangian Submanifolds}

Let us gi .. the statement of the theorem from [Guillemin and Sternberg. 1977]:

Theorem 16.6. Let $f: M \rightarrow N$ be a smooth map with df of constant rank and Ift A the a Lagrangian submanifold of $T^{*} M$. If 1 intersects $d{ }^{*} T^{*} N$ transversally: then $d f . A$ is a Lagrangian submanifold of $T^{*} N$. 


\subsubsection{Application of the Theorem to Waves}

This restricts to the casc above when $A$ is the graph of the tunrtun $S$ In the case of waves. we take $M$ to be the space of 211 paths, $X$ to be the 3 dimensional space in whirh we observe our waves, the projection to be that which sends a path to its eadpoint, and the action $S$ of a path te be the function to excremize. The the theorem says that the differeutials of the actions of those paths with extremal action for each endpoint form a Lagrangian submanifold in the cotangent space of obervation space $\boldsymbol{N}$. The fibers of the cotangent bundle are the derivative of action witb respect to the observation point and represent the wavevectors at a given point. This cotangent bundle is the wave phase space and the Lagrangian submanifold is the grapk of the wavevector at each point for an eikonal wave with wave phase $S$.

\subsection{T.4. Application of the Theorem to Thermodynamica}

In the case of statistical mecbanics, we take $M$ to be the space of probability distributions, $N$ to be the space of extensive observables that wic are studying, the projection to be that which seads a distribution to the mean value of the observables in that distribution. and the entropy $S$ of a distribution to be the function to maximize. The theorem then says that the differentials of the cntropnes of tiuse distributions witb maximal entropy for cacb mean walut of the whervables form a Lagrangian submanifold in the cotangent space of the extetusive vartathes. The hibers of the cotangent bundle are the derivative of enlowy sulh rentert th the pxtepsive variatyle and reprenent the rnigugate intmave variables The colangent 
bundly is the thermodynanic phase space and the Lagrangian subnanifold is the graph of the equation of state for an equilibrium system with entropy $S$. 
16.7. Theorem on the Pughforward of Legendre Sintemanifulds

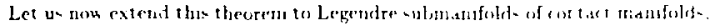
because the de a context in which we may underitand nore of the strueture of thermodyami - Assuming the same transversality condition as in the lant thesem (which is generically true). we find that for a projection $M-V$ and a function $S$ on $M$. the points in the first jer bunde $J^{t} N$ of $N$ which puil back to point= in $J^{1} M$ in the graph of $S$ and $d S$ wbere the derivative of $S$ along the fibers of the projection is zero, toget ber form a Legendre submanifold of $J^{\prime} N$. Let us locally use coordinates (q,a, S, p.a) on $M$ where a parameterizes the fibers of the projection and $q$ are coordinates on $Y, \alpha$ and $p$ are the correspondiug differentials. and $S$ represents the value of a function We assume that the coordinates $(q, S, p)$ agree with those of $J^{l} N$ on the set of pulled back vectors.

The canonical contact structure on $J^{\prime} M$ is given by the vectors annihilated by the form

$$
d S-p d q-a d a .
$$

A contact form on $J^{1} X$ is given by

$$
d S-\mathrm{d} d q
$$

We have see earlier that the one - ets of $S$ in $J^{\prime} M$ form a Legendre submanifold with respect to this contact structure. We are interested in its intersection with the set $a=0$ (i.e. those points where $S$ 's derivative vanishes along the fibers of the projection). From the expression for the contact form. we see that at these points $d S-p d q$ vanishes on $S$ one-jets a $d$ so the projected submanifold is contact on $Y$. 


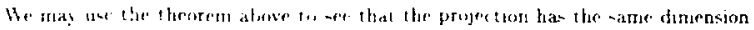

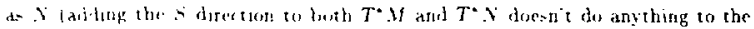
fimenoma Thus we man roncludre that the projection is a Legende submanifold of $J^{1} \cdot \mathrm{Y}$

\subsubsection{The Contact Structure for Thermodynamica}

Let us apply this result to the thermodynamic situation. Here the manifold $N$ is made of the asymptotically scaled extensive thermodynamic variables we are considering. We have been using $y$ for coordinates on this and they represent such quantities as the energy $U$, the volum.e $V$. the numbers of the various species of particles or molecules (not including the total as discussed above) $N_{1} \ldots N_{2}$, the magnetic momert $\mu$, the electric dipole moment $\Pi$ etc. On the first jet space $J^{1} N$, the function variable is the entropy $S$, and the derivative directions are coordinatized by the thermodynamically conjugate variables $\boldsymbol{x}$ to the $y$. The conjugate variable to $E$ is the inverse temperature: $\beta$, to $V$ is the pressure over the temperature: $\mu / T$, to $S_{1}$ is minus the $i$ 'th caemical potential over the temperature: $-\mu_{3} / T$, to the magnetic moment is minus the magnetic field strength over the temperature: $-H / T$, to the electric dipole moment is minus the electric field over the temperature: $-E / T$, etc. 


\subsection{1. The Contac' Form Ior Jets of Entropy}

The cont act [orm is then

$$
d S-\frac{1}{T}\left(d I^{r}+p d V-\sum_{V} \mu_{2} d H_{2}-H \cdot d M-E \cdot d P+\cdots\right)
$$

We bave seen that ou: asymptotic theory guarantes that thit form vanishes on the equation of state surface in $J^{1} N$ since it is a legendre submanifold. We recognize this as the first law of thermodynamics.

\subsubsection{The $U, V,(1 / T),(p / T)$ Symplectic Manifold}

For simplicity, from now on we shall cousider ouly $(S, U, V,(1 / T),(p / T))$ space. The other coordinaz as behave in exactly the same way if they are desised in a theiryWie bave seen that we ma: project our contact space along the $\$$ direction to obtain the symplectic manifold coordinatized by $\{U, V,\{1 / T\},\{p / T)\}$. The contact form given above goes into the canonical one form on this space (since it is constant on the fibers):

$$
\frac{1}{T}\left(d U+\rho d I^{\prime}\right)
$$

The corresponding symplectir structure is:

$$
d U \wedge d\left(\frac{1}{T}\right)-d \eta \wedge d\left(\frac{p}{T}\right)
$$

By our general theory. the equation of stale surface is a Lagrangan subnamifold with respect to this symplertu structure. 
16.7.1.3. The (S.I., . T) Symplectic Manifold

W. have seen carlier that a contact form gives the same contact structure when it in multiplied by any nouhere vasishing function. Let us use that freedom to get an equivalent contact form on our contact space by multiplying by $-T$ :

$$
d U-T d S+p d V .
$$

This tou vanishes on our Legendre submanifold. Now the form is constant along the $U$ direction and so we may project it to $\left(S, V, p_{r}-T\right)$ space, where it becomes the canonical one-form. The corresponding symplectic structure is exactly the one given by [Kijowski and Tulczyjew, 1979] that we listed in section 12.1. We may obtain this same symplectic structure as the canonical cotangent structure by viewing any of the pairs: $(l, S),(V, T),(p, T)$, or $(S, p)$ as the base and the other two variables as the cotangent fibers. Our Lagrangian submanifold is then represented as the graph of four different functions. As we have seen in great detail these are the Legendre transforms of one another, and are known as the internal energy, the Helmholz free energy, the Gibbs free energy, and the entbalpy.

\subsubsection{Legendre Transforms and Thermody rumic Potentials}

The reason for introducing these extra gellerating functions for our surface is that it is they that are extremized under different combinations of constraints. He sak that for given extensive variables the system maximizes its entropy. For adjahatu variation of a system, the entropy is an adiabatic constant of the motion (wr have wen that this is exactly the same situation as the adiabatic invariance of 


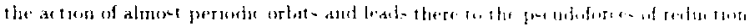

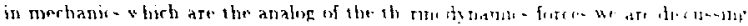

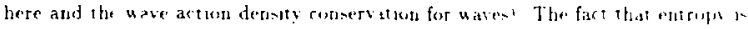
maximized when energs and volume are fixed ss equivalent to the fart that enewry 1 . minimized when entropy and volume are held fxed An andlaguns stuatum o that the shapes in three dimensions whith minimize ther surface arca for given volume. are the sam. as those which maximize their volume for given surfare area Thu by the same argument we used to show entropy was a concave function of the extenswe variables, we see thac the energy is a convex function of the or ther extensive variables and the entropy. As an example of a Legendre transform in both $S$ and $l$, wie sec that the Gitbs free energy $U-T S+P Y$ is minimized for given temperat ure and pres: ure. 
16.8. Phame Transitions and the Coometry of the Fquation of State

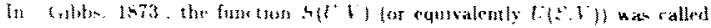

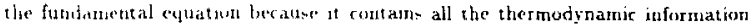

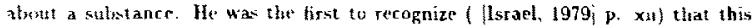
functorl contaim more information than the usual "equation of state which is a Jalition af the forju

$$
f(p, V, T)=0
$$

For example. in the rase of as ideal gas one nceds the relatica

$$
p t^{2}=\text { constant }
$$

in addition to the equation of state

$$
P V=N k T
$$

to specify the behavior of the gas.

1 will. nonetheless, call the expression of $S$ as a function of the mechanical variatles the equation of state, because it really describes the allowed relations between the intensive and extensive variables for a substance. For this example. the surfare descibing the possible states is a two dimensional surlace in $(U, V, T, p)$ space (or equivalently in $(S, V, T, p)$ space). The usual equation of state only says that $t$ is surlare lies in a three dimensiogal one given by $f=U$ and requires another constraint to obtain complete information. 
16.8.1. , tick and Phape Traneitiona

We have sten that in the cikonal wav' situation. places where the Lagrangua submanifold corresponding to an eikonal wave does not projert nirely onto y upare correspunt to caustin s of the wave field. These structures correspond wh higher order derivatives vanishing at critical parameters in our stationary phave. in the statigm tical mechanical context. the situation is simpler because only maxima contribute to the state as opposed to arbitsary critical points. In Rene Thom's cat astrophe theory such a condition is called the Maxwell condition. The places where the thermodynamic Lagrangian submanifold does not project nicely onto the intensive variables correspoud to first order phase transitions.

\subsubsection{Convexity and Firat Order Pbase Tranaitiona}

For definitesess, let $u$ use the extensive variables $U$ and $l$ to descibe the ineas of this section, though any set $y$ would do as well. |Gibbs, 1873b| consicers the form of the cotropy as a fuoction of $U$ and $V$. He showed that $S$ is a concave

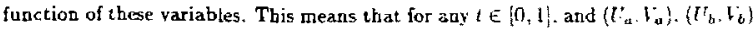
in the domain of interest, we have the inequality

$$
S\left((1-t) U_{a}+t U_{b},(1-t) V_{a}+t V_{b}\right) \geq(1-t) S\left(U_{a} \cdot V_{a}\right)-t S\left(U_{b}, V_{b}\right)
$$

If we think of the raph of $S$ as a two dituensional surfare m (r) $Y$ S) space, this just means that lue grapb of $S$ doen not fall below a hne segment joining ans inti points on it Equivalently. the region below this graph in comes (and we cone ay $-S$ is a ronvex function! 
Gible argument rum as follows Assume we had a point $\left(H_{c}, V_{r}, S_{c}\right)$ on the graph of $S$ which las below a line segenent joiniug the two allowable states: $\left(C_{a}, V_{a}, S_{a}\right)$ and $\left(U_{b}, V_{b}, S_{b}\right)$. As the system wants to maximize its entropy as much as possible, instead of going into a bomogeneous phase with $U_{r}, V_{t}$, it will split into two phases. one of $U_{a}, V_{a}$ and one with $U_{b}, V_{b}$ in such a way to have the total be $U_{r}, F_{c}$ and yet get greater entropy than $S_{c}$. In fact the system will try to do this in the way that gives the maximum total entropy. The combination of phases with the highest entrony will he on the convex tull of the region below $S$. (The convex bull of a region is the smallest convex region containing it. It contains at least all points of all line segnents whose ends lie in the original region.) Thus the actual entrar" fugction will be concave. "Flat parts" of its graph (where a tangent plane contains more than a point) correspoad to states which are linear combinations of the states corresponding to the extreme points which are at the boundary of the flat regions (and represent pure phases of the sutrstance).

Notics that if the graph of the eutropy contains a straight line segment, then the corresponding derivative along that direction is constant. Thus all points in a flat region have the same values for the intensive variables corresponding to the flat directions. If we chooge an underlying snooth entropy function arbitrarily, it is non-generic for it to contain any straight line segments (though one wouid bave to verify that this is true of entropies that arise from physically possible statistical mechanical situations). Therefore all the phase transition type bebavior comes from taking the convex hull, and we may classify the possibilities.

It wa' hawe only othe extensive quantity, say $F$ fas in an isothermal Van det 


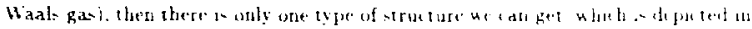

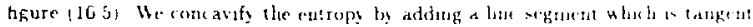

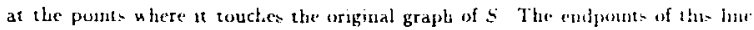
segement correspond to the liqud and gas phases (for instaure) Phycirally we think of a puddle of fluid in the hottom of a volume that we are expandis at constant temperature. As we increate the volume the fluid evapurates at constant pressurt (the vapor pressure) until it is all gas. Thus, at we move along the segment from one to the other, the proportion changes from all of one tu all of the other. The pressure is the slope. so the wbole change takes place at constant pressure.

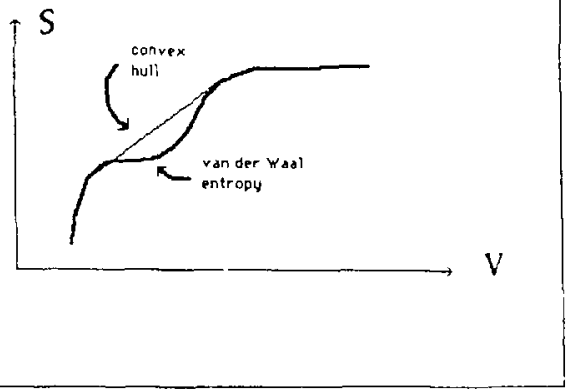

Figure 16.5: Isothermal edtropy as a function of volume for the van der Waals gas at the gas-liquid phase transition.

$W$ ben we consider 2 extensive variables. say $U$ and $V$. wi fint weval ums: 


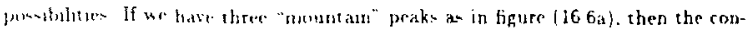

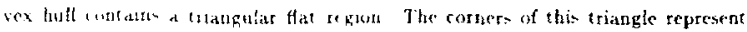

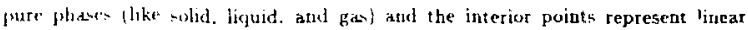
combinations of these pure states. Since there is a 2 -dimensional flat spot, there ate two intensive varialik, that are coustant, which are here the pressure and the temperature. This is thet: a triple point of the substance T'the edges of the triangle bound two dimenkional ruled surfaces which contan ouly 1-dimensional line segmeuts (since it is not generic for the original tertain to contain line segments). (A ruled surface may be thought of as a curve in the space of lines.) These represent first ordet phase transitions between two phases as discussed above. The width of the lines can get shorter as we move along the surfacc and go to zero as the square root of the parametrs labeling the line segments. This disappearance is called a critical point. The more usual picture of these phenomena is given in the intensive space of $T$ and $p$ as in $(16.60)$. We perform the Legendre transform to get to these variables and as we tave seen, points in the graph will correspond to tangent planes of the onginal graph. If the original is convex, then so is its Legendre transform, but if the original has flat spots, then the transform can have discontinuous first derivatives (i.e. corners). Since the first derivatives are discontinuous in this picture, it is called a first order phase transition. The ruled surfaces correspond to edges with a shaxp corner, the Hat truple print correspouds to three corvered edges coming together as in a letrahedron vertex, and the critical point is where an edge smootha out. 


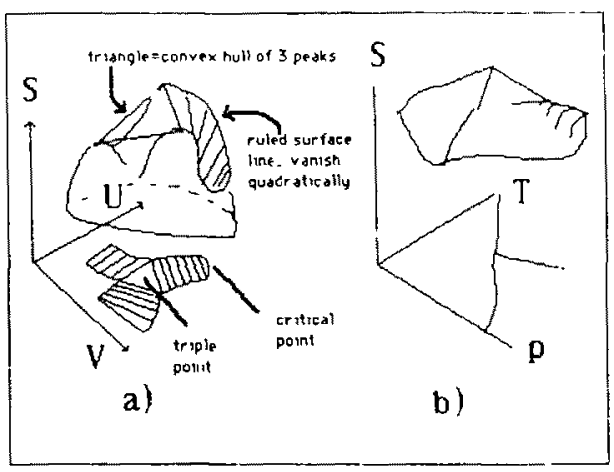

Figure 16.6: a) The entropy as a fuctioz of $U$ and $r$. A flat triangle represents the coexistence of the three phases represented by the corners of the triangle. The ruled surfaces emilating from the edges vepresent phase transitions of two states. The parabolic end of the ruled surface represents a critical peint. b) The entropy on $T$ and $p$ space giving the nore usual picture as the Legendre transform of a).

\subsubsection{A Generalization of Maxwell's Erual Area Ruit}

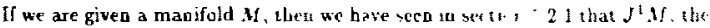
space of 1 -jets of functions on $M f$, has a natural contict structure. We saw an section 2.4.5 that $T \cdot M$. the cotan at bundle, has a natural symplectic structure ${ }^{*}$ Hhich is minus the differertial of the canonical ong-form $\theta$. There ts a batural profertum

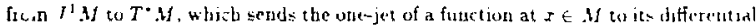

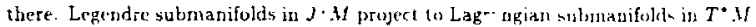


Any loop in $J^{: M}$ which lie- in a Legendre submanifold therefore projects to a loop in $T . M$ with zero atrion (i.c. the intagral of $\theta$ around the loop vanishes). We may gencralute this in

Lemma 16.7. Any piecenise smootb loop in $J^{1}$ M whose tangent vector at each point lies in the contact plape at that point projects to a loop with zero action in $T^{*} M$.

Eroof. In local coordinates on $J^{1} M$, the contast planes are given by the tangent vectors annililated by the one-form $d u-p d x$ (where $I$ are coordinates on $M$, $u$ is the value of the function whose jet the point in $J^{3} M$ represents, and $p$ its derivative). The integral of this one-form around our loop therefore vanishes (since the loop is tangent to the contart planes). The canonical one form on $T^{*} M$ pulls back to $p d z$ on $J^{i} M$. The integral of the casonical oneform is thus equal to the integral of du on cach local piece. But $u$ is a well defined function globally on the loop. Therefore the integral of $d z$ and therefore of $\theta=p d x$ around the loop is zero. Q.E.D.

Let us now use this lemma to generalize Max"ell's "equal arean rule for first order phase transitions, Let us be given asme smooth function $S$ of the variables 1. Which are linear coordinates on the linear state space $M$. This represents the "eatropy" as a function of the exlensive thermodynamic variables, but without regard for the therrnodynamic stabj' $y$ of the state it reprosents. We have scen that the entropy of the real state of the system, as a fuaction of $y$ will be the smallest concave fuurtiou $S_{e}$ that is everywhere greater than or equal to $S$. Equivalently, the graph of $S$, is the boundary of the convex hull of the region below the graph 


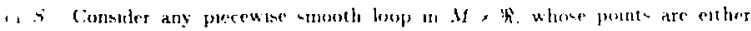
in the graph of $S$ or the graple of $S_{r}$ If we are at a poun contened in both graphs, then the differentuale 15 and $d S$, are alor equal. (lf the's werent equal

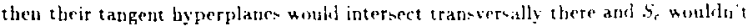
be everywhere geater thas "s equal to $S .1$ We may therefore lift ou: loop to beth $J^{\prime} M$ and $T^{*} M$ by sending each point to the jet or differential of th- functuon whese. graph it lies in. Now the tangent vertor to the original curve at each point is alour tangent to the graph of the fugction whose jet we use to lift. Thus the tangent vector to our curve in $J^{1} M$ at each pont lies in the corresponding contact plane. By the lemma above, the loop in $T^{*} M$ has zero action

Furthermore, if $S_{\varepsilon}$ is strictly greater than $S$, then its graph must contain a straight line segment (otherwise we could lower it and still keep it concave). Thr derivative along this line segment is therefore constant. The Lagrangian submanifold $d S_{x}$ in $T^{*} M$ will therefore have a singular projection onto the thermodynamically conjugatr (cotangent fber) variables along this direction. These many states with the same value for the conjugate variables represent different combinations of amounts of the various phases that can coexist with that value. The graph of $d S$ is a Lagrangian submanifold which agrees with $d S_{r}$ except in this singular region if we describe a loop consisting of the singular line in the graph of $d S$, from one rond to the other and then back to the beginning of the line inside the graph of dS, we have just seen that the symplectic area enclused by the loop in zero.

But this generalizen the usual Maxwell equal area rule. This rule conerne the situation where we hold the temperature fixed and consider the justlermal equation 


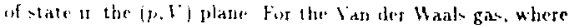

$$
(1-b)\left(r^{2}+\underset{(\because)}{a}\right)=R T
$$

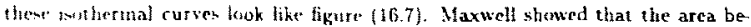
Twen the thu curve: lying above the phase transition line is equal to the area between them below 10 . Our construction generalizes the to arbitrary loops in the thermodynanic phase space. which ned not be isothermal.

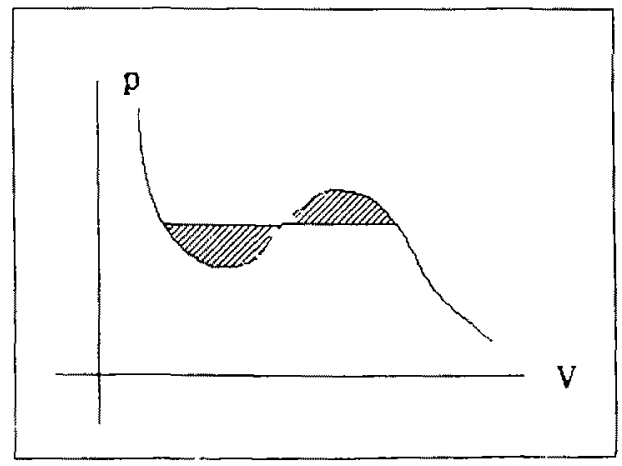

Figure 16,7: The Maxwell equal area construction for the Van der MaAls equation of statc. 


\subsection{Relationg Between symplectic Thermodynamica ond Merhanica}

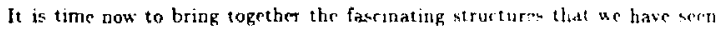
arise asymptotically out of tayes and sut of stafistical mecbancs. We have seces many tantalizing clues that these theorics have mucb in parallel and would like to make these structures explicit. Tha there should he a connertum letwen the sit theories and some of the parallels were first suggested to mo by Robert Littlejohr

\subsubsection{A) Eikonal Waves and Stationary Phase}

In wave theory we deal with waves it the cikonal limit where we study the propertics of waves represented by many wavelengths. We introduce asymptotics which siretch the scale leugth and work with quantitics defined in ternus of the slow spare $y=c x$. The method of stationary phase lets us aymptotically express quantities that a priori depend on the whole wave in terms of values only near a stationary phase point.

\subsubsection{B) Thermodynamic Limit and Steepest Descenta}

In statistical mechanics we deal with statisics in the thermodynamus limit Where we study the properties of the siatistics of macbanical spistems reprement by many degress of freedom. We introdure asymptome tw strech the wale of the extensive obscrvables and wotk whth quattitles detined in terms of the rescalert

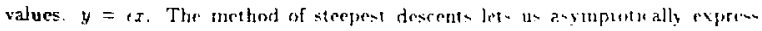

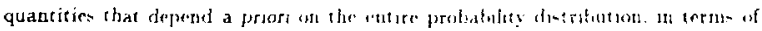
values only at a maximum 


\subsubsection{A) Wavea and the Feynman Path Integral}

W. may vew Have rnechanics in terms of the Feynman path integral. The value of the wave at a fiven point in the observation space typically a point in three mpace. but mort general things may orcur) is expressed at an integral, over all pacte to the pois of interer of the exponential of a times the action. The artion $S$ on a given path is the integral of the Lagrangran along that path. Asyruptotically. the rxponent scales as l/c. We apply stationary phase to see that only those paths whth extremal actions can contrihute asym totically.

\subsubsection{B) Probability and the Maximum Entropy Formaliam}

We may view statistical mechanics in terms of an integral ovet all observation path: (or equivaleatly all weighted probability distributions). The value of the probability density at a given point in the observation space (sometines the three dimensional spare of etserg $U$, volume $V^{*}$, and number $A$, but often more general) 12 expresued as an integral (the average) of the exponential of the entropy over all probability distributions runsistent with the abservation point of interest. The cntrops 5 of a given distribution is tbe integral of $-p \log p$ over that distributionAnymptutically the exponent scales as $1 / \%$. We apply steepest descents to see that 3ath that dentribution with maxirnum entropy can contribute asvinptotically. 
16.9.3. A) Wave Path Iniegrals over a Subapace

Offen in wave mechanico we don't do the entere path integral at one an rxample of a commmon problen is that of tinding a light wave at a poun in space given it. value on some initial surfare for volume, surfare. bue or puste, or many of them may be emiting raves reievant to the region of unterent: in the limit we mas have a continuum of sourre types) We may do this by integrating over all pathe. but we ofen like to first integrate over all paths between our point and a given point of the surface. and then integrate the resulting values over the surfare ()nly the extremal path between the two points will contribute and we may introduce an action defiued on the surfare. relative to the observation foint. which is just the action of the extremal patb to that point. The remainisg part of the integral :0 obtain the desired wave value is a finite dimensional untegral over the initial surface

\subsubsection{B) Probability Distribution Averages over a Subspace}

Oftec in statistical mecbanics we don't do the entire probabluty integral at once. An rxanple of a common problem is that of finding the probability ditrbution on the space of two thermodynamic systems in contact with one another (they may exchange any or all of the extensive quantitues and there may be inany such a upled systems: in the limit we may bave a continum of systrots. We may do this by integrating over all distributions consistent $\mathrm{w}$ ith the con-trant. but we often like to first integrate over all consistent distritulions on the prodert prace for whirh the first spaces thermodynamic quantit wh have fiven vilue atud the? integrate the resulting distributions over thes values For tach we of value un the 
for-t -pare, only the maximat entrofy com-istent distritutuon on the full space will contohbur and we maty in rudure an ent ropy detined on the space of thermedyamic atwertallen on the firse space, relative to the constraint condutions. which is just the maximat entuopy over all consistent distributions with the given values for the first =pare The remaining part of the integral is an integral over the observables of the thit system.

\subsubsection{A) Lagrange Multipliers and Canonical Conjugacy}

For the wave system we may also decide not to impose the constraint that the end of the integrated paths has to end at the point we are interested in. To make the stationary points of this unconstrained problem obey the constraints, we int roduce Lagrange multipliers $k$ that are in the dual space to the relaxed constraints. In the wave case, this asymptoticaily becomes the dual space of the tangent space at the point of observation (i.e. the cotangent space). We say that the variables $y$ and $k$ are canonically conjugate. Instead of the exponential just being of $i S / c$, it is of $i(S-\langle k, y\rangle) / c$. Here $S$ is a function of beth the initial and final endpoint of the paths. and the integral is over both. We use stationary phase and force the rrsult to apply to the point of iuterest by choosing $k$ so that the differential of the exponential vanishes at the desired point. This gives: $k=d S$, where this $S$ is a function unly of the observation point (the initial point integral already having been done). Thus we see chat $k$ is really the wavevector and the eikonal wave is naturally associated with the Lagrangian submanifold defined by $d S$ in the cotangent bundle of $y$-pare 
16.9.4. B) Lagrange Multipliera and Thermodynamic Conjugacy

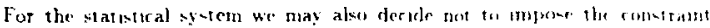

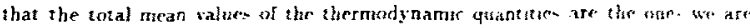
interested in. To make the thaximum entropy states th the warom-trand potslen ohey the constrants. we introduce Lagrange mulaplier $x$ that are in the duat

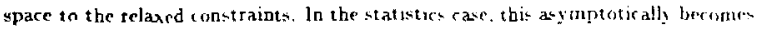
the dual space of the langent space of the space of extensive quantitic at the pont of observation (i.e. the cotangent space). We say that the variabirs $y$ and $X$ are thermodynamicaly coujugate Instead of the expotential just being of $5 / x$, it is of $\{S-(X, y)\}$. Here $S$ is a function of both the thermudyname quantitics of the Erst system and of the toral system, ard the integral is over bo.h. We use sterpest descrnts and force the result to have the total mean values of interest hy rhoosing $\mathrm{X}$ so that the differential of che exponential vanishes at the desired pont This gives $X=d S$, where this $S$ is only a function of the tot al mean waluet (the integra) over the values of the fist system alreaty havirg been done). Thus we set tlat $X$ is really the set of conjugate thermodynamic variables and the overall equ at iots of stete is naturally asociated with the Larangian subtranifold defined by ds in the cotangent bundle of $y$ space

\subsubsection{A) Fourier Transforms and Legendre Transforme}

In the wave cast, the effect of utilizing the Lagrange multipluer $f$ waw to antre

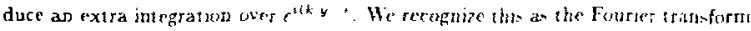

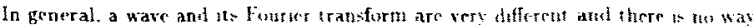


to define our wave an a function of both $k$ and $r$. We bave seen that asymptotw Ir all we may introduse the lural Fourier transorn as a function of $k$ and $y$ by

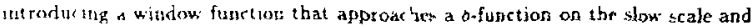
approarhes a commant in the fast wave scale. We find that approprately scaled

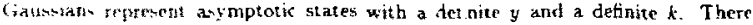
1: un al:tslufe uncertanty principle which prevents us from finding such states in $I$ and $k$ An eikona? wawe has a local Fourier transform that is supported on the Lagrangian submanifold $k=d S(y)$. The Fourier transform of an cikonal wave is anothey cikonal wave whase phasc function is the Legendre transform of the original phase function.

\subsubsection{B) Laplace Transforms and Legendre Tranoforms}

In the statigties case, the effect of utilizing the Lagrange multipliers $X$ was to introduce an mxtra integration over $e^{(X, v) / 4}$. We recognize this as the Lapiace transform. In general. a distribution and its Laplace transform are viry different and there is no way to define a probability distribution on both $x$ and $y$. We bave seen that asymptotically we may introduce the local Laplace transform as a function of $\boldsymbol{X}$ and $y$ by introducing a window function that is an intermediate scale exponential. We find that appropriately scaled Catussians represent asymptotic distributions with $x$ definte $X$ and a definite $y$. There is an absolute uncertainty principle that says that is anstotically the dispersion tensor in $\lambda$ and the dispersion teasor in $x$ are nfyrrses. A thermoryamic equation of state has a local Laplace transform that is sapported on the Lagrangiar su'manifold $\mathrm{X}=d S(y)$. The Laplare transform of a 


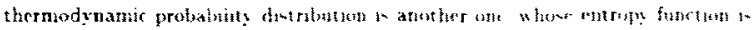

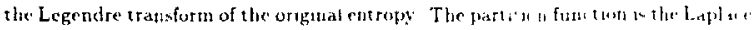

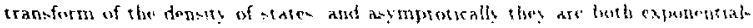
of quantities over' 


\section{Chapter 17: \\ Glossary of Terms}

itdiabat" invariaut

1.2.1. 2.9. 5.4

adjoint action

almost adiabatic invariant

5.4 .3

almost attractor

angular moment um generates rotations

asymptotic series

asymplotic stability

attractor

the method of averaging

$2.9 .2,2.9 .2 .1$

baker's transform 'on

basin of attractiou

BBGKY' hierarchy

2.9. $11.4,13.3 .4$

houndary layer

canonical one-form $\theta$ on a cotangent bundle

canonical transformations

Cantor set

Casimir functions

rentral linit theorem

cont rifugal force 
chaos

characteristi directuts:

ritrir articts

$\because 3$

roadjoint actuon

$2 \div 3.274$

coadjoint orbit:

conormal bundle

$7 \div 4.10$

contact elemeats

723.16

coutact form, $\boldsymbol{\theta}$, .

contart structure

contaci iransformation

convex function

cotangent bundle. $T \cdot M^{\prime}$

cotangent space. $T_{*}^{*}$ HF

covector.

critical point

deusity of stafe

deriative of a map. Tf

2.2 .8

diffeomorphisw. I

differential of a function

drift tangent bundle:

Duffing osrillator

dynamiral sistem 
E. 3 +3 5

rikmal मate

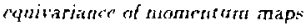

Euter : rugu body equatuons:

Finter: ffutd requatorn-

Fuler-Lagrazge equat'sin

Feynman path integral

firtitioum forres

first jet bundIe. $J^{1}$ M

frst order $P D E$.

flow. $\xi_{\text {f }}$

fuctuation

germs of ratbs. (;:M

graph of closed one-form is Lagrangian submanifold

f5romotion

Hamileonian, $H$

Hamiltonian vector feld, $x_{H}^{*}$

Hamiltonian, symmetry

Hamilton-Jarobi tbeory

hotsestone

14. 14.3

hyperplane

hypersurfare

inf rmatum 
intergrable

Istrg spin roode'

iturated tangent bundle. $T^{j} M$

Jacobi's identity

jet space Hamiltonian. $H$

jet space Poiskon brackef $\{$ I J,

jets of pat:s, JM

KA.M tori

Kirillov-Kostant-Souriou (KKS) ssmplecti structure

Koopmagism

Kruskal's perturbation theory

Lagrange multipliers 16

Lagrangias submanifold

Landau damping

Legendre map 16

Legendre submanifold

Legendre transform

Liapunov stability

Lie algebras

Lje bracket .

Lie groups

Lie-Poisson brackets .

Lie transform 
ma vimally won-integrable

non-singular perturbation thcory

non-uniform asyuptotic expansion

one-form

orcillatory stabilization

parametric resonance

partition function

path group, $P G$

patb -pace, $P_{1} M^{\prime}$

path-space Hamiltonian. $\tilde{H}$

4.3.1. 4.6 .1

nath-space symplectic strurture.

phase transitions.

Poincaré recurrence theorem 
Pointaré retusn map

Poincare st theoren

Poisson bracket, $\{$.

Poisson manifold

ponderontotive potestials

projective duality

projective space

pseutio-forces

pseudo-potenticls

reduced Hamiltoniah

reduced phase space.

reduced Poisson bracke:

reduction

reinsertion in area-preserving horseshoes

14.5

renormalization

resonance

$10.1 .2,13.3 .5$

Riemann-Lebesgue lemma

rings

roto-rate vector field

ruled surface

semi-direct product

simple me hanical systems

singular or seculas pe returbation theres 
space of loops

stable, iforences

stable manifold

statjonary phase

stcepest descents

Stirling

Stosszahlansatz 12

strange at tractor

sufficient statistics

symbolic dynamics

14. 1

symplectir bones

strmplectic jeaves

symplectic manufold

symplectic stricture, $\omega$

symplectomorpbisms

2.7.10, 3.2.1

tangent bundle, TM

tangent map $T f$ of $f$

tangent space, $T_{2} M$

$t_{n} \in n t$ vector, $t$

2.2.6.

triple point I6

two-timing

uncertainty princjple unkable manifolc . 
varation of parameter

vertor beld along a Ind

vertax of a comvex region

1041

uavefront set. . .

Whit'an s methos

7113

Windor function. $\mathrm{H}$; (y)

712

WhB theory , . . . . . . . . . 


\section{Chapter 18:}

\section{Bibliography}

[Abraham and Marsden, 1978]

R. Abrahan. ard J. E. Marsden, Foundations of Mechanics, 2nd edition, Ben. 4 min/Cummings, Reading, Mass., 1978.

[A braham, Marsden, and Ratiu, 1983]

R. Abraham, J. E. Marsden, and T. Ratiu, Manifolds, Teusor Analysis, and Applications, Addison-Wesley Publishing Company, Inc., Reading, Mass., 1983.

[Arnold, 1965]

v. 1. Arnold, "Sur les propriétées topologiques des applications globalement canoniques de la méchanique classique’. C. R. Acad. Sci. Paris, Paris, 1965.

\section{[Arnold, 1966]}

V. Arnold, "Sur la geometrie differentie!le des groupes de Lie de dimension infinie et ses applications a lh́ydrodynamique des tuids parfaits". Ann. Inst. Fourier Grenoble 16(1) (1966) 319-361.

[Arnold and Avez, 1968]

V. I. Arnold and A. Avez. Ergodic Problems of Classical Mechanics. W. A. Benjarnin. Inr.. New York. 1968. 
[Arnold, 1978]

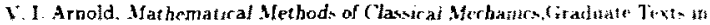
Math. vol, 60. \$, singer. Verlag, Berlin and .icu York. 1978

\section{[Aruold, 1983]}

V. Arnold, Geometrical Methods in the Theory of Ordinary Differential Equa. tions. Springer-Verlag. Yew York. 1983.

\section{[Arnold, 1984]}

v. I. Arnold, Catastropbe Theory, Springer-Yeriag. Berlin, 984.

[Banyaga, 1977]

A. Banyaga, "Furmes volumes sur les variétés à bord". Enscign. Math 20 . (1977), p. 127-131.

\section{[Birkhof, 1913]}

G. D. Birkhof, "Prool of Poincarés geometric theorem", Trans. Amer. Math. Soc. 14. (1913), p. 14-22.

\section{[Borp and Wolf, 1970]}

M. Born and E. Wolf. Pridciples or Optics, fourth edition. Permagmon Prow. Oxford, 1970.

\section{[Brush, 1983]}

S. G. Brush. Statstiral Physics azd the Atomir Theory of Water. from Buth

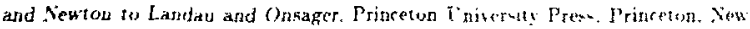
Jerwy. $198 \%$ 
[Burke, 1980]

II L Burke, Spacetine, Ceometry (\%osmology Viniversity Science Books. Mill Valley. California. 1980

[Chaperon, 1983]

$\$$ Chaperon, "A tbeorem on Lagrangian Intersections", preprint, École Poly* technique. Palaiscau (1983).

[Cary, 1981]

J. R. Cary. "Lic Transforn Perturbation Theory for Samiltonian Systems", Plyssics Reports 79 (198i), 131.

\section{[Chaprnan and Cowling, 1958]}

S. Chapman and T. Cowling. The Matbematical Theory of Nion-uniform Gases, Cambridge University Press, Cambridge, 1958.

\section{[Chernofi and Marsden, 1974]}

P. R. Chernof and J. E. Marsden, Properties of Infinite Dinensiogal Hamilto nian Systems, Lecture Notes in Math., vol. 425, Springer"Verlag. New York (1974).

[Conley and Zehnder, 1983]

C. C. Conley and E. Zebnder, "The Birkhoff-Lewis Exed point theorem and a conjerture of V. I. Annold", Jnventiones Math. 73 (1983), 33-49.

[Cornfeld, Fomin, and Sinai, 1982]

I. P. Conffeld, S. V. Fomin and Ya G. Sinai, Ergodir Theory. Springer-Veriag Sex York 1982 
[Courant, 1966!

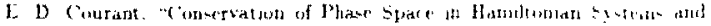

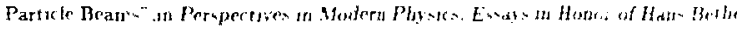

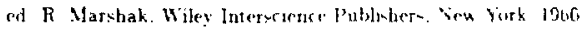

\section{[Crawforl and Omobundra, 1984]}

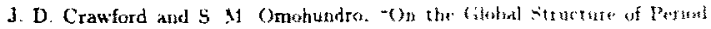
Doubling Flows", Physica 1301 \& 2, (1984). p. 161818.

\section{[de Bruijn, 1981]}

S. G. de Bruija. Asyruptotir Methods in Andtwis. Dower Publications Inc. New York. 1981.

\section{[Dewar, 1972a]}

R L. Dewar. "A Lagzangian Throry fos Nonlinear Mace Parketz in a Collisiontess Plasma". J. Plasma P'bysic: 7. (1972) pp. 267-284.

\section{[Dewar, 1972h'}

R. L. Detar. "A Lagraggian Derivation of the Artin (onservation Theorem for Density Waves-. The Astrophysical Journal 174, (1972) pp. 301-307

[Dewas, 1977]

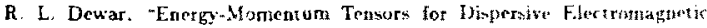
Wave:" Aust. J Phy 30. (1977)-pp. \$337-575.

[Dewar, 1983]

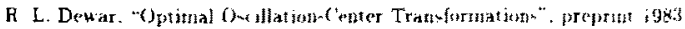


[Dirac, 1958]

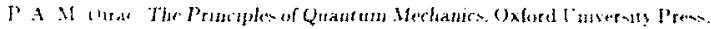
dyine

[Dirac, 1977]

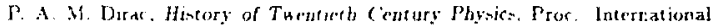

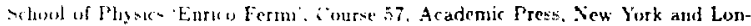
dor. 1977.

[Dubrovin and Novikov, 1983]

B. A. Dubrovin and S. F. Sovikov, "Hamiltonian formalism of one-dimensional systems of the hydrodynamic type and the Bogolyubov-Whitham averaging metthod". DokI. Akad. Nank SSSR 270, no. 4. p. 781-785. i983. English translation: Soviet Math. Dokl. 27, no. 3, p. 665-669. 1983.

[Faddeev and Zakharov, 1971]

L. Faddeev and t. E. Zakbarov, "Korteweg-de Vries ax a Completely Integrable Hamiltonian System", Funct. Anal. Appl. 5 (1971) 280.

[Fefferman, 1983]

C. L. Fefermas, "The Uncertainty Principle". Bulletin of the A merican Wathcmatical Socimty 9 (1983). 129206.

[Feynman, 1948]

R. P Feymutn, "Space-time approarh to non-relativistic quantum merhanics". Retich = of Modern Physies 20 (19.18) p.267. 
[Feynman, 1972]

R P. Feyman. Statistical Mechanics. A Set of Lectures. HA A Benjamu. inc.. Reading. Mass., 1972.

[Feynman and Hibbs, 1965]

R. P. Feynman and A. R. Hibbs, Quant nm Mechanics and Path Intcgrals, McGraw-Hill Book Company, Kew York, 1965.

[Freed and Uhlenbeck, 1984]

D. Freed and K. Ublenbeck, Instantons and Four-Manifolds, Mathernatical Sciences Research Instituce Publication 1, Springer-Verlag. Sew: York. 1984.

[Gibbons, Holm and Kupershmidt, i982]

J. Gibbons, D. D. Holm, and B. Kupersbmidt, "Gauge-lnvariant Poisson Brack. ets for Chromobydrody damics", Physics Letters 90A (1982), 281283.

[Gibbs, 1873]

jW. Gibbs, "Graphical Methods in the Thermodynamies of Fluids". Transactions of the Connectic ut Academy II(1873), p 309-342. (ull. Wirks I, 1.32.

\section{[Gibbs, 1873b]}

J. W. Cibbs. "A Method of Gcometric al Repreent at inn of the Thermodynamic Properties of Substances by Meass of Surfaces" Tratsucturs of the cimmetur Academy II(1873), p 382-404, Coll Works 33-54 
[Gilmore, 1981]

R. Gilluure. Catastrophe Throry for Scientsts and Enguneers, John Wiley and Suns, Inc. New York. 1981.

[Grebogi and Littlejohn, 1984]

C. Grelngi and R. G. Littlejohn, "Relativistic Ponderomotive Hamiltonian", preprint, 1984.

[Grebogi, Ott, and Yorke, 1984]

C. Grebogi. E. Ott, and J. Yorke, "Strange Attractors Without Chaos", poster session. American Physical Society Division of Plasma Physics meeting, Boston, 1984.

[Guckenheimer and Holmeg, 1983]

J. Guckenheimer and P. Holmes, Nonlinear Oscillations, Dyoamical Systems, and Bifurcations of Vector Fields, Springer-Verlag, New York, 1983.

[Guillemin and Sternberg, 197\%]

V. Guillemin and S. Sternherg. Geometric Asymptotics, American Matbematieal Society, Providence, Rbode Islind, 1977.

[Hermann, 1968]

R. :"rrninn. Differential Geometry and the Calculus of lariations. Academic Press, Sicw Yrotk. 1;wi3 
[Guillemin and Sternbere, 1984]

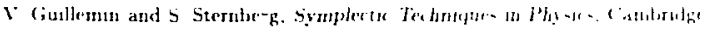
Inversity I'ress. Canbrugt. 198-1

[Hirscb, 1984]

M. W. Hirsch. -The Dynamical Systeme Approuth to Differental Fiquatum-". Bull. of Am. Matb. Soc 11 pps. 1-64

[Holm and Kupershmidt, 1984a]

D. Holm and B. Kupershmidt. "Poisson Brackets and Clebsch Representations for Sagnetohydradynamies. Multifluid Plasma., and Elasticity", Physica D.

[Holm and Kupershmidt, $1984 \mathrm{~b}$ '.

D. D Holm and B. A. Kupershmidt, "Poisson Structures of Superfuids". sub.nitted to Physics Letters A.

[Holn, Maraden, Ratiu, and Weinstein, 1984]

D. D. Holm. J. E. Marsden. T. Ratiu, and A Weirstein. "A Prion Estimates for Nonlinear Stability of Fluids and Planmas", to be published in Physics Reports. (1984).

¿Holmes and Whitley, 1984]

P. Holmes and D. Whitley, "Bifurcations of One and Two-Dinnemional Map" Preprint 1984. 
[Iagolnitzer, 1975]

D lagolunases "Mlerolocal emential support of a dotribution and deromposj-

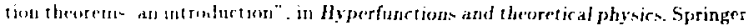
Lorture Sot th In Math. 419. 1975. pps. 121-132

[Hörmander, 1983]

L. Hörmauder. The Analysis of Linear Partial Differential Jperators I, Springer-lerlag. Berlin, 1983.

[Israel, 1979]

R. Isracl, Convexity in the Theory of Lattice Gases. Princeton University Press, Princeton, New Jersey. 1979.

[Jaynea, 1983]

E.T. Jayres, Papers on probability, statistics and statistical physics, ed. by R. D. Rosenkrantz, D. Reidel Publishing Company, Boston. 1983.

\section{[Jenner, 1983]}

W. E. Jenner, Rudiments of Algebraic Geometry, Oxford University Press, Inc., New York, 1963.

[Kac, 1959]

M. Kac, Probability and Related Topics in Pbysical Sciences. Lectures in Applied Mathematics. Volume 1A. American Mathematical Society. Providence. Rhode Island, 1959. 
[Kaufman, Cary, and Pereira, 1979]

A I haufman, I R. Cary, and X. R. Percera. "I niveral Furmula fur Qua-1.

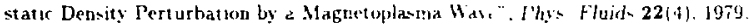
p. 790 .

[K aufman, 1979]

A. N. Kaufman, Regular and Stocha atic Particle Motion in Plasma Dyuamics". in The Proceedings of the International Worksbop on Intrinsir Srochichtirity in Plasmas, Cargese. Corsica. France, 1979.

[Kaufman, 1982]

A. X. Yaufman. "Elementary Derivation of Poisson Structures for Fluid Dy. namics and Electrodynamics", Phys. Fluids 25(1,). 199.. p. 1993.

[Kaufman and Holm, 1984]

A. K. Kaufman and D. D. Holm. "Thr Lie-Transformed Vlasov Action P. 'nc1ple: Relativistically Covariant Wave Pzopagation and Self-Conststent Pondromo tive Effects", Physics Letters 105 A \#G, (1984) p277-279.

[Kaufman end Boghosian, 1984]

A. X. Kaufman and B M. Boghosian. "Lite-transirm Derivation of the (ri-

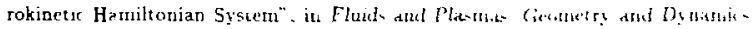
J. Marsden Ed.. American Mathematical Soriets Pturdence. Rl :98.4

[Kaufmsn, 1984]

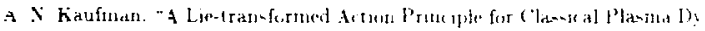




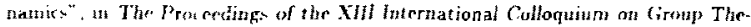
uretical Methods If J'hystes, (Nolle'ge' Park. MD. 1984.

[Keller, 1958]

J B Keller. Ant. Physics 4 (1958) p. 180. 188.

[Kevorkian and Cole, 1981]

J. Kevorkjan and J. D. Cole. Perturbation Methods in Applied Mathematics, Springer-Yerlag, New York. $199,1$.

[Kbincbin, 1949]

A. I. Khinchit, Matbematucal Foundations of Statistical Mechanics. Dover Publications. Inc., New York. 1919.

[Kijuwski and Tulczyjew, 1979]

J. Kiinowsi and $W$. Tulrzyjew, A symplectic framework for field theories, Lecture Notes in Physics 107. Springer, Sew York, 1979.

[Klein, 1928]

F. Klein. Voriesungen ub - die Eutricklung der Mathematik im 19 Jahrhundert. Springer-Verlag. Berlin, 1928, translation Development of Mathernatics in the 19th Crntury by Af Ackerman. Math Sci Press. Brouk!ne. Massarhusetts 1979.

[K ruskal, 1982]

11 Kruskal. "Anymptotic theory of Hamhonian and other systema with all uhutwit nearly permdic". J. Math Phys. 3. (1962) 806-828 
[Kubo et al., 1985]

R. Kubo. H. Ichimura, T. Usui, N. Hashitsume, Statistical Mechanics, NorthHolland, Amsterdam, 1965.

[Lagrange, 1808]

J. L. Lagtange, uMémoire sur la théorie des variations de éléments des plan'etes", Mémoires de la classe des sciences mathématiques et physiques de l'instiut de France (1808), pp. 1-72.

[Landau and Lifhitx, 1980-1981]

L. D. Laudau and E. M. Lifshitz, Course of Theoretical Physics, Volumes 1-10, Pergamon Press Ltd., New York (1960-1981).

[Littlejohn, 1079]

R. G. Littlejohn, "A guiding center Hamiltonian- A new approach", J. Math. Phys. 20(12) (1979) p2445-2458.

\section{[Littlejohn, 1981]}

R. G. Littlejobn, "Hamiltonian Formulation of Guiding Center Motion", Phys. Fluids. 24 (1981) p1730-1749.

[Littlejohn, 1983]

R. G. Littlejobn, "Variational Principles of Guiding Centre Motion". J. Plasma Physics 29 (1983) 111. 
[Littlejohn, 1983]

R. G. Littlejohn, "Geometry and Guiding Center Motion", in Fluids and Plasmas, Geometry and Dynamics, J. Marsden Ed., American Mathematical Society, Providence, RI, 1983.

[Mackey, 1978]

G. W. Mackey, Unitary Group Representations in Physics, Probability, and Number Theory, Beojamin/Cummings, Reading, Massachusetts, 1978.

[Mersden, 1981]

J. E. Marsden, Lectures on Geometric Methods in Mathematical Physics, CBMS-NSF Regional Conference Series 37, Philadelphia, PA:SIAM, 1981.

\section{[Marsden and Hughes, 1983]}

J. E. Marsden and T. J. R. Hughes, Mathematical Foundations of Elasticity, Prentice-Hall, Englewood Clifs, New Jersey, 1983.

[Marsden, Ratiu, and Weinstein, 1984]

J. E. Marsden, T. Rati' ', and A. Weinstein, "Semi-direct Products and Reduction in Mechanics", Transactions of the American Mathernatical Society 281 (1984), pp. 147-177.

[Maraden and Weinatein, 1974]

J. Marsden and A. Weinstein, "Reduction of symplectic manifolds with symmetry", Rep. Math. Phys. 5 (1974) 121-130. 
[Mareden end Welnstein, 1982]

J. Mareden and A. Weinstein, "The Hamiltonian Structure of the MaxwellVlasov Equations", Physica 4 (1982), 394-406.

[Maraden and Weinstein, 1043]

J. E. Maraden and A. Weinstein, "Coudjoint Orbits, Vortices, and Clebsch Variablea for Incompresaible Fluids", Physica 7D (1983), 315-323.

[Marion, 18as]

V. P. Masior, Theory of Perturbations and Asymptotic Method, Izdat. Motror. Gos. Univ., Moscow 1965; French tranol., Dunod, Paris, 1972.

[Maslov, 1970]

V. P. Mulov, Operationd Method, Mir Publishers, Moncow, 1976.

[Mioner, Thorne, and Fheler, 197s]

C. Misner, K. Thorne, and J. Wheeler, Gravitation, Freeman, San Francisco, 1973

[Morrieon, 1960]

P. J. Morrieon, "The Mexwell-Vlasov Equations as a Continuous Hamiltonian System", Phys. Lett. 60 A (1980), 393-396.

[Morrion and Greene, 1980]

P. J. Morrison and J. M. Greene, "Noncanonical Hamiltonian Density Formulation of Fydrodynas ics and Ideal Magnetohydrodynamics", Phys. Rev. Letters 4 (1980), 790-791. 
[Nayfeh, 197s]

A. H. Nayfeb, Perturbation Methods, Jobn Wiley and Sons, lnc., New York, 1973.

[Noether, 1018]

E. Noether, "Iovariante Variationsprobleme", Nachrichten kgl. Geell. Wissenachaft. Göttingen, Matb. Phys. Kl. 2 (1918), pp. 235-257

[Northrop, 196s]

T. G. Northrop, The Adisbatic Motion of Charned Particles, John Wiley and Sons, lnc, New York, 1963.

[Olver, 1984]

P. Olver, "Hemiltonian perturbation theory and water waver", in J. Meraden, ed., Fluids and Plasmas: Geometry and Dynamics, American Mathemutical Society series in Contemporsy Mathematich, Volume 28,1984, p. 231.

[Omohundro, 1094a]

S. M. Omohundso, "Hamiltonien Structures in Perturbation Theory", to be submitted to the Journal of Mathematical Physjco (1984).

[Omohundro, 1984b]

S. M. Omohundro, "Geometric Hamiltonian Struetures and Perturbation Theory" in Lecture Notes in Physics volume on Local and Global Methods in Nonlinear Dyzamics, ed. B. Cawley, Springer-Verlag, New York (1964). 
[Omohundro, 1904c]

S. M. Omohundro, "A Hamiltonian Approach to Wave Modulation", Poster presented at April 1984 Sherwood Plasma Theory Meeting, Lake Tahoc, in preparation for publication.

- [Omohundro, 19o4d]

S. M. Omohundro, "Modelling Cellular Automata with Partial Differential Equation" ${ }^{n}$ Physica 10D (1984) 128-134.

[Omohundro, 1964e]

S. M. Omohundro, "Book Review of The Lorenz Equations: Bifurcations, Cheos, and Strange Attrectors by C. Sperrown ${ }^{n}$, Mathematical Biasciences 68 (1984) p. 303-304.

[Ott, 1979]

E. Ott, "Goodneso of Ergodic Adiabatic Invariants", Physical Review Letters 12 (1979) p.1628-1631.

[Palnin, 1901]

R. S. Palaig, "Equivalence of nearby differentiable actions of a compact group", B.all. Amer. Math. Soc. o7, p. 362-364, 1961

\section{Paull, 1053]}

W. Pauli, General Principles of Quantum Mechanies, (1933) Reprinted in English Translation by Springer-Verlag, Berlin, 1981. 
[Polncaré, 1892]

H. Poincaré, Les méthodes nouvelles de la mécanique céleste, 1,2,3. GauthierVillars, Paris (1892); Dover, New York, 1957.

[Polncaré, 1012]

H. Poineare, "Sur un thtorème de gtométrie", Rend. Circ. Math. Palermo 3S(1912), 375-407.

[Poston and Stewart, 1078]

T. Poston and I. Stewart, Catastrophe Theory and its Applications, Pitman, Boston, 1978.

[Schulmen, 198t]

L. Schulman, Techniques and Applications of Path Integration, John Wiley and Sons, Inc., New York, 1981.

[Shannod, 1048]

C. E. Shannon, "A Mathematical Theory of Communication", The Bell System Technical Journd 27, (1948),p. 379.

[Bimon, 1070]

B. Simon, Functional Integration and Quantum Physics, Academic Press, New York, 1979.

[Smale, 1907]

S. Smale, "Differential Dynamical Systems", Buletin of the American Mathematical Society 73, (1967), pp. 747-817, reprinted in: S. Smale, The Mathematics 
of Time: Evays on Dyoumical Syatems, Econowic Processes, and Related Topics, Springer-Verlas, New York, 1980.

[Smith and Kaufmen, 1975]

G. R. Smith and A. N. Kaufman, "Stochastic Acceleration by a Single Wave in a Magnetic Field", Physical Review Letters 84 \#26 (1975) 1613-1616.

[Bmilh and Kaufman, 1978]

G. R. Smith and A. N. Kaufman, "Stochastic zeceleration by an obliquely proparating wave- An example of overlapping resc dences", Phys. Fludd 21(12), 1978, p. 2230.

[Sourlau, 1970e]

J-M. Souriau, Structure des Syst'emes Dynamiques, Dunod, Paris, 1970.

[Sourlau, 1970b]

J+M. Souriau, "Mbcanique Statiatique, Groupes de Lie et Cosmologie", in ColIoques Internationaux C.N.R.S., No. 237. Gtometrie symplectique et physique matbematique p. 59 .

[Spencer and Kaufman, 1082]

R. G. Spencer and A. N. Kaufman, "Eamitonian Structure of Two-Fluid Plasma Dynamics", Phys. Rev. A az (1982) 2437-2439.

\section{[Spival, 1079]}

M. Spivak, A Comprehensive Iotroduction to Differential Geometry, Volume One, Second Edition, Publish or Perish, lnc., Berkeley, 1979. 
[Stoer and Burlirab, 1980]

J. Stoer and R. Burbirsh, Introduction to Numerical Analysis, Springer-Verlag, New York, 1980.

[Taylor, 1981]

M. E. Taylor, Pseudodifferential Operators, Princeton University Preso, Princeton, New Jersey, 1981.

[Thirring, 1978]

W. Thiring, A Course in Mathematical Physics, I: Clasoical Dyanmios, Springer-Verlas, New York, 1978.

[Tikochinaky, Thbby, and Levles, 19e4]

Y. Tikochinaky, N. Z. Tishby, and R. D. Levine, "Alternative Approacb to Maximum-entropy Inference", Physical Review A 30, (1994), pp, 2638-2644.

[Thes, 1806]

L. Tiaza, Geperalied Thermodyanmics, The M.I.T. Prean, Cambridse, Ma sachusetts, 1906.

[ven Kampen, 1903]

N. van Kampen, "Constraints", preprint, Murch 1983.

[Wex, 1054]

N. Wax, editor, Selected Papers on Noise and Stochastic Procemer, Dower Publications, New York, 1954. 
[Welnutein, 1971]

A. Weinstein, "Symplectic Manifolds and their lagrangian submanifolds", Advances in Math. (1971), 329-346.

[Weinutein, 1977]

A. Weinotein, Lectures on Symplectic Manifolds, Regional Conferences in Math. 20, American Matb. Society (1977).

[Woinatein, 1978]

A. Weinstein, "The Order and Symbol of a Distribution", Transactions of the American Mathematical Saciety, 241 (1978) p.1-54.

\section{[Woinuteln, 19a1]}

A. Weinstein, "Symplectic Geometry", Bulletin of the American Mathematical Society of (1981), 1-13.

[Weinatein, 19ssa]

A. Weinstein, "The Local Structure of Poisson Manifolds", J. Differential Geometry 18 (1983) 523-557.

[Weinutein, 1983b]

A. Weinstein, "Sophus Lie and symplectic geometry", Expo. Math. 1(1983) 95-96.

[Weinutein, 1983e]

A. Weinstein, " $C^{0}$ perturbation theorems for symplectic fixed points and lagrangian intersections", Preprint, Univ. of California, Berkeley (1983). 
[Weinatein, 1986a]

A. Weinstein, "Critical Point Theory, Symplectic Geometry, and Hamilkonian Systems", Preprint, Univ, of California, Berkeley (1984).

[Weinntein, 1984b]

A. Weinstein, notes by S. M. Omohundro, "Equations of Plasma Physics", p. 359 in Seminar on Nonlinear Partial Differential Equations, edited by S.'S. Chern, Matberatical Sciences Research Institute Publication 2, Springer-Verlag, 1984.

[Whitham, 1974]

G. Whitham, Linear aod Nonlinear Waves, Joha Wiley \& Sons, Inc., New York, 1974.

[Wileon and Kogut, 1974]

K. G. Wilson and J. Kogut, "The Renormalization Group and the e Expansion", Pbysics Reports 12 No. 2 (1974) p. 75-200.

\section{[Wolfram, 1984]}

S. Wolfam, "Undecidability and Intractability in Theoretical Physics", Pre print 1984.

[Yafie, 1082]

L. G. Yaffe, "Large N Limito as Classical Mer'anics", Reviews of Modern Physics 54 No. 2 (1982) p. $407-435$. 